\title{
THE EFFECTS OF A SIX-WEEK PHYSIOTHERAPIST-LED EXERCISE AND EDUCATION INTERVENTION IN PATIENTS WITH OSTEOARTHRITIS, AWAITING AN ARTHROPLASTY IN SOUTH AFRICA
}

\author{
Melissa Michelle Saw \\ SWXMEL001
}

SUBMITTED TO THE UNIVERSITY OF CAPE TOWN in fulfilment of the requirements for the degree

MSC PHYSIOTHERAPY BY DISSERTATION

Faculty of Health Sciences

UNIVERSITY OF CAPE TOWN

January 2015

Supervisor: A/Professor Romy Parker

Co-supervisor: Mrs Naila Edries

Division of Physiotherapy, Department of Health \& Rehabilitation Sciences, University of Cape Town 
The copyright of this thesis vests in the author. No quotation from it or information derived from it is to be published without full acknowledgement of the source. The thesis is to be used for private study or noncommercial research purposes only.

Published by the University of Cape Town (UCT) in terms of the non-exclusive license granted to UCT by the author. 



\section{Declaration}

I, Melissa Saw, hereby declare that the work on which this thesis is based is my original work (except where acknowledgements indicate otherwise) and have used the Vancouver system of referencing. I declare that neither the whole work nor part of it has been, is being, or is to be submitted for another degree at the University of Cape Town or any other university.

I empower the University of Cape Town to reproduce for the purpose of research either the whole or any portion of the contents in any manner whatsoever.

\section{Signed by candidate \\ Signature: Signature Removed}

Date: $\underline{28-01-2015}$ 


\section{Acknowledgements}

Firstly, above all, I acknowledge my God for the gift of intelligence and for continually building up my character of faith,perseverance and humility.

To my supervisor, Associate Professor Romy Parker, for your constant support, encouragement and critique throughoutthis degree. For always guiding me towards the solution and grooming me into the physiotherapist and researcher I am today. For allowing me to realise the privilege of performing clinical research and impacting lives for the better. You are my inspiration.

To my co-supervisor, Mrs Naila Edries, for your valuable input and advice during this degree.

To my loving mother and father for always challenging me to be and do more. For willingly assisting whenever I asked for help. For instilling in me the confidence that I can achieve what I set out for myself and for always being the proud parents you are.

To my incredible boyfriend, Triegaardt, for your ongoing motivation and unfailing belief in me. For your faithful prayers and making any challenge possible by being by my side. For the laughs that maintained my sanity and simply for your love.

To my amazing friends for their keen interest, support and upliftment from beginning to end. Especially to my daily MSc followers, Susan and Lara, for your unending enthusiasm and for celebrating each step of the way.

To Shayne Fourie, the research assistance, for your valuable work towards this study.

To the Tygerberg orthopaedic and physiotherapy departments for making the study possible.

To the South African Society of Physiotherapy for the funding received during this study.

Lastly, to the patients who were involved in this study who gave of their time and who eagerly returned each week hungry for more. For touching my heart and fuelling my passion for physiotherapy and research. 


\section{Abstract}

The effects of a six-week physiotherapist-led exercise and education intervention in patients with osteoarthritis, awaiting an arthroplasty, in South Africa: a randomised controlled trial.

\section{Author: Melissa Michelle Saw}

Background: Osteoarthritis $(O A)$ is one of the leading causes of disability worldwide. A major challenge facing those with severe $O A$ is long waiting lists delaying access to joint replacements. Patients are known to wait more than five years for a joint replacement in the Western Cape of South Africa (SA). The main complaint in this population is pain and its consequences including activity limitations, participation restrictions and reduced quality of life. Hip or knee $O A$ is not merely joint degeneration but a condition requiring holistic management, even while waiting for surgery. Most of the literature in this field is available from high income countries exploring the effects of interventions during short waiting periods. Thus research is warranted in a low income country such as SA, in those waiting for long periods to explore the effects of a six-week physiotherapist-led exercise and education intervention.

Methods: A single blinded randomised controlled trial, aligned with CONSORT guidelines, was performed at Tygerberg Hospital in the Western Cape, SA. The experimental group attended a six-week group-based physiotherapist-led intervention including education, exercise and relaxation. The control group continued to receive usual care. The primary outcome measure was pain with secondary measures of disability, function, quality of life and self-efficacy. Measures were obtained at six weeks, 12 weeks and six months by a blinded physiotherapist. An open ended questionnaire was completed by the participants in the experimental group at month six. Analysis was by intention to treat. Two-way analysis of variance and post-hoc Tukey comparisons were used for parametric data, Pearson Chi squared calculations for categorical data. Effect sizes were established for significant differences between groups.

Results: The study recruited 42 participants from the waiting list for a hip or knee arthroplasty. Mean waiting time was $3.6 \pm 2.5$ years. 
Compared to the control group, the experimental group had significant improvements with large effect sizes at month six for pain interference $(3.49 \pm 2.63$ vs. $6.09 \pm 2.43 ; p=0.02$, $E S=1.15)$ and function ( $15 \mathrm{~m}$ fastest speed walk) $(15.09 \pm 6.04 \mathrm{~s}$ vs $20.10 \pm 8.79 \mathrm{~s} ; \mathrm{p}=0.03$, $E S=0.88)$. Furthermore, the experimental group displayed significant $(p<0.01)$ and sustained improvements at month six in pain severity, disability and function (15m normal speed walk, sit-stand, 6-minute walk). Subgroup analysis showed participants with knee OA responded better to the intervention than those with $\mathrm{OA}$ of the hip or combined hip and knee OA. Participants enjoyed the intervention reporting improved knowledge, function and activity, pain relief and improvement in psychosocial aspects.

Conclusions: A six-week physiotherapist-led exercise and education intervention brought about significant long term improvements in pain interference and functional walking ability in patients with osteoarthritis, awaiting a joint replacement compared with a control group. Such a programme also appears to have significant and sustained improvements in pain severity and disability. Further research with longer follow up is recommended to determine if results are sustained.

Keywords: Hip/knee osteoarthritis; arthroplasty/joint replacement; waiting list; physiotherapy; exercise; education; chronic pain.

Ethical approval: University of Cape Town Faculty of Heath Sciences Human Resource Ethics Committee: Ref 378-2013.

Funding: Received from South African Society of Physiotherapy 


\section{Table of Contents}

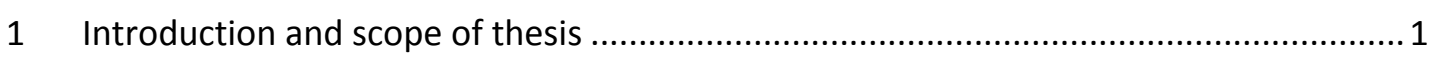

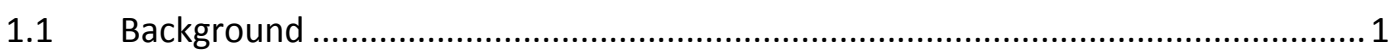

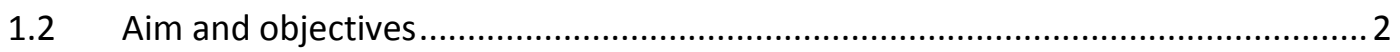

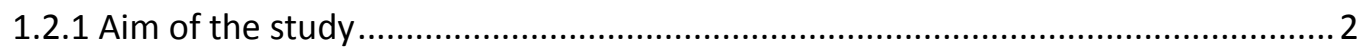

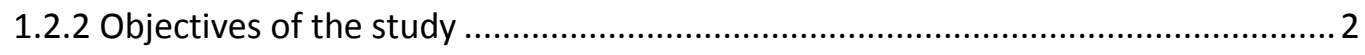

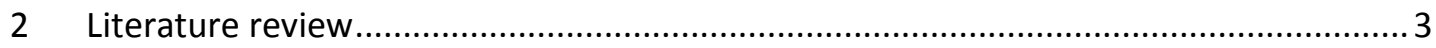

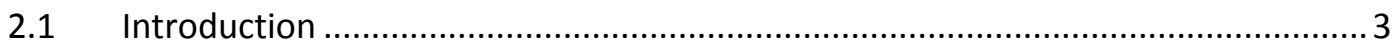

2.1.1 International Classification of Functioning, Disability and Health - a theoretical

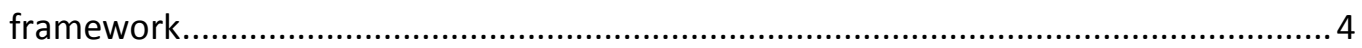

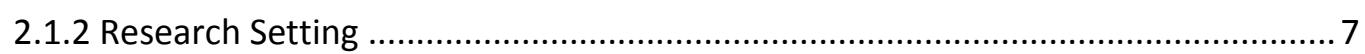

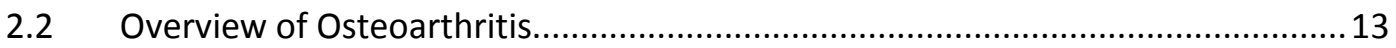

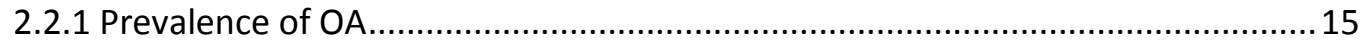

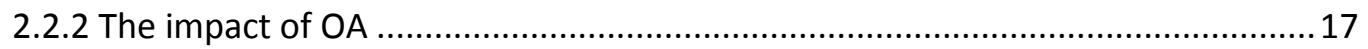

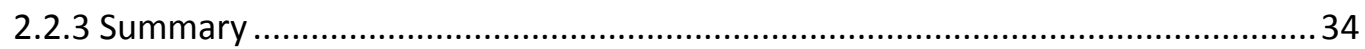

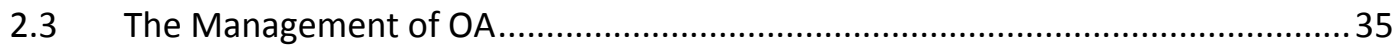

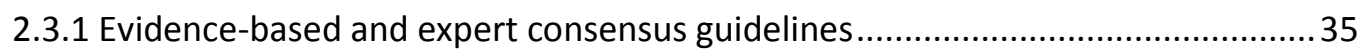

2.3.2 Summary of guidelines - The gold standard of management..............................47

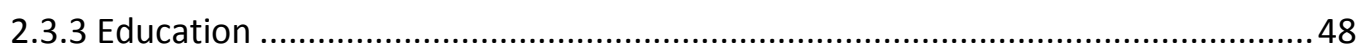

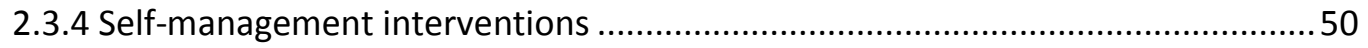

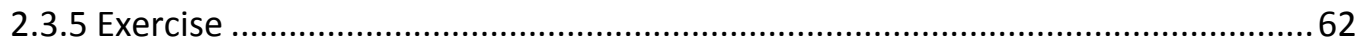

2.3.6 Combined Intervention: self-management intervention and exercise ................69

2.3.7 Relaxation therapy - guided imagery and progressive muscle relaxation ............76

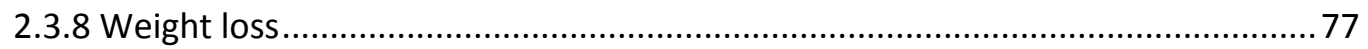

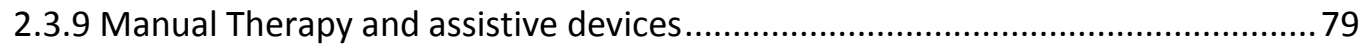

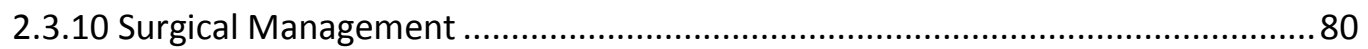

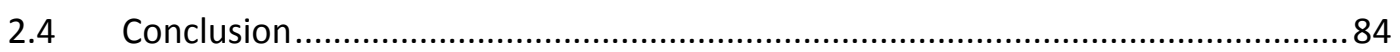

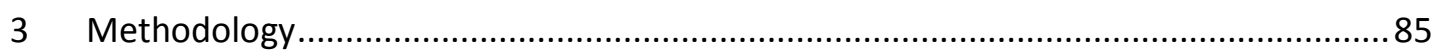

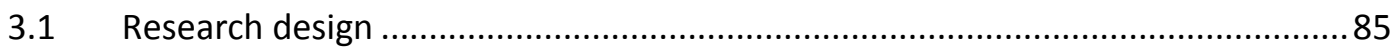

3.2 Sample

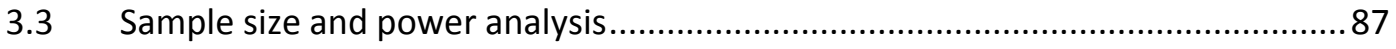

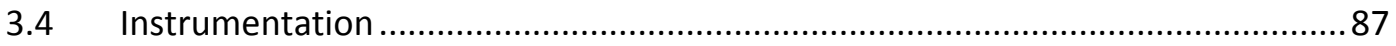

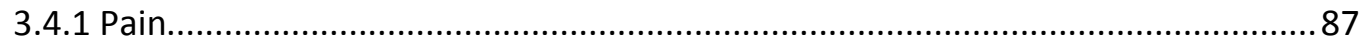




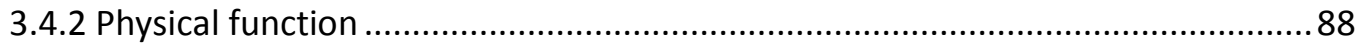

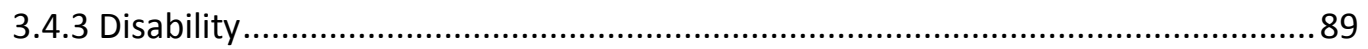

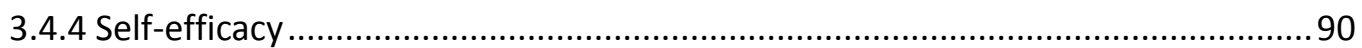

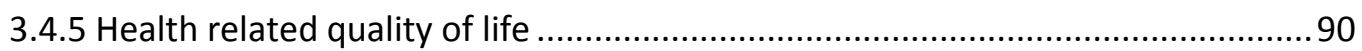

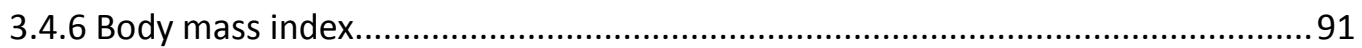

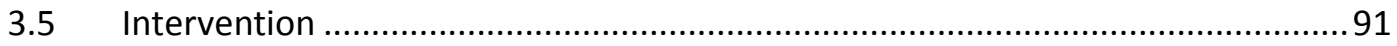

3.6 Procedure

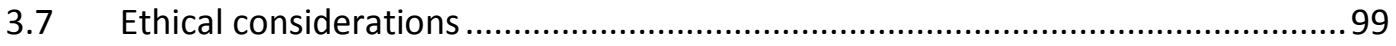

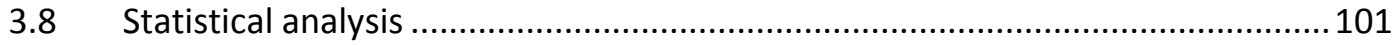

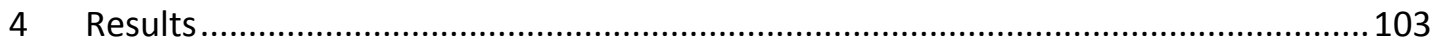

4.1 Sample

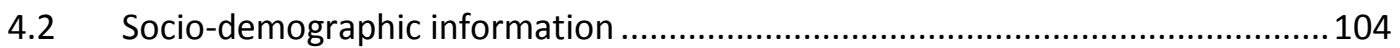

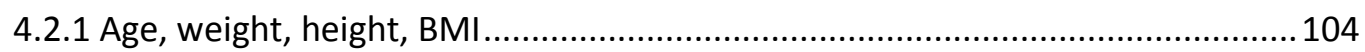

4.2.2 Gender, language, education level, employment status ................................. 105

4.2.3 Clinical information: time spent on the waiting list, co-morbidities, joint/s affected, assistive device used, medication used .....................................................106

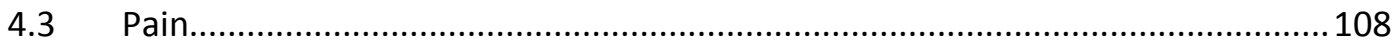

4.3.1 Pain Severity Score (PSS) and Pain Interference Score (PIS)............................. 108

4.3.2 Change in percentage pain relief from medication over time.............................116

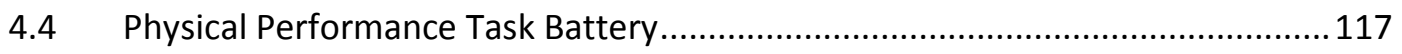

4.4.1 15 meter walk tests: fastest and normal speed..............................................117

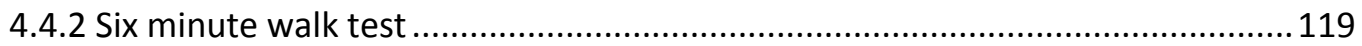

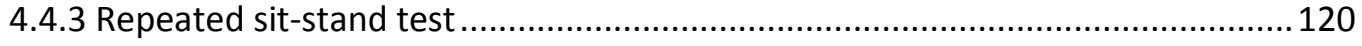

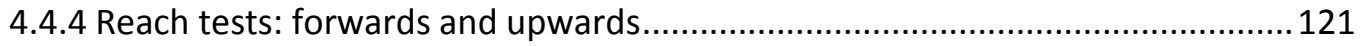

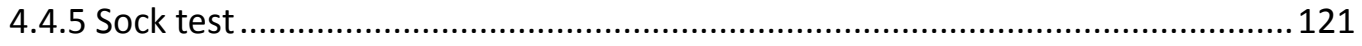

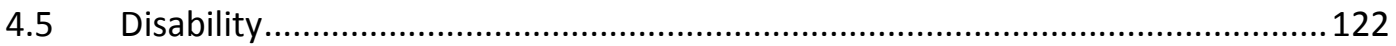

4.5.1 Health Assessment Questionnaire score ........................................................ 122

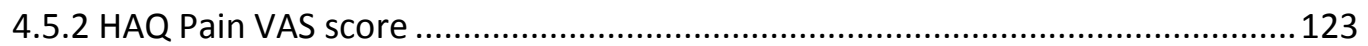

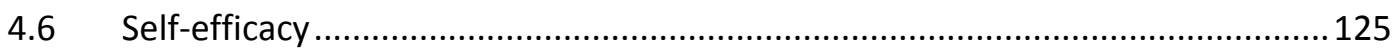

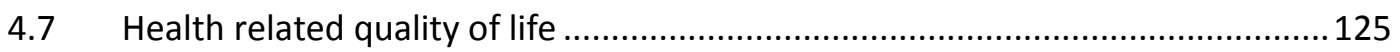

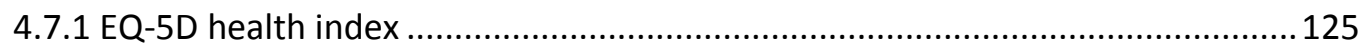

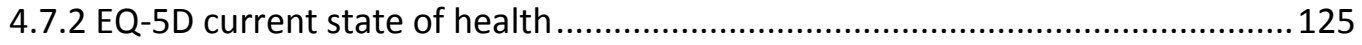

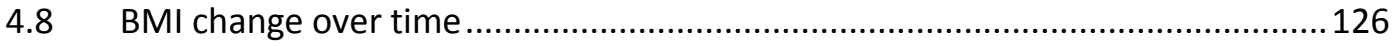

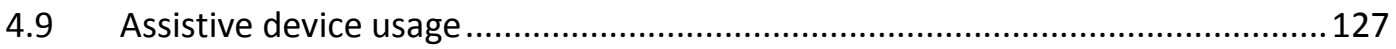




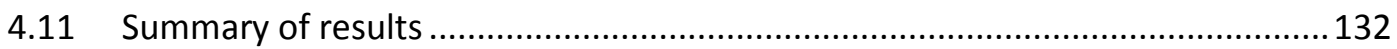

4.11.1 Primary outcome measure: pain as measured by the BPI............................ 132

4.11.2 Secondary outcome measures......................................................... 132

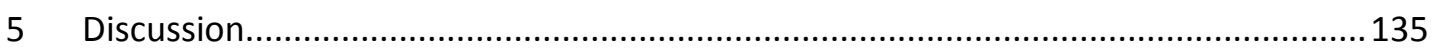

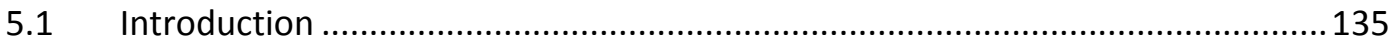

5.2 Primary outcome measure: pain (BPI) ............................................... 135

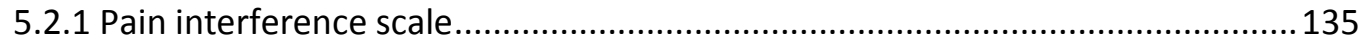

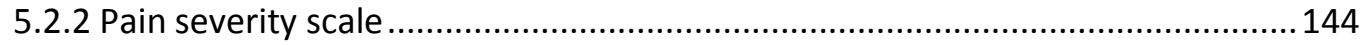

5.3 Secondary outcome measures: function (Physical performance task battery) ... 150

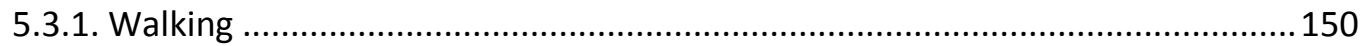

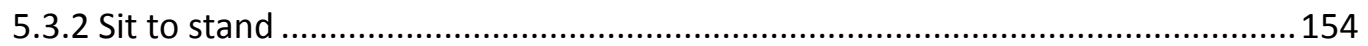

5.4 Secondary outcome: disability (HAQ) .................................................... 158

5.4.1 Baseline disability presentation of the sample ......................................... 158

5.5 Secondary outcome: personal factors (SE and HRQoL) ............................... 165

5.5.1 Baseline presentation of the sample in terms of personal aspects.................. 165

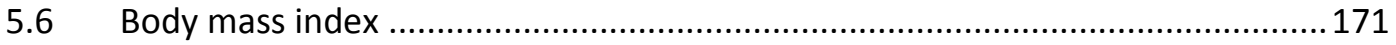

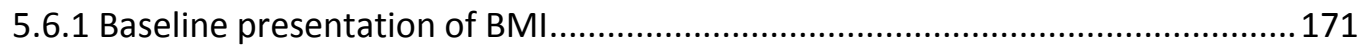

5.7 Open-ended questionnaire responses..................................................... 172

5.8 Strengths and limitations of the study................................................. 173

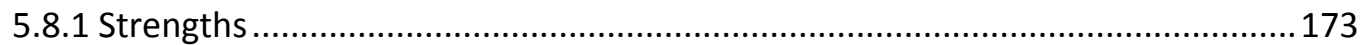

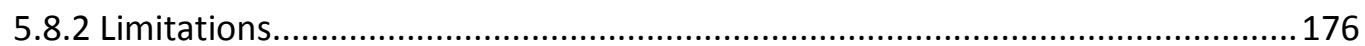

$5.9 \quad$ Further recommendations ................................................................... 179

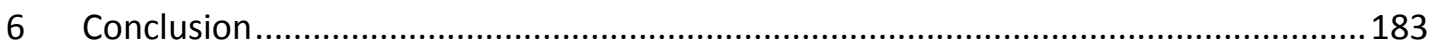

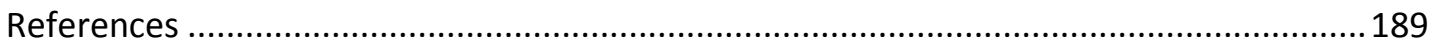

Appendix A: ESCEO algorithm for the management of knee OA...................................207

Appendix B: Ethical approval letters................................................................... 209

Letter of approval from the University of Cape Town's Faculty of Health Sciences, Human

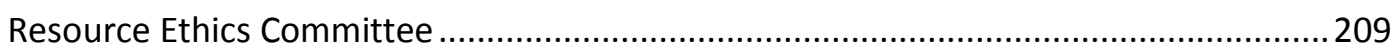

Letter of approval from Tygerberg Hospital's management of research projects ......... 210

UCT HREC amendment approval for the additional open ended questionnaire to be included at month six follow up 211

Appendix C: Study information and informed consent document..............................213

Appendix D: Telephonic questions and the ACSM screening for exercise guidelines ........ 219 
Appendix E: Data collection documents and outcome measure instruments

Demographic information document and outcome measures ....................................223

Appendix F: "Living with Osteoarthritis" patient booklet.....................................................237

Appendix G: Example of exercises and visualisation relaxation ..........................................329

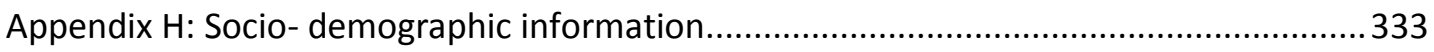

Attendance records for the six week intervention ........................................................33

Waiting time

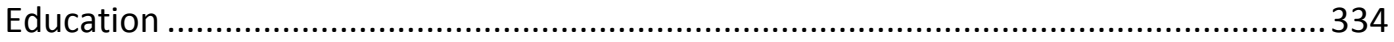

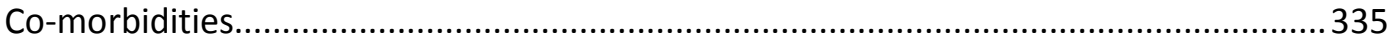

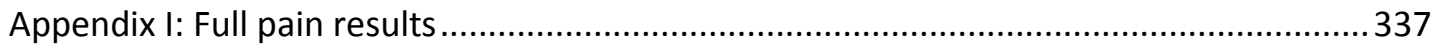

Pain severity score subgroup analysis: OA hip, OA knee and combined hip and knee OA

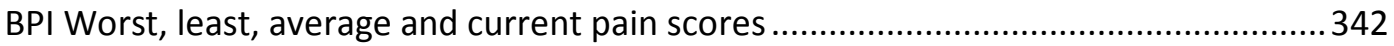

Pain interference score: subgroup analysis of OA hip, knee, both hip and knee .............347

Percentage pain relief from medication and medication usage......................................352

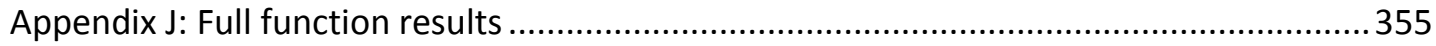

Physical performance task battery walk tests $-15 \mathrm{~m}$ fastest and normal speed ..............355

Physical performance task battery walk tests - fastest speed:.....................................356

Physical performance task battery walk tests - normal speed: ......................................362

Physical performance task battery six minute walk test ..................................................368

Physical performance task battery repeated sit to stand test........................................375

Physical performance task battery reach tests - forwards and upwards .......................382

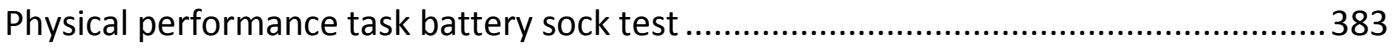

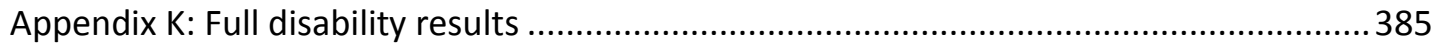

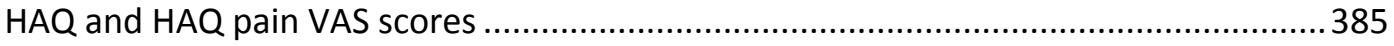

Correlation between the two pain severity scores..........................................................386

Correlation between the two pain severity scores for OA of the knee ............................387

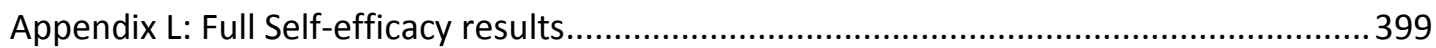

Appendix M: Full Health related quality of life results .....................................................401

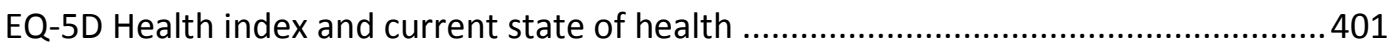

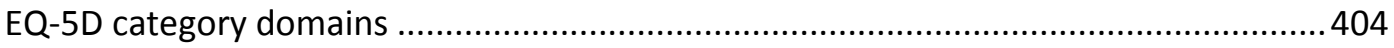

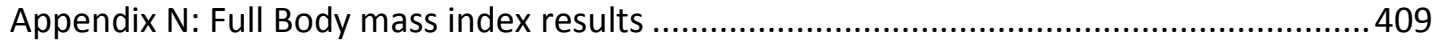

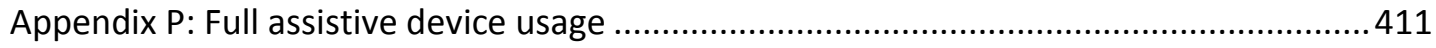

Appendix Q: Themes and full responses to open ended questionnaire...............................413 
Appendix R: CONSORT table 


\section{Table of Figures}

FIGURE 2-1 ICF LINKS SHOWING THE DIFFERENT COMPONENTS OF DISABILITY ................................................ 5

FIGURE 2-2 THE FIRST, SECOND AND THIRD LINE TREATMENT OPTIONS ......................................................... 48

FIGURE 2-3 COMPLEX RELATIONSHIP BETWEEN PAIN, DISABILITY AND PSYCHOSOCIAL ASPECTS ...............................66

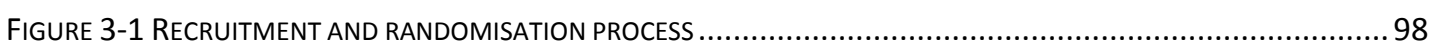

FIGURE 4-1 CONSORT FLOW DIAGRAM OF INITIAL CONTACT TO ANALYSIS .................................................. 103

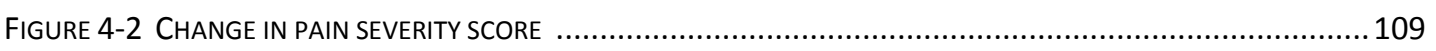

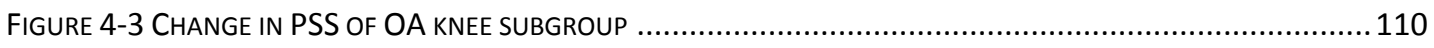

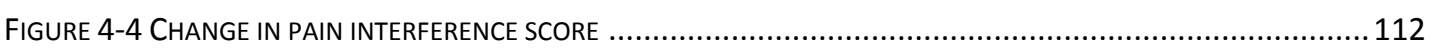

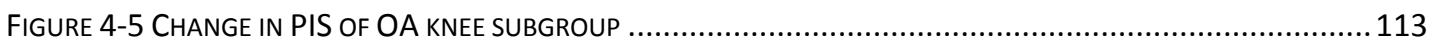

FIGURE 4-6 CHANGE IN TIME TAKEN TO WALK 15M AT FASTEST SPEED ....................................................... 117

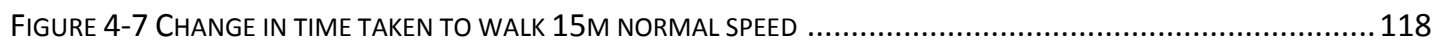

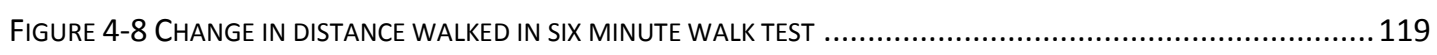

FIGURE 4-9 CHANGE IN TIME TAKEN FOR REPEATED SIT - STAND TEST .................................................. 120

Figure 4-10 ChANGE IN HEALTH ASSESSMENT QUESTIONNAIRE SCORE ................................................... 122

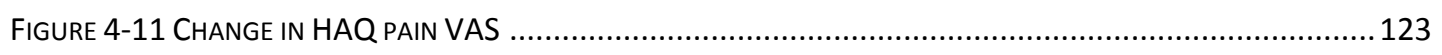

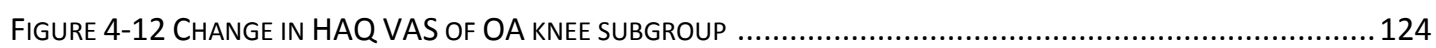

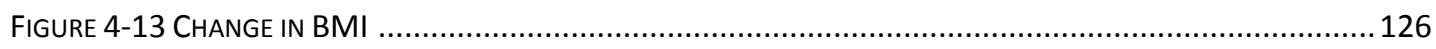

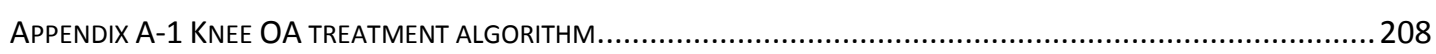

APPENDIX I -1 CHANGE IN PSS OF OA HIP, KNEE AND BOTH HIP AND KNEE SUBGROUPS IN EXPERIMENTAL GROUP ..... 338

APPENDIX I-2 CHANGE IN PSS OF OA HIP, KNEE, BOTH HIP AND KNEE SUBGROUPS IN CONTROL GROUP .................. 339

APPENDIX I-3 CHANGE IN PSS OF OA BOTH HIP AND KNEE SUBGROUP ........................................................ 340

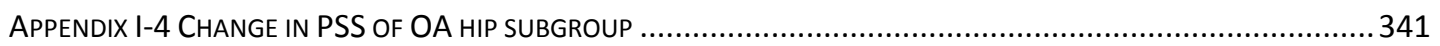

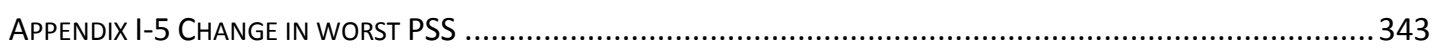

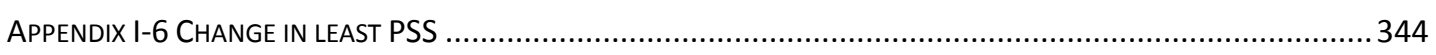

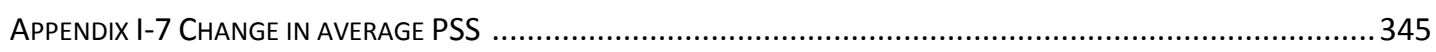

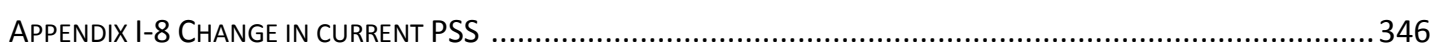

APPENDIX I -9 CHANGE IN PIS OF OA HIP, OA KNEE AND COMBINED HIP AND KNEE OA SUBGROUPS IN EXPERIMENTAL

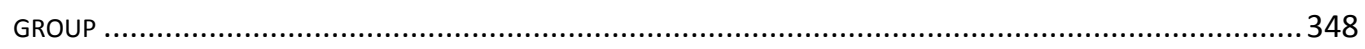

APPENDIX I-10 CHANGE IN PIS OF OA HIP, OA KNEE AND COMBINED HIP AND KNEE OA SUBGROUPS IN THE CONTROL

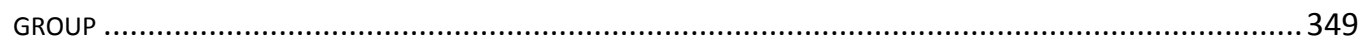

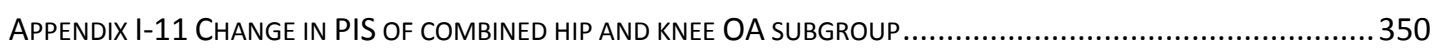

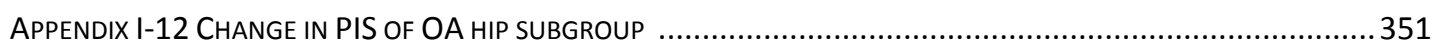

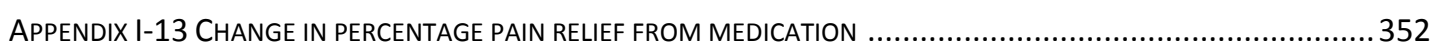

APPENDIX J-1 CHANGE IN OA HIP, KNEE, BOTH HIP AND KNEE SUBGROUPS IN THE EXPERIMENTAL GROUP .................357

APPENDIX J-2 CHANGE IN OA HIP, KNEE, BOTH HIP AND KNEE SUBGROUPS IN THE CONTROL GROUP........................358

APPENDIX J-3 CHANGE IN TIME TAKEN FOR 15M FASTEST SPEED WALK OF OA KNEE SUBGROUP ...........................359

APPENDIX J-4 CHANGE IN TIME TAKEN FOR 15M FASTEST SPEED WALK OF OA BOTH HIP AND KNEE SUBGROUP ..........360

APPENDIX J-5 CHANGE IN TIME TAKEN FOR 15M FASTEST SPEED WALK OF OA HIP SUBGROUP ..............................361

APPENDIX J-6 CHANGE IN OA HIP, KNEE, BOTH HIP AND KNEE SUBGROUPS IN THE EXPERIMENTAL GROUP ................363

APPENDIX J-7 CHANGE IN OA HIP, KNEE, BOTH HIP AND KNEE SUBGROUPS IN THE CONTROL GROUP.........................364

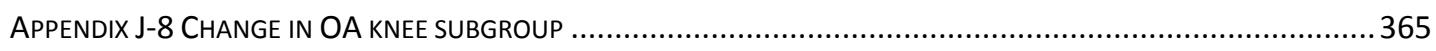

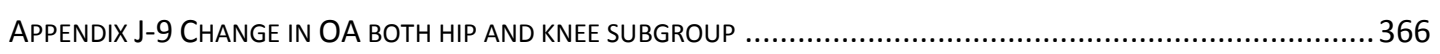

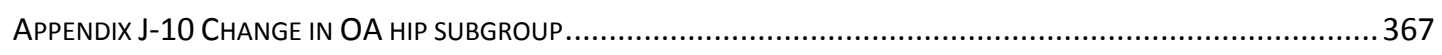

APPENDIX J-11 CHANGE IN OA HIP, KNEE, BOTH HIP AND KNEE SUBGROUPS IN THE EXPERIMENTAL GROUP ..............370

APPENDIX J-12 CHANGE IN OA HIP, KNEE, BOTH HIP AND KNEE SUBGROUPS IN THE CONTROL GROUP .....................371

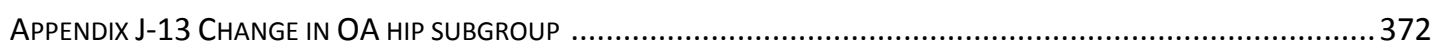


APPENDIX J-14 CHANGE IN OA KNEE SUBGROUP

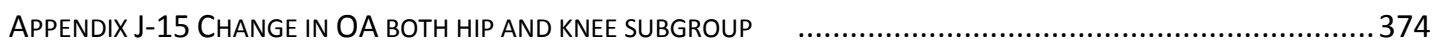

APPENDIX J-16 CHANGE IN OA HIP, KNEE, BOTH HIP AND KNEE SUBGROUPS IN THE EXPERIMENTAL GROUP ..............377

APPENDIX J-17 CHANGE IN OA HIP, KNEE, BOTH HIP AND KNEE SUBGROUPS IN THE CONTROL GROUP .....................378

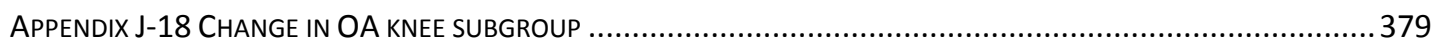

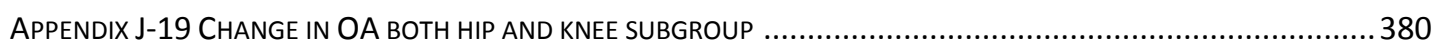

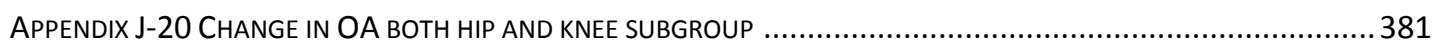

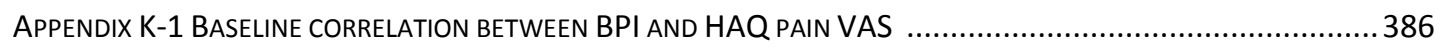

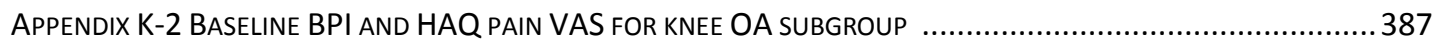

APPENDIX K-3 CHANGE IN HAQ OF OA KNEE, HIP AND BOTH HIP AND KNEE SUBGROUPS IN EXPERIMENTAL GROUP...389

APPENDIX K-4 CHANGE IN HAQ OF OA KNEE, HIP AND BOTH HIP AND KNEE SUBGROUPS IN CONTROL GROUP............ 390

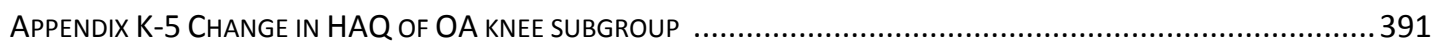

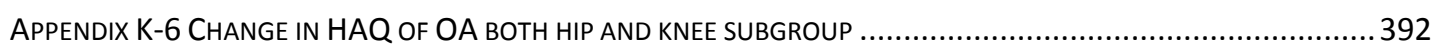

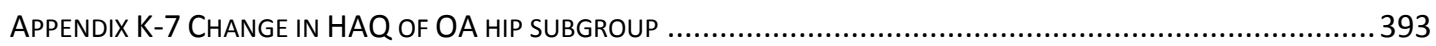

APPENDIX K-8 CHANGE IN HAQ VAS OF OA HIP, KNEE, BOTH HIP AND KNEE SUBGROUPS IN THE EXPERIMENTAL GROUP

APPENDIX K-9 CHANGE IN HAQ VAS OF OA HIP, KNEE, BOTH HIP AND KNEE SUBGROUPS IN THE CONTROL GROUP ...396

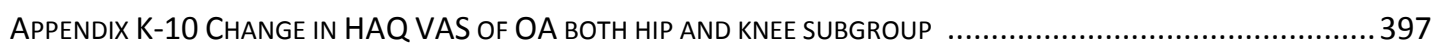

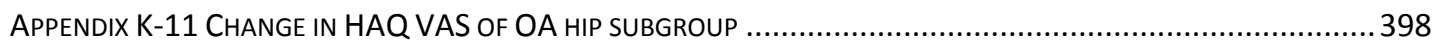

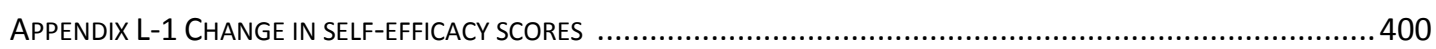

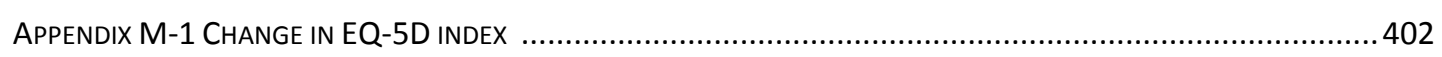

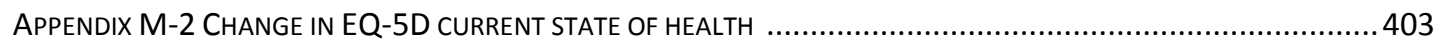




\section{List of Tables}

TABLE 2-1 DEFINITIONS AND CONCEPTS OF THE ICF COMPONENTS.............................................................. 5

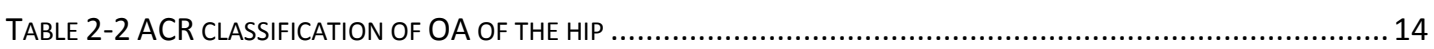

TABLE 2-3 EULAR RECOMMENDATIONS FOR THE MANAGEMENT OF OA OF THE KNEE AND HIP..............................38

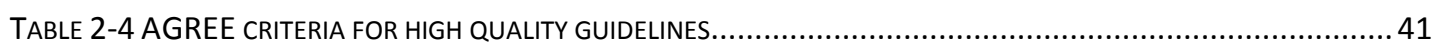

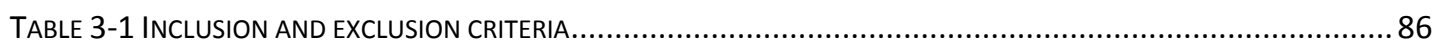

TABLE 3-2 EDUCATIONAL CONTENT COVERED ON A WEEKLY BASIS DURING THE INTERVENTION ...............................99

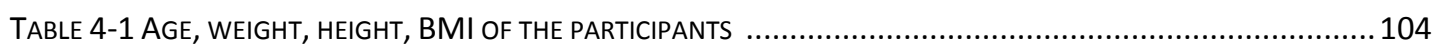

TABLE 4-2 GENDER, LANGUAGE, EDUCATIONAL LEVEL, EMPLOYMENT STATUS ................................................ 105

TABLE 4-3 CO-MORBIDITIES, JOINT/S AFFECTED, ASSISTIVE DEVICE AND MEDICATION USAGE ............................... 107

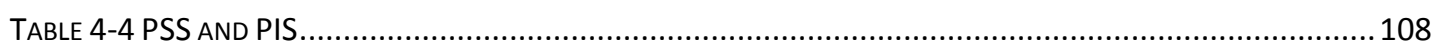

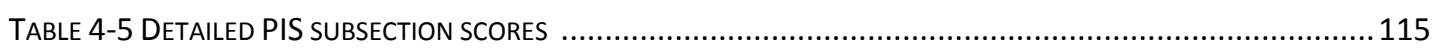

TABLE 4-6 PATIENT REPORTED VERSUS RA RECORDED ASSISTIVE DEVICE USAGE AT BASELINE ..............................127

TABLE 4-7 THEMES THAT EMERGED FROM PARTICIPANT'S ANSWERS TO OPEN ENDED QUESTIONNAIRE ....................129

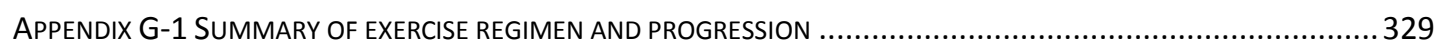

APPENDIX H-1 OTHER CO-MORBIDITIES RECORDED ON DEMOGRAPHIC INFORMATION DOCUMENT...........................335

APPENDIX I-1 DETAILED PSS FOR OA HIP, OA KNEE AND COMBINED HIP AND KNEE OA SUBGROUPS .......................337

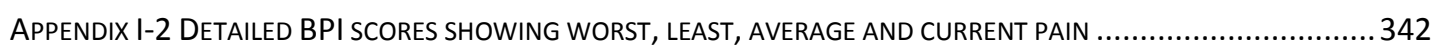

APPENDIX I-3 DETAILED PIS FOR OA HIP, KNEE, BOTH HIP AND KNEE SUBGROUPS ….........................................3 347

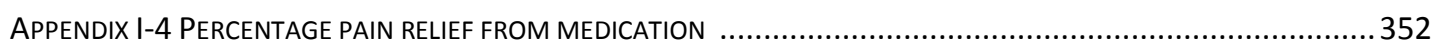

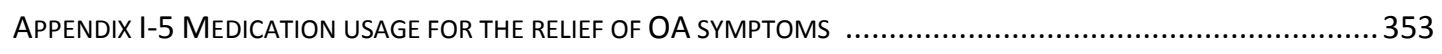

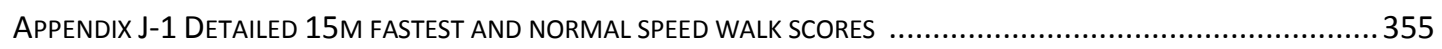

APPENDIX J-2 DetAILED TIME TAKEN TO WALK 15M AT FASTEST SPEED IN OA HIP, KNEE, BOTH HIP AND KNEE SUBGROUPS

356

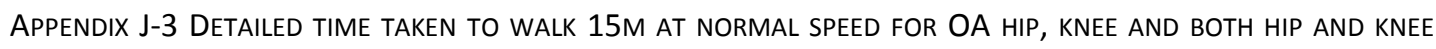

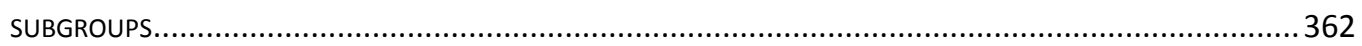

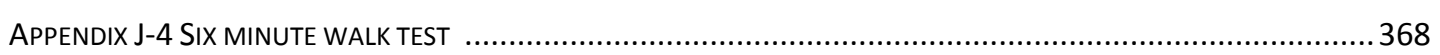

APPENDIX J-5 DETAILED DISTANCE WALKED IN 6 MINUTES FOR OA HIP, KNEE, BOTH HIP AND KNEE SUBGROUPS......... 369

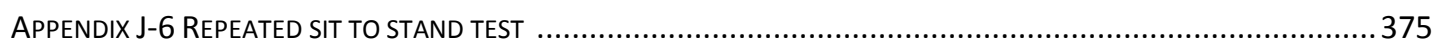

APPENDIX J-7 DETAILED TIME TAKEN FOR REPEATED SIT-STAND FOR OA HIP, KNEE AND BOTH HIP AND KNEE SUBGROUPS

376

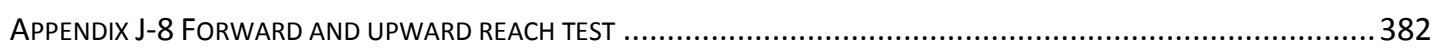

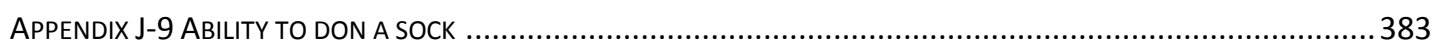

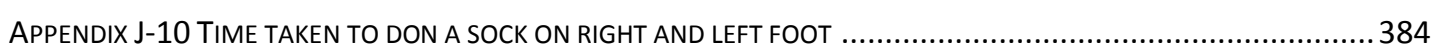

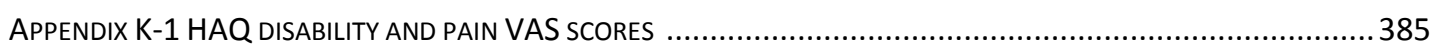

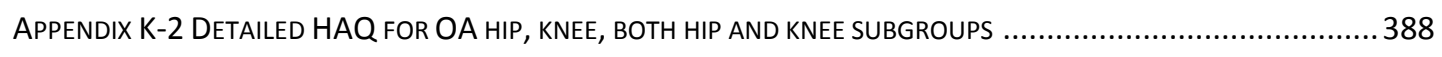

APPENDIX K-3 DETAILED HAQ PAIN VAS OF OA HIP, KNEE, BOTH HIP AND KNEE SUBGROUPS ............................ 394

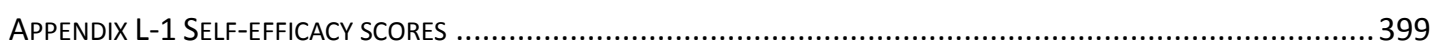

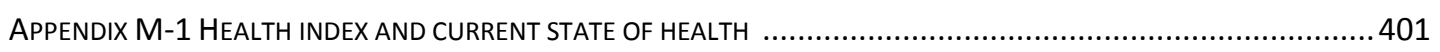

APPENDIX M-2 MOBILITY, SELF-CARE, ACTIVITY, PAIN/DISCOMFORT, ANXIETY/DEPRESSION ...............................404

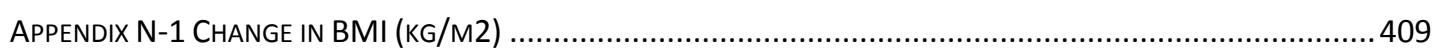

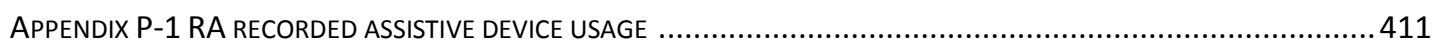

APPENDIX Q-1 THEMES EMERGED FROM PARTICIPANT'S RESPONSES TO OPEN ENDED QUESTIONS ......................... 413

TABLE R-1 CONSORT GUIDELINES AND INDICATION OF CHAPTER WHERE ITEMS WERE ADDRESSED ........................ 417 


\section{List of Abbreviations}

\begin{tabular}{|c|c|}
\hline 6MWT & 6 Minute Walk Test \\
\hline AAOS & American Academy of Orthopaedic Surgeons \\
\hline ACR & American College of Rheumatology \\
\hline ADL & Activities of Daily Living \\
\hline AGREE & Appraisal of Guidelines Research and Evaluation \\
\hline AIDS & Acquired Immuno-Deficiency Syndrome \\
\hline ANOVA & Analysis of Variance \\
\hline ASES & Arthritis Self-Efficacy Scale \\
\hline ASMP & Arthritis Self-Management Programme \\
\hline BOD & Burden of Disease \\
\hline BDI & Beck Depression Inventory \\
\hline BPI & Brief Pain Inventory \\
\hline BPQ & Brief Pain Questionnaire \\
\hline CDSMP & Chronic Disease Self-Management Programme \\
\hline CNS & Central Nervous System \\
\hline CONSORT & Consolidated Standards for Reporting Trials \\
\hline cox-2 & Cyclo-oxegenase-2 \\
\hline CP & Chronic Pain \\
\hline CRPS & Complex Regional Pain Syndrome \\
\hline CS-PFP & Continuous-Scale Physical Functional Performance test \\
\hline DALY & Disability Adjusted Life Years \\
\hline DM & Diabetes Mellitus \\
\hline EQ-5D & EuroQol 5 Dimensional outcome questionnaire \\
\hline ES & Effect Size \\
\hline
\end{tabular}




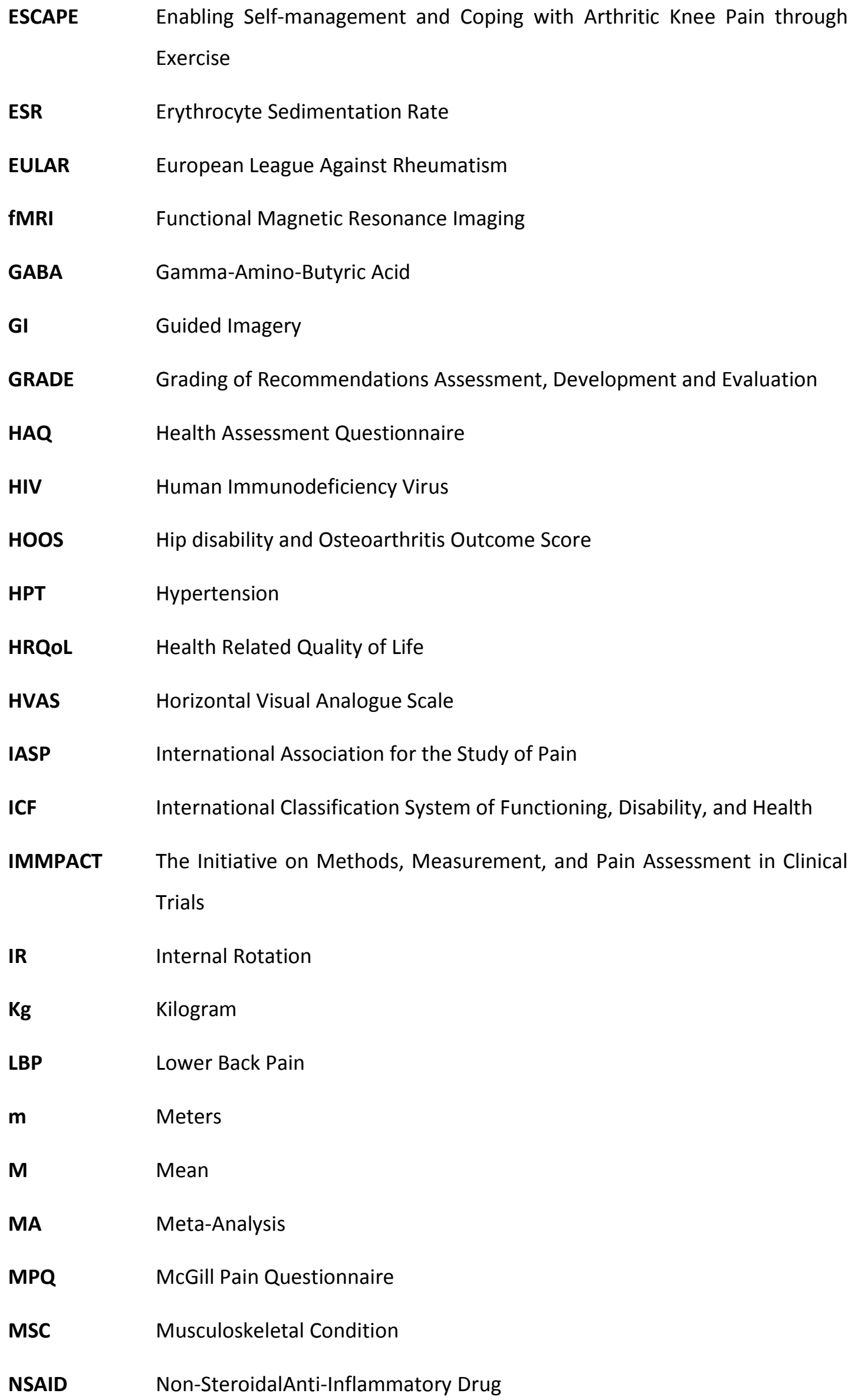




\begin{tabular}{|c|c|}
\hline NCD & Non-communicableDisease \\
\hline NICE & National Institute for Health and Clinical Excellence \\
\hline NRS & Numerical Rating Scale \\
\hline $\mathbf{O A}$ & Osteoarthritis \\
\hline OARSI & Osteoarthritis Research Society International \\
\hline OP & Osteoporosis \\
\hline POMS & Profile of Mood States \\
\hline PIS & Pain Interference Score \\
\hline PMR & Progressive Muscle Relaxation \\
\hline PPI & Proton Pump Inhibitor \\
\hline PPT & Physical Performance Test \\
\hline PSS & Pain Severity Score \\
\hline RA & Rheumatoid Arthritis \\
\hline RCT & Randomised Controlled Trial \\
\hline ROM & Range of Movement \\
\hline SD & Standard Deviation \\
\hline SE & Self-Efficacy \\
\hline Sec & Seconds \\
\hline SPPB & The Short Physical Performance Battery \\
\hline SR & Systematic Review \\
\hline SA & South Africa \\
\hline SF-12 & The Medical Outcomes Study 12-Item Short Form Health Survey \\
\hline SF-36 & Medical Outcomes Study 36-Item Short-Form Health Survey \\
\hline SF-MPQ & Short Form McGill Pain Questionnaire \\
\hline SMI & Self-management Intervention \\
\hline SYSADOA & Symptomatic Slow Acting Drugs for Osteoarthritis \\
\hline TB & Tuberculosis \\
\hline
\end{tabular}


TBH

TENS

UK

US

USA

VAS

VVAS

WHO

WOMAC

YLD

YLL

VRS
Tygerberg Hospital

Transcutaneous Electrical Nerve Stimulation

United Kingdom

Ultrasound

United States of America

Visual Analogue Scale

Vertical Visual Analogue Scale

World Health Organisation

Western Ontario and McMaster Universities Osteoarthritis Index

Years Lived with Disability

Years of Life Lost

Verbal Rating Scale 


\section{Glossary of Terms}

Acetabulum/acetabular: an anatomical term that refers to the socket of the hip joint in which the head of the femur articulates ${ }^{(1)}$.

Acupuncture: traditionally a type of Eastern medicine that involves the insertion of thin needles into the skin and muscle. It is used to treat a variety of symptoms and is known as the physiotherapy term "dry needling" in South Africa ${ }^{(1)}$.

Allodynia:experiencing pain in response to a stimulus that does not normally provoke pain $^{(2)}$.

Apartheid: a historical term meaning "separateness" used to describe a political periodof racial discrimination in South Africa that ended in 1994.

Arthroscopic lavage:asurgical procedure performed by thin telescopic instruments to examine and wash out an area or joint ${ }^{(1)}$.

Arthroplasty:a surgical procedure whereby the total joint surfaces areremodelled and replaced by a prosthesis ${ }^{(1)}$.

Assistive device:an artificial aid designed to assist a person to perform a task. In this study it refers to mobility assistive devices like walking sticks, crutches and walking frames that allows for transfer of weight from the legs through the arms ${ }^{(3)}$.

Chronic pain: pain that persists past the normal time of healing ${ }^{(4)}$.

Coxa valga: an anatomical term used to describe a deformity of the hip joint whereby the femur neck angle is abnormally large $\mathrm{e}^{(1)}$.

Coxa vara: an anatomical term used to describe a deformity of the hip joint whereby the femur neck angle is abnormally small ${ }^{(1)}$.

Delphi technique:a term used to describe a systematic method of analysis, decision making and forecasting in order to eliminate subjective views or opinions. Typically it lasts at least two rounds of experts answering questions and giving justification for their answers, providing the opportunity between rounds for changes and revisions. The multiple rounds, which are stopped after a pre-defined criterion is reached, enable the group of experts to arrive at a consensus forecast on the subject being discussed ${ }^{(5)}$. 
Electrotherapy: amanual branch of physiotherapy that makes use of electrical equipment for therapeutic purposes. The passage of electric currents through the body's tissues is used to stimulate the functioning of nerves and the muscles that they supply ${ }^{(1)}$.

Fear avoidance: the characteristic or belief based on the fear of pain and therefore the avoidance of activities that cause pain ${ }^{(6)}$.

Femur/femoral: an anatomical term that refers to the long bone of the thigh that superiorly articulates with the acetabulum to form the hip joint and inferiorly with the tibia to form the knee joint ${ }^{(1)}$.

Genu valgum: an anatomical term used to describe a malalignment of the knee joint whereby the knees are angulated inwards (knock kneed) ${ }^{(1)}$.

Genu varum: an anatomical term used to describe a malalignment of the knee joint whereby the knees are angulated outwards (bow legged) ${ }^{(1)}$.

Hyperalgesia:an abnormal state of increased sensitivity to pain whereby extreme pain is experienced in response to a normally painful stimulus ${ }^{(2)}$.

Innocuous: a term used to describe a stimulus which is harmless or does not usually evoke $\operatorname{pain}^{(2)}$.

Leptin: a protein produced by adipose tissue that plays an important role in regulating eating ${ }^{(1)}$.

Manipulation: a treatment term used in physiotherapy when a jointis moved in a brisk manner to facilitate more mobility at the joint ${ }^{(1)}$.

Morning stiffness: difficulty moving a joint through full range of movement experienced in the morning directly after lying for a long period with minimal movement. It usually lasts 30 minutes or less for $\mathrm{OA}^{(7)}$.

Nociceptor:a sensoryreceptor involved in the pain processing system, which if stimulated can result in central nervous system processing which produces a pain reaction to warn the body of actual or potential tissue damage ${ }^{(1)}$.

Nociception: refers to the process whereby nociceptor receptors are stimulated ${ }^{(1)}$.

Osteophyte: an additional piece of bone, often formed in an attempt to create stability at a site such where cartilage degeneration or destruction near joints occur ${ }^{(1)}$.

Osteotomy:a surgical procedure whereby a portion of bone is removed ${ }^{(1)}$. 
Pain:an unpleasant sensory and emotional experience associated with actual or potential tissue damage or described in terms of such damage ${ }^{(8)}$.

Pain intensity: describes the severity or amount of pain felt by an individual ${ }^{(9)}$.

Pain interference: describes how much pain interferes or affects an individual ${ }^{(9)}$.

Placebo effect:a therapeutic effect from the administration of a placebo intervention, aimed at achieving a non-specific benefit of treatment ${ }^{(1)}$.

Plasticity:the ability of the nervous system to change and adapt as necessary ${ }^{(2)}$.

Primary level care: a term used in South Africa to describe the first level in the public health care system, these clinic facilities are usually situated in a small community and deliver health care to the population of and surrounding that community ${ }^{(10)}$.

Prosthesis: an artificial implant used to replace a biological part of the body. In terms of this study, a hip prosthesis comprises of an acetabular cup and a femoral head and shaft. A knee prosthesis comprises of a femoral and tibial articulating surface ${ }^{(1)}$.

Rehabilitation: the process whereby the aim is to maintain, improve or restore normal health and function to prevent disability from worsening ${ }^{(1)}$.

Reliability: a term used in research to describe the consistency of an outcome measurement tool.

Self-management: wherebythe patient assumes an active part in the daily care of their health condition that involves three components namely; medical, behavioural and emotional management ${ }^{(11)}$

Secondary level care: a term used in South Africa to describe the second level in the public health care system, these small hospitals deliver health care and simple surgery ${ }^{(10)}$.

Self-efficacy:the belief in one self's perceived capability to perform a task or achieve a goal $^{(12)}$.

Thermotherapy: a therapeutic term used in physiotherapy to describe therapy based on temperature; either cold named cryotherapy to reduce pain or inflammation or heat named thermotherapy to relieve pain, stiffness and promote circulation ${ }^{(1)}$.

Tertiary level care:a term used in South Africa to describe the third level in the public health care system, these big hospitals deliver specialised health care and surgery ${ }^{(10)}$. 
Tibia/tibal: an anatomical term that refers to the long bone of the shin that superiorly articulates with the femur to form the knee joint and inferiorly with the talus bone to form the ankle joint ${ }^{(1)}$.

Transcutaneous electrical nerve stimulation: an electrotherapy modality used in physiotherapy which causes electrical impulses to pass into tissues to activate a muscle or inhibit pain ${ }^{(1)}$.

Ultrasound: an electrotherapy modality used in physiotherapy which sends high frequency sound waves into the tissues to stimulate a pro-inflammatory response ${ }^{(1)}$.

Validity: a term used in research to describe how sound research is or to what extent a sign or test is a true indicator of what is being tested ${ }^{(1)}$. 


\section{Introduction and scope of thesis}

\subsection{Background}

Osteoarthritis (OA) is known as the most common joint disease worldwide ${ }^{(13)}$. The prevalence of $\mathrm{OA}$ continues to rise exponentially in both high and low income countries $^{(14,15)}$, including South Africa (SA).The burden of OA leads to a large portion of the older population suffering with daily pain and disability ${ }^{(16-20)}$. This burden extends far beyond the physical aspects of a joint pathology alone as $\mathrm{OA}$ is considered a complex condition affected by and affecting various factors related to the individual ${ }^{(21,22)}$. These include and are not limited to personal, psychological, social and environmental factors ${ }^{(22)}$.

Due to the complexity of $O A$, effective management is necessary at all stages of the condition.As insight into this field of research is constantly improving, many evidencebased guidelines have been published for OA of the hip and knee ${ }^{(23-30)}$. The gold standard of treatment for OA begins withnon-pharmacological methods of education, exercise and weight control ${ }^{(29)}$. Pharmacological agents are subsequently added as required, in combination with first-line management ${ }^{(29)}$. Finally, surgery is indicated for those with late stage OA who do not respond to conservative treatment methods ${ }^{(29)}$.

A major concern identified internationally is the limited accessibility of the necessary management options, especially in the public health system ${ }^{(14)}$. This is true for many individuals with OA of the hip and/or knee in the Western Cape of SA, where delays in surgery are experienced as a result of long waiting lists for a joint replacement ${ }^{(31)}$. Tygerberg hospital (TBH) is one of eight health care facilities in the province that provides joint replacements to patients with late stage $O A^{(32)}$. It has been reported that waiting for surgery leads to negative impacts on the individual and thus adds to the burden of $\mathrm{OA}^{(33,34)}$.

In response to the increasing burden of $\mathrm{OA}$ and long waiting lists at Tygerberg hospital, research was warranted to establish the effects of an exercise and education intervention in patients with osteoarthritis on the waiting list. Once these effects are known, the results can be utilised to provide the appropriate evidence-based management to patients, while awaiting surgery. Additionally this study can lead to further valuable research in this field. 


\subsection{Aim and objectives}

\subsubsection{Aim of the study}

The aim was to determine the effects of a six-week physiotherapist-led exercise and education intervention in patients with $\mathrm{OA}$, awaiting a hip/knee joint replacement.

\subsubsection{Objectives of the study}

The objectives were to determine whether participating in a six-week physiotherapist-led exercise and education intervention, resulted in a significant change in the following outcomes, when compared to the control group,in patients with OA awaiting a hip or knee replacement:

- Pain severity and pain interference as measured on the Brief Pain Inventory (BPI) ${ }^{(35)}$

- Function as measured by the Physical Performance Task Battery ${ }^{(36)}$

- Disability as measured by the Stanford Health Assessment Questionnaire (HAQ) $)^{(37)}$

- Self-efficacy (SE) as measured by the Self-Efficacy for Managing Chronic Disease 6Item Scale ${ }^{(38)}$

- Health-related quality of life as measured by the EQ- $5 D^{(39)}$.

In addition, to determine whether body mass index (BMI) value ${ }^{(40)}$; orOA of the hip or knee or a combination of hip and knee OA, accounted for any significant differences in the above outcome measures. Finally, to determine the acceptability of a six-week physiotherapistled exercise and education intervention for patients on the waiting list for joint arthroplasty. 


\section{Literature review}

\subsection{Introduction}

According to research statistics in low- and middle income ${ }^{*}$ countries, the adult burden of disease (BOD) is shifting from infectious diseases to an increased prevalence of chronic non-communicable diseases $(N C D)^{(41,42)}$. This prevalence rate wasreported to be close to $45 \%$ in $2004^{(41)}$. Chronic NCD include musculoskeletal conditions (MSC), which are the most common causes of severe long term pain and disability ${ }^{(43)}$. According to studies by Murray and Lopez ${ }^{(44)}$, MSC account for $4.68 \times 10^{6}$ disability adjusted life years ${ }^{\dagger}$ (DALY) lost in higher income countries. This figure is expected to increase to $5.6 \times 10^{6}$ DALYs by the year $2020^{(45)}$. Similar statistics for lower income countries are scarce. Statistics available from SA in 2004 show that $28 \%$ of the total DALYs lost are attributed to $\mathrm{NCD}^{(46)}$. However, it is noted that risks for attaining NCD are higher in low to middle income countries than in higher income countries $^{(41)}$ and that the burden caused by these conditions is estimated at two to three times higher than in higher income countries ${ }^{(46)}$. The expected increase in burden in lower income countries is said to be due to a greater life expectancy of the population ${ }^{(45,46)}$, and increasing co-morbidities such as obesity, diabetes mellitus (DM) and cardiorespiratory conditions $^{(47,48)}$. Of the various musculoskeletal conditions, $O A$ is one of the four leading causes of disability worldwide, together with rheumatoid arthritis(RA), osteoporosis(OP) and low back pain (LBP) $)^{(19,49,50,50,51)}$.

Disability is not a simple term but rather a complex phenomenon ${ }^{(52)}$. Being "disabled" is not a category that a person falls into or not, as traditionally described ${ }^{(52,53)}$. For many years disability was defined by biomedical terms alone, due to the presence of pathology. However since 1994, disability has been recognised as the disparity between an individual's capability and their unique social or environmental demands ${ }^{(54)}$. The term "disability" differs from the term "functional limitation" by explaining the individual's functional inadequacyin terms of the person's social and cultural context ${ }^{(53,54)}$.

\footnotetext{
* The World Bank's definition of classifying country's economies is based on the country's gross national income per capita; low income, middle income (subdivided into lower middle and upper middle), or high income. South Africa is classified as an upper middle income country ${ }^{(362)}$.

+Disability-adjusted life years (DALYs) represent a health gap; it measures the state of a population's health compared to a normative goal. The goal is for individuals to live the standard life expectancy in full health. DALYs are the sum of two components: years of life lost due to premature mortality (YLLs) and years lived with disability (YLDs). YLLs = number of deaths at each age multiplied by a standard life expectancy at age $\mathrm{x}$. YLDs - the prevalence of different disease-sequelae and injury-sequelae multiplied by the disability weight for that sequelae. DALYs are an absolute measure of health loss; they count how many years of healthy life are lost due to death and non-fatal illness or impairment. ${ }^{(363)}$
} 
In order to accurately define and measure disability, assessment of the person's physical condition as well as their functional ability in their personal and social environment is necessary ${ }^{(52)}$. The information gained from an assessment such as the aforementioned, is then used to formulate a tailored approach to management options for the individual. This explains how two individualswith the same condition, such as $\mathrm{OA}$, may present with the same physical impairment, such as decrease range of movement (ROM) in a joint, yet function at different levels due to variances in their personal environments or social settings either facilitating or hindering their functional ability. Additionally, personal factors such as meaning or importance placed on the achievement of a certain task or participation in an activity has an influence on the way two people with the same condition experience a similar situation ${ }^{(55)}$. In order to provide a framework to define disability and facilitate the development of personalised treatment plans, the WHO released the International Classification System of Functioning, Disability, and Health (ICF) in $2001^{(56)}$. The ICF will be used in this thesis as a theoretical framework.

\subsubsection{International Classification of Functioning, Disability and Health - a theoretical framework}

The development of a theoretical framework for defining disability and health commenced in the 1960 's and over the years has been revised on a number of occasions ${ }^{(54,57,58)}$. In 2001, the World Health Organisation (WHO) released the newest classification called the ICF ${ }^{(56)}$. This classification has been accepted and utilised worldwide, as it reflects the best understanding of the complexities of disability, as mentioned above ${ }^{(52,59)}$. The ICF model not only describes disability in medical terms of physical pathology or impairments, but also incorporates personal and social aspects of disability. It does so by integrating the physical condition and personal or psychological aspects with functional and/or environmental restrictions that are experienced. This is done by identifying difficulties in specific activities or participation in the individual's social situation and the reasons therefore ${ }^{(53,56)}$. See Table 2-1 and Figure 2-1below for components of the ICF. 
Table 2-1 Definitions and Concepts of the ICF components

\begin{tabular}{|c|c|c|c|c|}
\hline Component & $\begin{array}{l}\text { Body functions } \\
\text { and structures }\end{array}$ & Activity & Participation & $\begin{array}{l}\text { Environmental } \\
\text { Factors }\end{array}$ \\
\hline Definition: & $\begin{array}{l}\text { Body functions } \\
\text { are the } \\
\text { physiological } \\
\text { functions of body } \\
\text { systems } \\
\text { (including } \\
\text { psychological } \\
\text { functions) } \\
\text { Body structures } \\
\text { are the } \\
\text { anatomical parts } \\
\text { of the body, such } \\
\text { as organs, limbs } \\
\text { and their } \\
\text { components }\end{array}$ & $\begin{array}{l}\text { Activity is } \\
\text { the } \\
\text { execution } \\
\text { of a task or } \\
\text { action by an } \\
\text { individual }\end{array}$ & $\begin{array}{l}\text { Participation is } \\
\text { the } \\
\text { involvement in } \\
\text { a life situation }\end{array}$ & $\begin{array}{l}\text { Environmental } \\
\text { factors make up the } \\
\text { physical, social and } \\
\text { attitudinal } \\
\text { environment in } \\
\text { which people live } \\
\text { and conduct their } \\
\text { lives }\end{array}$ \\
\hline Negative aspect: & Impairment & $\begin{array}{l}\text { Activity } \\
\text { limitation }\end{array}$ & $\begin{array}{l}\text { Participation } \\
\text { restriction }\end{array}$ & $\begin{array}{l}\text { Barriers or } \\
\text { hindrances }\end{array}$ \\
\hline \multicolumn{5}{|c|}{ (Dahl, T.H., 2002) } \\
\hline \multicolumn{5}{|c|}{$\begin{array}{l}\text { Health Condition } \\
\text { (injury/disorder/disease) }\end{array}$} \\
\hline $\begin{array}{l}\text { Body fun } \\
\text { struct } \\
\text { (Impairn }\end{array}$ & $\begin{array}{l}\text { Environmental } \\
\text { Factors }\end{array}$ & $\begin{array}{c}\text { Activities } \\
\text { (Limitation) } \\
\uparrow\end{array}$ & 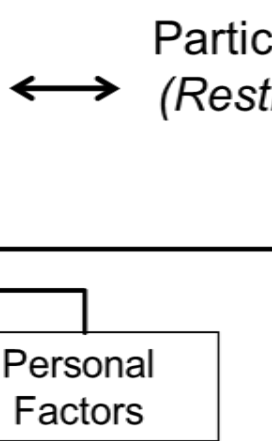 & $\begin{array}{l}\text { ipation } \\
\text { riction) }\end{array}$ \\
\hline
\end{tabular}

Figure 2-1 ICF links showing the different components of disability 
In rehabilitation medicine, the ICF is used to bring structure and clarity to the problems identified during assessment by the rehabilitative professional ${ }^{(60)}$. This is done by evaluating the many different components involved to give a clear picture of the entire impact of the condition ${ }^{(60)}$. Rehabilitation definitions have previously focused on solely "enabling the individual" by addressing their physical ailment but not necessarily attendingto personal factors or addressing function in a social or environmental context ${ }^{(59)}$. However, reeducation of function in rehabilitation according to the ICF, incorporates impairments, function, personal aspects and the social environment to enable the individual in their different contexts.

As with most frameworks or models, limitations of the ICF have been noted in the literature by various authors. Oncology researchers ${ }^{(61)}$ raise a few logistical concerns regarding the ICF and have put forward recommendations to improve these areas. Firstly, an issue has been raised regarding the term used as "health condition". Bornbaum and colleagues $^{(61)}$ suggest that a "health condition" is either present or absent which realistically is not the case in many chronic disorders; some conditions have different stages and progressions which are not fully described by the term above. Another point was made regarding the term "condition" being in the singular form; suggesting that this model does not account for the common occurrences of co-morbidities that result in further health, personal and social consequences ${ }^{(61)}$. The recommendation has been made to add in a component dedicated to co-morbidities and to rather make use of the term "health state" as this better reflects the presentation of different health concerns and their related aspects as they affect the individual.

Furthermore, it is noted that limitations exist surrounding the classification terms for personal factors and the subjective dimension of a patient's experience; more specifically highlighting the aspect of quality of life ${ }^{(55,61)}$. It is known that personal factors such as the perception of one's quality of life and psychological aspects have a great influence on a person's way of functioning and participating in a work or social context. The aim of the WHO and researchers is to improve this component of the framework by the addition of "personal perception" as a personal factor in order that quality of life is correctly accounted for when using this model ${ }^{(55)}$. 
Other authors criticise the model as being purely a useful classification system to detect deviations from the norm in those with a disability and is not always carried over into rehabilitation ${ }^{(62)}$.

Additionally, the layout of the ICF implies that disability is first and foremost focused on identifying concerns related to a condition with a physical impairment and not necessarily on the development of a condition or impairment due to personal or environmental factors. The structure infers that once the physical condition has been treated or cured, the components of activity restrictions and participation limitations should no longer be of concern. In this way it fails to account for issues prior to the development of a disorder or persistent problems after the impairment has been managed ${ }^{(61)}$.

Despite the above criticisms the ICF is a useful framework to ensure the importantaspects which impact an individual are assessed, treated and measured during rehabilitation. Essentially, the ICF provides a biopsychosocial framework for the analysis of the multiple factors affecting a person with $O A$.

\subsubsection{Research Setting}

As discussed, when using the ICF as a framework for the management of various conditions requiring rehabilitation, the patient's environment is therefore an important factor to consider. One individual can function well in one environment but can be disabled in another. For instance; a patient with OA of both knees walks safely on the smooth hospital floors with a walking aid, however at home the patient is unable to walk beyond his front door as the surface in front of his house is a sandy terrain. Therefore, the setting in which assessment and on-going management of this condition takes place should be considered and adapted accordingly to facilitate the highest level of functioning. Additionally, if the person is faced with an unknown environment as a barrier to function, it may be necessary for the person to adapt to this new setting by being able to evaluate the situation and problem solve accordingly. This draws focus to a very important aspect of effective management: empowerment through learning self-management skills to increase selfconfidence $^{(11)}$. 
Self-management is defined as the patient assuming an active part in the daily care of their health condition that involves three components namely; medical, behavioural and emotional management ${ }^{(11)}$. As a prelude to further discussion on $O A$ and related topics such as self-management, a brief overview is first given aboutthe population and health care setting under investigation.

\section{i. South African population and health care}

Data gathered from the most recently published burden of disease study in $2000^{(63)}$ gives a clear breakdown of the South African population. Black Africans account for the majority of the population (79.2\%), followed by whites $(9.2 \%)$, coloured $(9 \%)$ and Indian population (2.6\%). Economically, $10 \%$ of the wealthy population is considered rich and possess $51 \%$ of the country's income, whereas the majority of the population are poor $(45.5 \%$ of the population are classified as poor and $20.2 \%$ as living in extreme poverty $\left.{ }^{(64)}\right)$ with the poorest $10 \%$ accounting for only $0.2 \%$ of the country's income ${ }^{(65)}$.

The entire health system ofSA has had many struggles from the past apartheid era until the current day ${ }^{(66)}$. Despite SA being classified economically as a middle income country, the health outcomes reported are still not in accordance with this income level; instead it reflects results expected of a lower income country ${ }^{(66)}$. Differences seen in health statistics based on race clearly show the ever present carry over effects from apartheid times. Values recorded for morbidity and mortality rates in SA for the last decade are much higher in poorer black populations compared to middle-class white populations ${ }^{(66)}$. Since the emergence of the private health sector, public health care is presented with a multitude of challenges, as close to half of the country's expenditure on health care goes towards the minority of wealthier patients in the private sector ${ }^{(66)}$. Statistics emerging from the South African General Health Survey in $2011^{(67)}$ reported mostly black and coloured households utilise the public health care facilities in SA ( $81.3 \%$ and $63.1 \%$ respectively).

In 2009 the major problems affecting the health systems of the country were brought to the attention of the national government and major institutions with the aim to improve factors contributing to the ongoing issues ${ }^{(68)}$. 
The four main health concerns related to Human Immunodeficiency Virus (HIV)/Tuberculosis (TB), Chronic/mental health conditions, injury/violence and maternal/neonatal and child health ${ }^{(68)}$. As mentioned, particular issues originating from previous racial and gender discrimination remain a challenge and have longstanding influences on the health of the population today ${ }^{(66)}$. High levels of unemployment, inadequate housing and sanitation, and malnutrition aresome of the social factors that play a part in determining health and well-being ${ }^{(66,68)}$.

Three key suggestions were made to address the ongoing challenges in the country: firstly, emphasis was placed on better prevention of major health conditions, followed by the need for integration of public and private sectors at a primary level to effectively promote well-being and manage conditions at entry point into the health system. Subsequently implementation of evidenced-based strategies and interventions to successfully manage these conditions is essential ${ }^{(68)}$. Together with this, is the ongoing need for high quality research to continually produce relevant and scientifically sound material on which health care decisions can be based ${ }^{(68)}$.

\section{ii. Increasing rise in non-communicable diseases in SA}

It has been noted that, like many other countries, South Africa has also displayed a rising prevalence of NCD in the last 25 years and that chronic conditions present a large burden on the existing health care system ${ }^{(68)}$. The increase in NCD is predominantly in the large portion of the SA population living on low income and with low levels of education ${ }^{(68)}$. The national department of health set a goal to reduce NCDs like hypertension (HPT), diabetes mellitus and asthma by encouraging lifestyle changes, weight loss, regular physical activity and adherence to medication ${ }^{(69)}$. In order to aspire to this goal, among other prerequisites, is the need for further human resources within both the urban and rural areas of the public healthsector ${ }^{(66)}$. Furthermore, human resources required at all levels of the health system need to be adequately trained ${ }^{(66)}$. If the goal is to provide healthcare based on the ICF framework, all personnel (including rehabilitative personnel) require specific education in this model of care. 
With the increase in burden from chronic diseasesin SA, service delivery from community or district level to tertiary level has beenplaced under severe pressure ${ }^{(46)}$. Ongoing issuessuch as limited staffing capacity and growing numbers of patients presenting with both infectious and chronic conditions cause lengthy waiting periods at all levels ${ }^{(31)}$. At primarylevel care facilities the focus needs to be on prevention of illness or disability and promotion of health and self-management, so as to prevent a further burden on the higher levels of the public health system. A recent media release ${ }^{(31)}$ by the Western Cape's Minister of Health reported on the challenges faced in the province wherebed occupancy is over $100 \%$ in many of the major secondary hospitals. At tertiary level, both out-patient clinics and inpatient services are often delayed or re-scheduled. Elective waiting lists for various surgeries are extremely long as there are restricted resources in terms of surgery time, theatre staff, beds, nursing and rehabilitative personnel. Elective surgeries are also often pushed back to accommodate the unacceptably high emergency waiting lists. This leads to further time delays and inevitably patients are forced to wait long periods before receiving recommended surgical management. Concerted efforts have been made to reduce these waiting times but the population in the Western Cape has had a sudden increase in number since $2001(28.8 \%)$ due to migration from surrounding provinces compounding the issues ${ }^{(31)}$.

Of particular interest to this study is the waiting time for surgery at one of three tertiary hospitals in the province; Tygerberg Hospital. The hospital,located in Parow, Cape Town, services in- and outpatients primarily from, but not restricted to, the Western Cape. Patients are usually referred from secondary hospitals or primarylevel care facilities in the surrounding areas to TBH for tertiary level care. The population of interest for this study are persons with osteoarthritis who have been referred to the orthopaedic department for consultation by the doctors concerned with arthroplasty surgery. Those requiring surgery are placed on the waiting list to electively receive a hip or knee joint replacement. The waiting list at TBH consists of over 3000 patients ${ }^{(32)}$ and average waiting time for surgery for a hip or knee joint replacement is between one to eight years ${ }^{(31)}$. 
Literature from higher income countries such as Canada, United Kingdom (UK) and New Zealand show that waiting for an arthroplasty has negative effects on a person's function and quality of life ${ }^{(70-72)}$ and that conservative management, led by a physiotherapist, is necessary while waiting for surgery ${ }^{(70)}$. Literature on the effects of pre-operative treatment has inconsistent views; a systematic review (SR) and meta-analysis (MA)was performed in 2011exploring pre-operative conservative and non-pharmacological interventions, mainly exercise with some including education, in those with $\mathrm{OA}$ of the knee and hip awaiting joint replacement ${ }^{(73)}$.It showed low to moderate evidence from small randomised controlled trials (RCT) exist that support the use of pre-operative treatment, specifically exercise, to reduce pain ${ }^{(73)}$. While, a more recent SR on pre-operative interventions while on a waiting list stated that exercise can improve pain and function in those with hip OA but not with OA of the knee ${ }^{(74)}$. The limited literature available justifies the need for further research into OA management while on a waiting list as well as to ascertain whether the effects, particularly of exercise, are different for those awaiting a hip or knee replacement ${ }^{(74)}$.

Loirg and Holman advised that the most likely option for pre-operative intervention would be to provide management at out-patient primary level facilities in the community ${ }^{(11)}$. Additionally group-based treatments which attend to a larger number of patients at once could potentially address the issue of waiting for individual time with a health professional. Treatment in the interimduring the waiting period has been shown to improve fitness and quality of life as well as having the added benefit of possible improvements in activity ${ }^{(73)}$ and perceived disability post-operatively ${ }^{(70)}$. Whether these recommendations are practical to implement and more importantly of benefit to the South African population with osteoarthritis of the hip and/or knee, attending Tygerberg Hospital is of interest. 


\section{iii. An attempt to address the issue in SA}

In order to explore the many factors related to osteoarthritis, a literature review on the different aspects surrounding $O A$ and the current management options was conducted. The following search engines were used: Medline, (PubMed, Ebscohost, OVID), Science Direct, Google scholar, Cinahl, Scopus, and Web of Science. Keywords used: ICF, musculoskeletal conditions, osteoarthritis, chronic disease, disability, health systems in SA, burden of disease, non-communicable diseases, guidelines for management of osteoarthritis, self-management, education, physiotherapy, exercise, weight loss, surgery, arthroplasty, waiting lists, effects of waiting for surgery, pain, chronic pain, pain physiology and pathophysiology, central sensitization in OA. The available literature of evidence-based research on this topic is presented below. 


\subsection{Overview of Osteoarthritis}

Osteoarthritis is defined as a degenerative joint disease, primarily affecting the articular cartilage and subchondral bone of the joint ${ }^{(75,76)}$. In simple terms; it is the breakdown of previously healthy joint surfaces or the loss of cartilage, causing friction between the bones ${ }^{(77)}$. Unlike rheumatoid arthritiswhich affects both sides of the body, OA is often asymmetrical $^{(78)}$. The course of progression differs for each person affected, but most commonly the condition progresses as one ages to result in extensive joint damage with deformities ${ }^{(19)}$.

Osteoarthritis is most common in the hip and knee joints ${ }^{(50)}$ and the small joints of the hands ${ }^{(79)}$. Classification between primary and secondary OA is made according to the cause, either known or unknown. Primary OA is of unknown origin or is idiopathic, and secondary $\mathrm{OA}$ is due to a specific cause or event ${ }^{(7)}$. The development and progression of $\mathrm{OA}$ is very seldom due to one factor alone, but rather a combination of two or more factors acting together ${ }^{(79)}$. Known causes or risk factors include but are not limited to the following ${ }^{(19,76,78,79)}$ :

- Increasing age - which means that other co-morbidities may co-exist ${ }^{(80)}$

- Genetic predisposition to developing OA, especially in the hands ${ }^{(77)}$

- Gender - females have a higher incidence of OA than males

- Obesity

- Faulty biomechanics

- Certain occupations, sports and/or repeated stresses on the joints

- Previous trauma

Diagnosis is relatively simple and is made primarily by clinical diagnosis including history taking and completing a physical examination of the patient and their main symptoms ${ }^{(78)}$. Radiological imaging such as an X-ray can aid in the diagnosis, but is not necessary in order to diagnose $\mathrm{OA}^{(78)}$. Notably, there is no correlation between osteoarthritic changes seen on X-ray and severity of clinical symptoms. In other words, some individuals may present clinically with severe arthritis however, show minimal changes on X-ray and vice versa ${ }^{(81)}$. Changes which may be seen on X-ray include decreased or narrowed joint space, breakdown of joint surfaces, and osteophyte formation amongst others ${ }^{(82)}$. 
Kellgren \& Lawrence ${ }^{\ddagger}$ describe a radiological scoring system used to diagnose $O A$ in the 1950 's, which is still utilised today. The American College of Rheumatology (ACR) has further classified the diagnosis of $\mathrm{OA}$ of the hip to include purely clinical symptoms, or clinical and radiological symptoms which are present or absent (Table 2-2below) ${ }^{(7)}$. Further radiology or laboratory tests are not often necessary or recommended in the diagnosis of this condition. The fact that initial diagnosis can be done without complicated tests or specialised doctors should make early diagnosis and management $O A$ at primarylevel facilities possible in order to decrease the growing burden of OA on tertiary institutes.

Table 2-2 ACR classification of OA of the hip

\begin{tabular}{|c|c|}
\hline $\begin{array}{l}\text { Clinical signs and symptoms- } \\
\text { pain present in combination } \\
\text { with either : }\end{array}$ & $\begin{array}{l}\text { - hip internal rotation(IR) greater than or equal to } \\
15 \text { degrees } \\
\text { - } \text { pain on IR of the hip } \\
\text { - } \text { morning stiffness of the hip for less than or equal } \\
\text { to } 60 \text { minutes } \\
\text { - older than } 50 \text { years } \\
\text { OR: } \\
\text { - hipIR less than } 15 \text { degrees } \\
\text { - erythrocyte sedimentation rate (ESR) less than or } \\
\text { equal to } 45 \text { mm/hour } \\
\text { if no ESR was obtained, hip flexion less than or } \\
\text { equal to } 115 \text { degrees was substituted }\end{array}$ \\
\hline $\begin{array}{l}\text { Clinical and radiological - } \\
\text { pain with at least two of the } \\
\text { following threecriteria: }\end{array}$ & $\begin{array}{l}\text { - osteophytes (femoral or acetabular) } \\
\text { - joint space narrowing (superior, axial, and/or } \\
\text { medial) } \\
\text { - ESR less than } 20 \mathrm{~mm} / \mathrm{hour}\end{array}$ \\
\hline
\end{tabular}

\footnotetext{
${ }^{\ddagger}$ Kellgren \& Lawrence scoring: Grade $0-4$ is used to rate the severity of changes seen on X-rays; $0=$ absent. $1=$ doubtful. $2=$ minimal. $3=$ moderate. $4=$ severe $^{(364)}$
} 


\subsubsection{Prevalence of $O A$}

\section{i. Worldwide prevalence}

As mentioned, $\mathrm{OA}$ is the most prevalent joint disease in high, middle and low income countries and is reported to be a leading cause of pain and disability worldwide ${ }^{(16-20)}$. Globally, OA has been reported to affect $18.0 \%$ of females and $9.6 \%$ of males over the age of $60^{(75,83)}$. As previously stated, due to advances in medicine, the population's life expectancy is predicted to continue to rise, contributing to a larger number of aging people ${ }^{(43)}$. Research states that $50 \%$ of all chronic conditions in persons over 65 years, are as a consequenceof joint conditions; thus further research is warranted in this field ${ }^{(43)}$.

In higher income countries, OA has been ranked as the condition having the fourth and eighth greatest impact on health in women and men, respectively ${ }^{(41,42)}$. Considerably more studies have been performed on the prevalence of OA in countries of higher income levels; showing that OA was third on the list for years lived with disability (YLD) in high income countries but wasn't even part of the top ten contributors to YLDs in low to middle income countries $^{(84)}$. A more recent study by Brooks ${ }^{(51)}$ showed thatlower income countries make up the majority of the burden of MSC with figures such as 21076 DALYs lost in lower income areas versus 8723 in high income countries, of which 11049 and 5323 respectively, were due to $\mathrm{OA}^{(51)}$. Woolf ${ }^{(50)}$ suggested that this is a consequence of limited resources and access to surgical techniques, such as a joint replacement for those with severe $O A^{(50)}$. However, these high figures could be due to a lack of rehabilitative personnel at a community and/or primary health care level in lower income areas. Early rehabilitation could facilitate better management of pain and dysfunction, prevent worsening of the condition and slow down progression to severe OA; thus lowering the number of DALYs ${ }^{(51)}$. Further research is needed in low to middle income countries to produce much neededcurrent data for these regions ${ }^{(51)}$. The above mentioned rising prevalence of $O A$, especially in lower income areas, contributes toincreasing health and social burden ${ }^{(85,86)}$.

\section{ii. South African prevalence}

The burden of indirect health care costs related to OA in SA may be underestimated as the associated co-morbidities, such as cardiovascular conditions and obesity, caused by lowered levels of activity, are frequently present and costly to manage ${ }^{(76,85)}$. 
In a recent prevalence study of MSC in the Western Cape ofSA, the most frequentconditions seen were HPT, joint problems and DM; showing that MSC together with such co-morbidities are a common occurrence in the population examined ${ }^{(87)}$. It is noted that many of these chronic conditions share the same risk factors of inactivity, unhealthy diets and abuse of substances ${ }^{(46)}$. Additionally disability associated with OA has been seen to be worse if two or more comorbidities are present ${ }^{(80,88)}$. As mentioned, due to insufficient current statistics, the prevalence of OA and RA was estimated to be much lower $(150 / 100,000)$ in Sub-Saharan Africa when compared to $1,500 / 100,000$ in Europe ${ }^{(84)}$. However, as seen in the recent study by Parker and Jelsma ${ }^{(87)}, \mathrm{MSC}$ is suggestedto be more prevalent than previously estimated in low to middle income countries such as SA. Their findings show that the prevalence rate of MSC is more likely to be closer to $362 / 100000^{(87)}$ when compared to the OA and RA combined value of only 144/100 000 in the Global BOD study in $1990^{(84)}$. OA is further reported to be the most common joint disorder and the leading cause of disability in SA, with specific reference to the Cape Town Metropolitan $\operatorname{area}^{(89,90)}$. In the 2000 South African BOD study however, MSC didnot feature in the top 20 conditions causing YLLs and were not mentioned under the YLD section either ${ }^{(63)}$. Nevertheless, it was reported that these statistics were incorrectly calculated and figures were said to be underestimated ${ }^{(63)}$. The figures of YLD and DALYS for SA in this study were said to be initial estimates rather than definite values as data used for this study was not based on current data but that of $1996^{(63)}$. This emphasises the need for reliable and up to date statistics to be made available ${ }^{(51)}$. The general health survey performed in SA in $2011^{(67)}$ reported $13.9 \%$ of the population aged 65 or older to be affected with arthritis. Once again this supports the larger estimatesof OA asa contributor to burden of disease in $S A^{(51,87)}$.

The cost of health care involved in $\mathrm{OA}$, from services rendered to resources used, is an important factor to consider when dealing with this condition and choosing the necessary management options as it is seldom that OA occurs in isolation ${ }^{(85,86,91)}$. It is proposed that in an attempt to deal with the increasing negative economic and social impact caused by OA, an effective chronic disease management approach be used ${ }^{(92)}$. An extensive description of examples of these approacheswith a specific focus on OA follow in the text. Of important note forthe purposes of this study, is that these management options have the potential to be reliably delivered by a physiotherapist ${ }^{(93)}$. 


\subsubsection{The impact of $O A$}

The extent of disability caused by MSC such as OA, has not been comprehensively addressed prior to the year $2000^{(43)}$. MSCs have been viewed as less important than other infections or fatal conditions as they are not primarily life threatening. Some MSC conditions are often regarded as a normal degenerative part of aging, but this does not mean the associated morbidity should go unnoticed. Health systems throughout the world have not properly prioritised dealing with the growing burden caused by these conditions $^{(11)}$. A possible reason for this could be due to the WHO's focus traditionally being on prevention and control of infections and mortality ${ }^{(43,59)}$. At last the need to draw further attention to the growing burden of MSC was highlighted in 1998 by the European Orthopaedic and Rheumatology divisions, resulting in the years 2000-2010 being declared the "Bone and Joint Decade"(43). The desired goals were to raise awareness of the current issues and to improve the health related quality of life of those suffering fromchronic musculoskeletal conditions ${ }^{(43)}$. Once again, the association between MSC and fatal comorbidities was emphasised and supported by later literature showing the incidence of $O A$ and co-morbidity was as high as $73 \%$ with an average of two co-morbidities being present in patients requiring surgery for $O A$ of the hip or knee in Europe ${ }^{(88)}$. This is an essential association as preventing and managing the health issues such as inactivity due to a MSC can begin to break the cycle of disability.

The aim of the Bone and Joint Decade was to achieve a decrease in health problems by "empowering patients to participate in their own care; by promoting cost effective prevention and treatment; and by advancing understanding of musculoskeletal disorders through research to improve prevention and treatment" ${ }^{\prime(43)}$. As implied in this aim; better understanding of MSCs will enable better management thereof ${ }^{(43)}$. Not only does this endorse the need for on-going research studies in this field, but furthermore, highlights the need for soundly educated health personnel to be able to deliver comprehensive education and treatment to patients with such conditions ${ }^{(11)}$. 
As stated in the aim above, the need to manage OA with the purpose ofminimising its impact on the sufferer's life is essential. In order to provide effective management, understanding of the common clinical presentation and main complaints of those affected by $\mathrm{OA}$ is necessary. The common signs and symptoms are described below; commencing with pain.

\section{a. Pain in $O A$}

The most common reason for seeking medical attention and the main symptom of OA is pain; a physical impairment according to the $\mathrm{ICF}^{(79,94,95)}$. The International Association for the Study of Pain (IASP) first presented the definition of pain in $1979^{(96)}$. The terms and definitions related to pain have been revised since then in 1986 and 1994, and the most recent definition updated in 2012 defines pain as "an unpleasant sensory and emotional experience associated with actual or potential tissue damage or described in terms of such damage ${ }^{\prime(97)}$. OA initially presents as acute pain, mainly experienced with movement or use of the affected joint ${ }^{(76)}$. As the condition progresses, the pain becomes chronic and often leads to pain at rest, at night or even spontaneous pain ${ }^{(76,95)}$.Additionally sufferers may present with acute and chronic pain at the same time; with exacerbations in pathology occurringtogether with chronic pain changes in the nervous system.

Chronic pain(CP)is defined by the IASP as "pain which persists past the normal time of healing ${ }^{\prime(4)}$, while others have broadly described CP as pain that is experienced on most days for at least three consecutive months ${ }^{(98)}$.Essentially chronic painis described as pain that does not resolve as expected after the normal repair process has been completed; it has been said that this type of pain is no longer protective in nature ${ }^{(2)}$. The time for "normal healing" after acute injury or pathology is complex as a result of the nervous system being changeable or better known as "plastic" ${ }^{(2,99)}$.Plasticity of the central nervous system (CNS) can prolong and maintain the pain experience well after the normal time of healing.

Previously, the mechanisms contributing to chronic pain in OA have not been well ${ }^{(95,100)}$. Various suggestions of how acute pain progresses to chronic pain have recently been proposed based on maladaptive changes in the nervous system ${ }^{(95,100-103)}$. 
This is an area whereby knowledge of theories or mechanisms related to nociceptive processes are constantly changing and are expected to continue to be updated as research is ongoing ${ }^{(104)}$. Further research is still needed to ascertain how the mechanisms ofCP changes influence prevention and/or effective management in various pain syndromes ${ }^{(8)}$. Recent advances in research have led to explanations for the changes that take place in chronic pain disorders, including $\mathrm{OA}^{(2,22)}$.

Chronic pain is believed to be a multifaceted process:the role of the CNS (the spinal cord and brain)is key; with various neurotransmitters modulating nociceptiveinput at many levels ${ }^{(22)}$. The major change that is said to take place in CP disorders that leads to the exaggeration of pain is sensitization of the nervous system. This is caused by an increase in the membrane excitability and synaptic efficacy in both the peripheral and central nervous system, that leads to neurones firing at a lower threshold ${ }^{(2,22)}$. It is explained that peripheral sensitization and its consequences are limited to the peripheral site of nociceptive input, causing primary hyperalgesia at that site. This usually requires ongoing nociceptive input or peripheral pathology to continuously contribute to the changes that take place in the nociceptive system ${ }^{(2)}$. Whereas, central sensitization results in both primary and secondary hyperalgesia by amplified nociceptive stimuli not only at the periphery, resulting in abnormally high levels of pain at the site of insult, but also affects other receptors such as mechanoreceptors which cause pain beyond the site of injury ${ }^{(2)}$.

Furthermore, neurones are also more sensitive to previously normalstimuli of touch or pressure; resulting in allodynia ${ }^{(2,22)}$. This is demonstrated when a normally innocuous stimulus, such as movement, is a cause of pain. This has been shown in studies where heightened activity is seen onfunctional magnetic resonance imaging ( $\mathrm{fMRI}$ ) in the regions of the brain that process pain to normal stimuli that usually do not cause pain ${ }^{(22)}$. Central sensitization changes in the nervous system are present long after the original nociceptive input and it is said that the body no longer generates pain due to a potential or actual threat, but rather as a consequence of the ongoing sensitization of the nociceptive system $^{(2)}$. 
Additionally, anirregularity in the modulation of the descending pain pathways also exists in CP. It seems as if the ascending pathways are unopposedbecause the descending endogenous pain pathways that inhibit pain are less active ${ }^{(2,22)}$. A fairly new area of research in patients with chronic pain draws attention to a dysfunction in the downward endogenous analgesic responsefollowing exercise in some pain conditions, such as chronic fatigue syndrome ${ }^{(105-107)}$, chronic whiplash ${ }^{(108)}$ and fibromyalgia ${ }^{(109)}$. This is of particular interest as exercise usually activates the endogenous pain pathways in healthy individuals and is used as an effective treatment approach to many chronic pain disorders such as $O A^{(109)}$. This is discussed in more detail in section 2.3.5 (pg. 62).

A recent SR by Lluch and colleagues ${ }^{(110)}$ has shown that central sensitization takes place in a subgroup (30\%) of patients with OA. This leads researchers to believe that OA is not simply a peripheral joint condition; instead pain in OA can also be driven by central nervous system changes. Further research into this particular field is encouraged as interventions, such as neuroscience education, exercise and centrally acting pharmacological agents that have the potential to target the maladaptive changes of the CNS in this subgroup should be explored $^{(110)}$. Central sensitization can also explain why the physical impairment of OA in the periphery is not always synonymous to the amount or area where pain is felt or the overall effect on the person's life ${ }^{(22,111)}$. Statistics show that up to $50 \%$ of persons with moderate to severe changes on radiology display no symptoms whatsoever of $O A$, and roughly $10 \%$ of individuals with moderate to severe knee pain show no arthritic changes on radiographs ${ }^{(112,113)}$. This can be attributed in some ways to psychological factors but these play a limited role; however changes in the nociceptive system driven by ongoing pathological peripheral input and central sensitization, are more likely reasons to explain these occurrences ${ }^{(22)}$. A study by Wideman and colleagues ${ }^{(114)}$ suggest that "many individuals with knee OA show a prolonged, sensitized response to routine activities of daily living (ADLs), such as walking". This study attributes sensitivity to activity to psychological factors, such as pain catastrophizing, as well as to peripheral and central sensitization ${ }^{(114)}$. This research presents a possible reason for low adherence rates to exercise or activity-based treatment in people with $\mathrm{OA}^{(114)}$. 
Another change seen in those with central sensitization is in the levels of neurotransmitters such as norepinephrine, gamma-amino-butyric acid (GABA), serotonin, glutamate, and substance P. Alterations in these substances account for frequently seen issues that chronic pain sufferers experience such as altered sleep patterns, changes in cognition, decreased alertness and mood disorders ${ }^{(22)}$. All of which indirectly influence the individuals' quality of life and daily functioning ${ }^{(115)}$. Furthermore, Smith et al. ${ }^{(115)}$ also state that disturbances in sleep are very likely related to further sensitization of the nervous system as a "direct contributor to both hyperalgesia and impaired endogenous pain modulation."

As mentioned earlier in the text, it is possible to have acute and chronic pain present at the same time, a factor which may confound the assessment and management of pain in $\mathrm{OA}^{(22,29,116)}$. Although patients may have late stage chronic $\mathrm{OA}$, pain is not necessarily present at all times and individuals usually experience periods of less or worse pain. Periods of sudden worsening of acute pain on chronic pain is named a flare up ${ }^{(22)}$.

The above advances in research have begun to improve understanding of the processes that occur with long-standing pain. These explanations are fundamental in order to address disorders of $\mathrm{CP}$ including $\mathrm{OA}$. There is evidence that untreated $\mathrm{CP}$ can lead to further disability and decreased quality of lifeas well as being an important risk factor in the development of common co-morbidities ${ }^{(87,98,117)}$. Research suggests that changes in our nervous system leading to widespread pain conditions, are a result of "inadequately treated chronic focal pain problems such as osteoarthritis or myofascial pain"(118). The important factor to note is that pain is a far more complex process than previously believed and whether acute or chronic it is clear that pain has an enormous effect on an individual's life.In order to address these effects, the different aspects need to be correctly measured ${ }^{(119)}$.Furthermore, the management of pain needs to address the full impact of pain by targeting all components contributing to pain; the nociceptive, the peripheral and the central nervous system, as well as other psychosocial aspects which influence the pain experience resulting in activity and participation limitations ${ }^{(119)}$. 


\section{b. Measurement of Pain}

As important asunderstanding the definition of pain and pain-related aspects is the accurate measurement of pain. Research suggests that simple measures of pain often depict misleading information and thus a comprehensive assessment of the full pain experience is necessary ${ }^{(120)}$. The Initiative on Methods, Measurement, and Pain Assessment in Clinical Trials (IMMPACT) group state that when measuring pain, the instrument used should not only evaluate pain on a uni-dimensional level, instead it should assess the efficacy of the intervention under concern in terms of pain intensity, physical functioning, emotional functioning, patient ratings of treatments received, symptoms and adverse events, and participant disposition ${ }^{(121,122)}$. Many different instruments exist which are used to evaluate pain and its consequences. Goldenberg and colleagues ${ }^{(22)}$ state that "there is no single best instrument to evaluate pain in the rheumatic diseases" and others state that the optimal tool for monitoring pain is still unknown ${ }^{(123)}$. For the purpose of this study's review, relevant examples of some of the frequently used instruments are discussed in the text below.

Commonly used in current research, including rheumatological conditions, is a patient selfadministered tool called the pain visual analogue scale (VAS) ${ }^{(124)}$ to vertically (VVAS) or horizontally (HVAS) rate pain intensity ${ }^{(125)}$. The VAS is a simple single-item, subjective report of a $100 \mathrm{~mm}$ long line with a pain description on either end and the patient is required to mark what their pain severity is on the line ${ }^{(126)}$. The Numerical Rating Scale (NRS) ${ }^{(127)}$ is based on the same structure but is less abstract as it includes numbers from $0-10$ on the line and the Verbal Rating Scale (VRS) is similarly used to verbally rate pain severity. All three have been shown to be valid and reliable and none has been reported to be more sensitive to detect change than the other ${ }^{(128)}$. However, NRS and VRS have been stated to be preferred by patients over the VAS and it is noted that errors in correctly completing the VAS are more common than the former scales ${ }^{(128)}$. However, it has been documented that patients with hip or knee OA have described the NRS as too simplistic a rating scale for the complexities of their pain experiences ${ }^{(129)}$.

A tool developed in the 1970's named the McGill Pain Questionnaire (MPQ) $)^{(130)}$, has been commonly used to measure pain in more than one domain. It evaluates quantity and quality of pain in three domains:sensory, affective and evaluative ${ }^{(130)}$. 
Despite this measure being used in a variety of conditions, Cleeland ${ }^{(131)}$ examined the appropriateness of this tool and yielded results that proved the instrument to be complicated and time-consuming to complete as it isextremely long (78 pain descriptions). This lead to a compact version being developed, the Short-form McGill Pain Questionnaire $(\mathrm{SF}-\mathrm{MPQ})^{(132)}$, which was completed with greater ease but seemed to lack qualitative data in comparison to the multi-faceted original version.

Specific instruments for measuring pain and its effects in $O A$ also exist. The Western Ontario and McMaster Universities Osteoarthritis Index(WOMAC) ${ }^{(133)}$ is the most frequently used instrument developed for the measuring of pain intensityand functioning in $\mathrm{OA}$ and RA patients. Whereas the Constant and Intermittent Osteoarthritis Pain measure was designed to evaluate the intensity, frequency and impact of pain on mood, sleep and quality of life ${ }^{(129)}$. Similar tools were developed for both instruments for specific use in either hip or knee conditions, including questions on pain, stiffness, ADLs, sporting activities and quality of life. Issues in answering the pain severity section of these instruments have been noted in the WOMAC tool, as pain is not a constant, but rather an intermittent and variable measure ${ }^{(120,134)}$. A limitation also exists in the answering of these tools particularly in domains of ADLs because if a person avoided certain activities, they did not answer the questions related to that activity completely or accurately, resulting in missing or incorrect data ${ }^{(120)}$. Therefore a tool with more commonly executed tasks could be more appropriate.

The Brief Pain Inventoryhas now become a regular assessment tool for measuring the severity of pain and the effects thereof ${ }^{(135)}$. It was developed as a generic tool as far back as 1982; the initial version called the Wisconsin Brief Pain Questionnaire(BPQ) $)^{(136)}$. From the original $\mathrm{BPQ}$, a long version and subsequently the more commonly used short version of the BPI was developed ${ }^{(35,137)}$. The short form (BPI-sf) is widely used in both clinical and research environments ${ }^{(135)}$. To correctly assess the complexity of pain, three domains were seen as important (sensory-discriminative, motivational-affective and cognitiveevaluative $)^{(138)}$. Differences between these terms were not easy to distinguish so two dimensions were then used instead of three. Simply put they are the sensory and reactive aspects of pain ${ }^{(9)}$. The sensory domain evaluates pain according to intensity and the reactive domain assesses the effect/interference of pain. 
To address the limitation found with the WOMAC scale of pain being a variable factor, the BPI evaluates pain severity on the person's "worst", "least", "average" and "now" pain over the last 24 hours instead of one score for intensity of pain during different tasks. Pain interference is scored by evaluating the effect of pain on an activity sub-dimension (walking, general activity and work) and four other aspects of life (relationships with others, enjoyment of life, mood forming the affective sub dimension and sleep) $)^{(135)}$. These two dimensions (severity and functioning) are the first two dimensions, described by the IMMPACT panel, which should be included in all research dealing with chronic pain evaluation ${ }^{(122)}$.

Psychometric testing has been performed on the BPI's use in cancer and non-cancer pain, such as acquired immuno-deficiency syndrome (AIDS), complex regional pain syndrome (CRPS), Fabry's disease, LBP and $\mathrm{OA}^{(139,140)}$. Literature has been published to support the use of this tool in the assessment of pain to distinctly depict pain intensity and itseffects on function $^{(141-143)}$. The BPI has high internal consistency in various languages and does not display cultural bias; instead it maintains the meaning and assessment of pain in these two domains $^{(144,145)}$. It demonstrated high levels of test re-test reliability in many studies $^{(136,140,146,147)}$,although older studies do not discuss this aspect ${ }^{(143,148,149)}$. Of particular interest to this study, the BPI has been used in studies of OA which reported good internal consistencies; particularlyfor the subscale of the pain interference ${ }^{(148,149)}$. This is particularly relevant when selecting an instrument to measure change over time and therefore the BPI was chosen as the primary outcome measure.

Further research by Williams and colleagues ${ }^{(139)}$ presented evidence for the use and validation of the tool in $\mathrm{OA}$ where the validation, consistency and reliability of the pain interference scale and sleep item of the BPI in OA patients was explored. Results provide strong support for both the responsiveness and construct validity of the BPI interference and sleep item scales in OA related pain ${ }^{(139)}$. It also confirmed the high internal consistencies previously seen with optimal alpha values of 0.89 and $0.82^{(139)}$. Test re-test reliability (intraclass correlation value $=0.81$ ) for the interference scale is higher than the normally accepted value of $0.70^{(150)}$. 
The intra-class correlation value for the sleep item was lower at 0.65 but is still seen as "good" and in this case it is seen as an accepted value considering the value is usually calculated for a multi-item scale and this was for a one-item scale ${ }^{(139)}$.The IMMPACT panel has specifically singled out the pain interference scale from the BPI as a recommended scale to use for assessment of pain-related functional impairment ${ }^{(128)}$.

As seen by the above complexities of pain and the many aspects related to the impact of pain, measuring pain as accurately as possible in the different domains is necessary to fully understand the effect pain has on an individual, livingwith a chronic condition such as OA.

\section{c. Stiffness, reduced range of movement (ROM) and strength in $O A$}

Besides pain being the most common complaint in persons with $O A$, stiffness, reduced $\mathrm{ROM}^{\S}$ and a decrease in muscle strength are also common impairments. Stiffness is described as the inability to move a part with ease or comfort ${ }^{(151)}$. Stiffness is particularly felt in the affected joint following a prolonged period in one position of sitting or standing (called the gelling phenomenon)and is also frequently experienced in the early morning after waking, lasting less than 30 minutes $^{(78)}$. As a consequence of pain and stiffness in a particular area, reduced ROMat the affected joint is also a common occurrence ${ }^{(19)}$. Full ROM is not achieved as often as needed to maintain normal ROM and thus the available range decreases over time. Range of motion at different joints in the body is necessary for functional activities of daily living ${ }^{(152)}$. Following restricted ROM, the muscles and stability structures surrounding that joint are not as mobile as before and are unable to function as intended during use of the joint ${ }^{(152)}$. Consequently, because muscles are seen as the force generators of movement, if movement is restricted, the ability of muscle to contract and the strength of that contraction will also be limited and decrease if not used regularly. Kisner and Colby ${ }^{(152)}$ note that insufficient muscular strength leads to activity limitations and participation restrictions. These impairments surrounding a joint lead to feelings of instability, described as "giving way, buckling or slippage" and are reported to have a large impact on function ${ }^{(153)}$.

\footnotetext{
${ }^{\S}$ Range of movement is defined as the normal full motion possible at a joint and therefore anything less than this is considered decreased $\mathrm{ROM}^{(152)}$. In order to maintain a normal range of motion, a joint needs to move regularly through its full range, either actively by the muscles across that joint contracting or active assisted/passively by an outside source ${ }^{(152)}$.
} 


\section{d. The measurement of stiffness, reduced ROM and strength}

The standard measurement of stiffness or decreased ROM is done by determining the available range of motion at a joint with a goniometer (measured in degrees) ${ }^{(154)}$. The measurement can be recorded passively by an external force moving a joint through the available range, or by the person contracting the muscles across the joint independently, or with assistance, to produce movement at that joint ${ }^{(152)}$. Measuring muscle $\operatorname{strength}^{* *}$ is a topic of debate in recent literature as many factors are involved during assessment ${ }^{(155)}$. Various methods are available for muscle strength testing and can involve measuring force or torque and/or the rate of force or torque. It appears that a single standard measure of muscle strength does not exist as testing conditions and equipment can differ and measuring techniques vary greatly because a muscle can be tested in different positions and contraction states ${ }^{(156)}$. Additionally, a single muscle versus a single or multiple muscle group also produce different measures ${ }^{(155)}$. It has been proposed that a standard method of muscle testing be developed to minimise confounding variables and issues with reliability ${ }^{(155)}$. It is noted that an important reason for measuring muscle strength is to assess functional movement performance; however other variables can confound this purpose of strength testing. Measures such as ROM or muscle strength can be useful when tracking progress over time but whether these objective measures hold meaning regarding functional performance is questionable. Objective functional testing is therefore indicated as more meaningful in relation to the effect of impairments such as stiffness, reduced ROM and MP in OA sufferers ${ }^{(157)}$. This is described in more detail below.

\section{ii. Activity limitations and participation restrictions in $O A$}

As noted above, physical impairments are not the only factors to consider in such a chronic pain disorder, as the impairment itself is usually not the biggest issue, rather the resulting challenges experienced due to the impairment are the issue. A survey carried out by the IMMPACT group $^{(119)}$ revealed that besides pain; functioning, well-being and health-related quality of life (HRQoL) were the most important domains affected in chronic pain sufferers. Thus instruments that measure the effect the condition has on these three aspects as well as sleep, mood, and energy should also feature in research in order to grasp the full effects of a condition such as $\mathrm{OA}^{(22,119)}$. The first of these aspects according to the IMMPACT group, functioning, is discussed in the text to follow.

\footnotetext{
${ }^{* *}$ Muscle strength is defined as the maximumforce(in N) or torque (in Nm) developed during maximal voluntary contraction under a givenset of conditions ${ }^{(365)}$
} 


\section{a. Function and disability}

Physical impairmentsare known to result in a decrease in function and limitations performing certain activities and participating in life situations ${ }^{(95,158)}$. Pain, stiffness and decreased ROM and muscle strength limit activities of daily living such as climbing out of bed in the morning, walking to the bathroom for toileting and personal hygiene purposes, standing up from a toilet or a chair, and domestic tasksare a frequent struggle ${ }^{(74)}$. Difficulty completing daily tasks which require sitting or standing for extended periods is common ${ }^{(119)}$; examples include certain occupations such as a desk job, or labour work respectively. Problems are commonly experienced in activities that require bending down, for instance bending during household tasks or cleaning, as well as activities that require kneeling, such as gardening, playing with small children or praying ${ }^{(119)}$.Furthermore, social activities such as sitting in a movie or attending church and daily tasks like standing in a queue at the bank, post office or shops are not easily carried out ${ }^{(119)}$. Joint locking or feelings of instability are reasons for issues experienced during walking and climbing stairs and persons often experience difficultyin standing up from a chair without arm rests due to lack of strength ${ }^{(78,159)}$. It has also been reported that in those with lower limb joint issues, the ability to work is largely affected ${ }^{(160,161)}$. It has been predicted that by the year 2030, 25 million people living in the United States of America (USA) will have various activity limitations due to arthritis. This has been said to be quite a conservative prediction not taking into account the very possible increases in obesity and other risk factorswith comorbiditiesalso likely to rise ${ }^{(160)}$.

\section{b. Measurement of Function and Disability}

According to the ICF model, assessment of function is imperative for determining health and disability status ${ }^{(162)}$. Scarce documentation exists on the use of quantitative assessment tools to evaluate change in function in chronic pain studies ${ }^{(36)}$. Many studies evaluate function in terms of self-reported questionnaires. However, recent literature shows that results obtained from an objective performance test provide additional and valuable information ${ }^{(163)}$. The reason forlack of use of objective quantitative measures is possibly due to the need for extra space and specific training of personnel administering the tests to ensure intra- and inter-rater reliability ${ }^{(164)}$. 
To the researcher's knowledge, only one SR has been published which aimed to assess different objective measures of physical performance in terms of their content and psychometric properties ${ }^{(164)}$. This review focused on studies in older persons (over 60 years) living in a single community area. The sample could have caused a floor effect ${ }^{\dagger+}$ as it excluded those in old age homes or frail care facilities (who very possibly have higher disability scores) ${ }^{(164)}$. Importantly, the review was also limited to those with general chronic condition/s. In total 12 instruments were reviewed and only three (the Short Physical Performance Battery, Physical Performance Test and the Continuous Scale-Physical Function Performance) had published evidence on properties of validity (concurrent and/or predictive), reliability (intra-class correlation coefficient values ranged from $0.70-0.99$ ) and responsiveness (effect sizes ranged from $0.48-1.25$ ), which can explain why many tests are not yet widely accepted ${ }^{(164)}$

An objective measure of function, as mentioned above, that has been extensively studied and modified into different versions is the $\mathrm{SPPB}^{(163)}$. A possible reason why the SPPB achieved such positive psychometric ratings could be as a consequence of having fewer domains as it only assessed lower limb function. Other measures, namely the PPT ${ }^{(165)}$ and theCS-PFP(166) ${ }^{(16 v a l u a t e}$ function of upper and lower extremities during day to day tasks of varying difficulty. Both have been tested for psychometric properties and displayed good values for the different domains but these studies were only completed in older persons and were not specific to OA. Additionally, the CS-PFP consists of 15 tasks compared to five and seven out of nine tasks in SPPB and PPT respectively and is described as multifaceted and thus does not prove as quick and simple to administer as the others ${ }^{(164)}$.

Another simple and commonly used objective measure of mobility, usually used in cardiopulmonary conditions, is the six-minute walk test ${ }^{(167)}(6 \mathrm{MWT})$. The distance covered by walking on a flat hard floor for six minutes is recorded. This measure has been shown to be valid and reliable as a tool for measuring mobility in older adults in general, yet not specifically for those with OA. In 2011 it was suggested that further research be performed in order to validate the 6MWT in chronic pain patients and in 2014 a pilot study was performed to begin the validation of this tool in this population ${ }^{(168)}$.

\footnotetext{
${ }^{+\dagger}$ Floor effect: occurs when a measure possesses a distinct lower limit for potential responses and a large concentration of participants score at or near this lower limit. In the case above, disability scores were low as the sample excluded those with more disability who possibly would have produced higher scores ${ }^{(366)}$.
} 
Other work, specific to those with OA awaiting a joint replacement, suggested that a selfpaced walk test ${ }^{(169)}$ (walking two lengths of 20 meters $(m)$ without exertion), the well-known reliable and valid timed "up and go" test ${ }^{(170)}$ (rising from a standard arm chair, walking at a safe and comfortable pace to a line $3 \mathrm{~m}$ away, crossing the line, turning, and returning to a sitting position in the chair) and a 6MWT (as above) would provide useful information alongside self-reported pain and function measures ${ }^{(171)}$. Furthermore, an aggregate functional performance time (AFPT) is obtained from a series of tasks used to objectively measure function ${ }^{(172)}$. This consists of a fifty feet timed walk, a 'Get up and Go' test and a stairs ascent and descent test. It was stated that a single test of function provides minimal information about overall functional ability and therefore the four scores are aggregated to produce a score of general functional ability ${ }^{(172)}$. At the time of this write up, no studies had reported on the reliability and validity of the AFPT.

Following on from the above is a well-documented standard measure of function consisting of more than the four functional tasks of the AFPT; called the Physical Performance Task Battery $^{(36)}$. The physical performance task battery is readily available for clinical and research-related use. It was initially used in patients with lower back pain ${ }^{(173)}$, cancer $^{(36)}$ and HIV/AIDS ${ }^{(174)}$ The physical performance task battery has been shown to be responsive to change, valid (discriminative, concurrent, construct and predictive) and reliable(test-retest, intra- and inter-rater; with intra-rater correlation coefficients ranging from 0.7 $0.9)^{(157,173,175)}$. It involves a series of nine short tasks which are easily administered using minimal equipment, with underlying constructs of speed, co-ordination and endurance ${ }^{(36)}$. The tasks are namely: picking up coins, tying a belt, reaching upwards, reaching forwards, putting on a sock, standing up from sitting on a chair with armrests, a 50-foot walk at normal speed and at fastest speed and a 6MWT. It provides objective measures for individual tasks that are performed on a daily basis and valuable insight into how the task is performed instead of merely reporting that it is difficult to perform ${ }^{(36)}$. When used in clinical practice, the clinician can establish ways to adjust the task or correct the manner in which the patient is performing the task so as to improve function as well as monitor progress ${ }^{(174)}$. Important to note is that these quantitative measure of function do not aim to replace qualitative self-report measures, but should be used concurrently as both sets of information arevaluable ${ }^{(163,174)}$. 
Similar to measures of function is the less commonly used concept of assessing disability. As described earlier in the text, despite physical impairment and even functional abilities, persons can present with various disabilities depending on their individual environments. The Stanford Health Assessment Questionnaire(HAQ) was published in 1980 as a selfreported questionnaire of health status ${ }^{(37,176)}$. The $H A Q$ is a generic 22 -item scale which includes a disability index assessing disability observed during common daily activities and a VAS pain index assessing severity of pain if present ${ }^{(37,176)}$. The disability scale comprises of eight categories of tasks namely; dressing, rising,eating, walking, hygiene, reach, grip, and usual activities made up of aseries of 20 questions related to these tasks involving both fine and gross motor skills of the commonly used joints ${ }^{(37)}$. Later, a shorter version was developedcalled theHAQ 8-Item Disability Scale. Psychometric testing has been reported to show excellent responsiveness to change as well as minimal values ranging from $0.1-0.22$ needed for meaningful clinical difference ${ }^{(37)}$. A high internal consistency reliability of 0.85 was previously reported ${ }^{(177)}$ and validity in terms of construct, convergent and predictive has also been established ${ }^{(37)}$. The HAQ has been used in different languages and in a wide range of population groups in terms of income levels, age, health status and conditions including $\mathrm{OA}$ and was therefore chosen as an outcome measure in the current study. A shortcoming of this scale, not of direct importance to this study but worth noting, is that it does not depict disability relatedto sensory organ defects such as eyesight, hearing, touch etc. or disability associated with psychiatric dysfunction ${ }^{(37)}$.

\section{iii. Personal factors in $O A$}

\section{a. Psychosocial effects}

As seen above, each measure has a limit as to what it evaluates. This shows the importance of utilising various instruments to fullyunderstand the effects of the condition being investigated. Important factors have been acknowledgedin earlier text such as cognitive and emotive effects of chronic pain conditions which influence well-being and quality of life ${ }^{(50,119)}$. Hurley and colleagues ${ }^{(178)}$ have described the extent to which CP influences a person and highlighted the interactions between biological, psychological, socioeconomic and environmental factors (as depicted by the ICF model). Interestingly, these authors report that the emotive and cognitive changes appear to beunrelated to the physical aspects of $\mathrm{OA}$ and that changes in these dimensions could more reasonably explain activity limitations and participation restrictions in people with $O A^{(178)}$. 
Appealingqualitative research in persons with MSC, was performed in Europe to foster better understanding of persistent pain on the entire existence of an individual ${ }^{(179)}$. Three major themes wererevealed regarding responses to chronic pain: "failed adaption, identity restoration and finding a way out" ${ }^{\prime 179)}$. The first theme is described by the individual who has failed to successfully adapt to changes experienced while living with persistent pain; this seemed to cause frustration, depression, passive reliance on medication and avoidance of movement or exercise. The other two themes explain how a person can react to persistent pain in a more positive manner by re-evaluating their thoughts and attitudes towards pain to form a new identity within the condition despite radical changes that have occurred. This attitude encourages active coping mechanisms such as physical activity, helpful awareness of one's body, ongoing social interaction and personal growth ${ }^{(179)}$. Studies such as this underline the need to include psychosocial variables such as quality of life and SE for example, as a part of assessing the impact of OA on a person's life.

It has been noted that the inability to take part in normal daily tasks or being able to participate in usual happenings or recreational activities, has immenseeffects on a person's psychosocial health and overall quality of life ${ }^{(174,180)}$. Commonly frustration, anger and depression are experienced due to the inability to perform normal activities related to home and work life ${ }^{(179,181)}$. This causes a cascade of fear of movement, avoidance of activities, anxiety, dependence on others, shameand low SE; all of which are extremely demotivating for the individual ${ }^{(179)}$.Pain and disability impacts negatively on social interaction between friends, dynamics at home, putting strain on relationships between spouses and/or children, especially if these persons also assume the carer role ${ }^{(119)}$. As a consequence, individuals isolate themselves which then leaves them with lower selfesteem andminimal support structures to deal with the changes in their lives ${ }^{(179)}$. Therefore the combination of physical and psychosocial factors impacting on participation and function as one factor affects another which affects yet another, leading to a downward spiral in quality of life. 


\section{b. Psychosocial Measures}

Two emotional assessment tools that have been accepted and recommended by IMMPACT in chronic pain conditions are: the Beck Depression Inventory $(\mathrm{BDI})^{(182)}$ and the Profile of Mood States(POMS) ${ }^{(183)}$. These tools evaluate emotional distress and have been shown to be reliable and valid during psychometric testing in chronic pain trials ${ }^{(184)}$. The BDI isdesigned to measure the severity of depression and has been used in previous studies on chronic pain and HIV ${ }^{(185)}$. The POMS scale has specific scales for the three most commonly experienced emotional dysfunctions mentioned above; depression, anxiety and anger as well as other emotional distress associated with $\mathrm{CP}^{(128)}$. An interesting point has been raised regarding the commonly displayed symptoms of depression in this population; "decreased libido, appetite or weight changes, fatigue, and memory and concentration deficits" ${ }^{\prime 186)}$. Confusion concerning the direct cause of these symptoms exists, as the same symptoms can often present as a result of changes taking place in CNS from longstanding pain or as side-effects of medication commonly used ${ }^{(186)}$. Thus monitoring symptoms of depression as a result of a specific intervention in clinical trials can possibly provide insight into what may be contributing to these symptoms depending on the manipulated variables (either physical impairments, mood disturbances or a combination of these $)^{(187)}$. However for the purpose of this study, depression or anxiety was not the primary outcome measure and therefore monitoring of these emotional aspects were performed to a small degree as a part of the HRQoL (EuroQol 5 Dimension) outcome measure discussed below.

Well known generic measures of quality of life are: The Medical Outcomes Study 12-Item Short Form Health Survey (SF-12) $)^{(188)}$ derived from the commonly used Medical Outcomes Study 36-Item Short-Form Health Survey (SF-36) ${ }^{(189)}$ and the EuroQol 5 Dimension (EQ$5 D)^{(190)}$. The SF-12 and the EQ-5D comprise of 12 and five questions respectively. Questions are related to different physical and mental components, including pain and depression, to create a health status index ${ }^{(188)}$. Both of these measures are accepted in terms of reliability, validity and response to change in chronic conditions. They are easily administered and thus widely used in research and clinical practice to determine quality of life. It has been noted in a comparison study of the two instruments that the EQ-5D is more applicable and sensitive to population groups with higher morbidity and the SF-12 is better used in persons with less severe morbidity ${ }^{(191)}$. Furthermore, Brazier and colleagues found the EQ$5 \mathrm{D}$ to be more appropriate for the use in persons with $\mathrm{OA}$ of the knee awaiting surgery than the SF-36 ${ }^{(192)}$. For this reason the EQ-5D was utilised in the present study. 
The belief in one's self perceived capability to perform a task or achieve a goal has been defined as self-efficacy; the higher one's confidence is in the ability to carry out a task to completion, the higher one's SE ${ }^{(12)}$. This aspect has become increasingly more important in self-management strategies as people with chronic diseases have been shown to have lower SE than others, and therefore SE should be included as an outcome in clinical trials and practice ${ }^{(193,194)}$. Self-efficacy has a greater impact on behaviour than previously thought; it affects a person's physical activities as a person more confident in their ability to carry out a task, is more likely to attempt it and re-attempt it (compared to those who do not believe they can achieve the task at all) ${ }^{(195)}$.

Additionally, encompassed in SE are the skills to evaluate a situation, set desired goals and form expectations linked to goals made. Self-efficacy influences the amount of effort put into achieving a goal, reaction to challenges experienced, emotional well-being and beliefs held regarding individual's impairments and how this affects their activities in daily tasks and participation in social situations ${ }^{(196)}$. Studies have shown that probable correlations exist between SE and many different domains in arthritis patients such as: pain, coping strategies, stiffness, function, mood and mental well-being ${ }^{(197-199)}$. All of the above factors impact the behaviour of the patient and thus functioning. Recognition of the role of SE and the importance of enhancing SE during the management of $\mathrm{OA}$ is fundamental to bring about change in all arms of the ICF model ${ }^{(200)}$.

Various SE scales exist, most of which are specific for certain conditions or domains such as the exercise- or pain self-efficacy scales ${ }^{(201-204)}$. Different arthritis SE scales have been developed; beginning in 1989 with the Arthritis Self-Efficacy Scale (ASES) ${ }^{(205)}$ followed by a shorter version, the ASES-8 ${ }^{(206)}$. The 8-item version includes two items on pain, four items on other symptoms, and two items that relate to preventing pain and fatigue from interfering with daily activities. Importantly, the English version of ASES-8 has not been supported by psychometric evidence in literature and therefore it cannot be assumed that this scale is appropriate for use in English populations yet ${ }^{(204)}$.

From previously used scales, six items were selected to develop a short generic scale called the Stanford Self-Efficacy for Managing Chronic Disease 6-Item Scale ${ }^{(177)}$. 
This scale is seen as an improvement from previous scales as it covers appropriate aspects involved in determining SE in chronic diseases (setting and achieving goals, managing conditions in general and symptoms), as well as being easy to use $\mathrm{e}^{(177,204)}$. It was noted that not all questions may be appropriate for various chronic diseases; however with the rising trend in multiple chronic diseases of lifestyle or co-morbidities present in the global population, this factor does not seem to hold much weight as a negative for this scale and it is still recommended for use above disease- or task specific instruments ${ }^{(194)}$. Furthermore, this scale's limited psychometric results have yet to be formally published however some results have been made available online. Despite this, the 6 -item scale has been and is currently widely used for research purposes and was therefore chosen as a secondary outcome measure in this study ${ }^{(204)}$.

\subsubsection{Summary}

As discussed in the above section, effective assessment of the full impact caused by OA and the progression thereof, not only deals with the physical ailments or impairments, but evaluatesall areas of the ICF model; namely activity limitations, participation restrictions and personal and environment factors (psychosocial) as well. After including all domains affected by such a chronic pain condition in the assessment, it is even more essential to target each area during the management of OA. Having said this, the literature on best available management options will be extensively reviewed below. 


\subsection{The Management of OA}

\subsubsection{Evidence-based and expert consensus guidelines}

There is no known cure for $\mathrm{OA}$, nor one definitive solution to manage the many aspects of this condition ${ }^{(207)}$. Addressing the disease related factors and management thereof needs to be carefully balanced in order that the benefits of treating each factor outweighs the risks of others ${ }^{(76,207)}$.Since $O A$ is not a simple condition to treat, various evidence-based and expert consensus recommendations for management of the condition ${ }^{(207)}$ have been put forward over the last 20 years ${ }^{(23-25,27,208)}$.Guidelines were first published as far back as 1993 by the Royal College of Physicians ${ }^{(209)}$ and later by the ACR in 2000 on treatment of OA of the hip and $k_{n e}{ }^{(210)}$. The European League Against Rheumatism (EULAR), Osteoarthritis Research Society International (OARSI), National Institute for Health and Clinical Excellence (NICE), American Academy of Orthopaedic Surgeons (AAOS) and the newly revised ACR guidelines have been developed since then and are discussed below.

\section{i. EULAR guidelines}

The first of the guidelines is based on a review of evidence-based literature on treatment of $\mathrm{OA}$ in the knee that was conducted in $2000^{(211)}$. This research was subsequently updated in $2003^{(23)}$, along with a new publication on recommendations for management of OA of the hip in $2005^{(24)}$. These studies utilised research available from 1966 until 2004 and assessed the quality of literature reviewed using the evidence hierarchy ${ }^{\ddagger \ddagger}$. This means of literature appraisal distinguishes studies by ranking themaccording to methodological or design differences. Grading for how strongly a proposed treatment is recommended was also done by means of the letters $A-D^{\S \S}$. Conclusions drawn from their development process presented 10 recommendations for the management of OA of the knee and hip(see Table 2-3below-note the table shows both columns if the recommendation differedfor the knee and hip, otherwise the recommendation applies to both joints).

\footnotetext{
${ }^{\ddagger \ddagger}$ Hierarchy of evidence:la Meta-analysis of randomised controlled trials (RCTs), Ib RCT, Ila Controlled study without randomisation, Ilb Quasi-experimental study, III Non-experimental descriptive studies, such as, comparative, correlation, and case-control studies, IV Expert committee reports or opinion or clinical experience of respected authorities, or both. ${ }^{\text {(354) }}$ $\S^{\S}$ Strength of recommendation: A: Category I evidence. B: Category II evidence or extrapolated recommendation from category I evidence. C: Category III evidence or extrapolated recommendation from category I or II evidence. D: Category IV evidence or extrapolated recommendation from category II or III evidence ${ }^{(23)}$
} 
In summary, the foremost recommendation stated that both pharmacological and nonpharmacological methodsare to be combined for effective management of $\mathrm{OA}^{(23)}$. One of the most widely agreed upon guideline indicated that non pharmacological methods, with emphasis on education and exercise, are well supported by evidence ratings of $1 \mathrm{~A}$ and $1 \mathrm{~B}$ respectively ${ }^{(23)}$.Furthermore, weight loss and the use of supportive appliances, although only supported by weak evidence, are still strongly encouraged by expert opinion. This is due to the shortcomings noted in the evidence rating system used in these recommendations as discussed below ${ }^{(23)}$.

Concern has been raised over the suitability of using the evidence hierarchy when critically reviewing literature and providing recommendations for or against future use of different methods ${ }^{(24)}$. In some cases, especially studies on surgical procedures, ethical issues prevent high rated studies, such as a RCT, from being executedand thus research in such areas is rated as a lower category level $\mathrm{III}^{(24)}$. Suggestions have been made for the development of a similar rating system that would be more appropriate than the evidence hierarchy to be used in such cases so as to not exclude studies due to these ethical issues ${ }^{(24)}$. The strength of recommendation in the EULAR review was mainly based on the level of evidence for that specific treatment option, but was said to consider other aspects such as effect size (ES), side effect profile, applicability to population in question and practical and economic concerns $^{(23)}$. How much these other aspects were taken into account is debatable. Therefore, since the strength of these recommendations is based mainly on the evidence hierarchy and unknown weightings of other aspects, more recent guidelines with different methods of rating of evidence should be consulted in conjunction with the EULAR recommendations.

Despite the above, these guidelines were robust in their development through expert opinion using a 5-stage Delphi technique. An additionalstrength of the EULAR guidelines is that evidence-based literature included was not limited to English, but included European languages as 13-14 experts on the panel were from various European countries. A weakness however, was that the experts were solely European and this should be kept in mind when using this data internationally, especially in low to middle income countries such as SA. 
This shortcoming is acknowledged in the guideline with a statement that each country should utilise the recommendations to form their own country-specific guidelines, as so far only a limited representation of countries were involved in the development and the results should be applied to each different population and environment as appropriate ${ }^{(23)}$. Whether the same recommendations can be applied in lower income areas with limited resources and lower education levels is to be considered. It is also worthy to note that the research by EULAR examined the treatment effects of modalities on pain and/or function alone, and did not include effects on other aspects, such as psychosocial components, affected by OA. A further point raised is that data relating specifically to the hip is scarce and that further research is needed into this condition to provide more insight into OA affecting this joint. 
Table 2-3EULAR recommendations for the management of OA of the kneeand hip

\begin{tabular}{|c|c|c|}
\hline & Knee $^{(23)}$ & $\mathrm{Hip}^{(24)}$ \\
\hline 1 & \multicolumn{2}{|c|}{$\begin{array}{l}\text { The optimal management of } \mathrm{OA} \text { requires a combination of non-pharmacological and } \\
\text { pharmacological treatment modalities }\end{array}$} \\
\hline 2 & \multicolumn{2}{|c|}{$\begin{array}{l}\text { The treatment of knee and hip OA should be tailored according to: } \\
\text { (a) Knee and hip risk factors (obesity, adverse mechanical factors, physical activity and } \\
\text { dysplasia for hip) } \\
\text { (b) General risk factors (age, sex, comorbidity, polypharmacy). } \\
\text { (c) Level of pain intensity and disability/handicap. } \\
\text { (d) Sign of inflammation - for example, effusion. } \\
\text { (e) Location and degree of structural damage. } \\
\text { (f) Wishes or expectations of the patient }\end{array}$} \\
\hline 3 & \multicolumn{2}{|c|}{$\begin{array}{l}\text { Non-pharmacological treatment: regular education, exercise, appliances (sticks, insoles, knee } \\
\text { bracing), and weight reduction }\end{array}$} \\
\hline 4 & \multicolumn{2}{|c|}{$\begin{array}{l}\text { Paracetamol is the oral analgesic to try first and, if successful, the preferred long term oral } \\
\text { analgesic }\end{array}$} \\
\hline 5 & $\begin{array}{l}\text { Topical applications (Non-steroidal anti- } \\
\text { inflammatory drug (NSAIDs)and capsaicin) } \\
\text { have clinical efficacy and are safe in knee OA }\end{array}$ & $\begin{array}{l}\text { No mention of the topical applications for the } \\
\text { hip }\end{array}$ \\
\hline 6 & \multicolumn{2}{|c|}{$\begin{array}{l}\text { NSAIDs should be considered in patients unresponsive to paracetamol. In patients with an } \\
\text { increased gastrointestinal risk, non-selective NSAIDs and effective gastro-protective agents, or } \\
\text { selective COX- } 2 \text { inhibitors should be used }\end{array}$} \\
\hline 7 & \multicolumn{2}{|c|}{$\begin{array}{l}\text { Opioid analgesics, with or without paracetamol, are useful alternatives in patients in whom } \\
\text { NSAIDs, including COX-2 selective inhibitors, are contraindicated, ineffective, and/or poorly } \\
\text { tolerated }\end{array}$} \\
\hline 8 & $\begin{array}{l}\text { Symptomatic slow acting drugs for OA } \\
\text { (SYSADOA -glucosamine sulphate, } \\
\text { chondroitin sulphate, diacerein, hyaluronic } \\
\text { acid) have symptomatic effects and may } \\
\text { modify structure }\end{array}$ & $\begin{array}{l}\text { SYSADOA have a symptomatic effect and low } \\
\text { toxicity, but effect sizes are small, suitable } \\
\text { patients are not well defined, and clinically } \\
\text { relevant structure modification and pharmaco- } \\
\text { economic aspects are not well established }\end{array}$ \\
\hline 9 & \multicolumn{2}{|c|}{$\begin{array}{l}\text { Intra-articular injection of long acting corticosteroid is indicated for flare ups of pain, especially } \\
\text { if accompanied by effusion in the knee and those unresponsive to analgesics and NSAIDs. }\end{array}$} \\
\hline
\end{tabular}


10 Joint replacement has to be considered in patients with radiographic evidence of OA who have refractory pain/disability. Osteotomy and joint preserving surgical procedures should be considered in young adults with symptomatic hip OA, especially in the presence of dysplasia or varus/valgus changes. 


\section{ii. The OARSI guidelines}

Since the EULAR recommendations are now over 10 years old, more recent guidelines are discussed. The OARSI group ${ }^{(25)}$ published guidelines in 2008 which were anticipated to be more adaptable for countries worldwide. Firstly, a thorough appraisal of existing literature and new evidence was done, including literature as old as 1945 until more recently, 2006. A notable difference in the development of these guidelines compared to the EULAR guidelines was evident in the use of an assessment tool to review the quality of the guidelines developed. The appraisal of guidelines research and evaluation (AGREE) ${ }^{(212)}$ instrument was utilised. This tool was accepted by the WHO to appraise the guidelines which they regularly develop ${ }^{(212)}$. This tool was the first of its kind to be developed and tested internationally to assist with the increasing number of new guidelines being produced in clinical practice ${ }^{(212)}$. This tool is exclusively focused on the quality of the process of development and reporting of guidelines and does not evaluate the quality of subject matter or data being discussed ${ }^{(212)}$. See Table 2-4below for the AGREE criteria that must be met in order to classify a clinical practice guideline as high quality. 
Table 2-4 AGREE criteria for high quality guidelines

\begin{tabular}{|c|c|}
\hline $\begin{array}{l}\text { 1. Scope and } \\
\text { purpose }\end{array}$ & $\begin{array}{l}\text { Contain a specific statement about the overall objective(s), clinical } \\
\text { questions, and describes the target population. }\end{array}$ \\
\hline $\begin{array}{l}\text { 2. Stakeholder } \\
\text { involvement }\end{array}$ & $\begin{array}{l}\text { Provide information about the composition, discipline, and relevant } \\
\text { expertise of the guideline development group and involve patients in } \\
\text { their development. They also clearly define the target users and have } \\
\text { been piloted prior to publication. }\end{array}$ \\
\hline $\begin{array}{l}\text { 3. Rigour of } \\
\text { development }\end{array}$ & $\begin{array}{l}\text { Provide detailed information on the search strategy, the inclusion and } \\
\text { exclusion criteria for selecting the evidence, and the methods used to } \\
\text { formulate the recommendations. The recommendations are explicitly } \\
\text { linked to the supporting evidence and there is a discussion of the health } \\
\text { benefits, side effects, and risks. They have been externally reviewed } \\
\text { before publication and provide detailed information about the } \\
\text { procedure for updating the guideline. }\end{array}$ \\
\hline $\begin{array}{l}\text { 4. Clarity and } \\
\text { presentation }\end{array}$ & $\begin{array}{l}\text { Contain specific recommendations on appropriate patient care and } \\
\text { consider different possible options. The key recommendationsare easily } \\
\text { found. A summary document and patients' leaflets are provided. }\end{array}$ \\
\hline 5. Applicability & $\begin{array}{l}\text { Discuss the organisational changes and cost implications of applying the } \\
\text { recommendations and present review criteria for monitoring the use of } \\
\text { the guidelines. }\end{array}$ \\
\hline $\begin{array}{l}6 . \quad \text { Editorial } \\
\text { independence }\end{array}$ & $\begin{array}{l}\text { Include an explicit statement that the views or interests of the funding } \\
\text { body have not influenced the final recommendations. } \\
\text { Members of the guideline group have declared possible conflicts of } \\
\text { interest. }\end{array}$ \\
\hline
\end{tabular}

The same Delphi approach as used in the development of the EULAR guidelines was used to produce the OARSI recommendations. In this case a 6-rounded Delphi technique was executed which generated 25 recommendations; many of which were similar for the hip and knee joint. OARSI's recommendations have been expanded from the mere 10 pointsmade by EULAR. 
The main recommendation confirms earlier proposals of optimal management by including both pharmacological and non-pharmacological treatment methods ${ }^{(25)}$. Besides agreeing with the other recommendations made by EULAR, a few additional recommendations are discussed. Strong emphasis is again put on the importance of education and selfmanagement by the patient becoming actively involved in the management of their condition. Additionally, not only is commencement of self-management stressed but importance is put on the need for adherence to these strategies ${ }^{(25)}$. Telephonic communication with patients is also encouraged. Emphasis is placed on sessions with a physiotherapist for education and instruction in different types of exercise as well as shortterm relief from symptoms using various physiotherapy modalities if necessary ${ }^{(25)}$. Surgical options reiterate the role of arthroplasty and smaller joint preserving techniques, but add further suggestions of surgical options such as a uni-compartmental replacement orfusion of the joint if knee arthroplasty has failed.

A strength of the OARSI recommendations by Zhang and colleagues was the rigorous critical appraisal of existing guidelines and a review of current research. This was done prior to the updated guidelines being developed, and published as a full report ${ }^{(213)}$. The authors raised concerns as to the quality of the evidence-based literature upon which previous reviewers were basing their decisions. The need for internationally accepted guidelines was also made clear after the first part of this work was completed. To address this, a multinational team, not only from the European regions but also from USA and Canada were included on the expert panel. Detailed accounts of all methodological processes were clearly presented ${ }^{(25)}$.

A key difference was noted between the EULAR and OARSI's order of development; the EULAR method firstly performed the expert opinion consensus and then proceeded to research the literature available on those 10 treatment options, whereas the OARSI technique initially performed the evidence-based research review and subsequently undertook the Delphi consensus. This minor difference attributes the OARSI process as more "evidence-driven and clinically supported" instead of vice versa; making the OARSI process less biased ${ }^{(213)}$. Additionally, another two processes were carried out which were not done by the EULAR group during development. 
A small pilot survey was undertaken to assess the perceived usefulness of the existing guidelines, although it was limited this assisted in confirmation of the core treatment modalities used. Secondly, besides the expert panel, members of the public were able to review and comment on the proposed OARSI recommendations during the six rounded Delphi process. These two supplementary consultation steps make these recommendations more robust ${ }^{(25)}$.

Another strength to be brought to attention is the approach used to rate the level of evidence; a VAS is recorded instead of the evidence hierarchy method ${ }^{(214)}$. As indicated in earlier text, the issue of rating evidence is troublesome as appropriate evidence may not be strongly ranked due to ethical and practical issues preventing the gold standard of studies, a RCT, from being performed ${ }^{(24)}$. The VAS allows an RCT to be rated appropriately by creating a rating which includes both evidence and expert opinion from clinical practice ${ }^{(214)}$. This upgrades the strength of recommendation as it is based on overall effectiveness and not solely on study design ${ }^{(25)}$.

Despite the many strengths discussed above, a number of flaws exist within the OARSI guidelines $^{(25)}$. Firstly, the representation of multidisciplinary professionals was small with 11 of the 16 experts being rheumatologists. An attempt to minimize this limitation was made by including the perception of usefulness survey (available to other members of the health team) as well as allowing the OARSI members to share their opinions on the proposed guidelines during the Delphi process ${ }^{(25)}$. It was also mentioned that new evidence had become available after the closing date for review of literature in early 2006; whether this literature would have had any effect on the end guidelines is unknown. But this is the case with all research; new studies are constantly being performed, thus highlighting the need for updated guidelines and constant evidence based literature reviews.

\section{iii. NICE guidelines}

The National Institute for Health and Clinical Excellence ${ }^{(80)}$ is another group who drew up recommendations in 2008. Similar core standards of treatment, as those discussed above, are recommended together with minor changes. The use of electro-acupuncture and glucosamine and chondroitin as a SYSADOA was excluded from this guideline. 
The recommendation against the use of the above supplements has been questioned by Dalbeth and Arrol ${ }^{(215)}$ as high quality studies do exist to support the use of glucosamine and chondroitin in knee OA. Thus this remains unclear and is thought to be safer to rather exclude use until consensus is reached on this point.Further emphasis is needed on the use of topical NSAIDs in affected hands and/or knees, as this method of management was said to be underused despite evidence for clinical effectiveness and high safety ${ }^{(215)}$.Another difference was that surgical arthroscopic lavage and debridement is not recommended for all patients. Rather, this procedure should be recommended for specific patients who may benefit from this procedure ${ }^{(27)}$.

Other criticism of the NICE guidelines was laid out by Dalbeth and Arrol ${ }^{(215)}$ : interestingly the small effect size of most of the treatments recommended was mentioned. Only exercise and oral NSAIDs had a moderate effect size, the other recommendations were based on low efficacy or small effect sizes. The suggestion for patients to be questioned qualitatively to obtain their subjective opinions on the efficacy of such treatment options was also made ${ }^{(215)}$.

On a more positive note, these guidelines highlighted gaps in the effective implementation of previously recommended treatments. The authors identified the need for adequate funding, allied health professionals and surgeons who can deliver such treatment modalities to bridge those gaps ${ }^{(215)}$. Furthermore, physiotherapists, occupational therapists, dieticians and/or other appropriate personnel were noted as a prerequisite to provide education and exercise to successfully implement the suggested treatment options ${ }^{(215)}$.

\section{iv. The AAOS guidelines}

After the guideline by NICE was published, further work was done by the American Academy of Orthopaedic Surgeons ${ }^{(216)}$ in the same field. This academy published their guidelines for treatment of knee OA in 2010 which consisted of 22 recommendations with the focus steering more in the specific direction of self-management programmesprior to recommending similar modalities as the previous guidelines such as exercise, weight loss etc. According to Richmond et al. ${ }^{(216)}$ this guideline was "developed using systematic evidence-based processes designed to combat bias, enhancetransparency, and promotereproducibility." 
Differences between the AAOS and previous guidelineswere notedby a number of inconclusive recommendations reported.The use of a few modalities were neither supported nor discouraged namely; the use of bracing for either lateral or medial unicompartmental OA of the knee, acupuncture for pain relief, intra articular hyaluronic acid injection and an osteotomy in patella-femoral OA. This discrepancy leads one to question the quality of the original data that supported the use of these options, or the necessity to gain further information on the new data available. Until thorough evidence-based decisions have again been made on such modalities, recommendations for the use of these modalities remain inconclusive ${ }^{(216)}$. Also, as in the OARSI guideline, evidence has shifted since the EULAR recommendations were developed and some modalities that were earlier supported, are no longer recommended. Once again, SYSADOA such as glucosamine, chondroitin sulphate or hydrochloride, the use of lateral heel wedges or joint washouts are not recommended ${ }^{(216)}$. This further strengthens the use of the options that are supported by evidence-based literature such as education and exercise. Once again, these evidencebased modalities were reported to have the potential to be delivered by health professionals such as physiotherapists ${ }^{(93)}$. Bryant and colleagues ${ }^{(217)}$ state that physiotherapists "are well placed to deliver treatments that integrate physical and psychosocial elements."

\section{The ACR guidelines}

Even more recently revised evidence based treatment guidelines were published in 2012, by the $A C R^{(208)}$.The guidelines once again concur with previous guidelines; recommending a holistic approach for the management of $O A^{(208)}$. This work waspublished as an update of their first recommendations made for the management of $\mathrm{OA}$ of the hip and knee in $2000^{(218)}$. The need for this revision was expressed due to improvements in the methods of drawing up such guidelines. More importantly, new literature had become available on the current management for knee and hip OA. The revision also aimed to develop guidelines for OA of the hand which had not previously been conductedin such a manner. A strength of this work is once more noted by the rigorous method of development of these updated guidelines. This was also completed by a more balanced and diverse range of health experts (academic and practicing rheumatologists, primary care physicians, physiatrists, geriatricians, orthopaedic surgeons, and one occupational and two physical therapists)reaching consensus on the available evidence based research. 
This is seen as an improvement from the expert panel in 2000 (a mere four rheumatologists from the USA) and when comparing to the EULAR and OASRI guidelines which had limited interdisciplinary input.However, a weakness is noted in that it also only included stakeholders from the USA and Canadainstead of extending the panel to other countries. This should be taken into consideration when applying these guidelines internationally, especially to developing countries, such as SA.

In ACR this update, consensus was made by means of a formal process called the Grading of Recommendations Assessment, Development and Evaluation (GRADE) approach to ensure that the results were as evidence based as possible ${ }^{(207,219)}$. This approach has also been adopted by the WHO, as well as other organisations such as the Cochrane collaboration and American College of Physicians ${ }^{(208)}$. This process is based on rating treatments by best supported evidence available. Support of a modality by published evidence in the literature far outweighed professional opinion from clinical practice ${ }^{(208)}$. Theoretically, research favours evidence-based work, so this method is hypothetically very strong,but some could see it as undermining professional opinion gained from clinical practice $^{(208)}$. Another limitation is that the guidelines only discuss non-surgical options that are used in the USA and Canada and very importantly, the committee was unable to explore surgical opportunities as this was beyond their scope of charge ${ }^{(208)}$. We see that even though the ACR guidelines are one of the most recent and robust in their development process; this work also has a number of limitations to be taken into consideration.

Beyond management of those who already have $\mathrm{OA}$, further suggestions for strategies which encompass health promotion and preventative measures to reduce the development of OA have also been put forward ${ }^{(19)}$.Mody and Woolf ${ }^{(19)}$ state there is a large demand for improved prevention of OA by implementing strategies aimed at decreasing the risk factors associated with OA. Addressing the issues of obesity, reducing injuries which cause secondary $\mathrm{OA}$, avoidance of long-term participation in certain occupations and sports that cause excessive or repetitive loading on certain joints and regular participation in exercises to improve control around joints, is proposed to assist in lowering the burden caused by this condition ${ }^{(19)}$. 


\subsubsection{Summary of guidelines - The gold standard of management}

Roos and Juhl ${ }^{(29)}$ produced the most recent SR at the time of this literature reviewin 2012,on existing and new literature available on the treatment of OA of the hip and knee. Additionally, a task force from theEuropeanSocietyfor ClinicalandEconomicAspects of OsteoporosisandOsteoarthritis(ESCEO) published a report in 2014, which reviewed the above existing guidelines and expressed the need for a management algorithm, similar to Roos and Juhl's pyramid sequence, to prioritise the recommended practice guidelines ${ }^{(30)}$. The SR and algorithm report are used as a summary of the above OA management guidelines $^{(23-25,208)}$.

In summary, the previous guidelines' core recommendations are reinforced by again describing the best supported management options for osteoarthritis of the hip and knee as education/information access, exercise and weight control ${ }^{(29,30)}$. ESCEO specifically mention the important role of physiotherapy in the first steps of OA management, as well as the involvement of physiotherapy at later stages too ${ }^{(30)}$. In addition to this first-line of treatment, pharmacological modalities, passive treatments, and surgical interventions are then indicated where appropriate ${ }^{(29,30)}$. A helpful pyramid is presentedbelow (Figure 2-2) to depict the suggested timing of gold standards of treatment, starting with all patients receiving the first-line of treatment and then moving up the pyramid for those patients who require other modalities ${ }^{(25,29,207)}$. ESCEO reports finer details of which pharmacological agents should be used at different stages. The task force recognised the general limitation that exists regarding a lack of clinical trials for various treatment options, however firmly states that recommendations at each stage are based on sufficient evidence ${ }^{(30)}$. The full ESCEO algorithm can be found in Appendix A (pg. 207). 


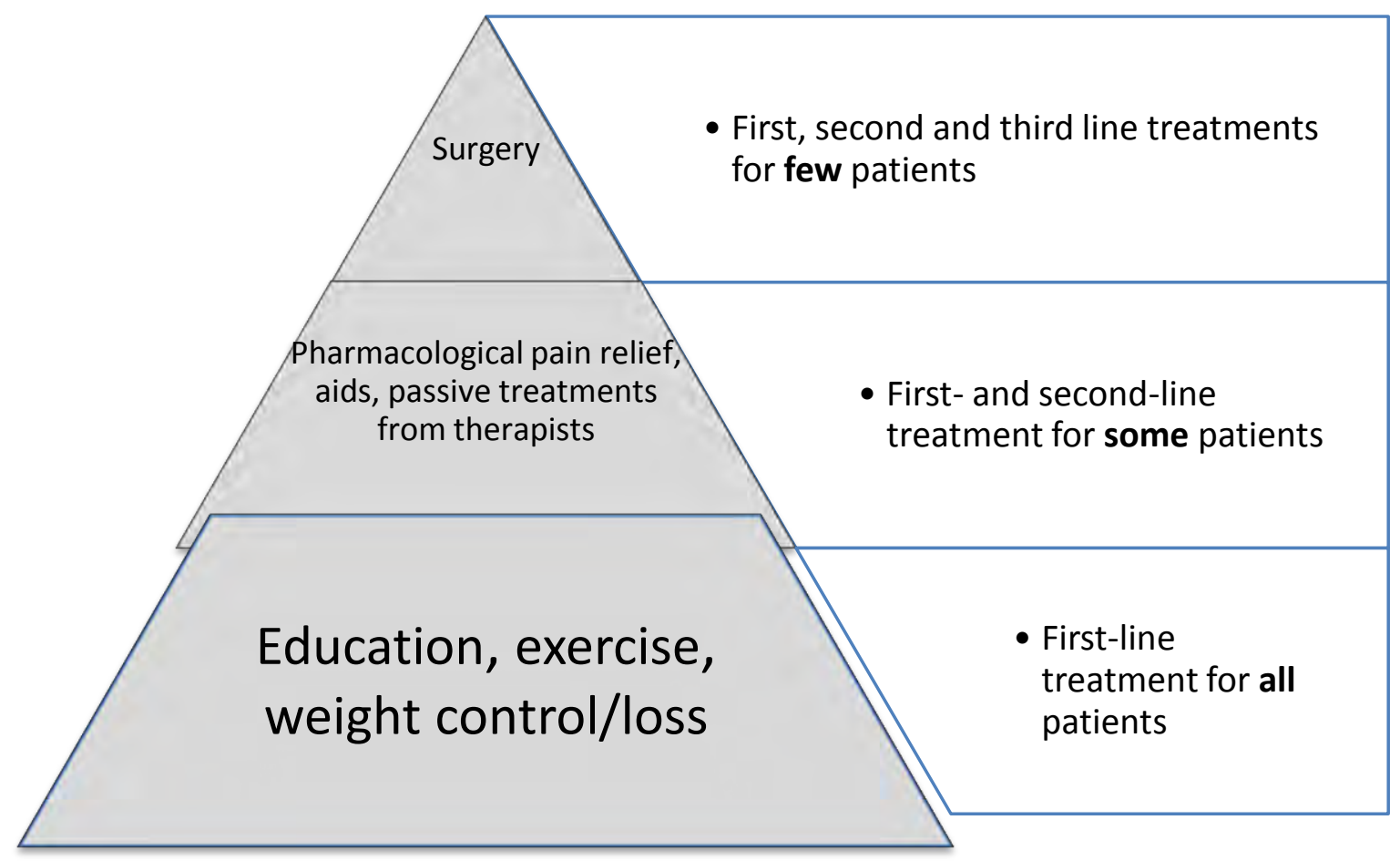

Figure 2-2 The first, second and third line treatment options

The extensive work in this field has produced various guidelines which all reinforce the core modalities of education, exercise and weight control or loss as the most effective management of $\mathrm{OA}$ of the hip and knee. This shows that this gold standard of core treatment is evidence-based and should be utilised in the management of the condition. An additional management approach that has shown benefits in persons with $\mathrm{OA}$ is relaxation therapy; consisting of guided imagery (GI) and progressive muscle relaxation (PMR) ${ }^{(220-222)}$. Although this management option does not form part of the gold standard of treatment for $\mathrm{OA}$ according to the evidence-based guidelines, the advantage of relaxation therapy is its ability to enhance treatment responses from the above-mentioned first-line modalities of education and exercise ${ }^{(222)}$. These physiotherapy specific management approaches, focussing mainly on variations of education and exercise, as well as relaxation therapy as an adjunctare discussed below.

\subsubsection{Education}

It is evident from all the guidelines discussed that education is imperative and usually one of the first recommendations to be made ${ }^{(22)}$. Noteworthy from the previously mentioned aim of the Bone and Joint Decade is the emphasis on empowerment of patients through education to promote more efficient self-management of their health ${ }^{(43)}$. 
Patient education is a well-known term in medicine. It has been described as an intervention that is aimed at increasing the patient's knowledge on a certain topic. The patient is usually a passivereceiver of information in order that they may better understand their condition, the treatment options and prognosis thereof ${ }^{(223)}$. The aim is then to use this information to make the necessary changes to manage their condition better than before ${ }^{(224,225)}$. However, this passive mode of educating patients has been criticised as it has not been shown to produce the desired change in behaviours ${ }^{(38)}$. Patients with OA who have chronic pain need to be educated in order to change their unhelpful thoughts, fears and beliefs regarding pain in order that they participate in exercise and correctly implement helpful coping skills i.e. change their behaviours ${ }^{(226,227)}$.

It has also been reported that patient education is often underutilised ${ }^{(225)}$. Reasons for this are not necessarily that education interventions are not available, but even in areas where they are readily available; the same underuse is still noted. An explanation is put forward reasoning that physicians do not see the value of such an intervention ${ }^{(225)}$ as merely educating the patient is not sufficient to cause the necessary changes needed to manage a chronic condition ${ }^{(223,228)}$. This supports the need for sufficient training of health professionals in effective patient education to bring about mind set and behaviour change rather than onlyproviding information ${ }^{(66,217)}$. Research has stated that due to the degree of involvement that a physiotherapist has in patient management, physiotherapists "have the potential to play a key role in applying a biopsychosocial model of health care" to patients with $\mathrm{OA}^{(217)}$. It goes on to describe how physiotherapists are trained to incorporate psychosocial components into treatment and with specific further training in a particular area, physiotherapists can provide specific interventions targeted at cognitive-behavioural changes $^{(217)}$.

A fairly new and advancing area of research regarding education as a management approach for those with chronic pain is that of neuroscience education ${ }^{(229)}$.This entails education onneurobiology and neurophysiology of pain and the changes that occur with central sensitization ${ }^{(229)}$. This approach is explained as a potential method to desensitise the CNS, by targeting the cognitive-emotional link to central sensitization. Further research is being undertaken in the field of OA but it is reported that one can theoretically treat the changes in the CNS by reconceptualising the meaning and reason behind chronic pain. 
The cognition that pain originates primarily from a local joint pathology ${ }^{(229)}$ and that pain is always directly related to the amount of tissue damage at that site is incorrect ${ }^{(230)}$. In fact unhealthy beliefs, fears related to pain and movement, catastrophizing and hypervigilance can lead to further central sensitization ${ }^{(229)}$. However, the correct pain cognition, in those with central sensitization, is that the pain experience is largely influenced by maladaptive changes in the nociceptive processes in the body as well as psychosocial aspects within each individual ${ }^{(230)}$. Thus if cognition surrounding pain and the associated behaviour is altered, processes leading to sensitization can too be altered ${ }^{(231)}$. Research has been performed on the effects of neuroscience education on LBP ${ }^{(232-234)}$ and limited MSC such as chronic fatigue syndrome, widespread pain and whiplash associated disorders ${ }^{(230)}$. Evidence shows that neuroscience education can have a positive effect on pain, disability, catastrophization, and physical performance ${ }^{(230)}$. How this should be incorporated into management of $O A$ is discussed later in the text (pg. 69).

\subsubsection{Self-management interventions}

To address the challenges of traditional education showing limited benefits, an expansion on education has been well researched in the past decade. Self-management interventions (SMI) focus on enabling the patient to be an active member in managing their day to day health by improving their perception of their ability to do so ${ }^{(11,235)}$. This is achieved through education about their condition, together with discussions on related aspects, goal setting and active monitoring of behaviour change progress. A key factor in physical behaviour change is encouraging activity and applying healthy psychosocial skills to certain life situations ${ }^{(11,236)}$. Sadly self-management is not a simple act to master ${ }^{(228)}$. For many patients with chronic illnesses, adherence to any sort of treatment plan is poor. This is a problemseen with adherence to self-management, patients often start off well making necessary changes but after a period of time, previous habits and behaviours are readopted ${ }^{(228)}$. Barlow et al. ${ }^{(237)}$ states in order for self-management to be effective, it should provide the patient with the "ability to monitor one's condition and to affect the cognitive, behavioural and emotional responses necessary to maintain a satisfactory quality of life." The importantcomponents in this statement are "monitor" and "maintain." Selfmanagement aims to teach these two aspects to patients, among others. 
As discussed earlier, unhealthy beliefs held by patients about pain and activity, can often lead to further disability ${ }^{(178)}$. To correct these beliefs, educating the person on an alternative to the unhealthy belief is the first step to adjust these thought patterns. This is based on the principles of cognitive behavioural therapy ${ }^{(238)}$ that with knowledge comes understanding and once both are gained, self-confidence can then improve and changes can be put in place to take on healthier behaviours; allowing patients to manage their condition better than before ${ }^{(178,239,240)}$. A key aspect to making the necessary changes is to improve the person's self-belief to achieve a goal or perform a task ${ }^{(158,239)}$. This term, as discussed earlier, is called self-efficacy ${ }^{(12)}$ and an important part of this management approach is aimed at improving SE. Better SE means being able to make informed decisions and therefore having more control of their condition ${ }^{(158,239)}$. Furthermore, belief in one's ability to performa task is increased by the person actually exercising or practising the task as this helps to align the knowledge gained and converts the false beliefs that movement causes pain and disability ${ }^{(179)}$.

In order to become a successful self-manager of health, certain helpful skills are taught during SMIto assist the person with living with their chronic condition ${ }^{(92)}$. These skills pertain to day to day medical management, changing behaviours and activities and managing the emotional aspects of the condition ${ }^{(11,92)}$. Topics such as problem solving, decision making, correct use of resources, forming healthy partnerships within the health services and action planning are discussed and applied to the individual's situation. Besides these core skills, content also deals with healthy lifestyle choices, incorporating exercise into daily life, pain and stress management techniques and general advice about their condition. This type of approach requires careful assessment of the problems in each person's or group's situation; explaining the necessity for discussing topics such as problem-based thinking and solving ${ }^{(92)}$. It has been noted that a successful SMI should attempt to educate the patient on all the above components in order to focus on the aspects that the patients perceive as their main problems (impairments, activity limitations and participation restrictions) and thus how to manage these ${ }^{(11)}$. 
Research states that SMI have been recognised as a basic component of health care for chronic disease management ${ }^{(241)}$. However, studies on the effectiveness and benefits of self-management programmes show varied results ${ }^{(92,242)}$. Although accepted guidelines ${ }^{(23,24,26,208)}$ recommend the use of self-management strategies, a recent $\mathrm{SR}^{(92)}$ and $\mathrm{MA}^{(243)}$ have indicated that this method has not had the expected significant effects in reducing pain, disability(effect sizes ranged from negative to negligible to small) and function (negligible to small effect sizes) ${ }^{(92,243)}$. Rather, improvements have been seen in other areas such as psychological well-being and SE (medium effect sizes were more common). One explanation for these results may be due to the types of outcomes used when assessing these programmes' effectiveness. Nolte shares that at the timethere were "no objective biological measures of disease severity in musculoskeletal conditions," and that the outcome measures used in these studies are reliant on a subjective measure of patient self-report and therefore may not indicate the actual effect of the $\mathrm{SMI}{ }^{(92)}$.

\section{a. Outcome measures in SMI}

The four most commonly reported outcomes to have been used during SMI studies in Nolte's SR are pain, disability, depression and $\mathrm{SE}^{(92)}$. Additionally, in the same SR, outcomes such as visits to physician, general health, fatigue, communication with physician, knowledge, anxiety, and physical functioning are also discussed ${ }^{(92)}$. The 18 studies included in the review had to pass strict inclusion criteria as all studies were either based on or similar to the Stanford principles of management ${ }^{(244)}$. The homogenous intervention structure is a strength of this review, however many different outcomes were used across the 18 studies and thus makes it difficult to compare studies side by side ${ }^{(92)}$. The results are unfavourable with regards to pain, disability, function and impairment and raises the questions of whether the outcome measures are being correctly administered to accurately detect changes in the above mentioned outcomes, or whether different primary outcome measures should be used instead ${ }^{(228)}$.

Newman and colleagues ${ }^{(228)}$ rightly state that it is vital to consider what the primary aim of a SMI is, together with the intention of the specific outcome measures used. He states that self-management programmes, alone, cannot achieve a reduction in the level of pain itself. 
However, the programme is designed to educate the person to improve their coping mechanisms in order to perceive the pain in a better manner, manage it appropriately and change the debilitating effect people equate with pain ${ }^{(228)}$. Thus, outcome measures used should measure these expected changes andevaluation of pain interference seems to have more importance than pain severity. Crotty and colleagues also advise in those waiting a joint replacement "that changes seen in exercise and activity are the primary selfmanagement improvements that would be desired from the self-management interventions"(242).

It is stated that improvements in the above areas are beneficial for those patients on a waiting list as this will help to prevent further stiffness and disability during the waiting period $^{(242)}$. A possible explanation has been put forward for those persons awaiting surgery that do not show significant improvements in pain or function after an SMI.This could be as a consequence of being put on a waiting list. The expectation of surgery as the following step in the management of their condition has been established and therefore it is possible that patients regard lifestyle changes and self-management as unnecessary ${ }^{(242)}$. Research by Moffet et al. ${ }^{(245)}$ supports the above rationale by attributing lower response rates to conservative treatment to beliefs in, or the need for surgery. Despite this negative aspect, lengthy waiting lists for joint replacement surgery remain a burden in both high and lower income countries ${ }^{(242)}$ and the patient's quality of life, while awaiting surgery, is poor and decreases over time with many frustrations due to their health concerns not being addressed $^{(33,246,247)}$. The detrimental effects of being on a waiting list is discussed later in the text, but what seems to be the essential factor pertains to the uncertainty of choice of outcome measures and the use of patient self-report in establishing the effectiveness of SMI.

\section{b. The Chronic Disease Self-management Programme}

Despite the above disparities in literature, a need for management of OA is evident both before surgery is required and while awaiting surgery. Therefore, individual studies that support the use of self-management programmes for improvements in a variety of outcomes, including pain and disability, should not be disregarded.Self-management programmes which follow prescribed outlines, have been widely used in high income countries, showing a range of positive results ${ }^{(241,248)}$. 
Two commonly utilised and well researched programmes from Stanford University in the USA are; the Chronic Diseases Self-Management Programme (CDSMP) ${ }^{(239)}$, which assumes that patients with any one or more chronic disease/s face similar issues and can take part in a general programme for a variety of chronic diseases. More specifically the Arthritis SelfManagement Programme(ASMP) ${ }^{(249)}$ targets people suffering specifically with arthritis. The two approaches are reviewedbelow.

The CDSMP was originally researched in two studies including persons with a variety of chronic conditions ${ }^{(177,239)}$.The self-management intervention initially consisted of seven weekly sessions and participants were followed up at month six, compared to a wait listed control group in the first RCT, and one and two years post intervention in the second longitudinal study (with no control group). The CDSMP showed after six months and one year respectively, significant improvements in health behaviours, status and SE as well as decreased health care usage. Significant improvements in health distress and SE were sustained at two years $(p<0.05)^{(240)}$. This was evident by participants' improved selfconfidence, exercise habits (number of minutes per week of stretching or strengthening and aerobic exercise), energy, less fatigue, better coping mechanisms, cognitive symptom management, communication and reduced hospitalisation ${ }^{(239,240)}$. The studies however, did not show any differences in pain or physical discomfort, shortness of breath or psychological well-being ${ }^{(239)}$. It is suggested however that this programme can reverse the trends seen in the control group towards a decline in health variables ${ }^{(239)}$.

At two years follow up, thecontrol group was included in the sample ( $N=533$ ) as the programme was offered to them. Despite the expected average increase in disability per year $(0.02-0.03 \text { on the } \mathrm{HAQ})^{(250,251)}$ different aspects of health status remained constant or improved with a decrease in the use of outpatient services ${ }^{(240)}$. The primary outcomes of these studies were change in health behaviours, health status, and health service utilization. Interestingly, the measures of these primary outcomes correspond with the suggestion by Newman et al. ${ }^{(228)}$ to focus on outcomes which possibly measure the effectiveness of such programmes more accurately. 
The original RCT was based on a large sample size of 952 participants and displayedhighly significance values for the improvement in outcomes for the treatment group were noted: $p<0.01$ for health behaviours, $p<0.02$ for health status improvements at month six ${ }^{(239)}, p<$ 0.01 for improvements in health distress and SE at one and two year visits ${ }^{(240)}$. However effect size was not calculated or reported at the time of the studies. The CDSMP was shown to be cost effective in terms of considerable savings per participant when compared to a control group ${ }^{(177,240)}$. These results support the use of the CDSMP as a management option for chronic diseases with the specific objective of increasing SE and reducing health service utilization.

However, even though level of education was not seen as a covariant when analysing results, in this particular sample, it is noteworthy that the mean level of education for these subjects was high (14-15 years) ${ }^{(239)}$. Results may differ in groups of lower educational levels such as those in SA where the majority of the older non-whitegeneration today received no or poor quality education in previous years ${ }^{(66)}$. In SA, adult literacy rates only increased by 4\% between 1995 and 2006 and in 2007 it was reported that persons who received secondary to tertiary education are still presenting with issues of basic numeracy, literacy and problem solving skills ${ }^{(66)}$.

Another factor that should be considered when generalising this study to other populations of patients with chronic conditions is that there is a recruitment bias. Patients volunteered to take part in this programme. This means that the results are based on a samplewho were highly motivated to improve and not on patients who did not volunteer and who may not be as determined to show improvements. Lastly, due to the variety of different chronic diseases and stages of each condition in the patients attending the CDSPM, the individual problems and needs differed. This showswhat is known as a ceiling effect ${ }^{* * *}$ by only having a small variability in improvements in the primary outcomes, as some patients did not have very low health status or behaviours initially. This meant their scores were near the upper limit to begin with, thus did not show large improvements but rather were maintained ${ }^{(239)}$.

${ }^{* * *}$ Ceiling effect: occurs when a number of scores are seen towards the highest available limit ${ }^{(367)}$ 
Further research by means of a MA on the effectiveness of CDSMP was conducted in $2005^{(252)}$. However, this review was limited by the presence of possible publication bias andthe studies used in the MA were noted to be of variable quality ${ }^{(252)}$. A total of 14 studies of OA, among other conditions, were reviewed from 1985 until 2004. Simply put, the MAresults concurred with Lorig's original work that showed CDSMPdid not cause a relevant clinical change in those with $\mathrm{OA}$, based on $\mathrm{ES}$ in pain and function $(\mathrm{ES}=-0.06)^{(252)}$.

More recently, a RCT was performed in the UK to establish the effects of a modified version of the CDSMP on a variety of chronic conditions, including arthritis ${ }^{(253)}$. This trial was designed as a pragmatic RCT; comparing a six week SMI to a wait listed control group. A realistic sample of 629 patients who commonly diagnose themselves with a chronic condition was used together with practical treatments delivered in a similar fashion to usual care ${ }^{(253)}$. This programme had small yet significant effects on $\mathrm{SE}(E S=0.44, p<0.01)$ and energy $(E S=0.18, p<0.01)$ in this group of patients at month six ${ }^{(253)}$.There were also improvements in exercise habits, relaxation, psychological and social well-being and health distress ${ }^{(253)}$. This result however is not specific to OA as it was performed on a range of chronic conditions. Other restrictions were also noted such as the approach to recruiting participants; participation was voluntary which means information on the population who did not volunteer is unknown. Additionally, the sample used in this study differed to a large extent when compared to the broader population's data gained in the national health survey in England ${ }^{(253,254)}$. Nonetheless, it provides valuable data on the effects of such a pragmatic RCT and leaves room for further research in this field.

\section{c. The Arthritis Self-management Programme}

Due to the above concerns of a heterogeneous group of chronic conditions, it is important to discuss the research available on similar programmes aimed at specific conditions, such as the ASMP ${ }^{(249)}$. The initial RCT on the effectiveness of the ASMP was performed over a period of four months and yielded significant benefits of improved knowledge and behaviours $(p<0.01)$ and a decrease in pain according to VAS $(p<0.04)^{(249)}$. It should be noted that despite randomisation, less pain $(p=0.03)$ was observed in the control group at baseline. These benefits were observed in a longitudinal study over 20 months, as well as decreased health care utilisationand improvements in depression $(p<0.01)^{(16,158,249,255,255)}$. 
Lorig continued follow up measures at four years showing further decreases in health care utilisation (40\%) and sustained benefits in pain ${ }^{(158)}$. Effect sizes were not attainable for the above mentioned studies and the long term studies were lacking a control group. Likewise, as with the CDSMP, these benefits occurred despite an increase in disability (9\%) over a longer period ${ }^{(158)}$. A 12 year review confirmed the initial results by concluding that the ASMP was effective, in the short- and long-term, at improving health behaviours, various aspects of health status and self-efficacy ${ }^{(256)}$. It was also reported that the duration of such a programme is important as a six week programme is better than only three weeks of $\operatorname{ASMP}^{(257)}$.

In spite of the above results, this original research was performed more than 20 years ago and mostly in high incomecountries; henceit is relevant to discuss more recent literature available on such an intervention. In 2000, a randomised pragmatic controlled trial was performed in the UK whereby the ASMP similarly supported positive findings at four and 12 months ${ }^{(235)}$. Significant improvements compared to the control group were reported in SE, self-management techniques and health status $(p<0.01$, ES was not calculated between groups) ${ }^{(235)}$. This RCT similarly showed no improvementin pain or disability at four months but showed a significant decrease in pain $(p<0.01)$ in the intervention group at 12 months compared to their baseline measures. However, it cannot be said that this is an accurate reflection of the intervention's effect as there was no control group with which to compare at 12 months ${ }^{(235)}$.

Another RCT was performed in the UK in 2006 on patients with OA who attended the "expert patient programme" (developed from the ASMP) ${ }^{(258)}$. This study was conducted in a primary care setting with follow up measures at four and 12 months. Similar results were evident whencompared to a control group;with psychological aspects such as improved SEanddecreased anxiety observed ( $p$ values or ES were not obtainable). As with previously mentioned studies, this RCT did not showany significant differences on pain or function. This study excluded patients who were requiring surgery and who had poor mobility; the reason for this was not stated. A possible reason could be attributed to the patients awaiting surgery not seeing the need for self-management techniques, as surgery is thought to be the solution to their condition. 
The study had a few methodological flaws and miscalculations in the required sample size and the relevance of the reported significant improvements is unknown due to a lack of $p$ and ES values ${ }^{(258)}$. Thereforedespite similar results on improved psychological factors, the results from this particular trialdo not seem of sufficient quality to substantially add to the literature available.

\section{d. Summary of the CDSMP and the ASMP}

These two variants of SMI are widely used in high income countries such as USA, UK, Australia, New Zealand, Canada, Japan, Singapore, Holland, Norway, Denmark, Sweden, Austria, Switzerland as well as middle income countries such as Mexico and China. When the ASMP and the CDSMP were compared to determine which is more effective than the other, it was seen that both programmes are beneficial in terms of decreasing activity limitation, health distress and fatigue at four months and additionally improving global health at one year $(p<0.05 \text {, ES were only reported on within group changes })^{(241)}$. The authors suggest for patients presentingwith arthritis, the more specific course be administered due to the slightly better results seen for the ASMP group at four monthsand one year post intervention (significant improvements in pain were noted in the ASMP group $E S=0.26, p<0.01$ at four months, $E S=0.27, p<0.01$ at one year; with differences between ASMP and CDSMP approaching significance at one year, $p=0.08)$. However, it was documented that the number of patients with arthritis in the CDSMP sample was less than half of those in the ASMP group and therefore significant values are not completely accurate due to the notably smaller sample in the CDSMP group ${ }^{(241)}$.

Furthermore, the study of comparison between ASMP and CDSMP (241) included predominantly females of once again, a high educational level(15 years), which should be taken into account when generalising its use to the broader arthritis population. This is particularly important in lower income countries such as SA, where according to the 2001 South African census, $14 \%$ of the population had no schooling and less than $1 \%$ had obtained a grade 12 certificate ${ }^{(259)}$. Educational statistics in South Africa have improved in the last decade and figures in 2011 show that $27.4 \%$ of the population older than 20 years had obtained a grade 12 certificate, however, this figure is still low ${ }^{(260)}$. 
It has been stated that interventions based on a cognitive behavioural approach, such as the above ASMP and CDSMP, have the potential to be reliably delivered by physiotherapists in urban and rural settings ${ }^{(93)}$. It is recommended that because physiotherapists recognise the need for a holistic approach, this profession is a rational choice for delivering such a psychosocially based treatment ${ }^{(261)}$. A qualitative study by Nielsen and colleagues ${ }^{(262)}$ explored the experiences of physiotherapists who were educated specifically to adopt a cognitive behavioural approach during treatment of patients with pain. It was reported that some of the components were already familiar to physiotherapy training, yet applying the theory into the clinical environment was reported to differ somewhat to traditional physiotherapy ${ }^{(262)}$. It was seen that physiotherapists, when trained in this field, experienced greater knowledge and ability to integrate such a psychological approach into management and were recognised as a profession who can confidently deliver such programmes ${ }^{(262)}$.

\section{i. Barriers to SMI implementation}

Individualstudies ${ }^{(228,243,263)}$ find that there are several benefits to participating in SMIs. These benefits are captured well in the quote "SMIs help people to understand and cope with their problems more effectively, improve adherence to management advice and reduce healthcare utilisation”(264). Despite research in favour of the use of SMI in management of chronic diseases such as $\mathrm{OA}$, this method of management is not often used and has been said to be not easily implemented ${ }^{(223)}$.

As the Bone and Joint Decade correctly emphasises; instead of being seen as an optional addition to regular care, this manner of management needs to be prioritised ${ }^{(11,43)}$. Health systems require the necessary resources to support such programmes, such as sufficient space and numbers of adequately trained staff to be able to deliver them ${ }^{(228)}$ as well as structures in place to sustain such interventions ${ }^{(11)}$. Bodenheimer and colleagues ${ }^{(193)}$ states that self-management is unavoidable. If one looks at how much time a clinician spends dealing with the patient's condition versus how much greater the time is that the patient is left to manage their condition without a clinician; self-management has to occur. 
Barriers have been identified which hinder the success of SMI which necessitate different suggestions to assist in successfully implementing SMIs ${ }^{(11,223)}$. Firstly, as mentioned, according to Lorig $^{(11)}$ the health system needs to prioritise SMIs and integrate theminto all aspects of health care. Initially instead of managing all patients with this strategy, specific groups of patients who would possibly benefit most from this management style should be distinguished ${ }^{(228)}$ and the type of SMIs which are to be delivered should be chosen for these groups $^{(11)}$. In order that patients accept this approach to chronic disease management, the expectation of passively receiving health care needs to change to individuals adopting an attitude of actively being involved in the management of their condition ${ }^{(11)}$. Adequate financial support is clearly also required in order to disseminate such management methods into health systems.

Glasgow and colleagues ${ }^{(223)}$ describe a strategy called "self-management support" which is used in primary health care settings throughout the USA. It consists of five main focus areas to aid in implementing SMIs in health care practices. The five areas are briefly described as assessment of whether or not SMIs are being used at all, as well as to determine if there are any support systems in place to allow for changes. This includes: advice regarding what changes need to be made and how to make them; agreement on the key focus of SMIs and working together to bring about successful implementation; assistance with identifying and addressing barriers that may arise and lastly arrangements to review the changes and progress of the new system need to be done ${ }^{(223)}$. These recommendations were initially implemented in the USA, a higher income country, and whether this strategy can be translated and implemented as successfully into the South African context is again questionable.

Examples to foster implementation of SMIs into primary care facilities in higher income areas are presented, followed by possible barriers experienced in the SA context ${ }^{(223)}$. Firstly, it is advised that communication should be made prior to the patient's visit to remind them of previous goals and to reflect on progress. In poorer areas in SA, communication pathways are often unreliable as many patients do not have fixed or constant telephonic means. This makes contacting patients a struggle. 
Additional human resources would also be necessary if extra telephone calls were to be made on a daily basis in preparation for those patients expected to arrive the next day. Limited physical resources of space, equipment and money is also an issue in health environments and should be considered.

Secondly, while in the waiting area patients should complete forms which assess their selfmanagement skills since the last visit, advice on further self-management tips and information on community support should be readily available for reading ${ }^{(223)}$. Illiteracy and low educational levels are a common problem in the patients serviced by the public community health clinics in $\mathrm{SA}^{(259)}$. Ongoing education and discussion on self-management progress should be commenced during interaction with nursing personnel ${ }^{(223)}$. Feedback on any changes in the patient's physical condition should be shared with the patient to keep the patient well informed and knowledgeable on their current condition. With low numbers of personnel available and the excessive number of patients attending any health setting daily in the SA environment, this interaction or in depth feedback is limited. As mentioned earlier, further human resources, either health professionals or lay persons, would be needed to facilitate such educational interactions or feedback sessions as more time would be needed on each patient, causing further delays and backlogs.

Specific areas of concern for the patient are to be addressed in the consultation period and alterations to goals or plans are advised ${ }^{(223)}$. Continued support by the consulting clinician to advocate for ongoing communication, problem-solving and behaviour change is needed to ensure self-management skills are sustained and improved. Once again, this advice requires specific attention to individual patients and ongoing support impliesongoing expenses, which are not readily available in lower to middle income countries ${ }^{(223)}$.

Glasgow et al. ${ }^{(223)}$ stated that implementation of self-management support principles should be trialled and tested to suit the different health care environments as changing health care principles to a patient centred self-management model is not a simple task or an immediate transformation but requires time and effort at any level of health care, in both high and low income countries. 
It is stressed that high quality research studies continue to be performed to confirm the effectiveness of self-management programmes in different groups of patients, so as to justify implementation of this health strategy ${ }^{(11)}$.

Due to previously mentioned discrepancies in the available literature on the effectiveness of SMIs in OA, a Cochrane review was released in 2014 concluding that low to moderate quality evidence exists showing no or small benefits in those with $O A^{(265)}$. Thus authors suggested no further trials such as those previously reviewed on SMI in isolation are needed, as good quality evidence supporting the use of SMI alone is unlikely to arise ${ }^{(265)}$. However the use of SMI techniques in OA individuals is unlikely to cause any harm and therefore the recommendation for further research has been made to evaluate the effects of other models of SMI and/or interventions combining SMI and other approaches.

\subsubsection{Exercise}

\section{i. Effectiveness of Exercise}

Literature refers to exercise as a key strategy in reducing disability in patients with OAand is one of the fundamental management options for those who have $O A^{(29)}$. Exercise for $O A$ ranges from general body conditioning (cardiovascular exercise), to specific joint ROM exercise, to strengthening and flexibility exercises to stretch muscles. The manner in which exercise is delivered can either be supervised by a physiotherapist (during individual or group-based sessions), or by self-directed means of a home programme ${ }^{(23)}$. It has been documented that exercise has various benefits for people living with this condition; although it is noted that many more studies exist on OA of the knee than of the hip. Having said this, the literature available on the importance of exercise in OA of the hip and knee is discussed below.

As discussed in the evidence-based guidelines for OA, exercise is strongly recommended as the primary management for all persons with $O A^{(207)}$. In several SRs exercise has been shown to be effective in providing pain relief and functional improvement in patients with mild to moderate $O A$ of the hip and knee ${ }^{(23,24,26,73,76,91,214,266-268)}$. Accepted guidelines show no favour in terms of benefits or safety for either land- or water-based exercise, but rather that this should be decided according to the patient's needs, abilities and preferences ${ }^{(208)}$. 
The same principle applies to choice of activity performed during strengthening and flexibility exercises. Promotion of exercise in general is gaining more support from recent literature, rather than specific exercises that target one muscle group.

However, due to the broad nature of the term "exercise", some authors do advocate for specific exercise programmes detailing type, intensity and duration. Fransen et al. ${ }^{(76)}$ explains why this is thought of as necessary: in order to applyevidence-based results into clinical practice, one needs particular details to correctly replicate the intervention used in that study ${ }^{(76,178)}$. Yet evidence for specified programmes is lacking in the available literature ${ }^{(23,269)}$ and specific programmes are even seen as a limitation to implementing this management. Specific protocols often result in problems due to population's needs and environments being so vastly different ${ }^{(178)}$. This rationale supports the use of exercise in general, rather than specific and detailed exercises as treatment.

The SR by Roos and Juhl, discussed earlier, summarised the literature made available during the period July 2011 to April 2012. The conclusion was made that high levels of evidence support the use of exercise and the benefits thereof, in cases of mild to moderate $\mathrm{OA}$ of the knee and hip ${ }^{(29)}$. It is recommended in all existing guidelines that symptomatic patients are to be seen by a physiotherapist and prescribed an appropriate exercise regime ${ }^{(213)}$. This highlights the important role the physiotherapist plays in the management of this condition. The benefits of exercise in this population according to available literature are discussed below.

At the time of Zhang's review in 2008, the use of exercise in persons with OA of the knee showed improvements in pain, function as well as health-related quality of life and clinical outcome ${ }^{(25)}$. High evidence (level la) supports aerobic walking and strengthening exercise in knee $O A$ to reduce pain and self-reported disability. Whereas lower level IV evidence supports aerobic walking and ROM exercise to reduce pain and stiffness in hip $\mathrm{OA}^{(25)}$. 
An overview of systematic reviews from $2000-2007$ (presenting high quality evidence according to the GRADE approach of rating studies ${ }^{\dagger++}$ ) supported exercise in OA of the knee to bring about decreases in pain and improved function. In 2012, Brakke and colleagues ${ }^{(267)}$ reviewed literature on physical therapy in those with $\mathrm{OA}$ and agreed with the above statement. They added that, as expected, strength training demonstrated improvements in muscle power in knee $\mathrm{OA}^{(267)}$. These reviews further confirm that general exercise is supported by literature as no difference was seen when using high or low resistance strengthening or dynamic versus static exercises ${ }^{(266,267)}$. It is stated that one concept behind the effectiveness of exercise lies in the "ability to reverse muscle sensorimotor dysfunction (weakness, fatigue, poor control) preventing abnormal movement and restore normal biomechanics, effecting better gait, relieving pain and improving function" ${ }^{(178)}$.

Most of the literature on exercise in OA thus far has focused on those patients with mild to moderate OA. This might explain the small effects sizes seen for the benefits of pain and function. Due to many studies being performed in early or mild OA only, it is speculated that a ceiling effect may occur because pain and function are not as affected as those with late stage $O A^{(76)}$. This means that the known effects of exercise in patients specifically with severe $O A$, is exceptionally limited ${ }^{(29)}$. This area has shown to be in need of further research as only a small number of studies addressing this topic exist.In response to the limited evidence, Wallis and Taylorpublished a SR and MA in 2011 ${ }^{(73)}$. In summary, after reviewing 23 small RCT's of which only 12 were considered higher quality trials of above $6 / 10$, the results showed low to moderate evidence to support exercise in those with severe OA of the hip or knee awaiting surgery, by reducing pain pre-operatively, and that exercise, together with education, could additionally improve function post-operatively ${ }^{(73)}$.

\footnotetext{
${ }^{++\dagger}$ High-quality evidence: based on more updated, high-quality systematic reviews that are based on at least 2 high-quality primary studies with consistent results

Moderate-quality evidence: based on One or more updated systematic reviews of high or moderate quality -based on at least 1 high-quality primary study or at least 2 primary studies of moderate quality with consistent results

Low-quality evidence: based on One or more systematic reviews of variable quality - based on primary studies of moderate quality/inconsistent results in the reviews/inconsistent results in primary studies
} 
Another scarce area of research exists in the effects of exercise in those with OA and central sensitization changes due to chronic pain. The work by Nijs et al. ${ }^{(231)}$ recommends the use of exercise in management of these individuals as exercise can "deactivate brainorchestrated top-down pain facilitatory pathways" and can activate the body's endogenous opioid system, thus reducing hypersensitivity in the $\mathrm{CNS}^{(231)}$. Exercise by means of a "timecontingent approach" is preferred over a "symptom-led approach"(231). By participating in exercise for a predetermined time despite pain, instead of exercising until the symptom of pain prompts the person to stop, facilitates the reconceptualization of pain's meaning during exercise and can reinforce the notion that pain is not only as a result of peripheral damage ${ }^{(231)}$. Other research by Nijs and colleagues ${ }^{(109)}$ examined the effects of central sensitization in the endogenous opioid system during exercise in chronic pain subjects. They found that not all chronic pain patients respond positively towards exercise as seen by a deactivation of their endogenous analgesia ${ }^{(109)}$. Further research is suggested, by means of RCT, whereby longer durations of therapeutic exercise and the effects thereof on the central nervous system are explored. This is necessary to determine whether patients, particularly with $O A$, also display centralised endogenous analgesic dysfunction ${ }^{(109)}$. This work has important implications for the use of exercise as a treatment option in chronic pain disorders with central sensitization. To allow for this dysfunction, it is recommended that for those with CS, aerobic and low intensity exercises suited to individual patients are used and progressed slowly according to each person's capability ${ }^{(109)}$.

\section{a. Outcome measures in exercise}

In the majority of the studies reviewed reporting on the effects of exercise in people with $\mathrm{OA}$, the primary outcome measures used were self-reported pain and function. However it was noted that these are not recommended to be used alone but that objective measures should also be used to strengthen the results obtained by self-report instruments ${ }^{(228)}$. Another point is that most of the studies reviewed may fail to reflect the full benefits of exercise as outcome measures should not be limited to merely pain and function but should include psychosocial and behavioural measures such as those identified by Newman and colleagues discussed earlier in the text ${ }^{(228)}$. 
Further studies have been published which havebroadened the scope of the benefits of exercise by not only investigating the physiological effects but furthermore the important psychosocial effects from exercise ${ }^{(21)}$. Hurley et al. ${ }^{(21)}$ speak of the "psychosocial sequelae" that arise from the ongoing experience of pain and disability. Again this is incorporated in the ICF model of how pain is not limited to a physical impairment but has many other complex effects. Figure 2-3belowwas obtained by permission ofCopyright Clearance CenterRightsLink and Wolters Kluwer Healthwhich depicts the complex association between all aspects $^{(21)}$.

\section{Biological changes}

Normal mechanical joint (ab)use; obesity; ageing; process of muscles and joint; disease; injury

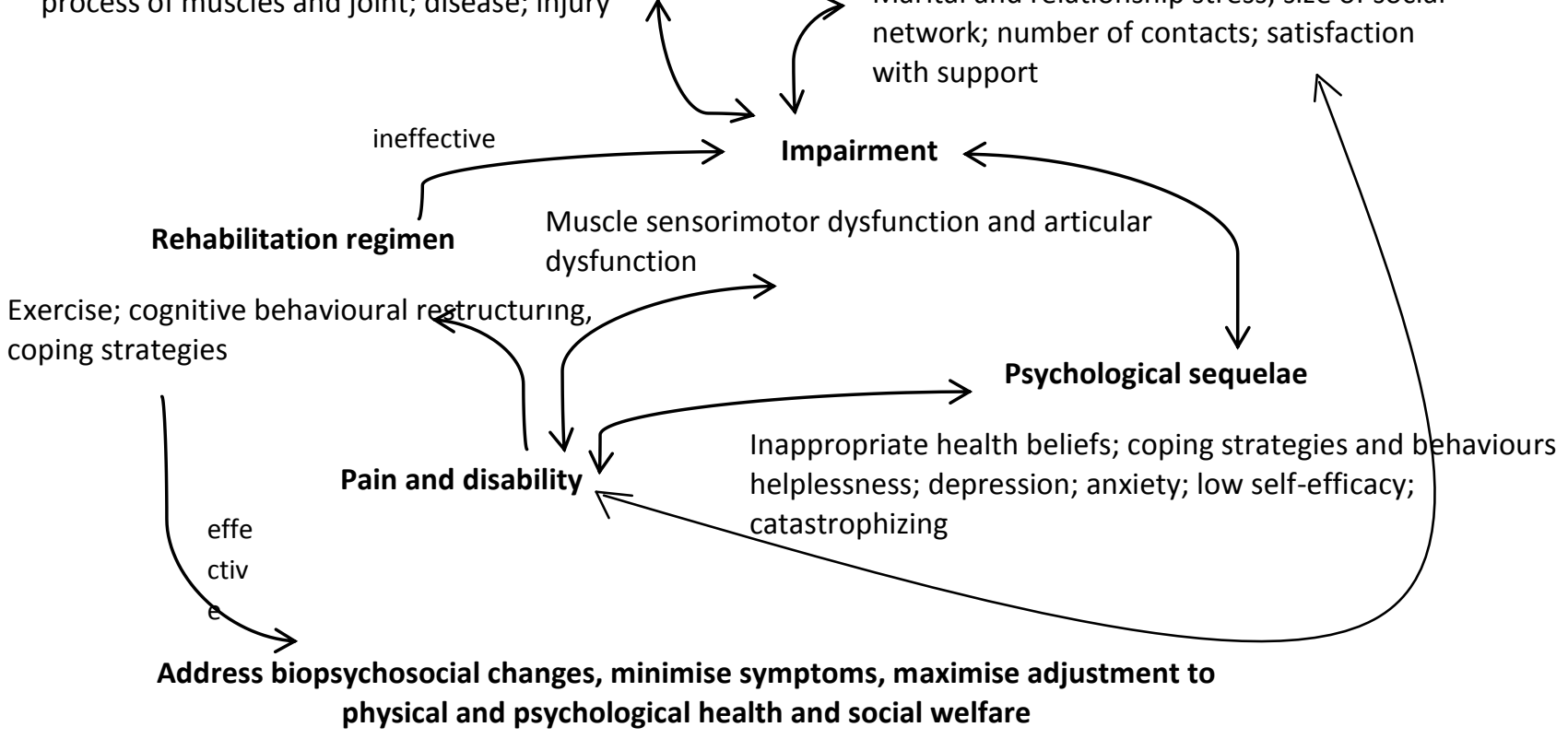

Figure 2-3 Complex relationship between pain, disability and psychosocial aspects

The complex relationships between the physical impairments or behaviour change and psychosocial traits that exist in persons with OA can either impact on the management of OA negatively or positively ${ }^{(21)}$. If the psychosocial sequelae are ignored, these factors will become more of an issue and further impact disability negatively. However, due to the associations illustrated in Figure 2-3above, exercise can be used as one of the means to address psychosocial effects. 
It has been proposed that the immediate benefits, of a reduction in pain and disability, seen after participating in exercise cannot solely be as a result of physiological changes taking place, as variables such as strength, stiffness, and endurance usually take time to change substantially. Thus, psychological variables are thought to have a distinct influence ${ }^{(21)}$.

Exercise has been shown to affect many different psychosocial aspects such as: incorrect pain beliefs, fearavoidance, catastrophizing, depression, anxiety, helplessness, social isolation, dependency, coping strategies, interpersonal relationships and self-efficacy ${ }^{(21)}$. The areas that are especially important in chronic pain patients with $O A$ are unhealthy pain beliefs, fear-avoidance and SE. By actively participating in exercise, patients experience for themselves that their pain does not dramatically increase or cause more joint damage at the site of $\mathrm{OA}^{(21)}$. Once the belief that movement causes more pain and destruction to the joint is challenged and the opposite results are felt, the person begins performing previously avoided activity as that fear has been overcome. By achieving healthier beliefs about movement and activity and an improvement in their physical symptoms, patients feel like they have more control over their disability and have acquired helpful coping mechanisms. This improves SE which has a knock on effect on helplessness, dependency and depression ${ }^{(21)}$. Physiotherapists have a key role in this field of chronic pain management, including patients with osteoarthritis, as physiotherapy encompasses strategies to alter incorrect thought patterns by actually performing the movement the person has been avoiding in order to overcome their fears. Physiotherapy has a major emphasis on "achieving health through movement" ${ }^{\text {(179) }}$.

\section{b. Summary of exercise in $O A$}

In conclusion, literature advocates for the use of general exercise/s in the management of $\mathrm{OA}$ to reduce pain and disability as well as having substantial effects on a number of psychosocial aspects associated with OA. This highlights the principles discussed in the ICF and leads to a suggestion for further research on the importance of exercise in patients with severe $O A$ to ensure exercise does not only improve body structures but translates into improved psychological and social traits thereby improving coping skills, ADLs and participation in social environments ${ }^{(29)}$. 


\section{i. Cost effectiveness of exercise}

The cost effectiveness of exercise as a treatment option of choice is still to be established in the literature ${ }^{(270)}$. A systematic review, which explored the cost effectiveness of first-line treatment options for $\mathrm{OA}$, showed that studies on exercise programmes were cost saving. However, there were possibilities of bias in predicting costs and cost effectiveness ${ }^{(270)}$. It was reported that such an intervention had a higher probability (81-100\%) of being more cost effective than other treatment options ${ }^{(85,271)}$. Even though,in a long-term follow up study, this research showsthat an exercise-based rehabilitation programme is more cost effective than usual primary care, flaws exist in this and other studies by Hurley and colleagues $^{(85,271,272)}$. The studies were performed in the health system of the UK and one cannot say if these results can be equally transferrable to other countries with different health systems, for example public versus private health systems in SA. Additionally, calculations to estimate health costs in cases that were lost to follow up may not be an accurate reflection of actual costs that patients would have incurred.Of interest to the current study is that the significant difference seen in community-based and medication costs alone may lower the health care costs as these are two resources often used by individuals with chronic pain ${ }^{(271)}$. This may be true in the public health setting of SA as community health services are the first entry point such patients are encouraged to use and may be more conducive to such management than at a tertiary level.

On a separate point, it could also be argued that the class-based exercise method provides a potentially more cost-effective alternative to individual sessions with a physiotherapist. This is especially true in lower income countries where waiting lists also exist for one on one treatment with physiotherapists. These group sessions, when introduced into community centres or clinics, could be more regularly accessed by older people or those who rely on public transport ${ }^{(264)}$. The social contact with others of a similar age, particularly those experiencing similar disease-related symptoms, is also proposed to encourage treatment adherence ${ }^{(76)}$.

In summary, the above section has discussed the cost effectiveness of exercise in persons with OA. This treatment approach is believed to more costeffective in group based management than individual sessions or usual care. 
In addition, the importance of on-going exercise has been recognised; as positive effects gained from exercise have been seen to decrease over time if exercise is not performed on a regular basis ${ }^{(76,273)}$. Peer support could further improve adherence to programmes but long-term adherence has been said to usually require stimulus of regular supervision or monitoring ${ }^{(274)}$.

\subsubsection{Combined Intervention: self-management intervention and exercise}

\section{i. $\quad$ Effectiveness of a combined approach}

The regular "stimulus" that Woodard and Berry ${ }^{(274)}$ speak of is not limited to outside supervision or monitoring by a health professional.Rather, keeping in line with a patient centred approach, motivation should be driven by an internal change of mind set and selfmonitoring of changes made, progression and accomplishments achieved. This is what should encourage chronic pain patients to continue with new beliefs and behaviours. It has now been clearly laid out that firstly; education through SMIs is effective in improving knowledge andSE, correcting fear-avoidance beliefs and encouraging goal setting and activity. Additionallydue to the CNS changes that have been reported in $30 \%$ of OA patients, neuroscience education targeting cognitive-emotional sensitization is recommended as a part of initial education on pain and can be continually reinforced throughout intervention ${ }^{(2,22,231)}$. Secondly, it is seen that exercise has benefits in reducing pain, improving function ${ }^{(275)}$ andinfluences psychosocial factors ${ }^{(21)}$, as well as affecting the sensitized CNS by reducing hypersensitivity and activating the endogenous opioid system $^{(231)}$.

Taking these separate points into consideration, as the management guidelines suggest, instead of merely educating on the importance of exercise, interventionsshould include both educationalandactive participatory exercise components ${ }^{(264,276)}$. It is proposed that by incorporating both aspects, the optimal benefits of these two management options combined could be achieved ${ }^{(178,264,276)}$. Education methods of providing information and instruction for change alone has been said to be of little value ${ }^{(38)}$. Therefore, a proposed more effective approach is to allow the person to incorporate new knowledge, problem solve and implement the advised changes in beliefs and behaviours, such as exercise, in order to experience the benefits first hand ${ }^{(21)}$. 
Hughes and colleagues ${ }^{(277)}$ performed one of the first RCTs to establish the effects of a combined exercise and education intervention in persons with mild to moderate lower limb $\mathrm{OA}$ in the USA. The intervention comprised of an eight-week programme combining progressive exercise (aerobic and strength) with educational content to improve selfmanagement ${ }^{(277)}$. Preliminary results from the first 150 participants were extremely encouraging reporting significant improvements from baseline to month six in exercise SE $(p<0.01)$, exercise participation $(p<0.01)$, function as per the 6MWT $(p=0.02)$, WOMAC stiffness $(p=0.03)$ and WOMAC pain $(p=0.02)$ (ES were not noted in the first study) ${ }^{(277)}$. A second study with a larger sample size $(N=215)$ was carried out which supported the above results of improved exercise $\mathrm{SE}(\mathrm{ES}=0.78, \mathrm{p}<0.01)$, exercise participation $(\mathrm{ES}=0.86$, $p<0.01)$ and stiffness $(E S=-0.33, p=0.02)$ seen at two months. These improvements were sustained at month six, with added benefits of reduced pain $(E S=-0.47, p=0.04)^{(278)}$. Long term follow up was also reported where exercise SE and exercise adherence improvements were maintained with large effect sizes at month 12 (ES $=0.905$ and 0.669 respectively, $p$ $<0.01)^{(278)}$. Yet improvements in stiffness and pain declined somewhat at month $12(p>$ $0.05)^{(278)}$. Further studies were recommended by the authors in this field of research, which was subsequently performed elsewhere and reviewed by a SR in 2006. Ten studies were included in this 2006 review to establish the effects of an integrated programme of exercise and education on pain and function in hip and knee $O A^{(276)}$. The review concluded that despite methodological flaws, such an approach was effective in bringing about significant improvements in pain and function. Again, further research was called for with fewer methodological flaws to establish these effects ${ }^{(276)}$.

Following on from the above studies, Lamb et al. ${ }^{(279)}$ assessed the effects of a six week programme, combining exercise and education via a cognitive behavioural approach, in individuals with severe knee OA. Outcome measures were taken at baseline, week six and 12. The intervention showed moderate significant improvements in pain, function and SE at week six which declined slightly at week 12. Interestingly, the effect sizes were larger (ES = 0.7 and 0.8 for pain at week six and 12 respectively) in those individuals who were not yet on a waiting list for arthroplasty compared to those who were $(E S=0.3 \text { and } 0.1)^{(279)}$. Evident improvements for pain, function and self-efficacy in those with severe $O A$ are encouraging but a limitation to this study was noted as there was no control group to compare improvements against. 
Hurley and colleagues ${ }^{(85,271,272)}$ performed the more robust"Enabling Self-management and Coping with Arthritic Knee Pain through Exercise" (ESCAPE) study and yielded positive results. This combination of approaches (SMI and exercise) is the basis on which the present study has been developed and is described in the text that follows.

\section{a. The ESCAPE programme}

ESCAPE is described as comprising of two components, namely; education and exercise. The intervention aims to actively involve participants in their condition as a self-manager of their own health and is achieved by providing knowledge of the condition and related aspects. The participants learn to engage in regular exercise to address pain and other common symptoms. As mentioned, actually performing the exercises instead of just discussing it boosts self-confidence in the ability to do it. The intervention is usually run in small groups (up to 12 people) with discussions regarding different topics such as what it means to be an effective self-manager, goal setting, planning and problem solving and coping strategies amongst others. Again, this group based treatment has been shown to be more beneficial than individual sessions. By teaching chronic pain sufferers the appropriate reasons for their symptoms, unhelpful thoughts and beliefs about pain are changed and by seeing a change in themselves and the other participants in the group further enhancestheir belief in their own ability to achieve their goals ${ }^{(264)}$. Importantly, the exercise component is supervised by a physiotherapist and can consist of a circuit of up to 10 exercises. These exercises are commenced at low intensity and repetitions and are adjusted and progressed according to the individual's abilities. This ensures safety in exercise and appropriate progression despite being in a group, one of the reasons to restrict group size.

Research to support this programme was performed by means of a six month RCT in patients with arthritis of the knee, which compared participation in this integrated intervention (ESCAPE-knee pain) with usual pragmatic care. The results showed that such a self-management intervention, which integrates self-management and exercise, led by a physiotherapist, was effective in improving function as well as having a higher probability of being more cost effective than usual care ${ }^{(85,272)}$ as well as being less costly than regular individual out-patient physiotherapy ${ }^{(264)}$. 
A second study was later conducted by the same group to establish the long-term outcomes of the intervention (up to 30 months). It was noted that initial improvements after the intervention had decreased somewhat. However, measures of function were still improved, and health care costs continued to be less when compared to the usual care group $^{(271)}$. Additional benefits of improved health beliefs and self-efficacy were also seen as a result of this combined approach. These two studies show that both short and long term benefits arise from combined intervention of exercise and education in knee OA for function and health costs.

A strength of the study by Hurley et al. ${ }^{(271)}$ was that a large sample size was used $(N=418)$ from various locations and socio-economic groups in the UK, which provided results that are likely to be generalizable to other areas in similar status countries. Research is warranted to establish if the same benefits are seen in lower income countries such as SA. Additionally, these results are very likely not due to chance as the required sample size of 200 in each group was achieved andthe trial was powered at $90 \% \mathrm{Cl}$, with $5 \%$ significance level and calculated to allow for attrition (only $18 \%$ withdrawal). The intervention's content and delivery is well explained to allow accurate replication and necessary adjustmentsfor implementation in the clinical setting. What makes this intervention attractive is that it is realistic: requiring minimal training of health personnel such as physiotherapists and simple equipment for the exercise component.Simple exercises and equipment have also been shown to further boost compliance and self-confidence in the ability to replicate the exercises at home. Thus it could be introduced into other clinical environments without these aspects being a major barrier. Additionally, despite the model of intervention being performed in knee pain patients (most labelled with OA)the sample could have included conditions other than OA of the knee. It is hoped that the principles behind such an intervention can be applied to other joints such as the hip and to those suffering from chronic pain and therefore it has been proposed that the programme could be used with people with general OA and chronic joint pain ${ }^{(271)}$.

As supported by previous evidence, the exercises used in the program focus on becoming more active, rather than specific strengthening of a group of muscles related to one joint. 
The advantage of the exercises being led by a physiotherapist and modified according to participant's abilities, means that persons with either hip or knee OA could participate in such an exercise programme as it is not specific or fixed. Exercises can be adapted not only to the abilities but also the needs of the participants by exercising in a certain way to address issues faced during activities of daily living. This allows for a clinically relevant programme which targets those arms of the ICF such as activity limitations and participation restrictions.

After the initial ESCAPE findings were published, Patel et al. ${ }^{(280)}$ delivered a similar intervention in persons primarily with knee OA awaiting a joint replacement. The authors took note of previous recommendations to extend such a combined intervention to other conditions $^{(226,271)}$ and did not exclude other joint pathologies or musculoskeletal conditions of the lower limbs from partaking in their study ${ }^{(280)}$. Likewise the intervention consisted of integrated education, self-management and exercise. Significant improvements were reported at 12 months in the Oxford Knee Score $(E S=0.27, p<0.01$ for severe $O A)$ and WOMAC scale (total WOMAC score ES $=0.42, p<0.01$ for severe $O A)^{(280)}$. A limitation noted for this study was the lack of a control group to compare improvements to. Therefore, although the findings are encouraging, the long term improvements reported in OA symptoms in a sample awaiting surgery should be considered with caution.

A few years later Jensen et al. ${ }^{(281)}$ proposed a trial in Denmark evaluating the effects of a combined exercise and education interventionin those with hip OA awaiting surgery. The study is currently being delivered and is due to end in 2015. In this study, the intervention is compared to a control group of education alone which makes the study more robust than the trial by Patel et al. ${ }^{(280)}$. The results of the study by Jensen and colleagues ${ }^{(281)}$ are eagerly awaited as it is hoped that the results will strengthen the above findings of an integrated intervention. Additional findings will add to the limited literature available in persons with hip OA. 


\section{b. Summary of combined intervention}

It has been recommended that this approach be used inOA and other conditions as it could result in lower health care usage and costs thus decreasing the burden on the health system. Interestingly a study on LBP pain managementin a group based format, had higher attrition than individual sessions with a physiotherapist ${ }^{(226)}$. The possible reason for thisis inflexibility of fixed group time session or difficulties travelling to sessions in disadvantaged persons.Also, such an intervention challenges the old ways of thinking and responding to pain. One of these challenges includes changing patient's thoughts about the meaning of pain i.e. that in chronic pain hurt does not always mean there is harm occurring; and this concept could be difficult for some patients to grasp ${ }^{(226)}$. Resistance to change further reinforces the need for this combined approach to correct this old mind set.

\section{ii. $\quad$ Barriers to application of combined management}

Despite strong evidence of the benefit and safety of exercise and self-management programs, only a minority of people are referred to these interventions because they continue to be erroneously considered ineffectual, expensive, and impractical. Consequently, few people benefit because of their poor provision and restricted access ${ }^{(271)}$. Review of the ESCAPE-knee pain programme shows otherwise. It was effective in improving long-term functional outcomes as well as improving secondary outcomes such as exercise health beliefs and SE. It was less costly and more cost effective than usual care as well as being practical in terms of implementation in a flexible clinical environment.

A possible barrier to the application of combined interventions is the lack of clear recommendations regarding precise indicationfor and timing of different treatment options. This explanation issupported by the low percentage (28\%) of patients who are first managed conservativelywith education, exercise and weight loss, as recommended by guidelines, prior to beingscheduled for a total joint replacement in developed countries such as the Netherlands ${ }^{(282)}$. To address this issue of unclear optimal timing of treatment alternatives and to improve decision making capacity, Smink and colleagues have developed the "stepped care strategy" in Europe whereby structure and timing of different treatment options are presented and discussed to enable the patient centred approach $^{(282,283)}$. 
A RCT is currently being undertaken in Denmark to assess whether a total joint replacement of the knee together with a 12 week multimodal intervention is more effective than a non-surgical 12 week intervention ${ }^{(284)}$. These results will assist in guiding evidence based practice regarding arthroplasty in patients suffering from OA of the knee (284).

It has been discussed how compliance to any intervention can and does become a barrier to treatment ${ }^{(285)}$. Different reasons have been put forward as to why adherence becomes an issue, most of which are psychosocial and not due to physical factors. Three suggestions have been reported by patients as to why behaviours revert and unhealthy thought patterns return ${ }^{(285)}$. The first mentions how patients experience difficulties in encompassing the educational advice and exercise into their normal day. The second speaks of patients requiring positive feedback when performing exercise as reassurance is required to continue taking part in it. Thirdly, patients feel helpless when faced with a challenge instead of being empowered. It is thus suggested that a multi-dimensional approach to changing beliefs and behaviours is used by the physiotherapist to build trust and confidence in what is being recommended so that patients can implement changes in their home lives ${ }^{(285)}$. Instead of passively teaching a patient the importance of movement, the physiotherapist should guide the patient through movements so that they can experience it themselves, ensuring they perform it correctly. Additionally by providing the patient with exercises in functional positions and alternatives or variations of exercise, they are able to implement activity more easily into their daily lives and feel confident in repeating it in their own environment ${ }^{(285)}$. These suggestions emphasise the combination of learnt selfmanagement skills and action through exercise as an approach which can be more beneficial when used together with SMI than when each is utilised separately ${ }^{(264)}$.

\section{iii. Summary of combined intervention}

By extensively reviewing the different aspects relating to education and exercise in persons with $O A$, it is clear that these two methods are continuously recommended as first-line management for sound reason. As seen by evidence-based literature, combining the two approaches is proposed to be more beneficial than either option used in isolation. Further research is being performed to provide evidence that physiotherapists are capable of delivering such a combined programme of education and exercise. 
Another RCT is currently underway in Australia whereby physiotherapy-led pain coping skills training and exercise in knee $\mathrm{OA}$ is being compared to education on pain coping skills or exercise alone ${ }^{(261)}$. Since the protocol for this RCT was approved, a pilot study has been reported on by Hunt and colleagues ${ }^{(286)}$ whereby a combined intervention delivered by a physiotherapist is seen as feasible and can potentially benefit patients with knee OA physically and psychologically. This emphasises the need for concrete evidence in this area of research.

\subsubsection{Relaxation therapy - guided imagery and progressive muscle relaxation}

As mentioned, the OA management guidelines have notdiscussed or included relaxation therapy as a recommended treatment option; this could be due to research relating to this approach in OA being scarce in the past. However, Baird and colleagues recognised the need for evidence-based studies in this field and produced encouraging findings from a number of studies performed on the effects of guided imagery (GI) and progressive muscle relaxation (PMR) in women with $\mathrm{OA}^{(220-222)}$. $\mathrm{Gl}$ is a technique using verbal suggestions to create a flow of relaxing thoughts based on an imagined scene, incorporating sight, sounds, smells and tastes. The process is aimed at refocusing attention on these imagined sensations instead of the pain and/or difficulties the person usually experiences ${ }^{(287)}$. During the Gl session, muscle relaxation is encouraged throughout the body, with emphasis on the affected sites. GI and PMR or guided imagery with relaxation (GIR) result in various psychological and physiological responses ${ }^{(287)}$. It is understood that these complex responses cause changes in pain, mobility and personal factors ${ }^{(221)}$.

Initially, Baird et al. ${ }^{(220)}$ pilotedthe effect of $\mathrm{GI}$ and PMR in OA in a small sample over 12 weeks. This was followed by a longitudinal study lasting four months ${ }^{(222)}$. The intervention consisted of listening to a 12-minute audio tape twice a day which guided the participant through visualisation scenes and progressive muscle relaxation. Results showed this management approach to significantly reducepainand improve mobility $(p<0.01)$ after 12 weeks $^{(220,222)}$. Improvements in mobility were seen to last for two months $(p=0.02)$ and reductions in pain were maintained at four months $(p=0.03)^{(222)}$. Additionally, $\mathrm{Gl}$ and PMRbrought about significant benefits in health related quality of life $(p=0.02)^{(221)}$. 
Although small female samples were used, the benefits demonstrate such a management approach holds the potential to bring about positive changes in this population. Further research, making use of standard outcomes for measuring mobility, was suggested in order to strengthen the results.Baird et al. suggested that such a self-management option, when used in isolation, is less complex than cognitive behavioural approaches, as it only requires one session to explain how and when relaxation should be performed ${ }^{(220)}$. Additionally GI and PMRwas stated to be less costly than cognitive behavioural interventions as attendance at multiple weekly classes is minimised.

Therefore the GI and PMR approach is described as simple to learn and use in the home environment. This makes it a very practical self-management technique for the population under investigation. Given that this management option is not part of the recommended first-line treatment for $\mathrm{OA}$, it was anticipated that a short relaxation component could be used as an adjunct to the previously discussed combined exercise and education intervention. The hope is that benefits from all three components would bring about desired changes in pain, disability, function and personal factors in a sample with $O A$ awaiting definitive surgery.

Furthermore, as suggested by previously discussed guidelines, other management options such as weight loss and manual therapy should also be encouraged and used in addition to education and exercise to further benefit the person living with this chronic condition ${ }^{(29,207)}$. These two treatment approaches are briefly discussed below.

\subsubsection{Weight loss}

The third aspect which forms a part of first-line treatment approach along with education and exercise is the need to address weight issues in those who are overweight or obese $^{(29)}$. Rationale behind this recommendation lies in the fact that higher body weight is a known risk factor for the development and early progression of $\mathrm{OA}^{(19,288)}$. Excess or abnormal mechanical loading of a joint appears to be one of the main factors leading to the development and progression of osteoarthritis, especially in the knee ${ }^{(19)}$. This risk factor applies to both primary and secondary osteoarthritis, where excess loading may be related to obesity or abnormal loading due to mal-alignment of the hip (coxa- vara or valga) and/or knee (genu- varum or valgum) ${ }^{(80)}$. 
The estimated risk for developing knee OA increases from about $40 \%$ to $60 \%$ in persons who are considered obese ${ }^{(289)}$ and a $40 \%$ and $80 \%$ increase in the odds of developing unilateral and bilateral hip OA respectively ${ }^{(290)}$. In the normal single leg standing phase of walking, the knee bears three to six times a person's body weight. Thus those that are overweight are exerting excessively more force through the knee joint which is proposed to cause damage to the joint surface ${ }^{(290)}$.

This association of obesity with the development and progression of OA, particularly at the knee and less so of the hip ${ }^{(291)}$, provides justification for weight reduction as part of the prevention and management of $\mathrm{OA}^{(80)}$. Weight loss can be accomplished by dietary changes together with increases in usual physical activity, as research has shown that if weight loss is targeted only by dietary changes without exercise, a decrease in leg muscle tissue and strength occurs whereas one would prefer to maintain or improve muscle mass and strength with a reduction in adipose tissue ${ }^{(292)}$. Other studies confirm the benefits of combined diet changes and exercise in improving self-reported function, pain as well as objective measures of function or mobility in patients with OA of the knee ${ }^{(293-295)}$. A MA on the effects of weight reduction in obese persons with knee OA showed that weight reduction of $5 \%$ body weight was associated with a significant decrease in self-reported disability $^{(296)}$.

In addition to excess weight causing $\mathrm{OA}$, studies have shown the negative effects of obesity on post-arthroplasty outcomes. Higher rates of post-operative complications and the need for revision were seen in knee replacement patients ${ }^{(297)}$. Additionally, longer hospital stay and systemic complications were reported in overweight and obese hip replacement patients ${ }^{(298)}$. Besides the effect of excess or abnormal loading on the structure of the affected joint, developingresearchhas identified that a chemical substance, called leptin, is produced in bodies with excess body fat. This substance circulates through the systemic system and it has been suggested that leptin can lead to joint damage ${ }^{(77)}$. If so, this would provide clear physiological support for the need for weight loss, not merely to decrease joint loading in this population. 


\subsubsection{Manual Therapy and assistive devices}

More and more literature is advocating for the first-line approaches such as education, selfmanagement, home exercises and weight loss programmesto address chronic diseases such as $O A^{(29,226,271)}$. This shows a clear shift in focus away from passive treatment delivered by a health care professional towards a more patient-centred management approach ${ }^{(299)}$. This has implications on manual modalities commonly used during physiotherapy such as electrotherapy, acupuncture and manipulations. Despite the lack of evidence supporting clinical efficacy for these techniques, they are clinically usedfortheir powerful placebo effects $^{(178,267,300,301)}$. Techniques such as ultrasound (US) or transcutaneous electrical nerve stimulation (TENS) and manual therapy like massage and joint mobilisation are seen as passive modalities which could result in the patient continually relying on the therapist, thus leaving the patient helpless if therapy is withdrawn ${ }^{(178,299)}$. It has however been recommended that manual techniques can be used as a second-line treatment option in conjunction with first-line techniques to further benefit the patient ${ }^{(207,208)}$. Literature on these modalities is controversial which leaves physiotherapists to decide on clinical expertise which manual therapy techniques are to be used in this group of patients. Instead, education on simple modalities such as thermotherapy, that can be used at home are helpful; as this requires self-evaluation and use of techniques such as heat or ice therapy to control their symptoms of pain and/or stiffness as they deem necessary are less controversial $^{(273)}$.

Completely different from manual therapy is the recommendation for the use of assistive aids to supply the patient with additional support from a walking stick or crutches in their own environment ${ }^{(78,208)}$. The manner in which this works is to assist with load reduction through the hip or knee by bearing weight through the upper limbs as well. An assistive device needs to be measured properly and the person should be taught how to correctly make use of the device by a health professional. However, once this initial step with the physiotherapist is completed, the patient is able to continue with self-management skills learnt such as daily use or modification of the device; encouraging the patient-centred approach $^{(282)}$. The benefits obtained from other orthotic devices (knee braces or wedged soles) or strapping techniques used to alter the position and biomechanics of the affected limb and to provide support to the unstable joint remains unclear ${ }^{(208)}$. 


\subsubsection{Surgical Management}

Besides the first- and second-line treatment options previously discussed and the pharmacological treatment methods which have not been covered in this review; patients who do not respond appropriately to the recommended conservative management options are advised to undergo surgical management ${ }^{(23,24,26,207,273,302)}$. Various joint preserving surgical methods are available for those suffering from OA of the hip or knee. An osteotomy involves excision of a part of bone and is usually done to influence the forces through the joint. Arthroscopic lavage or debridement (key-hole surgery to clean out the joint space of the affected joint)is performed to potentially change the course of the degenerative process ${ }^{(24,26)}$. The effectiveness of these procedures has not yet been established as the literature available is limited and based on poor quality studies ${ }^{(24,26,78)}$.

\section{i. Arthroplasty}

The surgical method prescribed for the population of interest in this study, which has been shown to clinically improve OA sufferers' pain and disability is an arthroplasty or joint replacement ${ }^{(19,24,269,302-305)}$. This method of management is reserved for those patients who, despite been treated with the above mentioned conservative and pharmacological methods, have persistent pain and dysfunction ${ }^{(78)}$.A hip replacement involves open surgery, usually via the outer side of the upper leg, where the head and neck of the hip joint is removed and a prosthetic head and stem is inserted into the femur. The hip socket is enlarged and a prosthetic cup is placed in the acetabulum to create a new hip joint ${ }^{(306)}$. A knee replacement involves open surgery via the front of the affected knee joint, both joint surfaces of the knee, superiorly from the femur and inferiorly from the tibia are removed and a metal joint surface is placed at the end of each bone to form a new knee joint ${ }^{(306)}$. The prostheses used in these operations are said to last between 15-20 years, yet studies comparing different brands of prosthetics is not yet available in order to say which is preferred $^{(78)}$. Goodman ${ }^{(305)}$ writes that arthroplasty surgery gives "excellent patient outcomes" and Hawker et al. ${ }^{(307)}$ report that improvements in function and pain post operatively can last as long 10 years. Universal guidelines support the surgical technique of an arthroplasty as a successful and cost-effective management option for severe $O A^{(25,247)}$. 


\section{a. A barrier to surgery: waiting lists}

Various barriers to implementation of suggested conservative management options have already been discussed in the text; however a major barrier to surgical management has been identified in both high and low income countries due to the rapidly increasing prevalence of older people living with $\mathrm{OA}$ requiring a joint replacement ${ }^{(33)}$. Waiting lists have been described as a growing issue causing delays in surgery as a consequence of high demand for this procedure ${ }^{(14)}$. Statistics from 2009 predict the demand for a joint replacement in Canada to increase by $30-40 \%$ in the following ten years ${ }^{(14)}$.

As mentioned, surgery such as a joint replacement is necessary for those patients who do not respond well to best practice management recommendations for $\mathrm{OA}^{(78)}$. It is almost seen as a last resort to effectively relieve the burden caused by pain and disability as per the ICF definition ${ }^{(308)}$. Therefore once a patient reaches the point of requiring an arthroplasty they should not have to wait years or even months to receive this treatment option ${ }^{(14)}$. From a surgeon's perspective; the current waiting time in a high income country such as Canada is unacceptable at 38 weeks. Mascarenhas and colleagues ${ }^{(14)}$ report that most patients are not satisfied with the period that they are required to wait before receiving surgical management. In addition to patients being unhappy about waiting, it has been said that delays in surgery can affect pre-operative health related quality of life, health status ${ }^{(15,34,309,310)}$ and can cause further disability, reduced function and prolonged pain $^{(33,71,246,311)}$. Yet, the literature surrounding this topic is controversial as a few studies report no or minimal effect on outcomes such as pain and function while being on a waiting list ${ }^{(312,313)}$; this is possibly due to variations in research methodology. Various studies are discussed below.

Effects of waiting lists on pre-operative health related quality of life have been documented with HRQoL deteriorating while on a waiting list ${ }^{(14)}$. Elsewherea decline in HRQoL and function has been reported in those waiting for a hip replacement forlonger than six months ${ }^{(15,309,311)}$ and in those waiting for a hip replacement an average of eight months ${ }^{(310)}$. The association between $\mathrm{OA}$ and co-morbidities is also highlighted, as research has shown that those on a waiting list with co-morbidities are likely to have lower HRQoL life scores than those without co-morbidities ${ }^{(88)}$. 
Kili et al. ${ }^{(314)}$ reported in a sample waiting an average of 11 months, that pain increased and function deteriorated in those awaiting a hip replacement and worse pain and disability was correlated to the time spent on the waiting list.However, Kelly et al. ${ }^{(312)}$ report minimal correlation between pain severity and function and time awaiting surgery, although this was based on a waiting list of only one month. It has also been suggested that the number of persons who experience a decline in different factors such as function and HRQoL due to waiting lists has been underestimated ${ }^{(313)}$.

Furthermore, there may also be adverse effects, based on health related quality of life measures, to post-operative outcomes if waiting periods are longer than 12 months. Kili et al. $^{(314)}$ also showed that significantly reduced post-operative quality of life was correlated with longer periods on a waiting list. Mahon and colleagues ${ }^{(311)}$ showed lower postoperative health related quality of life and mobility to be correlated to longer periods of waiting for an arthroplasty, yet this correlation was not proven to be significant in Mahon's study. It is suggested that these negative effects on HRQoL are compounded in those waiting for periods longer than six months ${ }^{(311)}$

Finally, a SR was performed in persons with OA requiring a joint replacement of the hip or knee, to establish what effect being placed on a waiting list has on pain and function ${ }^{(313)}$. Results yielded no effects of being on a waiting list on pain or function pre-operatively, but this was also limited to a waiting period of less than six months ${ }^{(313)}$. As mentioned, the normal waiting list at the hospital of concern in this study is at least one year and may be up to eight or more years ${ }^{(31)}$.A small number of studies of varied quality have been undertaken that investigated the effects of waiting for longer than six months and concluded that there was some evidence to show that functional state decreased in this group ${ }^{(315)}$. The lack of evidence in this field indicates the need for further high quality studies that analyse the effects of longer waiting periods in a more robust manner ${ }^{(313)}$. 


\section{b. A potential solution to waiting lists}

As the burden of waiting lists rises, governments in high income countries, such as Canada, are developing and slowly implementing strategies to help reduce the waiting periods for a joint replacement ${ }^{(14)}$. Various changes have been made and results documented from a province in Canada, whose waiting list consisted of over 2500 patients in 2006. It seems as though a coordinator, with the purpose of overseeing the list and its processes is helpful. In this developed country, upgrades to operating rooms were completed, new equipment was purchased and employment of more personnel was possible to help reduce the amount of people on the list by increasing the number of surgeries performed each week. However, these ideals are not easily implemented in low income countries, such as SA.

Another strategy that could hold the potential to reduce the burden caused by long waiting lists supports the rationale behind this present study: implementing pre-operative preventative and maintenance programmes including advice, exercise, nutritional adjustments and physiotherapy to modify known risk factors associated with $\mathrm{OA}^{(14)}$. Additionally, supporting the use of psychosocial instruments used in this study; it has been recommended that interventions should also target psychosocial aspects and aim to improve self-efficacy in those awaiting surgery ${ }^{(246)}$. A programme including selfmanagement, education, exercise and peer support was run as an RCT in Australian patients with OA awaiting surgery, which showed benefits of improvements in health related behaviours, skill acquisition and stiffness. However, these results were limited to a period of six months while on a list, supporting the need for further research on benefits for those on longer waiting lists and post-operative benefits ${ }^{(242)}$. 


\subsection{Conclusion}

In conclusion, after reviewing the available literature on the many factors related to osteoarthritis and its management, it is clear that due to the steady increase in the incidence of $\mathrm{OA}$, along with associated co-morbidities, this burden on the health system and more importantly, on the patient involved needs further attention. As discussed, the ICF framework for disability is of utmost importance when addressing a chronic pain condition such as OA. Not only does this common joint pathology cause several direct and indirect physical impairments but due to the pathophysiology of chronic pain contributing towards changes in the nociceptive pathways and the nervous system in a subgroup of this population; $\mathrm{OA}$ is not a simple condition to address. The condition also has a huge impact on psychosocial factors such as self-efficacy and quality of life among others, and as reported, the cumulative effects lead to severe disability in terms of daily function and hinder social participation for these individuals. In order to implement and provide effective management for those affected by this condition, further research is required in the South African public health setting to establish the effects of the recommended evidence-based guidelines from higher income countries. Of particular interest is the effect of first-line treatment guidelines of education and exercise in those patients with OA who have been placed on an extremely long waiting list for an arthroplasty, at Tygerberg Hospital in the Western Cape. 


\section{Methodology}

\subsection{Research design}

A single-blind randomised controlled trial was conducted.

\subsection{Sample}

The population of interest consisted of patients diagnosed with OA in SA. The sampling frame was made up of those patients who had been placed on a waiting list to receive a hip/knee joint arthroplasty, specifically those in the public health system in the Western Cape, SA. The principle investigator was allowed access to the waiting list at TBH: the waiting list is prioritised by the consultant in charge into three lists. The first list consists of those patients with extensive $O A$, whereas the second and third lists include those patients with less and the least severe symptoms/progression of the condition respectively. As research suggests that early non-surgical intervention is beneficial ${ }^{(208)}$, this study randomly utilised patients who had been assigned to the third priority waiting list and were on the list for at least three months. This three month period was to allow for necessary stabilisation to any new medications that may have been prescribed or adjustments to their treatment when the patient was first put onto the list. By using this method of selection, the aim was to target the population who may achieve the most possible benefits from the intervention, as they had the least severe form of OA.

The use of an assistive device was not a cause for exclusion from the study, but was rather documented and any change in assistive device usage was recorded. The study was not limited to participants of a certain BMI, as people with a wide range of different BMIs are seen as a better representation of the broaderpopulation and it was felt that the possible benefits from the study should not be withheld from patients with a particular BMI.

A random sample $(n=281)$ was drawn from the patients on the third priority list by means of random number selection in Microsoft Excel. The sample was contacted telephonically to inform them of the nature of the study and to invite them to participate at TBH(Appendix C; pg. 213). 
A large number of participants were unreachable $(n=147)$, the remainder who indicated that they werewilling to participate were asked to provide verbal consent for initial telephonic screening $(n=134)$. Initial telephonic screening involved questioning to ensure eligibility to participate in the study (Appendix D; pg. 219). Full inclusion and exclusion criteria are described in Table 3-1below. All inclusion criteria were met in order to participate $(n=52)$, however the presence of any one or more of the exclusion criteria immediately excluded the participant from the study $(n=82)$. See Figure 3-1.

Table 3-1 Inclusion and exclusion criteria

\begin{tabular}{|c|c|}
\hline Inclusion Criteria & Exclusion Criteria \\
\hline Willingness to commit to the study & $\begin{array}{l}\text { Previous trauma/surgery to the unaffected } \\
\text { leg }\end{array}$ \\
\hline Aged $50-70$ years & Cognitive impairment \\
\hline Diagnosed with OA of the hip/knee & $\begin{array}{l}\text { Do not meet the American College of Sports } \\
\text { Medicine (ACSM) guidelines for exercise } \\
\text { prescription (Appendix D; pg. 219) }\end{array}$ \\
\hline $\begin{array}{l}\text { On the waiting list for a minimum of three } \\
\text { months }\end{array}$ & $\begin{array}{l}\text { Those who have taken part in a six-week } \\
\text { programme aimed at improving self-efficacy } \\
\text { and management previously }\end{array}$ \\
\hline $\begin{array}{l}\text { Can comprehend and are literate in English } \\
\text { or Afrikaans or isiXhosa }\end{array}$ & \\
\hline
\end{tabular}




\subsection{Sample size and power analysis}

As pain was the primary outcome measure, data was used from a previous study on pain in people with $\mathrm{OA}$, in which the modified Brief Pain Inventory (m-BPI-sf) was validated, to ensure that the sample size would provide sufficient statistical power ${ }^{(140)}$. Change in pain was selected to determine the required sample size. Required sample size for change in pain was calculated using a smallest meaningful difference of 3 (on a scale of 0 to 10), and a standard deviation of $2.49^{(140)}$. Statistical significance was accepted as $p<0.05$. A medium to large $E S(E S>0.5)$ is accepted as meaningful. It was calculated that samples of 26,36 , and 45 participants would provide $80 \%, 90 \%$ and $95 \%$ statistical power for change in pain respectively. To allow for attrition, the target sample was a total of 48 participants (24 participants in each group). After recruiting a total of 52 participants, 10 did not arrive for baseline measures, which left 42 remaining as the sample.

\subsection{Instrumentation}

The primary outcome measure was pain as this is described as the main complaint in persons with $\mathrm{OA}^{(20)}$. Pain was measured by Brief Pain Inventory ${ }^{(35)}$, as described in Chapter Two (pg.22).See Appendix E for the BPI (pg. 223). In addition, secondary outcome measures included disability, function, self-efficacy and health related quality of life, as described below (Appendix E).

\subsubsection{Pain}

As discussed, recommendations from theIMMPACT group state that when measuring pain, the instrument used should not only evaluate pain on a uni-dimensional level ${ }^{(119)}$.Instead it should assess pain in terms of pain intensity, physical functioning, emotional functioning and patient ratings of treatments received ${ }^{(121)}$. The BPI is a measure that evaluates the first two of these four domains by measuring pain severity, as well as the impact/interference of pain on function ${ }^{(135)}$. As previously stated, it was initially validated for use in patients with cancer ${ }^{(137)}$ after which it has been validated and is widely used in non-cancer conditions in which pain is experienced, such as osteoarthritis ${ }^{(140,148)}$. It is a short selfadministered questionnaire designed for use in research ${ }^{(135)}$. The pain severity scale consists of four answers related to the severity of pain experienced on a scale of $0-10$; "worst pain", "least pain", "average pain" and "current pain". A mean pain severity score (PSS) is 
calculated from the four answers out of 10 (0 "no pain" and 10 "pain as bad as you can imagine").

The pain interference scale consists of seven questions related to the impact of pain on a scale of $0-10$; activity (4), emotional functioning (3) and sleep (1). A mean pain interference score (PIS) is calculated from the seven answers out of 10 (0 "no interference" and 10 "complete interference") ${ }^{(135)}$. The inventory also asked two questions regarding the percentage pain relief achieved from analgesics used. The BPI-sf has previously been translated into Afrikaans ${ }^{(316)}$ as well as isiXhosa; which has been shown to be valid for use in amaXhosa women living with HIV in SA ${ }^{(317)}$.

\subsubsection{Physical function}

The Physical Performance Task Battery is widely used in research studies to assess simple daily tasks, in order to characterize and quantify the effect of a condition on the patient's function ${ }^{(175)}$. This battery of tests has been validated and shown to be reliable in patients with back pain, cancer and HIV/AIDS ${ }^{(36,157,173,174)}$. While no literature has been found to validate its use in lower limb $O A$, the individual tests which make up the battery have been separately validated for use in patients with $O A$ and are appropriate for use in this sample. The content validity of the physical battery was explored in this study.

The original battery consisted of nine tasks of which only seven were used as described below:

- 50 foot $(15 \mathrm{~m})$ walk at preferred speed. Participants were timed as they walked 25 feet $(12.5 \mathrm{~m})$, turned around and walked back to the starting point at their normal walking pace.

- 50 foot $(15 \mathrm{~m})$ walk at fastest speed. Participants were timed as they walked 25 feet $(12.5 \mathrm{~m})$, turned around and walked back to the starting point as fast as they could.

- Forward reach test. Participants stood adjacent to a wall on which a tape measure was positioned horizontally at shoulder height. Participants reached as forward as far as they could and the distance reached was measured in $\mathrm{cm}$. 
- Timed, repeated sit-to-stand. Participants sat in a standard chair with arm rests and were timed as they stood up and then sat back down, twice.

- The test was repeated after a brief rest and the average time of the two trials was used.

- Sock test. Participants sat in a standard chair and were timed as they put on one loose-fitting sock on each foot. The time taken to complete each foot was recorded in seconds as well as the ability to don a sock or not.

- Timed, repeated reach-up. Participants stood facing a wall and reached up as high as they could with both hands. A mark was placed on the wall at the reach distance. Participants then reached up to the mark and returned their hands to their sides three times, as fast as they could.

- Six minute walk test. Participants walked as far and as fast as they could for six minutes. The distance walked was measured at six minutes (Participants were allowed to rest if and as necessary during the six minute period).

- Timed belt tie. Participants are to be seated. Using an elastic wrap bandage approximately four feet long, they are timed as they wrapped the bandage around their waists and tied it in front of them. This task was not included as a part of the current study as it does not relate to lower limb balance or function.

- Coin test. Participants sit at a table. They are timed as they picked up four coins (a quarter, a dime, a nickel, and a penny) and placed them in a cup. They were required to pick up each coin individually. Again this task was not included as a part of the current study as it does not relate to lower limb balance or function.

\subsubsection{Disability}

The widely used Health Assessment Questionnaire ${ }^{(37,37,176)}$ has been established in rheumatology as a comprehensive, valid and patient orientated outcome measure of health status in arthritis patients ${ }^{(318)}$. Since the HAQ has previously been used in this group of patients, it will allow for comparisons to be made with other studies in this field of research. The HAQ assesses the functional ability of a patient and comprises of two parts. Firstly, 20 questions are grouped into eight categories; dressing and grooming, arising, eating, walking, hygiene, reach, grip and common activities. 
The four possible responses to each question (no difficulty, some difficulty, much difficulty, unable to do) are scaled such that the average across functional categories produces a variable that ranges from 0 (no disability) to 3 (severe disability). The second part comprises of a pain VAS from 0 (no pain) to 100 (severe pain) which the participant completes as a self-report of their pain severity in the past week (Appendix E; pg. 223). Translation of this questionnaire has previously been done into South African English and Afrikaans by the Mapi Research Institute (HAQ (C) Stanford University 1980, All rights reserved).An isiXhosa translation was not available for the $\mathrm{HAQ}$; therefore a translator was used during administration of this outcome measure.

\subsubsection{Self-efficacy}

Assessment of self-efficacy has been reported as increasingly more important when evaluating the effectiveness of any self-management programme ${ }^{(194)}$. The Self-Efficacy for Managing Chronic Disease 6-Item Scale ${ }^{(177)}$ has been modified from the Chronic Disease Self-Efficacy Scale and has been previouslyusedin patients with arthritis ${ }^{(204)}$. This short scale was developed and tested for the use in chronic disease management studies ${ }^{(38)}$ and a study by German researchers have shown this 6-item scale to be valid and reliable for use in measuring self-efficacy in the management of chronic conditions such as $\mathrm{OA}^{(194)}$. The scale consists ofsix questions including several domains common to many chronic diseases: symptom control, role function, emotional functioning and communicating with physicians. The response to each question is scored on a scale of 0 (not confident at all) to 10 (totally confident) from which a mean SE score is calculated out of 10 (a higher score indicating a higher self-efficacy)(Appendix E; pg. 223). The instrument is available in isiXhosa and was translated via forward/back translation into Afrikaans for use in this study.

\subsubsection{Health related quality of life}

The EuroQol Instrument is one of the most commonly used generic measures of health status by summarising the patient's HRQoL into a single index from -0.59 to 1 (1 indicating a better health status) ${ }^{(39,190)}$.Participants report on their health status in five dimensions; mobility, self-care, usual activities, pain/discomfort, and anxiety/depression. Responses to the domains are presented in three levels of severity from no problems to some/moderate problems, or extreme problems. Each response is scored to calculate the disability index. 
The second part of the EQ-5D comprises of a self-reported state of health value from 0 100 (100 being the best health) on a vertical VAS. Participants were asked to rate their health for today on the thermometer type VAS (Appendix E; pg. 223).This outcome measure is currently widely used in a variety of research areas in different countries ${ }^{(190)}$ and has been deemed valid and reliable after translations into several indigenous South African languages, including South African English, Afrikaans ${ }^{(190)}$ and isiXhosa ${ }^{(319)}$.

\subsubsection{Body mass index}

Body mass index ${ }^{\ddagger \neq}$ is a measure of body fat in adults ${ }^{(40)}$.It is calculated as $\frac{\text { weig ht }(\mathrm{Kg})_{2}}{\text { height }\left(\mathrm{m}^{2}\right)}$.

Additionally, at month six the experimental group were asked to complete an open ended questionnaire which included seven questions about the six-week intervention and workbook used. This was conducted via a questionnaire with the research assistant reading the questions aloud and the participants were asked to write down their responses in their preferred language. This was to gain insight into the participant's thoughts or feelings around the intervention (Appendix E; pg. 223).

\subsection{Intervention}

The intervention comprised of a six week physiotherapist-led education and exercise programme. This programme was developed from previously used programmes such as the ESCAPE-knee pain programme ${ }^{(178,226,271)}$, the Arthritis Self-Management Programme $e^{(158,256)}$ and the Positive Living Self-Management programme ${ }^{(317)}$, developed for South African patients experiencing pain due to HIV/AIDS ${ }^{(317)}$. As mentioned previously, the intervention is based on the ICF framework and is a multi-faceted approach to addressing the participant holistically by means of education (including neuroscience education), selfmanagement strategies and an active exercise component. Theintervention approach and content allows the same intervention to accommodate participants with OA ofboth hip and knee.

${ }^{\ddagger \neq \ddagger} \mathrm{BMI}<18.5=$ underweight, $18.5-24.9=$ normal, $25-19.9=$ overweight, $>30=$ obese $^{(40)}$ 
The interventiontook place in an out-patient setting at Tygerberg Hospital and was led by the principle investigator, a qualified physiotherapist who had been trained in the respected field and who could deliver the programme in both English and Afrikaans. An isiXhosa translator was present for all intervention sessions. The same physiotherapist led all six sessions to ensure content and delivery of the intervention remained constant. The intervention session took place for a maximum of two hours, on a weekly basis for six successive weeks, in groups of no more than 12 participants. Each two hour session included an educational component of an hour, an exercise component of 20 to 30 minutes and a relaxation session of 10 minutes.

The education and discussion topics covered are outlined in Table 3-2below. The topics and model of delivery of the educational and exercise content is based on evidence supported by literature ${ }^{(11,178,228,320)}$. The educational content was evidence based using relevant medical textbooks and peer-reviewed articles on $\mathrm{OA}$ and related topics ${ }^{(7,30,207,230,241,321)}$ and was aimed at increasing knowledge and understanding on the condition of concern, pain processes, exercise and related topics affected by their condition. Important topics such as self-management strategies, goal setting, coping mechanisms, stress management, pacing, nutrition, medication were also discussed. Each participant received a "Living with Osteoarthritis" booklet (in their preferred language) which comprised of the outline and content of each educational session as well as sections for the participants to complete during the week that followed each session (Appendix F; pg.237). Participants were requested to record weekly goals and if these were achieved, as well as any changes in abilities and/or pain scores during the week following each session.

The exercise component comprised of various stretching, light aerobic exercise and different lower limb muscle group strengthening exercises ${ }^{(272,317)}$. Each participant was monitored throughout the session by the investigator and exercise was adapted to each participant's capability. Participants were required to set exercise and activity goals on a weekly basis and to record these in their workbooks. The exercise component was progressed weekly in duration by two minutes and intensity progressed as appropriate, depending on each participant's individual ability. If a patient reported an increase in pain of $>3 / 10$ during testing or during the intervention, the activity was stopped, the participant assessed and the exercise adjusted appropriately or stopped completely. 
The detailed exercise programme is described in Appendix G (pg. 329) The relaxation session consisted of the physiotherapist facilitating a relaxation visualisation while participants sat or lay comfortably with their eyes closed (Appendix G; pg. 329).

Attendance for the intervention was recorded and monitored weekly (Appendix $\mathrm{H}$; pg. 333). When a participant was absent for a session of the intervention, the participant was contacted telephonically and asked the reason for absenteeism and were encouraged to attend the next session. 
Table 3-2 Educational content covered on a weekly basis during the intervention

\begin{tabular}{|c|c|}
\hline Topic for the week & Content covered \\
\hline Week 1: & Pathology of OA \\
\hline \multirow{7}{*}{ Osteoarthritis, Self-management and Exercise } & What is meant by "self-management" \\
\hline & Self-management steps \\
\hline & Action plans, goal setting \\
\hline & Exercise dos and don'ts \\
\hline & Types of exercise \\
\hline & Steps to success with exercise \\
\hline & An exercise routine \\
\hline Week 2: & Physiology of acute and chronic pain \\
\hline \multirow{7}{*}{ Managing common symptoms } & Pain and flare ups of pain \\
\hline & Swelling \\
\hline & Joint protection, assistive devices \\
\hline & Pacing and activity/resting cycles \\
\hline & Fatigue \\
\hline & Frustration \\
\hline & Isolation \\
\hline Week 3: & What is stress? \\
\hline \multirow[t]{4}{*}{ Stress Management } & Managing stress \\
\hline & Sleep management \\
\hline & Communication with your health carer \\
\hline & Relaxation skills \\
\hline Week 4: & Balanced nutrition \\
\hline \multirow[t]{3}{*}{ Eating well } & Dealing with barriers to eating well \\
\hline & Food safety \\
\hline & Weight loss benefits \\
\hline Week 5: & Making informed treatment decisions \\
\hline \multirow[t]{3}{*}{ Medication and disease related problem solving } & Appropriate use of medications \\
\hline & $\begin{array}{l}\text { Link between a healthy lifestyle, good } \\
\text { nutrition and exercise }\end{array}$ \\
\hline & $\begin{array}{l}\text { Communicating effectively with family, } \\
\text { friends, and health professionals with } \\
\text { regards to your problems }\end{array}$ \\
\hline
\end{tabular}


Week 6:

Continuing as a successful self-manager
Recap of key components of successful

self-managing

Action planning for the future

Reflection on changes 


\subsection{Procedure}

Firstly, $10 \%$ of the sample size (five participants) was requested to attend the initial meeting to pilot the baseline procedures with the principle investigator and the RA. This was to establish the feasibility of the data collection process, including the information sheet, informed consent document and outcome measures. This pilot process went smoothly and brought to the attention of the investigator two practical considerations regarding one of the outcome measures; namely the physical performance task battery. For the sock test, it was not established which foot should be included in the test, so the decision was made to complete the test on both feet. Additionally, it was decided that due to the practical environment of the research setting and to avoid possible distractions, the six minute walk test was measured out as a 48 meter stretch in a corridor that would be walked back and forth for the six minute duration. The five participants who formed the pilot study were included in the main study.

Thereafter, eligible participants were requested to meet in person with the principle investigator and the RA during week 0 . Ten participants did not arrive for this meeting (Figure 3-1). Written informed consent was obtained and full screening for ACSM exercise criteria was completed for the remaining sample ( $N=42$ ) (Appendix C; pg. 213 and Appendix D; pg. 219). Demographic information was recorded with the help of the RA on the demographic information sheet (Appendix E; pg. 223). The participants completed the five outcome measures at baseline (the BPI, HAQ, SE for Managing Chronic Disease 6-Item Scale, EQ-5D and the Physical Performance Task Battery). The RA was blinded throughout the study. At baseline the RA was unaware of allocation as participants had not been allocated to groups yet. Additionally, at follow up measures participants were requested not to disclose which group they had been a part of and measurements of those in the experimental and control group were done concurrently. Self-report instruments were led by the RA and the battery was administered by the RA in the participants preferred language, using a translator for isiXhosa if necessary. The participants completed the tasks as they usually function, if they usually use an assistive device they completed the battery with the device and this was noted. If there was any change in the use of their device this was also recorded. If a participant was unable to complete a task for any reason this was documented and the previous score was carried forward. 
The RA measured each participant's weight on a digital scale and their height by a wall tape measure without shoes. BMI was then calculated and recorded. This measure wasrecorded at baseline, week 12 and month six to assess any change over the course of the study.

The process of randomisation to the intervention and control groups was explained by the principle investigator after outcome measures were completed, as well as the need to maintain the blinding of the RA to either group for follow up measures. The participants were stratified by the primary researcher, according to whether they have OA of the hip or knee. Following stratification participants' codes were randomly assigned into either the experimental or control group, by means of random number allocation generated by Excel by the principle investigator to ensure concealed allocation. Those selected for the control group ( $n=22$ ) were contacted telephonically in the week that followed and were instructed to continue receiving their usual care, determined by their primary doctor, while awaiting surgery. The decision to continue usual care was made as it was comparable to pragmatic control groups which received routine care in previous studies on similar integrated management interventions ${ }^{(226,242,271)}$. The experimental group $(n=20)$ were contacted telephonically and were requested to begin the six week education and exercise intervention commencing the following week (Figure 3-1). 


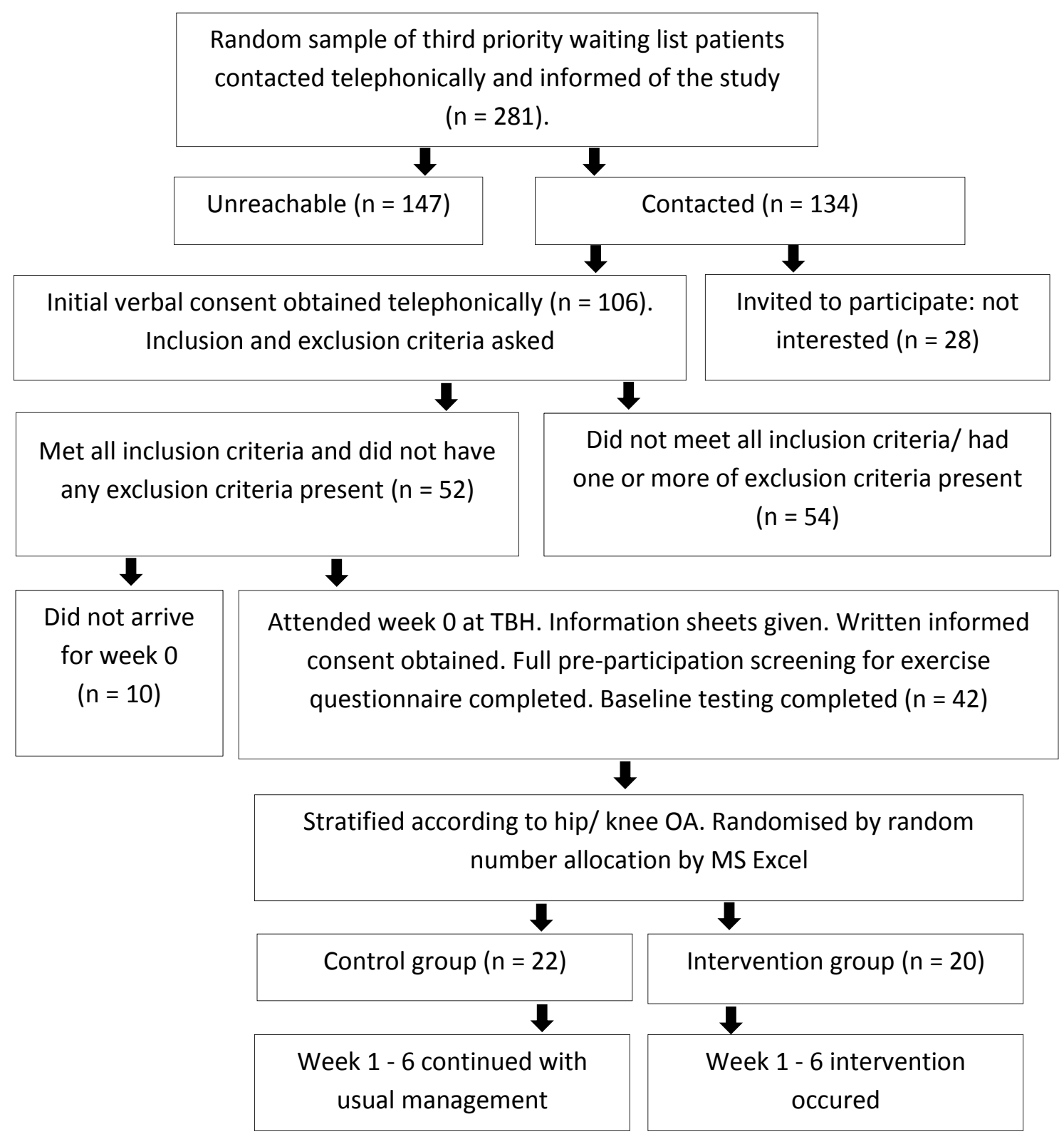

Figure 3-1 Recruitment and randomisation process 
The intervention took place as described above for six weeks. All outcome measures were taken at week 0 , week six, week 12 and month six by the same blinded RA. The RA was a qualified physiotherapist who was bilingual in English and Afrikaans. The instruments were available in the participant's first language, either English or Afrikaans or isiXhosa to foster better understanding of what was being asked, as well as to allow for differences in cultures. Additionally, an isiXhosa translator was at all data collection meetings to translate in person for isiXhosa first language speakers. Participants were provided with R30.00 to cover travel expenses for each visit to the hospital for the intervention and outcome measures, as these visits are in addition to their normal care.

\subsection{Ethical considerations}

Ethical approval was obtained from the University of Cape Town's Faculty of Health Sciences, Human Research Ethics Committee (HREC Ref no 378/2013) and from head of research projects at Tygerberg Hospital (Appendix B; pg. 209). An amendment was requested before the sixth month of follow up measures, to include an additional open ended questionnaire which the experimental group were to answer. This amendment was approved by the UCT HREC (Appendix B; pg. 209).

This study adhered to the principles outlined in the Declaration of Helsinki ${ }^{(322)}$. Autonomy was maintained throughout the study by providing the patients who were contacted and involved, with all the details pertaining to the study. Verbal consent was obtained telephonically prior to initial screening and written informed consent, in the participant's preferred language, was obtained prior to agreement of participation in the study.

Hypothesised advantages and any possible risks of the study were explained to the participants and it was made clear that, any participant had the right to withdraw from the study at any time for whatever reason, without prejudice or penalty. The principles of beneficence and non-maleficence were upheld throughout the study as previous studies have shown benefits of such an intervention and suggest further research on this topic. The intention was to replicate these positive results and bring about helpful, not harmful, changes to the participant's symptoms and lives. If the participant reported worsening symptoms due to the testing or intervention, the participants were assessed appropriately, the intervention adjusted accordingly or withheld if necessary. 
Referral to the required health professional to manage the matter further or if any other need arose for referral during the duration of the study was done as appropriate (one was referred to OT for a wheelchair assessment, two were referred to physiotherapy management for assessment and treatment of other conditions, one was referred to ophthalmologist for eye testing and two were referred to an orthotic centre for appropriate devices).

The study did not impact regular care. All other appointments the participants had prior to the study, continued as normal. There were no consequences to usual care if the participant was not interested in participating or chose to withdraw from the study at any time. Participation in the study did not change the participants place on the waiting list or chance of receiving surgery earlier or any other future medical care available to the participant. Allocation to the intervention or control group was performed by random number generation and identities were concealed for this process to avoid unfair allocation. The control group continued to receive their regular management while being on the waiting list; no aspect was removed/added to their usual care. However, the intervention produced significant positive effects in the experimental group; therefore the intervention was offered to the control group after the duration of the study, so as to maintain the principle of justice.

Confidentiality was maintained throughout the study by the allocation of a code for each participant. Personal information that was collected from the participants was not shared with others; instead the overall results of the group would be shared as the results of the study. Information gathered and data collected was stored on an external hard drive, protected and locked away. Coercion or undue influence was avoided as participants were not paid to participate in the study and the only possible cost to the participants was that of transport, which was covered by the researcher. Proposed benefits from the study was explained to participants before involvement; including decreased pain and disability, improved function, quality of life as well as self-efficacy and self-management of their chronic condition. Possible risks were also explained; including those risks associated with performing exercise. 


\subsection{Statistical analysis}

The primary outcome measure of pain severity according to the BPI at baseline showed that data were normally distributed [Week 0 BPI severity $(\mathrm{N}=42) \mathrm{K}-\mathrm{S} \mathrm{p}$ value $>0.20$ (K-S p > $0.05)]$. Therefore parametric statistics were used in the numerical analysis and Pearson Chisquared $\left(\chi^{2}\right)$ calculations were used for categorical data. Data was analysed using Statistica software (StatSoft, Inc. 2004. STATISTICA, Data Analysis Software System, Version 10. www.statsoft.com). Statistical significance for the two main effects of group and time, and the interaction (group $x$ time) for the BPI, the HAQ index and HAQ pain VAS, the physical performance task battery physical time and distance tests, the self-efficacy questionnaire and for HRQoL EQ-5D index was assessed using a two-way analysis of variance (ANOVA) with repeated measures. Tukey's post hoc comparisons were performed where necessary to determine significance within or between groups at different time periods.

Analysis was by intention to treat. Missing data was managed by carrying forward the last observed measurement. All numerical data was presented as the mean $(\mathrm{M}) \pm$ standard deviation (SD). Statistical significance was accepted as $p<0.05$. Effect sizes were calculated when there were significant differences between groups.Effect size ${ }^{\S \S \S}$ was calculated as Cohen's $\mathrm{d}^{(323)}$ using the following formula: $E S=\frac{\text { experimental mean change }- \text { control mean change }}{\text { pooled baseline } S D}$

\footnotetext{
${ }^{\S \S}$ Cohen's $d$ estimates the magnitude of the effect. 0.2 is considered a 'small' effect size. 0.5 represents a 'moderate' effect size. 0.8 a 'large' effect size.
} 


\section{Results}

\subsection{Sample}

The following Consolidated Standards for Reporting Trials(CONSORT) flow diagram ${ }^{(324)}$ reflects the processes from initial contact with the sample to those participants who were involved in the study and follow up measures (Figure 4-1).

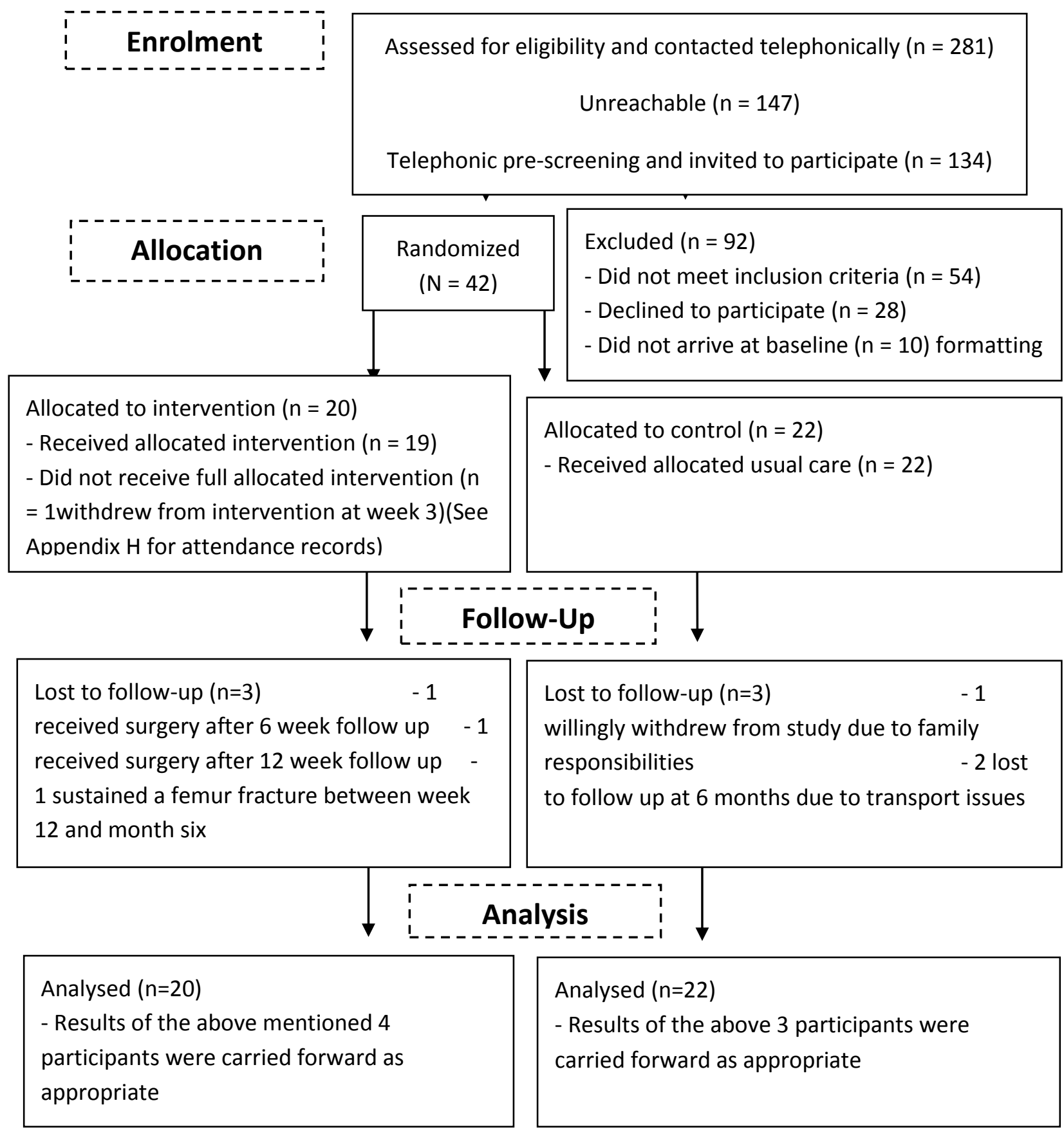

Figure 4-1CONSORT flow diagram of initial contact to analysis 


\subsection{Socio-demographic information}

Results are presented throughout for the sample $(N=42)$; the experimental group $(n=20)$ and the control group $(n=22)$.

\subsubsection{Age, weight, height, BMI}

Data was gathered by the demographic questionnaire and weight and height measures were taken at baseline to calculate BMI values for each participant.The mean age for the sample was59.52, SD $=4.75$ years. The sample had a very high mean BMI value of $35.79, \mathrm{SD}$ $=8.81$ which is classified as class llobesity ${ }^{(325)}$. There were no significant differences between groups ( $p>0.05$ ) for age, weight, height and BMI values (Table 4-1below).

Table 4-1 Age, weight, height, BMI of the participants ( $N=42)$

\begin{tabular}{|c|c|c|c|c|}
\hline & $\begin{array}{l}\text { Participants } \\
\qquad \mathbf{N}=\mathbf{4 2}\end{array}$ & $\begin{array}{c}\text { Experimental } \\
\text { Group }\end{array}$ & $\begin{array}{c}\text { ControlGroup } \\
\qquad n=22\end{array}$ & \\
\hline & & $\mathrm{n}=\mathbf{2 0}$ & & \\
\hline & $\begin{array}{c}\text { Mean } \pm \text { SD } \\
\text { (Range) }\end{array}$ & $\begin{array}{c}\text { Mean } \pm \text { SD } \\
\text { (Range) }\end{array}$ & $\begin{array}{c}\text { Mean } \pm \text { SD } \\
\text { (Range) }\end{array}$ & Significance test \\
\hline Age & & & & $t=0.55 ; p=0.74$ \\
\hline Years & $\begin{array}{l}59.52 \pm 4.75 \\
(51.0-70.0)\end{array}$ & $\begin{array}{l}59.95 \pm 4.98 \\
(51.0-68.0)\end{array}$ & $\begin{array}{r}59.14 \pm 4.62 \\
(53.0-70.0)\end{array}$ & \\
\hline Weight & & & & $t=-1.47 ; p=0.87$ \\
\hline Kilograms (Kg) & $\begin{array}{r}91.33 \pm 23.23 \\
(40.4-150.0)\end{array}$ & $\begin{array}{r}85.88 \pm 22.44 \\
(40.40-142.6)\end{array}$ & $\begin{array}{r}96.28 \pm 23.32 \\
(46.30-150.0)\end{array}$ & \\
\hline Height & & & & $t=0.09 ; p=0.34$ \\
\hline Meters (m) & $\begin{array}{l}1.60 \pm 0.10 \\
(1.41-1.8)\end{array}$ & $\begin{array}{l}1.60 \pm 0.11 \\
(1.41-1.8)\end{array}$ & $\begin{array}{l}1.60 \pm 0.09 \\
(1.49-1.8)\end{array}$ & \\
\hline Body mass index (BMI) & & & & $t=-1.49 ; p=0.59$ \\
\hline $\mathrm{Kg} / \mathrm{m}^{2}$ & $\begin{array}{r}35.79 \pm 8.81 \\
(17.96-59.4)\end{array}$ & $\begin{array}{r}33.69 \pm 9.22 \\
(17.96-59.4)\end{array}$ & $\begin{array}{r}37.69 \pm 8.16 \\
(18.11-50.0)\end{array}$ & \\
\hline
\end{tabular}




\subsubsection{Gender, language, education level, employment status}

For categorical variables, Pearson-Chi squared $\left(\chi^{2}\right)$ was calculated to determine any significant differences between groups.No significant differences were seen between groups forgender, first language spoken, educational level and employment status ( $p>$ $0.05)$. The majority of the sample was female $(n=32)$, Afrikaans speaking $(n=27)$, of low educational levels (grade 8-9: $n=16$ ) and unemployed $(n=36)$ (Appendix H; pg. 333).

Table 4-2 Gender, language, educational level, employment status ( $\mathbf{N}=42$ )

\begin{tabular}{|c|c|c|c|}
\hline & $\begin{array}{l}\text { Participants } \\
\qquad \mathbf{N}=42\end{array}$ & $\begin{array}{c}\text { Experimental } \\
\text { Group } \\
n=20\end{array}$ & $\begin{array}{c}\text { Control } \\
\text { Group } \\
n=22\end{array}$ \\
\hline & Number(\%) & Number(\%) & $\begin{array}{cc}\text { Number(\%) } & \begin{array}{c}\text { Significance } \\
\text { test }\end{array}\end{array}$ \\
\hline Gender & & & $\chi^{2}=0.81 ; p=0.37$ \\
\hline Male & $10(23.8)$ & $6(30.0)$ & $4(18.2)$ \\
\hline Female & $32(76.2)$ & $14(70.0)$ & $18(81.8)$ \\
\hline First language spoken & & & $\chi^{2}=5.19 ; p=0.16$ \\
\hline English & 5 (11.9) & $4(20.0)$ & $1(4.5)$ \\
\hline Afrikaans & $27(64.3)$ & $12(60.0)$ & $15(68.2)$ \\
\hline isiXhosa & $7(16.7)$ & $4(20.0)$ & $3(13.6)$ \\
\hline Sesotho & $3(7.1)$ & 0 & $3(13.6)$ \\
\hline Educational level & & & $\chi^{2}=17.06 ; p=0.15$ \\
\hline No schooling & $2(4.8)$ & $1(5.0)$ & $1(4.5)$ \\
\hline Grade $1-7$ & $10(23.8)$ & $5(25.0)$ & $5(22.7)$ \\
\hline Grade $8-9$ & $16(38.1)$ & $7(35.0)$ & 9 (40.9) \\
\hline Grade $10-12$ & $12(28.6)$ & $6(30.0)$ & $6(27.3)$ \\
\hline Tertiary level & $1(2.4)$ & $1(5.0)$ & 0 \\
\hline Not recorded & $1(2.4)$ & 0 & $1(4.5)$ \\
\hline Employment status & & & $\chi^{2}=1.22 ; p=0.54$ \\
\hline Employed & $6(14.3)$ & $4(20.0)$ & $2(9.0)$ \\
\hline Unemployed & $36(85.7)$ & $16(80.0)$ & $20(91.0)$ \\
\hline Pensioner & $21(58.3)$ & $10(62.5)$ & $11(55.0)$ \\
\hline Disability grant & $15(41.7)$ & $6(37.5)$ & $9(45.0)$ \\
\hline
\end{tabular}




\subsubsection{Clinical information: time spent on the waiting list,co- morbidities, joint/s affected, assistive device used, medication used}

There were no significant differences between groups for number of months on the waiting list, co-morbidities present, joint/s affected, subgroup stratification and assistive device usage at baseline (Table 4-3below).

The mean time on the waiting list was $3.61, S D=2.44$ years for the entire sample, with no significant difference between groups $(t=0.08, p=0.93)$. The mean number of comorbidities per participant in the sample was calculated to be 1.29 . Awide variety of comorbidities existed in the sample group, of which most were chronic diseases of lifestyle. The most common being HPT $(n=32)$ and HPT and DM co-existed in many of the participants $(n=11)$ (Table 4-3below). Refer to Appendix H (pg. 335)for full details regarding co-morbidities.

When the sample was stratified by OA of hip, knee or combined hip and knee OA, it was seen that there were slightly more OA knee participants $(n=19)$ than OA hip $(n=11)$ and combined hip and knee OA $(n=12)$ in the sampleyet there was no significant difference between groups after stratification.

With regards to the use of an assistive device: more than $70 \%$ of the sample used an assistive device, with more than half of those that used a device making use of a single crutch or walking stick and the rest used two crutches (Table 4-3below).

There was a significant difference between the groups' medication usage for controlling symptoms such as pain and inflammation as a result of OA $(\chi 2=6.25, p=0.04)$. The majority $(n=13)$ of the experimental group usedanalgesia alone, whereas the majority $(n=$ 13) of the control group was seen to use a combination of analgesia and anti-inflammatory drugs(Table 4-3). 
Table 4-3 Co-morbidities, joint/s affected, assistive device and medication usage ( $\mathrm{N}=42$ )

\begin{tabular}{|c|c|c|c|}
\hline & $\begin{array}{l}\text { Participants } \\
\qquad \mathbf{N}=42\end{array}$ & $\begin{array}{l}\text { Experimental } \\
\begin{array}{l}\text { Group } \\
n=20\end{array}\end{array}$ & $\begin{array}{l}\text { Control } \\
\text { Group } \\
\mathrm{n}=\mathbf{2 0}\end{array}$ \\
\hline & $\begin{array}{c}\text { Number } \\
\text { (\%) }\end{array}$ & $\begin{array}{c}\text { Number } \\
\text { (\%) }\end{array}$ & $\begin{array}{cc}\text { Number } & \text { Significance } \\
(\%) & \text { test }\end{array}$ \\
\hline Co-morbidities & & & $\chi^{2}=6.40 ; p=0.60$ \\
\hline None & $6(14.3)$ & $4(20.0)$ & $2(9.1)$ \\
\hline Not recorded & $1(2.4)$ & $1(5.0)$ & 0 \\
\hline Asthma & $1(2.4)$ & $1(5.0)$ & 0 \\
\hline Diabetes Mellitus (DM) & $2(4.8)$ & 0 & $2(9.1)$ \\
\hline Hypertension (HPT) & $16(38.1)$ & $8(40.0)$ & $8(36.4)$ \\
\hline Asthma \& HPT & $2(4.8)$ & $1(5.0)$ & $1(4.5)$ \\
\hline DM \& HPT & $11(26.2)$ & $4(20.0)$ & $7(31.8)$ \\
\hline Asthma \& HPT \& cardiac & $2(4.8)$ & $1(5.0)$ & $1(4.5)$ \\
\hline DM \& HPT \& cardiac & $1(2.4)$ & 0 & $1(4.5)$ \\
\hline $\begin{array}{l}\text { Total number of co-morbidities } \\
\text { (across the sample) }\end{array}$ & 54 & 22 & 32 \\
\hline $\begin{array}{l}\text { Mean number of } \\
\text { co-morbidities(per participant) }\end{array}$ & 1.29 & 1.1 & 1.45 \\
\hline OA Subgroup stratification & & & $\chi^{2}=0.05 ; p=0.98$ \\
\hline Hip & $11(26.2)$ & $5(25.0)$ & $6(27.25)$ \\
\hline Knee & $19(45.2)$ & $9(45.0)$ & $10(45.5)$ \\
\hline Both hip \& knee & $12(28.6)$ & $6(30.0)$ & $6(27.25)$ \\
\hline Assistive device used & & & $\chi^{2}=1.02 ; p=0.60$ \\
\hline None & $11(26.2)$ & $4(20.0)$ & $7(31.8)$ \\
\hline $1 \mathrm{crutch} /$ stick & $22(52.4)$ & $12(60.0)$ & $10(45.5)$ \\
\hline 2 crutches & $9(21.4)$ & $4(20.0)$ & $5(22.7)$ \\
\hline $\begin{array}{l}\text { Medication used to } \\
\text { manage OA symptoms }\end{array}$ & & & $\chi^{2}=6.25 ; p=0.04^{* *}$ \\
\hline None & 5 (11.9) & $2(10.0)$ & $3(13.6)$ \\
\hline Analgesia only & $19(45.2)$ & $13(65.0)$ & $6(27.3)$ \\
\hline $\begin{array}{l}\text { Analgesia\& } \\
\text { anti-inflammatories }\end{array}$ & 18 (42.9) & $5(25.0)$ & $13(59.1)$ \\
\hline $\begin{array}{l}* * \text { indicates a significant difference in } \\
\text { at baseline }\end{array}$ & tion use betw & experimental a & ontrol group \\
\hline
\end{tabular}




\subsection{Pain}

\subsubsection{Pain Severity Score (PSS) and Pain Interference Score (PIS)}

The primary outcome measure wasdetermined using the BPI and measures were obtained at baseline,week six, week 12 and month six. Full results of the PSS and PIS over time are presented in Table 4-4below.

Table 4-4 PSS and PIS ( $\mathrm{N}=42)$

\begin{tabular}{|c|c|c|c|}
\hline & $\begin{array}{c}\text { Participants } \\
\mathrm{N}=\mathbf{4 2}\end{array}$ & $\begin{array}{c}\text { Experimental Group } \\
\qquad n=20\end{array}$ & $\begin{array}{c}\text { Control Group } \\
n=22\end{array}$ \\
\hline & Mean $\pm S D$ & Mean \pm SD & Mean $\pm S D$ \\
\hline \multicolumn{4}{|l|}{ Baseline } \\
\hline PSS & $6.51 \pm 2.29$ & $7.23 \pm 2.19$ & $5.85 \pm 2.22$ \\
\hline PIS & $6.70 \pm 2.34$ & $6.75 \pm 2.50$ & $6.65 \pm 2.23$ \\
\hline \multicolumn{4}{|l|}{ Week 6} \\
\hline PSS & $5.10 \pm 2.47$ & $4.43 \pm 2.67^{*}$ & $5.72 \pm 2.15$ \\
\hline PIS & $4.57 \pm 2.46$ & $3.40 \pm 2.27^{*}$ & $5.63 \pm 2.16$ \\
\hline \multicolumn{4}{|l|}{ Week 12} \\
\hline PSS & $5.17 \pm 3.08$ & $4.50 \pm 3.38^{*}$ & $5.77 \pm 2.73$ \\
\hline PIS & $5.34 \pm 2.79$ & $4.72 \pm 3.04^{*}$ & $5.91 \pm 2.49$ \\
\hline \multicolumn{4}{|l|}{ Month 6} \\
\hline PSS & $5.32 \pm 2.92$ & $4.40 \pm 2.99 *$ & $6.16 \pm 2.65$ \\
\hline PIS & $4.85 \pm 2.82$ & $3.49 \pm 2.63 * *$ & $6.09 \pm 2.43^{* *}$ \\
\hline \multicolumn{4}{|c|}{ **indicates a significant difference in PIS between the experimental and control group } \\
\hline *indicates a si & vement in PSS and P & e experimental group & \\
\hline
\end{tabular}


There was no significant difference in the PSS between groups for the sample (Figure 4-2 below).There was a significant improvement in the PSS of theexperimental groupfrom baseline to week $\operatorname{six}\left(F_{(3,120)}=6.06, p<0.01\right)$. The significant improvement was sustained at week 12 and month six $(p<0.01)$. No significant changes were seen in the control group.

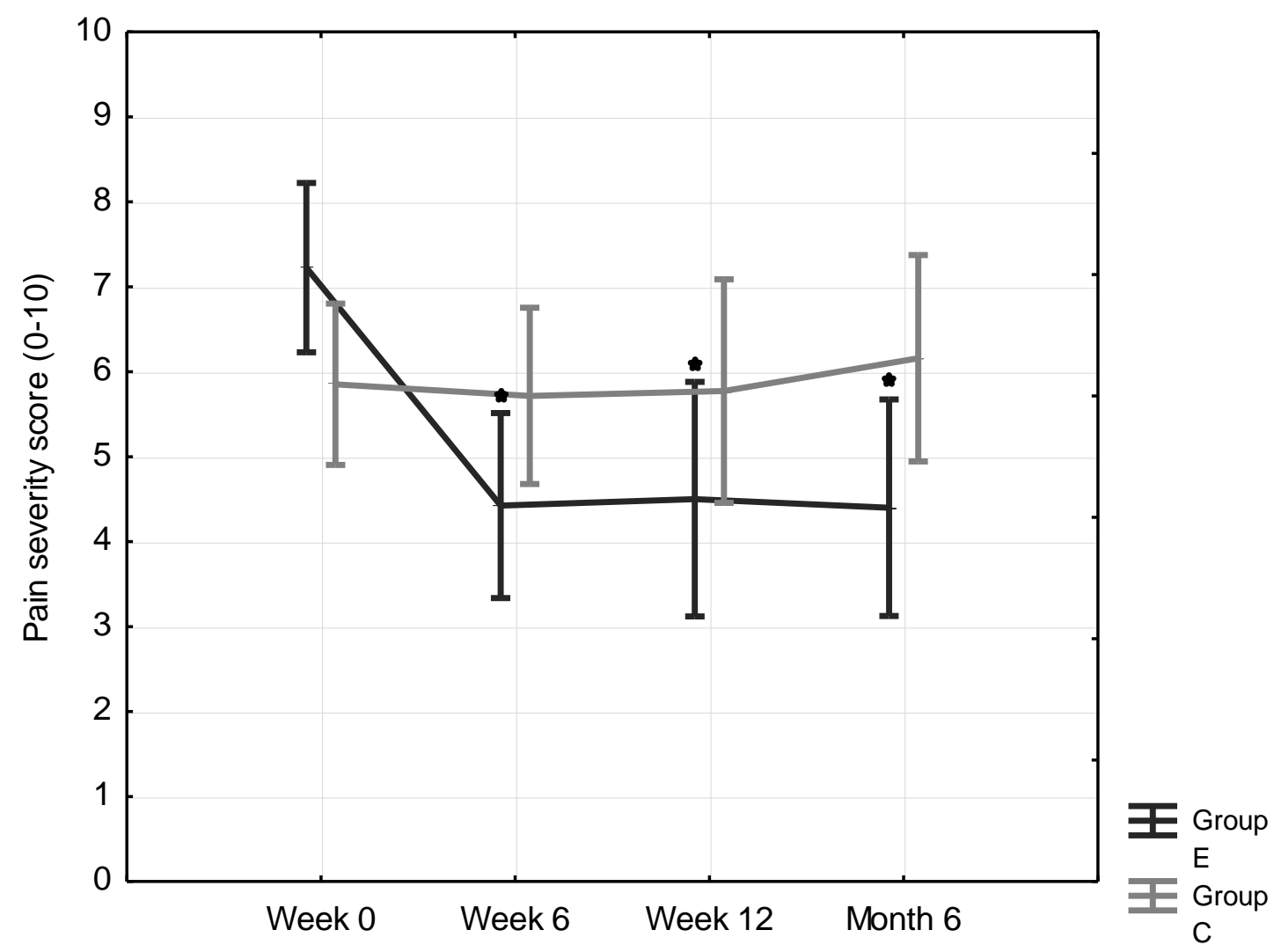

- Indicates significant improvement in PSS in the experimental group at week 6, 12 and month $6(p<0.01)$

Figure 4-2Change in pain severity score $(\mathrm{N}=42)$ 
When the subgroups of OA hip, OA knee and combined hip and knee OA were analysed it was found that for participants with OA knee there was a significant differencebetween groups at month six $\left(F_{(3,51)}=6.27, p<0.01\right)$ with a large ES (ES $\left.=2.64 ; p<0.01\right)$. The experimental OA knee group's PSS improved by 3.67 points on a scale of 0 to 10 , which is considered a clinically meaningful improvement in pain severity ${ }^{(121)}(M=6.86, S D=2.13$ to $M=3.19, S D=2.65)$ whereas the control group experienced worsening pain over the same period of time $(M=6.78, S D=1.48$ to $M=7.78, S D=0.88$ ) (Figure 4-3below) (Appendix I; pg. 337).

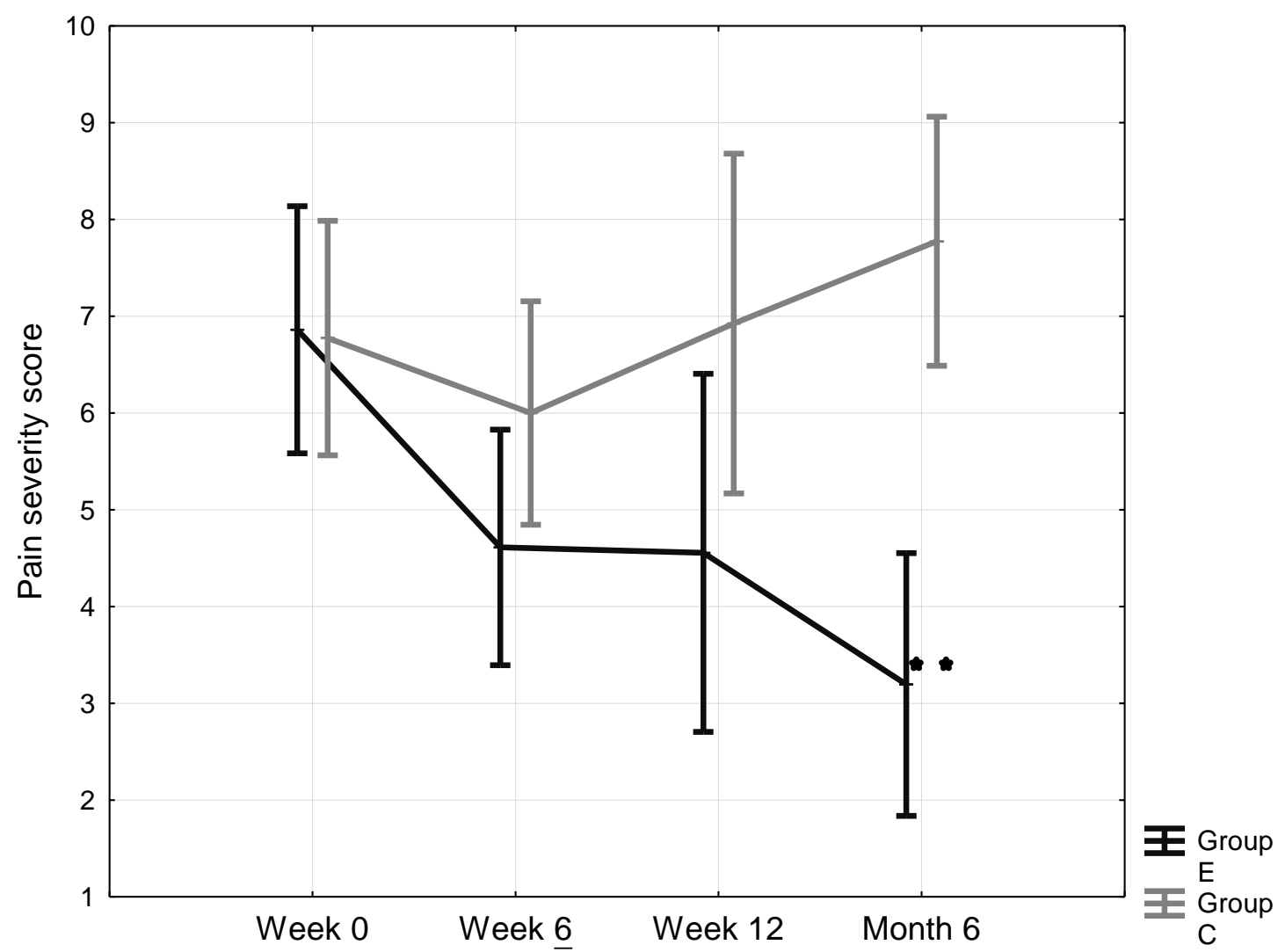

- Indicates a significant difference between groups for PSS at month $6(E S=2.64, p<0.01)$

Figure 4-3 Change in PSS of OA knee subgroup $(n=19)$

Those in the experimental group with combined hip and knee OA had the worst PSS at baseline ( $M=8.50, S D=1.27)$ and showed a significant improvement in PSS from their baseline measures to week $\operatorname{six}\left(F_{(3,30)}=4.20, p=0.02\right)$ by a reduction of 4.83 points on a scale of 0 to 10 . Yet there was no significant difference between groups. 
Participants in the experimental OA hip group had the lowest PSS ( $M=6.35, S D=2.84$ ) at baseline and did not show a significant improvement in PSS when compared to the control group $\left(F_{(3,27)}=1.12, p=0.36\right)$ (Appendix I; pg. 337).

\section{a. Worst, least, average and current pain score}

There were no significant differences for least, worst, average or current pain scores between groups or in the control group. There was a significant improvement in the BPI least pain score in the experimental group from baseline toweek six, week 12 andmonth six $\left(F_{(3,114)}=8.48, p<0.01\right)$. There was a significant improvement in the current pain score in the experimental group from baseline toweek six, week $12\left(F_{(3,117)}=6.13, p<0.01\right)$ andmonth six $(p=0.02)$. There were no significant changes for worst $\left(F_{(3,120)}=2.08, p>0.05\right)$ or average $\left(F_{(3,117)}=2.30, p>0.05\right)$ pain scores in the experimental group. Additional results gathered from the subsections of least pain, worst pain, average pain and current pain are presented in Appendix I (pg. 342). 


\section{ii. Pain Interference Score}

There was a significant difference in pain interference scores between the experimental and control group $\left(F_{(3,120)}=4.67, p=0.04\right)$ (Figure $4-4$ below). This significant improvement was seen at month six with the experimental group having significantly less pain interference than the control group $(M=3.49, S D=2.63$ vs. $M=6.09, S D=2.43 ; p=0.02)$ (Table 4-4above). ES calculations for BPI PIS showed a large ES for improvements in pain interference score between week 0 and month $\operatorname{six}(E S=1.15, p=0.02)$. Additionally there was a significant improvement in the PIS within the experimental group from baseline to week six $(p<0.01)$. The significant reduction in PIS was sustained over time, at week $12(p=$ 0.01 )and increased at month six $(p<0.01)$ (Figure 4-4below). There was no significant change in the control group.

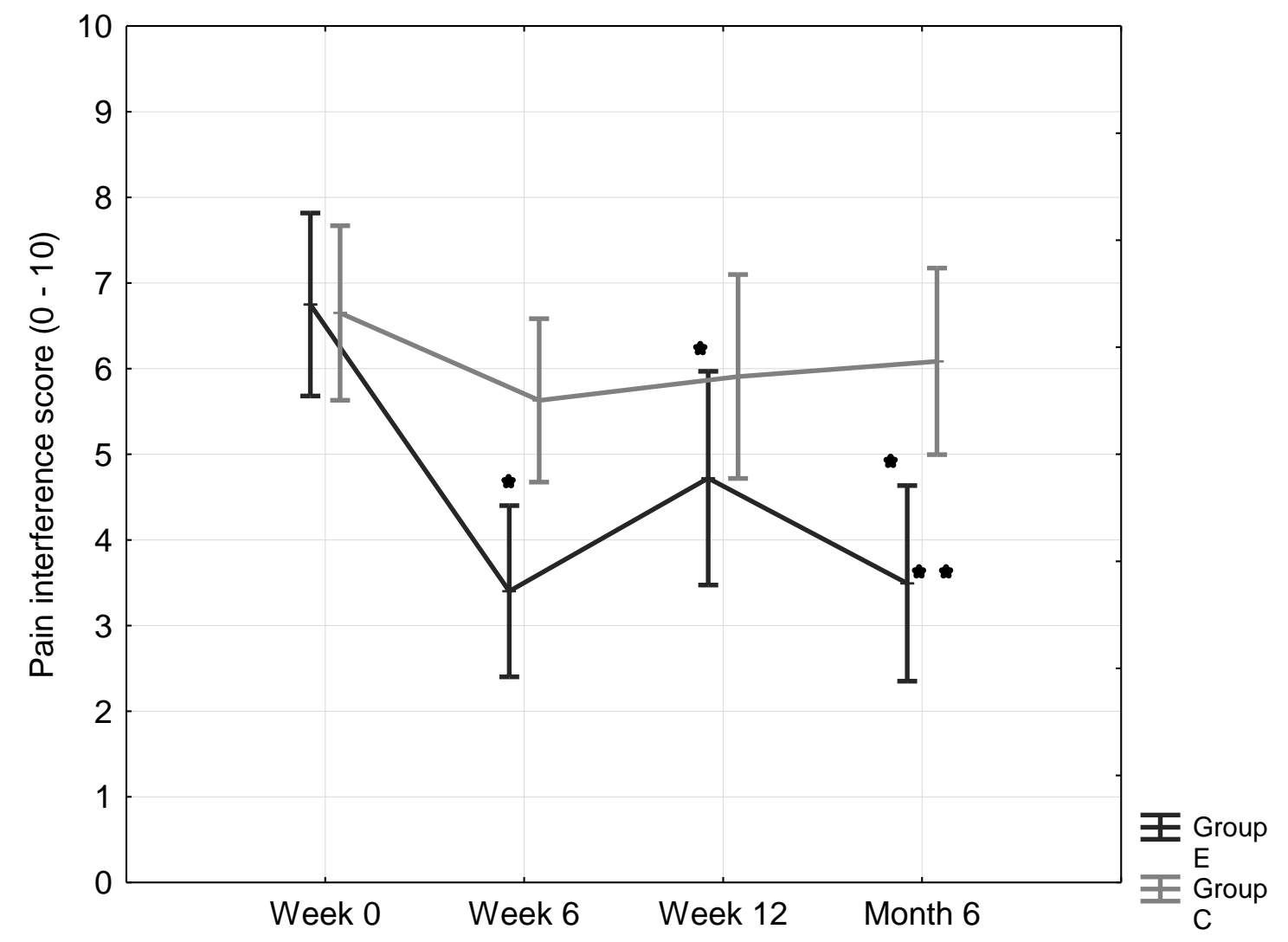

- Indicates a significant difference in PIS between experimental and control group at month $6(E S=1.15, p=0.02)$

- Indicates a significant improvement in PIS in the experimental group at week $6(p<0.01), 12(p=0.01)$ and month $6(p<$ $0.01)$

Figure 4-4 Change in pain interference score $(N=42)$ 
Subgroup analysis was performedrevealing a significantdifferencebetween groups for the $O A$ knee subgroup at month $\operatorname{six}\left(F_{(3,51)}=2.29, p<0.01\right)$. Those in the $O A$ knee group improved significantly when compared to the control group with a large ES from baseline to month six ( $E S=1.21 ; p<0.01$ ) (Figure 4-5 below). There was a significant improvement in those with combined hip and knee $O A$ in the experimental group from baseline to week $\operatorname{six}\left(F_{(3,30)}=5.06, p<0.01\right)$, week 12 and month six $(p=0.02)$, yet there were no differences when compared to the control group. There were no significant improvements in PIS for the OA hip group $\left(F_{(3,27)}=1.44, p=0.25\right)$ (Appendix l; pg. 347).

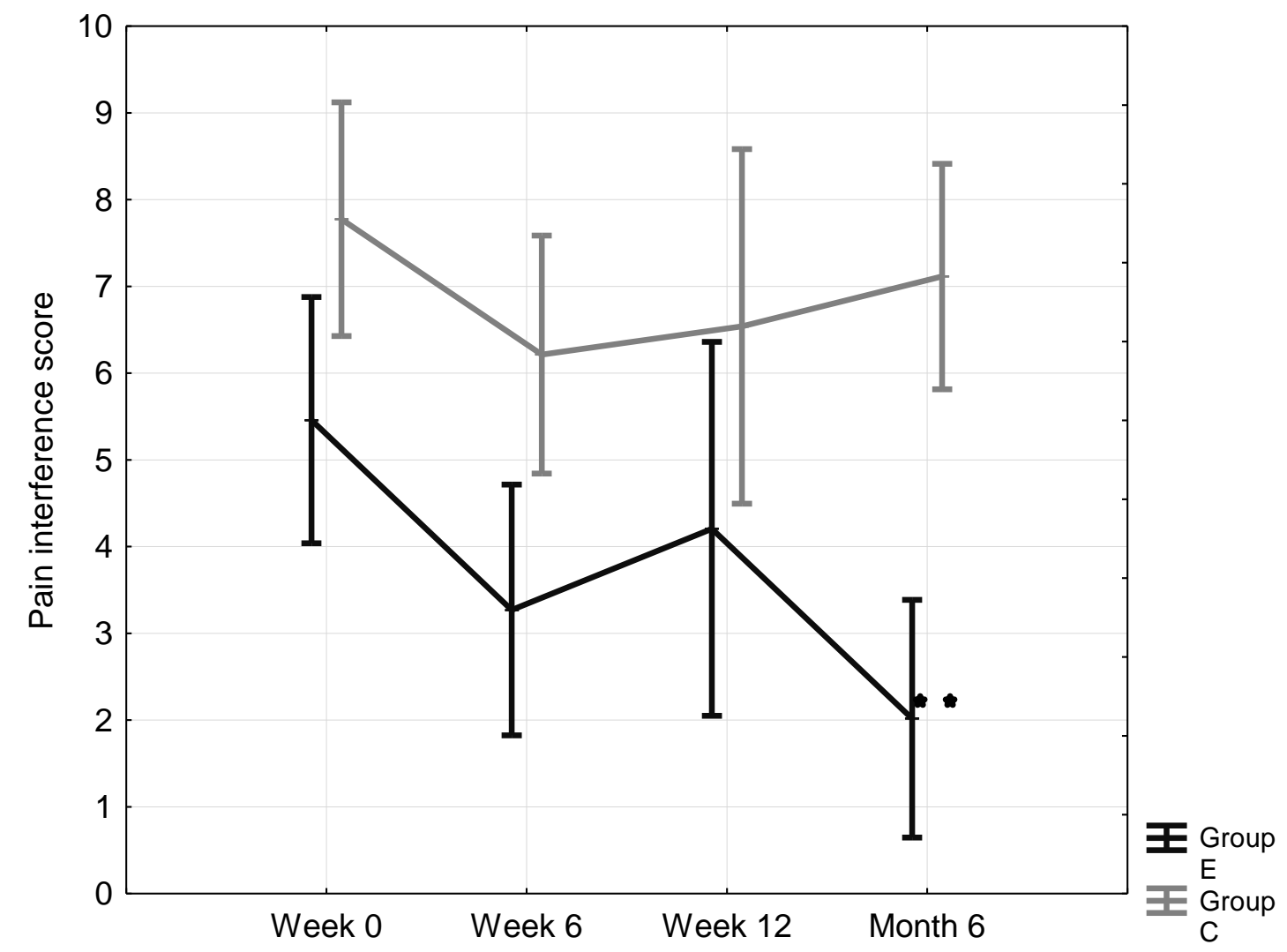

- Indicates a significant difference between groups in PIS of OA knee subgroup at month $6(E S=1.21, p<0.01)$

Figure 4-5 Change in PIS of OA knee subgroup $(n=19)$ 
Table 4-5displays the detailed mean \pm SD scores for each itemof the Pain Interference scale namely; general activity, mood, walking ability, normal work, relationships, sleep and enjoyment of life. There was a significant improvement in thepain interference subsection of mood in the experimental group from baseline toweek six, 12 andmonth $\operatorname{six}\left(F_{(3,117)}=4.13\right.$, $p<0.01)$. There was a significant improvement in the experimental group in the subsection of walking ability from baseline toweek six $\left(F_{(3,120)}=2.89, p<0.01\right)$, week $12(p=0.01)$ and month six $(p<0.01)$, in the subsection of normal work from baseline to week $\operatorname{six}\left(F_{(3,}\right.$, $\left.\left.{ }_{114}\right)=3.35, p<0.01\right)$ and month six $(p<0.01)$ and in the subsection ofsleep from baseline to week six $\left(F_{(3,120)}=2.75, p<0.01\right)$, week $12(p=0.04)$ and month $\operatorname{six}(p<0.01)$. There was no difference in the experimental group for the items of general activity, relationships with others or enjoyment of life $(p>0.05)$. There wereno significant differences for any item between experimental group and control group or any significant changes for any item in the control group. 
Table 4-5 Detailed PIS subsection scores ( $\mathrm{N}=42)$

\begin{tabular}{|c|c|c|c|}
\hline & $\begin{array}{l}\text { Participants } \\
\qquad \mathbf{N}=\mathbf{4 2}\end{array}$ & $\begin{array}{c}\text { Experimental } \\
\text { Group } \\
n=20\end{array}$ & $\begin{array}{l}\text { Control } \\
\text { Group } \\
n=22\end{array}$ \\
\hline & Mean \pm SD & Mean \pm SD & Significance test \\
\hline PIS general activity & & & $F_{(3,117)}=2.26 ; p=0.09$ \\
\hline Week 0 & $7.34 \pm 2.77$ & $6.95 \pm 2.93$ & $7.68 \pm 2.64$ \\
\hline Week 6 & $4.81 \pm 3.11$ & $3.95 \pm 3.22$ & $5.59 \pm 2.86$ \\
\hline Week 12 & $5.52 \pm 3.49$ & $4.70 \pm 3.76$ & $6.27 \pm 3.12$ \\
\hline Month 6 & $5.17 \pm 3.59$ & $3.40 \pm 3.20$ & $6.77 \pm 3.21$ \\
\hline PIS mood & & & $F_{(3,117)}=4.13 ; p=0.01^{*}$ \\
\hline Week 0 & $6.62 \pm 3.24$ & $7.08 \pm 2.66$ & $6.19 \pm 3.72$ \\
\hline Week 6 & $4.95 \pm 3.36$ & $3.40 \pm 3.33^{*}$ & $6.36 \pm 2.75$ \\
\hline Week 12 & $4.98 \pm 3.35$ & $3.95 \pm 3.33^{*}$ & $5.91 \pm 3.16$ \\
\hline Month 6 & $4.48 \pm 3.43$ & $3.10 \pm 3.21^{*}$ & $5.73 \pm 3.19$ \\
\hline PIS walking ability & & & $F_{(3,120)}=2.89 ; p=0.04^{*}$ \\
\hline Week 0 & $8.36 \pm 1.85$ & $8.45 \pm 1.70$ & $8.27 \pm 2.00$ \\
\hline Week 6 & $5.81 \pm 3.34$ & $4.70 \pm 3.39 *$ & $6.82 \pm 3.02$ \\
\hline Week 12 & $6.43 \pm 3.25$ & $6.05 \pm 3.58^{*}$ & $6.77 \pm 2.96$ \\
\hline Month 6 & $5.88 \pm 3.30$ & $4.80 \pm 3.66^{*}$ & $6.86 \pm 2.64$ \\
\hline PIS normal work & & & $F_{(3,114)}=3.35 ; p=0.02^{*}$ \\
\hline Week 0 & $7.83 \pm 2.38$ & $8.00 \pm 2.27$ & $7.65 \pm 2.54$ \\
\hline Week 6 & $4.79 \pm 2.95$ & $3.50 \pm 3.03^{*}$ & $5.95 \pm 2.38$ \\
\hline Week 12 & $5.83 \pm 3.06$ & $5.65 \pm 3.45$ & $6.00 \pm 2.73$ \\
\hline Month 6 & $5.81 \pm 5.26$ & $4.10 \pm 3.06^{*}$ & $7.36 \pm 6.35$ \\
\hline PIS relationships & & & $F_{(3,117)}=2.39 ; p=0.07$ \\
\hline Week 0 & $4.83 \pm 3.75$ & $4.80 \pm 3.93$ & $4.86 \pm 3.68$ \\
\hline Week 6 & $3.00 \pm 2.92$ & $1.60 \pm 2.11$ & $4.33 \pm 2.99$ \\
\hline Week 12 & $4.15 \pm 3.64$ & $3.65 \pm 3.70$ & $4.62 \pm 3.60$ \\
\hline Month 6 & $3.34 \pm 3.19$ & $2.10 \pm 2.94$ & $4.52 \pm 3.03$ \\
\hline PISsleep & & & $F_{(3,120)}=2.75 ; p<0.05^{*}$ \\
\hline Week 0 & $6.88 \pm 3.63$ & $6.90 \pm 3.70$ & $6.86 \pm 3.67$ \\
\hline Week 6 & $4.76 \pm 3.32$ & $4.00 \pm 3.45^{*}$ & $5.45 \pm 3.11$ \\
\hline Week 12 & $5.00 \pm 3.79$ & $4.70 \pm 3.93^{*}$ & $5.27 \pm 3.73$ \\
\hline Month 6 & $5.21 \pm 3.82$ & $3.85 \pm 3.95^{*}$ & $6.45 \pm 3.32$ \\
\hline
\end{tabular}




\begin{tabular}{|c|c|c|c|c|}
\hline & $\begin{array}{l}\text { Participants } \\
\qquad \mathrm{N}=42\end{array}$ & $\begin{array}{l}\text { Experimental Group } \\
\qquad n=20\end{array}$ & $\begin{array}{l}\text { Control Group } \\
n=22\end{array}$ & \\
\hline & Mean \pm SD & Mean \pm SD & Mean \pm SD & Significance test \\
\hline PIS enjoyment of life & & & & $f_{(3,117)}=2.31 ; p=0.08$ \\
\hline Week 0 & $5.83 \pm 3.81$ & $6.00 \pm 3.97$ & $5.67 \pm 3.75$ & \\
\hline Week 6 & $4.60 \pm 2.92$ & $3.55 \pm 2.94$ & $5.55 \pm 2.60$ & \\
\hline Week 12 & $5.40 \pm 3.49$ & $4.50 \pm 3.65$ & $6.23 \pm 3.21$ & \\
\hline Month 6 & $4.95 \pm 3.25$ & $3.60 \pm 3.23$ & $6.18 \pm 2.81$ & \\
\hline
\end{tabular}

\subsubsection{Change in percentagepain relief from medicationover time}

There was no significant difference forpercentagepain relief from medication $\left(F_{(3,81)}=0.90\right.$, $p=0.45$ ) within or between groups. There was a general improvement in percentage pain relief from baseline to month six in both groups but this was not significant(See Appendixl for full results; pg. 352).

Additionally, according to the BPI, participants recorded what type of medication they made use of each week. The responses were grouped as none, analgesia, antiinflammatories or analgesia and anti-inflammatories. The dosage was not recorded. There was no significant difference between groups at baseline $\left(\chi^{2}=3.71, p=0.45\right)$, week six $\left(\chi^{2}=\right.$ 9.17, $p=0.06)$, week $12\left(\chi^{2}=3.53, p=0.47\right)$ and month six $\left(\chi^{2}=5.90, p=0.12\right)$ (Appendix I; pg. 352). 


\subsection{Physical Performance Task Battery}

\subsubsection{5 meter walk tests: fastest and normal speed}

There was a significant difference between groups in the time taken to walk 15 meters at fastest speed $\left(F_{(3,117)}=3.82, p<0.01\right)$. This significant improvement was seen at month six with the experimental group walking significantly faster than the control group ( $M=15.09$, $S D=6.04$ s vs $M=20.10, S D=8.79 s ; p=0.03)$ (Figure 4-6below). $A$ large $E S$ was evident $(E S=$ $0.88, p=0.03)$.

There was a significant improvement in the time taken to walk 15 meters at fastest speed in the experimental group from baseline to week 12 and month $\operatorname{six}(p<0.01)$. There was no improvement in the control group (Appendix J; pg.355).

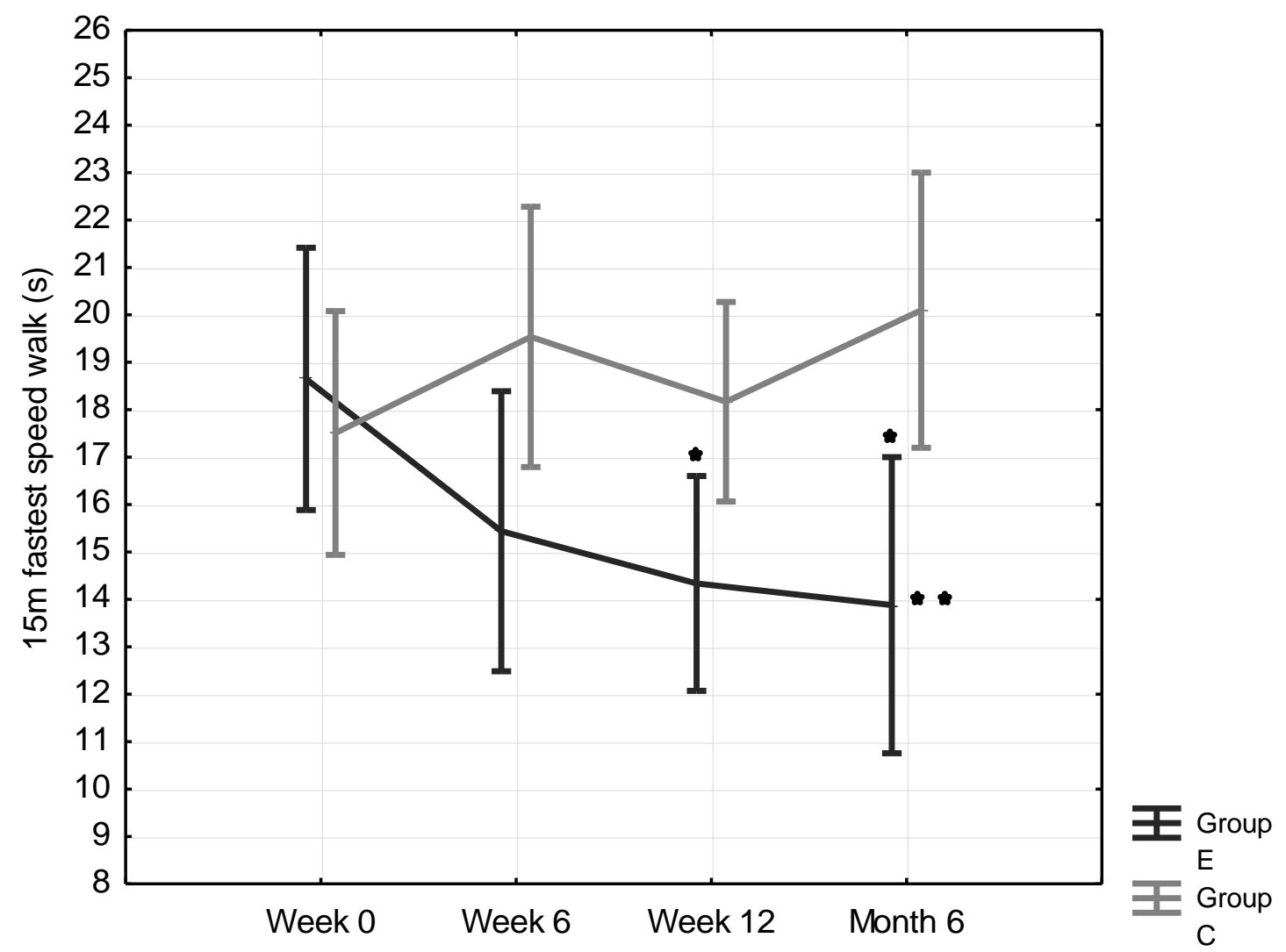

- Indicates a significant difference between groups for the time taken to walk $15 \mathrm{~m}$ fastest speed at month 6 (ES $=0.88$, $p=0.03)$

- Indicates a significant improvement in time taken to walk $15 \mathrm{~m}$ fastest speed in experimental group at week 12 and month $6(\mathrm{p}<0.01)$

Figure 4-6 Change in time taken to walk $15 \mathrm{~m}$ at fastest speed $(\mathrm{N}=42)$ 
When subgroup analysis was performed, there was a significant improvement in the experimental combined hip and knee OA subgroup from baseline to week $12\left(F_{(3,30)}=3.35, p\right.$ $=0.04)$. There were no significant improvements for the OA hip or OA knee subgroup in the experimental group. There was no significant difference for any of the subgroups when compared to the control group (Appendix J; pg. 356).

There was a significant improvement in the time taken to walk 15 meters at normal speed in the experimental group from baseline to week 12 and month $\operatorname{six}\left(F_{(3,117)}=7.06, p<0.01\right)$ (Figure 4-7below). There was no significant difference between groups or within the control group. Subgroup analysis showed there were no significant changes within or between groups for any of the subgroups OA (Appendix J; pg. 362).

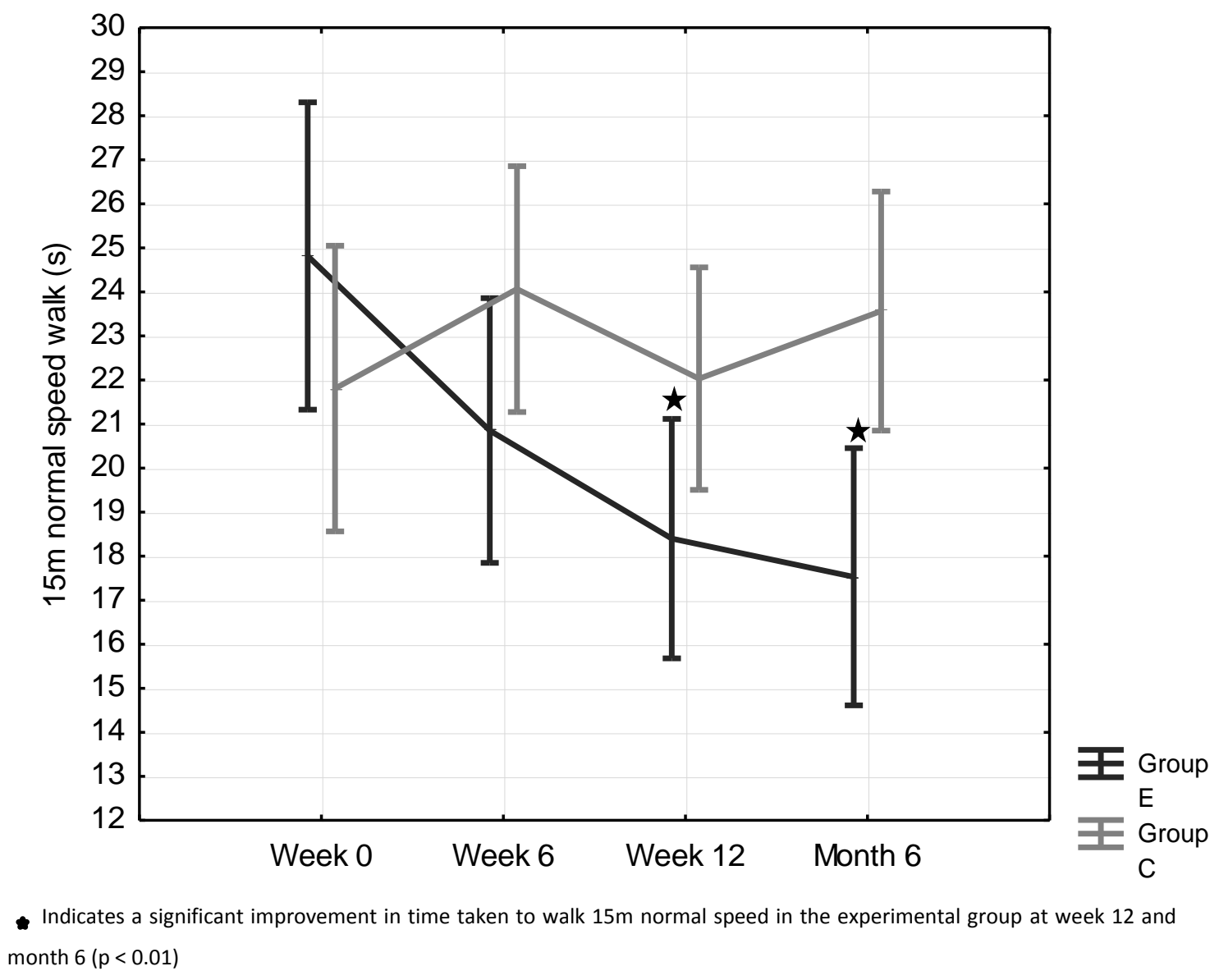

Figure 4-7 Change in time taken to walk $15 \mathrm{~m}$ normal speed $(\mathrm{N}=42)$ 


\subsubsection{Six minute walk test}

There were no significant differences between groups or change in the control group for the distance walked in a six minute walk test (Appendix J; pg. 368).There was a significant improvement in the distance in the experimental group from baseline to week 12and month six $\left(F_{(3,120)}=5.61, p<0.01\right)$ (Figure 4-8below).

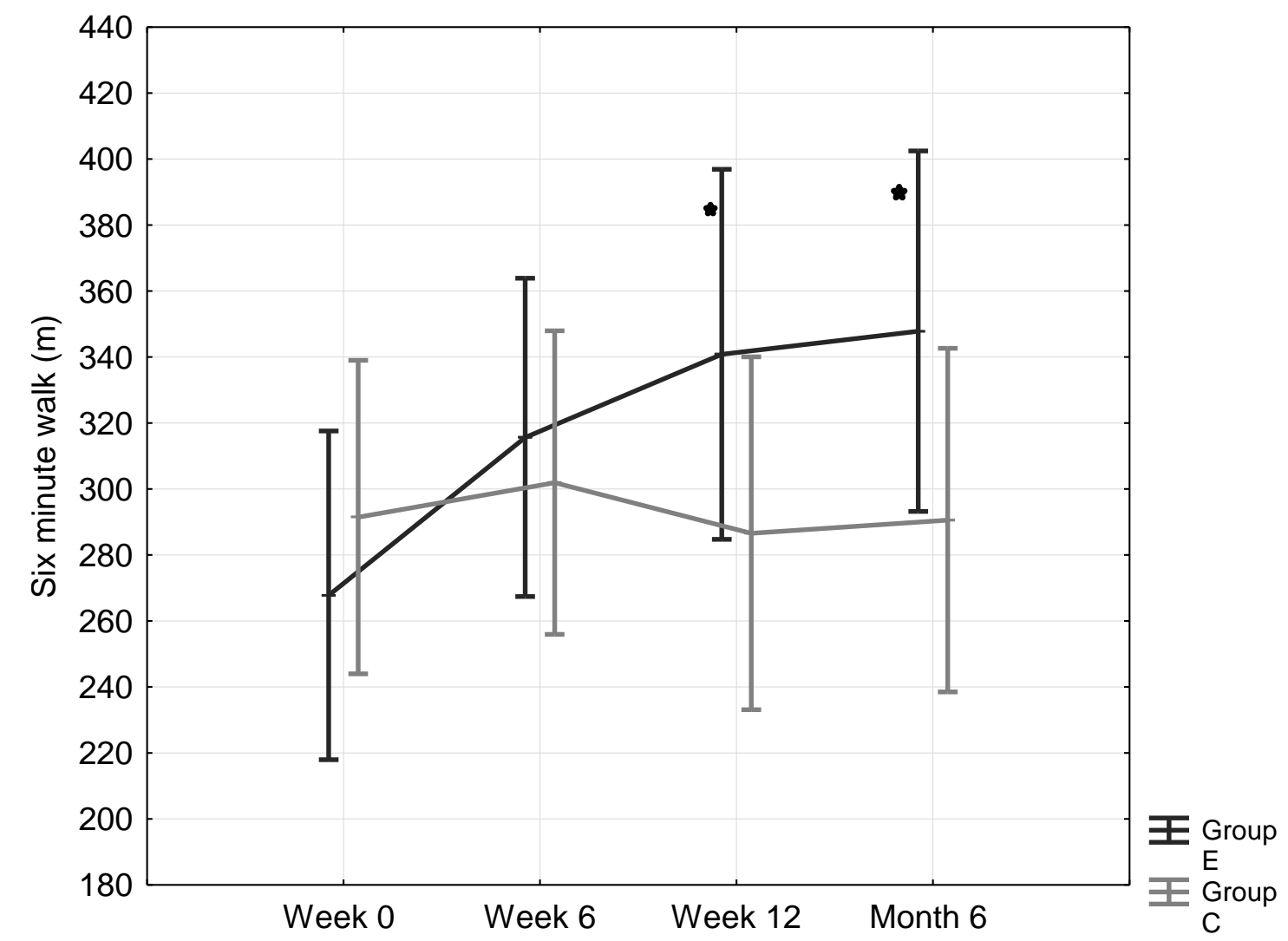

- Indicates a significant improvement in distance walked in six minute walk test in the experimental group at week 12 and month $6(p<0.01)$

Figure 4-8 Change in distance walked in six minute walk test $(\mathrm{N}=42)$

Subgroup analysis showed there was a significant improvement in the experimental OA knee group from baseline to month six $(p=0.01)$. There was a significant improvement in the experimental combined hip and knee OA group from baseline to week six $(p=0.02)$, week 12 and month six $(p<0.01)$. There were no differences in the OA hip subgroup $\left(F_{(3,27)}\right.$ $=0.22, p=0.88)$. There were no significant differences for either subgroup when compared to the control group (Appendix J; pg. 368). 


\subsubsection{Repeated sit-stand test}

There were no significant differences between groups in the time taken for the repeated sit to stand test. There was a significant improvement in the experimental group, from baseline to week six, week 12 and month six $\left(F_{(3,120)}=4.20, p<0.01\right)$ (Figure 4-9below). There was also a smaller but significant improvement in the control group from baseline to week $12(p=0.02)$ and month six $(p<0.01)$ (Appendix J; pg. 375).

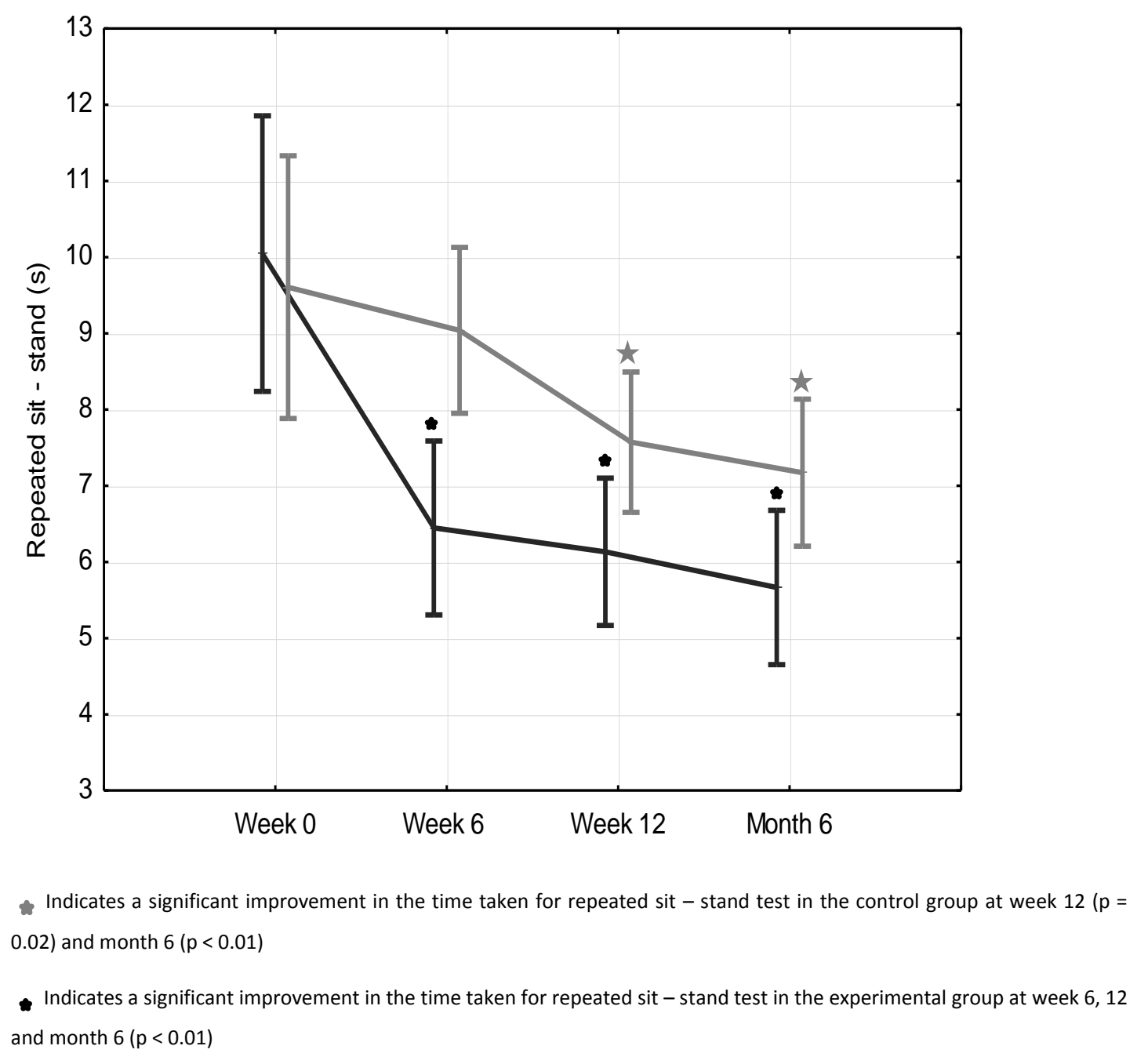

Figure 4-9 Change in time taken for repeated sit - stand test $(\mathrm{N}=\mathbf{4 2})$ 
Subgroup analysis showed a significant improvement in the experimental OA hip subgroup from baseline to week six, 12and month six $\left(F_{(3,27)}=3.61, p<0.01\right)$. There was no significant difference in the experimental subgroup ofcombined hip and knee $O A\left(F_{(3,30)}=2.25, p=\right.$ $0.10)$ and $O A$ knee $\left(F_{(3,51)}=0.84, p=0.48\right)$ or for either subgroup when compared to the control group (Appendix J; pg. 375).

\subsubsection{Reach tests: forwards and upwards}

There were no significant differences in the forward reach test $\left(F_{(3,120)}=1.45, p=0.23\right)$ or upward reach test $\left(F_{(3,111)}=1.30, p=0.28\right)$ within or between groups (Appendix J; pg. 382).

\subsubsection{Sock test}

There was no significant difference in the ability to don a sock $(p>0.05)$ within or between groups. There was a significant improvement in the time taken to don the left sock in the experimental group between baseline and week $\operatorname{six}\left(F_{(3,78)}=3.60, p=0.04\right)$ and month six $(p$ $<0.01)$. There was no significant difference in time taken to don a sock on the right foot within the experimental group. There was no significant change within the control group for either foot and no significant difference between groups for either foot (Appendix J;pg. 383). 


\subsection{Disability}

\subsubsection{Health Assessment Questionnaire score}

There was no significant change seen in the control group and no significant differences seen between groups in HAQ disability scores. Therewas a significant improvement in the experimental groupwhich was sustained over time from baseline to week six, week12and month six $\left(F_{(3,120)}=6.79, p<0.01\right)$ (Figure 4-10below). (Appendix K; pg. 385).

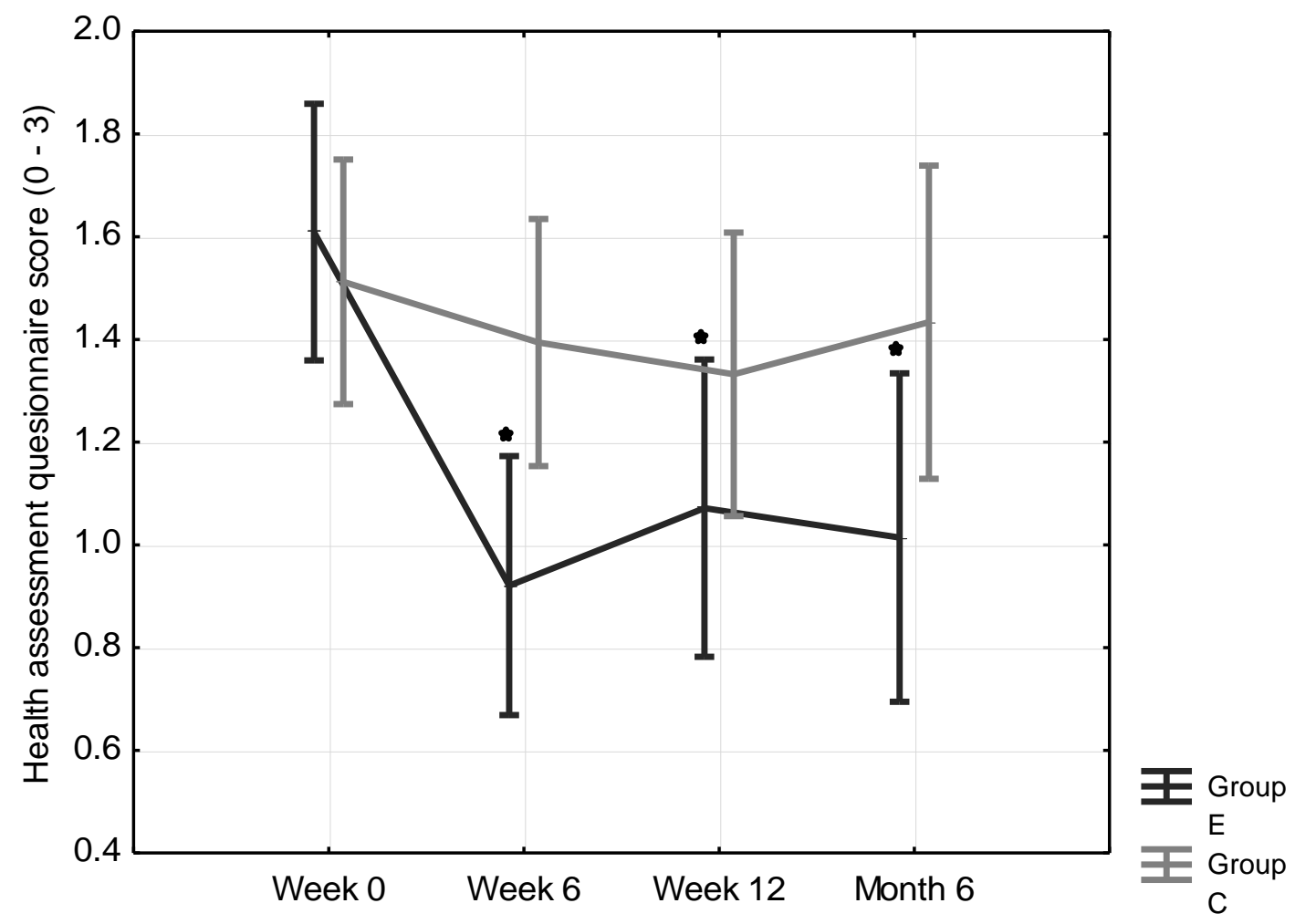

- Indicates a significant improvement in the HAQ sores in the experimental group at week 6, 12 and month $6(p<0.01)$

Figure 4-10 Change in Health Assessment Questionnaire score ( $\mathrm{N}=42)$

Subgroup analysis showed a significant improvement in the experimental OA knee subgroup from baseline to week six $\left(F_{(3,51)}=4.19, p<0.01\right)$, week $12(p=0.03)$ and month six $(p<0.01)$. There was a significant improvement in experimental combined hip and knee OA subgroup from baseline to week six $\left(F_{(3,30)}=4.34, p<0.01\right)$ as well as a significant improvement in the control combined hip and knee OA subgroup from baseline to week 12 $(p<0.01)$. 
There was no significant improvement for the OA hip subgroup $\left(F_{(3,27)}=2.29, p=0.10\right)$. There were no significant differences in either subgroup when compared to the control group (Appendix K; pg. 385).

\subsubsection{HAQ Pain VAS score}

There was a significant improvement in the HAQ pain VASin the experimental groupwhich wassustained over time, from baseline to week $\operatorname{six}\left(F_{(3,120)}=5.97, p<0.01\right)$, week $12(p=$ 0.03 ) and month six $(p<0.01)$ (Figure 4-11below). HAQ pain VAS decreased from $M=$ $73.35, S D=25.45$ to $M=48.30, S D=33.95$ in the experimental group. The reduction in pain on the VAS of 25.05 is considered a clinically meaningful difference in pain severity ${ }^{(128)}$. No significant change was seen in the control group and no significantdifferences were seen between groups for the sample(Appendix K; pg. 385).Additionally, there was a strong positive correlation between the baseline values for pain severity according to the HAQ pain VAS and BPI PSS $(r=0.74, p<0.01)$ (Appendix K; pg. 386).

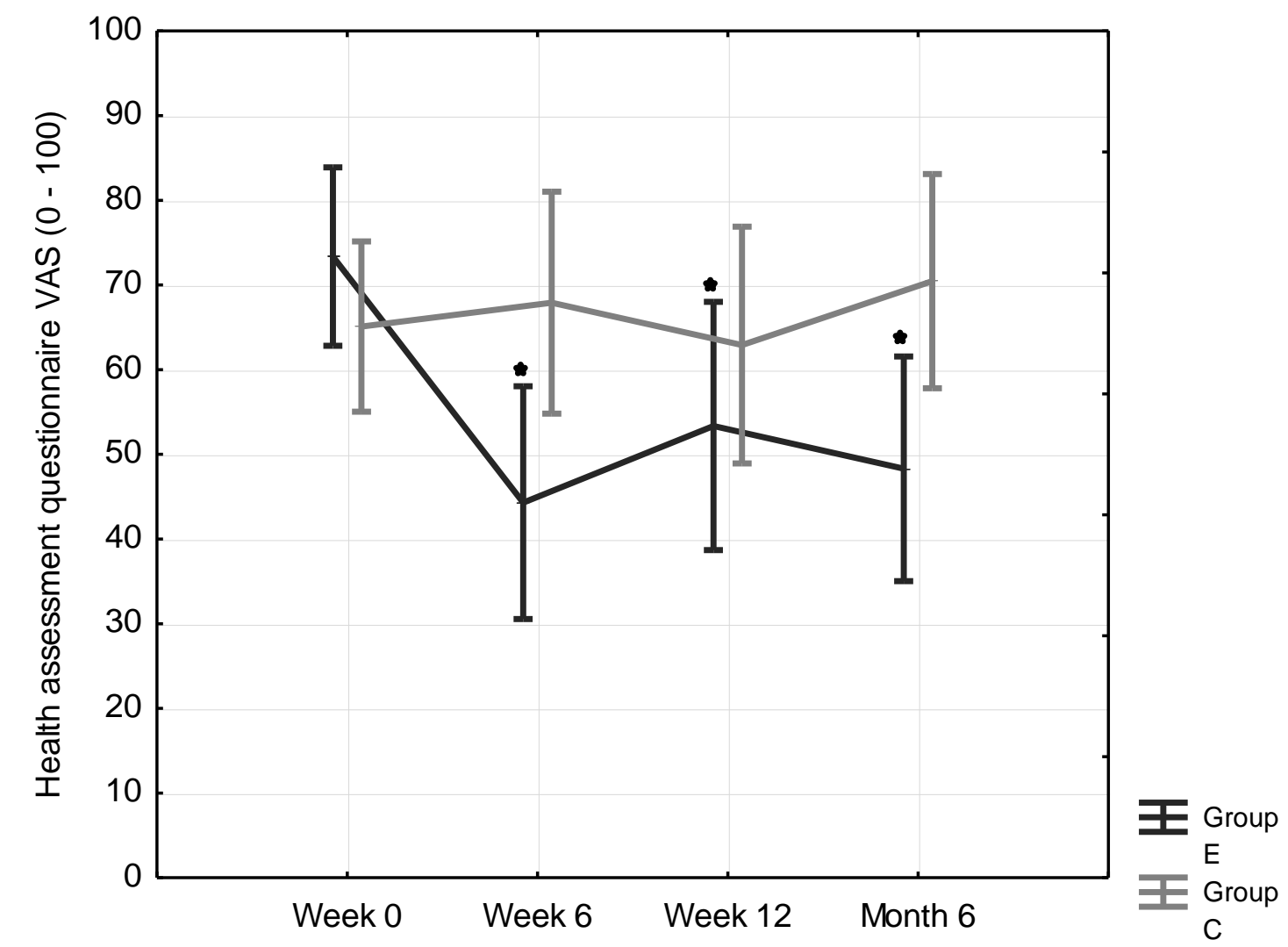

- Indicates a significant improvement in HAQ VAS in the experimental group at week $6(p<0.01), 12(p=0.03)$ and month 6 $(p<0.01)$

Figure 4-11 Change in HAQ pain VAS $(N=42)$ 
Subgroup analysis showed a significant difference between groups in the OA knee subgroup $\left(F_{(3,51)}=6.26, p<0.01\right)$. There was a significant improvement in HAQ VAS with asubstantial effect size from baseline to week $\operatorname{six}(E S=2.36, p=0.02)$ and month six ( $E S=$ 3.4, $p<0.01$ ) (Figure 4-12). A slightly weaker yet positive correlation is present between the HAQ pain VAS and BPI PSS for the subgroup of knee OA $(r=0.53, p=0.02)$. There were no significant improvements in the subgroup ofOA hip and combined hip and knee OA(Appendix K; pg. 387).

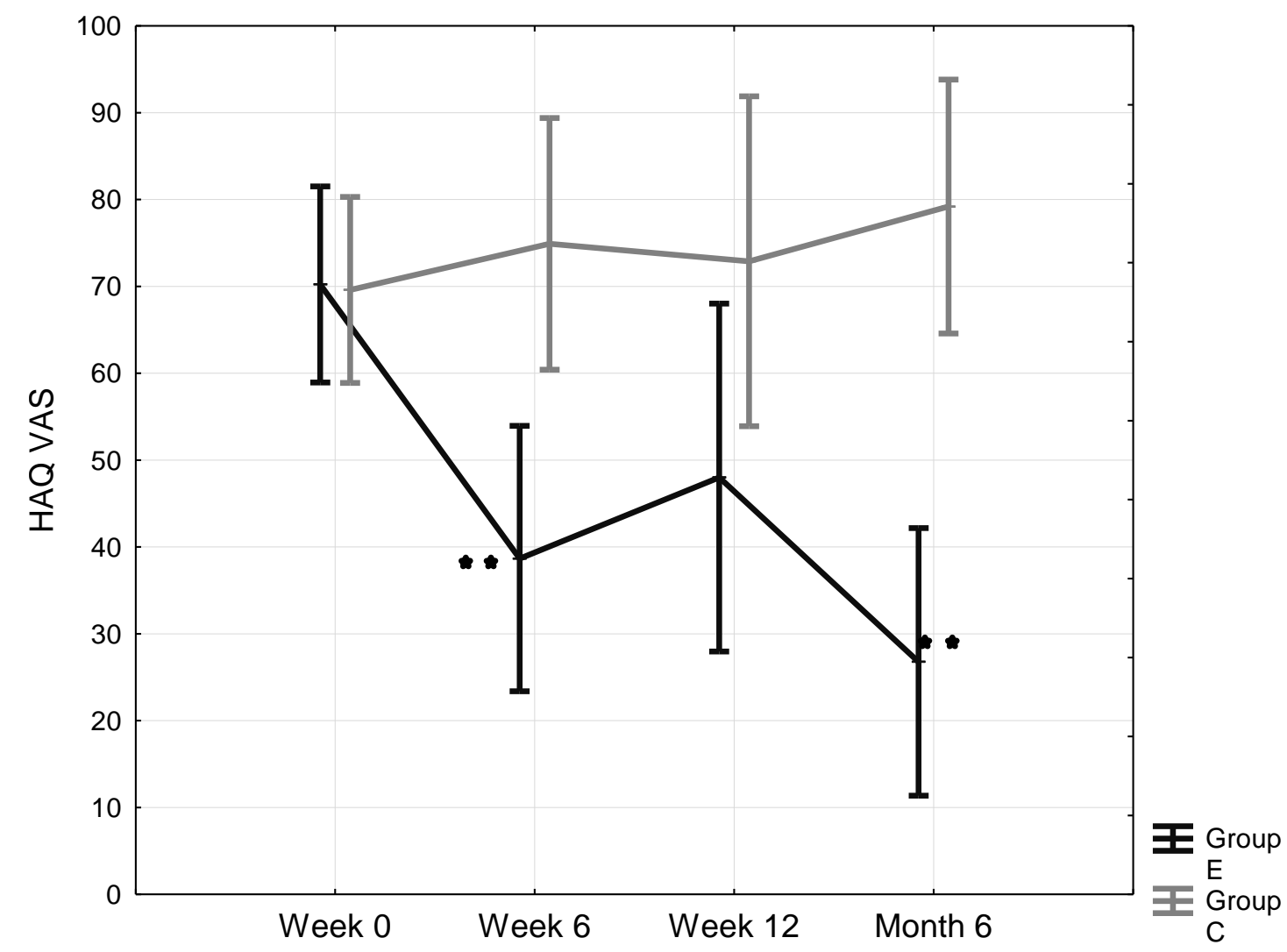

- Indicates a significant difference between groups in the HAQ VAS of OA knee subgroup at week $6(E S=2.36, p=0.02)$ and month $6(E S=3.4, p<0.01)$

Figure 4-12 Change in HAQ VAS of OA knee subgroup $(n=19)$ 


\subsection{Self-efficacy}

There were no significant differencesfor self-efficacy scoreswithin or between groups. While there appeared to be an improvementin the SE for the experimental group, it was not significant. A general worsening of SE scores was noted in the control group from baseline to week 12; however this was also not significant(Appendix L; pg. 399).

\subsection{Healthrelated quality of life \\ 4.7.1 EQ-5D health index}

There was nosignificant change in health related quality of life as measured by the EQ-5D index $\left(F_{(3,120)}=0.83, p=0.48\right)$ within or between groups(AppendixM; pg. 401).

\section{i. $\quad E Q-5 D$ category domains}

There were no significant changesin the five different category domains that make up the EQ-5D index score; namely mobility, self-care, activity, pain/discomfort and anxiety/depression (Appendix M; pg. 404).

\subsubsection{EQ-5D current state of health}

There were no significant changesin the current state of health as measured by the EQ-5D $\left(F_{(3,120)}=0.06, p=0.98\right)$, within or between groups. It appears there was a general improvement in current health statefor both groupsover time;however this was not a significantimprovement(Appendix M;pg. 401). 


\subsection{BMI change over time}

There was no significant change in $\operatorname{BMI}\left(F_{(2,80)}=0.59, p=0.56\right)$ within groups or between groups. BMI remained stable over the six months in both groups (Figure 4-13below) (Appendix N; pg. 409).

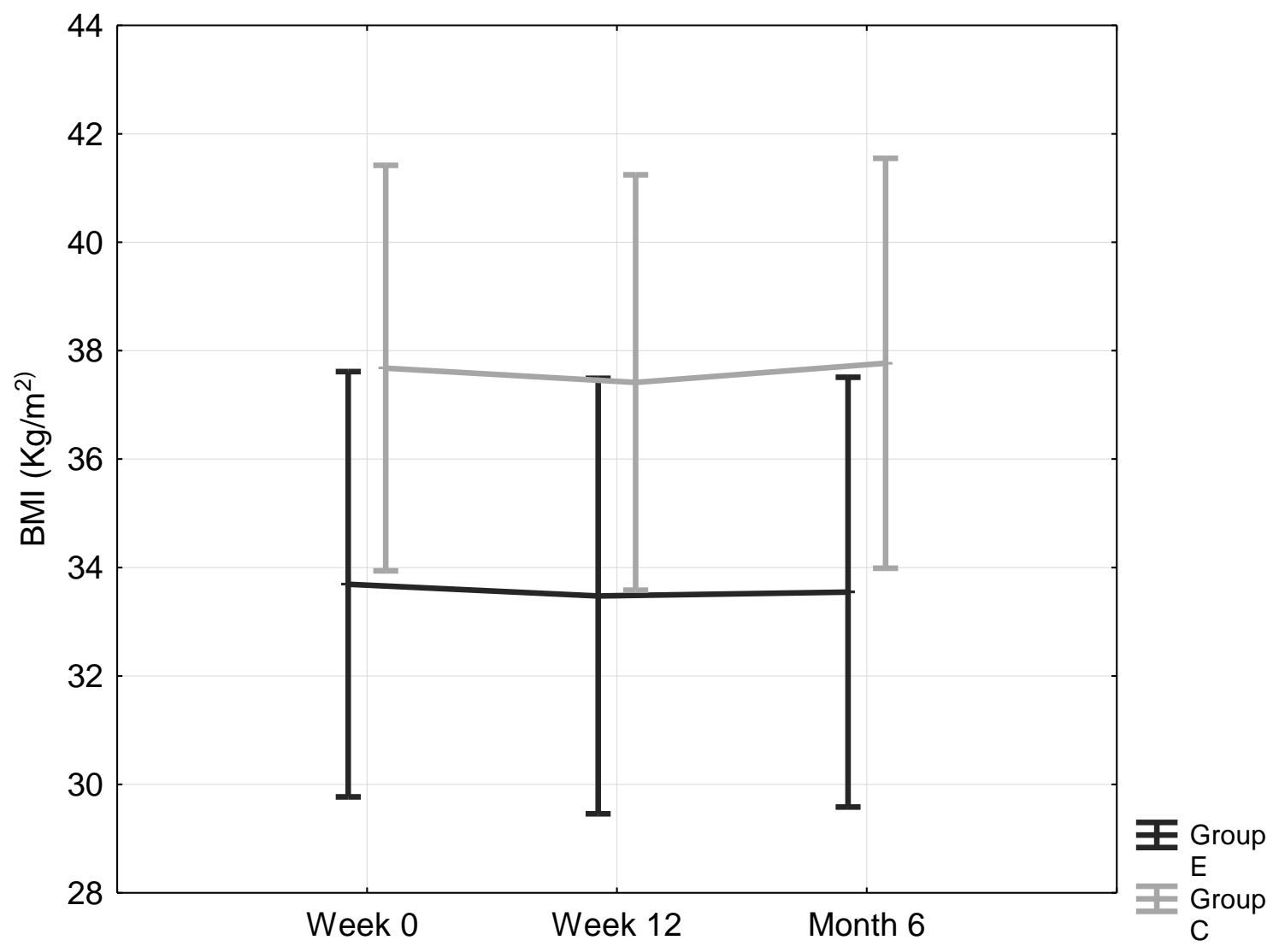

Figure 4-13 Change in $\mathrm{BMI}(\mathrm{N}=42)$ 


\subsection{Assistive device usage}

Use of assistive devices was reported by participants on the demographic questionnaire at baseline. However, the RA also recorded what devices participants used in person atbaseline and at follow up visits. There was a lack of agreement between patient reported and RA recorded device use at baseline $\left(\chi^{2}=50.13 ; p<0.01\right)$ (Table 4-6below).

Table 4-6 Patient reported versus RA recorded assistive device usage at baseline ( $N=42)$

\begin{tabular}{|c|c|c|c|c|c|}
\hline & \multicolumn{2}{|c|}{$\begin{array}{l}\text { Patient reported assistive } \\
\text { device used }\end{array}$} & \multicolumn{2}{|c|}{$\begin{array}{l}\text { RA recorded assistive } \\
\text { device used }\end{array}$} & \multirow[b]{2}{*}{$\begin{array}{c}\text { Significance } \\
\text { test }\end{array}$} \\
\hline & $\begin{array}{l}\text { Experimental } \\
\qquad(n=20)\end{array}$ & $\begin{array}{l}\text { Control } \\
(n=22)\end{array}$ & $\begin{array}{l}\text { Experimental } \\
\qquad(n=20)\end{array}$ & $\begin{array}{l}\text { Control } \\
(n=22)\end{array}$ & \\
\hline Baseline: & & & & & $\begin{array}{r}\chi^{2}=50.13 \\
p<0.01^{*}\end{array}$ \\
\hline Yes & 16 & $15^{*}$ & 14 & $9 *$ & \\
\hline No & 4 & $7^{*}$ & 6 & $13^{*}$ & \\
\hline \multicolumn{6}{|l|}{ Type: } \\
\hline None & 4 & $7 *$ & 6 & $13^{*}$ & \\
\hline 1 crutch/stick & 12 & $10 *$ & 10 & $6 *$ & \\
\hline 2 crutches & 4 & 5 & 4 & 3 & \\
\hline
\end{tabular}

There were no significant changes in $A D$ usage within the experimental group from baseline to week six $\left(\chi_{2}=3.81, p=0.05\right)$. There was a significant reduction in $A D$ usage within the experimental group from baseline to week $12\left(\chi^{2}=7.01, p<0.01\right)$ as five participants had stopped using an assistive device. This significant reduction was maintained at month six $(\chi 2=10.48, p<0.01)$ as three participants were still not making use of an assistive device after receiving the intervention.

There was a significant increase in $A D$ usage within the control group from baseline to week six $\left(\chi^{2}=18.28, p<0.01\right)$ as one more participant began using an AD. A further increase in $A D$ usage was seen in the control group at week 12 and maintained at month six $\left(\chi^{2}=\right.$ $15.23, p<0.01)$ as a total of two participants who previously did not make use of an $A D$ now were using an $A D$ (Appendix P; pg. 411). 


\subsection{Open ended questionnaire}

Four themes emerged from the seven open ended questions asked at month six to the experimental group ( $n=17$, due to three lost to follow up at month six). These themes were: increased knowledge; improvement in function and activity; pain relief and personal benefits (social support,fostering a positive attitude towards life andhaving fun). Table 4-7 presents these four themes by showing some examples of how the participants answered questions one, two and five.

In response to question three: "What didn't you like about the course?" most participants responded that there was nothing they did not like. Twoparticipants answered that they would like the course to be longer than six weeks. In response to question four: "Is there anything more you would like to learn that wasn't in the course?" most participants answered "no", while two requested to learn about falling and getting up after a fall and one requested to be taught additional exercises and helpful hints. In response to question six: "What didn't you like about the workbook?" all participants responded that there was nothing they didn't like. Lastly, in response to question seven: "What more would you add in the workbook that wasn't covered?" the majority answered there was nothing more to add, whereas one participant requested information on falling and one participant requested helpful information for underweight people.See the AppendixQ for all quoted responses to the open ended questions according to themes (pg. 413). 
Table 4-7 Themes that emerged from participant's answers to open ended questionnaire ( $n=17)$

Examples of the experimental group's answers to open ended questions

Theme: Question 1: Did you find the 6 week course helpful to you in any way?

If so how did it help you?

Knowledge

Function/

Activity

Pain relief

Personal

benefits
Participant K: "Yes the information about healthy eating and that I don't have to drink pills all the time."

Participant DD: "learnt more exercises, especially with equipment."

Participant A: "yes, I began with two crutches, now I walk most of the time with just one crutch and short distance with none."

Participant P: "This course helping me very much because I moving myself everyday"

Participant V: "All the pain was gone"

Participant AA: "it gave memotivation again because my life was all about pain before. Now I use my workbook and it helps a lot"

Participant L: "It gave me the opportunity to get out and meet people with the same sort of problem"

Participant WW: "it made me very positive... very motivating to exercise in a group instead of alone." 
Examples of the experimental group's answers to open ended questions

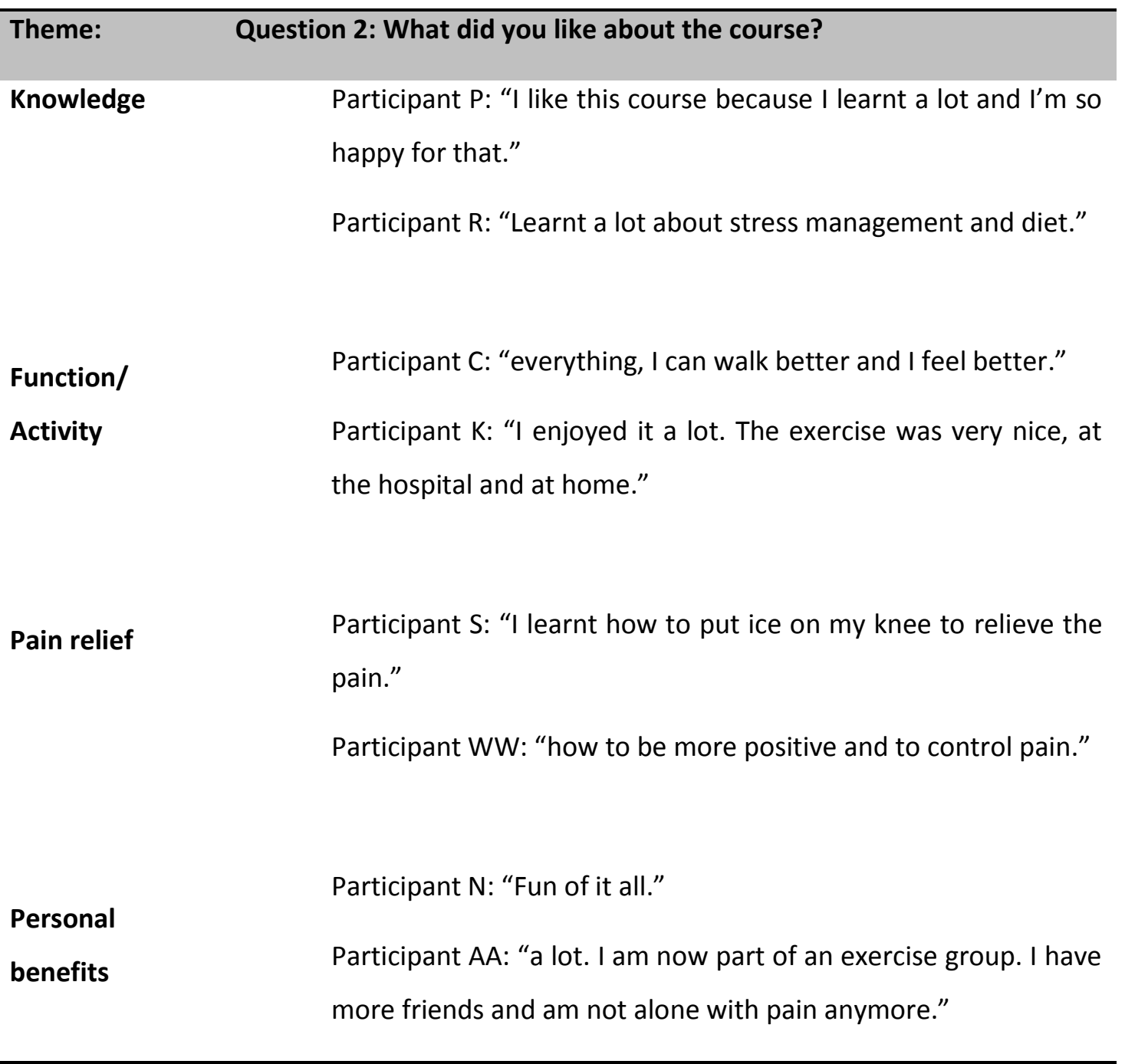

\section{Activity}




\begin{tabular}{|c|c|}
\hline & $\begin{array}{l}\text { Examples of the experimental group's answers to open ended } \\
\text { questions }\end{array}$ \\
\hline Theme: & Question 5: What did you like about the workbook? \\
\hline Knowledge & $\begin{array}{l}\text { Participant WW: "it was very interesting. Learnt a lot of } \\
\text { knowledge from the experience." } \\
\text { Participant P: "I'm just teaching others from the book." }\end{array}$ \\
\hline $\begin{array}{l}\text { Function/ } \\
\text { Activity }\end{array}$ & Participant A: “Exercise." \\
\hline Pain relief & $\begin{array}{l}\text { Participant R: "...also the section about pain management and } \\
\text { not having to rely on pills" }\end{array}$ \\
\hline \multirow[t]{2}{*}{$\begin{array}{l}\text { Personal } \\
\text { benefits }\end{array}$} & $\begin{array}{l}\text { Participant B: "Everything, I learnt a lot, especially about self- } \\
\text { confidence." }\end{array}$ \\
\hline & $\begin{array}{l}\text { Participant R: "It was helpful when it spoke about } \\
\text { communication with others and getting support." }\end{array}$ \\
\hline
\end{tabular}




\subsection{Summary of results}

In summary, the results showed the following effects of a six-week physiotherapist-led exercise and education intervention in patients with $\mathrm{OA}$, awaiting a hip/knee joint replacement:

\subsubsection{Primary outcome measure: pain as measured by the BPI}

There was a significant difference in pain interference scores between groups from baseline to month six $(p=0.02)$, with the experimental group experiencing less pain interference than the control group ( $3.49 \pm 2.63$ vs. $6.09 \pm 2.43 ; p=0.02)$. There was a large effect size for improvements in pain interference score $(E S=1.15)$. Although there was no significant difference between groups for PSS, there was a significant sustained reduction in pain severity score in the experimental group from baseline to week six, week 12 and month six $(p<0.01)$. Both the PIS and PSS showed significantly improved pain scores particularly for those in the subgroup of knee $O A$ (ES $=1.21, p<0.01$ and $E S=2.64, p<0.01$ respectively). No significant differences were seen for pain severity or interferencebetween groups for the subgroups of hip OA or combined hip and knee OA.

\subsubsection{Secondary outcome measures}

There was a significant difference in function, as measured by the 15 meter fastest speed walk test, between groups from baseline to month six $(p=0.03)$. The experimental group walked significantly quicker than the control group $(15.09 \pm 6.04 \mathrm{~s}$ vs $20.10 \pm 8.79 \mathrm{~s} ; \mathrm{p}=$ 0.03). There was a large effect size for improvement in time taken to walk 15 meters at fastest speed $(E S=0.88)$. Similarly, those with knee $O A$ in the experimental group improved their walking ability compared to baseline $(p=0.01)$ whereas participants with hip OA did not. Individuals with combined hip and knee $O A$ in the experimental group showed an improvement in walking ability $(p<0.01)$ yet this was not significant when compared to the control group.

Significant improvements were observed for the HAQ disability indexand pain VAS, for the experimental group from baseline to week six $(p<0.01)$, week $12(p=0.03$ for VAS) and month six $(p<0.01)$. HAQ pain VAS showed a strong positive correlation with the BPI PSS at baseline $(r=0.74, p<0.01)$. 
Subgroup analysis showed a significant difference for HAQ pain VAS in participants with knee OA compared to the control group, from baseline to week six ( $E S=2.36, p=0.02)$ and month six $(E S=3.4, p<0.01)$. No significant differences were seen in disability between experimental and control group for the subgroups of hip OA and combined hip and knee OA.

No significant differences were observed between or within groups for self-efficacy. There were no significant differences in the current state of health within or between groups for the EQ-5D VAS. There were no significant differenceswithin or between groups for health related quality of life, as measured by the EQ-5D index.

There was no significant difference between groups at baseline for BMI, or any significant change in BMI over time. BMI did not account for any differences in the above mentioned effects.

The addition of an open ended questionnaire for the experimental group at month six highlighted the benefits with four themes emerging. Namely: increased knowledge, an improvement in function and greater levels of activity, an improvement in pain relief as well as added psychological benefits. 


\section{Discussion}

\subsection{Introduction}

The results of this study support the use of a six-week physiotherapist-led exercise and education programme in patients with osteoarthritis of the hip or knee, on a long waiting list for a joint replacement. Clinically relevant findings were observed in the experimental group with significant long-term improvements in pain and function when compared to the control group.

In this discussion the current results will be discussed in relation to the existing literature covered in Chapter Two (pg. 3). Firstly, the focus will be on the results of the primary outcome measure of pain, as measured by the BPI, with specific reference to the pain interference scale followed by the pain severity scale. Thereafter the results of the secondary outcomes function, disability and personal factors will be discussed.

\subsection{Primary outcome measure: pain (BPI)}

\subsubsection{Pain interference scale}

As discussed in the literature review, pain is reported as the main complaint of persons with $\mathrm{OA}^{(79)}$. It is known that one of the recommended domains of pain measurement is pain interference ${ }^{(119,122)}$. For a chronic pain condition such as $O A$, it is not only how much pain one has but the debilitating effect that pain has on the person's daily life and interactions that requires evaluation ${ }^{(228)}$. Despite an average waiting period of 3.61 years (SD $=2.44$ ), with the longest waiting period of 11 years, the intervention was effective at significantly reducing pain interference in the experimental group compared to the control group $\left(F_{(3,}\right.$, 120) $=4.67, p<0.01)$. This statement emphasises the importance of first understanding to what extent the sample was affected at baseline by OA and by having to wait for such a length of time, before receiving surgery. Once this has been presented, full comprehension of the changes observed over time in such a sample is possible. 


\section{i. Baseline presentation for the sample according to the BPI:}

Previous research has shown debilitating effects of being on relatively short waiting lists (less than 12 months); with worse pain and function being correlated to longer periods of waiting $^{(71,314)}$. As a result of this sample's long waiting period, it was anticipated that this group would present at baseline with severe pain; defined on a scale of 0 - 10 as above 6 for knee OA and above 7 for hip OA awaiting surgery ${ }^{(326)}$. This was indeed the case with the present sample's baseline measures according to the BPI severity scale for worst pain beingM $=8.17, S D=2.24$. This is higher than worst pain scores reported by Mendoza and colleagues in their samples not on waiting lists, for hip $\mathrm{OA}(\mathrm{M}=7.53, \mathrm{SD}=1.84)$ and knee $\mathrm{OA}(\mathrm{M}=7.40, \mathrm{SD}=1.84)$ (Mendoza, personal communication,15 November 2014).

It was also expected that this sample would present with severe interference in physical and emotional functioning; defined on the BPI interference scale as above $\mathrm{M}=5.85, \mathrm{SD}=$ 2.04 for $\mathrm{OA}$ of the knee and above $\mathrm{M}=6.33, \mathrm{SD}=1.88$ for $\mathrm{OA}$ of the hip ${ }^{(327)}$. As expected, the present sample's baseline measures according to the BPI interference scale wereM = $6.70, S D=2.34$. The PIS in the current sample is slightly higher than those reported by Williams et al. ${ }^{(139)}$ in previous studies involving persons not on a waiting list with $\mathrm{OA}(\mathrm{M}=$ $6.60, S D=1.9$ and $M=6.33, S D=1.7$, for those with high levels of pain in studies one and two respectively $\left.{ }^{(139)}\right)$. Additionally, the PIS of the participants in the present study is higher than baseline PIS scores in Mendoza and colleagues' sample with hip OA ( $M=5.58, S D=$ 2.69) and knee $\mathrm{OA}(\mathrm{M}=5.23, \mathrm{SD}=2.7$ ) (Mendoza, personal communication, 15 November 2014). Furthermore, the current sample presented with a similar baseline VAS pain score on the HAQ ( $M=6.90, S D=2.34$ when converted to a score out of 10$)$ to that of a closer matched sample on a waiting list, who had been living with OA for an average of four to 11 years $(M=7.0, S D=2.2)^{(71)}$.

The current sample had the most severe pain interference with "walking ability" ( $M=8.36$, $S D=1.85)$ followed by "normal work" ( $M=7.83, S D=2.38)$. This finding is consistent with common difficulties experienced during walking, leading to various activity limitations associated with OA of the hip or knee, as discussed in Chapter Two (pg. 17) ${ }^{(78,314,315)}$. 
Although, this finding is in contrast to that of Williams et al.s' studies ${ }^{(139)}$ in persons with OA with moderate to severe pain, in which "normal work" was recorded as the most affected $(M=6.74, S D=2.5$ and $M=6.71, S D=2.2)$, "walking ability" followed closely behind as the second and third most affected in studies one and two respectively $(M=6.54, S D=2.7 ; M=$ $6.58, S D=2.4)$. The difference in the present study could be attributed to the wording of the instrument. According to the BPI, "normal work" is described as work outside of the house or housework. It could be that those participants who still work reported interference during work. However, if one considers the mean age of the sample involved in the present study was 59.52 years $(S D=4.75)$ and that most were unemployed (on a disability grant or on pension), participants probably reported on this subsection as interference with housework. It can also be assumed that in many circumstances "normal work", be it formal work or housework, consists of walking and therefore either way it reflects that pain has a large impact on both components of daily activity.

The third most affected subsection at baseline, following "walking ability" and "normal work" interference, was "general activity" ( $M=7.34, S D=2.77$ ). It is seen in this sample that all three aspects forming the activity sub dimension of the PIS ${ }^{(135)}$ were the most affected by pain. This was in agreement with Williamset al.s' sample in their second study where work, general activity and walking were the three most affected aspects ${ }^{(139)}$. This confirms that pain and pain interference with physical function are the most commonly experienced issues in the population with OA awaiting surgery ${ }^{(74)}$.

The next most affected subsection on the pain interference scale was that of sleep ( $M$ = $6.88, S D=3.63$ ). According to the literature, it has been stated that sleep disturbances are common in persons with $\mathrm{OA}^{(22,115,328)}$ and should be included as part of outcome measures in those with pain conditions ${ }^{(139)}$. Sleep has an important impact on daily life as disturbed sleep has a considerable influence on pain ${ }^{(115,329)}$, physical functioning ${ }^{(139)}$ and mood $^{(328)}$. As discussed in the literature review, emerging research states that disturbances in sleep have been associated with central sensitization, causing further pain and functional limitations ${ }^{(115)}$. The current study's results which report the functional activity dimension and sleep as the most affected subsections of the PIS, supports an association between sleep, pain and functioning ${ }^{(21,329,330)}$. 
Additionally, the common occurrence of sleep interference, in those on a waiting list is reinforced by a longitudinal study on persons with severe pain due to OA. It was found that in persons awaiting surgery for up to six months, $40 \%$ of the sample reported disturbances in sleep every night as a result of pain ${ }^{(71)}$.

Following on from the above, it was observed that the affective/emotive functioning subdimension of the PIS was similarly grouped together as seen by interference scores for "mood" ( $M=6.62, S D=3.24)$, "enjoyment of life" ( $M=5.83, S D=3.81)$ and "relationships with others" ( $M=4.83, S D=3.75)$. This could be explained by the length of time on the waiting list causing not only physiological effects, but also negative psychological effects $^{(246)}$. Interestingly, despite the present sample having higher pain related functional and sleep interference when compared to the two samples used by Williams et al. ${ }^{(139)}$; enjoyment of life was less affected in the current sample (Williams et al. sample 1: $M=6.2$, $S D=2.9$, sample $2: M=6.43, S D=2.6$ ). The subsection of "relationships with others" was the least affected by pain at baseline and throughout the current study. This finding is consistent with research by Williams et al. ${ }^{(139)}$, yet this subsection interference score was higher in the current sample when compared to these two studies $(M=4.1, S D=2.9$ and $M$ $=4.02, \mathrm{SD}=2.7$ in studies one and two respectively). The current sample also showed higher pain related mood interference when compared to both samples in the studies by Williams and colleagues not on a waiting list $(M=5.29, S D=2.8$ and $M=5.81, S D=2.5$ in studies one and two respectively $\left.{ }^{(139)}\right)$. The current finding of moderate pain interference on "mood" also reinforces the negative effect of disturbed sleep on psychological factors ${ }^{(139)}$, with specific mention to the association between sleep disturbances and depressed or anxious mood states ${ }^{(115,328)}$.

Therefore at baseline the sample was greatly affected in terms of all aspects of pain related functional interference as measured by the PIS on the BPI. The current sample's presentation confirmed that pain and function worsens when on a waiting list as seen by higher levels of pain related interference when compared to previous studies reporting on persons with OA not on a waiting list ${ }^{(139)}$. 


\section{ii. Change in PIS over time}

Remarkably, the significant improvement between groups for overall pain interference in the present study, showed a large effect size at month six ( $E S=1.15, p=0.02)$ (pg. 112). This is notably better than the ES of NSAIDS $[E S=0.32,(0.24,0.39)$ ]and acetaminophens [ES $=0.21(0.02,0.41)]$ for pain in people with OA as reported by OARSI ${ }^{(213)}$. Both NSAIDs and analgesics are prescribed to most, if not all, patients on a waiting list as part of the first-line management of OA despite small $\mathrm{ES}^{(25)}$.

Further, the ES of the combined treatment of exercise and education used in this study on pain interference was much larger than the ES on pain in hip and knee OA for aerobic exercise $[E S=0.52(0.34,0.70)]$, strength exercise [ES $=0.32(0.23,0.42)]$, water-based exercise $[E S=0.25(0.02,0.47)$ or education or self-management interventions alone $(E S=$ $0.06(0.02,0.10)]^{(213)}$. The large effect of the combined intervention seen in this study suggests that this may be a more effective treatment strategy for these patients than individual treatment approaches.

Furthermore, benefits observed in pain in the present study support the use of a combined intervention over a self-management intervention alone such as the CDSMP; which has not shown significant improvements in pain ${ }^{(239,240,252,253)}$. This could be due to a confounding factor such as a larger number of co-existing chronic conditions $(M=2.2-2.3$ per participant) in previous samples ${ }^{(239,240)}$ as opposed to the current study's average of $\mathrm{M}=$ 1.29 co-morbidities per participant. The rationale behind a generic intervention is based on the commonly shared problems in the rapidly increasing number of people with chronic diseases and that approaches to manage these are similar ${ }^{(252)}$. As discussed in the literature review, it seems that a generic intervention targeting a variety of chronic conditions is not as effective at reducing pain but rather leads to limited psychosocial benefits ${ }^{(92,243)}$.

There is a need for more than merely improved knowledge and psychological outcomes in those with chronic pain and disability. The current study aimed to meet this by incorporating active exercise into a self-management intervention to reduce pain and improve function. 
It appears a combined programme of exercise and education can and does impact pain related functional interference in the current sample. The results of the present study concur with the earlier SR by Walsh et al. ${ }^{(276)}$ where it was reported that a combined intervention has significant effects on reducing pain in patients with hip and knee OA (as seen in eight of ten trials reviewed with $p$ values ranging from $0.001-0.05)$. However, ES for the treatments were not documented in the SR and it was not stated whether the participants involved in these trials were on a waiting list. Therefore caution should be taken when comparing the results of the SR to the present study.

\section{iii. Change in PIS subsections over time}

Interestingly, the present findings report the most improved subsection of the interference scale in the experimental group to be on pain interference with "mood" $\left(F_{(3,117)}=4.13 ; p=\right.$ 0.01). The open ended questionnaire confirmed improvements in psychological aspects after the intervention by participants reporting the intervention had a positive impact on attitude, improved motivation and outlook on life. These self-reported psychological benefits appear to be reflected by the immediate and sustained significant improvement in mood interference in the experimental group at week six $(p<0.01)$. The finding may be directly related to the significant reductions observed in pain severity in the experimental group discussed in the following section. This link supports the previously documented bidirectional association between pain and mood disorders, such as depression and anxiety in $\mathrm{OA}^{(22,328,330)}$. The current association seen between pain and mood further supports the previously identified relationship between joint pain and depression in a study performed in a South African population ${ }^{(87)}$.

Another explanation of the current findings for improved mood interference can be as a result of the positive psychological effects of active participation in exercise ${ }^{(21)}$. This corroborates with Hurley et al.s' work on the psychosocial benefits of exercise; including improved mood, reduced stress, depression, anxiety and increased self-worth and esteem $^{(21)}$. If this explanation is the reason for change in mood seen throughout the duration of the study, it suggests that participants in the experimental group adhered to the exercises taught during the intervention, even at month six $^{(331)}$. 
This is in contrast to studies which report low adherence to exercise in those with OA, especially when the physiotherapist is no longer involved and able to reinforce exercise habits ${ }^{(276,331)}$. Adherence was not however recorded in this study therefore this is a speculation. A possible explanation as to why the current sample may have adhered to exercise in this study lies in the combined management approach of exercise and education; as discussed later in the text.

Leading on from the above, it is known that exercise can improve function in those with $\mathrm{OA}^{(29)}$. Improvements in function have been said to lead to improved mood states ${ }^{(21)}$. Therefore the current findings support the known relationship between dysfunction and mood disorder in $\mathrm{OA}^{(328,330)}$.

This is evident by significant improvements in functional interference [walking $\left(F_{(3,120)}=\right.$ 2.89; $p=0.04)$ and normal work $\left.\left(F_{(3,114)}=3.35 ; p=0.02\right)\right]$, coupled with significantly improved mood interference (pg. 115). Thistoo is confirmed by participants in the experimental group reporting improvements in their ability to function in the open-ended questionnaire (see Appendix Q, pg. 413).

An unlikely possibility for improvements in mood may be attributed to the Hawthorne effect ${ }^{(332)}$. This effect is described as an improvement in outcomes as a result of attention received by participating in clinical research ${ }^{(333,334)}$. Mood improvement could have been said to be due to the participants eventually receiving attention by some form of treatment, after being on the waiting list for years. Yet, if this was so, it would be expected that the control group would also show significant improvements in mood, which was not so, as they too received attention for the duration of the study by follow up measures. This further strengthens the above probability of mood benefits as a result of improvements in pain, ongoing exercise and better functional ability. 
As mentioned above, there appeared to be an association at baseline between poor sleep and mood in persons with $\mathrm{OA}^{(328)}$. As the results were analysed, this association remained with mood interference improvingtogether with improvements in sleep interference $\left(F_{(3,}\right.$, $\left.{ }_{120)}=2.75 ; p<0.05\right)^{(328)}$. Additionally, the findings of reduced sleep disturbances and the improvements in overall pain scores seen in the sample of the current study corroborates with the previously discussed association between pain and sleep. Not only do those with severe pain report common sleep disturbances, but when improvements in pain are experienced, so are improvements in sleep and vice versa ${ }^{(335)}$.

The significant improvement in overall pain interference in the current sample is encouraging. The four subsections that improved after the intervention support the known relationships between pain, physical function, sleep and mood. In order to further understand the improvements observed in the present sample, a previous research recommendation was applied to the current findings to establish whether differences existed in treatment responses between participants with hip or knee $O A^{(73)}$. Subgroup analysis for the effects seen in those with OA of the hip, knee or both hip and knee are discussed in the text that follows. It is acknowledged that the sample was limited for subgroup analysis and as such the subgroup results need to be viewed with caution. However, the distinct differences in results between the subgroups were felt to be worthy of mention.

\section{iv. Subgroup analysis for PIS}

The subgroup analysis showed that those with OA of the hip presented at baseline with the lowest pain interference score $(M=5.50, S D=2.71)$ compared to those with $O A$ of the knee $(M=6.68, S D=2.29)$ or both hip and knee $O A(M=7.82, S D=1.49)$. The results show that the participants with $\mathrm{OA}$ of the hip only participating in the intervention showed no significant improvements from baseline or compared to the control group $\left(F_{(3,27)}=1.44, p=\right.$ 0.25). To the researchers' knowledge, comparison studies performed using the BPI PIS in a sample of patients with hip OA awaiting surgery does not exist. Most studies report the effects of treatments on pain severity instead of pain interference, which limits comparisons in the discussion on changes in PSS. 
Since the subgroup with hip $O A$ had no overall significant improvements in pain interference, it implies the improvement must have occurred in one of the other two subgroups, those with OA of the knee only or OA of both the hip and knee.

Interestingly, those with $\mathrm{OA}$ in both the hip and the knee in the present study's experimental group showed a significant improvement in PIS, contributing towards the overall significant difference between groups. It was observed that this subgroup showed significant within group improvements from baseline to week six $(p<0.01)$, week 12 and month six $(p=0.02)$. As far as the researchers are aware, no literature is available on persons with OA of both the hip and the knee, therefore comparisons could not be made either regarding previous work utilising the BPI pain interference scale.

The largest difference was observed in the subgroup of participants with OA of the knee. This subgroup presented at baseline with severe pain interference $(M=6.68, S D=2.29)$ and showed a significant difference between groups from baseline to month six with a considerable effect size $(E S=1.21, p<0.01)$. This suggests that such an intervention was more effective in the participants with OA of the knee only rather than in those with OA of the hip or those with OA of the hip and the knee. This result should be taken into consideration for future research in those with lower limb OA and when applying such an intervention into clinical practice. Likewise for prior studies in OA of the hip, comparisons of effect sizes between the current study and previous work showing benefits for PIS in persons with knee OA could not be drawn due to different outcome measures used for pain. Suitable comparisons were able to be drawn for pain severity and will be discussed in the following section. 


\subsubsection{Pain severity scale}

\section{i. $\quad$ Change in PSS over time}

In addition to benefits of reduced pain interference, the intervention appears to have resulted in a sustained significant reduction in pain severity scores seen within the experimental group over time $(p<0.01)$. It is noted that this change is not between groups for the sample and could be as a result of the choice of measurement instrument. According to Newman et al. ${ }^{(228)}$, an intervention including self-management strategies should focus more on the effect of pain (as seen by the previously discussed significant between group improvements in PIS) rather than the amount of pain. Yet, most studies that are performed in OA assess pain severity as an outcome by a variety of measures. Therefore, a more likely reason as to why improvements in PSS were not noted between groups could be explained by subgroup analysis, as done for PIS results, to establish the effects according to OA of the hip, knee or both hip and knee joint.

\section{ii. Subgroup analysis for PSS}

According to a systematic review in 2011, studies support the use of various pre-operative interventions to moderately reduce pain severity in those with knee OA, yet, only low evidence exists for the use of these interventions in those with hip $O A^{(73)}$. Wallis and colleagues' finding of conflictingresults in pain severity for those with hip OA versus those with knee OA suggests exercise may produce different benefits in each group based on differences in biomechanics, progression and risk factors for OA of the knee or hip ${ }^{(73,336)}$. The current findings support the above statement as seen by the differences observed between the subgroups below.

The subgroup analysis for PSS yielded the same finding as that of the PIS for the subgroup of those with OA of the hip. Similarly, participants with OA of the hip presented with the lowest pain severity scores at baseline $(M=5.27, S D=2.89)$ and those in the experimental group showed no significant improvement compared to baseline measures or against the control group $\left(F_{(3,27)}=1.1200, p=0.36\right)(p g .337)$. This result is in contrast to those from a prior study ${ }^{(331)}$, on the effects of exercise and education in those with hip $\mathrm{OA}$, who reported significant reductions in pain severity $(p<0.05)$, although ES were not reported ${ }^{(331)}$. 
It was noted however that the intervention in de Jong at al.'s study ${ }^{(331)}$ differed somewhat from the integrated exercise and education programme used in the current study as the educational component was limited to one session and the exercise component mainly consisted of strength training with fitness equipment. Likewise, the participants with OA of both the hip and the knee in the present study showed no significant difference in PSS between groups. As reported in the PIS subgroup analysis, it was observed that participants with OA of both the hip and the knee showed significant within group improvements in PSS from baseline to week six $(p<0.01)$ and week $12(p=0.03)$. Once again, to the researchers' knowledge, no literature is available reporting on those with both hip and knee OA using the BPI PSS, thus limiting comparisons to previous work.

As in the PIS, the most significant results were observed in the participants with OA of the knee. This subgroup presented at baseline with severe pain $(M=6.82, S D=1.77)$ and showed a significant difference between groups atmonth $\operatorname{six}\left(F_{(3,51)}=6.27, p<0.01\right)$ with a considerable ES of 2.64. The current ES for pain severity is substantially larger than those reported in a prior study on a similar integrated intervention in knee OA $(p<0.01$ and $p=$ 0.03 with small $E S=0.2$ and $E S=0.27$, for two pain severity measures used $)^{(331)}$. The current finding disputes previous small minimally powered studies ${ }^{(276)}$ which showed no significant changes in pain after exercise and education interventions in participants with OA of the knee. Rather, the present results support a recent systematic review on the effects of a variety of combined exercise and education interventions in those with knee $\mathrm{OA}$, whereby improvements in pain, ranging from short (two months) to long term sustained benefits (two years), were reported in all eight studies included ${ }^{(178)}$. Although the SR did not state whether these samples included patients on waiting lists, nor were statistical p-values and effect sizes noted, the positive results in people with knee OA are considered encouraging.

The contrasting findings in the present study between improvements in pain severity seen in those with knee OA compared to those with hip OA, supports a study design in which participants with knee or hip OA are separated. This would avoid different treatment responses within one sample confounding the results ${ }^{(178,272,276,279)}$. 
In the present study, the lack of change in the PSS in the hip OA subgroup likely resulted in modulating the results for the entire sample.

As mentioned previously, most of the literature regarding waiting periods included short waiting lists of less than a year. A comparison study of integrated exercise and education by Lamb et al. ${ }^{(279)}$ was more in line with the longer waiting period of the current study. The sample used by Lamb et al. ${ }^{(320)}$ had symptoms of knee OA for an average of 10 years and were on the waiting list for over a year. The significant improvements in pain severity with a large ES for the knee subgroup in the present study supports Lamb et al.s' findings that moderate effect sizes for pain improvement are attainable in those with knee $\mathrm{OA}^{(279)}$. The lack of a control group was a major limitation of Lamb and colleagues' work as the significant benefits seen were not compared to those not receiving the programme. This points to a strength of the methodological design of the current study which allows significant improvements to be compared to a control group.

The present findings further support a more robust study in a large sample with symptoms of knee pain for an average of six to nine years ${ }^{(272)}$. The current study design was very similar to that of the RCT by Hurley and colleagues ${ }^{(272)}$, where a combined exercise, education and active coping strategies intervention was compared to a control group of usual care. Pain was used as a secondary outcome and showed sustained significant improvements in pain severity over the long term (six months) $(E S=0.27, p=0.02)^{(272)}$. The ES of Hurley and colleagues' results were small, although similar to those of previous studies reporting the effects of exercise on pain in persons with OA (ES $0.39-0.52$ ) ${ }^{(91,275,337)}$. In a study by Fransen et al. ${ }^{(338)}$, a larger ES reported on pain after an exercise intervention in persons with knee OA (ES $=0.94, p<0.01$ ), is better matched to the ES noted in the current study for reduction in pain severity in knee $O A(E S=2.64, p<0.01$ ). However, comparison to Fransen et al.s' study ${ }^{(338)}$ on exercise should be made with caution as they had a short follow up duration (two - four months). 
Along with the small ES it was also noted that the sample used by Hurley et al. ${ }^{(272)}$ was not specified to be on a waiting list for arthroplasty. Therefore Hurley and colleagues' sample ${ }^{(272)}$ could be considered less affected by OA as they were not yet assessed as having sufficient joint damage to require an arthroplasty and be placed on a waiting list. However, WOMAC pain scores at baseline in Hurley et al.s' sample ${ }^{(272)}$ showed high levels of pain severity $(M=7.6, S D=0.4)$ which can be compared to the current study's high baseline pain severity scores on the BPI. Despite comparative pain severity scores, caution should be taken when drawing contrasting these two studies as different outcome measures for pain were utilised. Further, the sample used by Hurley et al. ${ }^{(272)}$ had lower baseline WOMAC function scores $(M=27.2, S D=1.45)$ when compared to the normal WOMAC scores reported elsewhere for people with $O A$ of the knee $(M=41.3, S D=14.8)^{(133)}$. Additionally Hurley and colleagues' sample of participants with knee OA had lower scores when compared to baseline scores of persons with $O A$ on a waiting list $\left(M=40.3, S D=12.1^{(71)}, M\right.$ $\left.=28.3^{(280)}\right)$. This could suggest that despite high levels of pain, Hurley and colleagues' sample was more physically able than the sample in the current study and therefore the findings of a large effect for improvements in pain severity in those with knee OA in the present study are highly encouraging. Despite the worse levels of disability in the present sample with $\mathrm{OA}$ of the knee specifically, the combined intervention was effective in bringing about meaningful benefits in pain severity.

The benefits seen in PIS and PSS, particularly in those with knee OA rather than hip OA, were both long term improvements with the largest significant differences noted at six months. It has been suggested that an effective integrated exercise and educational programme should be considered as an alternative to joint replacement surgery if the significant benefits are sustained over the long term of more than 12 months ${ }^{(279)}$. It was never the intention of the current study to provide an intervention to substitute the need for an arthroplasty. However, since the wait for surgery is so lengthy, the long term benefits seen after the intervention are extremely positive and support the previously identified need to improve pain and related function in those whose waiting period is longer than a year ${ }^{(73)}$. The current intervention appears to have met this need by reducing pain interference and severity for the sample awaiting surgery. However, a previous study by Lamb and colleagues ${ }^{(279)}$ contradicts our findings by reporting that a combined intervention does not in fact cause the desired advantages for those on a waiting list. 
Interestingly, those on Lamb et al..$^{(279)}$ waiting list had significant short term improvements in pain with low ES at week six and 12 (ES $=0.3$ and 0.1 ) compared to those not on the waiting list who showed moderate to high ES which were sustained at 12 weeks (ES $=0.7$ $0.8)$. As mentioned, the current results contradict Lamb et al. $\mathrm{s}^{(279)}$ findings as the significant reductions in pain severity with a large ES (ES $=1.15)$ was most significant for the current sample on a waiting list, in the long term at month six. This is remarkable for a sample on such a long waiting list and has not yet been documented elsewhere in literature. Similar long term improvements in pain were seen in Barlow et al. $s^{(235)}$ RCT assessing the effects of the ASMP in a sample with arthritis symptoms for an average of $10-11$ years, whereby no improvements in pain severity were seen at four months, yet improvements were seen at 12 months.

Similar findings were also reported by Lorig et al. ${ }^{(241)}$ whereby the ASMP was compared with the CDSMP. In their sample it was only at 12 months post intervention that differences in pain severity between groups approached significance $(p=0.08)$. Furthermore, consistent slow improvements in pain severity are seen in a sample treated with a similar intervention to the one used in the current study, whereby exercise and education in participants with lower limb OA showed no significant changes in pain at two months but displayed significant improvements in pain at month six $(p=0.02$ on the WOMAC pain scale $)^{(277)}$. The delay in improvements in pain is speculated to be related to the length of time that it takes to adopt newly learnt beliefs and self-management strategies prior to obtaining marked improvements in pain ${ }^{(235)}$. This is supported by the recommendation that studies investigating the effects on pain in a chronic pain condition such as $O A$, should include long term follow ups of more than 12 months $^{(276)}$. Despite the current study following participants for shorter than the recommended duration, the results are encouraging as improvements in pain were observed after only six months.

Due to the discrepancy in pain improvements between Lamb et al..$^{\prime(279)}$ participants who were on a waiting list and those who weren't, Lamb and colleagues recommended that a six-week combined intervention only be utilised before being placed on a waiting list. The reason behind this suggestion lies in the low probability of symptoms improving from conservative management, once the need for surgery has been determined. 
Elsewhere it is explained that long term adherence to self-management and exercise is low and may be related to psychosocial aspects rather than physiological factors ${ }^{(276,285)}$. It could be that those who are not yet on a waiting list hold beliefs that conservative management options can result in benefits and therefore pain reductions are observed.

Yet for those who have been told they have severe arthritis for which only a joint replacement will help their symptoms, are less likely to have confidence that less invasive options can cause changes and therefore are not responsive to conservative management ${ }^{(242,245)}$. Contrary to Lamb et al. $\mathrm{s}^{(279)}$ recommendation; the current significant long term improvements in pain interference and severity from a conservative management option is effective in individuals with severe OA on a waiting list in a South African context.

To conclude the discussion on the primary outcome measure, the significant benefits reported in pain severity and pain interference in the current study support the statement by Williams and colleagues ${ }^{(139)}$ that the primary outcome measure used in the present study is sensitive enough to establish treatment advantages for pain. This was clearly demonstrated by the relationships observed between pain severity, pain interference in physical function, sleep disturbances, effect on mood and physical functioning. These links were not only evident at baseline but as different variables changed throughout the current study each of the variables followed a similar pattern of change over time. As discussed in the literature review, it is not sufficient to simply measure pain and changes in pain during trials related to $\mathrm{OA}$. Therefore secondary outcome measures were used to establish further effects of the intervention; these are discussed in the text that follows. 


\subsection{Secondary outcome measures: function (Physical performance task battery)}

\subsubsection{Walking}

Walking ability in the growing population affected with $\mathrm{OA}$ is a critical aspect on which to focus management ${ }^{(277)}$. Even though the condition continues to progress and disability has been said to increase each year ${ }^{(250,251)}$, the present findings show a short term intervention, without reinforcements, has lasting effects on daily function, particularly in terms of walking ability.

The second important finding of this study was observed in the significant functional improvements of the experimental group when compared to the control group at month six in their ability to walk at a fast speed $(E S=0.88, p=0.03)$. The ability to walk faster than before is a sound objective measure of improved functional ability or mobility ${ }^{(339)}$. This finding further reinforces the significant improvements in walking ability on the PIS of the BPI. As discussed in the section above on pain, it is observed in research evaluating the consequences of waiting for surgery, that deterioration in function has been reported in those on a waiting list ${ }^{(33,71,310,314)}$. The current findings indicate that even in a sample that has severe pain and functional limitations while waiting a long time for surgery, an exercise and education intervention is effective in bringing about long term improvements in walking ability with a large ES.

The encouraging finding of improved functional ability is supported by previous work to establish the effects of integrated interventions on function in individuals with $O A$ of the hip and knee. The SR by Walsh et al. ${ }^{(276)}$ discussed in the previous section on pain, reported that exercise and educational interventions brought about significant improvements in function in persons with hip and knee OA $(p<0.05$ in seven of the 10 studies reviewed yet no effect sizes documented). Again, the sample in the SR was not on a waiting list and baseline measures of functional ability were unavailable to draw comparisons against the current study. Additionally, methodological flaws existed across the studies included in the review and therefore caution should be taken when drawing conclusions from the results in relation to the present findings ${ }^{(276)}$. 
The results of the current study lead the researchers to speculate that the ability to walk could possibly continue to improve after month six, as the time taken to walk 15 meters at the fastest speed shows a clear steady decrease (improvement) from baseline to month six $(M=18.65, S D=6.27$ to $M=15.09, S D=6.04)$. Further follow up after a combined intervention would be needed to confirm this possibility.

In addition to walking faster, those in the experimental group could walk further than documented at baseline. It appears that the intervention could also lead to the improvement seen in distance walked as measured by the 6MWT. Distance walked in the experimental group was significantly further than baseline scores at week $12(27.3 \%$ improvement) and continued to increase at month six $(30 \%, p<0.01)$. This finding is supported by previous work ${ }^{(277)}$ using the $6 \mathrm{MWT}$ as an outcome measure in persons with OA who completed a combined exercise and education intervention. In a RCT by Hughes et al. ${ }^{(277)}$ the participants in the experimental group were able to walk $13.8 \%$ further at month six than they were able to at baseline $(p=0.02)$. It is noted that their sample was not on a waiting list, and comparisons of baseline distance walked during the 6MWT showed the sample in the current study to be more severely affected by OA than the sample in Hughes et al.s' study ${ }^{(277)}$. Hughes and colleagues' sample ${ }^{(277)}$ were able to walk considerably further than the current study's sample at baseline $(395.8 \mathrm{~m}$ in the experimental group of Hughes et al. compared to $267.8 \mathrm{~m}$ for the current study). This is a very encouraging comparison as even in a sample such as in the present study who had worse functional ability than previously reported $^{(277)}$, the current sample showed significant improvements with a higher percentage of change in distance walked in six minutes. Therefore the likelihood of the intervention resulting in improvements in walking distance should be assessed against a larger sample size to confirm whether the intervention produces significant improvements when compared to the control group.

Once again, based on the findings of the present study it may be hypothesised that walking distance shows the potential to improve further as participants displayed a steady increase in walking distance over time. The proposal put forward by Barlow et al. ${ }^{(235)}$ to explain the changes in pain occurring slowly in chronic conditions raises attention to whether the same applies for changes in function. 
Therefore, evaluation of walking ability at month 12 would provide useful information as to whether function in chronic pain conditions such as OA also takes longer to become significantly evident between groups. To establish whether differences existed in walking ability between those with OA of the hip or knee only or those with both hip and knee OA, analysis of subgroups is discussed below.

\section{i. Subgroup analysis for walking}

The results of changes in walking ability in the sub groups agrees with the sub analysis findings performed for the primary outcome measure of pain. Those in the experimental group affected with hip OA, showed no significant improvement in walking ability over time. Unfortunately, as previously mentioned, most studies reporting on the effects of a combined approach in managing OA are performed in persons suffering from knee OA. Therefore no studies were found that evaluated the effects of a combined intervention on function in individuals with hip OA only, which would allow comparisons to be made. However, it must be kept in mind that the current finding is based on a small sample $(n=$ 11) and it cannot be concluded that persons with hip OA in other settings would not benefit from an integrated intervention. Further research should be performed to ascertain the effects in this population which was only analysed as a sub-group of the current study. This recommendation is supported by a SR reporting other interventions, such as exercisebased interventions, can result in benefits for pain and function in participants with hip OA awaiting a joint replacement ${ }^{(74)}$.

Analysis of walking ability for the subgroup of those with knee OA was also consistent with the benefits seen in this subgroup for pain. The participants with knee OA had significant improvements in walking ability (6MWT) from baseline to month six $(70 \mathrm{~m}$ increase in distance, $27.5 \%$ improvement, $p=0.01$ ). This finding was slightly less evident than that of pain as a significant difference was not observed between groups, as was seen for the PIS and PSS. Yet, improvements in function for those in the current sample with knee OA agrees with a previous integrated walking and education programme whereby distance walked on the 6MWT was improved at month two by $70 \mathrm{~m}(18.4 \%)$ in those with OA of the knee when compared to their baseline distance walked ${ }^{(340)}$. 
Similarly, both the current control group and the one involved in Kovar et al.s' study displayed a worsening in function by a decline in walking distance of $39 \mathrm{~m}$ and $17 \mathrm{~m}$ respectively (13.3\% and 4.5\%). Furthermore, the current results also support the findings from a previous $\mathrm{SR}^{(178)}$ performed to establish the effects of a combined intervention on function in knee OA. All eight studies included in the review indicated improved functional abilities in those with OA of the knee, although details regarding what, when and how functional abilities were assessed and the magnitude of improvements were not specified in the review. The present findings can be better compared to a more recent study by Hurley et al. where the long term effect on function after participating in an integrated programme was established ${ }^{(271)}$. The results of the current study confirm Hurley and colleagues $^{(271)}$ significant improvements in function at month six $(p<0.01)$. Additionally, the benefits were maintained in the long term (month $30, p<0.01$ ) which supports the recommendation made by the researchers of the present study; to include longer follow up measures in future trials.

Unexpectedly, participants in the present study with both hip and knee OA showed the greatest improvements in walking ability during the 6MWT. The experimental group had significant improvements at week six $(37.4 \%, p=0.02)$, week $12(50.7 \%, p<0.01)$ and month six $(46.9 \%, p<0.01)$ and significant improvements in the $15 \mathrm{~m}$ fastest speed walk test at week $12(23.7 \%, p=0.04)$. This was initially thought to be attributed to this subgroup presenting with severe restrictions in walking ability at baselineand therefore having greater opportunity to improve than participants with only hip or knee OA. Yet, baseline measures for walking ability are contradictory to this proposed idea. Those with OA of both the hip and knee presented as the better of the three subgroups at baseline by being able to walk the fastest and furthest (Appendix J; pg. 355). This leads the researchers to believe that because this subgroup was slightly less affected in walking ability at baseline they were able to benefit more from the intervention in respect to walking ability. Although this finding was not anticipated, the significant improvement was seen within the experimental group only and no difference between groups was found. Similar to the changes recorded in this subgroup for PIS and PSS, these findings suggest that a combined intervention such as the one used in the present study is able to bring about improvements in walking in those with multiple lower limb joint OA. 
Once again, previous studies assessing the functional benefits in persons with both hip and knee $\mathrm{OA}$ are unavailable to draw comparisons and therefore a larger sample may provide valuable results to confirm the speculations around this subgroup.

\subsubsection{Sit to stand}

Another commonly reported difficulty in those with OA of the lower limbs besides walking, is the functional ability to stand up from a seated position ${ }^{(74)}$. Hughes et al. ${ }^{(277)}$ emphasise the importance of the ability to perform this task for functional independence in daily life. Significant improvements in the ability to stand up from a chair were reported in the experimental group with participants demonstrating large and steady improvements in time taken to perform the repeated sit - stand test. Time was significantly faster in the experimental group, compared to baseline, at week six and continued to improve until month six $(p<0.01)$. However, it cannot be said that the intervention was the only variable leading to these changes as there was no significant difference between groups and the control group also completed the task in significantly faster times at week $12(p=0.02)$ and month six $(p<0.01)$.

A possible explanation as to why both groups showed a significant improvement in time scores could be due to familiarisation with the task. Task familiarisation is defined as an improvement in a performance from one administration of the outcome measure to the next as a result of performing the test again and becoming more familiar with how to perform it each time ${ }^{(341)}$. Rather than this being a conscious process, it is a process of neural adaptation. It could be explained that the familiarisation effect was evident in this task test and not in the others as a consequence of the sit-stand test being the only task to be repeated twice at each follow up measure in order to get an average time for the functional task. This finding was in contrast to the study by Hughes et al. ${ }^{(277)}$ in persons with lower limb OA, where no significant improvements or difference between groups was reported for sit to stand. 
Hughes and colleagues ${ }^{(277)}$ also tested by means of a repeated sit - stand test and despite repeating the function five times at each reassessment, familiarisation did not impact the results. Instead the authors reasoned that no differences were reported due to the range for timed scores in both the experimental and control group being very large ${ }^{(277)}$. Hughes et al. ${ }^{(277)}$ suggested a larger sample be used to detect changes in this specific functional test.

\section{i. Subgroup analysis for sit - stand}

The functional measure of sit - stand was the only outcome measure which showed conflicting results when sub analysis was done to compare hip and knee OA participants. Those with hip OA in the experimental group showed significant improvements from their baseline scores to week six, 12 and month six $(p<0.01)$, although this was not significant when compared to the control group. This could be due to the participants with hip OA in the experimental group presenting at baseline with the longest time taken to complete the task ( $M=11.31, S D=2.91)$ and therefore it could be said that they had more chance for improvement. Whereas those participants with OA of the knee or both hip and knee showed no significant improvements.

The above finding is in agreement with the recent $\mathrm{SR}^{(73)}$ that stated moderate evidence exists for improvements in activity in those with hip OA on a waiting list and not in those with knee OA. However comparison between the SR and the results of the present study are difficult as the type of intervention reported on in Wallis and colleagues' review was exercise alone and not an integrated programme. Further compounding the comparison is the studies included in the review had large variations in the content of the exercise used $^{(73)}$. Additionally, the changes recorded in activity levels were not maintained in the long term and therefore assumptions regarding sustained functional improvements in hip or knee OA cannot be drawn from Wallis et al.s' systematic review ${ }^{(73)}$. Similarly, the findings from this functional test in the present study do not present clear effects in relation to the intervention as results from the test could be confounded by all subgroups, including those in the control group, showing a steady improvement in time taken to complete the task which was not observed in the other functional tests. 
A more relevant comparison can be made against the results obtained from previous integrated exercise and education studies ${ }^{(178,271,279)}$ reporting benefits on function. Contradictory to the present study's subgroup analysis results, are the positive findings of significant benefits achieved in function for those with knee OA only. Documented effect sizes for functional benefits were small $(E S=0.3-0.4)$ and moderate for functional SE (ES = $0.5)^{(279)}$. These benefits were observed from week six follow up until one year in a variety of studies ${ }^{(178)}$. Again the findings of the present study do not concur with the results from Lamb et al. ${ }^{(279)}$ regarding those who were on a waiting list. In the current study the large ES for functional improvements ( $E S=0.88$ ) compared to a smaller $E S(E S=0.2)$ for those on a waiting list in Lamb and colleagues study and those not on a waiting list $(E S=0.4-0.5)^{(279)}$ and $(E S=0.29)^{(271)}$ is notable. Furthermore, the ES from the current exercise and education intervention far exceeds the effect sizes reported for changesin function in persons with OA not on a waiting list receiving exercise only $(E S=0.34)^{(91,275,337)}$. As discussed in the previous section on pain, the differences in treatment response may be attributed to psychological factors causing limited improvements once patients have been put on a waiting list ${ }^{(242,245)}$. The positive findings in the present study demonstrate once again, that a conservative intervention combining exercise and education is an effective treatment strategy to improve function in those awaiting a joint replacement in a South African context.

In summary, despite the average age of the sample being 59.52 years ( $S D=4.75$ ), the participants who took part in the combined exercise and education intervention displayed the ability to reduce their functional interference relating to pain and increased their functional activity levels. These positive results support the use of a combined intervention such as the one used in the current study. Firstly, including education, specifically education on pain and the neurophysiological changes that occur in chronic pain, into the intervention has shown that unhealthy beliefs regarding pain and fear avoidance behaviours may be changed in persons with OA on a waiting list in a South African context. Secondly, by incorporating an active participatory exercise component into the intervention allows new concepts that have been taught during the educational sessions to be implemented in a practical manner by the participants ${ }^{(271)}$. 
New knowledge regarding the meaning behind pain and the importance of remaining active in spite of pain, can be tested by participants on a first hand basis by applying new beliefs and learning the benefits of exercise for themselves. Once individuals experience improvements in pain, function and psychological aspects in their daily lives, it is more likely that beliefs can be changed and new behaviours adopted ${ }^{(271)}$.

The above is further supported by Crotty and colleagues' ${ }^{\prime 242)}$ statement that the desired primary changes caused by approaches involving self-management strategies is not simply improved knowledge on management of OA but applying the knowledge to bring about increases in activity and exercise. Participants' responses to the open ended questionnaire confirmed these behavioural changes by nine of 17 (53\%) participants reporting an increase in activity, exercise and specifically an improved walking ability at month six. A possible reason as to why much smaller effect sizes were reported in previous studies which assessed the effects of combined interventions on pain and function, could be due to the larger variation in the age of participants in the previous studies and consequently their probable clinical presentation $\left(51-91^{(271)}\right)$ and $\left(38-81^{(73)}\right)$. 


\subsection{Secondary outcome: disability (HAQ)}

\subsubsection{Baseline disability presentation of the sample}

As discussed in Chapter Two (pg. 3), pain and functional difficulties in persons with OA cause substantial disability, contributing to the growing burden on the health care system in South Africa ${ }^{(50,89,90)}$. It was therefore expected that together with the severe pain and functional interference and limitations, the current sample would present at baseline with high disability scores $(M=1.56, S D=0.55)$. The baseline disability index for the sample was much larger than a population-based norm in Finland $(M=0.25, S D=0.03)$ for persons with a similar mean age of 55.4 years $\left(S D=14.9^{(342)}\right)$. A more appropriate comparison was made between the current sample and a South African sample used in a previous study on prevalence and functional impact of musculoskeletal conditions in a sample in the Western Cape ${ }^{(87)}$. The present sample's disability index was almost twice as large when compared to the SA sample $(M=0.8)$ of Parker and Jelsma. They considered a mean HAQ disability index of 0.8 as a mild to moderate disability; suggesting the HAQ disability index at baseline for the current sample was at least moderate to severe. Similarly low baseline measures were also documented in a sample of varied chronic diseases taking part in a SMI in the USA(M = $0.81)^{(240)}$ confirming the present sample was far worse off in terms of disability when compared to populations with chronic conditions. Furthermore, Parker and Jelsma's ${ }^{(87)}$ findings supported the known association between age and disability index $(r=0.31 ; p<$ $0.001)$; as age increased, so did disability ${ }^{(342)}$. Therefore the older age $(M=59.52$ compared to $M=51.7$ years ${ }^{(87)}$ ) of the participants in the current study with higher disability scores further supports this relationship.

As mentioned throughout the literature review, adding to the burden of disability is the rising prevalence of co-existing conditions such as obesity, hypertension, diabetes mellitus and cardiac conditions ${ }^{(47,48)}$. The study by Parker and Jelsma ${ }^{(87)}$ highlights the prevalence of common co-morbidities in the South African population suffering from musculoskeletal conditions. In their sample high percentages were documented for hypertension (59.1\%), diabetes $(24.8 \%)$ and heart problems $(18.9 \%)$ in persons with musculoskeletal disorders ${ }^{(87)}$. These percentages are similar to a study in the USA in a sample of persons with OA who took part in a combined exercise and education intervention (hypertension $60.4 \%$, diabetes $23.4 \%$ and cardiovascular $15.3 \%)^{(343)}$. 
The prevalence of co-existing conditions in the participants in the current study agrees with those reported previously. Besides suffering with $\mathrm{OA}, 76.2 \%$ of the participants presented with hypertension, $33.3 \%$ with diabetes and $7.1 \%$ with cardiac conditions. Additionally, 15 of the 42 participants (35.7\%) were no longer able to work and were on a disability grant; this too suggests the current sample has been greatly affected in terms of disability. Furthermore, the current sample presented with an average of 1.29 co-morbidities per participant. This is slightly less than theaverage number of co-morbidities reported in two studies using a combined exercise and education intervention in knee $O A(M=2.2)$ and hip $\mathrm{OA}(\mathrm{M}=2.6)^{(279)}$. However Lamb et al.s' two samples ${ }^{(279)}$ were considerably older $(\mathrm{M}=65$ and $M=67$ years respectively) than the current sample and therefore would be expected to have a higher number of co-morbidities ${ }^{(87)}$.

The findings from a separate pain VAS that forms part of the HAQ disability outcome showed strong positive correlation at baseline with the BPI PSS $(r=0.74, p<0.01)$. According to Pearson's correlation a value between $r=0.6$ and $r=0.79$ is considered a strong positive agreement ${ }^{(344)}$. This finding confirms the validity of the pain severity measures used in this sample. The extent to which the baseline disability and pain VAS scores, according to the $\mathrm{HAQ}$, changed throughout the study is discussed below.

\section{i. $\quad$ Change in disability over time}

Even though the current sample were markedly disabled at baseline, it appears that the exercise and education intervention resulted in significant and sustained reductions in disability in the experimental group at weeks six, 12 and month six $(p<0.01)$ when compared to baseline. The investigators are aware that disability was not the primary outcome for this study and power analysis was not calculated in terms of disability, yet the differences seen in disability supports those seen in the primary outcome. This probability is further strengthened by the participants in the experimental group's responses to the open ended questionnaire. Participants described improvements in the ability to perform various activities that were previously too difficult to execute. Examples of this include being able to do shopping and no longer being reliant on the aid of an assistive device in all activities. 
Similarly, participants expressed being able to participate in social aspects of life that were not possible before the intervention; such as being able to leave the house and be part of an exercise group in the community, as well as the ability to socialize and make friends with others (Appendix Q; pg. 413). Therefore the significant improvement seen within the experimental group, although not significant when compared to the control group, is an encouraging finding in relation to the significant improvements already discussed in pain and function. The finding that disability and function displayed variations in improvement in this sample, supports the description made in Chapter Two on how disability differs from limited function ${ }^{(53)}$.Even though disability is a complex phenomenon, the positive findings of this study suggest disability can be altered ${ }^{(52,53)}$.

The strong positive correlation $(r=0.74, p<0.01)$ between the BPI PSS and the HAQ pain VAS was present throughout the study as similar response curves were seen for both pain severity scores on the BPI and the HAQ VAS (pg. 386). Significant and sustained improvements in pain severity recorded on the HAQ mirroring those from the $\mathrm{BPI}$ in the experimental group from baseline to week six $(p<0.01), 12(p=0.03)$ and month six $(p<$ 0.01). The pain HAQ scores in the current study showed a clinically meaningful reduction in pain severity (25.05 out of 100$)^{(128)(128)}$. This correlates with the previously discussed results observed in the current sample for reduction in pain severity according to the BPI (2.83 out of 10).

The present results of decreased disability together with improvements in pain and function in the experimental group are contradictory to prior work on the effects of anSMI by Lorig and colleagues ${ }^{(158,240)}$. One study ${ }^{(240)}$ showed improvements in health status and self-efficacy while disability followed the usual trend of increasing with each year $(M=$ 0.025 in older persons with and without chronic conditions and $M=0.03$ in older persons with chronic conditions ${ }^{(250,251)}$. In another study ${ }^{(158)}$, despite a $9 \%$ increase in disability over a four year period, pain was still improved by $40 \%$ and activity and role function were maintained. Additionally, less health care utilisation was evident in both studies, despite increased disability in the participants ${ }^{(240)}$. This suggests that the participants in Lorig et al.s' studies implemented self-management strategies, taught in the SMI interventions, into their lives as they were less reliant on health professionals. 
Yet sadly, the disability continued to worsen( $M=0.035$ in the first year, $M=0.02$ after the second year). It appears that the combined intervention used in the present study is more beneficial than a SMI, as the current intervention shows the potential to reverse the anticipated increase in disability described elsewhere. It is noted that the decrease in disability in the current South African sample $(M=0.6)$ was only monitored until month six in the current study and not over a longer period as in previous work. Therefore it would be advantageous to extend the follow up period to 12 months in order to draw more likely comparisons with regards to the change in disability index. As previously discussed, the differences in treatment responses between subgroups are also of interest for the HAQ scale in order to support or contradict earlier findings. These results are discussed in the section that follows.

\section{ii. Subgroup analysis of disability index and pain VAS}

In accordance with the previously discussed subgroup analysis for pain, it was observed that participants with $\mathrm{OA}$ of the hip only in the experimental group did not show any significant improvements for disability as measured by the $\operatorname{HAQ}\left(F_{(3,27)}=2.29, p=0.10\right)$. Comparison studies of an integrated intervention on disability in individuals with hip OA alone are scarce as most studies have been performed in persons with knee OA, or if studies have been completed in those with hip or knee OA, separate data for the hip group is not documented. To the researchers' knowledge, a RCT is presently underway in response to this limited area of research. A combined education and exercise intervention is being compared to an educational control group in order to establish the ability of the intervention to delay the need for surgery in a sample, with $O A$ in need of a total hip replacement $^{(281)}$. Disability, according to the Hip disability and Osteoarthritis Outcome Score $(\mathrm{HOOS})^{(345)}$, will be assessed as a secondary outcome measure in Jensen and colleagues trial ${ }^{(281)}$ and once results are made available could prove a well suited comparison for the data in the present study.

Due to the lack of literature available using the HAQ to measure changes in disability in persons with $\mathrm{OA}$ of the hip following a combined intervention programme, a comparison was made with a study exploring the effects of an exercise intervention in those with lower limb $O A$, of which $35.5 \%$ of the sample had hip $O A^{(346)}$. 
Significant improvements in disability with small ES were reported in the study by van Baar et al. ${ }^{(346)}(E S=0.26, p=0.07)$. The improvements however, are not specified according to joint affected and therefore it could be said those participants with knee $O A$, which consisted of the majority of the sample (59.5\%), may have accounted for the improvements in disability noted, as seen in the results of the current study. This reinforces the need for further research in the specific field of hip OA.

The findings of improved disability in participants with knee OA in the experimental group, agree with the improvements seen for this subgroup in respect to pain and function. Once again, significant improvements were observed in those with OA of the knee only at week six $(p<0.01), 12(p=0.03)$ and month six $(p<0.01)$, when compared to baseline values. Furthermore, the results pertaining to this subgroup for the HAQ pain VAS strongly mirrors the significant between group differences in pain severity on the BPI for those with knee OA. Significant and sustained improvements in the HAQ pain VAS with a large ES for participants with OA of the knee, was observed between the experimental group and the control group at week six $(E S=2.36, p=0.02)$ and more so at month six $(E S=3.4, p<0.01)$. Again it is seen that even the subgroup analysis results for PSS and the pain VAS were positively correlated $(r=0.53, p=0.02)$, validating the use of the BPI in those with knee OA (pg. 387).

Interestingly, response to change in the subgroup of knee OA were reiterated by participants in the control group reporting worsening disability over the first 12 weeks (an increase of 0.05 on the $\mathrm{HAQ}$ )(pg. 385). Their disability index was maintained at this worse score at month six. This deterioration was not significant, however it is notable when compared to the previously discussed annual rate of worsening disability reported on the $H A Q^{(250,251)}$. The subgroup analysis finding further supports the previously discussed significant changes in those with knee OA for pain and function; suggesting this subgroup to be more responsive to change compared to participants of the hip OA subgroup. 
The disability findings relating to knee $\mathrm{OA}$ in the current study are consistent with previous studies making use of the $\mathrm{HAQ}$ as an outcome measure of disability. In a study comparing an integrated intervention of exercise and education to a control group of education alone in persons with knee $\mathrm{OA}$, significant reductions in disability scores were reported at three $(p=0.02)$ and six months $(p<0.01)$. ES were not available ${ }^{(347)}$. Dias et al. $s^{\prime}$ results ${ }^{(347)}$ for the disability index were observed between groups, whereas the disability index for the current study was seen within the experimental group compared to baseline. A possible reason for a lack of difference between groups in the present study could be that despite an older sample (65-89 year) in the study by Dias and colleagues ${ }^{(347)}$, who also presented with very severe pain at baseline (similar to the sample used in the present study); they were not yet specified to be on a waiting list for surgery and had better baseline disability scores (Median $=1.0)$ compared to the current sample $(M=1.56)$.

As previously mentioned, being placed on waiting list for surgery has potentially negative consequences for non-surgical interventions which could also explain why significant differences were not observed between groups in the present study but were observed in Dias et al.s' sample ${ }^{(242,245)}$ not on a waiting list. Once again, it is important to note that this study's power analysis was not calculated for disability and therefore may not be sufficiently powered to show improvements in this outcome measure.

A further comparison is made with caution against a study which found an exercise intervention in persons with $\mathrm{OA}$ of the knee gave positive results for disability measured with the $\mathrm{HAQ}^{(338)}$. Significant improvements in disability were reported with large effect sizes at two months $(E S=1.22, p<0.01)$ and further maintained at four months. Once again, the sample of participants with knee OA used in the study by Fransen et al., was not on a waiting list and presented with better baseline disability scores $(M=0.67)$ than the sample in the current study. Another comparison is made against a prior study on the effect of a self-care educational intervention in persons with moderate knee pain due to $\mathrm{OA}(\mathrm{M}=5.93, \mathrm{SD}=2.97 \text { on the } \mathrm{HAQ} \text { pain } \mathrm{VAS})^{(348)}$. Significant improvements were seen between groups for disability at four $(p<0.01)$ and eight months $(p=0.4)$ but these improvements were lost at month 12 . 
Once again these results were for a sample who were not on a waiting list and presented with better disability scores at baseline $(M=1.21, S D=0.61$ for the experimental group, $M$ $=1.13, \mathrm{SD}=0.66$ for the control group $)^{(348)}$ when compared to the current study. Nevertheless, in the present sample, with severe pain and disability and on a waiting list for a knee replacement, the conservative combined intervention used in the current study favoured the experimental group withimprovements in disability sustained until month six. A larger sample size of people with knee OA only, could confirm the positive treatment responses observed in disability score. A longer follow up evaluation would also provide data to substantiate whether these improvements were maintained or lost in the longer term.

Strangely, the participants with both OA of the hip and knee showed conflicting results with those in the experimental and control group improving at weeks six and 12 respectively ( $p$ $<0.01)$. Following which both groups' scores began worsening again, with the control group reverting back towards baseline disability scores more distinctly than the experimental group. As seen in the results on function, this subgroup of people with OA of both the hip and the knee presented at baseline with the worst scores on the disability index of the three subgroups $(M=1.89, S D=0.42)$. These findings corroborate the statement that combined joint involvement leads to higher disability than a single affected joint ${ }^{(87)}$. The reason for the improvements in both the experimental and control groups is unknown and leads one to believe there may be different treatment responses in those with more than one joint affected. Thus comparisons to those with either isolated hip or knee OA should be made with caution. Further research targeting the effects of such an intervention in this population is necessary to further understand varied responses.

In summaryregarding the outcome of disability, a conservative intervention such as the one used in this study, holds the potential to impact an individual beyond improving impairment, i.e. a painful joint. Moreover, it appears to have the ability to influence daily life in terms of decreasing activity limitations and participation restrictions, which is remarkable in a group severely affected by their condition and on a long waiting list. 


\subsection{Secondary outcome: personal factors (SE and HRQoL)}

According to the ICF model, besides the interactions between and the effect of impairment, activity limitation and participation restriction on the overall health of an individual, personal factors are included as having a bidirectional effect on the former components ${ }^{(53)}$. As alluded to in the above section on disability, intervention strategies need not only focus on physiological improvements such as pain and function, but should aim to impact the person's entire being, involving personal and psychological aspects too. Previous literature has emphasised the consequences of pain, function and disability on cognitive and emotional well-being ${ }^{(50,119)}$ as well as the impact that psychological factors and beliefs have on function ${ }^{(272)}$. Therefore understanding to what extent the current sample was affected in terms of these aspects and how this changed throughout the study was of interest and is discussed below.

\subsubsection{Baseline presentation of the sample in terms of personal aspects}

Besides the usual consequences on psychological factors due to $O A$, it is assumed that the current sample had further negative influences on emotional well-being and quality of life as a result of being on a waiting list for so long ${ }^{(15,33,246,309,310)}$. The baseline presentation of the participants' emotive functional interference was previously covered in the discussionof PIS results, showing pain largely affected the aspects of mood and sleep. As expected, it was illustrated by the result from the EQ-5D that the current sample presented with a low health related quality of life at baseline $(M=0.33, S D=0.33)$. This agrees with previous work reporting high psychological stress and low HRQoL while being on a waiting list for anything from one month to three years ${ }^{(246)}$. The mean HRQoL scores for two samples on a waiting list in Ackerman and colleagues' studies were measured by the assessment of quality of life tool $(M=0.39, S D=0.24$ and $M=0.37, S D=0.26$, compared to those with $\mathrm{OA}$ not on a waiting list, $\left.\mathrm{M}=0.52, \mathrm{SD}=0.22^{(15,246)}\right)$. This was also far lower than the norm without $\mathrm{OA}$, in an Australian population of $\mathrm{M}=0.83, \mathrm{SD}=0.20$ (scale ranges from -0.04 to 1 , with 1 representing the best quality of life $)^{(15,246)}$. Aside from the work by Ackerman et al. ${ }^{(15)}$,limited studies have reported on HRQoL in those awaiting surgery for more than one year. One study showed HRQoL to significantly deteriorate when waiting longer than a year, yet this was reported on changes in only three of the SF-36 domains of HRQoL and therefore generalizability and comparison is limited ${ }^{(34)}$. 
More suited for comparison to the current sample are studies that have made use of the EQ-5D to measure HRQoL ${ }^{(33,310,319,349)}$. The present sample showed a far worse EQ-5D index $(M=0.33, S D=0.33)$ when compared to the norm of the general population in the Khayelitsha district in the Western Cape, South African $(M=0.80, S D=0.22)^{(319,349)}$. The current sample also presented with a worse HRQoL index when compared to a sample of people living with HIV in the Khayelitsha district $(M=0.70, S D 0.28)$ (Jelsma, personal communication, 20 October 2014). It is noted that these two community samples has a much lower age $(M=37.7, S D=13.6$ and $M=33.6, S D=7.6$ years respectively) than the current sample and therefore it maybe postulated that the younger population may be expected to have a better health related quality of life.

It would therefore be more applicable to compare the HRQoL of the current sample to that of an older South African community. A study was performed in a widely diverse community in Woodstock in the Western Cape to establish the determinants of HRQoL using the EQ-5D in this older population $(M=50, S D=17.8 \text { years })^{(319)}$. However, this sample with a closer age match still had a better HRQoL index ( $M=0.79, S D=0.27$ )(Jelsma, personal communication, 20 October 2014). Further analysis of the Woodstock sample according to those with a disability or ill health and those without, showed that even those with a disability or ill health reported higher EQ-5D scores than the current sample with OA $(\mathrm{M}=0.51, \mathrm{SD}=0.39$ and $\mathrm{M}=0.68, \mathrm{SD}=0.32$ respectively)(Jelsma, personal communication, 20 October 2014). Another comparison was made against an older European population norm (age $70-74$ years) who similarly had a much higher $E Q=5 D$ index than the current sample $(M=0.76)^{(33)}$. This leads one to assume that age is not the main factor contributing to the lower HRQoL scores seen in the current sample but more likely as a consequence of the combined effects of having OA and being on a waiting list for such a long period.

In order to strengthen the above assumption, the current sample was also compared to an older ( $M=69.4, S D=9.7$ years) European sample awaiting a hip replacement for an average of six months ${ }^{(33)}$. 
The current sample presented with slightly worse HRQoL when compared to Ostendorf and colleagues' sample $^{(33)}(\mathrm{M}=0.39, \mathrm{SD}=0.31)$ and showed an even worse HRQoL when compared to another elderly European sample, awaiting a hip replacement for eight months (men: $\mathrm{M}=0.47, \mathrm{SD}=0.028$, and women: $\mathrm{M}=0.48, \mathrm{SD}=0.025^{(310)}$ ). The finding that the current sample has worse HRQoL scores while waiting a considerably longer time on a waiting list $(M=3.61, S D=2.44$ years), supports prior work reporting quality of life deteriorates with time while being on a waiting list and that this is the most likely reason for the lower HRQoL seen in the current sample ${ }^{(15,33,246,309,310)}$.

Similarly, the baseline EQ-5D VAS in the current sample $(M=58.26, S D=20.46)$ showed their self-rated state of health was far worse than both the younger and older South African community population norm values $\left[\mathrm{M}=77.8, \mathrm{SD} 22.7^{(319,349)}\right.$ and $\mathrm{M}=74.7, \mathrm{SD}=$ 14.9 (Jelsma, personal communication, 20 October 2014) respectively]. Similarly, the state of health was still worse than those in the older SA sample with disability and ill health ( $M=$ 62.9, $S D=19.9$ and $M=70.5, S D=17.5$ respectively)(Jelsma, personal communication, 20 October 2014). Furthermore, the present sample's baseline EQ-5D also revealed a slightly worse state of health when compared to the sample awaiting surgery in Ostendorf and

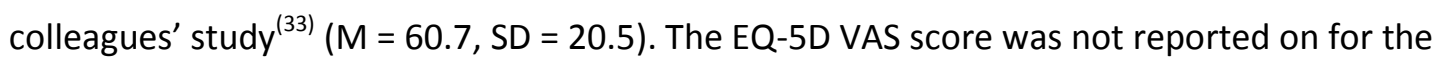
similar study awaiting eight months for surgery ${ }^{(310)}$.

The baseline EQ-5D scores show the participants in the current study on a long waiting list report their state of health to be much poorer than the younger and older South African population norms with and without health issues and as slightly worse than those on a short waiting list in Europe.

These findings emphasise just how remarkable the positive results of reduced pain and functional improvements in this study are; that in spite of a poor state of health and low HRQoL ratings in the current sample, improvements in pain and function are evident. Whether the study was also able to bring about benefits in the personal aspects of HRQoL, state of health and SE are discussed below. 


\section{i. Self-efficacy}

Another important factor previously discussed included under personal aspects relating to overall health and disability is self-efficacy. As mentioned in the literature review, selfefficacy is often lower in those with chronic conditions and has a role to play in cognition, behaviour and self-management ${ }^{(193-195)}$. The link previously spoken of between pain, dysfunction and low self-efficacy ${ }^{(197-199)}$ was not as evident in the present sample as anticipated. The baseline presentation for SE score, according to the chronic disease 6 item SE scale, was in fact not very low $(M=6.30 \pm 2.38$ ). This is surprisingly higher than the normal value for those with chronic diseases in the USA $\left(M=5.17, S D=2.22^{(177)}\right)$. It is noted that the use of the SE scale is intended for a measurement of SE in a variety of chronic conditions, therefore the lower SE score in the US sample could be justified as the score was obtained from a sample not specific to OA. Furthermore, the previously documented correlation $(r=0.49, p<0.01)$ between low SE and low quality of life in persons with OA awaiting surgery ${ }^{(246)}$ was not supported by the findings of the present study; as seen by poor HRQoL scores of the current sample despite high SE scores. However these unexpected findings related to SE may be appropriate for the current sample used and not necessarily generalisable to the entire population with OA in SA. The low level of education in the current sample could have resulted in the discrepancy in SE scores. Correct understanding of the SE scale may have contributed to the values not following the normative scores. This is supported by a previous study stating patient reports of health differed according to educational levels ${ }^{(350)}$.

\section{ii. Change in personal factors over time}

Although benefits seen in personal aspects as a result of participating in the intervention are less than those for pain, function and disability, it seems that the intervention can affect personal factors to some extent. Long term benefits in personal factors favoured the experimental group as seen by less interference in mood and sleep according to the BPI PIS. As discussed in the section on pain, these psychological benefits were also confirmed by participants' responses to the open ended questionnaire. The intervention helped participants to form new positive attitudes, build self-confidence and enhanced their mood by participating in an intervention they enjoyed, despite still waiting for surgery. 
Health related quality of life measured on the EQ-5D disability index showed no significant improvement throughout the study. It appeared that at week six both groups had improved somewhat but began deteriorating from then onwards. None of these changes were large enough to be called significant. The apparent improvement at week six could be said to be due to the Hawthorne effect ${ }^{(332)}$ as both the intervention and control groups could have responded to the attention received in the first six weeks of the study, after being on a waiting list for a long period. Subsequently less attention was given to groups as the study continued with participants only seen at the next two follow up points at week 12 and month six.

The finding of no significant change in the EQ-5D index is in agreement with the study by Hurley et al. assessing the effects of the ESCAPE-knee integrated programme in a UK sample with a higher baseline mean EQ-5D score to that of the current sample ( $M=0.60$, range 0.57 - 0.63). Their sample showed no significant changes in EQ-5D index, in either of the arms of the trial at month six $(E S=-0.01, P=0.07)^{(272)}$. Similar findings are reported in Barlow et al.s' study whereby another UK sample, with a closer matched baseline mean to the participants in the present study $(M=0.43$, range $0.35-0.54)$, reported no significant improvements in EQ-5D index after an arthritis self-management intervention ${ }^{(235)}$. The current EQ-5D findings, together with prior results showing no significant improvements in HRQoL, supports the recent conclusion drawn on the responsiveness of the EQ- $5 D^{(351)}$. It has been suggested that the three level responses can cause floor and ceiling affects and this instrument is not sensitive enough to changes that may take place ${ }^{(351)}$. The EQ-5D index was described as a poor reflection of health status in a study published after the outcome measure was chosen for use in the current study ${ }^{(351)}$.

Similarly, no significant change was reported for the EQ-5D VAS in the current sample. This is also in agreement with Barlow et al.s' previous study ${ }^{(235)}$ evaluating the effects of the ASMP $^{(235)}$, whereby a sample was utilised with a similar mean baseline EQ-5D VAS (M = 56.78 , range $=51.8-62.9)$ when compared to the current study $(M=58.26, S D=20.46)$. Likewise no significant improvement was observed in the EQ-5D VAS at month six $(p=$ $0.74)^{(235)}$. 
However, an interesting finding was reported in the same sample; a significant improvement in EQ-5D VAS was evident at month $12(E S=0.28, p<0.01)^{(235)}$. This finding could suggest that self-reported state of health may too take a longer time to demonstrate a significant change in chronic pain conditions. Therefore a longer follow up of the current sample may have revealed similar changes.

In line with the current HRQoL findings, no significant improvements were reported for SE

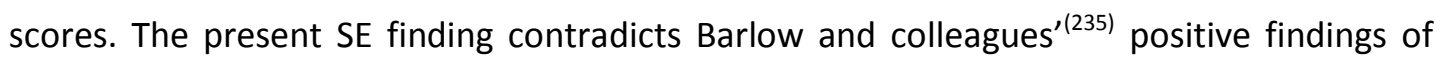
improved SE after a SMI in individuals with arthritis ${ }^{(235)}$. Results showed significant improvements in SE as measured by the ASE scale for pain and other symptoms at four months ( $p<0.01, E S=0.41$ and 0.43 respectively) as well as sustained long term benefits in the intervention group for pain and other symptom SE at month 12 ( $p<0.01, E S=0.35$ and 0.39 respectively). The findings of the current study also disagree with long term significant improvements in SE reported by Hurley et al. ${ }^{(271)}$ after a combined exercise and education intervention in knee conditions, where significant improvements in SE were still evident at 18 months post intervention $(p=0.01)$. Once again, Hurley and colleagues ${ }^{(271)}$ explain that by incorporating active exercise into the intervention participants were able to experience on a first hand basis exercise without pain aggravation. By integrating their positive experiences during exercise with advice and coping strategies, self-confidence can be restored in the ability to perform daily tasks ${ }^{(271)}$. However, even though the present intervention was based on these principles, SE was not significantly improved in the intervention group of the current study. This could be as a result of the SE scores being higher than expected at baseline, yet there was still room for improvement as scores were still lower than seven out of 10 . As mentioned above, a more likely explanation for the lack of change could be due misunderstanding of the instrument used to measure $\mathrm{SE}$, as a result of low educational levels in the current sample ${ }^{(350)}$. The RA also noted participants reported difficulties comprehending and completing this instrument at follow up meetings, despite translators being present. This leads the researchers to believe the outcome was not as appropriate for use in this particular sample as it has been in prior studies. 


\subsection{Body mass index}

\subsubsection{Baseline presentation of BMI}

As mentioned in Chapter Four (pg. 104) the average BMI in the current study at baseline was extremely high indicating the sample to be obese $(M=35.79, S D=8.81)$. Such a high $\mathrm{BMI}$ is a true representation of the growing obesity issue in South Africa ${ }^{(352)}$. The known association between obesity and $\mathrm{OA}^{(290)}$ is clearly illustrated by high $\mathrm{BMI}$ values in the present sample of persons with lower limb OA.

Furthermore BMI coupled with severe pain and disability at baseline, supports previous literature illustrating the detrimental cycle of obesity on the development of OA and the progression of pain and disability. $\mathrm{OA}$ is known to cause a less active lifestyle due to the associated impairments and lack of independence ${ }^{(50)}$. Sedentary behaviours can contribute towards further weight increases which in turn cause greater forces through the joints of the lower limbs and therefore more pain and disability and so the cycle continues ${ }^{(290)}$.

\section{i. $\quad$ Change in BMI}

It was no surprise that the present sample's BMI did not significantly improve over the six months as this was not the target of the intervention. Instead, an objective of the study was to observe whether any change in BMI affected changes seen in primary and secondary outcome measures. Previous research on weight loss in individuals with OA, particularly of the knee, was evaluated by means of a systematic review and metaanalysis ${ }^{(296)}$. Across five studies average age ranged from $63-69$ years and the maximum average weight loss was $6.7 \mathrm{~kg}$ over a period of six months. If weight loss was an aim of the present study, a much higher weight loss than $6.7 \mathrm{~kg}$ would be needed in order to change the mean BMI status of the sample from obese class II (>30) to overweight $(25-29.9)$.

However it has been previously stated that physical inactivity, not obesity, is the fourth leading cause of mortality globally ${ }^{(353)}$. Therefore it is not too concerning that BMI did not show a significant change over the six months, as more important changes that have previously been discussed, were evident in functional interference, physical function and activity levels in the current sample. 
This is extremely encouraging that in spite of high, unchanging BMI values in the present study, significant changes in pain and function, as well as improved disability and limited personal benefits, were reported in the study.

\subsection{Open-ended questionnaire responses}

As mentioned throughout the discussion, the open ended questionnaire provided valuable information regarding the participants' thoughts towards the intervention. All responses were positive reports of how participants thoroughly enjoyed the intervention and elaborated on the various benefits according to the four themes that emerged namely: knowledge, function/activity, pain relief and personal benefits. These findings strengthened the findings on the primary and secondary outcome measures used: the BPI, the walking tests of the physical performance task battery and the HAQ.

The theme of increased knowledge has not yet been discussed in relation to the significant findings in the present sample. Regardless of the fairly low educational levels, with the majority (28 of 42 ) of participants not completing schooling further than grade nine, the responses to the open ended questionnaire indicate that participants retained the knowledge that they learnt from the intervention over the six months. This is also supported by the long term significant improvements in pain and function observed in the intervention group when compared to the control group, as, if participants had not retained the knowledge they had learnt one would not expect the improvements that were most evident at month six. Additionally, it was reported by more than one participant that the intervention and booklet supplied during the study made it possible for the participants to share their newly attained knowledge with others in their daily lives (Appendix $Q$, pg. 413). Lastly, despite being on such a long waiting list with no indication of when surgery would be taking place in the future, a sample that was greatly affected by OA still responded positively to a combined intervention of exercise and education led by a physiotherapist. 


\subsection{Strengths and limitations of the study}

In all research, one is required to highlight the strengths and limitations of the study in order to fully understand the findings and how they may relate to the broader population and clinical practice. The CONSORT group have developed guidelines which specify what should be reported in a $\mathrm{RCT}^{(324)}$. These guidelines were used as part of the analysis of the strengths and weaknesses of the study (Appendix R, pg.417). First the strengths of the study are discussed in the text that follows below; this is followed by the analysis of limitations and their implications of interpreting the data.

\subsubsection{Strengths}

Firstly, the intervention design of exercise and education compared against a control group of usual care in the present study is viewed as a strength. According to Hurley and colleagues a safe, effective, accessible and affordable intervention that can be delivered to a large number of individuals is required ${ }^{(178)}$. The benefits of integrating exercise with education and self-management skills has previously been discussed in depth and appears to be more advantageous than isolated interventions in persons with OA.

The intervention utilised in the current study comprises two of the most commonly recommended treatment approaches to the management of $\mathrm{OA}$ by internationally accepted evidence-based guidelines; education and exercise ${ }^{(23-25,28,30,207,213,299)}$. The goal of the present study was to provide a patient centred approach to the ongoing management of individuals withOA on a long waiting list. This was achieved by basing the intervention on the ICF's understanding of health; targeting physiological impairments (pain, muscle weakness, joint stiffness, low fitness levels), personal factors (knowledge, health beliefs, self-efficacy, stress management) and behaviours during daily activities (avoidance of movement or exercise, eating and sleeping habits, goal setting) ${ }^{(21,271)}$. This intervention has the potential to meet all the aspects required to attempt managing this chronic noncommunicable disease, if supported and implemented in the South African public health system. 
Secondly, although the intervention's exercise component in the current study was general (aerobic, range of movement and strengthening exercises), specific exercise components were tailored to address individuals' needs throughout the study. This distinction in exercises varied depending on each participant's ability and progressed accordingly over time. Tailoring treatment reflects usual clinical practice and justifies the size of the group being small, thus allowing for individual attention. Such aspects improve the likelihood that such an intervention and its effects can be replicated in an outpatient setting ${ }^{(272)}$.

Besides the intervention design, the research setting where the intervention was delivered is also seen as a strength of the study. The suggestion has previously been made that research trials should be delivered in an outpatient setting as this allows for better implementation into clinical practice ${ }^{(276)}$. As mentioned in Chapter Two and Three, the current study was performed in a typical outpatient setting in a tertiary hospital; although if delivered in a primary health care setting it may be more accessible and affordable to those living in the surrounding communities. Some participants had to travel far distances to attend the study and if such an intervention is implemented for clinical use, transport costs would not be covered. This might result in lower adherence than was recorded for the present study as transport fees were subsidised.

The use of a group based intervention is supported by Hurley and colleagues' study which showed no differences between an individual or group based combined intervention for function or pain ${ }^{(272)}$. Due to the high numbers awaiting surgery in the Western Cape, an intervention that is delivered in a group rather than multiple one-on-one sessions with a physiotherapist is more practical to reach a large population. Furthermore, added benefits of utilising a group based intervention were noted in participants' response to the open ended questionnaire in terms of improved support and social benefits.

To ensure intra-rater reliability during the study, one research assistant measured outcomes at follow up meetings for participants, with the presence of the sametranslator throughout the study. To ensure the content and delivery of the intervention was the same for both small groups who received the intervention, one physiotherapist provided the intervention with the same translator at all sessions. 
Additionally delivery of such an intervention does not require lengthy training and the exercise and relaxation component does not involve expensive equipment or specific facilities, therefore replication of the intervention will be possible by other physiotherapists in the resource constrained SA health care system.

The use of the primary outcome measure to assess pain severity was shown to be valid in this South African sample, as it showed a strong positive correlation $(r=0.74, p<0.01)$ with the secondary outcome measure of pain severity as measured by the HAQ. This finding strengthens the results of the primary outcome measure used in the current study. Validity of the BPI in a South African population with OA on a long waiting list for a joint replacement has not been documented prior to this study.

Furthermore, the use of both a self-reported and objective measures of function is seen as another strength of the present study. It has been stated in literature that although the BPI gives valid information about the interference of pain on physical function, the scale provides no specific insight into where, when and how the difficulty in function is experienced $^{(174)}$. Hence, the BPI was the primary outcome measure of pain and pain related functional interference and secondary scales were used to assess function and disability. The self-reported HAQ was used to gain the participant's view of their functional abilities or disabilities, and the objective physical performance task battery was used to quantify functional ability. Multiple outcome measures were able to complement each other and strengthen findings as it is known that "performance tests do not duplicate self-reports of function $^{\prime(174)}$.

Despite the sample size appearing quite small $(N=42)$, the necessary calculations for a sufficiently powered sample were performed prior to the study commencing. As mentioned in Chapter Three (pg. 87) a sample of 36 participants was required to provide $90 \%$ statistical power for change in BPI scores between groups and 45 participants was required for $95 \%$ power. Therefore 42 participants providedbetween 90 and $95 \%$ power for the current study. 
The adequate sample size used to produce the significant differences in pain and function in the present sample disagrees with the majority of small underpowered samples reviewed in the SR by Walsh et al. on the effectiveness of combined interventions in hip and knee $O A^{(276)}$. Therefore the present study allows for generalizability of the sample's findings to the broader population of OA sufferers awaiting a joint replacement, in the Western Cape public health system. Concurrently, the same study was performed in the province of Gauteng in SA which can be used to further strengthen results if samples are combined to produce a larger sample size for SA. Additionally, findings of both provinces can be compared to establish whether the intervention brought about the same effects in two different regions in SA.

\subsubsection{Limitations}

Following on from the current study's strengths, several limitations of the study are also discussed. A double- blinded randomised controlled trial is considered the most valuable type of trial in human research ${ }^{(354)}$. However, according to Hughes at al..$^{(277)}$ it is impossible to undertake a double blinded RCT in a study involving an active participatory intervention versus a control group, as participants are aware they are part of the intervention arm of the trial instead of the control arm. This is applicable to the present study which allowed a single-blinded RCT to be performed. Since the participants were aware they were receiving the intervention self-reported outcome measures might reflect respondent bias for those in the intervention group. However, objective distance and timed tests were also used to evaluate responses to the intervention and a large effect size was recorded in the objective measure of walking ability during the $15 \mathrm{~m}$ fastest speed test. Researchers do not believe this limitation greatly affects the validity of self-reported results.

Importantly, this sample represented a low educational group attending a public facility in the Western Cape of SA. This is representative of the large portion of the previously disadvantaged SA population, who consequently also have low income levels and therefore attend public health facilities ${ }^{(68)}$. Caution should be applied as these findings might not be transferable to the private population, waiting shorter periods for surgery, with higher educational levels in SA. 
It was observed that despite efforts to recruit participants with less severe OA from the third priority waiting list, the participants who were selected displayed signs and symptoms of severe $O A$ at the time of the study. This is thought to be a consequence of the waiting list being so long. Additionally, it was presumed that the third priority list would present with a low number of co-morbidities, as research states that with increasing age and progression of the condition, the risk for development of co-morbidities also increases ${ }^{(87)}$. However this was not so as each participant had an average of 1.29 co-morbidities. Therefore, it is thought that the positive findings of the present study could be even more evident in a sample truly representative of earlier OA changes with less co-morbidity.

The response findings from the subgroup analysis should be interpreted with caution due to the samples in each subgroup being small. Despite encouraging results as seen by the significant differences in those with knee $\mathrm{OA}$, the power is not necessarily strong enough to draw clear inferences from these findings.

It is important to note that a few patients were lost to follow up and data was carried forward in these cases ( $n=6$ at different follow up times). Hurley et al. ${ }^{(271)}$ explains this is a common problem during research. This may have led to blunting of results and therefore could possibly explain why secondary measures of disability, functional abilities other than walking, HRQoL and SE were not significantly different when compared to the control group $^{(355)}$. A suggestion has previously been made to rather use "multilevel modeling and multiple imputation to generate robust predictions of the effect of missing data" ${ }^{\prime(355)}$. This approach will be explored when the data are pooled with the data from the trial being conducted in Gauteng.

A minor limitation is illustrated by the discrepancy between patient reported assistive device usage and RA observed or recorded assistive device usage at baseline $(p<0.01)$. It appeared that use of an assistive device was recorded by the RA as significantly lower than what participants initially reported on the demographic questionnaire. It is possible that this could be true for each follow up session after baseline measures yet this is unknown as participants did not report on their use of assistive devices after the initial session. The information recorded at weeks six, 12 and month six by the RA may not be a true reflection 
of normal daily assistive device usage in the participants' home environment. It is likely that participants portrayed themselves as less or more reliant on an assistive device during follow up sessions, depending on what message was being relayed to the RA. Similar discrepancies in patient reports have been previously documented whereby pain responses and associated disability is influenced by social and environmental context ${ }^{(356)}$. Therefore whether true assistive device usage during the six months was accurately reflected is questionable.

After results of the present study showed no significant changes in HRQoL and SE scores for either group at any time, the researchers questioned whether the instruments used to evaluate these outcomes were in fact sensitive enough to detect change in the current sample. Despite both being widely used in research, the EQ-5D and the Self-Efficacy for Managing Chronic Disease 6-Item Scale are generic outcome measures used in a variety of conditions. As mentioned in Chapter Two (pg. 32), the psychometric properties of SE scale used in the present study have previously been questioned around its appropriateness for condition specific use; yet these doubts were not deemed worthy enough to only utilise the instrument as a generic outcome ${ }^{(194)}$. The current findings seem to support the previous uncertainties regarding use of the instrument in condition or task specific cases ${ }^{(194)}$. Since the present study commenced, a study providing psychometric properties for the condition specific English SE scale (ASES-8) has been published ${ }^{(357)}$. Validity and reliability of the ASES8 is now available and therefore a recommendation for use of this instrument is made, as it could be more sensitive to detecting treatment responses in a sample so greatly affected by OA.

As mentioned earlier in the discussion, critique has previously been put forward on the three level version of the EQ-5D used in the current study; whereby authors argue that levels of response (e.g., no problems, some problems, severe problems) are not sufficient to detect more subtle changes in health related quality of life ${ }^{(351)}$. Use of the five level EQ5D (no problems, slight problems, moderate problems, severe problems, and extreme problems) may have provided a more accurate reflection of the HRQoL for the sample used. South African studies of the EQ-5D have also found that the instrument "does not capture all those elements of HRQoL that are important to the population under 
study..."(319). Furthermore, despite rigorous translation processes for different SA languages, it was stated that further validation studies are needed for the isiXhosa version. Criticism regarding the appropriateness of the category domains used was raised as meanings behind concepts of "anxiety/depression" differ in the isiXhosa culture ${ }^{(349)}$.

Important to note is that the EQ-5D was not validated specifically for apopulation with OA either. However, the EQ-5D was not the primary outcome measure therefore use of this instrument was chosen as it has been described as quick and easy to administer in chronic conditions. Perhaps an OA-specific instrument for HRQoL, such as the full WOMAC, even though it is more time consuming to complete, may be more appropriate to detect change in a SA sample, including isiXhosa individuals with OA. Additional recommendations for future research in this field are noted below and draw the discussion section of this study to a close.

\subsection{Further recommendations}

While various self-report functional outcomes have been used to assess function following an integrated programme, the most commonly used is the WOMAC scale of function $^{(73,264,271,272,279,358)}$. Dworkin et al. ${ }^{(121)}$ suggest that the BPI interference scale can be used for function assessment unless a validated disease specific tool is available. Since the WOMAC functional scale is specific to $O A$ and frequently used in research, it may be a useful addition to provide data to strengthen the results of improved functional interference. Additionally, Brazier et al. found the WOMAC functional scale to be more valid and responsive to change when compared to the patient reported $\mathrm{HAQ}^{(192)}$. Therefore, even though the HAQ was developed for the use in arthritis conditions, future research could consider replacing the HAQ with the OA specific WOMAC functional scale. However, the reliability and validity of the WOMAC in a South African context and OA population would need to be established.

It is observed that in previous studies, objective measures of daily functional activities have not been utilised as much as self-reported instruments. 
Some studies have made use of walking tests and repeated sit - stand tasks ${ }^{(264,276,277,359)}$, as well as stair climbing ${ }^{(264)}$ and a series of tasks making up a battery of tasks ${ }^{(281)}$. It was stated in Chapter Three (pg. 87) that the content validity of the physical performance task battery would be explored as a part of the present study. Notably, the walking tests were important determinants of treatment response in this sample, yet sit-stand and the sock test were not helpful in ascertaining the effects of such an intervention on physical functioning. Despite the forward and upwards reach tests predominantly involved upper limb functioning, it was presumed that lower limb components during balance may be affected by the intervention and therefore was included as a part of the physical battery. However, no treatment response was observed throughout the study in either group for either reach test and therefore these two tests were deemed unnecessary in a sample affected by lower limb OA.

As mentioned in Chapter Two (pg. 27), the aggregate functional performance time (AFPT) obtained from a series of four daily tasks ${ }^{(172)}$ is similar to the physical performance task battery. However it combines times for the four functions to produce an aggregated score of function. This outcome could be used in future research to possibly give a broader result of functional performance for those with lower limb OA, during daily tasks of walking, rising from a chair and stair climbing. Hurley et al. states that performance in a single task test does not provide accurate information about functional ability in another task and therefore a general functional score could be useful ${ }^{(172)}$. This measure has been used previously in the integrated ESCAPE trials by Hurley et al. ${ }^{(264,271,272)}$ showing significant improvements in function after six months $(p=0.02, E S=0.17)^{(272)}$. Validation of the AFPT in $\mathrm{OA}$ against another objective functional measure such as the physical performance task battery and self-reported measures of function and functional interference used in the present study is recommended.

Another recommendation regarding instrumentation is made for further research into this field. Although the relaxation component of the present study may have contributed towards the benefits reported in the current sample, no specific outcome measure was utilised to establish whether this adjunct to the exercise and education components was useful or necessary. 
An additional open-ended question concerning the relaxation component could be a simple way of gaining insight into this aspect of the intervention. Future studies should consider whether an instrument evaluating the effects of the relaxation component is worthwhile.

Continuing on from the above concerning instrumentation; this study's aim was not to remove the patient from the waiting list and therefore did not assess whether the intervention could result in participants choosing to remove their name from the waiting list. While avoidance of surgery was not used in the current study, it could be a valuable outcome to monitor in future studies. This could substantially add to the evidence-based research in this field if such an intervention has the potential to improve a patient's symptoms efficiently enough as to no longer require surgery.

Furthermore, monitoring of adherence and compliance to the principles learnt in the intervention was not included in this initial study. However, recording whether or not participants incorporated principles such as goal setting, pain management, coping skills, daily exercises and relaxation techniques etc. into their lifestyles may be of benefit. It is recommended that monitoring of adherence could be done during the six week intervention as well as during follow up periods. This could provide valuable insight into whether the sustained improvements seen in the study were due to the participants adhering to cognitive behavioural changes, as a result of the intervention.

As mentioned earlier in the discussion, once results were analysed for the six month period and compared with previous research, the current researchers propose that future trials extend the follow up period to at least 12 months. Walsh et al. states that long lasting benefits are especially needed in persons on a long surgical waiting list ${ }^{(276)}$. This will provide valuable comparative data whereby the current findings can further support or contradict prior long term research concerning persons with OA awaiting surgery.

Previous research has shown that pre-operative personal and psychological aspects have effects on post-operative outcomes in persons with $\mathrm{OA}^{(15)}$. Despite no significant change in HRQoL and SE in the current sample, the significant improvements in mood and sleep 
interference, as well as the positive responses towards the intervention as seen by the open ended questionnaire are not to be overlooked. If a short term intervention such as the one used in the present study, can bring about certain personal or psychological benefits, then it is thought that a combined exercise and education intervention could possibly have carry over effects post-operatively too. Therefore recommendation is made for research whereby participants are also followed up after they have received a joint replacement to confirm this assumption. According to de Jong et al. "Implementation studies are recommended to assess the feasibility and effectiveness in real-life of programmes which have been tested in randomized controlled trials"(331). Following this statement, it was reasoned that more than a single RCT is necessary to implement an effective intervention into any health care system ${ }^{(360)}$. Therefore it is recommend that further RCTs be performed replicating the present intervention, together with the suggestions discussed above in order to confirm the effectiveness of such an integrated intervention in the SA public health facilities. 


\section{Conclusion}

The rationale behind undertaking the present study was primarily due to the rising burden of osteoarthritis ${ }^{(14,15)}$ and delays in receiving surgery as a result of long waiting lists ${ }^{(14,31)}$. As mentioned in Chapter One, OA is one of the leading causes of pain and disability worldwide ${ }^{(19,20)}$. As a consequence of the expected increase in aging of the population and thus an increase in prevalence of the condition, effective management is essential at all stages of OA to minimise burden on an already stressed health care system ${ }^{(21,22)}$.

It is widely accepted that late stage $\mathrm{OA}$ requires surgical management by means of a joint replacement ${ }^{(19,24,29)}$. Yet a common issue in local and international health systems is restricted access to this elective surgery ${ }^{(50)}$. Relatively short waiting lists for a joint replacement have been reported for high income countries, whereas longer waiting times of a few years are experienced in lower income countries, such as South Africa ${ }^{(31,32)}$. As a consequence of $\mathrm{OA}$ being a progressive condition, increases in pain and deterioration in functional ability are common as time goes by ${ }^{(75,76)}$. Understandably so, research has shown debilitating effects of waiting for surgery without appropriate management in the interim $^{(33,34)}$.

To address this issue, particularly in the Western Cape of SA, it was proposed that providing evidence-based first-line treatment options for OA could ease the burden for patients awaiting an arthroplasty. To the researchers' knowledge, this is the first RCT to be performed in individuals with $\mathrm{OA}$ comparing an integrated exercise and education intervention to usual care, while on a waiting list in a South African context. Previous research has reported on the varying effects of exercise in $O A^{(266-268)}$, education in $O A^{(226,227)}$ and self-management in $\mathrm{OA}^{(237,249,258)}$, mostly in higher income countries. More recent work $^{(271,272,277-279)}$ has shown the positive effects of combining these approaches in one way or another in the management of OA. However, the effects of an integrated intervention have not been evaluated in the South African population with OA. Therefore this study aimed to evaluate the effects of a combined exercise and education intervention in a population of individuals affected by OA awaiting a joint replacement. Conclusions are drawn below on each specific objective of the present study. 
To determine, whether participating in a six-week physiotherapist-led exercise and education intervention resulted in a significant change in...pain severity and pain interference...

The findings of the primary outcome showed that participating in a combined exercise and education intervention was able to cause distinct changes in both pain severity and pain interference. The most meaningful difference was observed in pain interference at month six in participants who received the intervention. Despite commencing the study with severe levels of pain, the participants were able to enjoy the intervention and reported apparent reductions in pain intensity and less interference with physical and emotional functioning after completing the six-week intervention. Remarkably pain severity was reduced throughout the follow up period in those who took part in the intervention compared to previous pain ratings. Furthermore, at month six the extent to which pain interfered in daily life showed the largest difference when compared to the control group whose pain interference did not show any significant change. It is astounding that a short six-week intervention can markedly reduce pain severity and interference in individuals who have been experiencing chronic pain for many years.

To determine, whether participating in a six-week physiotherapist-led exercise and education intervention resulted in a significant change in...function...

The results for the objective functional performance tests demonstrated that partaking in this intervention caused sustained improvements in function; specifically walking ability. It was observed that many participants showed physical signs of difficultly during walking; with the majority making use of a walking aid to assist with this daily function. The main difference was again noted at month six whereby the participants who received the intervention were able to walk significantly faster when compared to the control group. Additionally, those in the experimental group were able to walk significantly further than prior to participating in the intervention. Both objective walking scores $(15 \mathrm{~m}$ fastest speed walk and six minute walk test) showed evident improvements during the study duration without reaching a plateau. Whether time and distance scores could possibly further improve would be useful information if participants are followed up for longer durations. 
This intervention made it possible for participants who have been struggling for years with walking and activities requiring the ability to walk, to function at a better level in their daily lives.

To determine, whether participating in a six-week physiotherapist-led exercise and education intervention resulted in a significant change in...disability...

The findings from the self-reported disability outcome indicated that such an intervention appears to have resulted in improvements in disability. The results illustrated that despite participants presenting with severe disability after being on a waiting list for a long time, those who received the intervention displayed significantly reduced disability compared to disability scores prior to the intervention. This reduction in disability was evident directly after the six week intervention and was similarly maintained at month six. For these individuals awaiting surgery, the burden of OA can cause a loss of independence and in many cases a person's purpose. Remarkably, it seems that an exercise and education intervention, led by a physiotherapist can begin to reverse the effects of disability.

To determine, whether participating in a six-week physiotherapist-led exercise and education intervention resulted in a significant change in...self-efficacy...

Self-efficacy results for this sample proved interesting, as it was noted that in spite of immense pain, functional difficulties and disability, the sample did not display the low levels of SE anticipated at the beginning of the study. Furthermore, the results implied that the six-week physiotherapist led intervention did not cause any significant effect on SE. Since SE was not the primary focus of this study, the results do not discourage the use of this intervention for clinical practice or future trials. As discussed in Chapter Five, these unexpected results could be due to misunderstanding of the instrument used to measure this outcome and further studies should consider an OA-specific outcome to measure SE. 
To determine, whether participating in a six-week physiotherapist-led exercise and education intervention resulted in a significant change in...health related quality of life...

Similarly, the findings of the last outcome used suggested that the exercise and education intervention did not produce significant changes in health related quality of life. Again this was not the primary outcome of the study and the findings could be due to choice of tool used to evaluate HRQoL. However, a more appropriate assessment of HRQoL following an intervention in future trials could be achieved if the generic instrument was replaced with a tool specific to $O A$.

To determine whether body mass index...accounted for any significant differences in the above outcome measures.

The extremely high values calculated for BMI for the present sample correctly depicts the obesity concern experienced in both low and high income countries. Despite small amounts of weight loss in a few participants who received the intervention, BMI did not account for any differences in the above outcomes as the BMI for either group remained constant throughout the study.

To determine whether...OA of the hip or knee or a combination of hip and knee OA... accounted for any significant differences in the above outcome measures.

Although the subgroups consisted of small numbers, analysis according to OA of the hip, knee or combined hip and knee OA provided substantial information regarding the treatment responses of each group. It appears that the subgroups did account for differences in the primary and secondary outcome measures. The differences accounted for by the group with OA of the knee only were most obvious. 
Findings showed that those participants with knee OA improved far more than those with OA of the hip only or those with a combination of hip and knee OA. This significant difference was particularly observed in pain interference and pain severity scores according to both the BPI and HAQ pain VAS. These results are particularly important to consider when planning further trials in this field. Subgroup findings are also useful when implementing this study into clinical practice, as targeting persons with OA of the knee may result in better treatment effects, instead of delivering an intervention to a individuals with hip OA only who might respond poorly to the management.

Finally, to determine the acceptability of a six-week physiotherapist-led exercise and education intervention for patients on the waiting list for joint arthroplasty.

Based on the various positive responses to the open-ended questionnaire by the participants involved in the current study, such an intervention is considered to be acceptable.

It was surprising that persons from the third priority waiting list would present at baseline with such a poor depiction of health as in the current sample. Yet as a result of being on the waiting list for such along duration of time, the third priority label given when initially assessed for a joint replacement was no longer appropriate; thus the baseline presentation is better understood. Despite high levels of pain severity, pain interference and disability across the sample, the exercise and education intervention was most effective in significantly reducing levels of pain interference as well as significantly improving functional ability during walking. Furthermore, it was noted that the intervention favoured those participants with knee OA as seen by significant differences in pain interference and pain severity on both pain scales when compared to the control group.

Thousands of individuals in the Western Cape are suffering from the burden of OA for far too long while awaiting surgery. This study provides an acceptable and effective evidencebased short term non-pharmacological and non-surgical intervention that can be easily delivered by a physiotherapist. 
The intervention has the ability to reduce this population's suffering by particularly improving long term pain and function. An integrated intervention of exercise and education is beneficial and should be urgently implemented for people with OA receiving treatment at public health facilities in South Africa. 


\section{References}

(1) Martin EA. Oxford Concise Medical Dictionary. 8th ed ed.: Oxford University Press; 2010.

(2) Latremoliere A, Woolf CJ. Central sensitization: a generator of pain hypersensitivity by central neural plasticity. J Pain 2009 Sep;10(9):895-926.

(3) MedicineNet. Definition of Assistive Device. 2013; Available at: http://www.medterms.com/script/main/art.asp?articlekey=2372. Accessed August 31, 2014.

(4) Bonica JJ. The management of pain. Philadelphia: Lippincott; 1953.

(5) Business Dictionary. Delphi technique. 2014; Available http://www.businessdictionary.com/definition/Delphi-technique.html. Accessed August 31, 2014.

(6) Lethem J, Slade PD, Troup JD, Bentley G. Outline of a Fear-Avoidance Model of exaggerated pain perception-I. Behav Res Ther 1983;21(4):401-408.

(7) Altman R, Alarcon G, Appelrouth D, Bloch D, Borenstein D, Brandt K, et al. The American College of Rheumatology criteria for the classification and reporting of osteoarthritis of the hip. Arthritis Rheum 1991 May;34(5):505-514.

(8) Loeser JD, Treede RD. The Kyoto protocol of IASP Basic Pain Terminology. Pain 2008 Jul 31;137(3):473-477.

(9) Cleeland CS. Measurement of pain by subjective report. In: Chapman CR, Loeser JD, editors. Issues in Pain Measurement New York: Raven Press; 1989. p. 391-403.

(10) The Presidency department: National planning commission. National Development Plan 2030:Our future make it work. 2012.

(11) Lorig KR, Holman HR. Self-Management Education: History, Definition, Outcomes, and Mechanisms. Annals of Behavioral Medicine 2003 07;26(1):1.

(12) Bandura A. Self-efficacy: The exercise of control. New York: Freeman.; 1997.

(13) Peat G, McCarney R, Croft P. Knee pain and osteoarthritis in older adults: a review of community burden and current use of primary health care. Ann Rheum Dis 2001 Feb;60(2):91-97.

(14) Mascarenhas R. The Manitoba arthroplasty waiting list: impact on health-related quality of life and initiatives to remedy the problem. J Eval Clin Pract 2009 Feb;15(1):208-211.

(15) Ackerman IN, Bennell KL, Osborne RH. Decline in Health-Related Quality of Life reported by more than half of those waiting for joint replacement surgery: a prospective cohort study. BMC Musculoskelet Disord 2011 May 23;12:108-2474-12-108.

(16) Lorig K, Holman HR. Long-term outcomes of an arthritis self-management study: effects of reinforcement efforts. Soc Sci Med 1989;29(2):221-224.

(17) Badley EM, Rasooly I, Webster GK. Relative importance of musculoskeletal disorders as a cause of chronic health problems, disability, and health care utilization: findings from the 1990 Ontario Health Survey. J Rheumatol 1994 03;21(3):505-514.

(18) March LM, Bachmeier CJ. Economics of osteoarthritis: a global perspective. Baillieres Clin Rheumatol 1997 $11 ; 11(4): 817-834$

(19) Mody GM, Woolf AD. The Global Burden of Musculoskeletal Disorders. Business briefing: European Pharmacotherapy 2003:1-5.

(20) Anita YN, Doherty M. What of guidelines for osteoarthritis? International Journal of Rheumatic Diseases 2011;14:136-144.

(21) Hurley MV, Mitchell HL, Walsh N. In osteoarthritis, the psychosocial benefits of exercise are as important as physiological improvements. Exerc Sport Sci Rev 2003 Jul;31(3):138-143. 
(22) Goldenberg DL, Clauw DJ, Fitzcharles MA. New concepts in pain research and pain management of the rheumatic diseases. Semin Arthritis Rheum 2011 Dec;41(3):319-334.

(23) Jordan KM, Arden NK, Doherty M, Bannwarth B, Bijlsma JW, Dieppe P, et al. EULAR Recommendations 2003: an evidence based approach to the management of knee osteoarthritis: Report of a Task Force of the Standing Committee for International Clinical Studies Including Therapeutic Trials (ESCISIT). Ann Rheum Dis 2003 Dec;62(12):1145-1155.

(24) Zhang W, Doherty M, Arden N, Bannwarth B, Bijlsma J, Gunther K, et al. EULAR evidence based recommendations for the management of hip osteoarthritis: report of a task force of the EULAR Standing Committee for International Clinical Studies Including Therapeutics (ESCISIT). Ann Rheum Dis 2005 05;64(5):669-681.

(25) Zhang W, Moskowitz RW, Nuki G, Abramson S, Altman RD, Arden N, et al. OARSI recommendations for the management of hip and knee osteoarthritis, Part II: OARSI evidence-based, expert consensus guidelines. Osteoarthritis Cartilage 2008 Feb;16(2):137-162.

(26) Zhang W, Nuki G, Moskowitz RW, Abramson S, Altman RD, Arden NK, et al. OARSI recommendations for the management of hip and knee osteoarthritis: part III: Changes in evidence following systematic cumulative update of research published through January 2009. Osteoarthritis Cartilage 2010 Apr;18(4):476-499.

(27) Conaghan PG, Dickson J, Grant RL, Guideline Development Group. Care and management of osteoarthritis in adults: summary of NICE guidance. BMJ 2008 Mar 1;336(7642):502-503.

(28) Nelson AE, Allen KD, Golightly YM, Goode AP, Jordan JM. A systematic review of recommendations and guidelines for the management of osteoarthritis: The Chronic Osteoarthritis Management Initiative of the U.S. Bone and Joint Initiative. Semin Arthritis Rheum 2013 Dec 4.

(29) Roos EM, Juhl CB. Osteoarthritis 2012 year in review: rehabilitation and outcomes. Osteoarthritis Cartilage 2012 12;20(12):1477-1483.

(30) Bruyere O, Cooper C, Pelletier JP, Branco J, Luisa Brandi M, Guillemin F, et al. An algorithm recommendation for the management of knee osteoarthritis in Europe and internationally: A report from a task force of the European Society for Clinical and Economic Aspects of Osteoporosis and Osteoarthritis (ESCEO). Semin Arthritis Rheum 2014 May 14.

(31) Western Cape Government media release [online]. Analysis of service pressures at health facilities across the province. 2014; Available at: http://www.westerncape.gov.za/news/analysis-service-pressures-healthfacilities-across-province. Accessed February, 21, 2014.

(32) Cloete S. Operasies kry databasis. Die Burger 201420 November.

(33) Ostendorf M, Buskens E, van Stel H, Schrijvers A, Marting L, Dhert W, et al. Waiting for total hip arthroplasty: avoidable loss in quality time and preventable deterioration. J Arthroplasty 2004 Apr;19(3):302309.

(34) Desmeules F, Dionne CE, Belzile E, Bourbonnais R, Fremont P. The burden of wait for knee replacement surgery: effects on pain, function and health-related quality of life at the time of surgery. Rheumatology (Oxford) 2010 May;49(5):945-954.

(35) Cleeland CS, Ryan KM. Pain assessment: global use of the Brief Pain Inventory. Ann Acad Med Singapore 1994 Mar;23(2):129-138.

(36) Simmonds MJ. Physical function in patients with cancer: psychometric characteristics and clinical usefulness of a physical performance test battery. J Pain Symptom Manage 2002 Oct;24(4):404-414.

(37) Bruce B, Fries JF. The Stanford Health Assessment Questionnaire: dimensions and practical applications. Health Qual Life Outcomes 2003 Jun 9;1:20.

(38) Lorig K, Stewart A, Ritter P, González V, Laurent D, Lynch J. Outcome Measures for Health Education and other Health Care Interventions. Thousand Oaks CA: Sage Publications; 1996. p. 24-25-41-45.

(39) The EuroQol Group. EuroQol-a new facility for the measurement of health-related quality of life. Health Policy 1990;16(3):199-298.

(40) Messier SP. Obesity and osteoarthritis: disease genesis and nonpharmacologic weight management. Med Clin North Am 2009 Jan;93(1):145-59, xi-xii.

(41) World Health Organisation. The Global Burden of Disease: 2004 Update. 2008 Geneva. 
(42) WHO Scientific Group on the Burden of Musculoskeletal Conditions at the Start of the New Millennium. The burden of musculoskeletal conditions at the start of the new millennium. World Health Organ Tech Rep Ser 2003;919:i-x, 1-218, back cover.

(43) Woolf AD. The Bone and Joint Decade 2000-2010 Ann Rheum Dis 2000;59:81-82.

(44) Murray CJ, Lopez AD. Global Burden of Disease and Injury Series Global health statistics. Vol II. ed. Geneva: Harvard School of Public Health, World Bank, and World Health Organization; 1996.

(45) Murray CJ, Lopez AD. Alternative projections of mortality and disability by cause 1990-2020: Global Burden of Disease Study. Lancet 1997 May 24;349(9064):1498-1504.

(46) Mayosi BM, Flisher AJ, Lalloo UG, Sitas F, Tollman SM, Bradshaw D. The burden of non-communicable diseases in South Africa. Lancet 2009 Sep 12;374(9693):934-947.

(47) Dawson J, Linsell L, Zondervan K, Rose P, Randall T, Carr A, et al. Epidemiology of hip and knee pain and its impact on overall health status in older adults. Rheumatology (Oxford) 2004 Apr;43(4):497-504.

(48) Dunlop DD, Lyons JS, Manheim LM, Song J, Chang RW. Arthritis and heart disease as risk factors for major depression: the role of functional limitation. Med Care 2004 Jun;42(6):502-511.

(49) Dreinhofer K, Stucki G, Ewert T, Huber E, Ebenbichler G, Gutenbrunner C, et al. ICF Core Sets for osteoarthritis. J Rehabil Med 2004 Jul;(44 Suppl)(44 Suppl):75-80.

(50) Woolf AD, Pfleger B. Burden of major musculoskeletal conditions. Bull World Health Organ 2003;81(9):646656.

(51) Brooks PM. The burden of musculoskeletal disease--a global perspective. Clin Rheumatol 2006 Nov;25(6):778-781.

(52) CERNIAUSKAITE M, QUINTAS R, BOLDT C, RAGGI A, CIEZA A, BICKENBACH JE, et al. Systematic literature review on ICF from 2001 to 2009: its use, implementation and operationalisation. Disability \& Rehabilitation 2011 02/15;33(4):281-309.

(53) Dahl TH. International classification of functioning, disability and health: an introduction and discussion of its potential impact on rehabilitation services and research. Journal of rehabilitation medicine : official journal of the UEMS European Board of Physical and Rehabilitation Medicine JID - 1010881692002.

(54) Verbrugge LM, Jette AM. The disablement process. Soc Sci Med 1994 01;38(1):1-14.

(55) Huber JG, Sillick J, Skarakis-Doyle E. Personal perception and personal factors: incorporating health-related quality of life into the International Classification of Functioning, Disability and Health. Disabil Rehabil 2010;32(23):1955-1965.

(56) World Health Organization . International Classification of Functioning, Disability, and Health (ICF).; 2001; Geneva: World Health Organisation; 2001.

(57) World Health Organisation. The International Classifications of Impairments, Disabilities and Handicaps - a Manual Relating to the Consequences of Disease.; 1980; Geneva: World Health Organisation; 1980.

(58) FOUGEYROLLAS P,et al. Quebec classification: disability creation process. : International Network on the Disability Creation Process INDCP] | International Network on the Disability Creation Process INDCP/CSICIDH], PO Box 225, Lac St-Charles, Quebec, G3G 3C1, Canada; 1999.

(59) Stucki G. International Classification of Functioning, Disability, and Health (ICF): a promising framework and classification for rehabilitation medicine. Am J Phys Med Rehabil 2005 10;84(10):733-740.

(60) Stucki G, Ewert T. How to assess the impact of arthritis on the individual patient: the WHO ICF. Ann Rheum Dis 2005 May;64(5):664-668.

(61) Bornbaum CC, Doyle PC, Skarakis-Doyle E, Theurer JA. A critical exploration of the International Classification of Functioning, Disability, and Health (ICF) framework from the perspective of oncology: recommendations for revision. J Multidiscip Healthc 2013;6:75-86.

(62) Hammel KW. Deviating from the Norm: A Sceptical Interrogation of the Classifactory Practices of the ICF. The British Journal of Occupational Therapy 2004;67(9):408-411.

(63) Bradshaw D, Groenewald P, Laubscher R, Nannan N, Nojilana B, Norman R, et al. Initial burden of disease estimates for South Africa, 2000. S Afr Med J 2003 Sep;93(9):682-688. 
(64) Lehohla P. Poverty Trends in South Africa: An examination of absolute poverty between 2006 and 2011. Pretoria: Statistics South Africa; 2014.

(65) Statistics South Africa. Income and expenditure of households 2005/2006: analysis of results. Pretoria: Statistics South Africa; 2008.

(66) Coovadia H, Jewkes R, Barron P, Sanders D, Mclntyre D. The health and health system of South Africa: historical roots of current public health challenges. Lancet 2009 Sep 5;374(9692):817-834.

(67) Lehohla P. The use of health facilities and levels of selected health conditions in South Africa: Findings from the General Household Survey, 2011. Statistics South Africa 2013;03 - 00 - 05 (2011).

(68) Mayosi BM, Lawn JE, van Niekerk A, Bradshaw D, Abdool Karim SS, Coovadia HM, et al. Health in South Africa: changes and challenges since 2009. Lancet 2012 Dec 8;380(9858):2029-2043.

(69) Strategic Plan for the Prevention and Control of Non-Communicable Diseases 2013-17. 2011;RP06(ISBN: 978-0-621-41510-0):37.

(70) Aiken $A B$, Harrison MM, Atkinson M, Hope J. Easing the burden for joint replacement wait times: the role of the expanded practice physiotherapist. Healthc Q 2008;11(2):62-66.

(71) McHugh GA, Luker KA, Campbell M, Kay PR, Silman AJ. Pain, physical functioning and quality of life of individuals awaiting total joint replacement: a longitudinal study Journal of Evaluation in Clinical Practice ISSN 2008;14:19-26.

(72) Derrett S, Paul C, Morris JM. Waiting for elective surgery: effects on health-related quality of life. Int J Qual Health Care 1999 Feb;11(1):47-57.

(73) Wallis JA, Taylor NF. Pre-operative interventions (non-surgical and non-pharmacological) for patients with hip or knee osteoarthritis awaiting joint replacement surgery--a systematic review and meta-analysis. Osteoarthritis Cartilage 2011 Dec;19(12):1381-1395.

(74) Gill SD, McBurney H. Does exercise reduce pain and improve physical function before hip or knee replacement surgery? A systematic review and meta-analysis of randomized controlled trials. Arch Phys Med Rehabil 2013 Jan;94(1):164-176.

(75) World Health Organization. Chronic diseases and health promotion. Chronic rheumatic conditions fact sheet. September 2008; Available at: http://www.who.int/chp/topics/rheumatic/en/. Accessed 09/24, 2012.

(76) Fransen M, McConnell S. Exercise for osteoarthritis of the knee. Cochrane Database Syst Rev 2008 Oct 8;(4):CD004376. doi(4):CD004376.

(77) Arthritis Foundation. Osteoarthritis: What is it? 2013; Available at: http://www.arthritis.org/diseasecenter.php?disease id=32. Accessed 05/21, 2013.

(78) Sinusas K. Osteoarthritis: Diagnosis and Treatment. . Am Fam Physician 2012;85(1):49-56.

(79) Zhang Y, Jordan JM. Epidemiology of osteoarthritis. Clin Geriatr Med 2010 Aug;26(3):355-369.

(80) National Collaborating Centre for Chronic Conditions (UK). 2008.

(81) Hannan MT, Felson DT, Pincus T. Analysis of the discordance between radiographic changes and knee pain in osteoarthritis of the knee. J Rheumatol 2000 Jun;27(6):1513-1517.

(82) Kellgren J, Lawrence J. Atlas of standard radiographs. The epidemiology of chronic rheumatism. Vol. 2 ed. Oxford: Blackwell Scientific Publications; 1963.

(83) Murray CIL, Lopez AD editors. The Global Burden of Disease. A Comprehensive Assessment of Mortality and Disability from Diseases, Injuries and Risk Factors in 1990 and Projected to 2020. Harvard School of Public Health on behalf of the WHO and the World Bank ed. Cambridge: Harvard University Press; 1996.

(84) Murray CJL, Lopez AD. Global and regional descriptive epidemiology of disability; Incidence, prevalence, health expectancies and years lived with a disability. In: Murray CJL, Lopez AD, editors. The Global Burden of Disease. 1st ed. Cambridge: Harvard School of Public Health; 1996. p. 201-246.

(85) Hurley MV, Walsh NE, Mitchell HL, Pimm TJ, Williamson E, Jones RH, et al. Economic evaluation of a rehabilitation program integrating exercise, self-management, and active coping strategies for chronic knee pain. Arthritis Rheum 2007 Oct 15;57(7):1220-1229. 
(86) Lawrence RC, Felson DT, Helmick CG, Arnold LM, Choi H, Deyo RA, et al. Estimates of the prevalence of arthritis and other rheumatic conditions in the United States: part II. Arthritis Rheum 2008;58(1):26-35.

(87) Parker R, Jelsma J. The prevalence and functional impact of musculoskeletal conditions amongst clients of a primary health care facility in an under-resourced area of Cape Town. BMC Musculoskelet Disord 2010 01/04;11:2-2.

(88) Tuominen U, Blom M, Hirvonen J, Seitsalo S, Lehto M, Paavolainen P, et al. The effect of co-morbidities on health-related quality of life in patients placed on the waiting list for total joint replacement. Health Qual Life Outcomes 2007 Mar 15;5:16.

(89) Brown GM, Dare CM, Smith PR, Meyers OL. Important problems identified by patients with chronic arthritis. S Afr Med J 1987 07/18;72(2):126-128.

(90) Department of Health: Chronic Diseases, Disabilities and Geriatrics. Arthritis: South Africa's leading disabling disease. 24 April 1998; Available at: http://www.doh.gov.za/show.php?id=81. Accessed 09/24, 2012

(91) Roddy E, Zhang W, Doherty M. Aerobic walking or strengthening exercise for osteoarthritis of the knee? A systematic review. Ann Rheum Dis 2005 Apr;64(4):544-548.

(92) Nolte S, Osborne RH. A systematic review of outcomes of chronic disease self-management interventions. Qual Life Res 2012 Oct 31.

(93) LeFort SM, Gray-Donald K, Rowat KM, Jeans ME. Randomized controlled trial of a community-based psychoeducation program for the self-management of chronic pain. Pain 1998 02;74(2-3):297-306.

(94) Reginster JY. The prevalence and burden of arthritis. Rheumatology (Oxford) 2002 04;41 Supp 1:3-6.

(95) Enohumah KO. Pain in osteoarthritis. African Journal of Biomedical Research 2008 01/01;11(2):12-12.

(96) Pain terms: a list with definitions and notes on usage. Recommended by the IASP Subcommittee on Taxonomy. Pain 1979 Jun;6(3):249.

(97) Loeser JD. IASP Taxonomy. 2012; Available at: http://www.iasppain.org/Content/NavigationMenu/GeneralResourceLinks/PainDefinitions/default.htm. Accessed January, 05, 2014.

(98) Pharmacological management of persistent pain in older persons. J Am Geriatr Soc 2009 08;57(8):13311346.

(99) Woolf CJ. Evidence for a central component of post-injury pain hypersensitivity. Nature 1983 Dec 1521;306(5944):686-688.

(100) Arendt-Nielsen L, Fernández-de-las-Peñas C, Graven-Nielsen T. Basic aspects of musculoskeletal pain: from acute to chronic pain. J MANUAL MANIPULATIVE THER (MANEY PUBL) 2011 11;19(4):186-193.

(101) Klein T, Magerl W, Hopf H, Sandkühler J, Treede R. Perceptual correlates of nociceptive long-term potentiation and long-term depression in humans. J Neurosci 2004 01/28;24(4):964-971.

(102) Jacobson L, Mariano AJ. General considerations of chronic pain. In: Loeser JD, Butler SH, Chapman CR, Turk DC, editors. Bonica's Management of Pain Philadelphia: PA: Lippincott Williams \& Wilkins; 2001. p. 241254.

(103) Vanderah TW. Pathophysiology of pain. Med Clin North Am 2007;91(1):1-12.

(104) Jensen TS, Gebhart GF. New pain terminology: a work in progress. . Pain 2008;140(3):399-400.

(105) Whiteside A, Hansen S, Chaudhuri A. Exercise lowers pain threshold in chronic fatigue syndrome. Pain 2004 Jun;109(3):497-499.

(106) Meeus M, Roussel NA, Truijen S, Nijs J. Reduced pressure pain thresholds in response to exercise in chronic fatigue syndrome but not in chronic low back pain: an experimental study. J Rehabil Med 2010 Oct;42(9):884-890.

(107) Van Oosterwijck J, Nijs J, Meeus M, Lefever I, Huybrechts L, Lambrecht L, et al. Pain inhibition and postexertional malaise in myalgic encephalomyelitis/chronic fatigue syndrome: an experimental study. J Intern Med 2010 Sep;268(3):265-278.

(108) Van Oosterwijck J, Nijs J, Meeus M, Van Loo M, Paul L. Lack of endogenous pain inhibition during exercise in people with chronic whiplash associated disorders: an experimental study. J Pain 2012 Mar;13(3):242-254. 
(109) Nijs J, Kosek E, Van Oosterwijck J, Meeus M. Dysfunctional endogenous analgesia during exercise in patients with chronic pain: to exercise or not to exercise? Pain Physician 2012 Jul;15(3 Suppl):ES205-13.

(110) Lluch E, Torres R, Nijs J, Van Oosterwijck J. Evidence for central sensitization in patients with osteoarthritis pain: A systematic literature review. Eur J Pain 2014 Apr 3.

(111) Bradley LA, Kersh BC, DeBerry JJ, Deutsch G, Alarcon GA, McLain DA. Lessons from fibromyalgia: abnormal pain sensitivity in knee osteoarthritis. Novartis Found Symp 2004;260:258-70; discussion 270-9.

(112) Duncan R, Peat G, Thomas E, Hay E, McCall I, Croft P. Symptoms and radiographic osteoarthritis: not as discordant as they are made out to be? Ann Rheum Dis 2007(66):86-91.

(113) Lee YC, Lu B, Bathon JM, Haythornthwaite JA, Smith MT, Page GGea. Pain sensitivity and pain reactivity in osteoarthritis. Arthritis Care Res (Hoboken) 2010 (Epub ahead of print).

(114) Wideman TH, Finan PH, Edwards RR, Quartana PJ, Buenaver LF, Haythornthwaite JA, et al. Increased sensitivity to physical activity among individuals with knee osteoarthritis: relation to pain outcomes, psychological factors, and responses to quantitative sensory testing. Pain 2014 Apr;155(4):703-711.

(115) Smith MT, Quartana PJ, Okonkwo RM, Nasir A. Mechanisms by which sleep disturbance contributes to osteoarthritis pain: a conceptual model. Curr Pain Headache Rep 2009 Dec;13(6):447-454.

(116) Meyr AJ, Saffran B. The pathophysiology of the chronic pain cycle. Clin Podiatr Med Surg 2008 07;25(3):327-346.

(117) Gagliese LF, Melzack R. Chronic pain in elderly people. Pain JID - 75086860623.

(118) Bliddal H, Danneskiold-Samsoe B. Chronic widespread pain in the spectrum of rheumatological diseases. Best Pract Res Clin Rheumatol 2007 Jun;21(3):391-402.

(119) Turk DC, Dworkin RH, Revicki D, Harding G, Burke LB, Cella D, et al. Identifying important outcome domains for chronic pain clinical trials: an IMMPACT survey of people with pain. Pain 2008 Jul 15;137(2):276285.

(120) Gooberman-Hill R, Woolhead G, MacKichan F, Ayis S, Williams S, Dieppe P. Assessing chronic joint pain: Lessons from a focus group study. Arthritis Care \& Research 2007;57(4):666-671.

(121) Dworkin RH, Turk DC, Wyrwich KW, Beaton D, Cleeland CS, Farrar JT, et al. Interpreting the clinical importance of treatment outcomes in chronic pain clinical trials: IMMPACT recommendations. J Pain 2008 Feb;9(2):105-121.

(122) Turk DC, Dworkin RH, Allen RR, Bellamy N, Brandenburg N, Carr DB, et al. Core outcome domains for chronic pain clinical trials: IMMPACT recommendations. Pain 2003 Dec;106(3):337-345.

(123) Chaffee A, Yakuboff M, Tanabe T. Responsiveness of the VAS and McGill pain questionnaire in measuring changes in musculoskeletal pain. J Sport Rehabil 2011 May;20(2):250-255.

(124) Woodforde JM, Merskey H. Some relationships between subjective measures of pain. J Psychosom Res 1972 Jun;16(3):173-178.

(125) McCormack HM, Horne DJ, Sheather S. Clinical applications of visual analogue scales: a critical review. Psychol Med 1988 Nov;18(4):1007-1019.

(126) Hawker GA, Mian S, Kendzerska T, French M. Measures of adult pain: Visual Analog Scale for Pain (VAS Pain), Numeric Rating Scale for Pain (NRS Pain), McGill Pain Questionnaire (MPQ), Short-Form McGill Pain Questionnaire (SF-MPQ), Chronic Pain Grade Scale (CPGS), Short Form-36 Bodily Pain Scale (SF-36 BPS), and Measure of Intermittent and Constant Osteoarthritis Pain (ICOAP). Arthritis Care Res (Hoboken) 2011 Nov;63 Suppl 11:S240-52.

(127) Farrar JT, Young JP,Jr, LaMoreaux L, Werth JL, Poole RM. Clinical importance of changes in chronic pain intensity measured on an 11-point numerical pain rating scale. Pain 2001 Nov;94(2):149-158.

(128) Dworkin RH, Turk DC, Farrar JT, Haythornthwaite JA, Jensen MP, Katz NP, et al. Core outcome measures for chronic pain clinical trials: IMMPACT recommendations. Pain 2005 Jan;113(1-2):9-19.

(129) Hawker GA, Davis AM, French MR, Cibere J, Jordan JM, March L, et al. Development and preliminary psychometric testing of a new OA pain measure--an OARSI/OMERACT initiative. Osteoarthritis Cartilage 2008 Apr;16(4):409-414. 
(130) Melzack R. The McGill Pain Questionnaire: major properties and scoring methods. Pain 1975 Sep;1(3):277299.

(131) Cleeland CS. The impact of pain on the patient with cancer. Cancer 1984 Dec 1;54(11 Suppl):2635-2641.

(132) Melzack R. The short-form McGill Pain Questionnaire. Pain 1987 Aug;30(2):191-197.

(133) Bellamy N, Buchanan WW, Goldsmith CH, Campbell J, Stitt LW. Validation study of WOMAC: a health status instrument for measuring clinically important patient relevant outcomes to antirheumatic drug therapy in patients with osteoarthritis of the hip or knee. J Rheumatol 1988 Dec;15(12):1833-1840.

(134) Bellamy N, Sothern RB, Campbell J. Rhythmic variations in pain perception in osteoarthritis of the knee. J Rheumatol 1990;17:364-372.

(135) Cleeland CS. The Brief Pain Inventory User Guide. ; 2009.

(136) Daut RL, Cleeland CS, Flanery RC. Development of the Wisconsin Brief Pain Questionnaire to assess pain in cancer and other diseases. Pain 1983 Oct;17(2):197-210.

(137) Cleeland CS. Pain assessment in cancer. In: Osoba D, editor. Effect of Cancer on Quality of Life. Boca Raton: CRC Press, Inc; 1991. p. 293-305.

(138) Melzack R, Casey KL. Sensory, motivational, and central control determinants of pain: a new conceptual model. In: Kenshalo DR, editor. The Skin Senses Proceedings Springfield IL: Thomas; 1968. p. 423-439.

(139) Williams VS, Smith MY, Fehnel SE. The validity and utility of the BPI interference measures for evaluating the impact of osteoarthritic pain. J Pain Symptom Manage 2006 Jan;31(1):48-57.

(140) Mendoza T, Mayne T, Rublee D, Cleeland C. Reliability and validity of a modified Brief Pain Inventory short form in patients with osteoarthritis. European Journal of Pain 2006 5;10(4):353-361.

(141) McDowell I, Newell C. Measuring health: A guide to rating scales and questionnaires. 2nd ed. New York: Oxford University Press; 1996.

(142) Jensen MP. The validity and reliability of pain measures in adults with cancer. J Pain 2003 Feb;4(1):2-21.

(143) Tan G, Jensen MP, Thornby JI, Shanti BF. Validation of the Brief Pain Inventory for chronic nonmalignant pain. J Pain 2004 Mar;5(2):133-137.

(144) Cleeland CS, Gonin R, Hatfield AK, Edmonson JH, Blum RH, Stewart JA, et al. Pain and its treatment in outpatients with metastatic cancer. N Engl J Med 1994 Mar 3;330(9):592-596.

(145) Serlin RC, Mendoza TR, Nakamura Y, Edwards KR, Cleeland CS. When is cancer pain mild, moderate or severe? Grading pain severity by its interference with function. Pain 1995 May;61(2):277-284.

(146) Mendoza TR, Chen C, Brugger A, Hubbard R, Snabes M, Palmer SN, et al. The utility and validity of the modified brief pain inventory in a multiple-dose postoperative analgesic trial. Clin J Pain 2004 SepOct;20(5):357-362.

(147) Radbruch L, Loick G, Kiencke P, Lindena G, Sabatowski R, Grond S, et al. Validation of the German version of the Brief Pain Inventory. J Pain Symptom Manage 1999 Sep;18(3):180-187.

(148) Keller S, Bann CM, Dodd SL, Schein J, Mendoza TR, Cleeland CS. Validity of the brief pain inventory for use in documenting the outcomes of patients with noncancer pain. Clin J Pain 2004 Sep-Oct;20(5):309-318.

(149) Keller S, Bann CM, Dodd SL, editors. Drug Information Agency 8th Annual Symposium. Power of the Brief Pain Inventory to document changes in arthritis and low back pain.; March 25-27; SC: Hilton Head; 2001.

(150) Shrout PE, Fleiss JL. Intraclass correlations: uses in assessing rater reliability. Psychol Bull 1979 Mar;86(2):420-428.

(151) American Heritage Publishing Company. The American Heritage ${ }^{\circledR}$ Dictionary of the English Language. 4th ed. Boston: Houghton Mifflin Company; 2009.

(152) Kisner C, Colby LA. Therapeutic Exercise. Foundation and Techniques. 4th ed. Philadelphia: F.A Davis company; 2002.

(153) Fitzgerald GK, Piva SR, Irrgang JJ. Reports of joint instability in knee osteoarthritis: its prevalence and relationship to physical function. Arthritis Rheum 2004 Dec 15;51(6):941-946. 
(154) Norkin CC, White DJ. Measurement of joint motion: a guide to goniometry. 4th ed. Philadelphia: F.A. Davis Company; 2009.

(155) Jaric S. Muscle strength testing: use of normalisation for body size. Sports Med 2002;32(10):615-631.

(156) Keating JL, Matyas TA. The influence of subject and test design on dynamometric measurements of extremity muscles. Phys Ther 1996 Aug;76(8):866-889.

(157) Novy DM, Simmonds MJ, Lee CE. Physical performance tasks: what are the underlying constructs? Arch Phys Med Rehabil 2002 Jan;83(1):44-47.

(158) Lorig KR, Mazonson PD, Holman HR. Evidence suggesting that health education for self-management in patients with chronic arthritis has sustained health benefits while reducing health care costs. Arthritis Rheum 1993 04;36(4):439-446.

(159) Guccione AA, Felson DT, Anderson JJ, Anthony JM, Zhang Y, Wilson PW, et al. The effects of specific medical conditions on the functional limitations of elders in the Framingham Study. Am J Public Health 1994 Mar;84(3):351-358.

(160) Hootman JM, Helmick CG. Projections of US prevalence of arthritis and associated activity limitations. Arthritis Rheum 2006 Jan;54(1):226-229.

(161) Wilkie R, Blagojevic-Bucknall M, Jordan KP, Pransky G. Onset of work restriction in employed adults with lower limb joint pain: individual factors and area-level socioeconomic conditions. J Occup Rehabil 2013 Jun;23(2):180-188.

(162) Cieza A, Stucki G. The International Classification of Functioning Disability and Health: its development process and content validity. Eur J Phys Rehabil Med 2008 Sep;44(3):303-313.

(163) Guralnik JM, Simonsick EM, Ferrucci L, Glynn RJ, Berkman LF, Blazer DG, et al. A short physical performance battery assessing lower extremity function: association with self-reported disability and prediction of mortality and nursing home admission. J Gerontol 1994 Mar;49(2):M85-94.

(164) Freiberger E, de Vreede P, Schoene D, Rydwik E, Mueller V, Frandin K, et al. Performance-based physical function in older community-dwelling persons: a systematic review of instruments. Age Ageing 2012 Nov;41(6):712-721.

(165) Reuben DB, Siu AL. An objective measure of physical function of elderly outpatients. The Physical Performance Test. J Am Geriatr Soc 1990 Oct;38(10):1105-1112.

(166) Cress ME, Buchner DM, Questad KA, Esselman PC, deLateur BJ, Schwartz RS. Continuous-scale physical functional performance in healthy older adults: a validation study. Arch Phys Med Rehabil 1996 Dec;77(12):1243-1250.

(167) ATS Committee on Proficiency Standards for Clinical Pulmonary Function Laboratories. ATS statement: guidelines for the six-minute walk test. Am J Respir Crit Care Med 2002 Jul 1;166(1):111-117.

(168) Peppin JF, Marcum S, Kirsh KL. The chronic pain patient and functional assessment: use of the 6-Minute Walk Test in a multidisciplinary pain clinic. Curr Med Res Opin 2014 Mar;30(3):361-365.

(169) Kennedy DM, Stratford PW, Wessel J, Gollish JD, Penney D. Assessing stability and change of four performance measures: a longitudinal study evaluating outcome following total hip and knee arthroplasty. BMC Musculoskelet Disord 2005 Jan 28;6:3.

(170) Podsiadlo D, Richardson S. The timed "Up \& Go": a test of basic functional mobility for frail elderly persons. J Am Geriatr Soc 1991 Feb;39(2):142-148.

(171) Stratford PW, Kennedy DM, Woodhouse LJ. Performance measures provide assessments of pain and function in people with advanced osteoarthritis of the hip or knee. Phys Ther 2006 Nov;86(11):1489-1496.

(172) Hurley MV, Scott DL, Rees J, Newham DJ. Sensorimotor changes and functional performance in patients with knee osteoarthritis. Ann Rheum Dis 1997 Nov;56(11):641-648.

(173) Simmonds MJ, Olson SL, Jones S, Hussein T, Lee CE, Novy D, et al. Psychometric characteristics and clinical usefulness of physical performance tests in patients with low back pain. Spine (Phila Pa 1976) 1998 Nov $15 ; 23(22): 2412-2421$ 
(174) Simmonds MJ, Novy D, Sandoval R. The differential influence of pain and fatigue on physical performance and health status in ambulatory patients with human immunodeficiency virus. Clin J Pain 2005 MayJun;21(3):200-206.

(175) Simmonds MJ. Measuring and managing pain and performance. Man Ther 2006 8;11(3):175-179.

(176) Fries JF, Spitz P, Kraines RG, Holman HR. Measurement of patient outcome in arthritis. Arthritis Rheum 1980(23):137-145.

(177) Lorig KR, Sobel DS, Ritter PL, Laurent D, Hobbs M. Effect of a self-management program on patients with chronic disease. Eff Clin Pract 2001 Nov-Dec;4(6):256-262.

(178) Hurley MV, Walsh NE. Effectiveness and clinical applicability of integrated rehabilitation programs for knee osteoarthritis. Curr Opin Rheumatol 2009 Mar;21(2):171-176.

(179) Lundberg M, Styf J, Bullington J. Experiences of moving with persistent pain--a qualitative study from a patient perspective. Physiother Theory Pract 2007 Jul-Aug;23(4):199-209.

(180) Sprangers MA, de Regt ,E.B., Andries F, van Agt ,H.M., Bijl RV, de Boer ,J.B., et al. Which chronic conditions are associated with better or poorer quality of life? J Clin Epidemiol 2000 09;53(9):895-907.

(181) Fernandez E, editor. Advanced Psychological Resources. Anxiety, depression, and anger in pain: research findings and clinical options. Texas; 2002.

(182) Beck AT, Ward CH, Mendelson M, Mock J, Erbaugh J. An inventory for measuring depression. Arch Gen Psychiatry 1961;4:561-571.

(183) McNair DM, Lorr M, Droppleman LF. Profile of Mood States. San Diego, CA: Educational and Industrial Testing Service; 1971.

(184) Kerns RD, editor. Initiative on Methods, Measurement, and Pain Assessment in Clinical Trials (IMMPACTII). Assessment of emotional functioning in pain treatment outcome research.; April 2003; .

(185) R. E. Parker. Pain in HIV/AIDS: Characteristics contributing factors and the effects of a six-week peer-led exercise and education interventionUniversity of Cape Town; 2013.

(186) Gallagher RM, Verma S. Mood and anxiety disorders in chronic pain. In: Dworkin RH, Breitbart WS, editors. Psychosocial aspects of pain: a handbook for health care providers. Seattle: IASP Press; 2004. p. 589606.

(187) Wilson KG, Mikail SF, D’Eon JL, Minns JE. Alternative diagnostic criteria for major depressive disorder in patients with chronic pain. Pain 2001;91:227-234.

(188) Ware J,Jr, Kosinski M, Keller SD. A 12-Item Short-Form Health Survey: construction of scales and preliminary tests of reliability and validity. Med Care 1996 Mar;34(3):220-233.

(189) Ware JE. SF-36 Physical and Mental HealthSummary Scales: A User's Manual. Boston, MA: Health Assessment La; 1994.

(190) Rabin R, de Charro F. EQ-5D: a measure of health status from the EuroQol Group. Ann Med 2001 Jul;33(5):337-343.

(191) Johnson JA, Coons SJ. Comparison of the EQ-5D and SF-12 in an adult US sample. Qual Life Res 1998 Feb;7(2):155-166.

(192) Brazier JE, Harper R, Munro J, Walters SJ, Snaith ML. Generic and condition-specific outcome measures for people with osteoarthritis of the knee. Rheumatology (Oxford) 1999 Sep;38(9):870-877.

(193) Bodenheimer T, Lorig K, Holman H. Patient Self-management of Chronc Disease in Primary Care. JAMA 2002 11/20;288(19):2469-2475.

(194) Freund T, Gensichen J, Goetz K, Szecsenyi J, Mahler C. Evaluating self-efficacy for managing chronic disease: psychometric properties of the six-item Self-Efficacy Scale in Germany. J Eval Clin Pract 2013 Feb;19(1):39-43.

(195) Bandura A. Guide for Constructing Self-Efficacy Scales. In: Pajares F, Urdan TC, editors. Self-Efficacy Beliefs in Adolescents USA: IAP; 2006. p. 307-337.

(196) Bandura A. Self-efficacy in changing societies. New York: Cambridge University Press.; 1995. 
(197) Lefebvre JC, Keefe FJ, Affleck G, Raezer LB, Starr K, Caldwell DS, et al. The relationship of arthritis selfefficacy to daily pain, daily mood, and daily pain coping in rheumatoid arthritis patients. Pain 1999 Mar;80(12):425-435.

(198) Cross MJ, March LM, Lapsley HM, Byrne E, Brooks PM. Patient self-efficacy and health locus of control: relationships with health status and arthritis-related expenditure. Rheumatology (Oxford) 2006 Jan;45(1):92-96.

(199) Brekke M, Hjortdahl P, Kvien TK. Self-efficacy and health status in rheumatoid arthritis: a two-year longitudinal observational study. Rheumatology (Oxford) 2001 Apr;40(4):387-392.

(200) Allegrante JP, Marks R. Self-efficacy in management of osteoarthritis. Rheum Dis Clin North Am 2003 Nov;29(4):747-68, vi-vii.

(201) Lorig K, Chastain RL, Ung E, Shoor S, Holman HR. Development and evaluation of a scale to measure perceived self-efficacy in people with arthritis. Arthritis Rheum 1989 01;32(1):37-44.

(202) Bijl JV, Poelgeest-Eeltink AV, Shortridge-Baggett L. The psychometric properties of the diabetes management self-efficacy scale for patients with type 2 diabetes mellitus. Journal of Advanced Nursing 1999;30(2):352-359.

(203) Webel AR, Okonsky J. Psychometric properties of a symptom management self-efficacy scale for women living with HIV/AIDS. Journal of Pain and Symptom Management 2011;41(3):549-557.

(204) Brady TJ. Measures of self-efficacy: Arthritis Self-Efficacy Scale (ASES), Arthritis Self-Efficacy Scale-8 Item (ASES-8), Children's Arthritis Self-Efficacy Scale (CASE), Chronic Disease Self-Efficacy Scale (CDSES), Parent's Arthritis Self-Efficacy Scale (PASE), and Rheumatoid Arthritis Self-Efficacy Scale (RASE). Arthritis Care Res (Hoboken) 2011 Nov;63 Suppl 11:S473-85.

(205) Lorig K, Chastain RL, Ung E, Shoor S, Holman HR. Development and evaluation of a scale to measure perceived self-efficacy in people with arthritis. Arthritis Rheum 1989 Jan;32(1):37-44.

(206) Lorig K, Holman H. Arthritis Self-Efficacy Scales measure self-efficacy. Arthritis Care Res 1998 06;11(3):155-157.

(207) Moskowitz RW. The 2012 ACR guidelines for osteoarthritis: Not a cookbook. Cleveland Clinic Journal of Medicine 2013;80(1):26-32.

(208) Hochberg M, C., Altman R, D., April K, Toupin, Benkhalti M, Guyatt G, McGowan J, et al. American College of Rheumatology 2012 recommendations for the use of nonpharmacologic and the pharmacologic therapies in osteoarthritis of the hand, hip, and knee. ARTHRITIS CARE RES (2151464X) 2012 04;64(4):465-474.

(209) Scott DL. Guidelines for the diagnosis, investigation and management of osteoarthritis of the hip and knee. Report of a Joint Working Group of the British Society for Rheumatology and the Research Unit of the Royal College of Physicians. J R Coll Physicians Lond 1993 Oct;27(4):391-396.

(210) American College of Rheumatology subcommittee on osteoarthritis guidelines. Recommendations for the medical management of osteoarthritis of the hip and knee. Arthritis Rheum 2000(43):1905-1915.

(211) Pendleton A, Arden N, Dougados M, Doherty M, Bannwarth B, Bijlsma JW, et al. EULAR recommendations for the management of knee osteoarthritis: report of a task force of the Standing Committee for International Clinical Studies Including Therapeutic Trials (ESCISIT). Ann Rheum Dis 2000 Dec;59(12):936-944.

(212) AGREE Collaboration. Development and validation of an international appraisal instrument for assessing the quality of clinical practice guidelines: the AGREE project. Qual Saf Health Care 2003 Feb;12(1):18-23.

(213) Zhang W, Moskowitz RW, Nuki G, Abramson S, Altman RD, Arden N, et al. OARSI recommendations for the management of hip and knee osteoarthritis, part I: critical appraisal of existing treatment guidelines and systematic review of current research evidence. Osteoarthritis Cartilage 2007 Sep;15(9):981-1000.

(214) Roddy E, Zhang W, Doherty M, Arden NK, Barlow J, Birrell F, et al. Evidence-based clinical guidelines: a new system to better determine true strength of recommendation. J Eval Clin Pract 2006 Jun;12(3):347-352.

(215) Dalbeth N, Arroll B. Commentary: controversies in NICE guidance on osteoarthritis. BMJ 2008(336):504.

(216) Richmond J, Hunter D, Irrgang J, Jones MH, Snyder-Mackler L, Van Durme D, et al. American Academy of Orthopaedic Surgeons clinical practice guideline on the treatment of osteoarthritis (OA) of the knee. J Bone Joint Surg Am 2010 Apr;92(4):990-993. 
(217) Bryant C, Lewis P, Bennell KL, Ahamed Y, Crough D, Jull GA, et al. Can Physical Therapists Deliver a Pain Coping Skills Program? An Examination of Training Processes and Outcomes. Phys Ther 2014 Jun 5.

(218) Recommendations for the medical management of osteoarthritis of the hip and knee: 2000 update. American College of Rheumatology Subcommittee on Osteoarthritis Guidelines. Arthritis Rheum 2000 Sep;43(9):1905-1915.

(219) Atkins D, Best D, Briss PA, Eccles M, Falck-Ytter Y, Flottorp S, et al. Grading quality of evidence and strength of recommendations. BMJ 2004 Jun 19;328(7454):1490.

(220) Baird CL, Sands L. A pilot study of the effectiveness of guided imagery with progressive muscle relaxation to reduce chronic pain and mobility difficulties of osteoarthritis. Pain Manag Nurs 2004 Sep;5(3):97-104.

(221) Baird CL, Sands LP. Effect of guided imagery with relaxation on health-related quality of life in older women with osteoarthritis. Res Nurs Health 2006 Oct;29(5):442-451.

(222) Baird CL, Murawski MM, Wu J. Efficacy of guided imagery with relaxation for osteoarthritis symptoms and medication intake. Pain Manag Nurs 2010 Mar;11(1):56-65.

(223) Glasgow RE, Davis CL, Funnell MM, Beck A. Implementing practical interventions to support chronic illness self-management. Jt Comm J Qual Saf 2003 Nov;29(11):563-574.

(224) Parcel GS, Bartlett, E.E. \& Bruhn, J.G. The role of health education in self-management. In: Holroyd, K.A. \& Creer, T.L., editor. Self-management of chronic disease: handbook of clinical interventions and research London: Academic Press; 1986. p. 3-27.

(225) Superio-Cabuslay E, Ward MM, Lorig KR. Patient education interventions in osteoarthritis and rheumatoid arthritis: a meta-analytic comparison with nonsteroidal antiinflammatory drug treatment. Arthritis Care Res 1996 Aug;9(4):292-301.

(226) Critchley DJ, Ratcliffe J, Noonan S, Jones RH, Hurley MV. Effectiveness and Cost-Effectiveness of Three Types of Physiotherapy Used to Reduce Chronic Low Back Pain Disability. A Pragmatic Randomised Trial with Economic Evaluation. SPINE 2007 Lippincott Williams \& Wilkins, Inc;32(14):1474-1481.

(227) Heuts PH, de Bie R, Drietelaar M, Aretz K, Hopman-Rock M. Self-management in osteoarthritis of hip or knee: a randomized clinical trial in a primary healthcare setting. J Rheumatol 2005;32:543-549.

(228) Newman S, Steed L, Mulligan K. Self-management interventions for chronic illness. The Lancet 2004 10/23-29;364(9444):1523-1537.

(229) Nijs J, Paul van Wilgen C, Van Oosterwijck J, van Ittersum M, Meeus M. How to explain central sensitization to patients with 'unexplained' chronic musculoskeletal pain: practice guidelines. Man Ther 2011 Oct;16(5):413-418.

(230) Louw A, Diener I, Butler DS, Puentedura EJ. The effect of neuroscience education on pain, disability, anxiety, and stress in chronic musculoskeletal pain. Arch Phys Med Rehabil 2011 Dec;92(12):2041-2056.

(231) Nijs J, Malfliet A, Ickmans K, Baert I, Meeus M. Treatment of central sensitization in patients with 'unexplained' chronic pain: an update. Expert Opin Pharmacother 2014 Aug;15(12):1671-1683.

(232) Moseley GL, Nicholas MK, Hodges PW. A randomized controlled trial of intensive neurophysiology education in chronic low back pain. Clin J Pain 2004 Sep-Oct;20(5):324-330.

(233) Moseley GL. Evidence for a direct relationship between cognitive and physical change during an education intervention in people with chronic low back pain. Eur J Pain 2004 Feb;8(1):39-45.

(234) Moseley GL. Widespread brain activity during an abdominal task markedly reduced after pain physiology education: fMRI evaluation of a single patient with chronic low back pain. Aust J Physiother 2005;51(1):49-52.

(235) Barlow JH, Turner AP, Wright CC. A randomized controlled study of the Arthritis Self-Management Programme in the UK. Health Educ Res 2000 Dec;15(6):665-680.

(236) Clark NM, Becker MH, Janz NK, Lorig K, Rakowski W, Anderson L. Self-Management of Chronic Disease by Older Adults: A Review and Questions for Research. Journal of Aging and Health 1991 February 01;3(1):3-27.

(237) Barlow J, Wright C, Sheasby J, Turner A, Hainsworth J. Self-management approaches for people with chronic conditions: a review. Patient Educ Couns 2002 Oct -Nov;48(2):177-187.

(238) Keefe, F.J. \& Caldwell, D.S. Cognitive Behavioral Interventions. In: Wegener ST, Belza, B.L. \& Gall, E.P., editors. Clinical care in the rheumatic diseases. Atlanta: American College of Rheumatolog; 1996. 
(239) Lorig KR, Sobel DS, Stewart AL, Brown BW,Jr, Bandura A, Ritter P, et al. Evidence suggesting that a chronic disease self-management program can improve health status while reducing hospitalization: a randomized trial. Med Care 1999 Jan;37(1):5-14.

(240) Lorig KR, Ritter P, Stewart AL, Sobel DS, Brown BW, J., Bandura A, et al. Chronic disease self-management program: 2-year health status and health care utilization outcomes. Med Care 2001 11;39(11):1217-1223.

(241) Lorig K, Ritter PL, Plant K. A disease-specific self-help program compared with a generalized chronic disease self-help program for arthritis patients. Arthritis \& Rheumatism 2005 12/15;53(6):950-957.

(242) Crotty M, Prendergast J, Battersby MW, Rowett D, Graves SE, Leach G, et al. Self-management and peer support among people with arthritis on a hospital joint replacement waiting list: a randomised controlled trial. Osteoarthritis Cartilage 2009 Nov;17(11):1428-1433.

(243) Warsi A, LaValley MP, Wang PS, Avorn J, Solomon DH. Arthritis self-management education programs: a meta-analysis of the effect on pain and disability. Arthritis Rheum 2003;48:2207-2213.

(244) Lorig KR, Gonza'lez VM, Laurent DD. The Chronic Disease Self-Management Program: Leaders Manual. Stanford University: Palo Alto; 1999.

(245) Moffett JA, Richardson PH, Frost H, Osborn A. A placebo controlled double blind trial to evaluate the effectiveness of pulsed short wave therapy for osteoarthritic hip and knee pain. Pain 1996 Sep;67(1):121-127.

(246) Ackerman IN, Graves SE, Wicks IP, Bennell KL, Osborne RH. Severely compromised quality of life in women and those of lower socioeconomic status waiting for joint replacement surgery. Arthritis Rheum 2005 Oct 15;53(5):653-658.

(247) Fortin PR, Penrod JR, Clarke AE, St-Pierre Y, Joseph L, Belisle P, et al. Timing of total joint replacement affects clinical outcomes among patients with osteoarthritis of the hip or knee. Arthritis Rheum 2002 Dec;46(12):3327-3330.

(248) Nolte S, Elsworth GR, Sinclair AJ, Osborne RH. The extent and breadth of benefits from participating in chronic disease self-management courses: a national patient-reported outcomes survey. Patient Educ Couns 2007 Mar;65(3):351-360.

(249) Lorig K, Lubeck D, Kraines RG, Seleznick M, Holman HR. Outcomes of self-help education for patients with arthritis. Arthritis Rheum 1985 06;28(6):680-685.

(250) Vita AJ, Terry RB, Hubert HB, Fries JF. Aging, health risks, and cumulative disability. N Engl J Med 1998 Apr 9;338(15):1035-1041

(251) Leveille SG, Wagner EH, Davis C, Grothaus L, Wallace J, LoGerfo M, et al. Preventing disability and managing chronic illness in frail older adults: a randomized trial of a community-based partnership with primary care. J Am Geriatr Soc 1998 Oct;46(10):1191-1198.

(252) Chodosh J, Morton SC, Mojica W, Maglione M, Suttorp MJ, Hilton L, et al. Meta-analysis: chronic disease self-management programs for older adults. Ann Intern Med 2005 Sep 20;143(6):427-438.

(253) Kennedy A, Reeves D, Bower P, Lee V, Middleton E, Richardson G, et al. The effectiveness and cost effectiveness of a national lay-led self care support programme for patients with long-term conditions: a pragmatic randomised controlled trial. J Epidemiol Community Health 2007 Mar;61(3):254-261.

(254) Charlton BG. The future of clinical research: from megatrials towards methodological rigour and representative sampling. J Eval Clin Pract 1996 Aug;2(3):159-169.

(255) Holman H, Mazonson P, Lorig K. Health education for self-management has significant early and sustained benefits in chronic arthritis. Trans Assoc Am Physicians 1989;102:204-208.

(256) Lorig K, Holman H. Arthritis self-management studies: A twelve-year review. Health Educ Q 1993 Spring93;20(1):17.

(257) Lorig K, Gonzalez VM, Laurent DD, Morgan L, Laris BA. Arthritis self-management program variations: three studies. Arthritis Care Res 1998 Dec;11(6):448-454.

(258) Buszewicz M, Rait G, Griffin M, Nazareth I, Patel A, Atkinson A, et al. Self management of arthritis in primary care: randomised controlled trial. BMJ 2006 Oct 28;333(7574):879.

(259) Lehlola P. Statistics South Africa 2013. 2013; Available at: www.statssa.gov.za. Accessed April, 07, 2014. 
(260) Statistics South Africa. General household survey (GHS) 2011. P0318 . 2012; Available at: www.statssa.gov.za. Accessed April, 07, 2014.

(261) Bennell KL, Ahamed Y, Bryant C, Jull G, Hunt MA, Kenardy J, et al. A physiotherapist-delivered integrated exercise and pain coping skills training intervention for individuals with knee osteoarthritis: a randomised controlled trial protocol. BMC Musculoskelet Disord 2012 Jul 24;13:129-2474-13-129.

(262) Nielsen M, Keefe FJ, Bennell K, Jull GA. Physical therapist-delivered cognitive-behavioral therapy: a qualitative study of physical therapists' perceptions and experiences. Phys Ther 2014 Feb;94(2):197-209.

(263) Holman H, Lorig K. Patient self-management: a key to effectiveness and efficiency in care of chronic disease. Public Health Rep 2004 2004;119(3):239-243.

(264) Jessep SA, Walsh NE, Ratcliffe J, Hurley MV. Long-term clinical benefits and costs of an integrated rehabilitation programme compared with outpatient physiotherapy for chronic knee pain. Physiotherapy 2009 Jun;95(2):94-102.

(265) Kroon FP, van der Burg LR, Buchbinder R, Osborne RH, Johnston RV, Pitt V. Self-management education programmes for osteoarthritis. Cochrane Database Syst Rev 2014 Jan 15;1:CD008963.

(266) Jamtvedt G, Dahm KT, Christie A, Moe RH, Haavardsholm E, Holm I, et al. Physical therapy interventions for patients with osteoarthritis of the knee: an overview of systematic reviews. Phys Ther 2008;88(1):123-136.

(267) Brakke R, Singh J, Sullivan W. Physical therapy in persons with osteoarthritis. PM R 2012 05;4(5):S53-S58.

(268) Fransen M, McConnell S, Hernandez-Molina G, Reichenbach S. Exercise for osteoarthritis of the hip. Cochrane Database Syst Rev 2014 Apr 22;4:CD007912.

(269) Benito Peinado PJ, Cupeiro Coto R, Calderón Montero FJ. [Physical exercise as non pharmacologic therapy in knee osteoarthritis]. Reumatol Clin 2010 2010;6(3):153-160.

(270) Pinto D, Robertson MC, Hansen P, Abbott JH. Cost-effectiveness of nonpharmacologic, nonsurgical interventions for hip and/or knee osteoarthritis: systematic review. Value Health 2012 Jan;15(1):1-12.

(271) Hurley MV, Walsh NE, Mitchell H, Nicholas J, Patel A. Long-term outcomes and costs of an integrated rehabilitation program for chronic knee pain: a pragmatic, cluster randomized, controlled trial. Arthritis Care Res (Hoboken) 2012 Feb;64(2):238-247.

(272) Hurley MV, Walsh NE, Mitchell HL, Pimm TJ, Patel A, Williamson A, et al. Clinical Effectiveness of a Rehabilitation Program Integrating Exercise, Self-Management, and Active Coping Strategies for Chronic Knee Pain: A Cluster Randomized Trial Arthritis \& Rheumatism 2007;57(7):1211-1219.

(273) Brander V. Changing the Treatment Paradigm: Moving to Multimodal and Integrated Osteoarthritis Disease Management. J Fam Pract 2011 11/03:S41-S47.

(274) Woodard CM, Berry MJ. Enhancing adherence to prescribed exercise: structured behavioral interventions in clinical exercise programs. J Cardiopulm Rehabil 2001 Jul-Aug;21(4):201-209.

(275) Fransen M, McConnell S, Bell M. Therapeutic exercise for people with osteoarthritis of the hip or knee. A systematic review. J Rheumatol 2002 Aug;29(8):1737-1745.

(276) Walsh NE, Mitchell HL, Reeves BC, Hurley MV. Integrated exercise and self-management programmes in osteoarthritis of the hip and knee: a systematic review of effectiveness. Physical Therapy Reviews 2006 $12 ; 11(4): 289-297$.

(277) Hughes SL, Seymour RB, Campbell R, Pollak N, Huber G, Sharma L. Impact of the fit and strong intervention on older adults with osteoarthritis. Gerontologist 2004 Apr;44(2):217-228.

(278) Hughes SL, Seymour RB, Campbell RT, Huber G, Pollak N, Sharma L, et al. Long-term impact of Fit and Strong! on older adults with osteoarthritis. Gerontologist 2006 Dec;46(6):801-814.

(279) Lamb SE, Toye F, Barker KL. Chronic disease management programme in people with severe knee osteoarthritis: efficacy and moderators of response. Clin Rehabil 2008 Feb;22(2):169-178.

(280) Patel S, Hossain FS, Paton B, Haddad FS. The effects of a non-operative multimodal programme on osteoarthritis of the knee. Ann R Coll Surg Engl 2010 Sep;92(6):467-471.

(281) Jensen C, Roos EM, Kjaersgaard-Andersen P, Overgaard S. The effect of education and supervised exercise vs. education alone on the time to total hip replacement in patients with severe hip osteoarthritis. A randomized clinical trial protocol. BMC Musculoskelet Disord 2013 Jan 14;14:21-2474-14-21. 
(282) Smink AJ, van den Ende CH, Vliet Vlieland TP, Swierstra BA, Kortland JH, Bijlsma JW, et al. "Beating osteoARThritis": development of a stepped care strategy to optimize utilization and timing of non-surgical treatment modalities for patients with hip or knee osteoarthritis. Clin Rheumatol 2011 Dec;30(12):1623-1629.

(283) Smink AJ, Bierma-Zeinstra SM, Dekker J, Vliet Vlieland TP, Bijlsma JW, Swierstra BA, et al. Agreement of general practitioners with the guideline-based stepped-care strategy for patients with osteoarthritis of the hip or knee: a cross-sectional study. BMC Fam Pract 2013 Mar 11;14:33-2296-14-33.

(284) Skou ST, Roos EM, Laursen MB, Rathleff MS, Arendt-Nielsen L, Simonsen OH, et al. Total knee replacement plus physical and medical therapy or treatment with physical and medical therapy alone: a randomised controlled trial in patients with knee osteoarthritis (the MEDIC-study). BMC Musculoskeletal Disorders 2012 01;13(1):67-77.

(285) Hengeveld E, Banks K. Self-managment strategies: compliance and behavioural change. In: Wells P, editor. Maitland's Peripheral Manipulation. fourth ed.: Elsevier Ltd; 2005. p. 617-623.

(286) Hunt MA, Keefe FJ, Bryant C, Metcalf BR, Ahamed Y, Nicholas MK, et al. A physiotherapist-delivered, combined exercise and pain coping skills training intervention for individuals with knee osteoarthritis: a pilot study. Knee 2013 Mar;20(2):106-112.

(287) Lang PJ. Presidential address, 1978. A bio-informational theory of emotional imagery. Psychophysiology 1979 Nov;16(6):495-512.

(288) Felson DT, Lawrence RC, Dieppe PA, Hirsch R, Helmick CG, Jordan JM, et al. Osteoarthritis: new insights. Part 1: the disease and its risk factors. Ann Intern Med 2000 Oct 17;133(8):635-646.

(289) Murphy L, Schwartz TA, Helmick CG, Renner JB, Tudor G, Koch G, et al. Lifetime risk of symptomatic knee osteoarthritis. Arthritis Care \& Research 2008;59(9):1207-1213

(290) Felson DT. Does excess weight cause osteoarthritis and, if so, why? Ann Rheum Dis 1996 Sep;55(9):668670.

(291) Lievense AM, Bierma-Zeinstra SMA, Verhagen AP, van Baar ME, Verhaar JAN, Koes BW. Influence of obesity on the development of osteoarthritis of the hip: a systematic review. Rheumatology 2002 October 01;41(10):1155-1162.

(292) Henriksen M, Christensen R, Danneskiold-Samsoe B, Bliddal H. Changes in lower extremity muscle mass and muscle strength after weight loss in obese patients with knee osteoarthritis: a prospective cohort study. Arthritis Rheum 2012 Feb;64(2):438-442.

(293) Messier SP, Loeser RF, Miller GD, Morgan TM, Rejeski WJ, Sevick MA, et al. Exercise and dietary weight loss in overweight and obese older adults with knee osteoarthritis: the Arthritis, Diet, and Activity Promotion Trial. Arthritis Rheum 2004 May;50(5):1501-1510.

(294) Rejeski WJ, Focht BC, Messier SP, Morgan T, Pahor M, Penninx B. Obese, older adults with knee osteoarthritis: weight loss, exercise, and quality of life. Health Psychol 2002 Sep;21(5):419-426.

(295) Messier SP, Loeser RF, Mitchell MN, Valle G, Morgan TP, Rejeski WJ, et al. Exercise and weight loss in obese older adults with knee osteoarthritis: a preliminary study. J Am Geriatr Soc 2000 Sep;48(9):1062-1072.

(296) Christensen R, Bartels EM, Astrup A, Bliddal H. Effect of weight reduction in obese patients diagnosed with knee osteoarthritis: a systematic review and meta-analysis. Ann Rheum Dis 2007 Apr;66(4):433-439.

(297) Amin AK, Patton JT, Cook RE, Brenkel IJ. Does obesity influence the clinical outcome at five years following total knee replacement for osteoarthritis? J Bone Joint Surg Br 2006 Mar;88(3):335-340.

(298) Sadr Azodi O, Bellocco R, Eriksson K, Adami J. The impact of tobacco use and body mass index on the length of stay in hospital and the risk of post-operative complications among patients undergoing total hip replacement. J Bone Joint Surg Br 2006 Oct;88(10):1316-1320.

(299) Walsh NE, Hurley MV. Evidence based guidelines and current practice for physiotherapy management of knee osteoarthritis. Musculoskeletal Care 2009 03;7(1):45-56.

(300) French H, Brennan A, White B, Cusack T. Manual therapy for osteoarthritis of the hip or knee - a systematic review. MANUAL THER 2011 04;16(2):109-117.

(301) Davis AM. Osteoarthritis year in review: rehabilitation and outcomes. Osteoarthritis Cartilage 2012 Mar;20(3):201-206. 
(302) St. Clair S, Higuera C, Krebs V, Tadross NA, Dumpe J, Barsoum WK. Hip and knee arthroplasty in the geriatric population. Clin Geriatr Med 2006 08;22(3):515-533.

(303) Scott DL, Shipley M, Dawson A, Edwards S, Symmons DP, Woolf AD. The clinical management of rheumatoid arthritis and osteoarthritis: strategies for improving clinical effectiveness. Br J Rheumatol 1998 05;37(5):546-554.

(304) Towheed TE, Hochberg MC. Health-related quality of life after total hip replacement. Semin Arthritis Rheum 1996 08;26(1):483-491.

(305) Goodman S. Osteoarthritis. In: Yee A, Paget S, editors. Expert Guide to Rheumatology Philadelphia, Pa: American College of Physicians; 2005. p. 269-283.

(306) Harris WH, Sledge CB. Total Hip and Total Knee Replacement. N Engl J Med 1990 09/20; 2014/04;323(12):801-807.

(307) Hawker G, Wright J, Coyte P, Paul J, Dittus R, Croxford R, et al. Health-related quality of life after knee replacement. J Bone Joint Surg Am 1998 Feb;80(2):163-173.

(308) Williams JI, Llewellyn Thomas H, Arshinoff R, Young N, Naylor CD. The burden of waiting for hip and knee replacements in Ontario. Ontario Hip and Knee Replacement Project Team. J Eval Clin Pract 1997 Feb;3(1):5968.

(309) Desmeules F, Dionne CE, Belzile E, Bourbonnais R, Fremont P. Waiting for total knee replacement surgery: factors associated with pain, stiffness, function and quality of life. BMC Musculoskelet Disord 2009 May 20;10:52-2474-10-52.

(310) Bachrach-Lindstrom M, Karlsson S, Pettersson LG, Johansson T. Patients on the waiting list for total hip replacement: a 1-year follow-up study. Scand J Caring Sci 2008 Dec;22(4):536-542.

(311) Mahon JL, Bourne RB, Rorabeck CH, Feeny DH, Stitt L, Webster-Bogaert S. Health-related quality of life and mobility of patients awaiting elective total hip arthroplasty: a prospective study. CMAJ 2002 Nov 12;167(10):1115-1121.

(312) Kelly KD, Voaklander DC, Johnston DW, Newman SC, Suarez-Almazor ME. Change in pain and function while waiting for major joint arthroplasty. J Arthroplasty 2001 Apr;16(3):351-359.

(313) Hoogeboom TJ, van den Ende CH, van der Sluis G, Elings J, Dronkers JJ, Aiken AB, et al. The impact of waiting for total joint replacement on pain and functional status: a systematic review. Osteoarthritis Cartilage 2009 Nov;17(11):1420-1427.

(314) Kili S, Wright I, Jones RS. Change in Harris hip score in patients on the waiting list for total hip replacement. Ann R Coll Surg Engl 2003 Jul;85(4):269-271.

(315) Noseworthy TW, Sanmartin C, Bohm E, Connor-Spady B, DeCoster C, Dunbar Mea. Toward Canadian benchmarks for health service wait times - evidence, application and research priorities. 14-10-2005;1e56.

(316) The University of Texas MD Anderson Cancer Center. The Brief Pain Inventory (BPI). Available at: http://www.mdanderson.org/education-and-research/departments-programs-and-labs/departments-anddivisions/symptom-research/symptom-assessment-tools/brief-pain-inventory.html. Accessed 04/02, 2013.

(317) Parker R. The effect of a 6-week peer-led exercise and education intervention on pain in amaXhosa women living with HIV/AIDS. University of Cape Town 2013.

(318) Bruce B, Fries JF. The Health Assessment Questionnaire (HAQ). Clin Exp Rheumatol 2005 Sep-Oct;23(5 Suppl 39):S14-8.

(319) Jelsma J, Ferguson G. The determinants of self-reported health-related quality of life in a culturally and socially diverse South African community. Bull World Health Organ 2004 Mar;82(3):206-212.

(320) Lamb SE, Toye F, Barker KL. Chronic disease management programme in people with severe knee osteoarthritis: efficacy and moderators of response. Clin Rehabil 2008;Email communication with K Barker on 02-10-14(2):169-178.

(321) Nijs J, Meeus M, Van Oosterwijck J, Roussel N, De Kooning M, Ickmans K, et al. Treatment of central sensitization in patients with 'unexplained' chronic pain: what options do we have? Expert Opin Pharmacother 2011 May;12(7):1087-1098. 
(322) WMA Declaration of Helsinki - Ethical Principles for Medical Research Involving Human Subjects. 59th WMA General Assembly, Seoul, Korea, October 2008; Available at: http://www.wma.net/en/30publications/10policies/b3/. Accessed 04/02, 2013.

(323) Cohen J. Statistical Power Analysis for the Behavioural Sciences. 2nd ed. USA: Lawrence Erlbaum Associates; 1988.

(324) Moher D. CONSORT: an evolving tool to help improve the quality of reports of randomized controlled trials. Consolidated Standards of Reporting Trials. JAMA 1998;279:1489-1491.

(325) World Health Organisation. Fact file: 10 facts of obesity. 2014; Available at: http://www.who.int/features/factfiles/obesity/facts/en/. Accessed November, 17, 2014.

(326) Kapstad H, Hanestad BR, Langeland N, Rustoen T, Stavem K. Cutpoints for mild, moderate and severe pain in patients with osteoarthritis of the hip or knee ready for joint replacement surgery. BMC Musculoskelet Disord 2008 Apr 21;9:55-2474-9-55.

(327) Cleeland C, Mendoza T. The Brief Pain Inventory: meaningful changes in pain interference: Presented at the fourth meeting of the Initiative on Methods, Measurement, and Pain Assessment in Clinical Trials (IMMPACT-IV); June. 2004; Available at: www.immpact.org/immpact4/Cleeland.pdf. Accessed October, 13, 2014.

(328) Hawker GA, Stewart L, French MR, Cibere J, Jordan JM, March L, et al. Understanding the pain experience in hip and knee osteoarthritis--an OARSI/OMERACT initiative. Osteoarthritis Cartilage 2008 Apr;16(4):415-422.

(329) Hawker GA, French MR, Waugh EJ, Gignac MA, Cheung C, Murray BJ. The multidimensionality of sleep quality and its relationship to fatigue in older adults with painful osteoarthritis. Osteoarthritis Cartilage 2010 Nov;18(11):1365-1371.

(330) Holla JF, van der Leeden M, Knol DL, Roorda LD, van der Esch M, Voorneman RE, et al. The association of body-mass index and depressed mood with knee pain and activity limitations in knee osteoarthritis: results from the Amsterdam osteoarthritis cohort. BMC Musculoskelet Disord 2013 Oct 17;14:296-2474-14-296.

(331) de Jong OR, Hopman-Rock M, Tak EC, Klazinga NS. An implementation study of two evidence-based exercise and health education programmes for older adults with osteoarthritis of the knee and hip. Health Educ Res 2004 Jun;19(3):316-325.

(332) Franke RH, Kaul JD. The Hawthorne experiments: First statistical interpretation. . Am Sociol Rev 1978;43:623-643.

(333) Braunholtz DA, Edwards SJ, Lilford RJ. Are randomized clinical trials good for us (in the short term)? Evidence for a "trial effect". J Clin Epidemiol 2001 Mar;54(3):217-224.

(334) Peppercorn JM, Weeks JC, Cook EF, Joffe S. Comparison of outcomes in cancer patients treated within and outside clinical trials: conceptual framework and structured review. Lancet 2004 Jan 24;363(9405):263-270.

(335) Goldenberg DL. The interface of pain and mood disturbances in the rheumatic diseases. Semin Arthritis Rheum 2010 Aug;40(1):15-31.

(336) Bennell KL, Hinman RS. A review of the clinical evidence for exercise in osteoarthritis of the hip and knee. J Sci Med Sport 2011 Jan;14(1):4-9.

(337) van Baar ME, Assendelft WJ, Dekker J, Oostendorp RA, Bijlsma JW. Effectiveness of exercise therapy in patients with osteoarthritis of the hip or knee: a systematic review of randomized clinical trials. Arthritis Rheum 1999 Jul;42(7):1361-1369.

(338) Fransen M, Margiotta E, Crosbie J, Edmonds J. A revised group exercise program for osteoarthritis of the knee. Physiother Res Int 1997;2(1):30-41.

(339) Wijlhuizen GJ, Ooijendijk W. Measuring disability, the agreement between self evaluation and observation of performance. Disabil Rehabil 1999 Feb;21(2):61-67.

(340) Kovar PA, Allegrante JP, MacKenzie CR, Peterson MG, Gutin B, Charlson ME. Supervised fitness walking in patients with osteoarthritis of the knee. A randomized, controlled trial. Ann Intern Med 1992 Apr 1;116(7):529534.

(341) Sampson JA, McAndrew D, Donohoe A, Jenkins A, Groeller H. The effect of a familiarisation period on subsequent strength gain. J Sports Sci 2013;31(2):204-211. 
(342) Krishnan E, Sokka T, Hakkinen A, Hubert H, Hannonen P. Normative values for the Health Assessment Questionnaire disability index: benchmarking disability in the general population. Arthritis Rheum 2004 Mar;50(3):953-960.

(343) Hughes SL, Seymour RB, Campbell RT, Desai P, Huber G, Chang HJ. Fit and Strong!: bolstering maintenance of physical activity among older adults with lower-extremity osteoarthritis. Am J Health Behav 2010 NovDec;34(6):750-763.

(344) Evans JD. Straightforward statistics for behavioral sciences. Pacific Grove, CA: Brooks/Cole publishing; 1996.

(345) Nilsdotter AK, Lohmander LS, Klassbo M, Roos EM. Hip disability and osteoarthritis outcome score (HOOS)--validity and responsiveness in total hip replacement. BMC Musculoskelet Disord 2003 May 30;4:10.

(346) van Baar ME, Dekker J, Oostendorp RA, Bijl D, Voorn TB, Lemmens JA, et al. The effectiveness of exercise therapy in patients with osteoarthritis of the hip or knee: a randomized clinical trial. J Rheumatol 1998 Dec;25(12):2432-2439.

(347) Dias RC, Dias JM, Ramos LR. Impact of an exercise and walking protocol on quality of life for elderly people with OA of the knee. Physiother Res Int 2003;8(3):121-130.

(348) Mazzuca SA, Brandt KD, Katz BP, Chambers M, Byrd D, Hanna M. Effects of self-care education on the health status of inner-city patients with osteoarthritis of the knee. Arthritis Rheum 1997 Aug;40(8):1466-1474.

(349) Jelsma J, Amosun Dea. The reliability and validity of the Xhosa version of the EQ-5D. Disability and Rehabilitation 2004;26(2):103-108.

(350) d'Uva TB, O'Donnell O, van Doorslaer E. Differential health reporting by education level and its impact on the measurement of health inequalities among older Europeans. Int J Epidemiol 2008 Dec;37(6):1375-1383.

(351) Tordrup D, Mossman J, Kanavos P. Responsiveness of the EQ-5D to clinical change: is the patient experience adequately represented? Int J Technol Assess Health Care 2014 Jan;30(1):10-19.

(352) Steyn L, editor. Exercise is Medicine. South African National Health and Nutrition Examination Survery (SANHANES-!); March 2014; ; 2014.

(353) Kohl HW,3rd, Craig CL, Lambert EV, Inoue S, Alkandari JR, Leetongin G, et al. The pandemic of physical inactivity: global action for public health. Lancet 2012 Jul 21;380(9838):294-305.

(354) Shekelle PG, Woolf SH, Eccles M, Grimshaw J. Clinical guidelines: developing guidelines. BMJ 1999 Feb 27;318(7183):593-596.

(355) Sterne JA, White IR, Carlin JB, Spratt M, Royston P, Kenward MG, et al. Multiple imputation for missing data in epidemiological and clinical research: potential and pitfalls. BMJ 2009 Jun 29;338:b2393.

(356) Hadjistavropoulos T, Craig KD, Duck S, Cano A, Goubert L, Jackson PL, et al. A biopsychosocial formulation of pain communication. Psychol Bull 2011 Nov;137(6):910-939.

(357) Wilcox S, Schoffman DE, Dowda M, Sharpe PA. Psychometric properties of the 8-item english arthritis selfefficacy scale in a diverse sample. Arthritis 2014;2014:385256.

(358) Heuts PH, de Bie R, Drietelaar M, Aretz K, Hopman-Rock M, Bastiaenen $\mathrm{CH}$, et al. Self-management in osteoarthritis of hip or knee: a randomized clinical trial in a primary healthcare setting. J Rheumatol 2005 Mar;32(3):543-549.

(359) Gill SD, McBurney H, Schulz DL. Land-based versus pool-based exercise for people awaiting joint replacement surgery of the hip or knee: results of a randomized controlled trial. Arch Phys Med Rehabil 2009 Mar;90(3):388-394.

(360) King L, Hawe P, Wise M. Review of the Literature on Dissemination and Uptake of New Information and Research relating to Health Promotion and Illness/Injury Prevention Activities. University of Sydney 1995 National Centre for Health Promotion.

(361) Max M editor. Pain clinical update: Pain and performance: what are the measures and what do they mean? Simmonds MJ. Seattle, WA: IASP Press; 1999.

(362) The World Bank. How we classify countries. 2013; Available at: http://data.worldbank.org/about/countryclassifications. Accessed 02/06, 2013. 
(363) Murray CJ, Vos T, Lozano R, Naghavi M, Flaxman AD, Michaud C, et al. Disability-adjusted life years (DALYs) for 291 diseases and injuries in 21 regions, 1990-2010: a systematic analysis for the Global Burden of Disease Study 2010. Lancet 2012 Dec 15;380(9859):2197-2223.

(364) KELLGREN JH, LAWRENCE JS. Radiological assessment of osteo-arthrosis. Ann Rheum Dis 1957 Dec;16(4):494-502.

(365) Sale DG. Testing strength and power. In: MacDougal JD, Wenger HA, Green HJ, editors. Physiological testing of the highperformance athlete IL: Champaign; 1991. p. 21-75.

(366) Hessling RM, Schmidt TJ, Traxel NM. Floor Effect. 2004; Available at: http://srmo.sagepub.com/view/thesage-encyclopedia-of-social-science-research-methods/n344.xml. Accessed April, 21, 2014.

(367) Hessling RM, Traxel NM, Schmidt TJ. Ceiling Effect. 2004; Available at: http://srmo.sagepub.com/view/the-sage-encyclopedia-of-social-science-research-methods/n102.xml. Accessed March, 6, 2014. 


\section{Appendix A: ESCEO algorithm for the management of knee $\mathrm{OA}$}

\section{Basic principle and core set}

Combination of treatment modalities, including nonpharmacological and pharmacological therapies is strongly recommended
Core set: Information/education, weight loss if overweight,exercise program (aerobic, strengthening)

\section{STEP 1: Background treatment}

If symptomatic:

(Paracetamol on a regular basis) OR Chronic SYSADOA: prescription glucosamine sulfate and/or chondroitin sulfate \pm as needed paracetamol
Referral to physiotherapist for: to control malalignment: Knee braces, insoles.

If symptomatic ADD at any time:

Walking aids, thermal agents, manual therapy, patellar taping, Chinese acupuncture, TENS

\section{STEP 2: Advanced pharmacological management in the persistent symptomatic patients}

\section{If still or severly symptomatic:}

Intermittent or continuous (longer cycles) oral NSAIDs. NORMAL GI RISK - Non selective NSAID with PPI-Cox-2 selective NSAID (consider PPI). INCREASED GI RISK* - Cox-2 selecve NSAID with PPI-Avoid non-selective NSAIDs *Including use of low dose aspirin. INCREASED CV RISK - Prefer naproxen, avoid high-dose diclofenac and ibuprofen (ifonlow-dose aspirin), caution with other non-selective NSAIDs, avoid Cox-2 selecve NSAIDs. INCREASED RENAL RISK - Avoid NSAIDs hyaluronate and intraarticular corticosteroids 


\section{STEP 3: Last pharmacological attempts}

Short-term weak opioids

Duloxetine

STEP 4: End-stage disease management and surgery

If severely symptomatic and poor quality of life: Total joint replacement

(Unicompartmental knee replacement)
If surgery contraindicated:

Opiod analgesics

\section{AppendixA-1 Knee OA treatment algorithm}




\title{
Appendix B: Ethical approval letters
}

Letter of approval from the University of Cape Town's Faculty of Health

Sciences, Human Resource Ethics Committee

HREC Ref no 378/2013 - 15Jul2013

UNIVERSITY OF CAPE TOWN

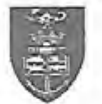

Faculty of Health Sciences Room E52-24 Groote Schuur Hospital Old Main Building observatory 7925 Telephone [021] 4066338 . Facsimile [021] 4066411 e-mail: shuretta.thomas@uct.ac.z

15 July 2013 Website: www, health.uct.ac.za/research/humanethics/forms

HREC REF: $378 / 2013$

\author{
Ms M Saw \\ c/o Dr R Parker \\ Physiotherapy \\ Health \& Rehab \\ OMB, F-Floor
}

Dear Ms Saw

PROJECT TITLE: THE EFFECTS OF A SIX-WEEK PHYSIOTHERAPIST-LED EXERCISE AND EDUCATION INTERVENTION IN PATIENTS, WITH OSTEOARTHRITIS AWAITING AN ARTHROPLASTY, IN THE WESTERN CAPE

Thank you for submitting your study to the Faculty of Health Sciences Human Research Ethics Committee for review.

It is a pleasure to inform you that the HREC has formally approved the above-mentioned study.

Approval is granted for one year till the $30^{\text {th }}$ July 2014

Please note that a witness signature is only required if participants are not able to provide written consent.

Please submit a progress form, using the standardised Annual Report Form if the study continues beyond the approval period. Please submit a Standard Closure form if the study is completed within the approval period.

(Forms can be found on our website: www.health.uct.ac.za/research/humanethics/forms)

Please note that the ongoing ethical conduct of the study remains the responsibility of the principal investigator.

Please quote the HREC. REF in all your correspondence.

Yours sincerely

PROFESSOR M BLOCKMAN

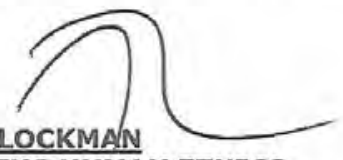

CHAIRPERSON, FHS HUMAN ETHICS

Federal Wide Assurance Number: FWA00001637.

Institutional Review Board (IRB) number: IRB00001938

s.thomas 
ETHICS NO: HREC REF - 378/2013 (UNIVERSITY OF CAPE TOWN)

The effects of a six-week physiotherapist-led exercise and education intervention in patients with osteoarthritis awaiting an arthroplasty in the Western Cape.

Dear Ms M Saw

PERMISSION TO CONDUCT YOUR RESEARCH AT TYGERBERG HOSPITAL

In accordance with the Provincial Research Policy and Tygerberg Hospital Notice No 40/2009, permission is hereby granted for you to conduct the above-mentioned research here at Tygerberg Hospital.

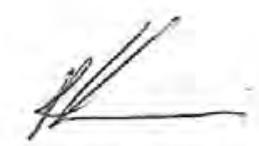

DR RR THOMSON

MANAGER: MEDICAL SERVICES

Date: $22 / 8 / 2013$ 
UCT HREC amendment approval for the additional open ended questionnaire to be included at month six follow up

FitsG! Annua! Prograss IRejort/ Renewal

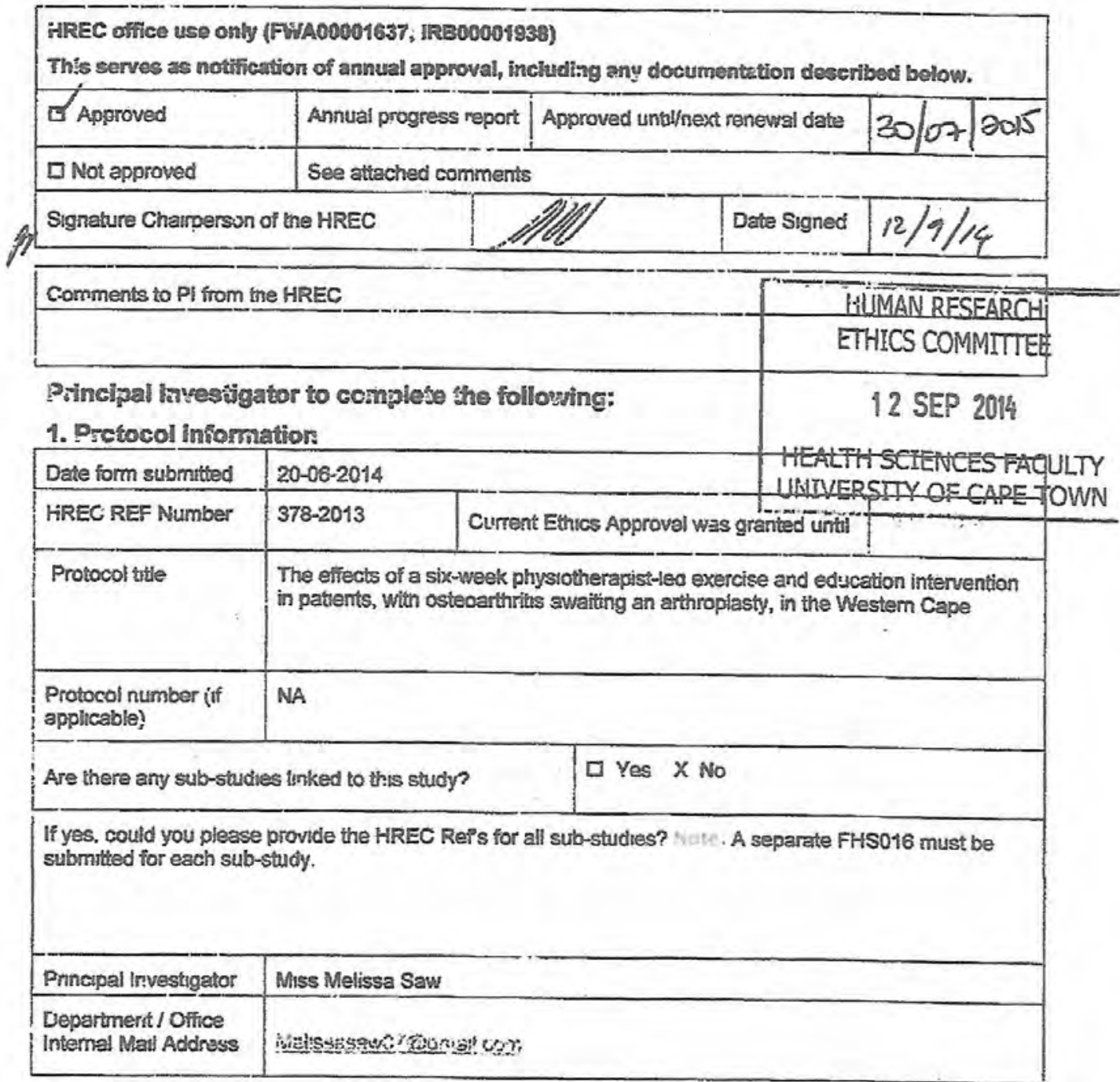

\begin{tabular}{|l|l|l|}
\hline 11 Does this protocol recenve US Federal funding? & $\square$ Yes & $X$ No \\
\hline $\begin{array}{l}12 \text { If the study recerves US Federal Funding, does the annual report } \\
\text { requre full committee apuroval? }\end{array}$ & $\square$ Yes & $X$ No \\
\hline
\end{tabular}

11 Fabnuary $2014 \quad$ Page 1 of 7 FHSO16

( f : Please complete the Closure form (FI:Ss: 6 ) if the study is completed within the approval pariod) 


\section{Appendix C: Study information and informed consent document}

English version (Initially explained telephonically and then explained in person and given to patients at week 0 session)

The effects of a six-week physiotherapist-led exercise and education intervention, in patients with osteoarthritis, awaiting an arthroplasty in the Western Cape.

\section{What are we trying to do?}

I am a master's student doing research from the University of Cape Town and I am interested in finding out whether a six week long course for people with arthritis will make a difference to your health. The course aims to increase your knowledge about arthritis, as well as teaching you various light exercises and relaxation techniques. I want to answer questions such as does increasing your knowledge of arthritis affect the way you cope with your condition and take part in daily activities? In order to answer these questions we would like to interview people whom we know have arthritis and invite them to commit to taking part in a six-week exercise and education course.

\section{Why have we contacted you?}

We have contacted you because you are on the waiting list for a joint replacement at Tygerberg hospital and this is the group of people we are looking at.

\section{What will you be asked to do?}

- If you are interested in the study, you will be asked to come into the hospital for the first visit. At this initial visit you will be asked a few questions which will check if it is safe for you to take part in exercise. If it is not safe, you will not be asked to continue. 
- You will be told about the study and any questions you may have will be answered.

- You will be assisted to complete four short questionnaires (in your preferred language; English, Afrikaans or Xhosa) which ask general questions about you and your health.

- You will be asked to do seven short tasks which test how well you do everyday activities. This consists of three short walking tests at different speeds and distance, two tests whereby you reach with your arms. One test which looks how you stand up and sit down and one which looks at how you put a sock on your foot. These will be timed to see how much time you need for each one. This will find out more about whether you can cope with your condition, and what things are difficult for you to do.

- This first visit should take about an hour to complete. We know that this is a long time but we want to get as much information as possible so that we can better understand the problems that you face.

- You will then be placed in one of two groups. The control or the course group. You will have an equal chance of being placed in either of these groups.

- If you are placed in the group for the course you will be asked to come back to the Tygerberg Physiotherapy department at the hospital the following week for the course which will happen once a week for two hours. This course lasts for six weeks. You will take part in this course with a group of 12 people who all have arthritis either on a Wednesday or a Thursday afternoon between $1 \mathrm{pm}$ and $3 \mathrm{pm}$.

- The course will include discussions on arthritis, diet and other health related issues aimed at helping you cope with your condition as well as gentle exercises and a time of relaxation. You will be asked to sign an agreement to respect the privacy of those attending the group, by not sharing group member's information outside of the group.

- At the last weekly session you will be asked to fill out the questionnaires and do the short tasks again like you did at the first visit.

- You will be asked to return to do these measurements after another six weeks and lastly after six months since beginning the study. This is so that we can see if anything has changed since the start. 
- If you are placed in the control group you will continue with your usual care that your doctor has prescribed. You will be asked to return to fill out the questionnaires and do the tasks again after six weeks and 12 weeks and lastly, after six months, so that we can see if anything has changed. This group is just as important as the other group and gives us essential information that we need to compare the six week programme with your usual care programme.

Should there be a difference in the two groups and the course shows that it helps people with arthritis, if you were in the control group, you will be invited to be a part of the six week course and you may join this group for six sessions after the study. We will want to stay in contact with you for six (6) months from the beginning to the end of this project so that we can see how you are doing over a period of time. To help us keep in contact with you we will ask you to give us a telephone number where you can be contacted.

\section{Is there any payment for taking part in the study?}

There is no cost to you to take part in this study and there is no payment to you for taking part in the study. We will give you R30 to cover transport costs each time you visit the hospital for this study.

\section{What are the benefits to you?}

We hope that you will learn more about arthritis by doing this course and that this might help you to cope better with your condition. We hope that you will learn the importance of exercise and how it may benefit you by making you fitter or stronger and possibly make it easier to do certain tasks. We hope that you will learn more about setting goals and achieving these and how to believe more in your abilities. 


\section{What are the risks to you?}

We aim to minimise any risks to you which may be caused by exercise having a qualified physiotherapist lead all your exercise sessions and by checking beforehand if it is safe for you to exercise. Exercise is known to cause some stiffness or mild ache in the muscles the day/two afterwards if exercising is new to you , but we will try minimise this by seeing what you can do in the beginning and starting off at a slow pace. Each week as you get used to exercising, we will see if you can do slightly more exercise. If for some reason, you experience any marked increase in pain during the study you will be assessed and if necessary you will be advised to do less exercise or stop and will be referred to the appropriate doctor at your nearest day hospital to manage this.

\section{What will the results be used for?}

We will make the information which we gather known (but of course not your personal details) to the local authorities, to the local institutions that provide assistance to people living with arthritis and to provincial and central government. We hope that the information we get might lead to changes being made in the treatment of patients with arthritis, but we cannot promise this. In the short term we cannot say for definite that there will be any direct benefit to you or your family.

\section{Will people know what answers you give to the questions?}

You will be given a code in the beginning of the study and this code will be used instead of your name. All the answers will be put together and no-one will know who gave any specific answer except the researchers. Your name will not be given to anyone and will not be listed anywhere. The results of the project will be made available to organizations involved in assisting people living with arthritis, local and government authorities and the scientific community but no names will be linked to any results.

Your usual care and appointments will continue as normal if you are in this study. Nothing bad will happen to you if you do not want to take part. It is completely voluntary. Even if you do take part, you can stop at any time and you can refuse to answer specific questions. 
If you wish to stop attending this will have no impact on your normal care. Taking part or not taking part in the study will not affect your chances of getting a joint replacement nor change your position on the waiting list. If you have any questions during the course of the study you are welcome to contact myself or my supervisors or ask during any of the sessions.

If you would like, the researcher will refer people who take part to whatever services they need which may be available in the area. Your participation is appreciated.

\section{Melissa Saw (physiotherapist)}

Tygerberg Hospital B5 west physiotherapy department

(021) 9385152

\section{Romy Parker (supervisor)}

UCT Dept of Health and Rehabilitation Sciences: Division of Physiotherapy

(021) 4066431.

If you have any questions or concerns about your rights as a research participant, please contact:Prof Marc Blockman: 0214066492

Please note that UCT does offer a no-fault insurance that will cover all participants in the event that something may go wrong. This insurance will provide prompt payment of compensation for any trial-related injury in accordance with the Association of the British Pharmaceutical Industry (ABPI) guidelines (1991). These guidelines recommend that UCT, without any legal commitment, should compensate you without you having to prove that UCT is at fault. An injury is considered trial-related if, and to the extent that, it is caused by study activities. You must notify the study investigators immediately of any injuries during the trial, whether they are research-related or other related complications. UCT reserves the right not to provide compensation if, and to the extent that, your injury came about because you chose not to follow the instructions that you were given while taking part in the study. Your right in law to claim compensation for injury where you prove negligence is not affected. 


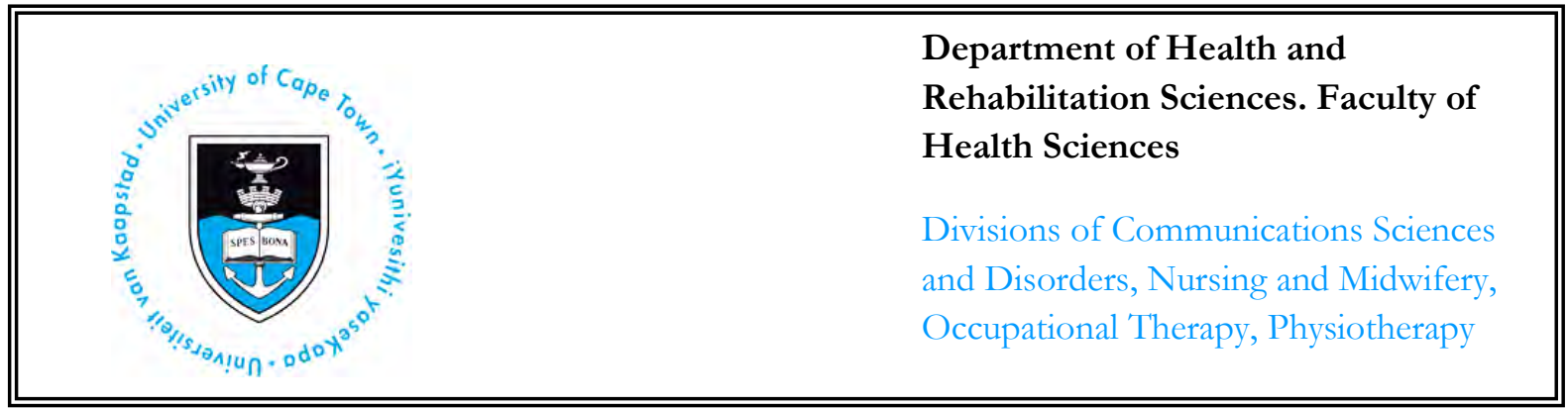

Dear participant

Please read the attached information sheet for the study titled: The effects of a six-week physiotherapist-led exercise and education intervention, in patients with osteoarthritis, awaiting an arthroplasty in the Western Cape.

We hope that this research will help health professionals to better understand whether a six-week course (including exercise and education) on arthritis helps people to cope with living with the condition. All questionnaires are anonymous and records will be kept strictly confidential.

You are welcome to contact the Investigator, Melissa Saw on (021) 9385152, or the supervisor, Romy Parker on (021) 4066431 or Professor Blockman on (021) 4066492 for further details about the research and your rights. This research is voluntary and refusal to participate or decision to withdraw at any time will involve no penalty or loss of benefits to which you, the participant, are otherwise entitled.

I, have read and understand the above information sheet,

I have had all my questioned answered. I understand what is required of me and have willingly chosen to participate in the study. I know that my usual care continues as normal and I am not forced into taking part and that I can withdraw at any time and that I do not have to answer all of the questions if I do not want to. I know that there will be no consequences to my usual care if I wish to withdraw from the study at any time. I give permission for the researchers to contact me to interview me. I agree to attend the course for six (6) weeks if I am placed in the course group, and I agree to visit the hospital and take part in the study for a period of six (6) months.

Participant

Date

Witness

Date

Researcher

Date 


\section{Appendix D: Telephonic questions and the ACSM screening for exercise guidelines}

In order for us to use the information we get from you we need to have a group of people who are similar to each other in age, language and health. So I need to ask you a few questions first to make sure you fit in this group that we want to look at in order to be a part of this study.

1. How old are you?

2. Can you understand, read and write either English or Afrikaans or isiXhosa?

3. Do you have osteoarthritis in your legs? Which joint/s is affected?

4. Have you had any accidents/previous surgery for problems in your legs before?

5. Do you have any other joint problems in your legs?

6. Do you have any condition which affects your ability to understand concepts?

7. Have you participated in any self-management programme for arthritis before?

As a part of this study you will be asked to do exercises. I need to ask you some questions now to make sure that it will be safe for you to do these exercises.

a. Do you have or have you had any of the following? (Category 1 - immediate exclusion. Yes to any of these, stop the interview and thank them for their time. They are not eligible for the study)

A heart attack

Heart surgery

_ Cardiac catheterization

__ Coronary angioplasty (PTCA)

Pacemaker/implantable cardiac defibrillator/rhythm disturbance

Heart valve disease

Heart failure

Heart transplantation

Congenital heart disease 
b. Screening question (see specific responses)

- Do you have diabetes?

IF YES, Is it controlled by medication? Yes - OK; No - end interview, thank you but not eligible

- Do you have asthma other lung disease?

IF YES, Is it controlled by medication? Yes - OK; No - end interview, thank you but not eligible

YES to $\mathbf{2}$ or more of these 4 following questions not eligible, end interview, thank you for your time

1. __ Do you have burning or cramping in your lower legs when walking short distances.

2. __ Do you experience chest discomfort with exertion.

3. __ Do you experience unreasonable breathlessness.

4. ___ Do you experience dizziness, fainting, blackouts.

5. __ Are you pregnant? 


\section{AHA/ACSM Health/Fitness Facility Preparticipation Screening Questionnaire}

\section{Assess your bealth status by marking all true statements}

\section{History}

You have had:

a heart attack

heart surgery

_ candiac catheterization

coronary angioplasty (PTCA)

_ pacemakerfimplantable cardiac

defibrillator/rhythm disturbance

heart yalve disease

heart failure

- heart transplantation

congenital heart disease

\section{Symptoms}

You experience chest discomfort with exertion.

You experience unreassonable bre athlessness.

You experience dizziness, fainting or blackouts

You tale heart medications.

\section{Other health issues}

You have diabetes.

You have asthma or other lung disease.

You have burning or cramping sensation in your lower

legs when walking short distanoes.

You have musculoskelatal problems that limit your

physical activity.

You have concerns about the safety of exercise.

You take prescription medication(s).

You are pregnant.

If you marked any of these statements in this section, consult your physician or other appropriate health care provider before engaging in exercis. You may need to use a facility with a medically qualified starf.

Cardiovascular risk factors

You are a man older than 45 years

You are a woman older than 55 years,

have had a hysterectomy, or are postmenopausal.

You smoke, or quit smoking within the previous 6 months

Your blood pressure is $>140 / 90 \mathrm{~mm} \mathrm{Hg}$.

You do not know your blood pressure.

You take blood pressure medication.

Your blood cholesterol level is $>200 \mathrm{mg} / \mathrm{dL}$.

You do not know your cholesterol level.

You have a close blood relative who had a heart attack

or heart surgery before age 55 (father or brother) or age 65 (mother or sister).

You are physically inactive (i.e., you get $<30$ minutes of physical activity on at least 3 days per week).

You are $>20$ pounds overweight.

If you marked two or more of the statements in this section you should oonsult your physician or other appropriate health care provider before engaging in exercise. You might be nefit from using a facility with a professionally qualified exercise staff to guide your exercise program.
You should be able to exercise safely without consulting your physician or other appropriate health care provider in a selfguided program or almost any facility that meets your exercise program needs. 


\section{Appendix E: Data collection documents and outcome measure instruments}

Demographic information document and outcome measures

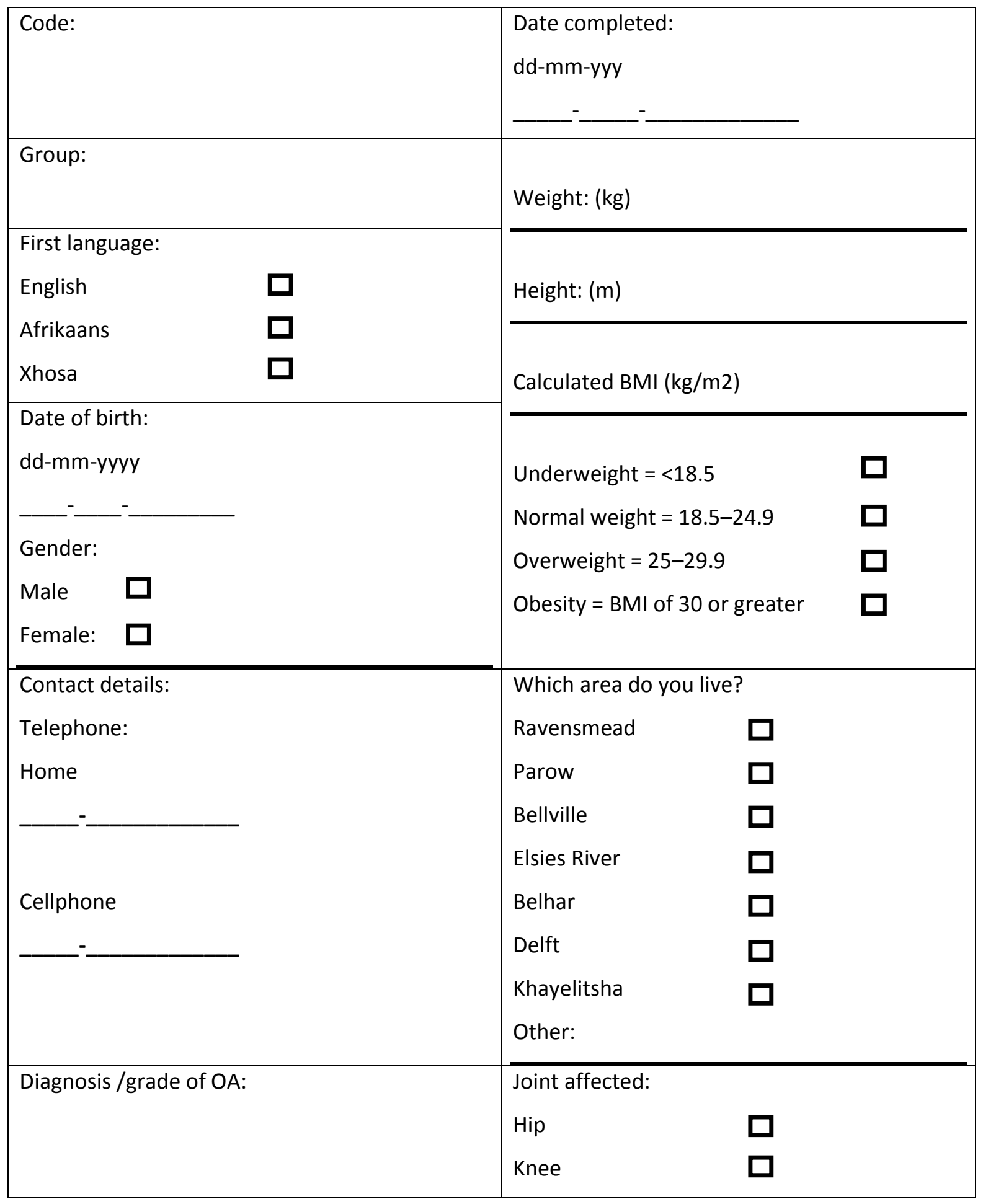




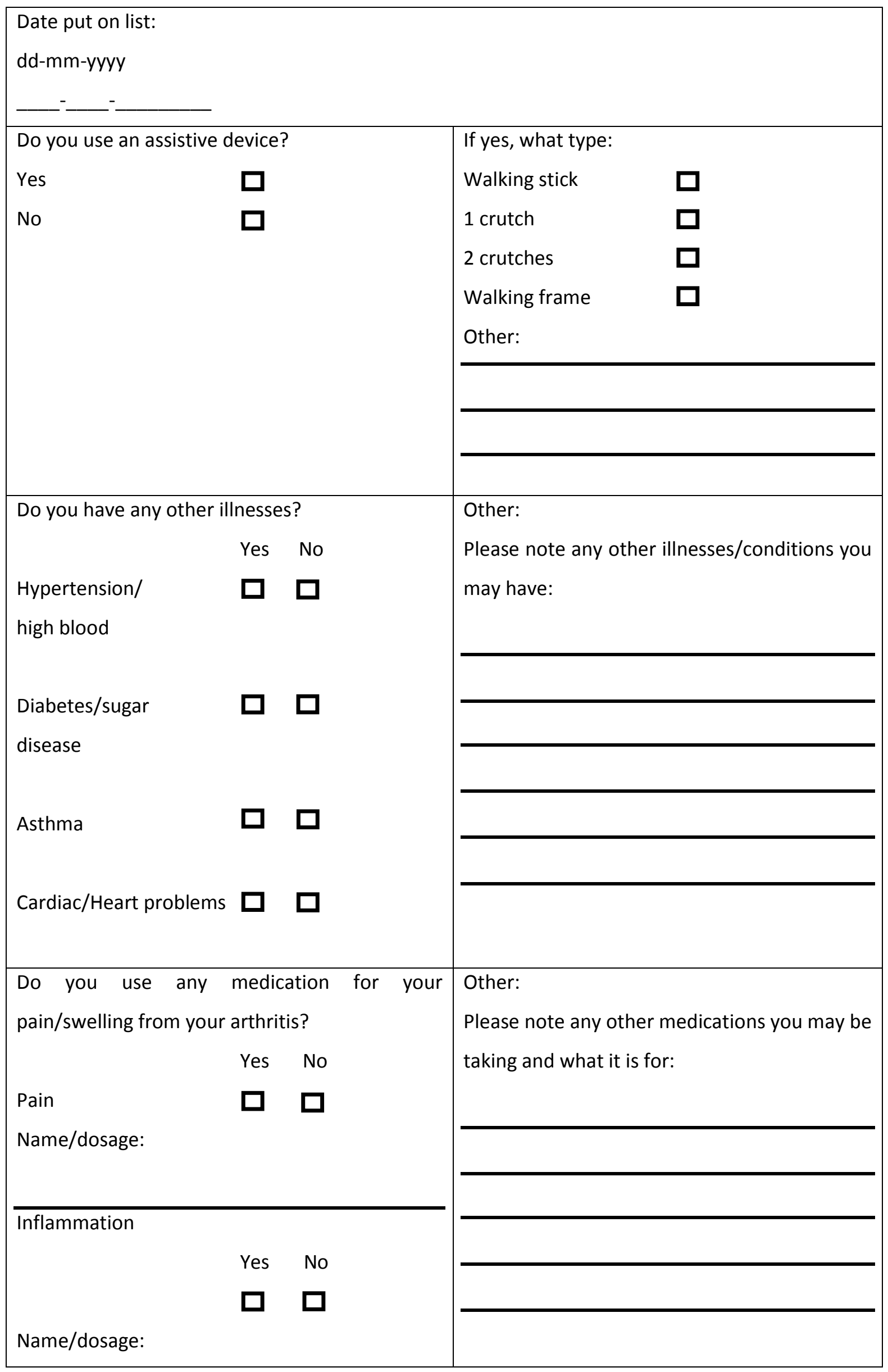




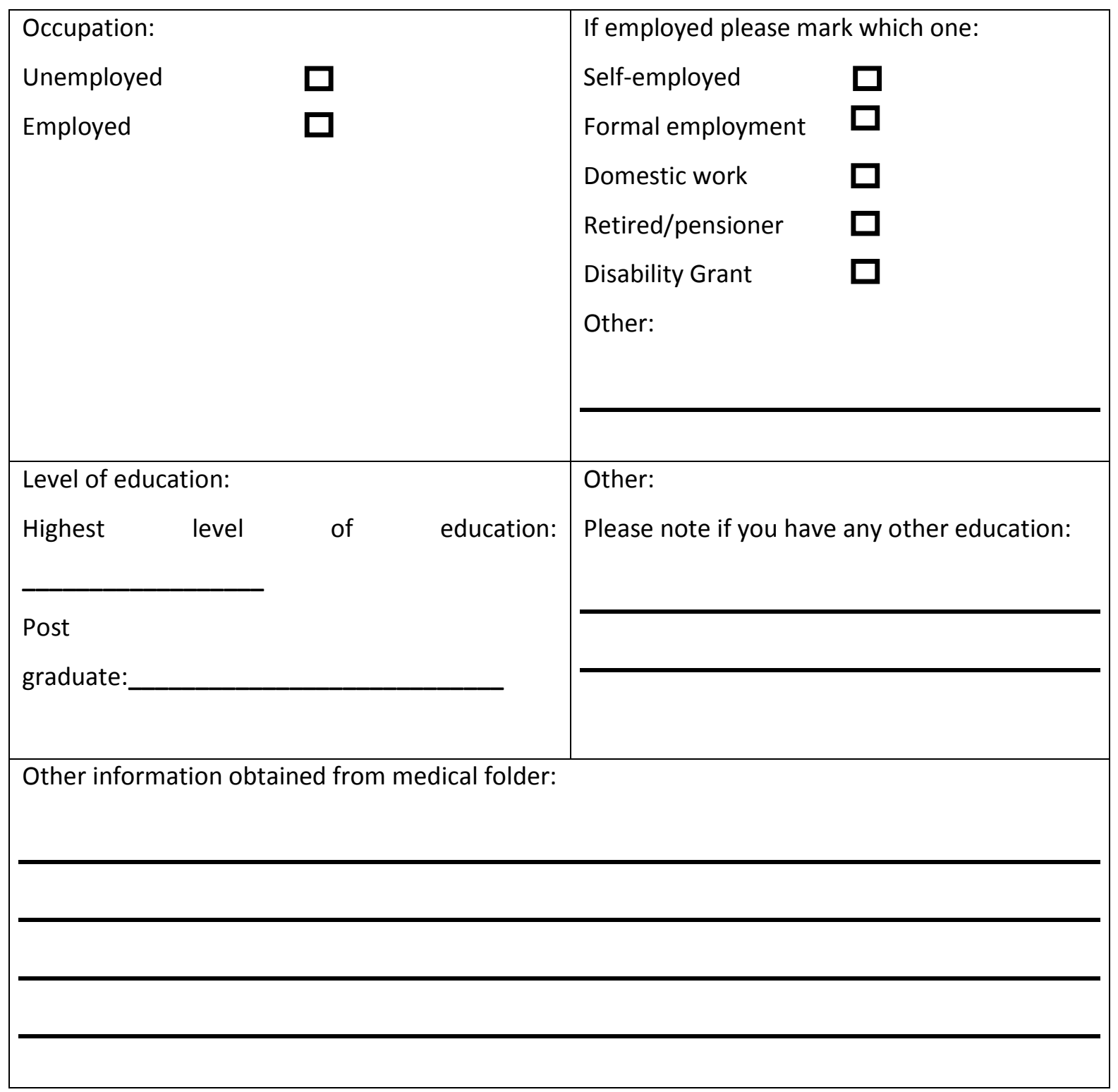




\section{Brief Pain Inventory}

1. Throughout our lives, most of us have had pain from time to time (such as minor headaches, sprains, and toothaches). Have you had pain other than these everyday kinds of pain during the last week?

Yes

$$
\text { No }
$$

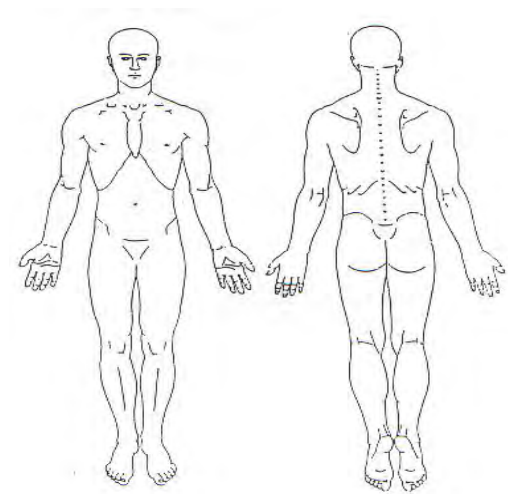

2. On the diagram, shade in the areas where you feel pain. Put an $\mathbf{X}$ on the area that hurts the most.

3. Please rate your pain by circling the one number that best describes your pain at its worst in the last week.

$$
1
$$

2

$$
3
$$

4

5

6

7

8

9

10

No

Pain as bad as

Pain you can imagine

4. Please rate your pain by circling the one number that best describes your pain at its least in the last week.

0

1

2

34

5

6

7

8

9

10

No

Pain as bad as

Pain you can imagine

5. Please rate your pain by circling the one number that best describes your pain on the average.

1

2

34

5

6

7

8

9

10

No

Pain as bad as

Pain

you can imagine

6. Please rate your pain by circling the one number that tells how much pain you have right now.

$\begin{array}{lllllllllll}0 & 1 & 2 & 3 & 4 & 5 & 6 & 7 & 8 & 9 & 10\end{array}$

No

Pain as bad as

Pain

you can imagine 
7. What treatments or medications are you receiving for your pain?

8. In the last week, how much relief have pain treatments or medications provided? Please circle the one percentage that most shows how much relief you have received.

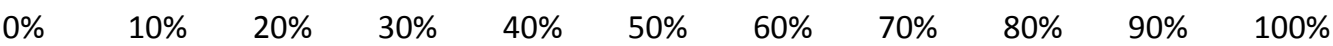

No Relief

Complete relief

9. Circle the one number that describes how much, during the past week, pain has interfered with your:

\section{A. General Activity}

$\begin{array}{lllllllllll}0 & 1 & 2 & 3 & 4 & 5 & 6 & 7 & 8 & 9 & 10\end{array}$

Does not interfere

Completely interferes

\section{B. Mood}

$\begin{array}{lllllllllll}0 & 1 & 2 & 3 & 4 & 5 & 6 & 7 & 8 & 9 & 10\end{array}$

Does not interfere

Completely interferes

\section{Walking Ability}

$\begin{array}{lllllllllll}0 & 1 & 2 & 3 & 4 & 5 & 6 & 7 & 8 & 9 & 10\end{array}$

Does not interfere

Completely interferes

D. Normal Work (includes both work outside the home and housework)

$\begin{array}{lllllllllll}0 & 1 & 2 & 3 & 4 & 5 & 6 & 7 & 8 & 9 & 10\end{array}$

Does not interfere

Completely interferes

\section{E. Relations with other people}

$\begin{array}{lllllllllll}0 & 1 & 2 & 3 & 4 & 5 & 6 & 7 & 8 & 9 & 10\end{array}$

Does not interfere

Completely interferes

\section{F. Sleep}

$\begin{array}{lllllllllll}0 & 1 & 2 & 3 & 4 & 5 & 6 & 7 & 8 & 9 & 10\end{array}$

Does not interfere

Completely interferes

\section{G. Enjoyment of life}

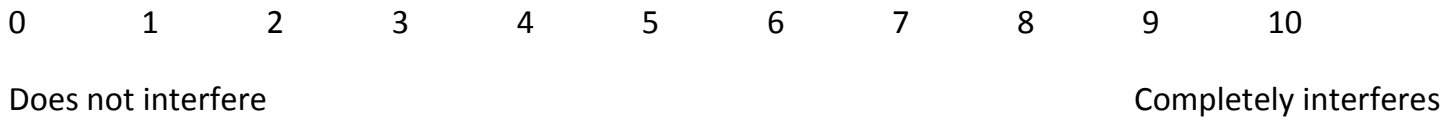




\section{HEALTH ASSESSMENT QUESTIONNAIRE}

Name

Date

In this section we are interested in learning how your illness affects your ability to function in daily life. Please feel free to add any comments on the back of this page.

Please tick $(\checkmark)$ the response which best describes your usual abilities DURING THE PAST WEEK:

\section{DRESSING \& GROOMING}

$\begin{array}{llll}\text { Without } & \text { With } & \text { With } & \text { UNABLE } \\ \text { ANY } & \text { SOME } & \text { MUCH } & \text { To Do } \\ \text { Difficulty } & \text { Difficulty } & \text { Difficulty } & \end{array}$

Were you able to:

- Dress yourself, including tying shoelaces and doing buttons?

- Wash your hair?

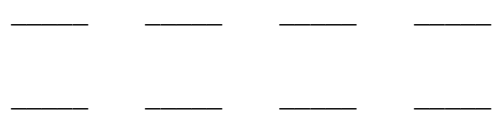

\section{GETTING UP}

Were you able to:

- Stand up from a chair without armrests?

- Get in and out of bed?
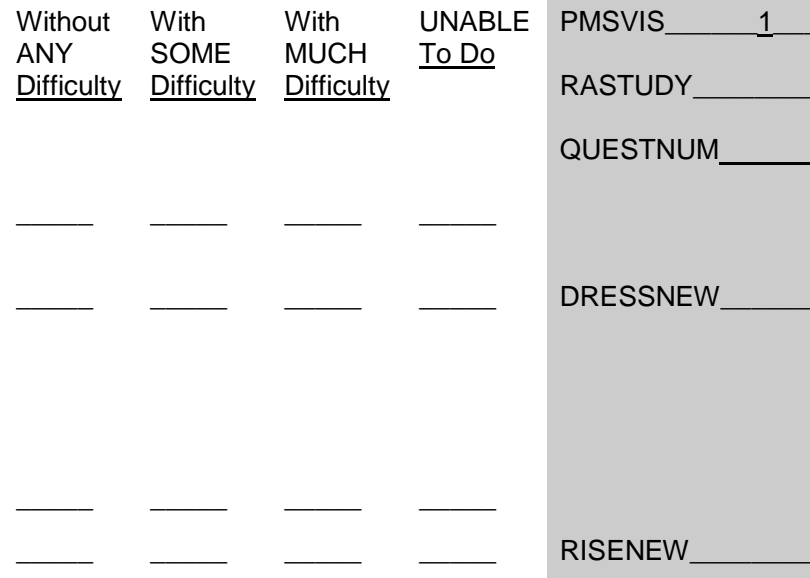

\section{EATING}

Were you able to:

- Cut up your meat?

- Lift a full cup or glass to your mouth?

- Open a new milk carton?

\section{WALKING}

Were you able to:

- Walk outdoors on flat ground?

- Climb up five steps?
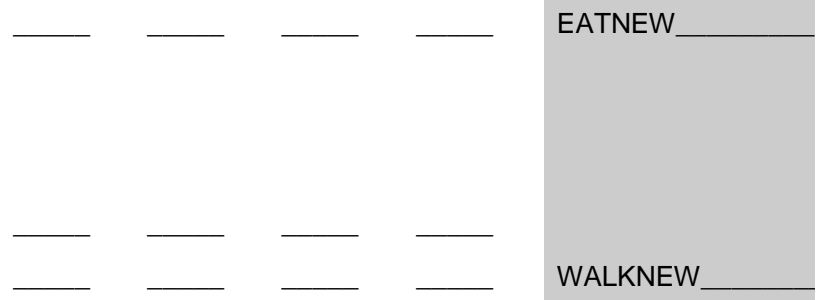

Please tick $(\checkmark)$ any of the following AIDS OR EQUIPMENT that you usually use for any of the activities mentioned above:

Walking stick
Walking frame
Crutches
Wheelchair

\begin{tabular}{|c|}
\hline \begin{tabular}{l}
\multicolumn{1}{c}{ Aids used for } \\
dressing \\
hook, (button \\
long-handled sholler, \\
horn, etc.)
\end{tabular} \\
\hline $\begin{array}{l}\text { Specially adapted } \\
\text { utensils (such as for } \\
\text { eating and cooking) }\end{array}$ \\
\hline $\begin{array}{l}\text { Specially } \\
\text { chair }\end{array}$ \\
\hline $\begin{array}{l}\text { Other } \quad \text { (Please } \\
\text { specify: }\end{array}$ \\
\hline
\end{tabular}

RISENEW

WALKNEW

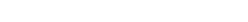

$$
\begin{array}{cc}
\text { Other } \quad \text { (Please } \\
\text { specify:___ }
\end{array}
$$

DRSGASST

RISEASST

Please tick $(\checkmark)$ any of the following categories for which you usually need HELP FROM ANOTHER PERSON: 
Please tick $(\checkmark)$ the response which best describes your usual abilities DURING THE PAST WEEK:

\section{HYGIENE}

$\begin{array}{llll}\text { Without } & \text { With } & \text { With } & \text { UNABLE } \\ \text { ANY } & \text { SOME } & \text { MUCH } & \text { To Do } \\ \text { Difficulty } & \text { Difficulty } & \text { Difficulty } & \end{array}$

Were you able to:

- Wash and dry your body?

- Have a bath?

- Get on and off the toilet?

\section{REACHING}

Were you able to:

- Reach up for and take down a
$2,5 \quad \mathrm{~kg} \quad$ object
(e.g. a bag of sugar) from just above your head?

- Bend down to pick up clothing from the floor?
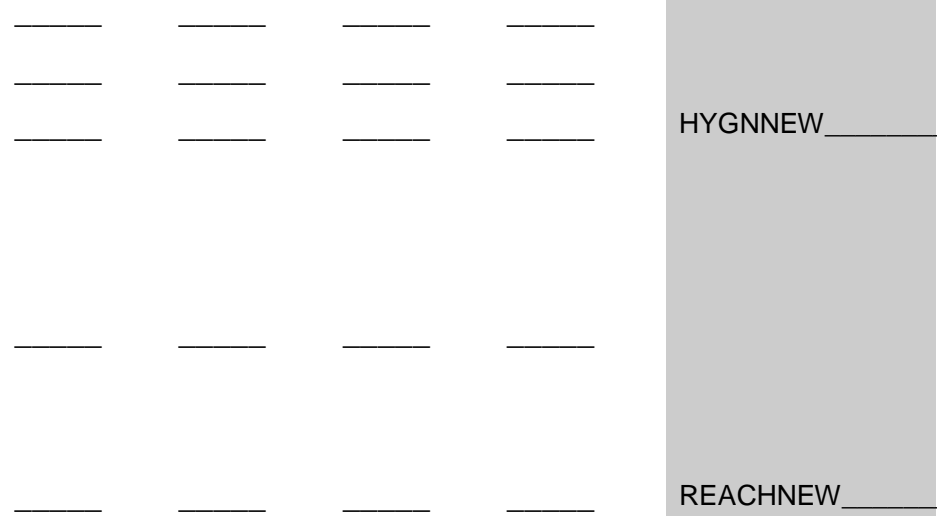

\section{GRIPPING}

Were you able to:

Sample copy - Do not use without permissi

- Open car doors?

- Open jars that have been previously opened?

- Turn taps on and off?

Sampiple copy - Do not use without permis
on and off?

\section{ACTIVITIES}

Were you able to:

- Go shopping (supermarket, post office, bank, etc.)?

- Get in and out of a car?

- Do domestic tasks such as

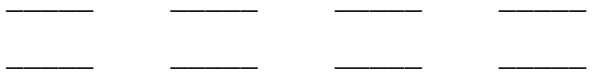
vacuuming or gardening?

GRIPNEW

Please tick $(\checkmark)$ any of the following AIDS OR EQUIPMENT that you usually use for any of the activities mentioned above:

Raised toilet seat
Bath seat

Jar opener (for jars not previously opened) (brush for the body)
Bath rail

Long-handled aids for reaching things

Long-handled aids bathroom Other

(Please specify 
Please tick $(\checkmark)$ any of the following categories for which you usually need HELP FROM ANOTHER PERSON:

$\begin{array}{lll}\text { Hygiene } & - & \begin{array}{l}\text { Gripping and } \\ \text { opening things }\end{array} \\ \text { Reaching } & \quad \begin{array}{l}\text { Shopping and } \\ \text { domestic tasks }\end{array}\end{array}$

We are also interested in learning whether or not you are affected by pain because of your illness.

How much pain have you had because of your illness DURING THE PAST WEEK:

PLACE A VERTICAL (I) MARK ON THE LINE TO INDICATE THE SEVERITY OF THE PAIN.

NO

SEVERE

PAIN

PAIN

0

100

HAQ ๔ Stanford University, 1980, All rights reserved.South Africa/English - 31 January 2001 - Mapi Research Institute. ID1465 /HAQ_AU2.0_engZA.doc 


\section{Physical Performance Task Battery}

The original performance battery consisted of a series of nine tasks. Tasks were aimed at testing arm and total body movements in seated and standing positions. Since this study's focus was on lower limb OA, tasks testing fine motor skills of the upper limbs were intentionally omitted. Distances were converted to metres instead of feet for the use in an SA context. The majority of the tasks were timed as usually a decline in performance of a task is seen by decreased reaction time or speed of the movement. ${ }^{(361)}$

The tasks are described as follows:

1. 15 metre $(\mathrm{m})$ walk at preferred speed. For this test, participants were timed (sec) as they walk $7.5 \mathrm{~m}$ turn around, and walk back to the starting position at their preferred walking speed.

2. $15 \mathrm{~m}$ walk at fastest speed. Participants were again timed (sec) as they walk $7.5 \mathrm{~m}$, turn around, and walk back to the start at their fastest walking speed.

3. Forward reach. For this test, participants stood adjacent to a wall on which a tape measure was positioned horizontally at shoulder height. Participants reached forward as far as they could and the distance reached was measured in centimetre $(\mathrm{cm})$.

4. Timed, repeated reach-up. Participants stood facing a wall and reached up as high as they could with both hands. A mark was placed on the wall at the reached distance. Participants were then times (sec) as they reached up to the mark and returned their hands to their sides three times.

5. Timed, repeated sit-to-stand. Participants sat in a standard chair with arm rests and were timed as they stood up and sat back down, twice. The test was repeated after a brief rest and the average time (sec) of the two trials was used.

6. Distance walked in six minutes. Participants walked as far and as fast as they could for six minutes. The distance walked is measured in metres at six minutes (Participants were allowed to rest if and as necessary during the six minute period).

7. Sock test. Participants sat in a standard chair. They were timed (sec) as they put on one loose-fitting sock on each foot separately.

Participants were instructed to complete each task above and if any worsening of symptoms occurred during the tests the participants were allowed to slow down or stop the task and this was noted. If the participant could not complete a task this was documented. 
Result sheet for Physical Performance Task Battery test and BMI measures

Patient code:

\begin{tabular}{|c|c|c|c|c|}
\hline Test: & Result week 0: & Week 6: & Week 12 & Month 6 \\
\hline $\begin{array}{l}15 \text { metre }(\mathrm{m}) \text { walk at } \\
\text { preferred speed. }\end{array}$ & $\mathrm{Sec}$ & & & \\
\hline $15 \mathrm{~m}$ walk at fastest speed & $\mathrm{Sec}$ & & & \\
\hline Forward reach & $\mathrm{cm}$ & & & \\
\hline $\begin{array}{l}\text { Timed, repeated sit-to- } \\
\text { stand }\end{array}$ & $\begin{array}{l}1^{\text {st }} \\
2^{\text {nd }} \\
\text { Average: } \\
\text { Sec }\end{array}$ & & & \\
\hline Sock test & $\mathrm{Sec}$ & & & \\
\hline Timed, repeated reach-up & $\mathrm{Sec}$ & & & \\
\hline $\begin{array}{l}\text { Distance walked in } 6 \\
\text { minutes }\end{array}$ & $\mathrm{m}$ & & & \\
\hline Assistive device used & & & & \\
\hline Other notes: & & & & \\
\hline BMI measurements & $\begin{array}{l}\text { Height: } \\
\text { Weight: }\end{array}$ & & $\begin{array}{l}\text { Height: } \\
\text { Weight: }\end{array}$ & $\begin{array}{l}\text { Height: } \\
\text { Weight: }\end{array}$ \\
\hline
\end{tabular}




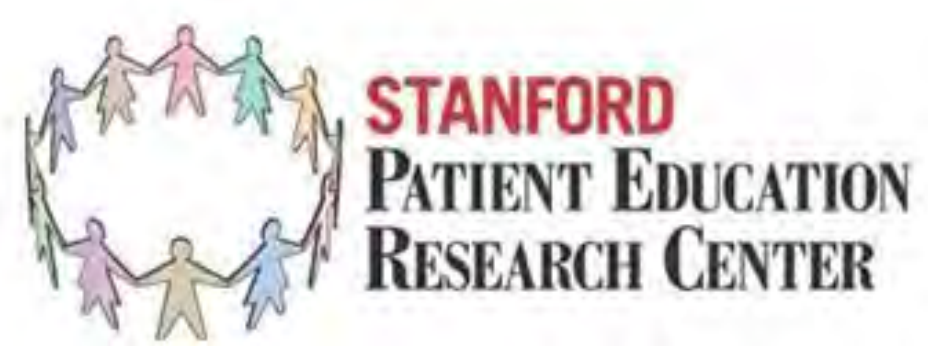

\section{Self-Efficacy for Managing Chronic Disease 6-Item Scale}

We would like to know how confident you are in doing certain activities. For each of the following questions, please choose the number that corresponds to your confidence that you can do the tasks regularly at the present time.

1. How confident are you that you can keep the fatigue caused by your disease from interfering with the things you want to do?

\begin{tabular}{lllllllllllll}
\hline Not at all & $\mid$ & $\mid$ & $\mid$ & $\mid$ & $\mid$ & $\mid$ & $\mid$ & $\mid$ & $\mid$ & $\mid$ & totally \\
confident & 1 & 2 & 3 & 4 & 5 & 6 & 7 & 8 & 9 & 10 confident
\end{tabular}

2. How confident are you that you can keep the physical discomfort or pain of your disease from interfering with the things you want to do?

\begin{tabular}{lllllllllllll|l}
\hline Not at all & $\mid$ & $\mid$ & $\mid$ & $\mid$ & $\mid$ & $\mid$ & $\mid$ & $\mid$ & $\mid$ & $\mid$ & totally \\
confident & 1 & 2 & 3 & 4 & 5 & 6 & 7 & 8 & 9 & 10 \\
confident & & & & & & & & & & &
\end{tabular}

3. How confident are you that you can keep the emotional distress caused by your disease from interfering with the things you want to do?

\begin{tabular}{llllllllllll|l}
\hline Not at all & $\mid$ & $\mid$ & $\mid$ & $\mid$ & $\mid$ & $\mid$ & $\mid$ & $\mid$ & $\mid$ & $\mid$ & totally \\
confident & 1 & 2 & 3 & 4 & 5 & 6 & 7 & 8 & 9 & 10 \\
confident & & & & & & & & & & &
\end{tabular}

4. How confident are you that you can keep any other symptoms or health problems you have from interfering with the things you want to do?

\begin{tabular}{lllllllllllll}
\hline Not at all & $\mid$ & $\mid$ & $\mid$ & $\mid$ & $\mid$ & $\mid$ & $\mid$ & $\mid$ & $\mid$ & $\mid$ & totally \\
confident & 1 & 2 & 3 & 4 & 5 & 6 & 7 & 8 & 9 & 10 \\
confident & & & & & & & & & &
\end{tabular}

5. How confident are you that you can do the different tasks and activities needed to manage your health condition so as to reduce your need to see a doctor?

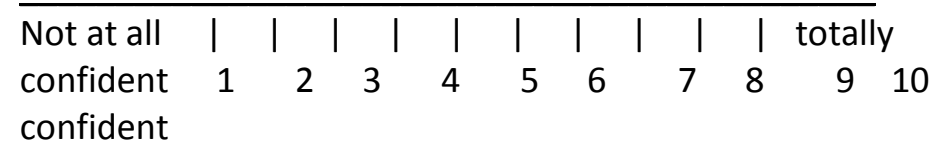

6. How confident are you that you can do things other than just taking medication to reduce how much your illness affects your everyday life?

\begin{tabular}{lllllllllllll}
\hline Not at all & $\mid$ & $\mid$ & $\mid$ & $\mid$ & $\mid$ & $\mid$ & $\mid$ & $\mid$ & $\mid$ & $\mid$ & totally \\
confident & 1 & 2 & 3 & 4 & 5 & 6 & 7 & 8 & 9 & 10
\end{tabular}

$\begin{array}{lllllllllll}\text { confident } & 1 & 2 & 3 & 4 & 5 & 6 & 7 & 8 & 9 & 10\end{array}$ confident 


\section{EQ5D Health Questionnaire - South African English version}

By placing a tick in one box in each group below, please indicate which statements best describe your own state of health TODAY.

\section{Mobility}

I have no problems in walking about

I have some problems in walking about

I am confined to bed

\section{Self-Care}

I have no problems with self-care

I have some problems washing or dressing myself

I am unable to wash or dress myself

Usual Activities (e.g. work, study, housework, family or

leisure activities)

I have no problems with performing my usual activities

I have some problems with performing my usual activities

I am unable to perform my usual activities

\section{Pain/Discomfort}

I have no pain or discomfort

I have moderate pain or discomfort

I have extreme pain or discomfort

\section{Anxiety/Depression}

I am not anxious or depressed

I am moderately anxious or depressed

I am extremely anxious or depressed

Compared with my general level of health over the past 12 months, my state of health today is:

Better PLEASE TICK

Much the same

ONE

Worse

BOX 
To help people say how good or bad their state of health is, we have drawn a scale on which the best state you can imagine is marked 100 and the worst imaginable state you can imagine is marked 0 .

We would like you to indicate on this scale, in your opinion, how good or bad your own health is today. Please do this by drawing a line from the box below to whichever point on the scale indicates how good or bad your state of health is today.

\section{Your own}

state of health

(C) 1990 EuroQol Group. EQ-5D ${ }^{\mathrm{TM}}$ is a trade mark of the EuroQol Group.

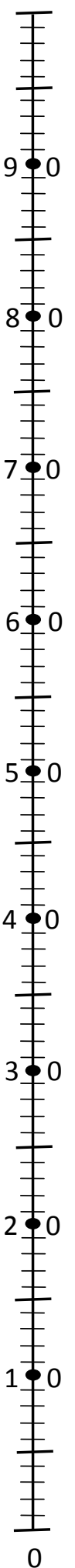

Worst 


\section{Participant open ended questionnaire}

1. Did you find the 6 week course helpful to you in any way? If so how did it help you?

2. What did you like about the course?

3. What didn't you like about the course?

4. Is there anything more you would like to learn that wasn't in the course?

5. What did you like about the workbook?

6. What didn't you like about the workbook?

7. What more would you add in the workbook that wasn't covered? 
Appendix F: "Living with

Osteoarthritis" patient booklet

\section{Living with}

\section{OSTEOARTHRITIS}

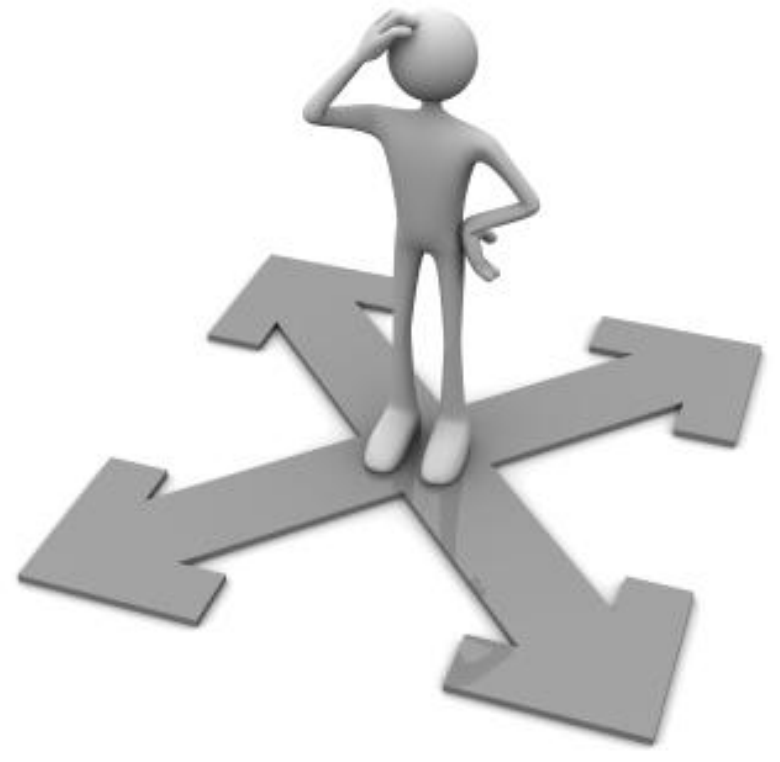

Patient Workbook 


\section{Welcome to the "Living with Osteoarthritis" patient workbook.}

This is a workbook designed to be used over 6 weeks. We hope that by using the workbook people will learn more about osteoarthritis and develop self-management skills for living with the condition. Using this workbook is not about sitting and reading or listening. In order to get the most out of this course you will be asked to share your experiences, you will need to set goals and share those goals with others and you will need to take part in activities. This workbook is NOT a substitute for any other medical care that has been recommended for the treatment of your condition but an addition to see if it helps you in any way.

You will benefit most from this workbook if you commit yourself to completing all the sessions within a 6 week period of time. Scientific research tells us that these courses are of great benefit to people living with chronic diseases such as diabetes, arthritis and HIV/AIDS. But to benefit from the course, using the workbook regularly over 6 weeks and participating in activities is essential. The workbook is divided into six sections:

- Week 1: Osteoarthritis, Self-management and Exercise

- Week 2: Managing common symptoms

- Week 3: Stress Management

- Week 4: Eating Well

- Week 5: Medication and disease-related problem solving

- Week 6: Continuing as a successful self-manager

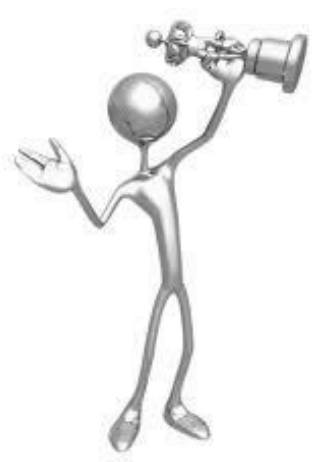


Your course leader is. She is a qualified physiotherapist and has been trained in all the information you will be going through in the discussions. She is also an expert in safe ways to exercise and relaxation techniques. 


\section{Week 1: Osteoarthritis, Self- management and Exercise}

\section{What is Osteoarthritis?}

What is osteoarthritis? It is a degenerative joint disease which we often call OA. In simple terms, it is the breakdown of previously healthy joint surfaces, causing the two bones that make up the joint to rub against each other. This wears away the bone's surface (the cushioning of the joint) and can become painful.
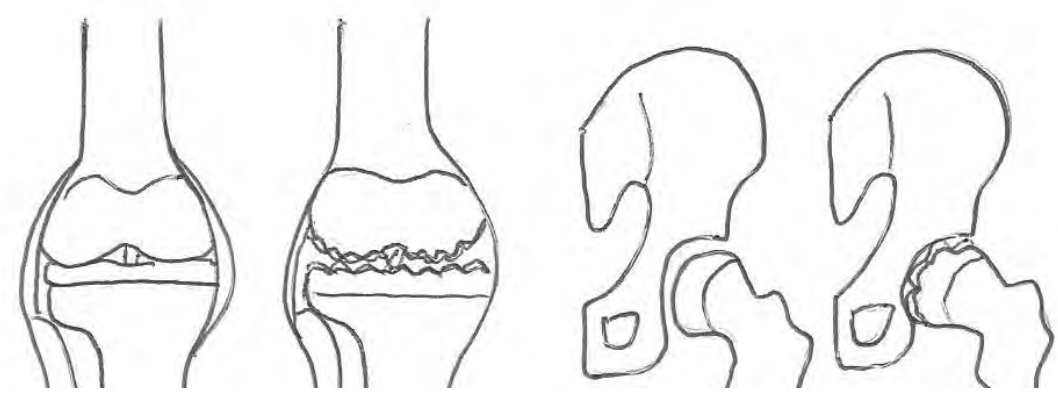

Normal knee. OA of the knee. Normal hip. OA of the hip

$\mathrm{OA}$ is different in every person and the way it progresses differs for each person too. Usually, as a person ages the condition progresses until in some people there is a lot of joint damage with deformities. OA is most common in the hips, knees and hand joints. The cause of $O A$ is very seldom due to one factor alone, but rather a combination of things acting together usually brings about this condition. Known causes/risk factors for OA include but are not limited to:

- age

- inherited or genetic predisposition to developing OA, especially in the hands

- gender - females are seen to have OA more commonly than males

- obesity and being overweight

- faulty alignments or having poor posture

- certain occupations/sports/repeated stresses on the joints

- previous trauma 


\section{Diagnosis:}

It is fairly simple for a doctor to make a diagnosis of OA. The doctor makes the diagnosis by examining you and by taking a history of the main symptoms. X-rays can help in correctly diagnosing $\mathrm{OA}$, but these are not always necessary. Changes which we look for on the X-rays include narrowing of the space between the two bones, evidence of wear and tear of joint surfaces and extra bone forming at some areas.

People who have OA mainly complain of:

- $\quad$ pain in the affected joint or limb

- stiffness, particularly in the morning for less than two hours

- muscle weakness

- difficulties in performing daily tasks like walking or climbing the stairs

\section{Management options:}

Scientific research shows that educating people about their condition in order that they may understand it better and manage it better, is the best way to treat it and that this works well. People are advised to become more active by exercising and balancing this with rest.

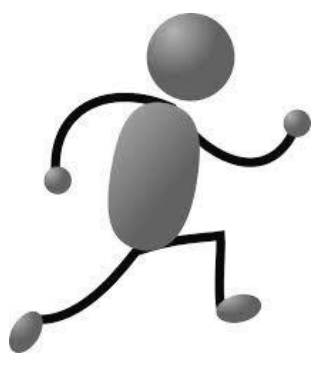

Physiotherapy, which focusses on education and exercise, can also help. There are medicines that your doctor will can prescribe for you to treat your pain or inflammation. Different surgical methods can also be used to treat this condition, the most effective being a joint replacement. Joint replacements are big operations though, so they are only done when the OA is very bad.

Now that you know a bit more about what the condition is, we will go through what we mean by self-management. 


\section{What is meant by "self-management"?}

Self-management does not mean that you are expected to look after your health on your own with no help. No, someone who is a successful self-manager takes responsibility for their health. This means that they choose to work with the health team, with their medication and with themselves to live a healthy life (just like a manager in a business they don't do everything themselves, they work with a team).

There are lots of things you can learn to do which will help you to be a successful selfmanager. First of all it is important to understand osteoarthritis. This is what we have just covered. You need to understand what the condition is, why it happens, how it changes and how it impacts on your life. You also need to know about the ways to treat it and medications which may be used.

The next step in being a self-manager is being able to think about this information in terms of how it affects you. The final step in being a self-manager is to think about what it is that you want to be able to do, decide how you are going to do it and then to learn and practice the skills you need to be able to do it. Some of the things you will learn about and practice every day when you do this course include exercising, relaxation techniques and healthy eating.

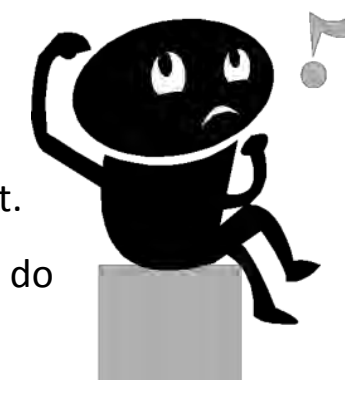

By using this workbook you will learn about exercise and its benefits, in the second section you will learn a bit about the common symptoms of osteoarthritis and how to manage these. The third section will focus on stress management, the final sections focus on eating well and medications. Some people using this workbook may already know a lot about these topics, others may not know very much. It is important to share information and make sure that everyone has the knowledge they need to become a self-manager, even if you think you know a lot about these topics it is still worth your going through the workbook to make sure you have not missed out on any information. Scientific research tells us that people who are well informed about their health manage better and have a 
better quality of life. Using this workbook, you will also learn about and discuss the steps that are needed to become a good self-manager. Let's look at these steps here.

\section{Self-management steps}

\section{Step 1:}

To be good at self-management you need to learn and practice several skills which you will practice through using this workbook. The first step is to decide what it is you want to be able to do. This can be the hardest step to think about. For example you might be feeling very sad and depressed. First you need to think about why you are feeling that way. Perhaps one of the reasons you are feeling that way is that you have lost touch with your friends. Your first step might be to decide that you need to reconnect with your friends or to meet people and make friends. This will help you to feel less sad and depressed.

Write down here three things that you want to be able to do:

1)

2)

3)

Step 2:

But deciding that you are going to meet people and make friends doesn't mean it will happen. You have to make it happen. The second step in being a self-manager is to decide how you are going to do it. Sometimes the thought of doing something new can seem too much and we don't even try. If you want to meet people to make friends you need to think about all the different options you have to do this. For example you could invite your neighbours for tea, or you could decide you would meet people by going to church, by joining a support group or an exercise group. Never assume that what you want to be able to do is impossible. Always look for every option and look at it from every angle. 
Write down here three different ways that you could try to achieve what you want to do:

1)

2)

3)

Now that you have decided on how you can try to achieve what you want, you need to make an action plan. It is important that this plan is realistic otherwise it is likely you will not succeed. How do you do this?

First decide what you are going to do this week

Now make a specific plan

\section{- Action plans, goal setting}

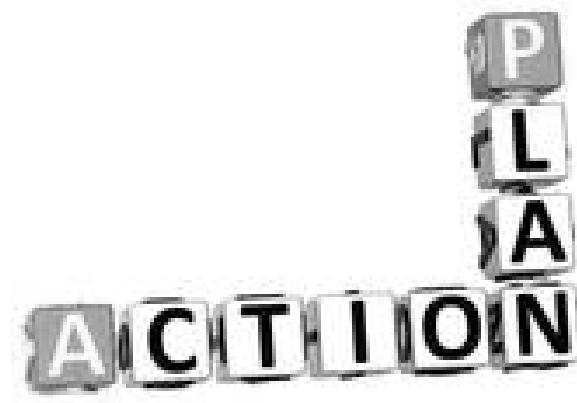

Saying that this week I'm going to try to meet some people is NOT a specific plan. To be specific, the plan must have different parts. It is useful to ask yourself some questions to help develop a specific plan. Questions like:

- What?

Exactly what are you going to do? For example you could decide that to meet people you are going to invite your neighbour for tea.

- How much?

Then you must decide how much you are going to do. For example are you going to invite one neighbour for tea or are you going to invite lots of neighbours over. Lots of people are much more tiring than one person. Or do you want to invite your neighbour for lunch? But lunch means a lot more preparation and time and will make you more tired. So you have to decide how much you can do.

- When will you do it?

Then you must decide on exactly which day you are going to do the activity and at what time of the day. Maybe it is better to invite your neighbour for tea in the morning because you get tired in the afternoon. Or if you feel sick in the morning from your medicines maybe it is better to invite your neighbour for afternoon tea. Or maybe your neighbour works and you need to invite them for tea the weekend. 
- How often?

This is always the hardest part. We all would like to be able to do more things every day. But we are human and this is not always possible. When people want to start exercising, we often say we are going to do it every day. But this is often just not possible and if we then miss a day we feel that we have failed and we give up. How often will you invite your neighbour for tea? Not every day but maybe once a week. You know that you won't become friends immediately and that it will take time.

- Is it a good plan?

To test whether you have come up with a good plan you need to ask yourself this question:

"If I give myself a score from 0 -10 for how confident I am that I will achieve my plan this week, where 0 is not at all confident, and 10 is totally confident. What score will I give to show how confident I am that I can complete this plan?"

If your answer is 7 or more out of 10 then this is probably a very good plan. If your score is less than 7 you need to think about why you are not confident. What are the problems or barriers? Can you change the plan or solve the problems to make yourself feel more confident?

\section{Step 3:}

Now, you need to write your plan down and put it somewhere you will see it every day. There is an action plan form at the end of this section and more in this book. Use them every week while you are using this workbook. You can always draw more of them to keep working on your plans in the future.

\footnotetext{
A good action plan is:

- Something I want to do

- Something I can expect to do this week

- Is specific

- Answers the questions: What? How much? When? How often?

- I am confident that I can achieve with a score of at least 7 out of 10.
} 
Now you need to carry out your action plan. If it is a good plan then doing it is usually fairly easy. It helps to tell family or friends what your plan is and to report back to them on how you are doing. On this course you are going to make a plan every week and record how you get on. It helps to report back on things because you can then have an idea on how well you are doing. If you haven't been able to keep to the plan you can discuss the problems you might have had and make plans to cope with them.

\section{Step 4:}

Always check your results and give yourself a reward for having achieved your plan. Also think about how achieving your plan is making you feel. In the example we talked about, you could congratulate yourself for having invited your neighbour for tea, you would also think about how you now feel. Is the plan helping you to achieve what you want?

\section{What happens if something doesn't go according to plan?}

What if your plan doesn't work? Are you going to give up and decide you had a bad plan? There are seven steps to solving problems. These are:

Deciding what the problem is (you might need friends and family to help here)

List ideas to solve the problem

Select one idea to try

How did it go?

If it didn't work, try another idea

If your ideas don't work, ask friends, family, counsellors, professionals for ideas

Finally you might have to accept that you can't solve the problem now.

At the end of each section and at the back of the workbook there are "Action Plan Forms". Use these forms to plan what you want to do and how you are going to do it. We are now going to discuss exercise and we are going to use the "Action Plan Form" at the end of this section to plan what exercise you are going to do this week. 


\section{A successful self-manager is someone who:}

- Sets goals

- Makes a list of ways to achieve those goals

- Makes action plans to achieve the goals

- Carries out the action plans

- Checks on their progress every week

- Can change the action plan if there are problems

- Gives themselves a reward for achieving their goals

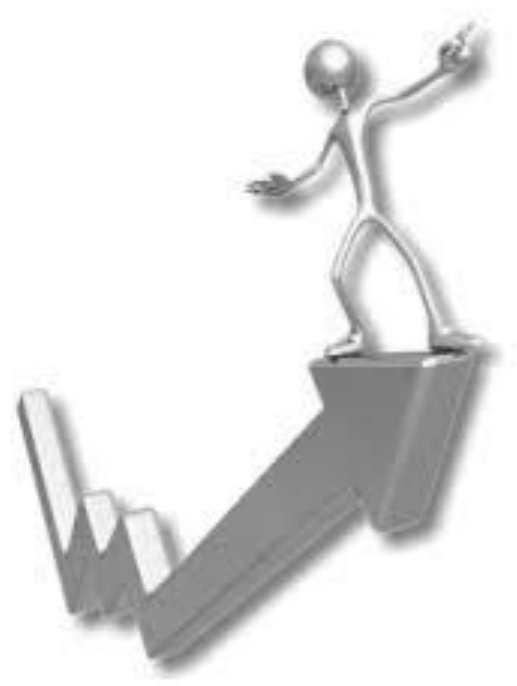




\section{Exercise dos and don'ts}

Exercise is a very important way to keep healthy. Scientific research tells us that exercise has a lot of good effects on our bodies like helping our digestive system absorb and process food; it trains our hearts so that they are strong and healthy and keeps our lungs working well. Exercise makes our muscles and bones strong and our joints flexible so that we can keep moving. Exercise also helps to make us feel happy, improves concentration and memory, improves sleep and exercise helps to decrease the chances of developing chronic illnesses like high blood pressure and cancers.

In the past, when people became ill with a chronic illness like high blood pressure or diabetes or arthritis, medical care focused on helping them when their symptoms became worse. Treatment focused on using medicine and people were often advised to rest or decrease their activity. Today we know that if we teach people who develop chronic illnesses about their disease and encourage them to do the right exercise we can prevent a lot of the problems which used to be treated with medicines. We also know that

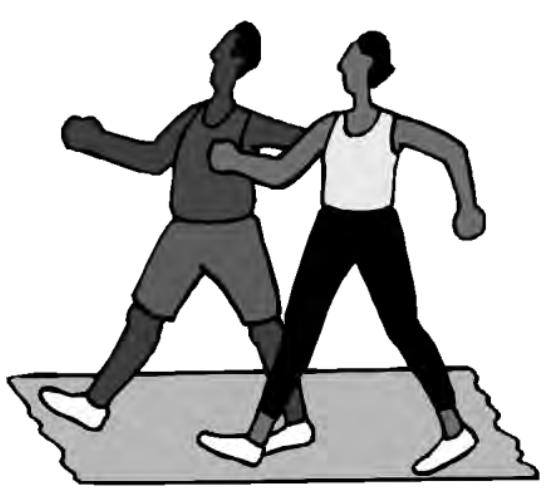
exercise can help to treat a lot of the symptoms which people with chronic diseases develop. Symptoms which may be caused by the disease or by the drugs used to treat the disease.

Exercise is good for:

- Improving mood

- Strength

- Improving sleep

- Concentration and memory

- Heart and lung health

- Decreasing body fat

- Digestion

- Increasing confidence to self-manage chronic illness. 
You may be wondering if it is safe for you to exercise when you have osteoarthritis. Research tells us that exercise has been shown to be beneficial in people with osteoarthritis, as well as in those who need a joint replacement. Exercise can help slow down the degenerative processes that happen with arthritis when you aren't exercising. Exercise can also help you to lose weight which helps with osteoarthritis of the legs as there are less forces through the knee joint. We know that people who are physically fit get fewer colds and take fewer days off work because of illness. One of the biggest benefits of exercise is that exercising regularly makes you feel more in control of your life.

Although exercise is good for you and safe for you to do, sometimes your body will give you clues that you need to cancel your exercise for the day. If you have a fever, feel dizzy, have vomiting or diarrhoea, if your joints have suddenly become swollen, or if you have a pain which is new and you are not sure what is causing it, it is better to miss an exercise session until you can speak to a doctor or physiotherapist.

\section{Do not exercise if:}

- You have a fever

- You are dizzy

- You have been vomiting

- You have diarrhoea

- Your joints have suddenly become swollen

- You have a new pain which you don't know the cause of

Miss one exercise session if you have one of these problems until you can speak to a nurse or doctor. This does not mean you should never exercise but you need to make sure you are not becoming ill. 


\section{Types of exercise}

You do not have to join a gym or a club to get exercise. There are lots of ways of exercising from formal sports like running, playing football or netball, swimming or playing tennis. But, walking is also a very good way to exercise. Any activity which makes your heart beat faster and makes you breathe a little harder is exercise. Dancing is exercise, walking up the stairs is exercise, gardening is exercise. There are lots of ways that we can exercise every day without having to go to a class or join a club. You could walk a little further before catching the bus or the taxi or you could play with your children or grandchildren!

There are three general kinds of exercise you can do.

Endurance exercise: like walking, running, dancing or swimming. Endurance exercise is sometimes called aerobic exercise which means that you will be breathing faster and your heart will be beating faster too. We know that this kind of exercise is very important to keep healthy and we need to do 30 minutes of this kind of exercise three times a week to keep healthy.

Strengthening exercise: this kind of exercise focuses on making us stronger. To make muscles stronger we have to do exercises which make the muscles work hard against a resistance, like weight training but you can also do strength training by working with heavy bags of shopping!

Stretching exercise: this focuses on keeping us mobile and flexible.

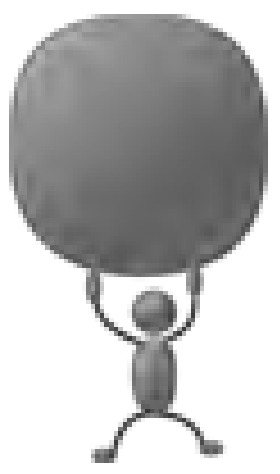

\section{Types of exercise:}

- Endurance exercise which makes you breathe harder (sometimes called aerobic)

- Strengthening exercise which makes you stronger

- Stretching or flexibility exercise which makes you more mobile and supple 
We know one of the hardest things about exercise is not doing it once, but doing it again and again. There are several steps we can follow to make sure that when we start to exercise we stick to it. We all make lots of excuses why we can't exercise. Let's look at the most common excuses.

\section{"I don't have time"}

It doesn't take a lot of time to start exercising. Five minutes a day is a good start. We make time to take medicine because we know without it we would become ill. Exercise is as important as medicine to help us remain healthy (remember it can never replace your drugs). If we know that it is that important we can make time for it.

\section{"I'm too tired"}

When people become ill they often become less active. As you become less active, your body loses fitness and you become weaker, you may feel stiffer

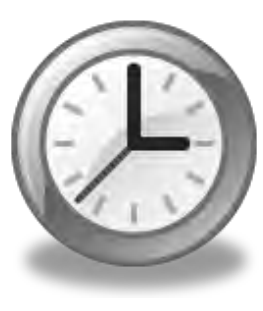
and you tire more easily. This means that exercising might feel harder and so you exercise less. This often results in a downward spiral of activity and people often get to the point where even walking down the street to visit the neighbour can feel like too much. Being active or doing exercise when you are feeling tired will give you more energy and make you feel less tired.

\section{"I'm too sick"}

You may be too sick to undertake very vigorous exercise but you can still aim to be more active. You can even break your exercise into one minute sessions which you repeat several times through your day. The fitter you get, the better you will be able to cope with your illness

\section{"I get enough exercise already"}

You may be getting a lot of exercise already in your job or simply walking around doing your daily chores. But for most people if we add this time up, it still isn't enough exercise to keep them fully fit. This kind of exercise also doesn't include one of the most important components that make exercise good for us - fun!

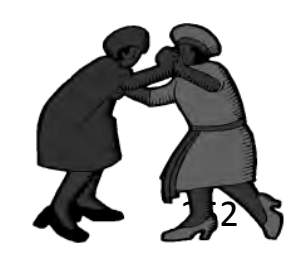




\section{"Exercise is boring"}

You don't have to do the exercises that everyone else does if they are boring. Choose something that is fun, exercise with a friend or with your favourite music or listen to the radio. You can also keep your exercises fun by changing them regularly.

\section{"Exercise is painful"}

Exercise may be uncomfortable but it shouldn't be painful. If you have pain before you start to exercise, it should not get worse while you are exercising. If you do not have pain before you start to exercise and you start to feel pain while you exercise you need to stop exercising and evaluate your pain using the guidelines in Week 2. If you have muscle or joint pain for more than two hours after you exercise then you have probably done too much. Next time do a little less, either exercise for less time or less vigorously.

\section{"It's too dangerous, it's too hot, it's too cold"}

There are always reasons like this not to exercise. Remember that exercise can be done anywhere and anytime. You can put on music in your home and dance, if it's too hot you could walk around shops which have airconditioning. Finding a group of people to exercise with will not only make it safer but also more fun!

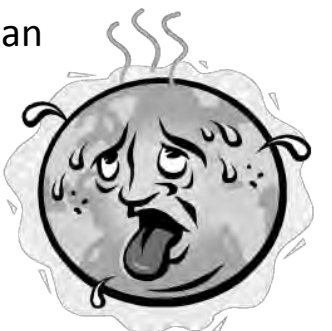

\section{"I know I won't stick to it so there is no point in starting"}

First review the steps we discussed on how to be a successful self-manager. If you set your exercise goals using these steps you have more chance of sticking to your exercises. Remember too, the important step of rewarding yourself for achieving your goals; this makes it easier to move on to your next goal. We are now going to have a look at the important steps to take to be successful at putting your exercise plan into action. 


\section{Steps to success with exercise:}

- Set a clear goal using the steps outlined in "How to be a successful self-manager"

- Choose exercise or activity that you want to do and that is fun

- Set a specific time and place to do your exercise

- Decide how long you are going to stick to the plan before you think about changing it (6 to 8 weeks is a good time to work on things)

- Keep an exercise diary to keep track of how you are doing (there is one at the back of this booklet for you to use)

- Keep track of your progress using the exercise diaries in this workbook.

- Start - don't wait, start now. Begin gradually and proceed slowly

- Revise your programme. At the end of the $6-8$ weeks make a new plan for the next 6 weeks

- Reward yourself. It is a reward to feel better and healthier but also give yourself a reward for achieving your goal, like eating a favourite meal, or visiting a friend or taking a walk somewhere special. 


\section{Your exercise programme:}

An exercise programme should include the three different types of exercise; remember they were endurance, flexibility and strength exercise. Following the steps in the box "Steps to success with exercise", you need to decide on what you want to be able to do and what exercise you would like to do. Now that you know what exercise you are going to do, you need to decide how much to do. The amount of exercise you are going to begin with will depend on a lot of different things. If you have not done any exercise for a long time or have been feeling unwell, have had difficulty breathing or been short of breath, if you have had stiffness or pain or weakness that interferes with your daily activities then you need to start your exercise slowly. You can begin slowly by starting with some flexibility and strengthening exercises. Do these exercises every other day for 5 minutes. Once you can do that comfortably and without feeling stiff or sore the next day, increase it to 10 minutes.

Once you can do 10 minutes comfortably, you can start doing the exercises every day (when we say exercise every day, we usually mean exercise for 5 days of the week; it can be very hard to keep a routine to exercise on weekends when activities are different). Once you can do at least 10 minutes every day then you are ready to begin endurance exercises. Choose your exercises from the ones set out in the sections below. Follow the instructions in the box to make sure you get the most out of the exercises and do them safely. 


\section{Getting the most out of your flexibility and strength exercises:}

- Move slowly and gently. Do not use jerking or bouncing movements as these will make your muscles shorter and tighter.

- Stretch to the point of tension in a muscle and hold for 20 seconds before you relax

- Don't push until it hurts, stretch to tension not pain

- Start off with 5 repetitions of each exercise. After one week increase it to 7, after another week increase to 10 .

- Always do the same number of exercises on the left side and the right side of your body

- Keep breathing; do not hold your breath when you exercise. Think about breathing out as you move to make sure you do not hold your breath.

- Use the two hour rule. If you have increased symptoms (like pain) for more than two hours after you exercise you have probably done too much. Don't stop doing the exercises but decrease how much you do next time.

- If you find an exercise difficult this does not mean you should not do it at all. You should adapt it, do it as completely as you can.

\section{Flexibility Exercises:}

Remember, these exercises are aimed at improving your ability to move. There is a long list of exercises that could be included here and you might not be able to do them all every time you exercise. Try to ensure that you do flexibility exercises at least once a week.

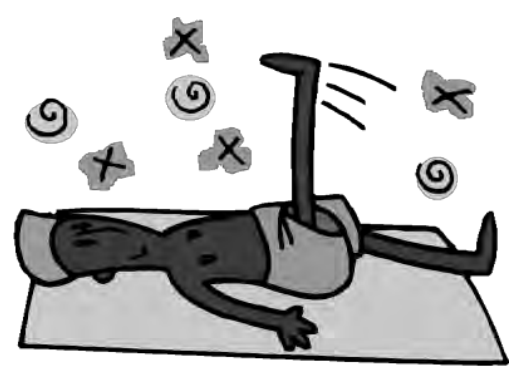

\section{Strengthening Exercises:}

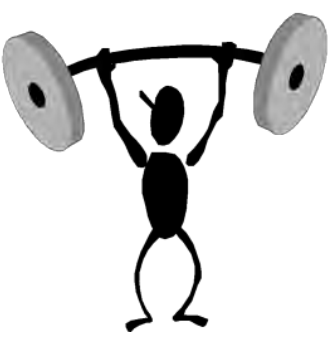

You do not need to go to a gym to do strength exercises, the exercises described here can be done at home. To make muscles stronger you must make the muscles work against a resistance or a force - they have to push or pull. You should not do strength exercises every day, rather they should be done every second day. Your muscles need a day of rest to adapt and get stronger. To make a muscle stronger you need to repeat each exercise 5 times to start with. Once you can do an exercise 10 times you will not get stronger by doing more exercises. Now you will need to add more resistance to the exercise to get stronger. 


\section{Endurance Exercises:}

The most difficult thing for most people is deciding how much exercise to start with. The easiest starting point is to ask yourself the question: "how much do I think I can do without suffering for it tomorrow?" If you feel you can do 5 minutes, then do 5 minutes. Remember that any exercise is better than none. You don't have to do 30 minutes from the first day. It is important to start slowly and increase very gradually. It is better to start off by doing less than you think you can and increase it from there.

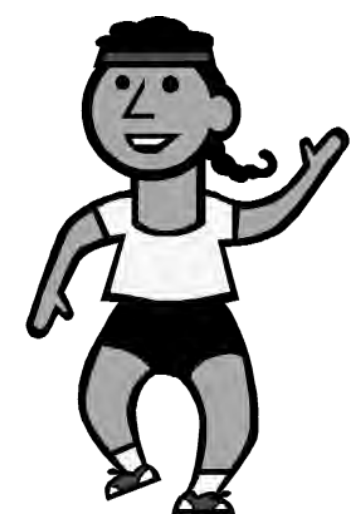

There are three things you need to think about when you do endurance exercise. These three things are frequency (how often am I going to do this exercise); duration (how long am I going to exercise for when I do exercise) and intensity (how hard am I going to work when I exercise).

\section{Frequency:}

Try to do endurance exercise 3 or 4 times a week. By doing this you can rest every second day and allow your body to recover. All athletes have at least one day a week when they rest. Rest does not mean that they lie in bed all day though, it means that they do not do their exercises.

\section{Duration:}

How much can I do without suffering for it tomorrow? That is your starting point. If you are starting with just a few minutes you can gradually increase it over time until you can do 30 minutes at a time. The easiest way to increase the time is to use intervals of exercise. For example to walk hard for 3 minutes, then walk slowly for 2 minutes, then walk hard again for another 3 minutes. Slowly over time cut down the slow walking and increase the hard walking. You could also break your exercise into separate sessions. You could walk for 10 or 15 minutes in the morning and do it again in the evening. This would still count as 30 minutes of exercise.

\section{Intensity:}

How will you know that you are exercising hard enough to be doing some good? How will you know if you are exercising too hard? When doing endurance exercise the easiest way 
to check the intensity is to use the "Talk Test". When you are doing moderate intensity exercise you should be able to talk comfortably, but if you tried to sing it would be a little difficult and you would have to stop singing to take bigger breaths. Moderate intensity means you should feel that you are breathing a little faster and a little harder but you can still talk. It may take you a while to find the right intensity for you for the whole of your exercise session. This is normal; take your time to get to know how your body will respond.

How will you know you are improving in your exercises? For the flexibility and strength exercises it is easy to feel the improvements as you will feel that moving is easier and you are stronger and can lift heavier items. For some people it is harder to know if you are improving with the endurance exercises. One way to see if you are improving is to do a test. One of the easiest tests to do is a timed test. Decide on a route that you can walk near your home. Walk this route at a moderate intensity and time how long it takes. After several weeks of exercise walk the route again and time it again. You may see that you can walk the same route faster within 4 weeks, but it may take 8 to 12 weeks before you see that you can do the route in a faster time. The goal is to complete the same route faster or in the same time but at a lower intensity (breathing much easier).

Use the exercise diary at the end of each section to record your goals and your progress in achieving them.

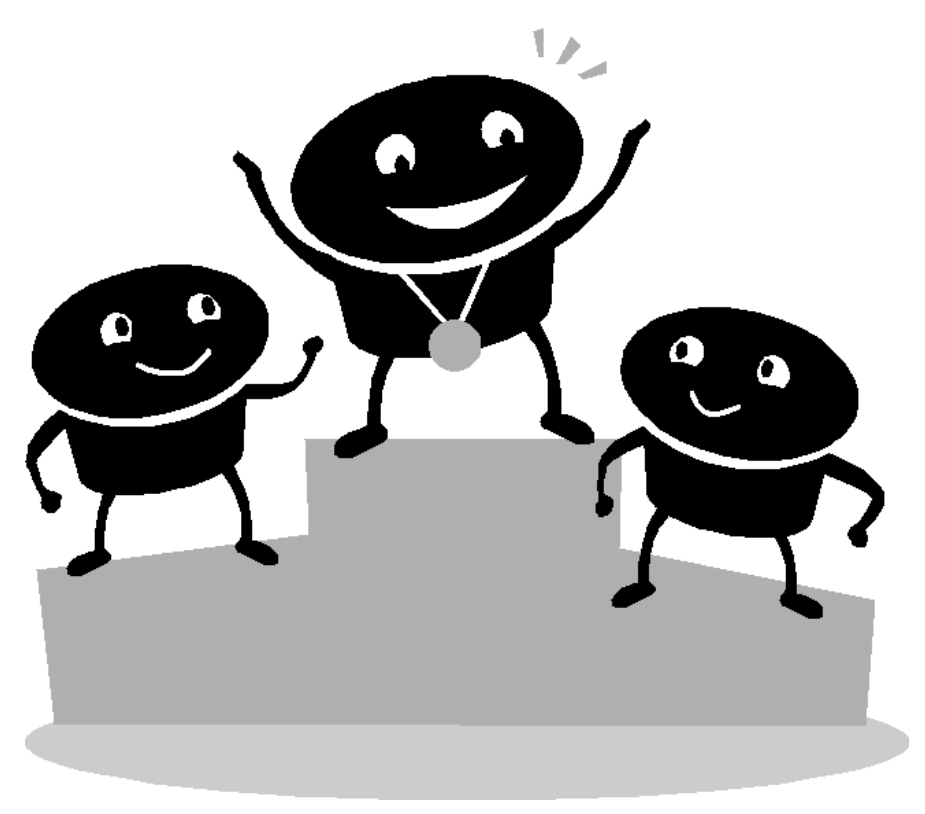




\section{An exercise routine}

This is a $20-30$ minute exercise routine which is safe for people living with osteoarthritis. This routine includes exercises which make you stronger (strength exercises), more flexible (stretching exercises) and fitter (endurance exercises).

Start by standing up straight and tall, feel your weight across your feet, relax your shoulders and open your chest, hold your head straight. Take a deep breath in and breathe out.

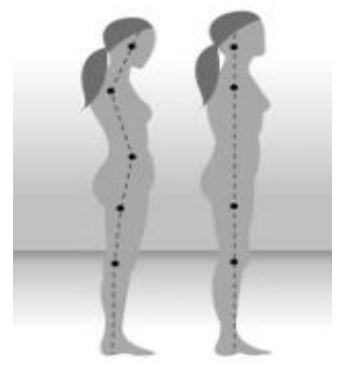

March on the spot for 2 minutes. March at a steady pace - that is a pace which you can maintain for 2 minutes. Do not start fast and get slower or start slowly and get faster. Pace yourself, start and finish at the same speed. You should be marching so that you can feel you are breathing a little bit harder than normal, you should be able to talk but not be able to sing.

Now stretch your neck - keep your shoulders relaxed and turn to look over your right shoulder - hold it for 20 seconds. Bring your head back to the middle, then turn to look over your left shoulder - hold it for 20 seconds and then bring your head back to the middle. Now put your left ear on your left shoulder - hold it for 20 seconds and then bring your head back to the middle. Repeat to the right. Now put your chin on your chest - hold it for 20 seconds and then bring your head back to the middle.

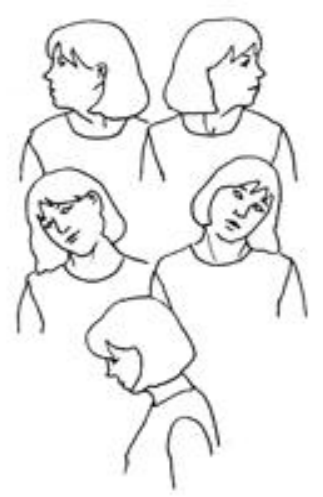

Roll shoulders forwards 5 times, then roll your shoulders backwards 5 times. Then stretch your arms by stretching your right arm across your body to the left and holding for 20 seconds and then repeat with the other arm.

March on the spot for another 2 minutes - 30 steps normal, 30 steps lift your knees up as high as you can. Keep changing every 30 steps.

Stretch your quadriceps muscles by bending your right leg backwards and holding your foot if possible below your buttock. You will feel the stretch down the front of your thigh. Hold it for 20 seconds and then do the same on the left. 
Stretch your hamstring muscle by putting your right heel on the ground and pulling your toes upwards, put your hands above your knee and lean forward to feel the stretch behind your knee. Repeat on the left for 20 seconds too.

Sit on a chair - make both your knees straight and then bend again. Do this 30 times. This works your front thigh muscles.

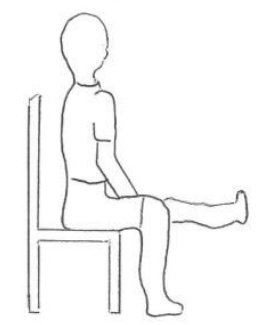

Then with your arms folded on your chest, stand up from the chair and sit down again. Keep sitting down and standing up for 2 minutes. Do this at a steady pace - that is a pace which you can maintain for 2 minutes.

March on the spot for 2 minutes - 30 steps normal, 30 steps lift your

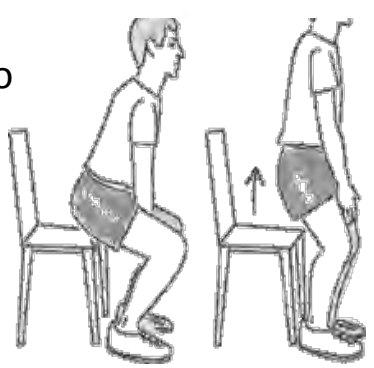
feet up as high as you can (try to kick your buttocks). Keep changing every 30 steps.

Stand on one leg at a time for 30 seconds each: use support by putting your hand on a wall or chair if necessary but try balance without holding on. Do this twice on each leg

Step ups: Step onto and off of a low step for 2 minutes. Start with the right leg and then step up with the left leg and alternate. This works both your front and back legs muscles as well as your hip muscles.

March on the spot for 2 minutes - 30 steps normal, 30 steps lift your knees up as high as you can. Keep changing every 30 steps.

End off the session by stretching your trunk: Slide your hands down the front of your thighs to see if you can touch your toes. Bend sideways by sliding your hand down the side of your leg. Hold this for 30 seconds and then do it to the other side.

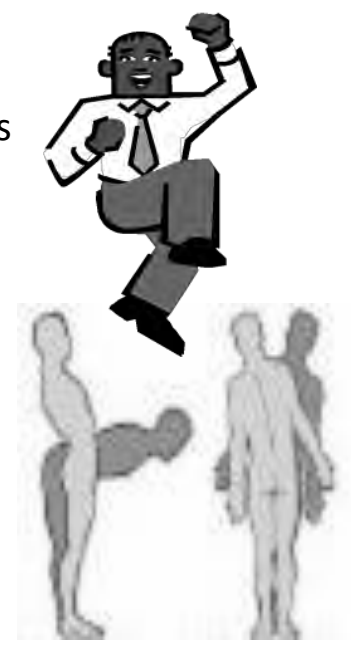


Finish by standing up straight and tall, feel your weight across your feet, relax your shoulders and open your chest, hold your head straight. Take a deep breath in and breathe out. 


\section{Action Plan Form - Exercise}

Use this form to develop an action plan on exercise. What exercise would you like to do?

Be sure your action plan includes:

What you want to do

How much you are going to do

When you are going to do it

How many days a week you are going to do it

For example: This week, I will walk (what) around the block (how much) before lunch (when) three times (how many).

This week I will:

(what)

(how much)

(when)

(how many?)

How confident are you that you can complete this action plan?

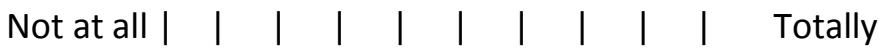

confident $122345 \quad 678910$ confident

Keep a record of how you did:

\begin{tabular}{|l|l|l|}
\hline & I Plan to..... & I did..... \\
\hline Monday & & \\
\hline Tuesday & & \\
\hline Wednesday & & \\
\hline Thursday & & \\
\hline Friday & & \\
\hline Saturday & & \\
\hline Sunday & & \\
\hline
\end{tabular}




\section{Week 2: Managing common symptoms}

Osteoarthritis is known to greatly affect a person's quality of life, with pain being the most common reason for seeing a doctor or physiotherapist. Other symptoms which are often present are:

- joint stiffness (especially following a period of rest or early morning stiffness for less than two hours),

- swelling and problems moving the joint through the whole range of motion

- difficulty in performing daily tasks

- Fatigue

- Frustration, isolation, depression

We will look at what each of these are and how you can manage them at home if you experience any of the above.

\section{What is pain?}

It is important for you to understand what pain is and what type of pain you may experience with $\mathrm{OA}$ in order to manage these symptoms.

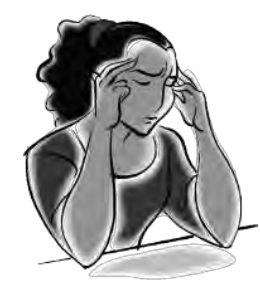

Acute pain is a normal sensation triggered in the nervous system when nerves in the body are stimulated and send messages up to the brain to say that there may be damage to your body. Acute pain comes on suddenly (e.g. after banging your finger in a door), and lasts for a few days or even weeks (usually less than six weeks) while the body heals itself. This pain is useful as it protects the part while the body heals. Once the body is healed, there is no more pain.

OA initially presents as acute pain, which means that pain starts suddenly and lasts for a short time. This usually happens when the joint is moved or used and then the pain subsides. OA doesn't always remain as acute pain but sometimes changes to what we call chronic pain. This can cause pain when you aren't moving or even at night or during movements/activities that don't usually cause pain. Chronic pain is described as pain that is experienced on most days for at least three months. The reason behind why the pain in OA starts out as acute and then becomes chronic is not yet known but research is being done to explain this better. 
Chronic pain is not helpful as acute pain is. It occurs after the body has healed itself and this means that there shouldn't be a reason why we need to protect that area anymore. But often we listen to the pain and don't use that joint or do certain things in case we cause damage. In OA the "damage" in the joint is not something new that we can injure more by using it or moving it but it is caused by the wear and tear as talked about before.

The reason why chronic pain is different from acute pain is because there are changes that take place in your nervous system when you have had chronic pain for some time. These changes can explain a lot of why you may experience pain more than someone else. When acute pain happens, the normal messages that are sent via your nerves to and from the brain are often changed in people who experience chronic pain. The pathways often make the messages bigger than they usually are and this can cause a person to be more sensitive to normal feelings and feel pain more easily than someone whose messages are not sensitive. There is also a "map" in the brain which locates each area of the body and in people with chronic pain, the areas which represent the painful area gets bigger and smudged so the brain focuses on this and can confuse some normal areas with the painful areas. This explains why the pain can feel like it is spreading from just one area as the map in your brain isn't as clear as it was before.

Here is the take-home message about pain:

- Hurt does not always mean harm. There can be pain wit something being wrong due to the changes that take pla system.

- There are physiological reasons why there is pain withou

- Your pain is NOT imaginary or in your head or psycholog

- Chronic pain is NOT the same as acute pain.

- Chronic pain is NOT a sign of ongoing damage

- Chronic pain cannot be "switched off."

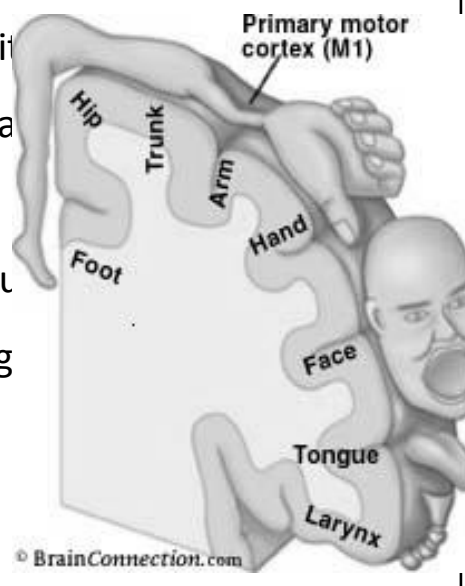

- Non-pain messengers (e.g. stretching or pressure) may send pain signals rather than Stretching or pressure messages to the brain.

- An increase in your pain (with or without exercise) does not mean a new injury. 


\section{Flare ups of pain}

It is possible to have both pains (acute pain and chronic pain) at the same time; this can confuse the picture. Although a person with OA may have chronic pain, pain is not necessarily present at all times and you can experience periods of less or worse pain. When pain becomes worse this is called a flare up. This means you will have acute pain at this time and there are ways to deal with this. It is important to note what causes such a flare up so you know what and how those activities can be modified in an attempt to reduce the flare up of pain again.

\section{Common signs of a flare up are:}

- $\quad$ a sudden increase in pain

- redness

- a warm or hot joint

- swelling

\section{What can I do about pain in a flare up?}

A flare up can be managed by taking a short time to rest from whatever has caused the flare up to allow it to settle. We don't want to rest TOO much either as then your joints can get stiff. It is helpful to take painkillers as prescribed by your doctor if your pain gets worse but we will talk about medication later on too. Putting ice onto the joint can also help to settle the flare up. Use ice for no more than 10 - 20 minutes at a time on the painful area. You can use a small bag of ice wrapped in a towel, a gel-filled ice pack or wrap a towel around a bag of frozen vegetables. Don't put ice directly onto bare skin.

\section{What is stiffness?}

Stiffness is when your joints feel like they aren't able to move easily or they feel stuck. This is common in the morning just after waking up as your joints have not been moving much while you sleep. The joints get used to being in the one position and then when you try to get up and start moving it feels difficult to move (stiff).

\section{What can I do about stiffness?}

After such a period of rest/sleep or limited movement the joints need to slowly get moving in order to get used to moving again. So before getting out of bed in the morning move your ankles up and down 20 times. Then bend each knee up and down 20 times and open and close your legs 20 times to get the joints warmed up. 
Warmth also helps the muscles around the joint to relax so a warm water bottle before rising or a warm shower in the morning will help get you moving. Make sure you can feel the warmth but it should not be directly on your skin or too hot that it will burn you. It's like the parts in a machine that need oil to work well; we need to "oil" our joints first before they work without being so stiff.

\section{What is swelling?}

Swelling is a common symptom of a flare up or may be a regular symptom experienced during OA. Swelling is caused by a build-up of fluid in the tissues in and around the joint. This causes the area to feel bigger than usual, hard, painful and it's difficult to move the swollen area.

\section{What can I do about swelling?}

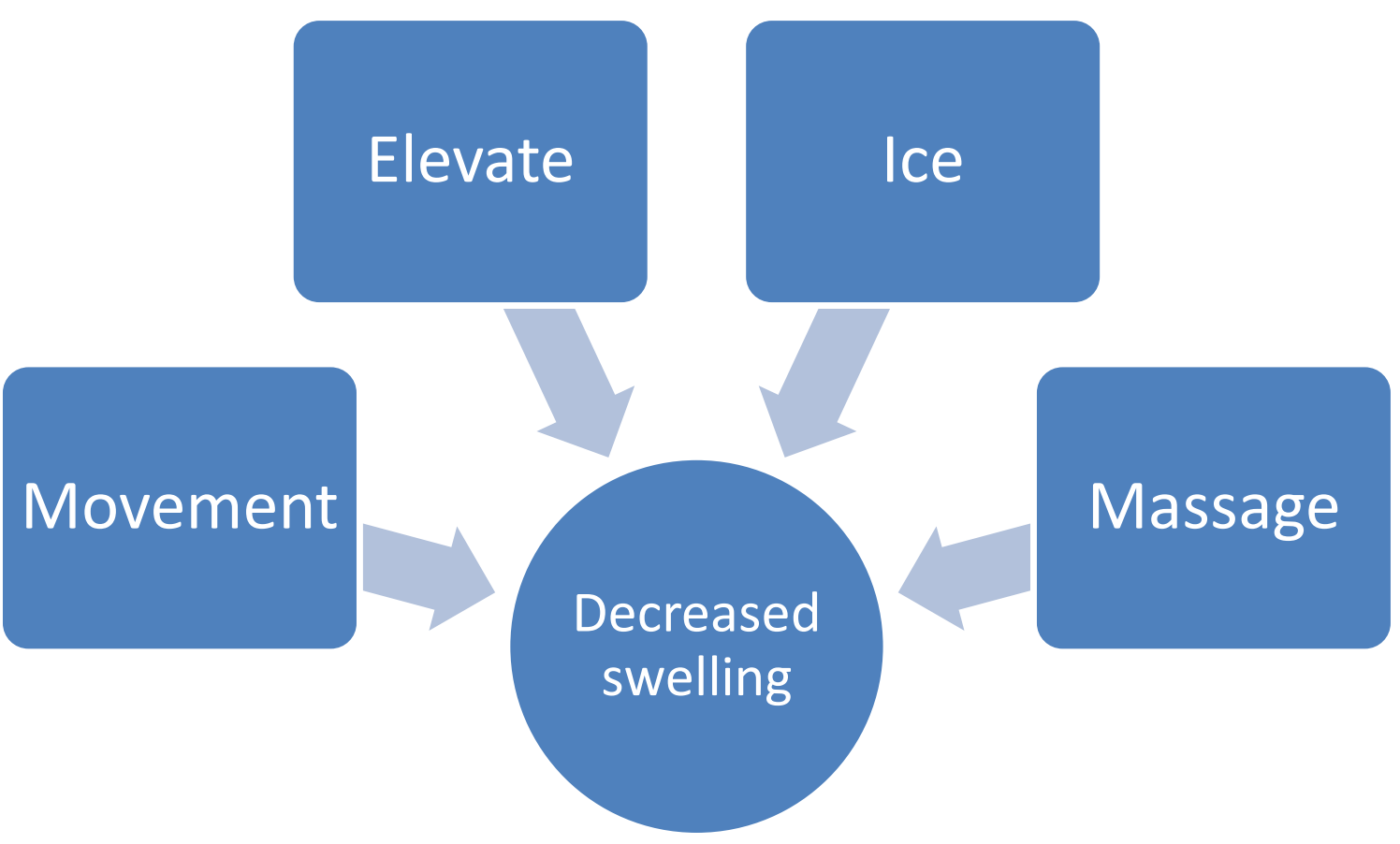


Movement is the best option to deal with swelling as it causes blood (rich in oxygen) to flow to the area and flush out the build-up of fluid and waste in the area of swelling. Staying in one position is probably the worst thing for swelling so we want you to start moving. You can also move your feet up and down and bend your hips and knees while sitting/lying down and go for a walk to get your body active.

It can help to elevate the area when sitting or lying so that gravity can help move the fluid back to the body instead of collecting in the leg. You can put your leg up on a small chair or two pillows when in a chair or the bed.

Rubbing/massaging the area from the furthest point towards the body can assist the fluid moving back to the body too. Normal body cream or baby oil will make it smooth to rub with your hands around the area. Never push the swelling downwards.

Putting ice on the affected area for no more than $10-20$ minutes at a time also helps with swelling, as explained above.

\section{Difficulties doing certain activities:}

Sometimes you may feel that you are unable to do a specific task at home or outside of the house. You may feel too stiff, weak or sore to do a certain activity. You may just need to "warm up" in the morning before trying something or you may be having a flare up and then you should follow the management tips as given above. It is alright to ask for help from a family member if you are struggling with something in the home but be careful not to be asking for your family to do everything for you.

\section{What can I do when I am struggling with doing an activity?}

First try and think about WHY you are struggling with a certain activity and then how you might be able to deal with this. There are some ways to help yourself when you feel like there are certain activities you are struggling with:

\section{Joint protection, assistive devices}

To reduce the weight and stresses through the joint, an assistive device such as a walking stick, crutch or walker can be very helpful to protect extra strain on your

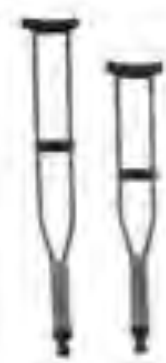
joints. This allows your arms to bear some of the

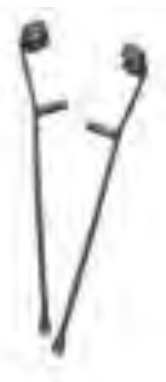
weight of your body when stepping on the affected leg. Using one stick or crutch gives you a little support and allows one of your arms to take the full

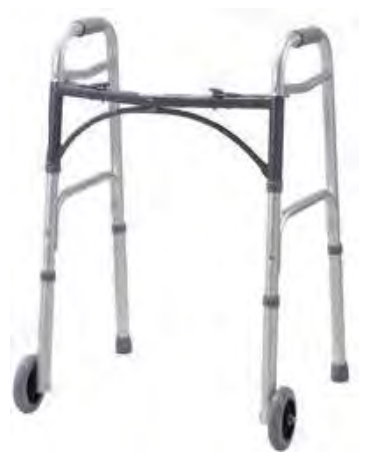


weight of your body off your leg but also allows your other arm to be free to use during activities such as preparing food or carrying items. Two crutches or a walking frame uses both arms to push through and gives more support but often take up more space while walking and both hands are being used on the device instead of being able to use them for daily activities. Try and start with the least supportive if you do feel like you need something like this.

\section{Activity modifications:}

There are certain things that can be changed in the way you do activities in order to protect the joint.

- Avoiding certain activities that put a lot of strain on the joint, like kneeling or climbing steps can be done.

- Use a padded pillow under your knee if you insist on kneeling for certain reasons

- Sit on a chair instead of kneeling

- If you struggle to stand up after sitting for some time then

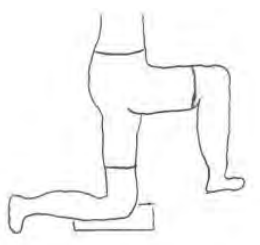
try sitting on a harder chair so you are not deep in the chair.

- Using a chair with armrests makes it easier to stand from.

- Avoid sitting on a low chair as it is more difficult to stand up from a lower chair than from a higher one.

- Walking up a ramp or using the elevator is better than struggling to climb steps if this is available.

Wheelchairs are useful to use if you are going to be doing a lot of walking at one time. Like going to the shops or taking a day's outing somewhere. This may just be too much walking at one time for your joints. Most big shopping malls have wheelchairs that you can borrow for the time you are there. Or another option is hiring a wheelchair or buying one from a pharmacy if you have the money. It is important to not rely on the wheelchair for everyday activities in the home if it's not necessary but just to use it for pacing or long distances if needed. Remember we want to keep you as able as possible.

Wheelchair hiring contact details:

Orthocare Medical Hire and sales (Bellville) 0219461717

Solutions Medical (Goodwood) 0215923370

M-Kem pharmacy (Bellville) 0219485706 


\section{Pacing and activity/resting cycles:}

During exercise and daily life, you need to learn to pace yourself. When you finish an activity/exercise you need energy at the end of it for your body to recover easily to enable you to carry on for the rest of the day. Exercise should not cripple you for 2 days after you do the exercise. When you start an activity you need to pace yourself at a speed at which you can maintain throughout the activity without getting faster or slower. At the end you should still have energy left over to be able to do more.

\section{How to pace?}

Pace your activities during the day so that they are spread out with adequate rest periods in between so you don't tire yourself out in one session of too many activities.

Find a balance between completing a task and resting. You should not do an activity for too long that you feel exhausted afterwards and you should also not rest too long so that you feel too stiff to get moving again.

For instance if you have washing and cleaning and ironing to do in a week, instead of doing all three on one day, spread them out over three days or if needed, with a day in between. Or while exercising or if you have a long day of shopping ahead of you, start exercising or walking with the trolley at a pace that you know you can keep the same until the end, without stopping or slowing down. If you find you are too tired then you must start slower or do less at one time and find the balance.

\section{What is fatigue?}

If too many activities are attempted at one time or in a short time without break periods of rest, your body will feel extremely tired and you will experience fatigue. It is important to slowly start small activities and short times of exercise to allow your body to adjust accordingly. If you do experience fatigue then it is wise to stop the activity and rest for a short while (1-2 days) until you have recovered from this

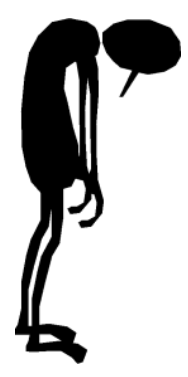
tiredness. This does not mean you need to lie in bed for this time; you can continue with your day but take a break from the exercise and then once you begin again, listen to your body and look for signs of doing too much in one go. This could be getting short of breath or your muscles feeling weak or shaky, your legs feeling like they want to give way etc. and slow down. 


\section{- What is frustration/isolation/depression?}

It is common for people living with osteoarthritis to become frustrated and to have feelings of isolation/depression. This could be due to not being able to do everything as they could before, being reliant on others for normal tasks, not being able to participate in activities amongst friends that leads to feelings of being alone and often useless/a burden on others.

\section{Frustration:}

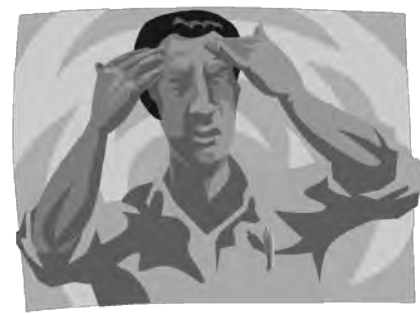

As we said above, try to understand why you struggle with a certain activity and use the tips to try and help yourself in these situations before feeling frustrated or useless and giving up. It's important to look at your situation positively and see how you can solve a problem instead of feeling frustrated about it.

\section{Isolation:}

Don't stop seeing your family or friends in social gatherings because of your condition. As we mentioned, there are ways to still be social (like using a walking device or a wheelchair when necessary) and having OA should not cause you to be alone or lose your friends.

\section{Depression:}

People with chronic pain can also be depressed due to the large effect such a condition can have on daily life. This is often not picked up by the nurses and doctors at the clinic. It is important for you to tell your nurse or doctor if you think you have depression. Depression is an illness; it is not simply being sad, scared, lonely and stressed feelings. Depression develops over a few weeks and is a general feeling of depressed mood which happens with physical symptoms. This is caused by an imbalance of chemicals in the brain. See the

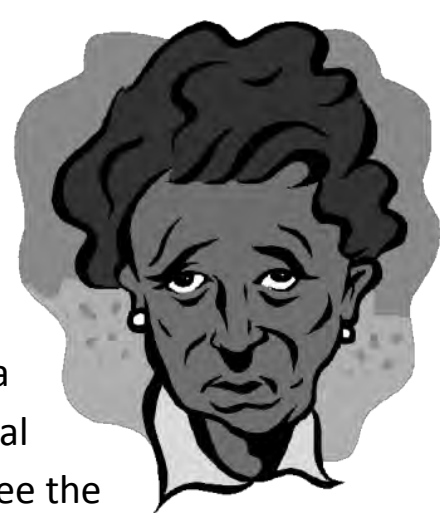
depression checklist below - if you think you have depression and you have many of these symptoms then you probably have some degree of depression. This can be treated with medicine and psychological support. It is not something to be ashamed of. Go to the clinic and tell the nurse or doctor how you are feeling so that they cans start you on treatment. 


\section{Depression Check List:}

- Do you feel down most of the time?

- Do you lack enjoyment with fun things like music, soccer or chocolate?

- Do you try to find peace by overeating?

- Or do you lack appetite and lose weight?

- Do you sleep badly at night?

- Do you struggle to get up in the mornings?

- Do you feel angry and agitated very quickly?

- Do you feel very passive?

- Do you lack energy every day?

- Do you struggle to concentrate?

- Is it difficult to make decisions about simple matters?

- Do you feel guilty?

- Do you feel worthless sometimes?

- Do you think of death a lot?

- Do you think of killing yourself?

If you answer yes to many of these questions, then you may have some degree of depression. Speak to the doctor or nurse at the clinic about how you are feeling.

If you answer yes to one or two of these questions then you may have depressed mood which you can manage with some of the techniques described in the section on stress management. 


\section{Home treatment for Depression:}

There are many things you can do to help manage depression. Make sure that you get help straight away if you feel like hurting yourself or someone else. Often talking to a person who understand or to a health professional will help you through this mood. Cut back on alcohol, although it might make you feel better in the short term. In the long term it affects the way your brain works and you will not be able to escape the depression. Keep active, make you sure you get up every day, get dressed and get out of the house. Even if you don't feel like doing things, it's important to keep active, visit friends, and join a group. If you start to lose contact with people and withdraw your mood will only get worse. Make plans for the future, for tomorrow, for next week, for next month. Make sure you do 20 to 30 minutes of exercise every day. As we said in Week 1, exercise is very important to keep us healthy and help our moods. Depression feeds on depression, when you believe that things will get better, they will start to change. Use the suggestions in the section on Week 3: Stress Management to help you manage your symptoms.

\section{Recap: What happens when you have pain and other symptoms?}

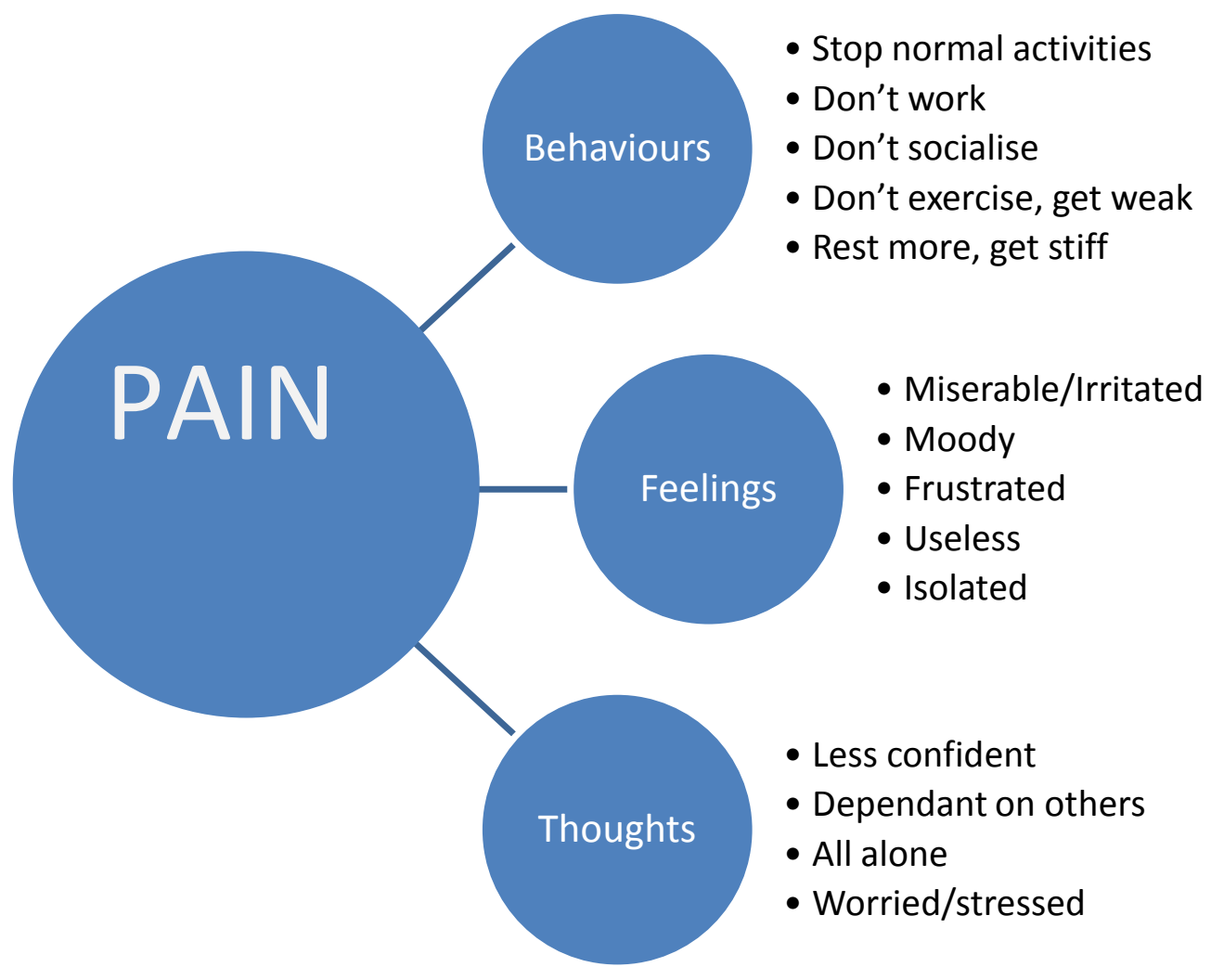


What to do to manage with this?

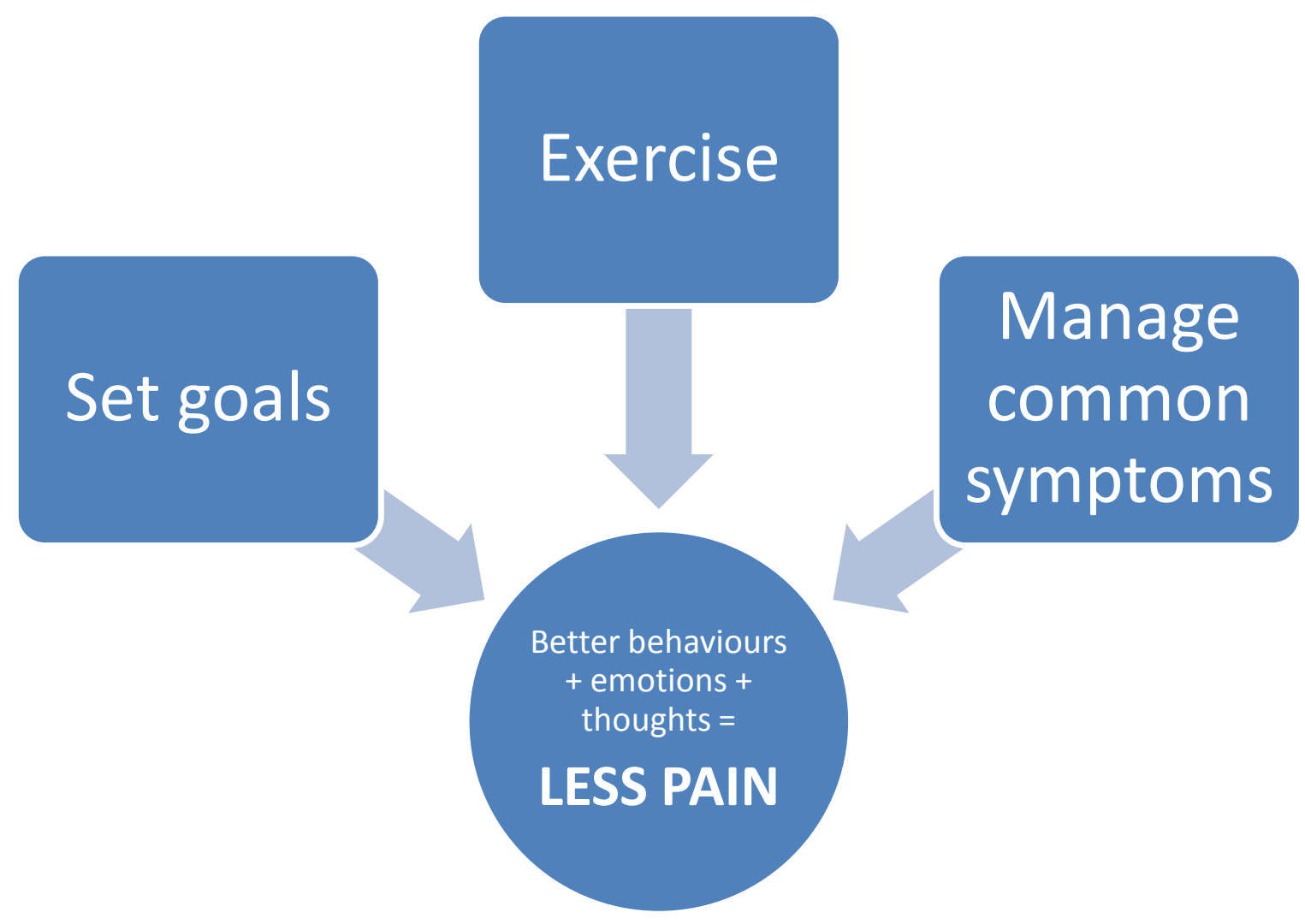




\section{Action Plan Form - Managing common symptoms}

Use this form to develop an action plan on exercise. What exercise would you like to do?

Be sure your action plan includes:

What you want to do

How much you are going to do

When you are going to do it

How many days a week you are going to do it

For example: This week, I will use an assistive device (what) like 1 crutch (how much) when walking outside (when) at least three times (how many).

This week I will:

(what)

(how much)

(when)

(how many?)

How confident are you that you can complete this action plan?

Not at all $|\quad| \quad|\quad| \quad|\quad| \quad|\quad| \quad|\quad|$ Totally

confident $122345 \quad 678910$ confident

Keep a record of how you did:

\begin{tabular}{|l|l|l|}
\hline & I Plan to..... & I did..... \\
\hline Monday & & \\
\hline Tuesday & & \\
\hline Wednesday & & \\
\hline Thursday & & \\
\hline Friday & & \\
\hline Saturday & & \\
\hline Sunday & & \\
\hline
\end{tabular}




\section{Week 3: Stress Management}

\section{- What is stress?}

In our society, we talk about stress a lot. We might say that it is stressful to live in South Africa. That it is stressful to worry about our children or our families, it is stressful to worry about money or it is stressful worrying about getting a job or coping with my job. We use the word stress a lot, but what does it mean? Stress is a feeling; it is a combination of feeling tense and worried. When we feel stressed we may be irritable, and find it difficult to concentrate or remember things, stress can affect our sleep, our appetite and our relationships.

The most common reason why we feel stressed is a lack of control. We tend to feel that things are stressful if we don't have any control over them. We feel stressed if we are going to be late for work because the trains are late - this is out of our control. We feel stressed about where we live if we don't feel safe there - those who commit crimes against us are also out of our control. In the same way, we may feel stressed when we have a chronic illness like arthritis or diabetes or high blood pressure. If you feel that your illness is out of your control and there is nothing you can do to affect it, this makes you feel stressed.

Stress is not always bad. We know that stress can be useful too. For many people if we feel some stress, we might feel under pressure to perform better. You might feel stressed because your family is coming to visit, but this stress makes you tidy up your home - a good effect of the stress. Students who are studying will only complete their studies if there are exams and deadlines for assignments, without the stress of the deadline, the students would not complete the work.

Sometimes we wish for a "stress-free" life. But, we know that if there was no stress in our lives, if we did not have to do anything all day long, this would not be good for us either. If I lay in bed all day and did not do anything, my muscles would get weak, my joints would get stiff and I would become ill. We need some stress in our lives to keep us healthy. The important thing is to keep the amount of stress at a level that we feel we can manage. This is why we talk about stress management, not stress elimination! 
There are many different things we can do in our lives to manage stress. The first step is to understand why we are feeling stressed. There are usually three things which affect how stressed we feel.

\section{The stressful situation:}

Usually the less you expect the situation and the less familiar you are with a situation, the more stressful it will be. If you needed to take the train to work but you knew the day before that the trains would be late, this would be less stressful than finding out after you have got onto the train that it is going to be late. If you think about having pain, if you know the cause of the pain is it more or less stressful? If you don't know what is causing your pain and you are worrying that there is something seriously wrong, is this more or less stressful?

\section{How you see the situation and how you cope with it:}

If the situation you are in is not important to you, you are likely to feel less stressed about it. If you are on a train which is going to be late, but you are going shopping on your own, then you are likely not to get so stressed about it. If you are on a train which is going to be late and you are going to work this might be more stressful, but if you have a cell phone with you and you have airtime on the cell phone and you telephone your boss to explain why you will be late, then this might be less stressful. Your ability to cope with the situation, affects the amount of stress you feel. While it is stressful to live with a chronic disease like arthritis, diabetes or high blood pressure, if you thought you could cope with it and it would not interfere with your job and your life, would it be more or less stressful? Having knowledge about your condition allows you to think about it in a different way and will change the way that you cope.

\section{Support from family and friends:}

Friends and family who understand and support you will affect your levels of stress. Feeling alone and like you have no support will probably make you feel more stressed. If you think about living with osteoarthritis, would it be more or less stressful if there were no one to support you? But, we do need to be careful about support from family and friends. If they take over doing everything for us (because they care about us and are trying to help), we might feel useless and like we don't have a purpose. Supporting me does not mean doing everything for me. 
Stress is not just the things that happen to us. The amount of stress that we feel depends on a lot of different things which can change every day. There are many different things we can do to manage stress every day.

\section{Dealing with the cause of the stress}

The first step in dealing with stress is to identify why you are feeling this way. Use the self-management steps to help you identify the problem. Once you know why you are feeling this way then you need to decide what you can do about it. Sometimes dealing with the things that stress us is easy, if you are friends with your neighbours and the noise from their television is irritating you, it might be easy to ask them to turn down the volume. If you are not friends with your neighbours, or you are very shy, it might be quite difficult to ask them to turn down the volume. Sometimes we can identify the things that stress us and do something about it. But, often we either cannot deal with it or it is out of our control. If you cannot deal with it or it is out of your control, the next step is to change the way you are looking at the problem.

\section{Look at the problem in a different way}

Think about how you are feeling. Are your thoughts and feelings about the problem inaccurate? Maybe you are very worried about your health, this is stressing you. Are you worried that you will be very ill and unable to work soon? Are these thoughts and feelings accurate? On what information are you basing these thoughts and feelings? Have you spoken to experts about your health or are you basing your thoughts and feelings and stress on poor information?

\section{Plan your life}

Do you get stressed by the same things over and over again? Or do you find yourself getting stressed because there are times when your life is very busy? If you are doing the same things over and over and getting stressed, you might want to look at how you are dealing with it and see if you can try a different plan. What about a busy life? This is also about planning, being very busy and having no time for ourselves, can be very stressful. Plan things over time carefully, make sure you have time to at least do some relaxation or exercise even when you are very busy. Do not leave things for the last minute. 


\section{Get help from family, friends and support groups}

These are a great way to decrease stress. If we want support from people though, we have to tell them clearly what the problem is and what we would like from them. Often we do not communicate clearly and this might make the stress worse! If you find your family or friends are not very helpful or supportive, it might be worth sitting down with them when you are not feeling stressed to talk about these things. It might be that they see things differently to you, this does not mean they are right and you are wrong, or that you are right and they are wrong. It just means that you see things differently and you can discuss how to handle things better. If having a discussion like this is difficult, it might be useful to ask a counsellor to help with the conversation. You can ask for assistance at a clinic or you can go to an organisation like FAMSA who specialise in family and relationship counselling.

\section{Exercise}

Exercise is a very effective way of managing stress. People who exercise regularly doing at least 20 to 30 minutes of exercise, 3 times a week have less risk of suffering from stress related illnesses. Go back to the section on exercise for more on how to exercise safely and effectively.

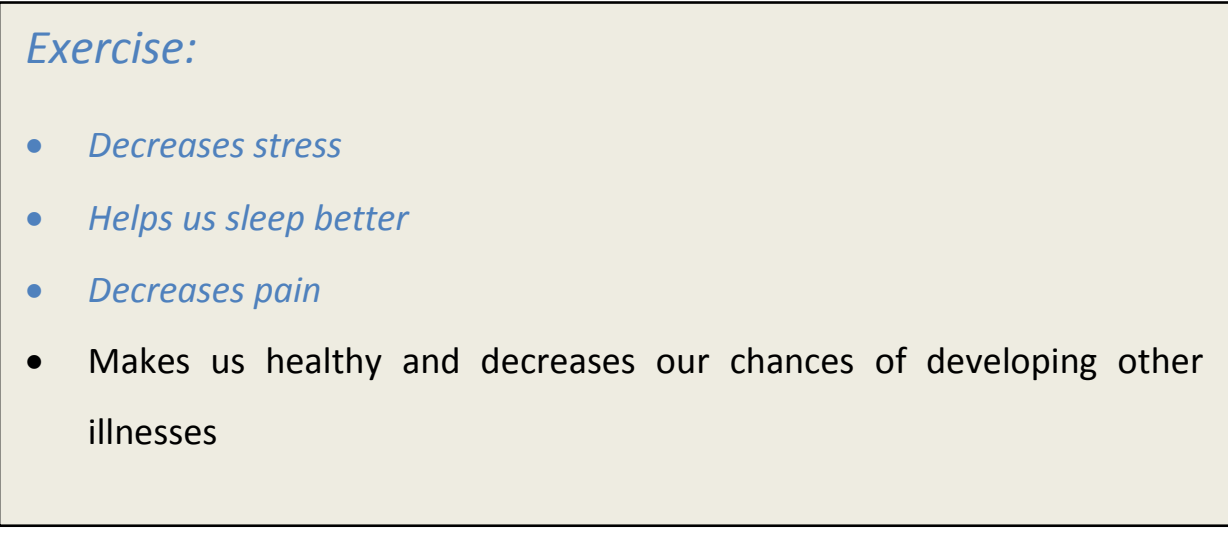

\section{Relaxation skills}

When we feel relaxed, we feel calm. Sometimes if we are relaxed and we are tired, we might feel sleepy. At other times we might feel relaxed and alert and be able to concentrate calmly on tasks. Relaxation can help us to concentrate and it can help us to unwind and go to sleep. Relaxation is a very useful way to manage stress and some of the symptoms of chronic diseases such as pain. 
If we are stressed, this can make our muscles tense, our hearts beat faster and we breathe faster, if we are also feeling unwell and have pain we will feel worse. Relaxation can decrease the tension in muscles and slow down our hearts and breathing and help to make us feel better. If we are stressed we often become irritable and moody, relaxation helps to calm you and make you feel more in control of your life. When we are stressed sometimes it is difficult to fall asleep as we are worrying about things out of our control, if you are also unwell, not sleeping will make you feel worse. Relaxation will help you get to sleep, this will help manage your stress and improve your health.

Just like learning to play a new sport or doing exercise, relaxation takes practice. The specific way that you relax doesn't matter; we are all different and might relax in different ways. The important thing is to practice it regularly. There are two different ways of relaxing described at the end of this section. You can do these at home in a quiet and comfortable safe place to begin with. But, once you get good at relaxation, you can relax in a crowded waiting room, on a train or a taxi. You can do relaxation anywhere!

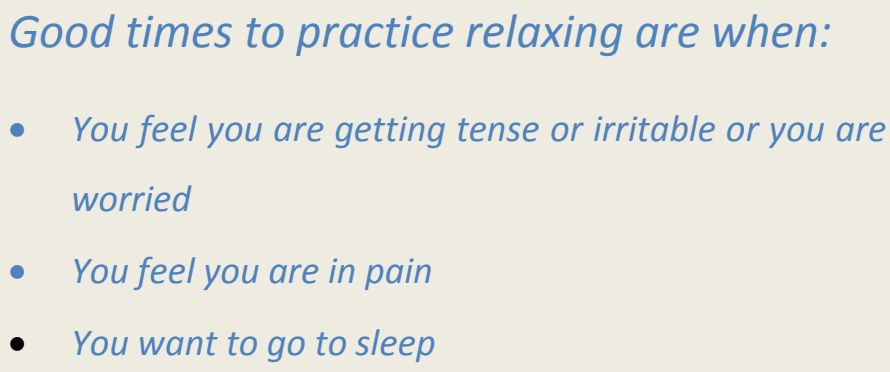




\section{Relaxation techniques:}

\section{Long relaxation:}

- $\quad$ Find a comfortable position. Lie on your back or sit in a chair with your back supported.

- $\quad$ Place your hands at your sides, palms up. Close your eyes if you wish.

- Now begin to become aware of your breathing.... Focus on slowing down the rhythm of your breathing.... Your chest and tummy will expand outward with each breath, like a balloon gently filling with air....

- Imagine your ribcage moving out to the sides when you breathe in.... and gently inward as you breathe out...

- Slowly take a deep breath in.... Pause for a moment.... and then slowly breathe out. Let the tension melt away as you relax more deeply with each breath...

- Continue breathing slowly and gently....

- Now think about the top of your head. Feel the skin on the top of your head beginning to relax, and spreading slowly downwards....

- Even your ears are becoming relaxed and heavy.... Feel your eyebrows resting....

- Your forehead is becoming relaxed and smooth.... all the lines on your face are becoming smooth.

- Let your jaw relax by allowing your mouth to be slightly open.... Allow your tongue to relax..

- Feel your throat relaxing.... relax your cheeks, nose, and eyes... Feel your eyelids becoming very heavy.... and very relaxed.... more and more relaxed....

- Enjoy the feeling of relaxation you are experiencing

- Now think about your neck... allow a feeling of relaxation to begin at the top of your neck, and flow downward...

- Feel the relaxation as your shoulders become relaxed and loose... Let your shoulders gently sink downward.... as they become relaxed.... and heavy.... very heavy.... and very relaxed.... deeper and deeper.... relaxed....

- Feel your collar bones becoming relaxed as your shoulders move gently back, and your chest widens slightly....

- Allow all the muscles in your shoulders to feel smooth... and relaxed.... as the muscles give up their hold completely...

- Notice your breathing once agdin... see how regular it has become... continue to take slow.... smooth... deep breaths... Breathe in the feeling of relaxation... and breathe out any tension... your breathing allows you to become mok and more relaxed.... deeply relaxed..... Now turn your

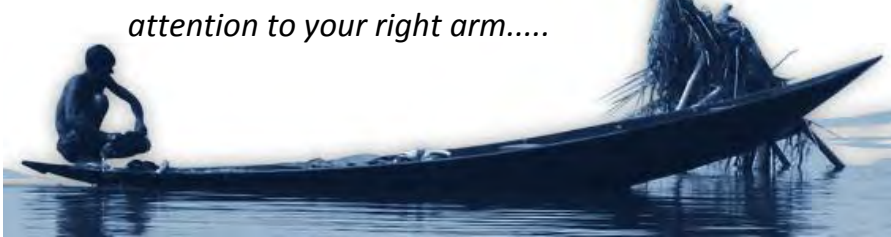


- $\quad$ Feel the relaxation flowing down from your right shoulder... allow your upper arm to relax... your elbow... lower arm... and wrist become loose and relaxed....

- Enjoy the feeling of relaxation as the muscles of your right arm give up their hold.... Feel the relaxation flowing into your hand... Let all the tension drain out of each finger tip and flow away.... the relaxation spreads to your thumb... index finger.... middle finger... ring finger... and little finger....

- Feel the relaxation flowing down your left arm... Let the muscles of the left upper arm relax.... Relax your elbow... lower arm.... and wrist....

- Enjoy the feeling of relaxation you are experiencing.

- Let the tension melt away.... imagine the tension flowing right out of your finger tips... Allow your left hand to relax completely... relax your thumb... index finger... middle finger... ring finger... and little finger....

- Both of your arms are now totally relaxed... allow them to be free and limp... pleasantly relaxed...

- Enjoy the feeling of relaxation you are experiencing...

- Allow the feeling of relaxation to continue to your chest and stomach....feel the relaxation there... becoming deeper with each breath....

- Now turn your attention to your upper back... Feel the relaxation flow down your spine... Let all the muscles give up their hold.... relax your upper back... middle and lower back.... allow your back to relax completely.... Feel the relaxation in your whole upper body ....

- $\quad$ Relax more deeply with each breath.... more and more relaxed.... deeply relaxed and calm....

- Let your hip muscles relax... Relax all the way from your buttocks (bottom), down the back of your thighs... relax the muscles on the front of your thighs...Feel the relaxation in your upper legs moving down to your knees... your calves and shins.... your ankles.... and your feet.... allow all the muscles to relax and go limp....

- Allow any last bits of tension to flow right out of the soles of your feet....Feel the relaxation flowing through your body... From the top of your head... down to the bottoms of your feet... become more relaxed with each breath.... enjoy the feeling of total relaxation.....

- You are now as relaxed as you want to be.... Experience the feeling of deep relaxation... enjoy the feeling.... relaxed.... calm..... at peace

- $\quad$ Focus on the feeling of relaxatidn throughout your body.... Notice your breathing.... Your relaxed muscles... Your calm thoughts... Memorize this feeling so you can re-create this relaxed state whenever you wish....

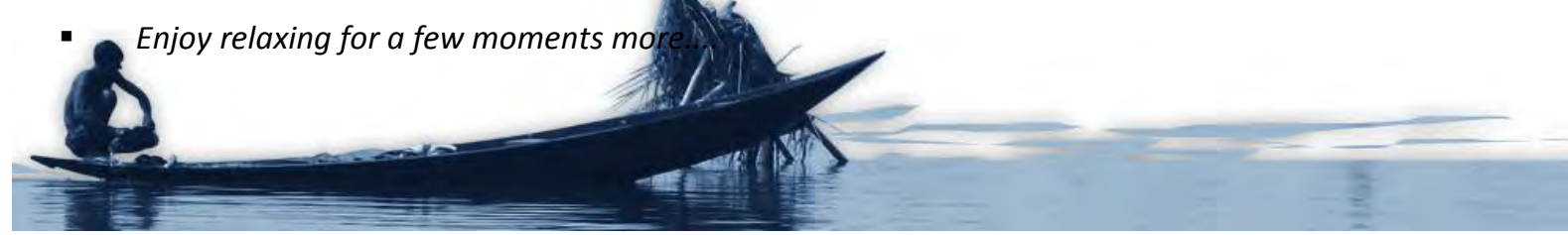


- When you are ready to return to your day, reawaken your body slowly... gently move your muscles... roll your shoulders slowly forward....then slowly backward.... lean your head gently to the left... return to centre.... lean your head gently to the right... turn your head...

- Wriggle your fingers and toes....Gently open your eyes.... Feeling alert... calm.... and full of energy.

\section{Short relaxation:}

- Deep breathing not only helps to cure anxiety and stress, it also triggers relaxation. Here's how to breathe deeply.

- Breathe in slowly to the count of four (count slowly; to the pace of one-one-thousand, two-onethousand....). Pause to the count of three.

- Breathe out slowly to the count of five.

- The breathing process goes like this:

- Inhale... two, three, four...pause...two, three....exhale...two, three, four five....

- Inhale... two, three, four...pause. two, three....exhale...two, three, four five....

- Repeat for a minute or two.

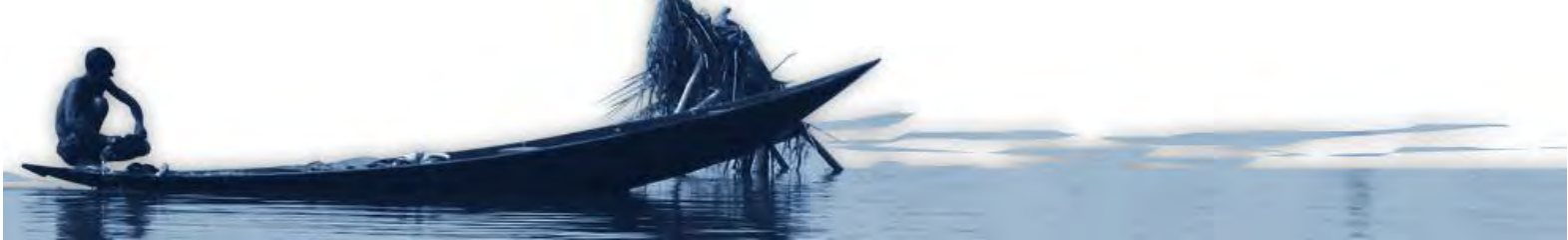


People with chronic illnesses often struggle to sleep because they are stressed and worried about their condition, they worry about what this means for them, for their family, for their future. People also often struggle to sleep because of the illness itself, perhaps you have

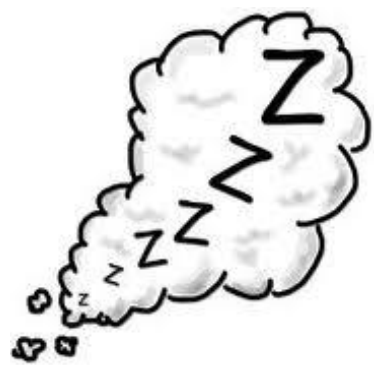
pain, you feel sick or you may even be so tired you can't sleep. Some people find it difficult to get to sleep and only fall asleep very late at night, others find that they fall asleep but then wake up during the night and can't get back to sleep. Some people find it difficult to sleep at all at night and sleep during the day.

Sleep is very important to keep healthy. We all need different amounts of sleep. Some people need 8 hours of sleep a night, some may need 10 hours and some people only need 5 hours of sleep. We are all different. We have been learning how to fall asleep and sleep well since we were babies. If you do not sleep well, following these steps will help you to learn how to fall asleep and sleep well. Remember that like learning anything new, this will take time. It might take up to 3 months to learn to sleep well if you have been struggling with sleep for a while.

\section{Suggestions for Improving Sleep}

Have a bedtime routine: try to go to bed at around the same time every night and always do the same things before getting into bed. A bedtime routine could be to lock the house, get undressed, wash your face, clean your teeth, get into bed and do a relaxation session.You can't sleep because of worrying: write down your problems or the things that are worrying you, then write down the next step that you think could help sort out the problem. If you wake up during the night worrying about the problem, remind yourself that you've gone over it and you have a plan. If you wake up with a new worry, write down that problem to deal with in the morning. Practice your relaxation to take your mind off the worry. If you still can't sleep, it may be better to get up and do something relaxing like reading, watching TV, listening to relaxing music or doing relaxation.

Your bed and bedroom are for sleeping: try not to use your bedroom during the day. Do not watch TV in bed. If you are not asleep within 30 minutes of going to bed, get up and do something else. Do not lie in bed and worry that you have not fallen asleep. This will only make you feel stressed and lessen the chance of falling asleep.

Have a morning routine: get up at the same time every day, even if you don't feel like it. Our bodies like to work on regular patterns to fall asleep and get up at the same time every day. 
Avoid drinks containing caffeine for at least 4 hours before going to sleep (drinks like coke, tea or coffee).

Never use alcohol to help you sleep. It might make you feel relaxed at first, but once this wears off it is likely to make you feel jumpy and you are likely to wake up during the night.

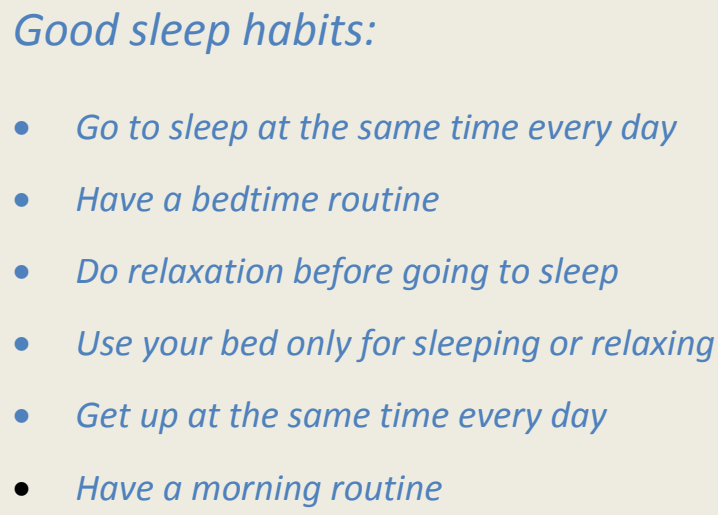

\section{Communication with your health carer}

Anyone living with a long term health problem, whether it is arthritis or high blood pressure or diabetes will have to visit their clinic regularly. Visiting the clinic regularly can be stressful because it takes time, you have to plan ahead, you might not be sure how long you are going to have to wait, you might be worrying about what the health carers are going to tell you. One of the most important ways of managing the stress associated with visiting clinics and seeing health carers is to think about and plan how to communicate with them.

When visiting the clinic to see a health care practitioner it is important that you feel comfortable asking questions (any questions, even if you feel they are "silly" or "stupid" questions) and comfortable expressing how you feel. It is also important that you feel you can negotiate your treatment with your health care provider so that both you and the carer feel that you are receiving the best care for you. It is important that you not feel that your health care provider is ignoring you, "puts you down" or treats you like a child. We know that doctors and nurses have a lot of patients to see and they have little time to spend with each person. One helpful way to make sure that you get the most out of your appointments with the doctor or nurse is for you to take PART - Prepare, Ask, Repeat, Take action. 


\section{Take PART:}

\section{Prepare:}

Before your appointment at a clinic it is important to prepare. Think about the reason for your appointment and whether there are any issues in particular that are worrying you. Write down your questions or the things that are worrying you. You need to be realistic about the list you write down, there will probably only be time to answer one or two of the things on your list. Make sure the most important problems are at the top of the list. Take your list with you to your clinic appointment, then when the doctor or nurse asks if there is anything you want to ask, you can use your list.

If there are particular symptoms or health issues you want to discuss, prepare for your appointment by writing down specific information the doctor or nurse will want to know. Things that are helpful are: when did it start, how long do the symptoms last, where are they in your body, what makes you feel better or worse, have you had a problem like this before and how was it treated; have you changed anything such as your diet, exercise, medicines. If you have already received treatment for a problem, be ready to report back on how well it has worked, or on whether it has not worked at all.

Be open about how you are feeling and about the things that are worrying you. The more open you are, the more the health care provider can help you. Finally, give feedback. If you don't like the way you have been treated you can tell the doctor or nurse. If you do not want to tell them directly then you can speak to someone else in the clinic or to someone in a support group. Remember too that doctors and nurses and other health care providers also appreciate being complimented. If you feel that you have been treated well and are happy with your treatment, it is acceptable to compliment the health carer.

\section{Ask:}

Another important step in having good communication and decreasing stress is to ask questions. Having good information is essential to you being successful in self-managing your health. Ask questions about your diagnosis such as what is wrong, what has caused it, is it contagious and what is going to happen now? Then ask questions if you have had tests, what is the test for; what if I don't have the test and what will the test involve? Remember to ask questions about your treatment options, what are the benefits of treatment and what are the risks and side effects? 
Finally ask questions about follow-up, when should you return to the clinic, what should you watch out for and what should you do next? If you find you have difficulty remembering information it is a good idea to write things down during your visit. Or you could ask someone you trust to come to the appointment with you to help with remembering.

\section{Repeat:}

One of the important things to do to help with remembering things is to repeat it. So if the nurse or doctor explains something to you, repeat back to them in your own words what you have understood. This is very useful to make sure there are no misunderstandings.

\section{Take Action:}

At the end of your appointment, it is important that you know exactly what you will need to do next. It might be that you need to make another appointment, or that you need to go home and change something or get new medicine from the pharmacy. Make sure that you are clear about what you need to do next, and then do it! 


\section{Action Plan Form - Stress management}

Use this form to develop an action plan on exercise. What exercise would you like to do?

Be sure your action plan includes:

What you want to do

How much you are going to do

When you are going to do it

How many days a week you are going to do it

For example: This week, I will relax (what) using long relaxation (how much) at bedtime (when) at least three times (how many).

This week I will:

(what)

(how much)

(when)

(how many?)

How confident are you that you can complete this action plan?

$\begin{array}{llllllllll}\text { Not at all } & \mid & \mid & \mid & \mid & \mid & \mid & \mid & \mid & \mid\end{array}$

confident $122345 \quad 678910$ confident

Keep a record of how you did:

\begin{tabular}{|l|l|l|}
\hline & I Plan to..... & I did..... \\
\hline Monday & & \\
\hline Tuesday & & \\
\hline Wednesday & & \\
\hline Thursday & & \\
\hline Friday & & \\
\hline Saturday & & \\
\hline Sunday & & \\
\hline
\end{tabular}




\section{Week 4: Eating well}

You are probably wondering why we talk about nutrition at this course. What has food got to do with osteoarthritis or the pain you are having? Well there are many good reasons for that, but if you think about it; if managing your pain is all about lifestyle changes and making decisions that are good for your health and body, surely wise food choices fit in to that as well. This section is not about weight loss - there are many people out there who are not overweight, but still suffer from terrible chronic diseases such as diabetes, high cholesterol and hypertension, on the other hand there are also overweight people, who exercise and eat a well-balanced diet, but don't suffer from chronic illnesses. So it is really about how we are built and how our bodies burn what we decide to feed it. What is common for all, is the fact that when we eat a well-balanced diet that is good for us, we feel better, we have more energy and we are happier. We will try and explore why that is now.

\section{The Diesel Diet}

Think of the Human Body as a Diesel car. The Human body needs to work and chug along at a constant pace throughout the day; it can't be like a Formula One car that goes very fast for a short amount of time and then stops!

Therefore we must re-fuel our bodies constantly so we are able chug along for the whole day without running out of energy. But we also have to be careful about what kind of fuel we put into our bodies. It has to be diesel that will last us for a long time. If we give ourselves high octane petrol fuel it will give us a quick burst of energy but will run out quickly.

So, to make it easy for everyone, the "diesel" we need to feed our bodies is food that will keep us full for long and keep the body's blood sugar levels steady. It is the high octane fuel we need to keep clear of. These are for example sugary and highly processed foods, such as cakes, pies, chocolates and any "quick fixes"; the kinds of foods which most parents know that if they gave it to small children would give them lots of energy to run around a lot before they collapsed exhausted!

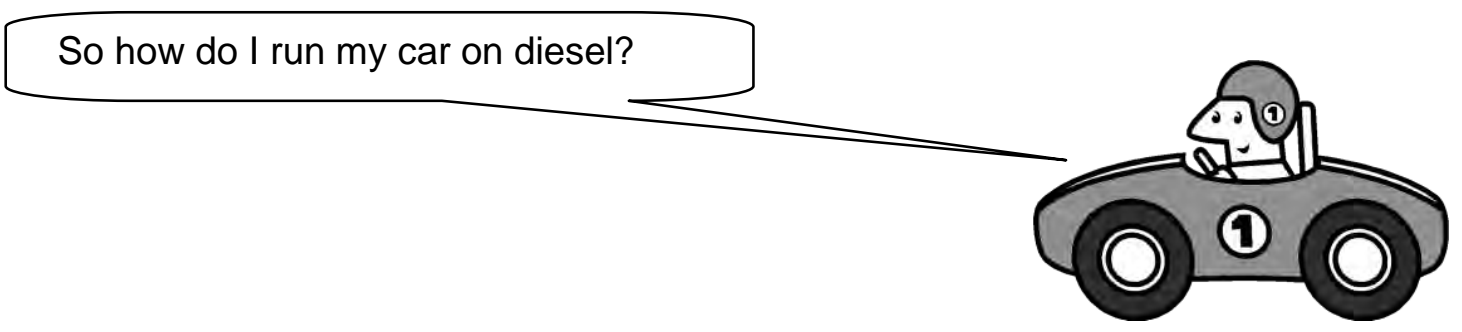




\section{Two Schools of Thought}

At the moment there is a lot of scientific research about what kind of foods we can categorise as healthy. There are generally two schools of thought. One favours a low fat and high carbohydrate diet and the other favours high protein and low carbohydrate diet. In this booklet there will be a choice of the two. With your doctor or a dietician, you can decide which one would suit you better. It is very important to emphasise that whichever suits you, neither one should contain high simple carbohydrates, that is foods containing a lot of sugar and starch. So in order to understand the options, we need to look at the food groups, from which we make our food choices. Let us have a look at what these food groups are:

\section{Carbohydrates}

Carbohydrates are often called starches or carbs. There are two kinds of carbohydrates simple and complex. They both provide the body with energy. But the simple carbs give you a quick fix (lots of energy very quickly and a subsequent high rise in blood sugar, followed by a rapid drop and you feel tired and hungry again quickly). The complex carbs raise your blood sugar more slowly and so you feel more energised for longer. It is safe to say, we need more of the complex carbs than the simple ones. Let's look at the difference.

Examples of complex carbs

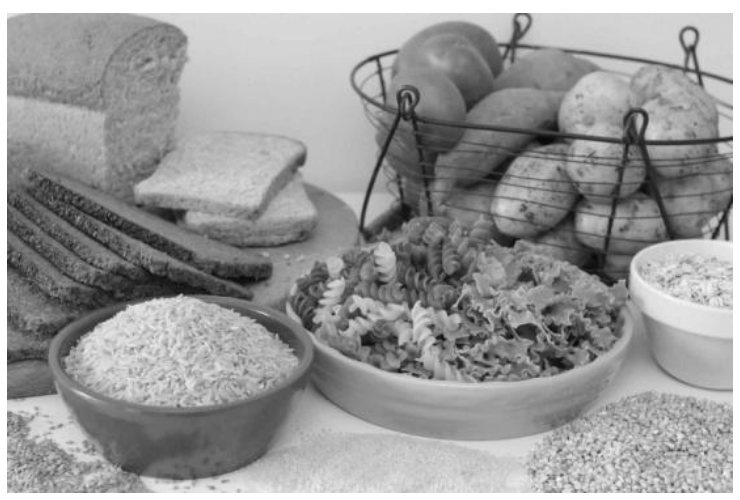

\section{Examples of simple carbs}

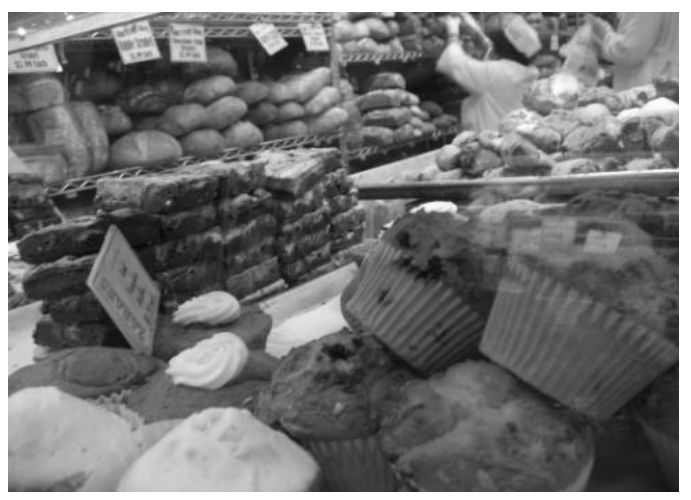


Why do we need Complex Carbohydrates?

Carbohydrates are your most important source of energy.

Where do the Complex Carbohydrates (diesel fuel) come from?

- Grains

- Brown Rice

- Oats

- Whole Wheat

- Lentils

- Vegetables - mainly green and leafy (broccoli, cabbage, lettuce)

- Whole wheat Pasta

- Whole Wheat/Brown Bread/rye bread

- Potatoes

What are the Simple Carbohydrates (high octane fuel)?

- Sweets

- Cake

- Sugar

- Biscuits

- Syrup

- Sweetened Cereals (e.g. Fruit Loops, Coco Pops)

- White Rice

- Wheat based pasta

- White Bread

- Chips/Crisps

- $\quad$ Pies \& Pastries

- Fizzy drinks like Coca Cola

NB! Even on a high carbohydrate diet, you need to keep clear of the simple carbohydrates! Simple carbs are very addictive - the more you eat the more your body wants. They are easily accessible, take no time to prepare and unfortunately are often cheap choices. 


\section{Protein}

Why do we need Protein?

Proteins are important for building and repairing muscle, blood cells, bone, hair, organs, other bodily cells and making hormones. They are also an important source of energy.

Where does the Protein come from?

- Animal Products

- Meat (Red meat, Fish, Poultry)

- Eggs

- Milk

- Cheese

- All other Dairy Products

- Beans

- $\quad$ Seeds \& Nuts

- Grains

- Soy Products

Fibre

Why do we need Fibre?

Fibre is important for the smooth running of our stomachs.

It helps prevent constipation. It helps prevent Stomach Cancer It is especially important if you are taking lots of pain killers and anti- inflammatory drugs, which slow the stomach down.

Where does the Fibre come from?

- Vegetables

- Fruit

- Whole-Grain Bread, Pasta \& cereals

- Oats, Oat bran

- Brown Rice, beans, lentils

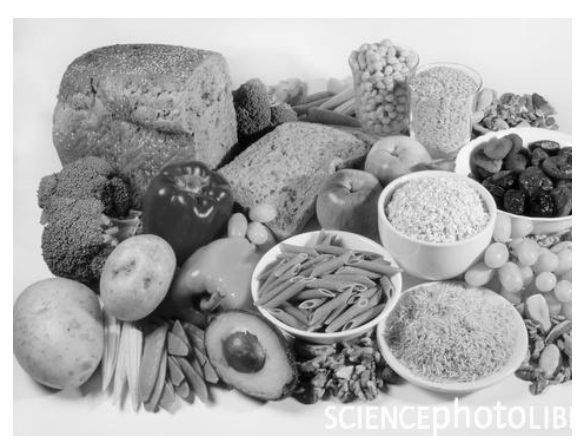


Why do we need Fat?

A source of fuel for muscles and brain. Primary source of energy when exercising hard.

Helps with constipation

Where does the Fat come from?

\section{Good (unsaturated) Fat}

- Vegetables

- Fish Oil

- Olive oil

- Nuts\& Seeds

- Avocados

Bad (Saturated) Fat

- Animal Foods

- Dairy Food

- Butter

- Milk

- Cream

- Margarine

- "Snack" Foods

- Chips/Crisps

- Samoosas

- Pastries

- Fried Foods

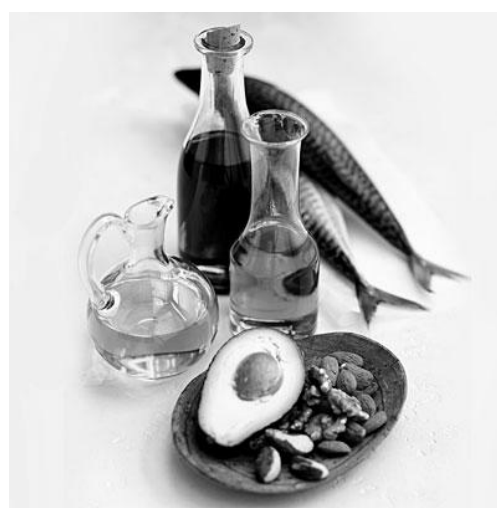

- Trans Fats

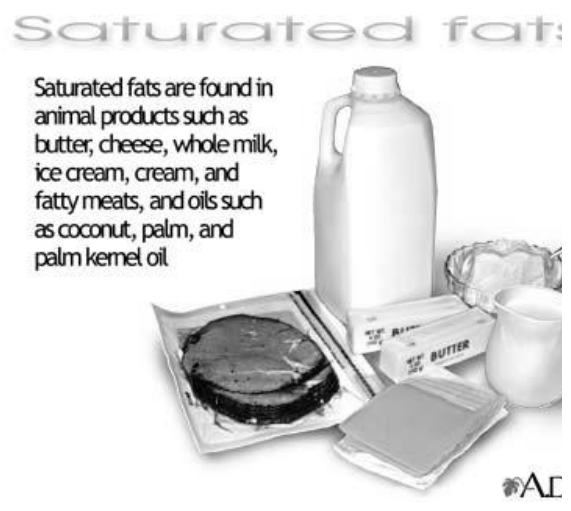




\section{Some helpful Guidelines:}

Bad (saturated) fat and increased fat consumption can increase your chance of having a Heart Attack, Stroke and Diabetes.

Avoid hard fats such as butter, margarine and animal fats. Focus on olive oils, grape seed oil and oil from avocados, nuts and seeds.

Don't fry or roast food, rather, steam or microwave the food or eat it raw.

Rather Grill meat than fry it or roast it.

Use Olive oil rather than butter, Canola Oil or Lard.

Read the food labels and eat low fat food! The total fat should be less than $10 \mathrm{~g}$ per $100 \mathrm{~g}$.

Use non-stick pans instead of oil. 
So now that we know all about the food groups, how do we put it all together? Let us have a look at the two different kinds of diets described earlier:

\section{LOW FAT - HIGH CARBOHYDRATE DIET}
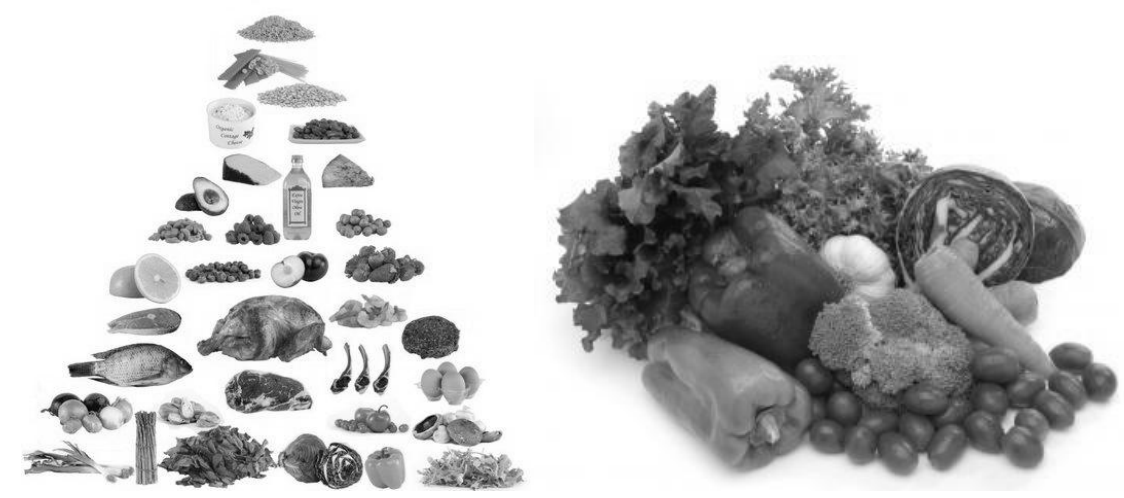

If you burn carbohydrates well, or feel like you need to lose some weight or at least want to benefit from better nutrition, a balanced diet of very little saturated fats, complex carbohydrates and some protein might do the trick.

On this kind of diet, it is important to keep the diesel going throughout the day and in doing so eating 5-6 smaller meals is the best way. There are more carbohydrates in this diet, which will not keep you full for as long as the protein rich diet, so you will need to eat more often. It is therefore suggested that your daily meals consist of:

- Breakfast

- Mid Morning Snack

- Lunch

- Afternoon snack

- Supper

- Late night snack (if you go to bed late). 
you crave more foods like the bad carbs often do. In other words they are not quick fixes. Table 2"The Not so Good Carbs" are carbs that you should be aware of not eating too many of and finally Table 3"The Bad Carbs" should be avoided as much as possible. Here is a recap:

Table 1: Good Carbs - Eat most of your carbs from this group

Table 2: Not so Good Carbs - Eat in moderation from this group

Table 3: Bad Carbs - Avoid eating from this group 


\begin{tabular}{|c|c|}
\hline TABLE 1 & GOOD CARBS \\
\hline Food Group & Examples of foods \\
\hline Dairy & Low fat/fat free: Milk, Yoghurt, heart foundation margarine spreads, cottage cheese. \\
\hline Starches & $\begin{array}{l}\text { All dried and canned beans, Peas, Lentils } \backslash \text { Dahl, Baked beans, Boiled barley wheat, Pasta made from whole wheat or } \\
\text { durum wheat-semolina, Sweet potato, Mealies/corn, cold samp, brown rice. }\end{array}$ \\
\hline Bread & $\begin{array}{l}\text { Provita, Seed loaf bread no added sugar, Pimpernickel bread, rye bread, any other bread with lots of whole kernels, } \\
\text { crushed wheat, and oat bran inside. }\end{array}$ \\
\hline Cereals & $\begin{array}{l}\text { Bokomo Pronutro Wholewheat, Hi Fibre Bran, Cold Mealie meal, Oat Bran, All-bran flakes, Fine Form muesli no added } \\
\text { sugar, shredded all bran. }\end{array}$ \\
\hline Vegetables & All vegetables not mentioned in table 2 and 3 . Stick to leafy and green ones. \\
\hline Fruit & $\begin{array}{l}\text { All deciduous fruit e.g. apricots, cherries, peaches, plums, pears, apples. All citrus fruit e.g. oranges, naartjies, } \\
\text { grapefruit and lemons. Kiwi and grapes - watch portions there is a lot of sugar in fruit - if in doubt stick to berries. } \\
\text { Avoid: See fruits in Table } 2 \text { and } 3\end{array}$ \\
\hline Snacks & Sugar-free jams and sweets, low fat popcorn. \\
\hline Drinks & WATER! Sugar free cool drinks, no more than 2 glasses of fruit juice per day. \\
\hline
\end{tabular}




\begin{tabular}{|c|c|}
\hline TABLE 2 & NOT SO GOOD CARBS \\
\hline Food Group & Examples of foods \\
\hline Dairy & \\
\hline Starches & Sweetcorn, potatoes with skin, coarse mealie meal porridge, white rice, couscous. \\
\hline Bread & Ryvita, brown bread \\
\hline Cereals & $\begin{array}{l}\text { Kellogg's fruitful bran, Bokomo pronutro flakes, Tastee wheat Kellogg's corn pops, Kellogg's frosties, Kellogg's choc's, } \\
\text { Mealie meal -reheated or with added corn }\end{array}$ \\
\hline Vegetables & Beetroot \\
\hline Fruit & $\begin{array}{l}\text { Tropical fruit e.g. banana, mango, sultanas, paw-paw, pineapple and litchis, grapes, melons. Dried fruit: sultana, dates } \\
\text { and raisins. }\end{array}$ \\
\hline Snacks & $\begin{array}{l}\text { Bakers Home-wheat digestive biscuits, Low fat biscuits containing oat bran, Low fat bran/fruit muffins/pancakes, Low } \\
\text { fat oat bran crumpets, Raw honey, Jam Sugar, Fine form canned peaches, Fine Form apricot jam }\end{array}$ \\
\hline Drinks & Juice of fruits in this table -1 glass only, Regular cold drink-cordials and soft drinks \\
\hline
\end{tabular}




\begin{tabular}{|c|c|}
\hline TABLE 3 & $B A D C A R B S$ \\
\hline Food Group & Examples of foods \\
\hline Dairy & \\
\hline Starches & Potatoes: boiled, mashed, baked and fried, Minute noodles, Rice especially sticky rice, Samp, Mealie rice. \\
\hline Breads & $\begin{array}{l}\text { All white bread, all bread rolls and anything made with cake flour, bread flour and nutty wheat flour, Rice Cakes, } \\
\text { Snackbreads }\end{array}$ \\
\hline Cereals & $\begin{array}{l}\text { Mealie meal - refined and sugar added, Puffed wheat, Maltabella, Instant Oats, Kellogg's strawberry pops and frosties } \\
\text { (any sugary cereal). }\end{array}$ \\
\hline Vegetables & Carrots and carrot juice, Pumpkin, Hubbard Squash, Butternut, Parsnips \\
\hline Fruit & Watermelon, Dried fruit rolls \\
\hline Snacks & Sweets -boiled and jelly type, Bakers Marie biscuits, Commercial honey, Glucose, Maltose \\
\hline Drinks & Game, Energade, Powerade, Lucozade \\
\hline
\end{tabular}




\section{Protein}

\section{How much do I need?}

50-65g per day.

\section{Some helpful Guidelines!}

Lean meat, poultry or fish (the size of a deck of cards) contain 25 to 35 grams of protein. Remember this is a low fat diet and all animal produce contain saturated fats, therefore you need to avoid fatty meats and processed meats.

One cup of cooked beans or lentils contains about 18 grams of protein.

One cup of low fat cottage cheese contains 28 grams of protein.

60 grams of solid cheese contains about 16 grams.

One cup of low fat milk contains 8 grams.

Two tablespoons of peanut butter contain 8 grams.

One serving of grain foods (barley, pasta, cereals, whole wheat bread, for example) generally contains 3 to 6 grams of protein.

One serving of vegetables ranges from 1 to 3 grams

\section{FIBRE}

\section{How much do I need?}

You need 25-35g of fibre per day

\section{Some helpful Guidelines!}

Choose whole-grain breads and pasta. (Flour from whole wheat contains three times as much fibre as refined white flour.)

Choose brown rice instead of white. (Brown rice has three times as much fibre).

Eat the whole fruit instead of juice. (One orange contains about six times as much fibre as a glass of juice).

Stir a few tablespoons of wheat germ into yogurt or hot cereal; add a couple of tablespoons of bran to pancake or waffle batter. 
Start your morning with cold or hot cereals that contain at least 4 grams of fibre in a serving. Read package labels and compare fibre content. Add fruit to your breakfast cereal.

Pack cold cereal, fruit or cut-up vegetables in your briefcase or handbag for nibbles.

Buy cut-up raw veggies at a salad bar or vegetable stand if time is short.

\section{FAT}

Remember there are saturated and unsaturated fats. On this diet you need to focus on the unsaturated fats which come from non-animal sources such as olive oils and low-fat spreads or your avocado, nut and seed oils, as well as fish oils.

How much do I need?

For one meal the total fat should amount to the same size as the tip of your thumb or one to two teaspoons. 


\section{HIGH PROTEIN - LOW CARBOHYDRATE DIET}

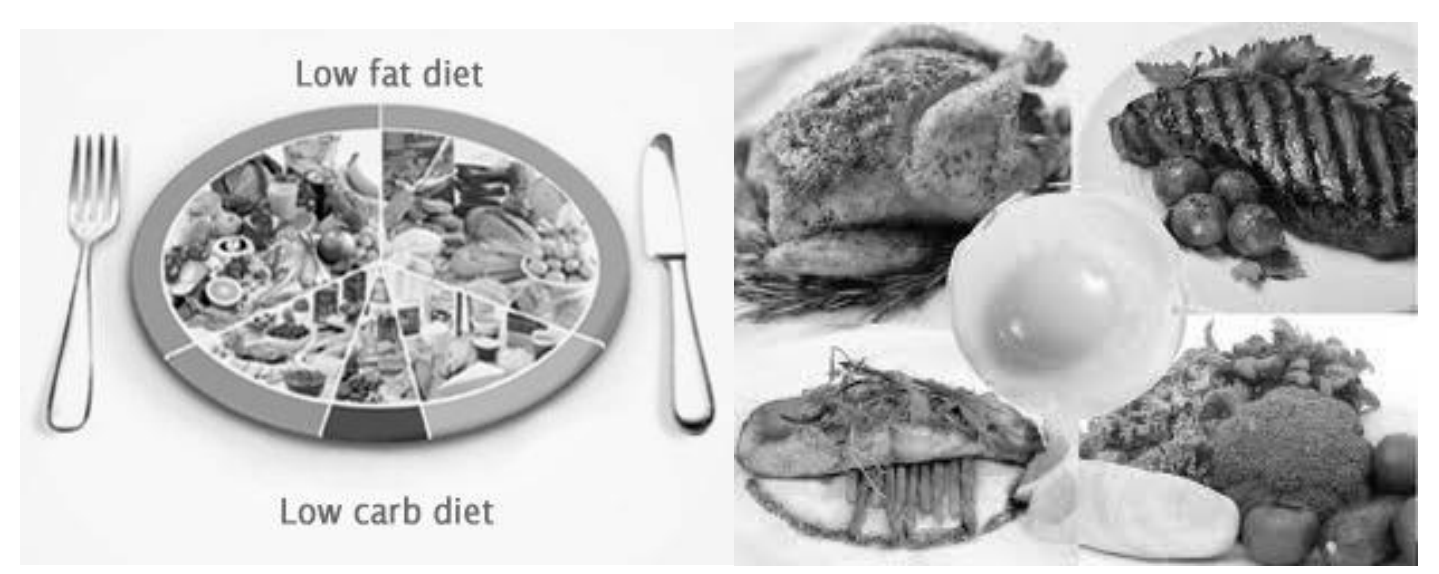

There is good scientific research showing that if you suffer from a sluggish metabolism, that is your body doesn't burn food very efficiently, you may benefit from a high protein - low carbohydrate diet. People who suffer from this have a hard time losing weight, you gain weight easily, get easily bloated when eating bread, rice, pasta and cereal. The researchers think this is because some people's bodies do not metabolise carbohydrates that well - even some of the carbohydrates that are more complex, such as pasta and rice.

On the high protein diet the main focus is on eating good and wholesome protein, such as lean meat, chicken, ostrich and fish. The kind of protein that you should keep clear of are processed meats like salami, poloni and spreads. They often contain a lot of saturated fats, sugar, flour and additives that are not good for us. With this kind of diet you are allowed to eat dairy foods too, for example cheese, eggs, full cream milks and yoghurts. Like any other diet it is encouraged that you choose nuts and seeds and good oils for fats and use less saturated fats such as cream and butter.

In terms of complex carbohydrates, these are very important but in this diet you get these from leafy vegetables. These leafy vegetables include broccoli, cabbage, lettuce, sprouts - basically most vegetable are acceptable BUT you need to avoid starchy vegetables such as potatoes, pumpkin, sweet potatoes and parsnips. These vegetables have a lot of natural sugars in them and if your body doesn't need them, they are converted into fat and your blood sugar levels rise.

You can eat some grains - but stick to brown rice, barley wheat, bulgur and quinoa for example. Like any diet it is important that you get fibre to maintain your stomach health. Most of your fibres you will get from the vegetables. Although it is not recommended to have lots of fruit, some is ok, especially berries as they are high in fibre and low in sugar content. Don't drink fruit juice as it will overload you with sugar (many pieces of fruit go into one glass of juice). 
The following foods are recommended for this kind of diet:

- Whole Grain foods

- (Oats, brown/whole wheat bread, Low GI bread, Oat cereals, Whole wheat cereals)

- Protein - lean fish and chicken, only red meat occasionally

- Fruit

- Vegetables

- Fats - no saturated fats. Stick to olive oils, nuts, avocado, seeds

This kind of diet should contain a balanced variety of the following food groups:

- Carbohydrates (complex!)

- Proteins

- Fibre

- Fat

- Vitamins and minerals

\section{How much carbohydrate?}

$25-50 \%$ of all food intake

At one meal the carbohydrates should amount to the size of your first.

Keep clear of simple (high octane) carbs

\section{Some helpful Guidelines!}

Cut Back on High Octane Foods; avoid fizzy drinks (A single can of fizzy drink has up to 3 tablespoons of sugar in it!) Try eating as much whole grain and whole wheat as possible Eat your carbs with help of The Tables 1, 2, 3 below

\section{How do I choose the right carbs?}

The tables below will help you choose the right kind of carbs. Table 1 we call "The Good Carbs". You should choose most of your carbs from this table. The carbs from Table 1 will help you sustain your energy levels - this is food that is good for your brain and muscles. These foods help prevent tiredness and give you a nice even flow of energy throughout the day. They are also ideal in maintaining your weight in the sense that they don't make 
Eating Guidelines

\begin{tabular}{|c|c|c|c|c|c|}
\hline PROTEIN & Amount & Avoid & CARBS & Amount & Avoid \\
\hline $\begin{array}{l}\text { Lean meats: Beef, pork, } \\
\text { chicken, fish, ostrich, } \\
\text { ham, tofu }\end{array}$ & $\begin{array}{l}\text { About 150- } \\
250 \mathrm{~g} / \text { daily }\end{array}$ & $\begin{array}{l}\text { Processed meat like } \\
\text { Salami,pollony, } \\
\text { sausages }\end{array}$ & Green vegetables & $\begin{array}{l}\text { A good fist size } \\
\text { portionwith every } \\
\text { meal }\end{array}$ & $\begin{array}{l}\text { Starchy vegetable like } \\
\text { potatoes, sweet pot. } \\
\text { Parsnip, }\end{array}$ \\
\hline $\begin{array}{l}\text { Diary eggs, cheese or } \\
\text { full cream yoghurt or } \\
\text { milk }\end{array}$ & $\begin{array}{l}\text { About } 50- \\
\text { 100g/daily }\end{array}$ & $\begin{array}{l}\text { Avoid too much } \\
\text { cream }\end{array}$ & $\begin{array}{l}\text { Other vegetables i.e. } \\
\text { tomatoes, cucumber, } \\
\text { peppers, carrots }\end{array}$ & In moderation & Pumpkin, butternut \\
\hline Nuts and seeds & 50g/daily & & $\begin{array}{l}\text { Grains brown rice, } \\
\text { barley wheat, bulgur, } \\
\text { quinoa }\end{array}$ & Small portions & $\begin{array}{l}\text { Bread in general, but } \\
\text { especially wheat } \\
\text { based, white pasta } \\
\text { and rice }\end{array}$ \\
\hline Legumes and lentils & 50g/daily & $\begin{array}{l}\text { Too many - they } \\
\text { also high in carbs }\end{array}$ & $\begin{array}{l}\text { Fruit } \\
\text { stick to berries }\end{array}$ & $\begin{array}{l}\text { 1-2 pcs per day } \\
\text { (large)or handful } \\
\text { of berries }\end{array}$ & $\begin{array}{l}\text { Sugary fruits like } \\
\text { grapes and bananas }\end{array}$ \\
\hline FATS & Amount & Avoid & & & \\
\hline $\begin{array}{l}\text { Omega } 6 \text { oils: Olive oil, } \\
\text { grape seed oil, rape } \\
\text { seed oil, avo oil }\end{array}$ & $\begin{array}{l}\text { In moderation } \\
\text { with all meals }\end{array}$ & $\begin{array}{l}\text { Margarine spreads. } \\
\text { Sunflower oils }\end{array}$ & & & \\
\hline butter & $\begin{array}{l}\text { Only small } \\
\text { amounts } \\
\text { (thumb sized) }\end{array}$ & $\begin{array}{l}\text { Mayonnaise (made } \\
\text { from sunflower oil) }\end{array}$ & & & \\
\hline Avocado, nuts and seeds & $\begin{array}{l}1 / 2 \text { avo per day, } \\
50 \mathrm{~g} \text { of nuts and } \\
\text { seeds per day }\end{array}$ & & & & \\
\hline
\end{tabular}




\section{What about drinking habits?}

On all healthy diets we need fluids. Often when we feel hungry, it is the body telling us we are in fact thirsty. So we need to drink enough fluids and our main source of good fluid is of course water. We have some of the cleanest tap water in the world here in South Africa - and it is free! SO go ahead and have a free treat! But in all seriousness, why is it important to drink water and avoid some of the other choices of drinks out there? Let us explore:

\section{Water}

Why do we need water?

- Water makes up 50-55\% of your body weight.

- Water and Oxygen are the most needed elements for life.

- Carries nutrients to, and waste away from cells

- Cools the body

- Necessary for proper body and organ function.

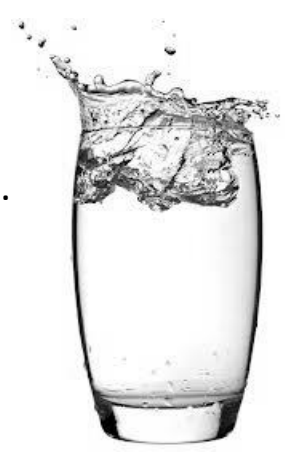

Where does the water come from?

- The fluid you drink

- The water in the food you eat

- Chemical reactions inside your body

\section{How much do I need?}

We usually recommend that adults drink 6 to 8 glasses of water a day but this water includes the water in the food we eat so you don't have to be drinking a whole 8 glasses.

If it is very hot or you are doing lots of exercise you need to drink more than 8 glasses.

\section{Some helpful Guidelines!}

Drink when you are thirsty. Drink at least one glass of water/juice every morning with breakfast. Drink 2 glasses one hour before exercising and another after exercising.

Caffeine (Coffee, tea \& Coke) and Alcohol make you lose water. These are poor sources of water. 
Often when you take a lot of medication you lose your appetite and you might resort to coffee and tea with lots of sugar. Try to avoid this and stick to 1-2 cups per day. Also try and substitute normal tea with rooibos teas.

Alcohol really doesn't go well with medication. But if you really enjoy it, women shouldn't drink more than 1.5 glasses per occasion and men 2 glasses. You should also know that alcohol has been shown to worsen the symptoms of osteoarthritis.

In general, juices from supermarkets are highly processed and contain a lot of sugar even when labels say $100 \%$ fruit juice. If you have juice, one glass a day is plenty - and when possible, rather a piece of fruit and then a glass of water. 


\section{Losing Weight}

Now that we know about healthy diet options, the question remains: Why do I actually need to lose weight? There are several answers to this, but first and foremost, not all of us need to lose weight. It is only when

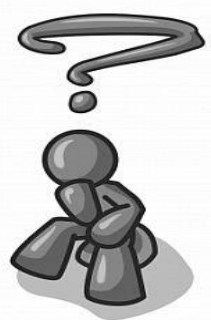
you are heavily overweight or what doctors call obese, that your weight becomes a big problem. Remember when you first came to the hospital to do questionnaires and tests? We also measured your BMI (Body Mass Index), which is a measurement of your weight according to your height. If you have a BMI of more than 25 , you should be concerned. If you don't know whether your weight is a problem, here is a way to calculate your BMI: Your weight in kg divided by your height in meters times your height in meters. To give you an example: If a person weighs $88 \mathrm{~kg}$ and she is $1.70 \mathrm{~m}$ tall, her $\mathrm{BMI}=88 / 1.70 \times 1.70=$ 30. This person would be classified as obese 1 according to the table below.

\begin{tabular}{|l|l|}
\hline BMI Class & Range \\
\hline Underweight & $<18$ \\
\hline Normal & $18-25$ \\
\hline Overweight & $25-30$ \\
\hline Obese 1 & $31-35$ \\
\hline Obese 2 & $35-40$ \\
\hline Obese 3 & $>40$ \\
\hline
\end{tabular}

What we know from research is that osteoarthritis is closely linked to obesity and being heavily overweight. What this means, is that when you weigh a lot more than you should, there are added stresses and strains on your body's joints. In particular the big joints like the hips and knees. This means that when doing daily activities or exercise your symptoms of pain increase and the natural reaction is to stop being active. Therefore, if you are heavy, losing weight will improve your condition by decreasing the loading on your joints and it will feel a lot easier for you to move around, do exercise and daily activities. It is never too late to change your mind set and lose weight - and no matter how old, young, tall or short - it will make you feel good about yourself.

Therefore, should you choose to lose weight, you can follow the guidelines for diets, which we have already gone through. Remember losing weight is not about starving yourself, but making sure that the food we eat, fills us up, gives us energy throughout the day and makes us shed the unwanted kilos.

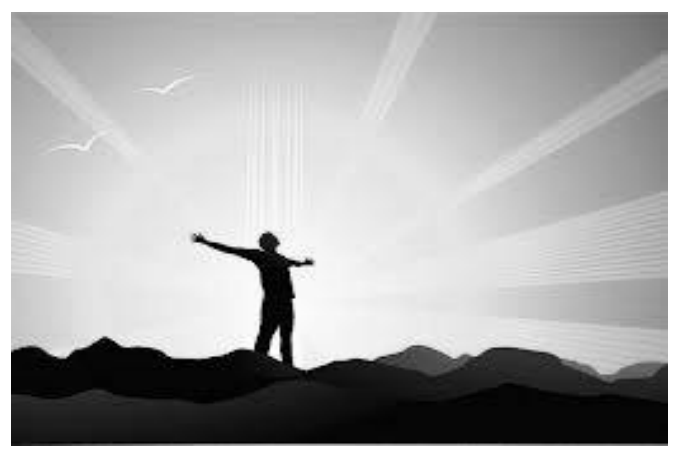


What about those who's BMI is normal? Well, as we have already talked about - you can be thin or normal and still benefit from a healthy diet. It will still give you the improved energy and make you feel less fatigued and most importantly help with your medicine uptake (we will talk more about this in a later chapter).

\section{"I find it hard to eat well!"}

Now that you know what you should be eating let's talk about why people living with OA may be struggling to eat enough. The reasons for not eating enough could be that they do not want to eat because they just don't feel hungry or because they are too tired to eat or they are too worried to eat or they feel like they will vomit if they eat, or food just doesn't taste good any more. The next section gives ideas on ways to manage these problems.

\section{"I'm not hungry"}

On the days when you do feel like eating, make sure you eat well to make up for days when you might not be eating so well. On the days when you do not feel like eating try to eat small meals more often, maybe 6 times a day. Eat in a relaxing place, maybe with a friend. Keep small snacks (healthy) with you in your bag or next to your bed so that if you wake up or suddenly feel hungry you can eat straight away. Make sure these snacks have lots of energy in them (are complex carbs). Make sure you have your favourite foods to eat, even if it's just a little bit, it helps.

\section{"I get full too quickly"}

You might be trying to get all your food at one meal. Try to spread it out more by eating five or six times a day. When you do eat, make sure it is food with lots of energy and protein. Don't eat foods without energy first and then feel too full for the important foods.

\section{"Food doesn't taste so good"}

Medicines can change the way food tastes. Sometimes you may have a bitter taste or a taste of metal in your mouth. Try cleaning your teeth and your tongue before you eat. If you have a taste of metal in your mouth, try to drink orange juice or another tart drink. 
A dry mouth might be a side effect of medications. You can help this by avoiding smoking and drinking alcohol as well as sugary drinks as these irritate your mouth and throat and make you even more thirsty. Eat softer food, if you mash your food or make soup as this will be easier to swallow. Try not to eat food with a lot of spices or drink fizzy drinks. Keep a bottle of water next to your bed so that you can drink during the night. 


\section{Action Plan Form - Nutrition}

Think about your eating habits. Use this form to come up with a plan to improve one thing about your nutrition. Be sure your action plan includes:

What you want to do

How much you are going to do

When you are going to do it

How many days a week you are going to do it

For example: This week, I will eat less simple carbs (what) by cutting out one (how much) during dinner (when) everyday (how many).

This week I will:

(what)

(how much)

(when)

(how many?)

How confident are you that you can complete this action plan?

Not at all $\mid \begin{array}{llllllllll} & \mid & \mid & \mid & \mid & \mid & \mid & \mid & \mid & \text { Totally }\end{array}$

confident 122345678910 confident

Keep a record of how you did:

\begin{tabular}{|l|l|l|}
\hline & I Plan to..... & I did..... \\
\hline Monday & & \\
\hline Tuesday & & \\
\hline Wednesday & & \\
\hline Thursday & & \\
\hline Friday & & \\
\hline Saturday & & \\
\hline Sunday & & \\
\hline
\end{tabular}




\section{Week 5: Medication and disease related problem solving}

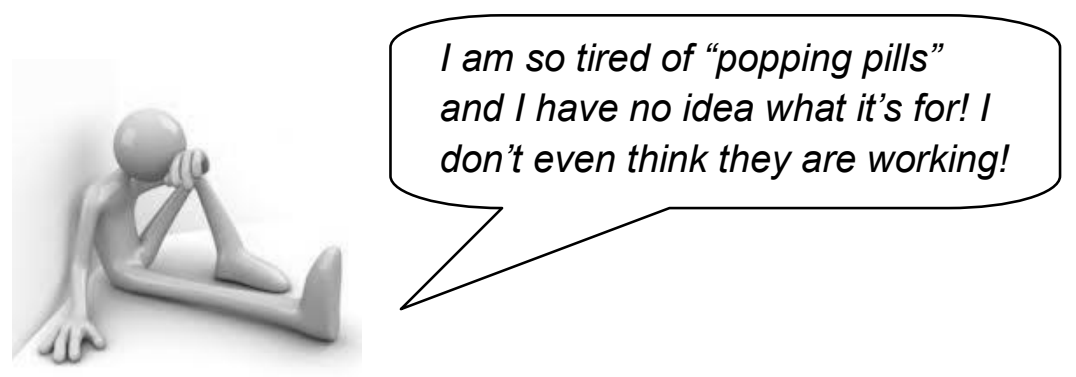

Surely you can recognise the above? At this stage, "popping pills" is not an unusual occurrence to you. Unfortunately with a chronic condition like osteoarthritis, taking medication is a huge part of trying to keep the symptoms at bay. Unfortunately it often comes with a lot of physical negatives and unanswered questions. One of the most common experiences for OA patients is that after taking the medications for many years - they seem to lose their effect. Why is that? Also, many OA patients take many different kinds of medication, without always understanding how they work and what they are for - what is each medication meant to do? In this section we will look at the different kinds of groups of medications and try to understand it all a little better. There are a few things that research has found out as well, and that is if a patient understands the medication and its purpose, the medication becomes more effective.

Many kinds of drugs are used for osteoarthritis and its symptom management. The majority of them fall into one of 4 categories.

- Analgesic Drugs

- Anti-Inflammatory Drugs

- Anti-spasmodic Drugs

- Antidepressants and Antiepileptic Drugs.

\section{Analgesic Drugs}


The name is derived from the Greek word analgia: an-, "without", and -algia, "pain". In other words analgesic drugs are what we commonly refer to as "painkillers." The analgesic drugs act in various ways on the nerves. These are the nerves in our brains (the central nervous system) and our bodies (the peripheral nervous system) peripheral and central nervous system. There are 3 main types of analgesic drugs, let us have a look at them:

\section{Mild Pain Killers}

These painkillers don't treat the cause of the pain but they work on the nerves in the peripheral nervous system which are telling you that you are in pain. They basically tell the nerve to stop sending messages. I.e. they tell the nerve to shut up! This is exactly what is needed for people with osteoarthritis and associated chronic pain, because the painkillers decrease the number of signals in the peripheral nervous system - remember when pain has been ongoing for a long time, it often intensifies and "gets louder" even though your tissue is not getting more damaged. In other words the nervous system is malfunctioning and sending mixed up messages. The mild pain killers have very few side effects, which makes them attractive compared to some of the stronger medications. These medications are available over the counter. Examples of such drugs are: Panado, Paracetemol, Grandpa, Disprin, Asprin, Panadol, Calpol, Panaleve

\section{Strong Pain Killers - Opioid Analgesic Drugs (MORPHINE BASED)}

These drugs are a lot stronger than the normal pain killers. They are similar to a hormone that the body produces, called Opiates...which is why we call them Opioid Analgesics. These drugs work straight on the nerves by blocking the messages in the brain and spinal cord. These medications need to be used carefully as there is a small chance that they can produce dependence, addiction and tolerance. Other side effects include drowsiness, decreased alertness (take care when driving a car), sedation, elation (sudden joy) or dysphoria (unhappiness). Respiratory side effects can include feeling short of breath and not coughing as much as you should. People with asthma or chronic obstructive lung disease should be careful when using these drugs. Other side effects from these drugs can be nausea, dry mouth and vomiting, constipation and difficulty in passing urine and itching. If you take opioids for a long time, the effects often fade. Many oral opioids are used in the treatment of chronic pain. Combining opioids with other painkillers such as paracetamol and NSAIDs involves attacking the pain in the different areas of the nervous system. This often decreases your opioid requirements, which then leads to improved pain relief and a reduced risk of side effects. These medications require a prescription from your doctor. Examples of these drugs are: Morphine, Fentanyl, Methadone, Pethidine, Tramaset, Tramadol, Codeine 


\section{Medium Strong Painkillers + Codeine}

These drugs are a combination between the Mild Pain Killers and the Opioid Analgesics. The dosage of codeine is much lower than the strong pain killers. They are stronger than the Mild Pain killers but weaker than the Opioid Analgesics. Anything with a "co" or a "codeine" added to the name will fall into this category. For Example: Panado Co, Myprodol and Empacod. These medications are sometimes available over the counter and you need to be careful if you are taking these and one of the other mild or strong painkillers as you might be taking too much of one drug without realising it. 


\section{Anti-inflammatory Drugs}

These drugs reduce inflammation. Inflammation is the body's response to injury. For example when you twist your ankle, it will go hot and red and become swollen and painful. These are all signs of inflammation. Like pain, inflammation can be acute or chronic. Acute inflammation last only for a few days, whereas chronic inflammation lasts longer. Anti-inflammatory drugs treat both acute and chronic inflammation. Unlike analgesics they treat the cause; Anti-inflammatory drugs help to decrease the heat, redness and swelling. There are 2 types of Anti-inflammatory drugs.

\section{Non-Steroidal Anti-inflammatory drugs (NSAIDs).}

This group of drugs is effective against mild to moderate pain and inflammation. They are widely used for arthritis and pain

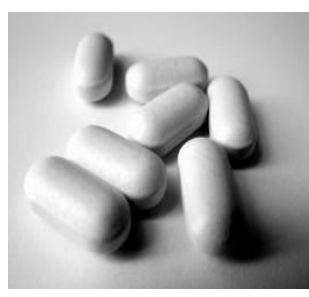
conditions. "Non-steroidal" means that they are not steroids (i.e. they do not belong to the "cortisone" family). Steroids are very effective against inflammation, and the term NSAID is used to tell the difference between this group of drugs from the steroid family of drugs.

"Anti-inflammatory" means that they are effective against inflammation. Some are better against pain and some better against inflammation. It is also important to know that different people react differently, so that one product might work well for one person, but may not be as effective in another person. It is important you talk to your doctor about these issues.

Some of these drugs also have negative effects on the stomach and digestive system. They can cause constipation, ulceration in the stomach and even bleeding in the stomach. It is therefore important to take NSAIDs with or after food. If you have a sensitive stomach with these drugs your doctor will prescribe one of the new NSAID drugs which do not have the same effect on the stomach. With chronic pain it is important that you take these drugs regularly for them to be effective. Examples of NSAIDS are: Ibuprofen, Brufen, Voltarin, Asprin/Disprin, Paracetamol, Pyroxicam, Betacin, Naproxen, Ketoprofen Sometimes doctors will combine anti-inflammatory drugs with Mild Pain Killers because they work better together than each on its own. Some pills come with them already together in one pill. E.g. (Mypradol).

\section{Steroidal Anti-inflammatory Drugs}

These drugs are made to mimic natural steroids that your body produces. They are very strong anti-inflammatories and are often used for arthritic conditions. However there are negative effects of short term and long term use. In the long term it can cause high blood 
pressure, high blood sugar levels, eye problems, fragile skin, osteoporosis and muscle wasting. Steroidal injections have fewer side effects than orally taken steroidal antiinflammatory drugs and are used more often for arthritic conditions. Examples are: Cortisone, Cortisol

\section{Anti-spasmodic Drugs}

When a person is in pain, their muscles become very tense and can often spasm. These drugs are used to relieve the spasm. However they do come with severe side effects if taken for longer than recommend - especially if taken together with other chronic medication. It is therefore important to understand these when your doctor prescribes them. Commonly used antispasmodic medication used for chronic pain is: Diazepam, Baclofen

\section{Anti-depressant \& Anti-Epileptic Drugs}

The brain is very sensitive to chemicals therefore, there is something called a Blood-Brain Barrier, which filters all the blood going to the brain to make sure there are no chemicals in the blood that would harm the brain. Because of this, drugs like mild painkillers cannot reach the brain. Antidepressants and Antiepileptic drugs are able to pass through the barrier and act on the brain. For this reason they are given to patients with chronic pain. You remember all the changes to the nerves that happen in the brain in a person with chronic pain. These drugs can help to normalise the pain signals in the brain. They are given to chronic pain patients in much smaller doses than they would be given to a clinically depressed person or a person who has epilepsy.

It is important that these drugs are taken regularly and for at least 2 weeks before they begin to be effective. Side effects can include: Scratchy eyes, drowsiness, dry mouth, constipation and blurred vision. But these side effects usually decrease with time.

Examples of ANTIDEPRESSANTS are: Amitriptyline, Doxepin, Desipramine, Imipramine. Examples of ANTIEPILEPTICS are: Gabapentin, Topiramate, Vigabatin, Phenytoin, Oxcarbazepine, Sodium Valporate, Carbamazepine, Lamotrigine

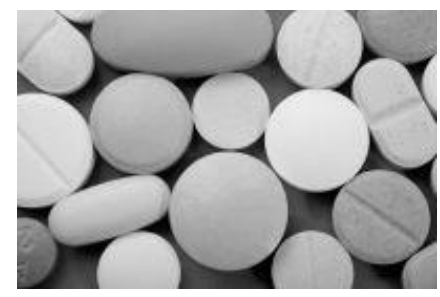




\section{The Analgesic Ladder}

The analgesic ladder was designed by the World Health Organisation (WHO) to assist the doctors when prescribing analgesic drugs by suggesting a logical way for managing pain in chronic conditions.

The ladder takes into consideration the analgesics we have already described earlier. At every step of the analgesic ladder non-opioid analgesics form the basis of the pain management. Paracetamol and NSAID should therefore always be prescribed with opioid analgesia (weak or strong). This is known as multi-modal analgesia and is the concept that pain is best managed, not by a single drug or therapy, but by combinations, with maximum effect whilst keeping side-effects low. Scientific research has demonstrated that when this happens pain relief is better, smaller amounts of pain killers are needed and fewer side effects occur.

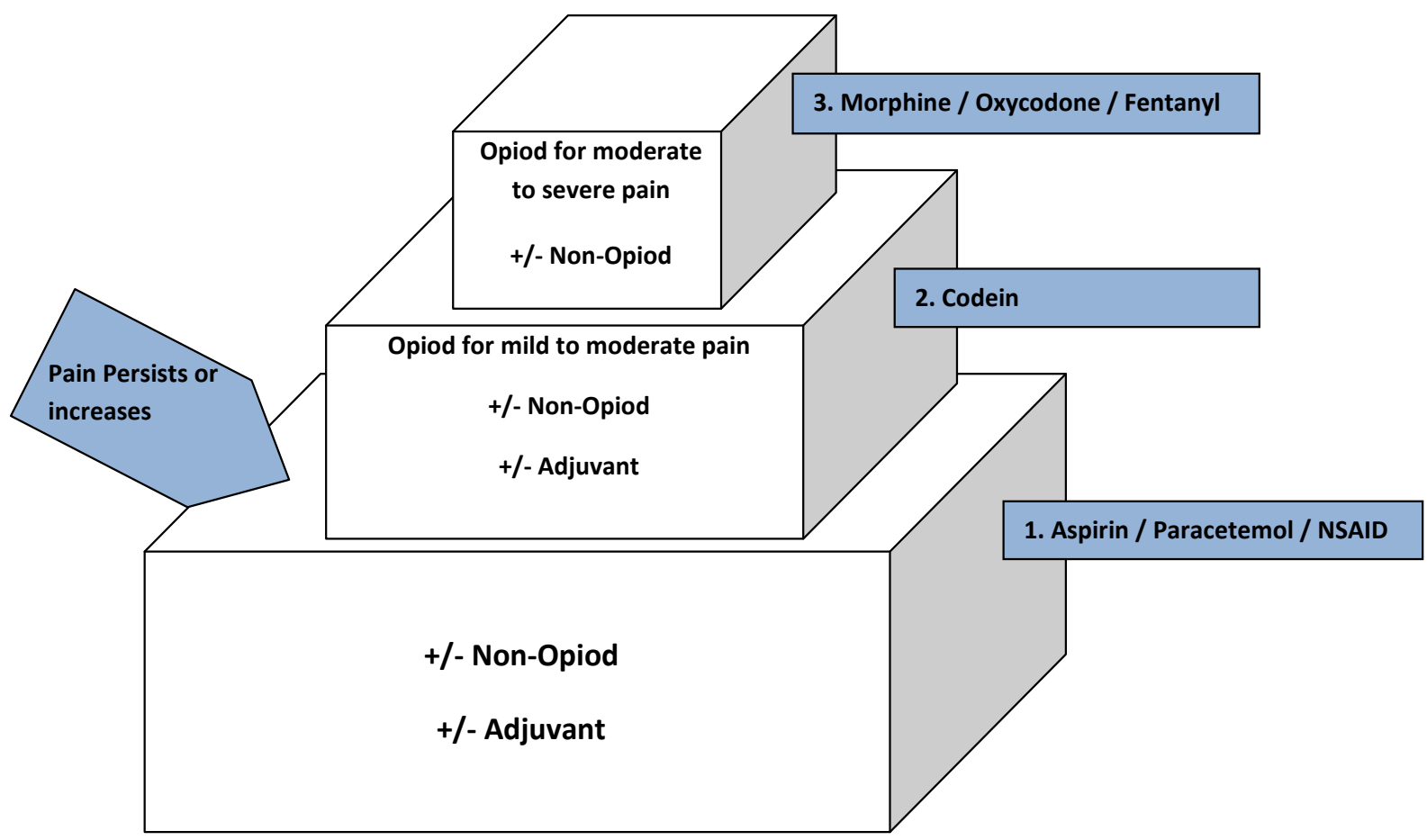




\section{Making informed treatment decisions}

By understanding the medication you are being prescribed, it will help you to understand its action as well as why it is to be taken together with other drugs or at specific times. For example it makes sense to take NSAIDS with healthy food, as we have learned that they can be rather rough on the stomach. If you were to take the NSAIDS with coffee and or very acidic foods, you have a high chance of getting an irritated stomach and or heart burn. In relation to the stronger pain killers, which might make you drowsy, they can be taken at a time where you don't have to be so alert or have to drive.

By understanding the medication you are on, research has shown us that the medication actually works better. Knowing more about your medicines also helps you understand, why you might at times have new and strange symptoms, these being related to some of the side effects we have discussed earlier. In this way your conversations with your doctor can improve, as you will be in a better position to tell him or her what does or does not seem to work for you.

By understanding the medicinal options for osteoarthritis, it becomes easier for you to be part of the decision making process, when deciding on which treatment option to go for. You are slowly becoming your own expert.

\section{Appropriate use of medications}

What is really important to understand well, is the analgesic ladder. Here you can see how and why the different groups of medications are prescribed at what stage of your pain. Therefore you must make sure that whatever level you are on in terms of medication, that you don't go ahead and start "prescribing" your own medication. You have to make these decisions together with your doctor by understanding them and communicating with him or her.

\section{Link between a healthy lifestyle, good nutrition and exercise}

It is really important to understand that medication is "only" one of the ways in which we can try and control your OA. That is also why we are doing this course. By a combination of your medication, a healthy lifestyle with good eating and exercise, decreasing the stresses in your life and maintaining your social life, you are on a good path to being the "manager" of your disease.

Healthy food helps the uptake of your medication - remember you have more energy, which in turns will help you handle the many side effects. Also by eating a fibre rich diet, the side effects of NSAIDS will be less. 
By adding exercise, activities and social engagements to your life, you become happier remember the body's own production of endorphins (the happy hormone)? The more we can produce of these, the better we feel, the more we want to do and the less we think about the pain and the downsides to living with OA. Also, by becoming an active "manager" of our lives, we can decide which stresses we can do something about, and which we cannot do anything about. We make the choices and make sure that when life does become a bit too stressful and hectic, to take a step back and say: I need to take a break from that, and focus on myself. This ultimately makes us better at communicating with our surroundings.

\section{Communicating effectively}

If we understand ourselves better, we can communicate better - it is as simple as that. We will be able to explain what is going, we will be able to show we are in charge of our own body and mind and thereby condition. We will be able to say, that although we are making good lifestyle choices for ourselves, there are days and times when things aren't that great, where the pain is really bad, but we can still communicate and get ourselves back on track when the next "good" day comes along. This makes is easier for our loved ones, friends and colleagues to help and support us. In this way, it becomes easier to say: I need a bit of space to cope, so I can get back on track again. Can you help me with th is today? When they see you are making the positive changes, they are more willing to eager to help and understand. 


\section{Week 6: Continuing as a successful self-manager}

\section{Recap of key components of successful self-managing}

Over the last six weeks you have learnt many skills which will help you to live positively with your condition. Research tells us that people living with any chronic disease who follow these steps have better quality of life, have fewer sick days and have better disease control. This is true for people living with high blood pressure, cancer or depression. You have learnt how to be a positive self-manager by being able to solve problems and set goals for yourself so that you can move forward with your life. You have learnt about the importance of exercise. How exercise can make you feel better, what exercises you should do and you have been doing those exercises too! You have learnt about the common symptoms that trouble people living with arthritis and you have learnt how to manage these symptoms. You have learnt about pain, what might be causing pain and how to treat and manage any pain you may have. You have learnt about food and eating well and how to make sure that your food is safe. With all of these you have also had the chance to practice doing things differently and to think about how this has made you feel.

\section{- Action planning for the future}

Now it is time to think about the future. People with long term illnesses often worry about what will happen if they get very sick, how they will manage their lives; how they will they look after themselves or their families. Worrying about these things can also make people feel sad, angry or depressed and helpless. These emotions may make everything feel even more difficult than they are. By working through this book you have already started to deal with these emotions. You have increased your knowledge and this is one of the main ways that we manage fear. If we are afraid of something, knowing more about it helps us to tackle the fear. If you know more about it, you can make a plan around it and making a plan helps us to get a sense of control over the very thing that we are afraid of.

Planning for the future means thinking about the things that might happen to you in the future and planning for them. You may never ever need to use the plan as the things that you worry about may not happen, but, having a plan will help you to worry less about 
these things and stay in control should they happen. You can use the action planning forms you have been using in this workbook to think about the things which worry you about the future. You can then start making a plan about what you want to do if these things happen. If you are not sure about making a plan, you may want to talk to different people who might be able to help you with this.

\section{Step 1:}

To be able to plan for the future, you need to decide what it is that you are worried about happening. This can be the hardest step to think about. For example you might be feeling very sad and depressed. First you need to think about why you are feeling that way. It might be that you are worried about not being able to look after your family if you become ill, or you may be worried about making someone else ill, or you may be worried about not being able to look after yourself, or you may be worried about dying. Once you have identified what it is that worries you and makes you feel sad, depressed, angry or afraid then you can start to make a plan to deal with it. This will help you to feel less sad, depressed, angry or afraid.

Write down here some of the things that might happen in the future that you worry about:

1)

2)

3) 


\section{Step 2:}

Now that you have identified some of the things which worry you, you can start to think about different ways to manage these things. If you were worried about becoming ill and not being able to look after yourself, write down a list of things that you would need help with. Then write down who you could ask to help you with those things. The people who can help might be family, friends, social workers, counsellors, nurses, physiotherapists, occupational therapists or doctors. If you are not sure who could help you, you may want to talk to someone you trust to help you identify who could help.

Write down here three different things you could do to help plan for the things in the future that you worry about:

1)

2)

3)

There are many organisations and people who you can approach for help in planning for the future. These organisations include the Treatment Action Campaign (TAC), the Family and Marriage Society of South Africa (FAMSA), your church as well as the health care practitioners at your local clinic. The contact details for these organisations are included at the end of this section.

Once you have completed Step 2 and written down three different things you could do to help plan for the things in the future that you worry about, choose the one which seems to suit you the best (this might be one which is easier or is cheaper or you know has worked for someone else). Now use this action plan form to work out what you will do if the thing which you worry about happening should happen. You can use this method to plan for any of the things which worry you. 


\section{Action Plan Form for Future Worries}

I am worried that in the future I will not be able to:

My plan to manage this if it happens is to:

(what, who, how, when?)

How confident are you that you can complete this action plan? (Remember you are aiming for 7 out of 10 on the confidence line)

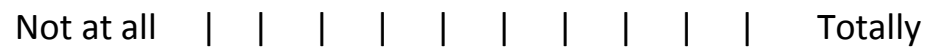

confident $12345 \quad 678910$ confident 


\section{- Reflection on changes}

Over the last 6 weeks you have learnt many things about how to live with a chronic illness like osteoarthritis. You have learnt about the disease and how to be a successful selfmanager. This does not mean that you should be managing your health on your own. What it does mean is that you now have the ability to manage your health and your life as part of the health team. Remember you are not alone but are part of a team of people whose aim is to help you get the most out of life. We encourage you to keep using the skills you have learnt in this workbook to live positively. There are extra "Action Plan Forms" and "Exercise Diaries" in the back of the workbook for you to use. Tryto keep using these forms to help you remain active and give you a sense of purpose and accomplishment in your life. Being active and involved, using the skills you have learnt in this workbook are important steps in helping you achieve the best quality of life you can. In the box write down some of the important changes you have made in your life over the past few weeks.

\section{Changes I have made in my life:}

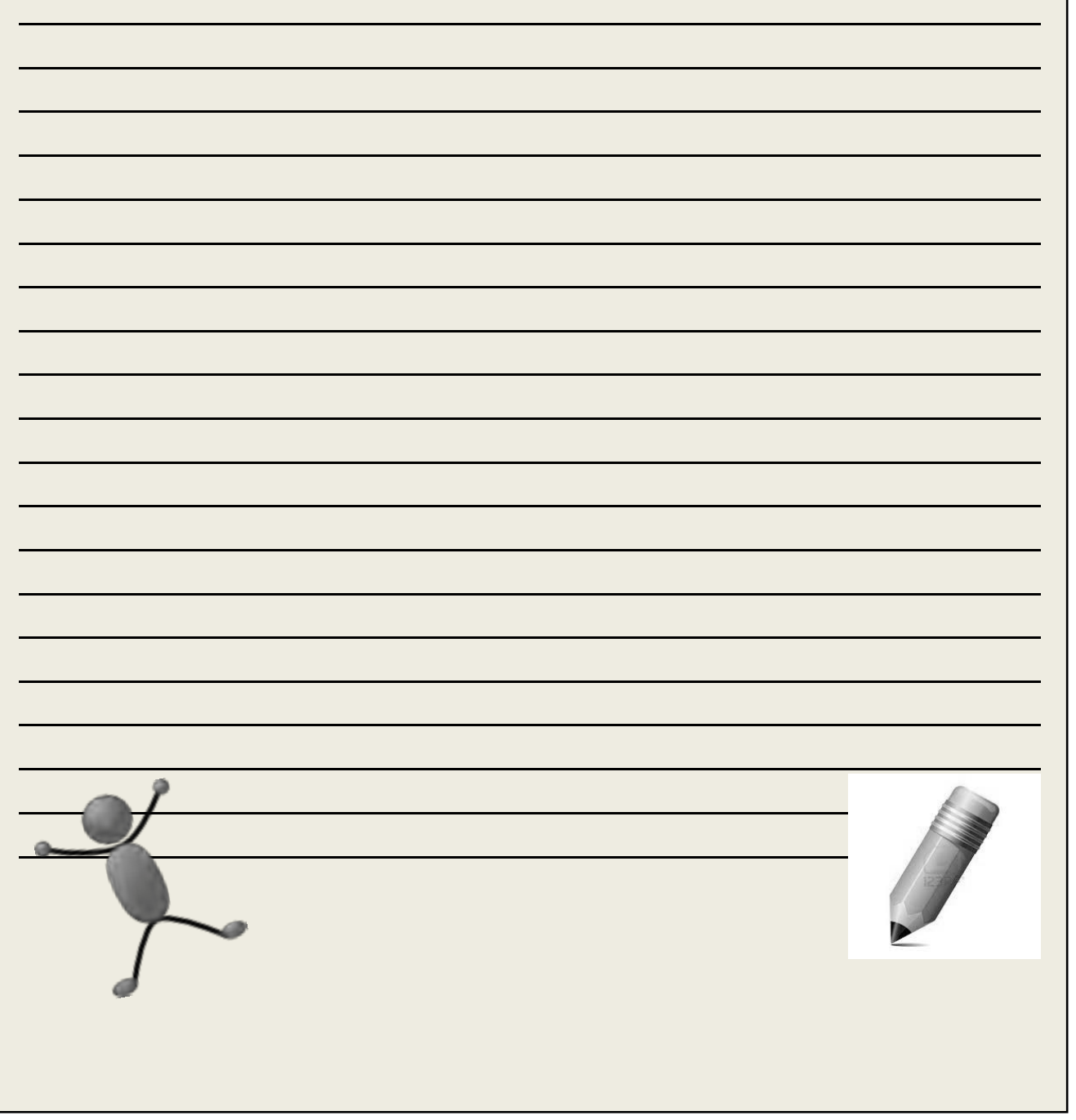


Now, your final task is to think about changes you still want to make in your life. Remember, this is about getting the most out of life, increasing your quality of life. Are there still some things missing in your life, things that you would still like to be able to do? In the box below, write down some of the things that are still missing. You can use this list to keep working on your goals from now on.

Things that are still missing in my life: 


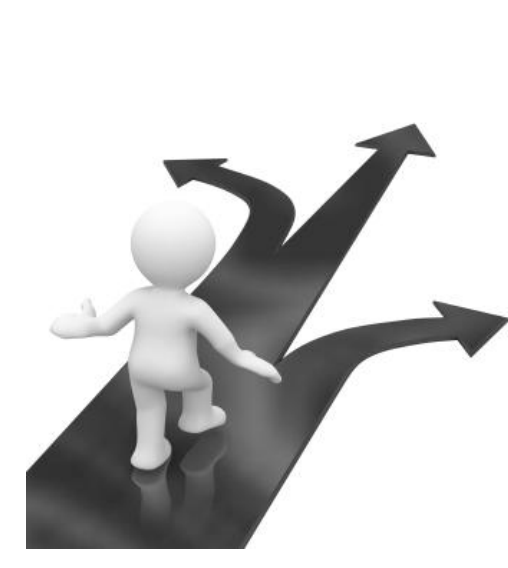

You have now completed this workbook. By working through this workbook you made an important commitment to yourself. You have chosen to spend time looking after yourself and you have taken steps to overcome the many challenges that people living with osteoarthritis face. Do not put this workbook away; keep it somewhere safe where you will be able to review it from time to time. We all forget things at times and it is useful to be able to look back and remind ourselves of things we may have forgotten. We can also look back and see how far we have come. We hope that the knowledge and the skills you have learnt by using this workbook will continue to have a positive effect on your life.

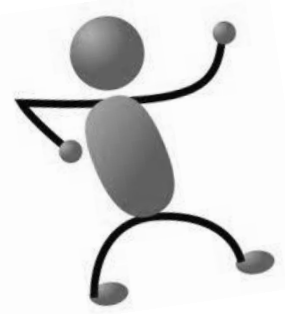




\section{Exercise Diary}

Start off by writing down your goal.

Write down here what you want to be able to do:

Now, what do you want to be able to do this week which will help you to reach your goal?

Remember from your action plan to include:

What you want to do

How much you are going to do

When you are going to do it

How many days a week you are going to do it

For example: This week, I will walk (what) around the block (how much) before lunch (when) three times (how many).

This week I will:

(what)

(how much)

(when)

(how many?)

\begin{tabular}{|l|l|l|l|}
\hline & Exercise Planned & Exercise I did... & $\begin{array}{l}\text { How did I feel? Do you need to } \\
\text { change anything? }\end{array}$ \\
\hline e.g. & $\begin{array}{l}\text { 20 mins in a.m. after } \\
\text { breakfast and in p.m. } \\
\text { after supper }\end{array}$ & $\begin{array}{l}\text { Very tired by the second session, I'm } \\
\text { going to cut it down to morning only } \\
\text { for this week. }\end{array}$ \\
\hline Monday & & & \\
\hline Tuesday & & & \\
\hline Wednesday & & & \\
\hline Thursday & & & \\
\hline Friday & & & \\
\hline
\end{tabular}




\section{Appendix G: Example of exercises and visualisation relaxation}

AppendixG-1 Summary of exercise regimen and progression

\begin{tabular}{|c|c|c|c|}
\hline Exercise & Aim & Activities & Progression \\
\hline Walking & $\begin{array}{l}\text { General warm up } \\
\text { Improve cardiovascular } \\
\text { fitness }\end{array}$ & $\begin{array}{l}\text { Walking on the spot for } \\
2 \text { minutes }\end{array}$ & $\begin{array}{l}\text { Increase speed and } \\
\text { duration } \\
\text { Incorporate arm swings } \\
\text { and boxing } \\
\text { Lift knees higher }\end{array}$ \\
\hline $\begin{array}{l}\text { Quadriceps } \\
\text { Strength }\end{array}$ & Improve strength & $\begin{array}{l}\text { Sitting with knees at 90, } \\
\text { extend the knee. }\end{array}$ & $\begin{array}{l}\text { Increase speed and } \\
\text { repetitions } \\
\text { With back against a wall, } \\
\text { flex hips and knees into a } \\
\text { squat }\end{array}$ \\
\hline $\begin{array}{l}\text { Standing on } \\
\text { one leg }\end{array}$ & $\begin{array}{ll}\text { Balance } & \text { and } \\
\text { coordination } & \end{array}$ & $\begin{array}{l}\text { Single leg stance, } \\
\text { supported by hand on a } \\
\text { wall if necessary }\end{array}$ & $\begin{array}{l}\text { Increase duration } \\
\text { Stand on soft matt } \\
\text { Remove wall support }\end{array}$ \\
\hline Sit to stand & $\begin{array}{l}\text { Strength, control, } \\
\text { function }\end{array}$ & $\begin{array}{l}\text { With arms folded, sit to } \\
\text { stand from chair or } \\
\text { bench }\end{array}$ & $\begin{array}{l}\text { Decrease height of chair } \\
\text { Increase reps }\end{array}$ \\
\hline Step ups & $\begin{array}{l}\text { Strength, control, } \\
\text { function }\end{array}$ & $\begin{array}{l}\text { With one foot on step, } \\
\text { step on/off step }\end{array}$ & $\begin{array}{l}\text { Increase height of step } \\
\text { Increase repetitions }\end{array}$ \\
\hline Stretches & $\begin{array}{l}\text { Range of motion and } \\
\text { flexibility }\end{array}$ & $\begin{array}{l}\text { Stretches of major } \\
\text { upper and lower limb } \\
\text { muscles groups: neck, } \\
\text { shoulder, arms, torso, } \\
\text { legs and feet }\end{array}$ & $\mathrm{N} / \mathrm{A}$ \\
\hline $\begin{array}{l}\text { Interactive } \\
\text { Activities }\end{array}$ & $\begin{array}{l}\text { Social and fun, } \\
\text { cardiovascular, balance, } \\
\text { concentration } \\
\text { coordination }\end{array}$ & $\begin{array}{l}\text { Large } \quad \text { gym-ball } \\
\text { bouncing between } \\
\text { participants. } \\
\text { Stepping/dancing } \\
\text { activities }\end{array}$ & $\begin{array}{l}\text { Use smaller ball and } \\
\text { throw to each other. } \\
\text { Stand in line and pass } \\
\text { ball backwards }\end{array}$ \\
\hline
\end{tabular}

(Hurley, M. et al, 2007) 


\section{Start reading the beach visualization relaxation script here:}

Get comfortable. Sit in a supportive chair or lie on your back. Relax your body by releasing any areas of tension. Allow your arms to go limp... then your legs....

Feel your arms and legs becoming loose and relaxed...

Now relax your neck and back by relaxing your spine.... release the hold of your muscles all the way from your head, down your neck....along each vertebra to the tip of your spine...

Breathe deeply into your diaphragm, drawing air fully into your lungs.... and release the air with a whooshing sound.... Breathe in again, slowly.... pause for a moment.... and breathe out.....

Draw a deep breath in.... and out....In..... out..... Become more and more relaxed with each breath....

Feel your body giving up all the tension.... becoming relaxed.... and calm.... peaceful....

Feel a wave of relaxation flow from the soles of your feet, to your ankles, lower legs, hips, pelvic area, abdomen, chest, back, hands, lower arms, elbows, upper arms, shoulders, neck, back of your head, face, and the top of your head....

Allow your entire body to rest heavily on the surface where you sit or lie. Now that your body is fully relaxed, allow the visualization relaxation to begin.

Imagine you are walking toward the ocean.... walking through a beautiful, tropical forest....

You can hear the waves up ahead.... you can smell the ocean spray.... the air is moist and warm.... feel a pleasant, cool breeze blowing through the trees....

You walk along a path....coming closer to the sea....as you come to the edge of the trees, you see the brilliant aqua colour of the ocean ahead....

You walk out of the forest and onto a long stretch of white sand.... the sand is very soft powder.... imagine taking off your shoes, and walking through the hot, white sand toward the water....

The beach is wide and long.... Hear the waves crashing to the shore.... Smell the clean salt water and beach.... You gaze again toward the water.... it is a bright blue-green....

See the waves washing up onto the sand..... and receding back toward the ocean.... washing up.... and flowing back down..... enjoy the ever-repeating rhythm of the waves... 
Imagine yourself walking toward the water.... over the fine, hot sand.... you are feeling very hot.... As you approach the water, you can feel the mist from the ocean on your skin. You walk closer to the waves, and feel the sand becoming wet and firm....

A wave washes over the sand toward you.... and touches your toes before receding...

As you step forward, more waves wash over your feet... feel the cool water provide relief from the heat....

Walk further into the clear, clean water.... you can see the white sand under the water.... the water is a pleasant, relaxing temperature.... providing relief from the hot sun... cool but not cold....

You walk further into the water if you wish.... swim if you want to.... enjoy the ocean for a few minutes..... allow the visualization relaxation to deepen.... more and more relaxed... enjoy the ocean....

Now you are feeling calm and refreshed... You walk back out of the water and onto the beach...

Stroll along the beach at the water's edge.... free of worries... no stress... calm..... enjoying this holiday.... Up ahead is a comfortable lounge chair and towel, just for you...

Sit or lie down in the chair, or spread the towel on the sand.... relax on the chair or towel.... enjoying the sun.... the breeze.... the waves.....

You feel peaceful and relaxed.... allow all your stresses to melt away....

When you are ready to return from your vacation, do so slowly....

Bring yourself back to your usual level of alertness and awareness....

Keep with you the feeling of calm and relaxation.... feeling ready to return to your day....

Open your eyes, stretch your muscles... and become fully alert... refreshed... and filled with energy. 


\section{Appendix H: Socio- demographic information}

Attendance records for the six week intervention

The six week intervention saw an average of $90.83 \%$ attendance by the 20 participants

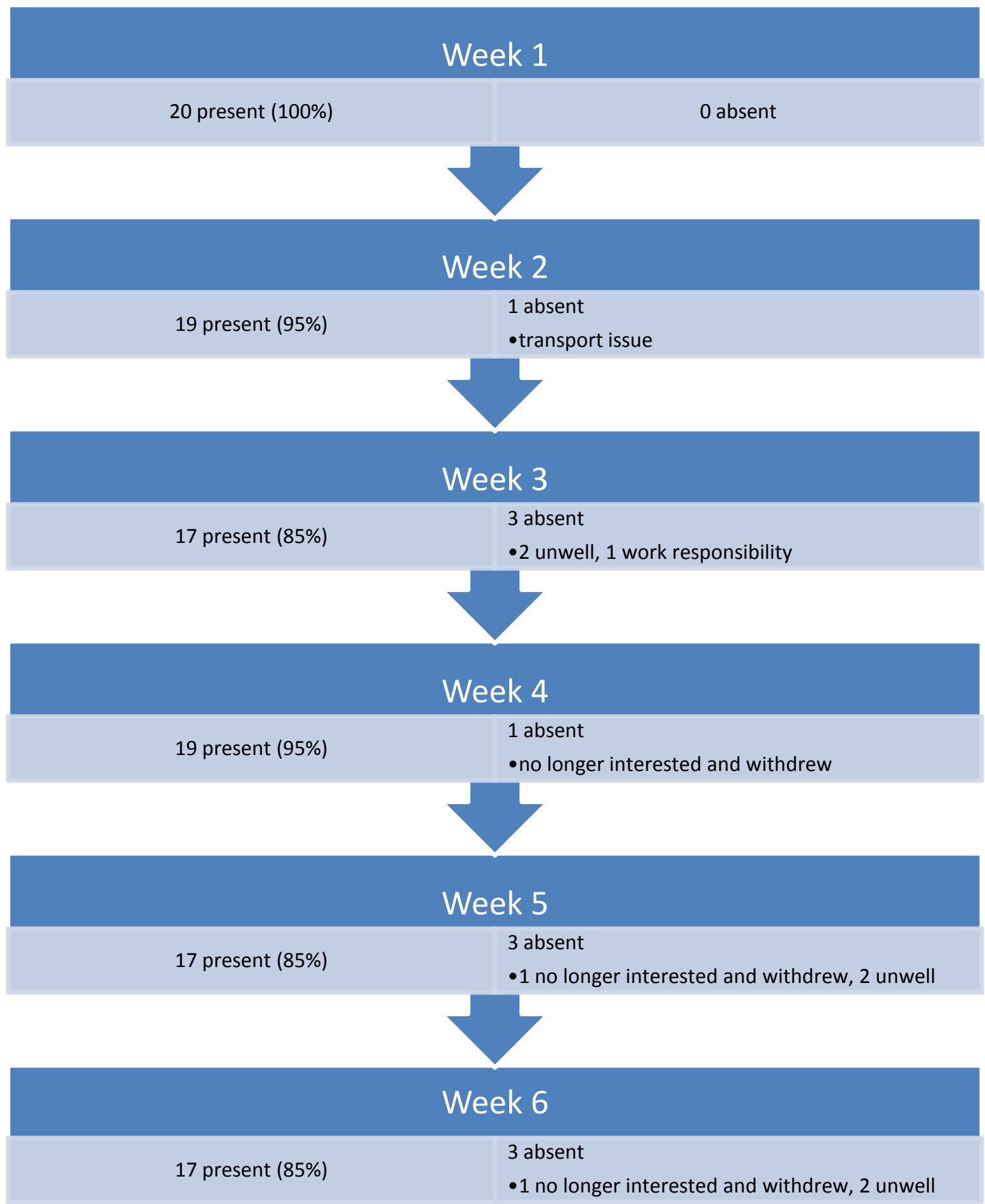




\section{Waiting time}

Waiting time for the experimental group was $M=3.64, S D=2.66$ years and for the control group $\mathrm{M}=3.58, \mathrm{SD}=2.28$ years.

\section{Education}

Only one of the participants completed formal tertiary education (Accounting) and only two of the participants completed short informal courses (secretary administration and upholstery skills) after school. 


\section{Co-morbidities}

Besides the four main co-morbidities listed in the demographic questionnaire, participants were asked to record if they had any other co-morbidity.

Appendix H-1 Other co-morbidities recorded on demographic information document

\begin{tabular}{|c|c|c|c|}
\hline & $\begin{array}{l}\text { Participants } \\
(\mathrm{N}=19)\end{array}$ & $\begin{array}{l}\text { Exp } \\
\text { Group } \\
(n=9)\end{array}$ & $\begin{array}{l}\text { Cont } \\
\text { Group } \\
(n=10)\end{array}$ \\
\hline \multicolumn{4}{|l|}{ Co- morbidities } \\
\hline Cholesterol & 8 & 4 & 4 \\
\hline Cancer - prostate & 1 & 0 & 1 \\
\hline Cancer - breast & 1 & 0 & 1 \\
\hline Anxiety/depression & 1 & 1 & 0 \\
\hline Sinusitis & 2 & 2 & 0 \\
\hline Allergies & 1 & 0 & 1 \\
\hline Spinal OA & 1 & 0 & 1 \\
\hline Bullous pemphigoid & 1 & 1 & 0 \\
\hline Previous stroke & 1 & 0 & 1 \\
\hline Previous abdominal surgery/ & 1 & 0 & 1 \\
\hline \multicolumn{4}{|l|}{ Kidney cancer/ spinal surgery } \\
\hline Previous brain surgery/goitre surgery & 1 & 1 & 0 \\
\hline
\end{tabular}




\section{Appendix I: Full pain results}

Pain severity score subgroup analysis: $O A$ hip, $O A$ knee and combined hip and knee $O A$

Appendix I-1 Detailed PSS for OA hip, OA knee and combined hip and knee OA subgroups

\begin{tabular}{|c|c|c|c|}
\hline & $\begin{array}{l}\text { Participants } \\
\mathrm{N}=42\end{array}$ & $\begin{array}{l}\text { Experimental Group } \\
n=20\end{array}$ & $\begin{array}{l}\text { Control Group } \\
n=22\end{array}$ \\
\hline & Mean \pm SD & Mean \pm SD & Mean \pm SD \\
\hline \multicolumn{4}{|l|}{ Baseline } \\
\hline Hip & $5.27 \pm 2.89$ & $6.35 \pm 2.84$ & $4.37 \pm 2.86$ \\
\hline Knee & $6.82 \pm 1.77$ & $6.86 \pm 2.13$ & $6.78 \pm 1.48$ \\
\hline Combined & $7.14 \pm 2.15$ & $8.50 \pm 1.27$ & $5.79 \pm 2.04$ \\
\hline \multicolumn{4}{|l|}{ Week 6} \\
\hline Hip & $4.59 \pm 2.80$ & $5.00 \pm 3.41$ & $4.25 \pm 2.47$ \\
\hline Knee & $5.34 \pm 1.83$ & $4.61 \pm 1.93$ & $6.00 \pm 1.53$ \\
\hline Combined & $5.19 \pm 3.12$ & $3.67 \pm 3.27 *$ & $6.71 \pm 2.26$ \\
\hline \multicolumn{4}{|l|}{ Week 12} \\
\hline Hip & $4.86 \pm 3.22$ & $4.55 \pm 3.52$ & $5.13 \pm 3.27$ \\
\hline Knee & $5.80 \pm 2.83$ & $4.56 \pm 3.50$ & $6.93 \pm 1.48$ \\
\hline Combined & $4.44 \pm 3.39$ & $4.38 \pm 3.72 *$ & $4.50 \pm 3.39$ \\
\hline \multicolumn{4}{|l|}{ Month 6} \\
\hline Hip & $5.30 \pm 2.98$ & $5.45 \pm 3.13$ & $5.17 \pm 3.14$ \\
\hline Knee & $5.61 \pm 3.01$ & $3.19 \pm 2.65^{* *}$ & $7.78 \pm 0.88^{* *}$ \\
\hline Combined & $4.90 \pm 2.94$ & $5.33 \pm 3.20$ & $4.46 \pm 2.89$ \\
\hline \multicolumn{4}{|c|}{ *Indicates a significant improvement in PSS of OA both hip and knee subgroup in the experimental group } \\
\hline
\end{tabular}




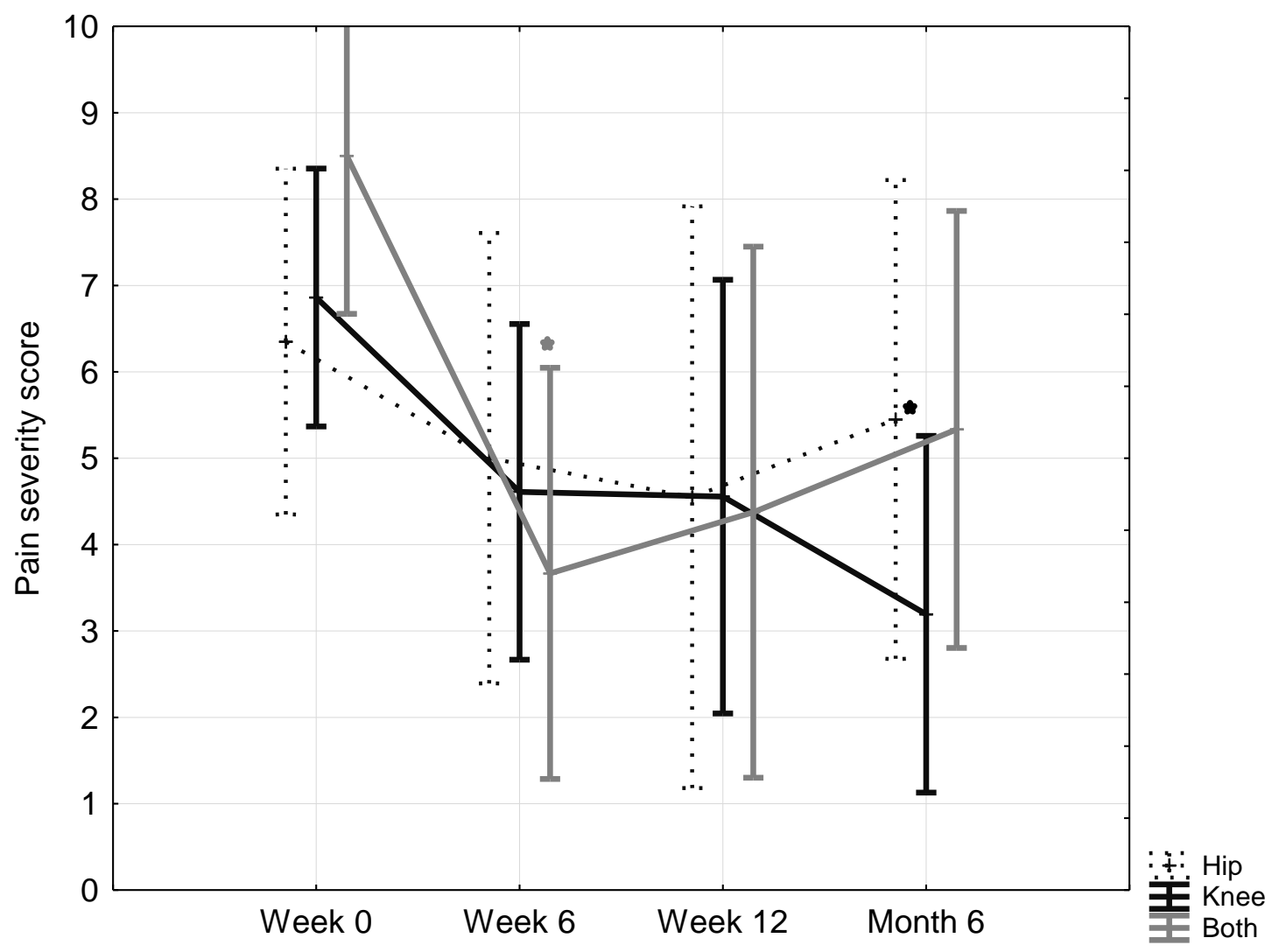

Indicates a significant improvement in PSS of OA both hip and knee subgroup at week $6(p=0.02)$

- Indicates a significant improvement in PSS of OA knee subgroup at month $6(p=0.03)$

Appendix I -1 Change in PSS of OA hip, knee and both hip and knee subgroups in experimental group $(n=20)$ 


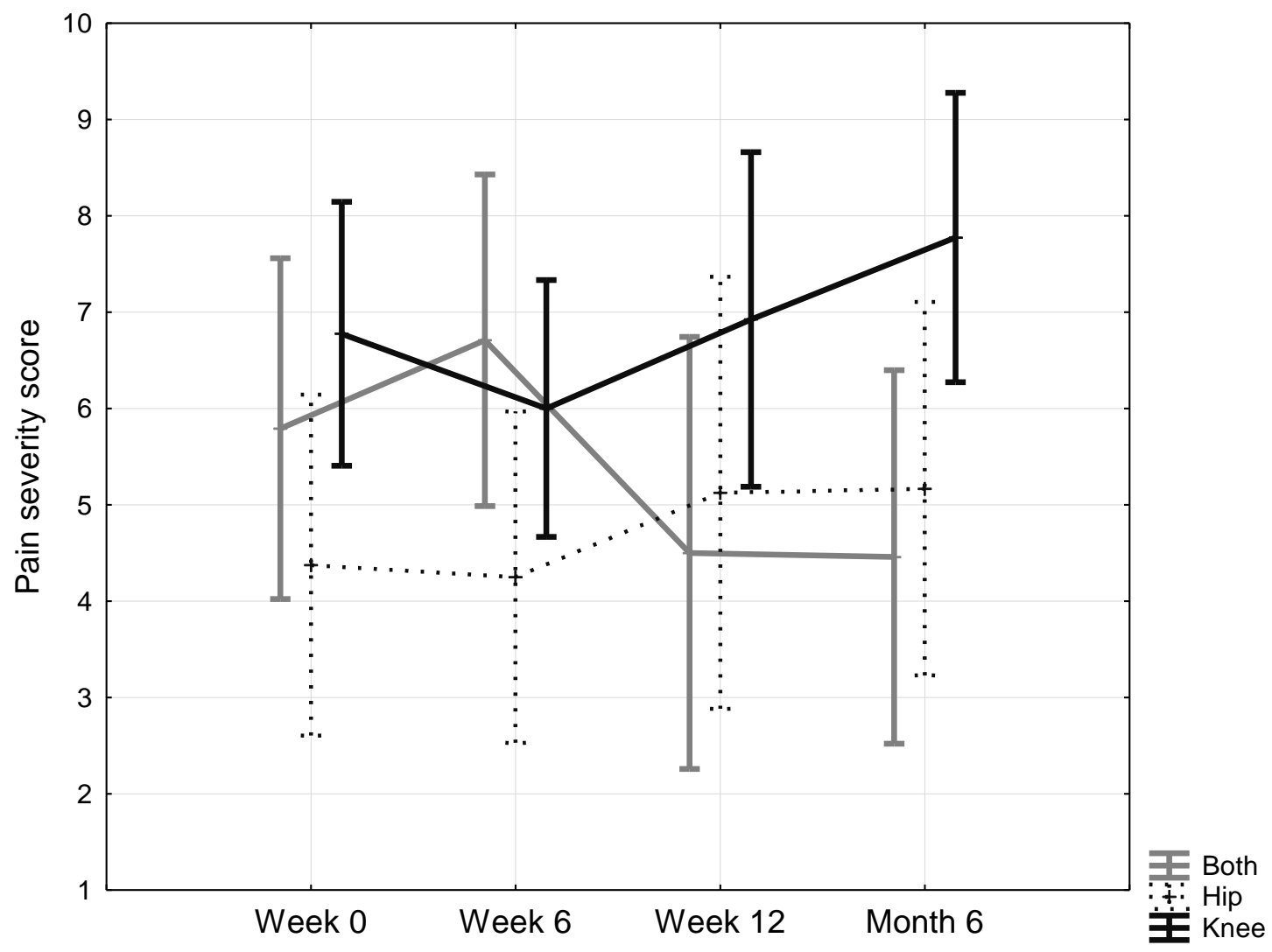

Appendix 1-2 Change in PSS of OA hip, knee, both hip and knee subgroups in control group $(n=22)$ 


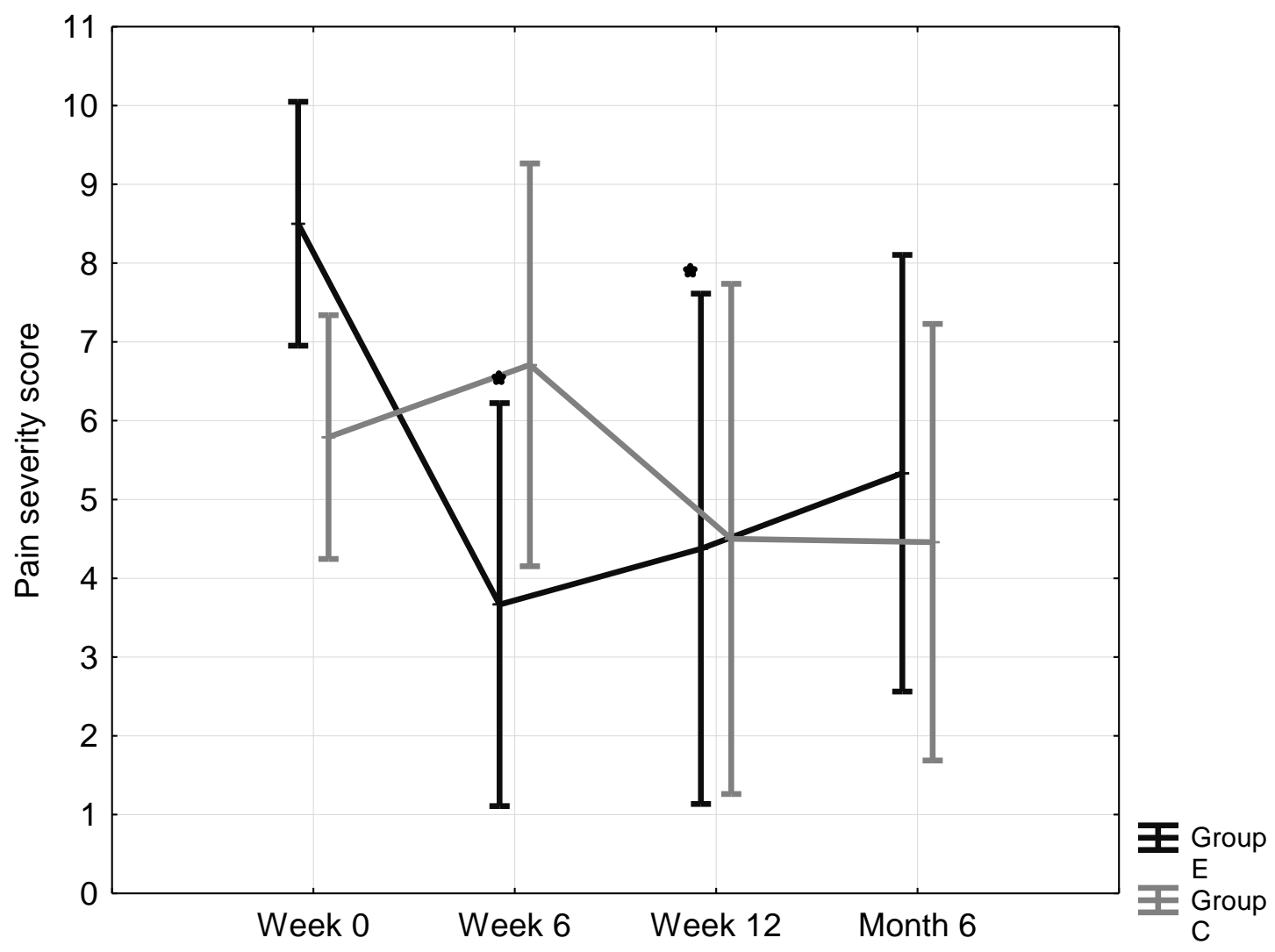

- Indicates a significant improvement in PSS in OA of both hip and knee subgroup within the experimental group at week $6(p<0.01)$ and $12(p=0.03)$

Appendix I-3 Change in PSS of OA both hip and knee subgroup $(n=12)$ 


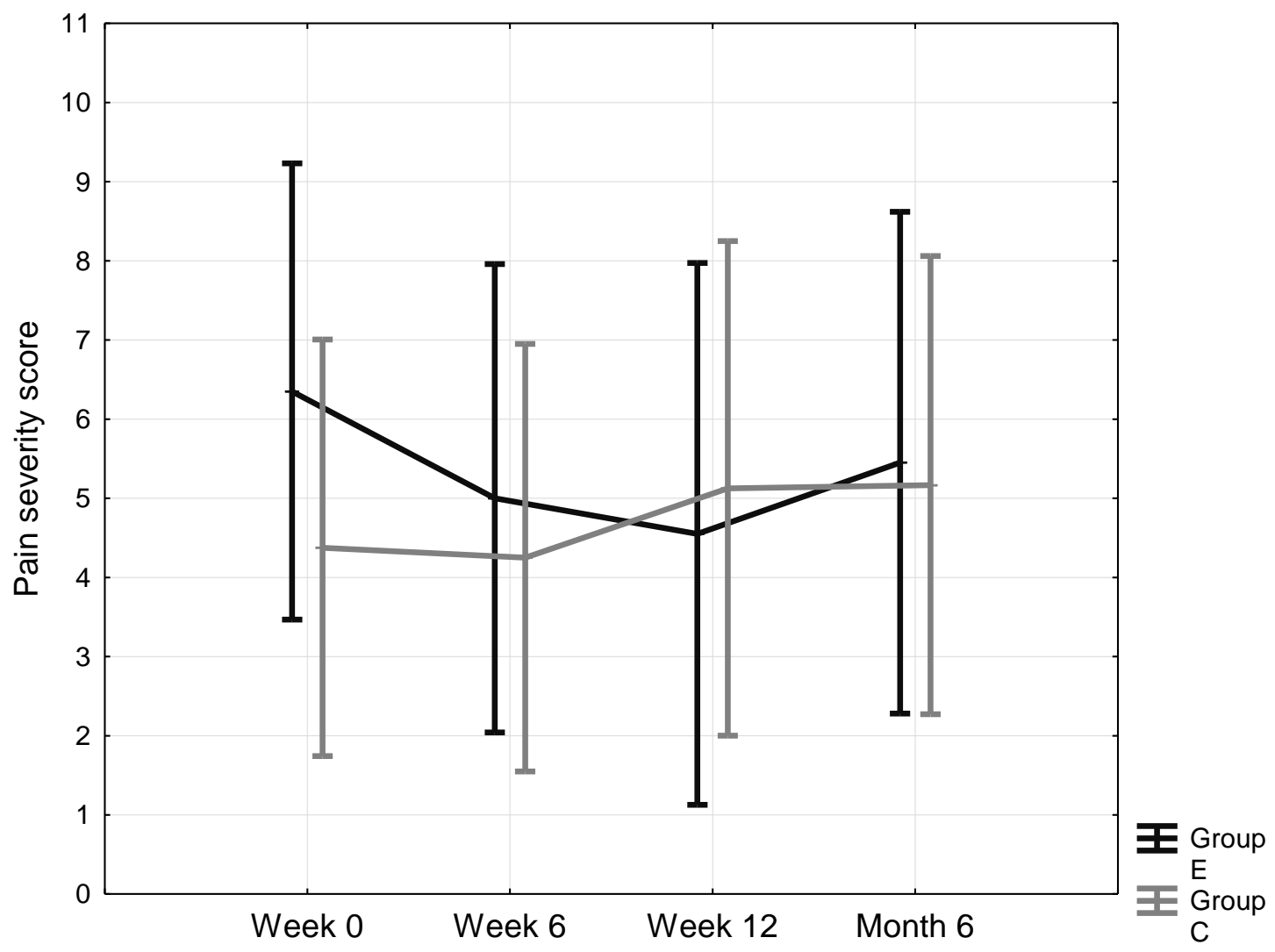

Appendix I-4 Change in PSS of OA hip subgroup $(n=11)$ 
Appendix I-2 Detailed BPI scores showing worst, least, average and current pain ( $N=42)$

\begin{tabular}{|c|c|c|c|}
\hline & $\begin{array}{l}\text { Participants } \\
\mathrm{N}=42\end{array}$ & $\begin{array}{l}\text { Experimental } \\
\text { Group } \\
\mathrm{n}=20\end{array}$ & $\begin{array}{l}\text { Control Group } \\
n=22\end{array}$ \\
\hline & Mean $\pm S D$ & Mean \pm SD & Mean \pm SD \\
\hline \multicolumn{4}{|l|}{ Baseline PSS } \\
\hline PSS worst & $8.17 \pm 2.24$ & $8.35 \pm 1.90$ & $8.00 \pm 2.54$ \\
\hline PSS least & $5.63 \pm 2.95$ & $6.90 \pm 2.75$ & $4.35 \pm 2.62$ \\
\hline PSS average & $6.41 \pm 2.51$ & $6.85 \pm 2.37$ & $6.00 \pm 2.63$ \\
\hline PSS current & $5.75 \pm 3.10$ & $6.80 \pm 3.00$ & $4.76 \pm 3.11$ \\
\hline
\end{tabular}

Week 6

$\begin{array}{lccr}\text { PSS worst } & 6.64 \pm 2.70 & 5.95 \pm 3.07 & 7.27 \pm 2.21 \\ \text { PSS least } & 4.07 \pm 2.61 & 3.60 \pm 2.70^{*} & 4.52 \pm 2.50 \\ \text { PSS average } & 5.27 \pm 2.49 & 4.80 \pm 2.80 & 5.71 \pm 2.12 \\ \text { PSS current } & 4.29 \pm 3.27 & 3.35 \pm 3.13^{*} & 5.14 \pm 3.23\end{array}$

Week 12

\begin{tabular}{cccc} 
PSS worst & $6.45 \pm 3.19$ & $5.75 \pm 3.31$ & $7.09 \pm 3.01$ \\
PSS least & $4.17 \pm 3.07$ & $3.80 \pm 3.53^{*}$ & $4.52 \pm 2.60$ \\
PSS average & $5.54 \pm 3.23$ & $5.45 \pm 3.41$ & $5.62 \pm 3.12$ \\
PSS current & $4.52 \pm 3.83$ & $3.30 \pm 4.07^{*}$ & $5.64 \pm 3.32$ \\
Month 6 & $6.40 \pm 2.95$ & & $7.14 \pm 2.59$ \\
PSS worst & $4.34 \pm 3.03$ & $5.60 \pm 3.17$ & $5.33 \pm 3.12$ \\
PSS least & $5.66 \pm 2.93$ & $3.30 \pm 2.62 *$ & $6.38 \pm 2.82$ \\
PSS average & $4.93 \pm 3.52$ & $4.90 \pm 2.92$ & $5.64 \pm 3.16$ \\
\hline
\end{tabular}

*Indicates a significant improvement in PSS least and current pain in the experimental group 


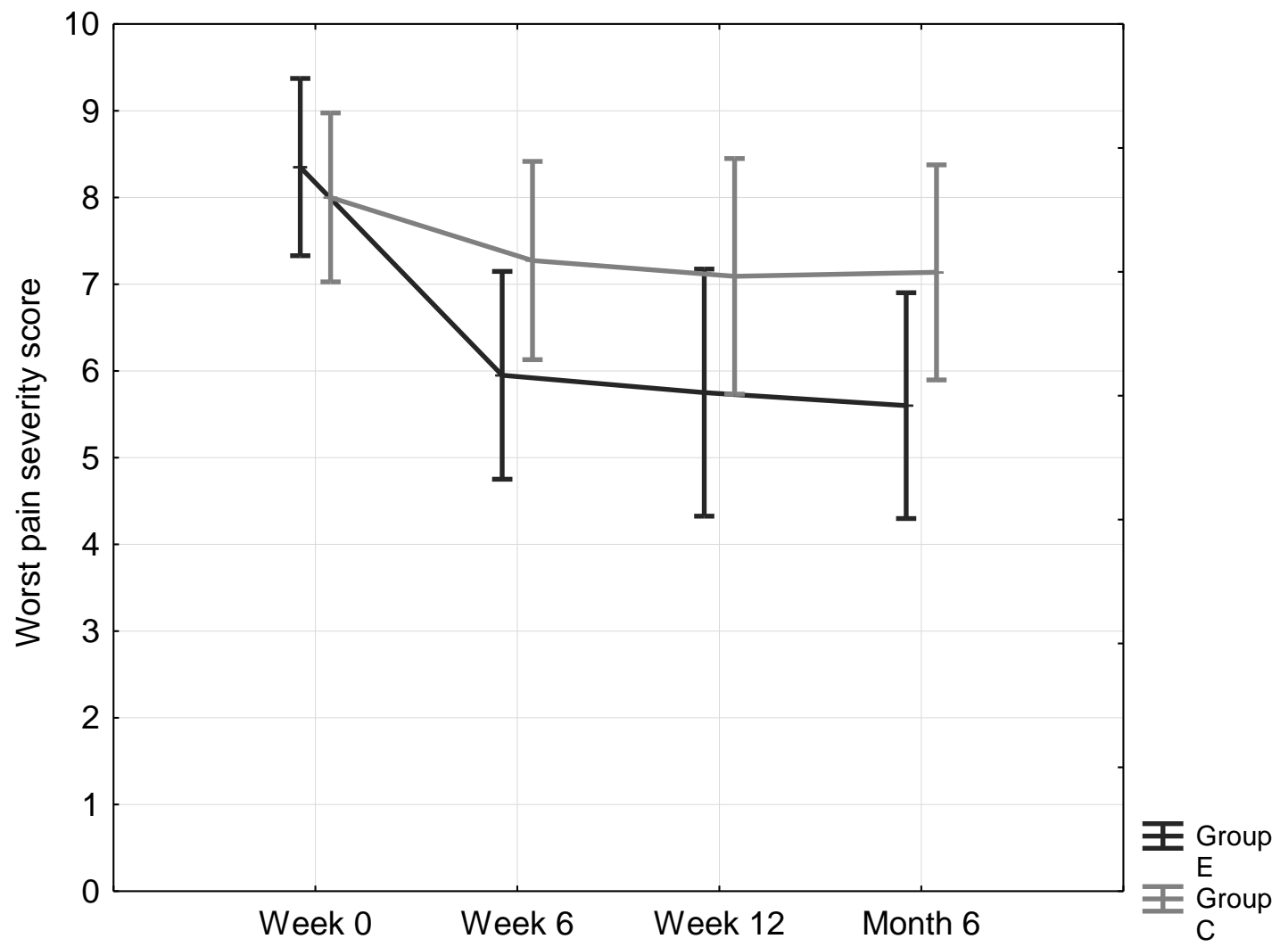

Appendix I-5 Change in worst PSS ( $N=42)$ 


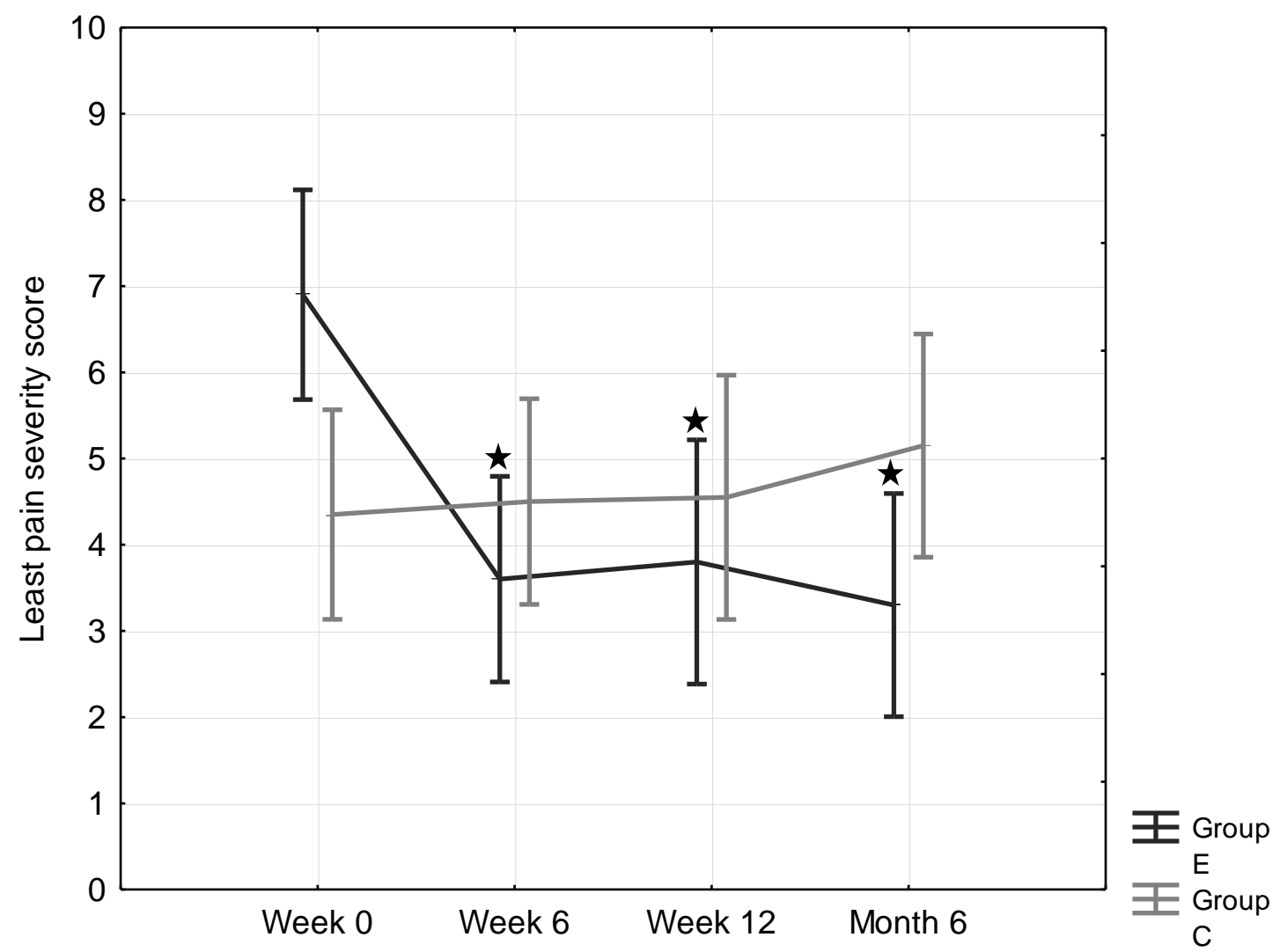

Indicates a significant improvement in the least PSS in the experimental group at week 6, 12 and month 6 (p<0.01)

Appendix I-6 Change in least PSS ( $N=42)$ 


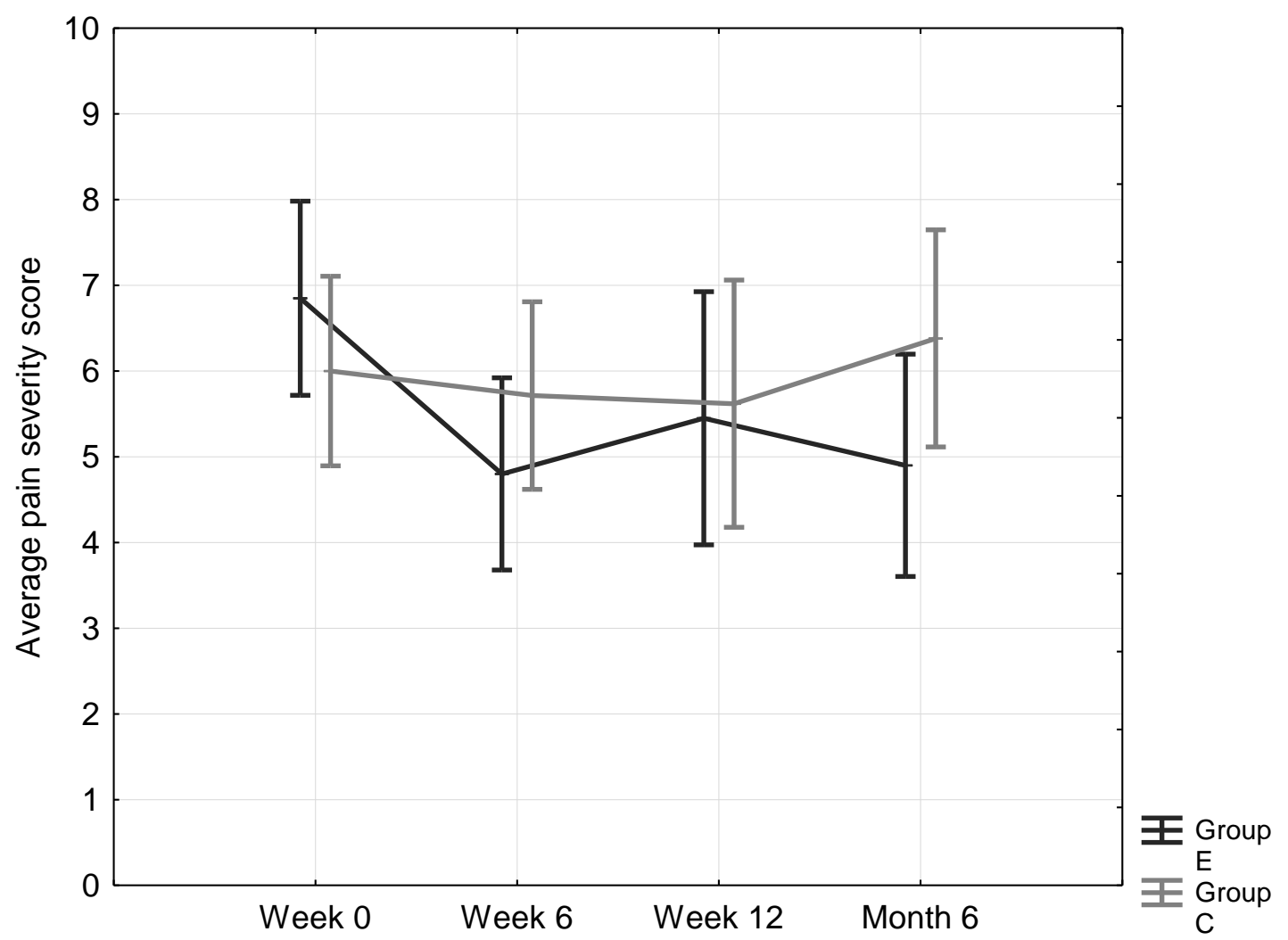

Appendix I-7 Change in average PSS ( $N=42)$ 


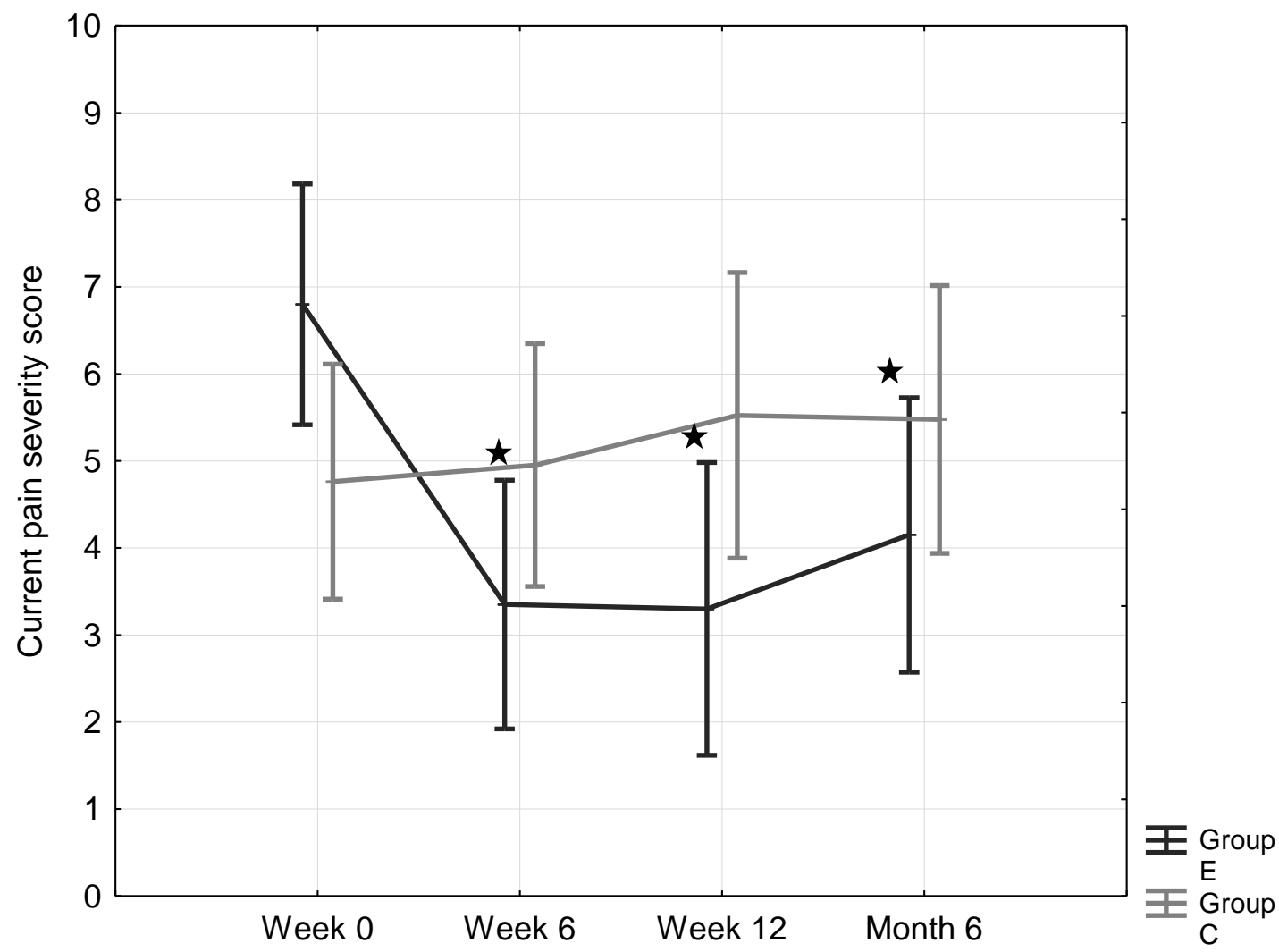

$\star$ Indicates a significant improvement in the current PSS in the experimental group at week 6 and $12(p<0.01)$ and month $6(p=0.02)$

Appendix I-8 Change in current PSS ( $\mathrm{N}=42)$ 
Pain interference score: subgroup analysis of OA hip, knee, both hip and knee

Appendix I-3Detailed PIS for OA hip, knee, both hip and knee subgroups ( $\mathrm{N}=42)$

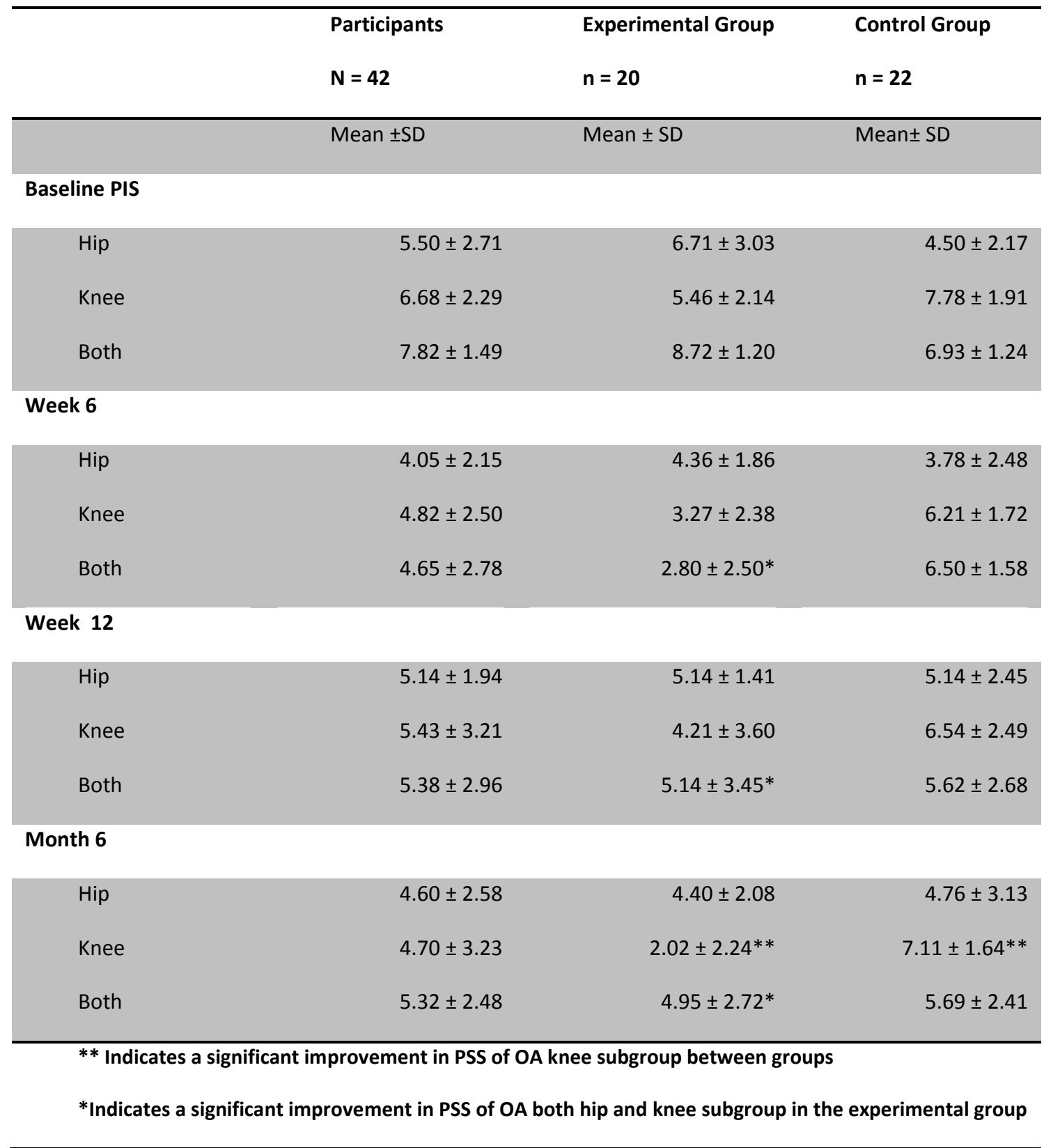




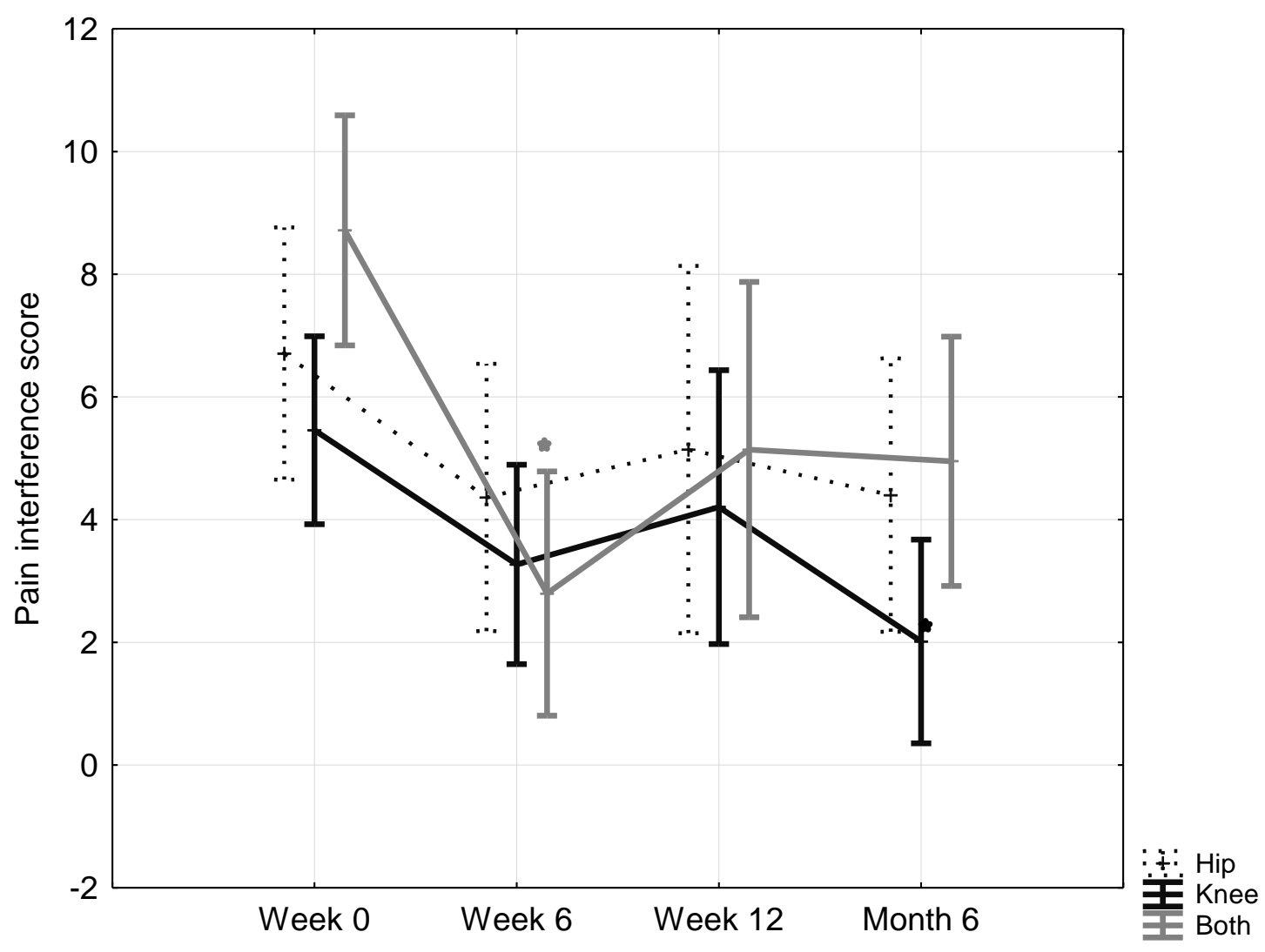

- Indicates a significant improvement in PIS of OA knee subgroup $(p=0.04)$

Indicates a significant improvement in PIS of OA both hip and knee subgroup ( $p<0.01)$

Appendix I -9 Change in PIS of OA hip, OA knee and combined hip and knee OA subgroups in experimental group $(n=20)$ 


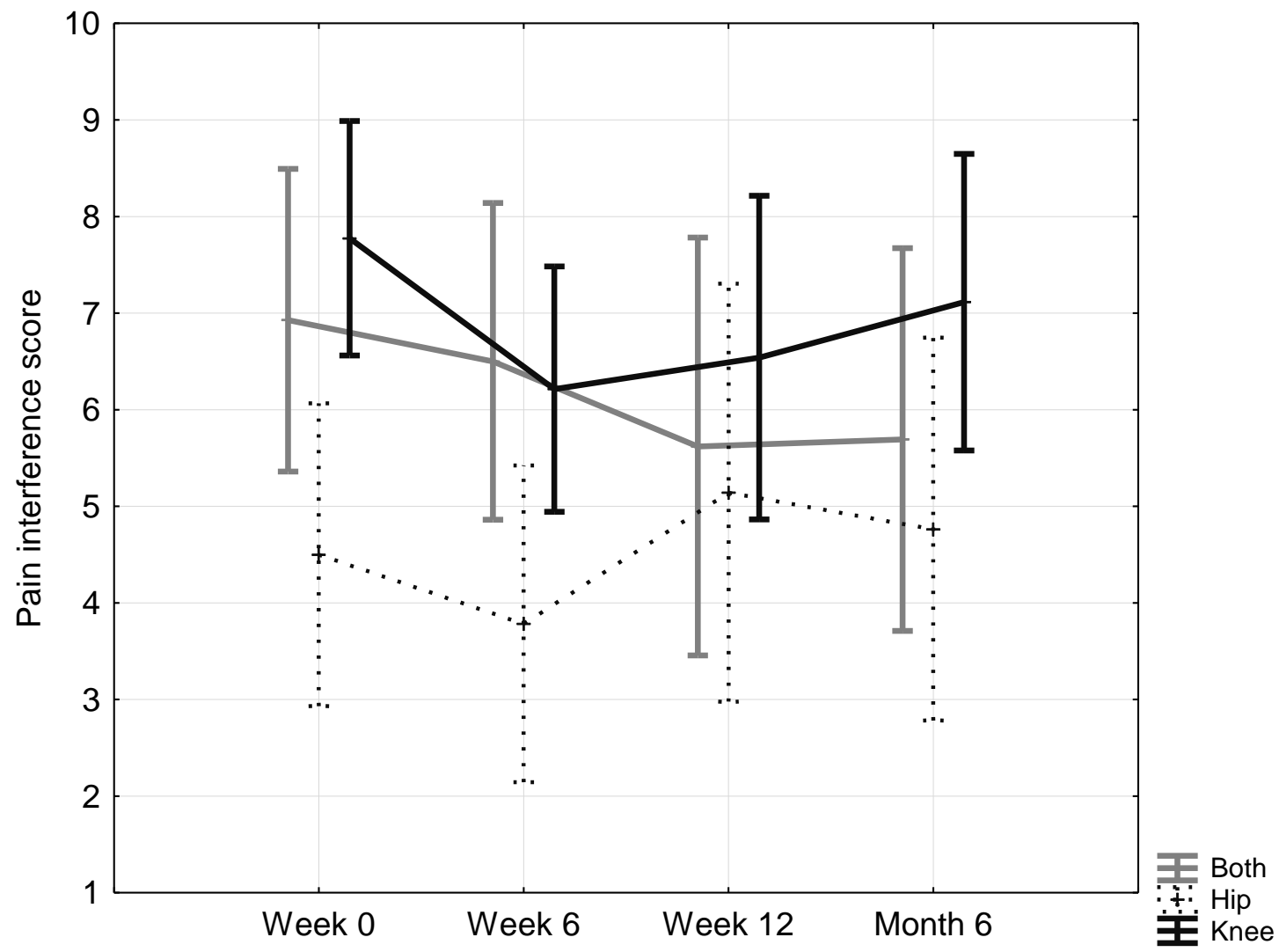

Appendix I-10 Change in PIS of OA hip, OA knee and combined hip and knee OA subgroups in the control group $(n=22)$ 


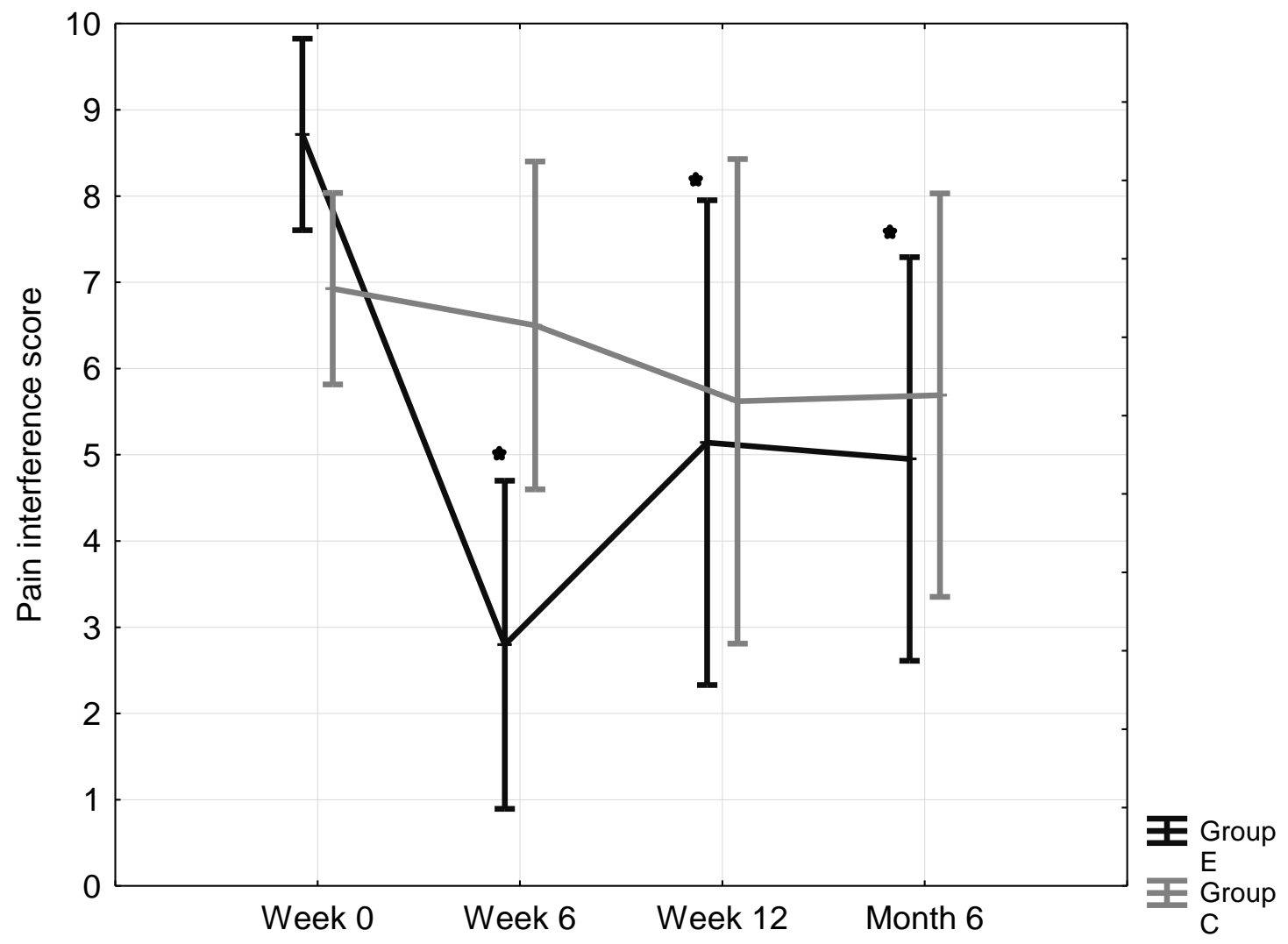

- Indicates a significant improvement in PIS of OA both hip and knee subgroup in the experimental group at week 6 (p < $0.01)$, week 12 and month $6(p=0.02)$

Appendix I-11 Change in PIS of combined hip and knee OA subgroup $(n=12)$ 


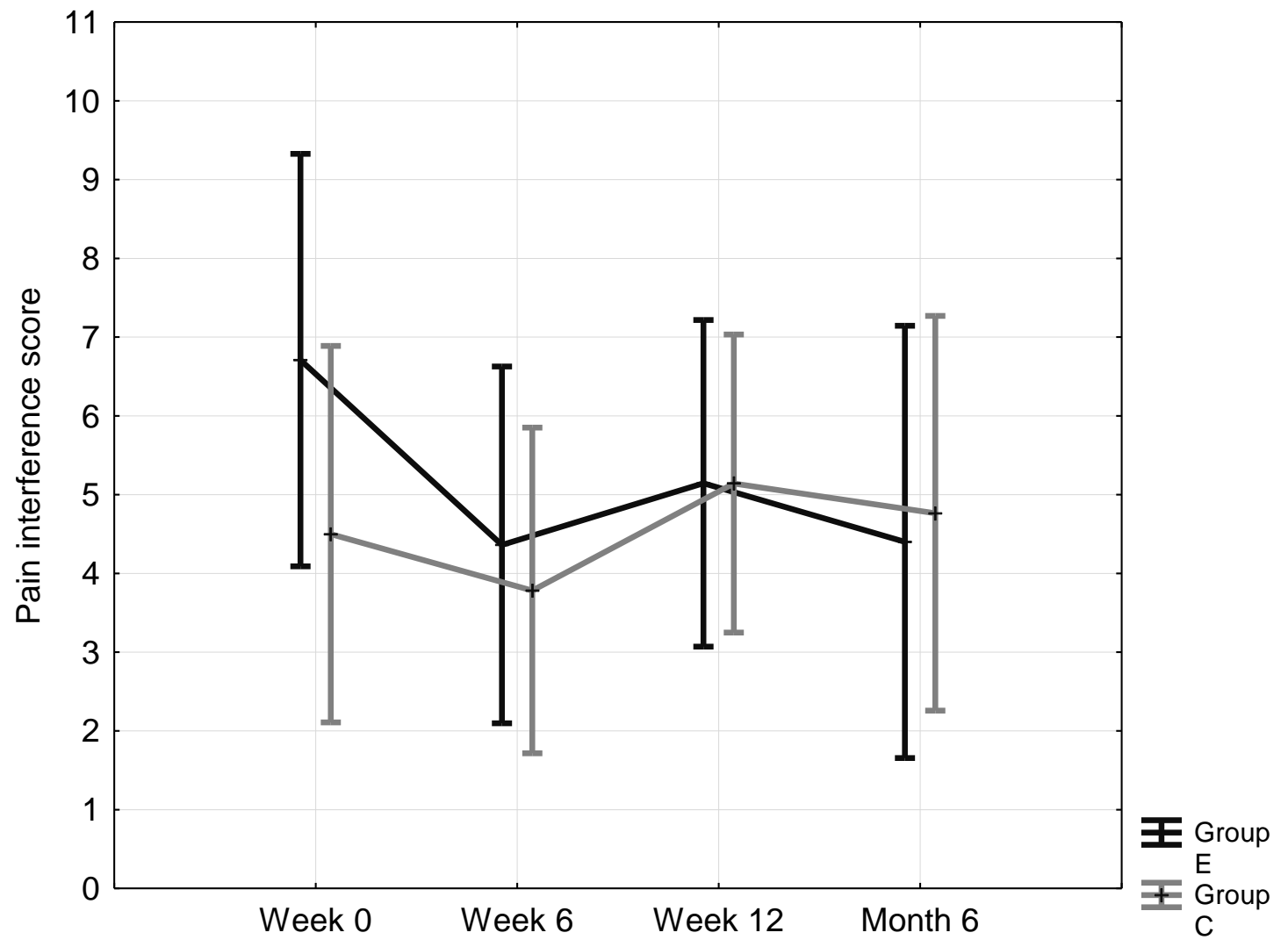

Appendix I-12 Change in PIS of OA hip subgroup $(n=11)$ 
Appendix I-4 Percentage pain relief from medication $(N=42)$

\begin{tabular}{|c|c|c|c|}
\hline & $\begin{array}{l}\text { Participants } \\
\mathbf{N}=42\end{array}$ & $\begin{array}{l}\text { Experimental Group } \\
n=20\end{array}$ & $\begin{array}{l}\text { Control Group } \\
n=22\end{array}$ \\
\hline & Mean \pm SD & Mean \pm SD & Mean \pm SD \\
\hline \multicolumn{4}{|c|}{ Percentage pain relief from medication } \\
\hline Baseline & $45.50 \pm 27.64$ & $38.42 \pm 25.88$ & $51.90 \pm 28.22$ \\
\hline Week 6 & $62.43 \pm 22.41$ & $60.59 \pm 24.87$ & $64.00 \pm 20.62$ \\
\hline Week 12 & $54.86 \pm 22.93$ & $56.67 \pm 23.20$ & $53.50 \pm 23.23$ \\
\hline Month 6 & $61.17 \pm 20.89$ & $58.07 \pm 24.68$ & $63.50 \pm 17.85$ \\
\hline
\end{tabular}

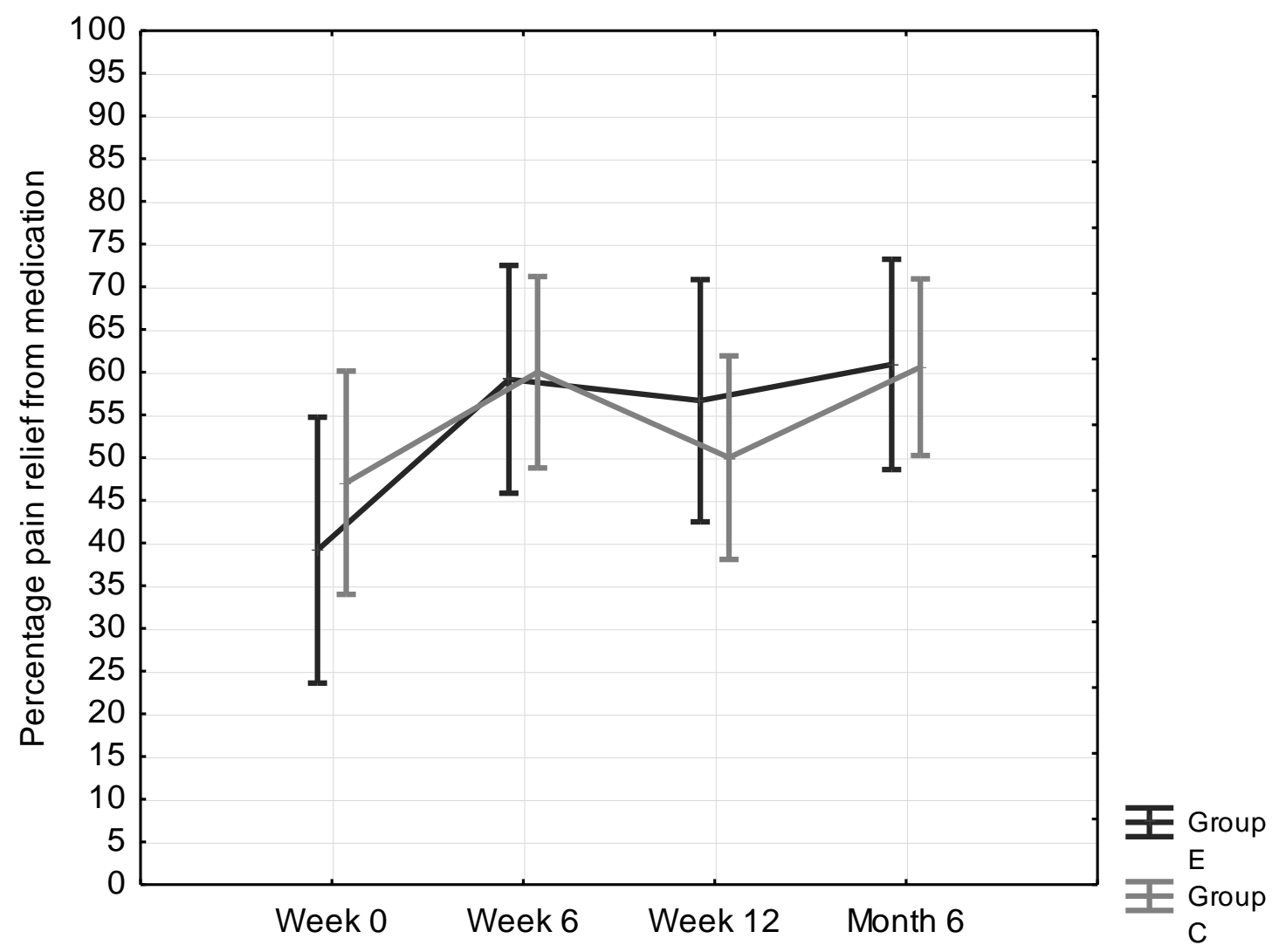

Appendix I-13 Change in percentage pain relief from medication $(N=42)$ 
It is noted that considerably more participants from the control group use analgesia and anti-inflammatories than the experimental group, however there is no significant difference between groups; although at week six the difference approached significance ( $p$ $=0.06)$.

Appendix I-5 Medication usage for the relief of OA symptoms ( $N=42)$

\begin{tabular}{|c|c|c|c|}
\hline & $\begin{array}{l}\text { Participants } \\
\mathrm{N}=42\end{array}$ & $\begin{array}{l}\text { Exp Group } \\
n=20\end{array}$ & $\begin{array}{l}\text { ContGroup } \\
n=22\end{array}$ \\
\hline & Number & Number & Number \\
\hline \multicolumn{4}{|l|}{ Medication usage baseline } \\
\hline None & 4 & 3 & 1 \\
\hline Analgesia & 15 & 9 & 6 \\
\hline Anti-inflammatories & 5 & 2 & 3 \\
\hline Analgesia \& Anti-inflammatories & 12 & 4 & 8 \\
\hline Not indicated & 6 & 2 & 4 \\
\hline \multicolumn{4}{|l|}{ Week 6} \\
\hline None & 5 & 3 & 2 \\
\hline Analgesia & 11 & 7 & 4 \\
\hline Anti-inflammatories & 6 & 5 & 1 \\
\hline Analgesia \& Anti-inflammatories & 18 & 4 & 14 \\
\hline Not indicated & 2 & 1 & 1 \\
\hline \multicolumn{4}{|l|}{ Week 12} \\
\hline None & 7 & 5 & 2 \\
\hline Analgesia & 13 & 6 & 7 \\
\hline Anti-inflammatories & 6 & 3 & 3 \\
\hline Analgesia \& Anti-inflammatories & 16 & 6 & 10 \\
\hline Not indicated & 0 & 0 & 0 \\
\hline
\end{tabular}




\begin{tabular}{lccc}
\hline & Participants & Exp Group & Cont Group \\
& $\mathbf{N}=\mathbf{4 2}$ & $\mathbf{n = 2 0}$ & $\mathbf{n}=\mathbf{2 2}$ \\
\hline Medication usage month 6 & Number & Number & Number \\
None & 9 & 7 & 2 \\
Analgesia & 16 & 8 & 8 \\
Anti-inflammatories & 5 & 2 & 3 \\
Analgesia \& Anti-inflammatories & 12 & 3 & 9 \\
Not indicated & 0 & 0 & 0 \\
\hline
\end{tabular}




\section{Appendix J: Full function results}

Physical performance task battery walk tests $-15 \mathrm{~m}$ fastest and normal speed

Appendix J-1 Detailed $15 \mathrm{~m}$ fastest and normal speed walk scores $(\mathrm{N}=\mathbf{4 2})$

\begin{tabular}{|c|c|c|c|}
\hline & $\begin{array}{l}\text { Participants } \\
\mathrm{N}=42\end{array}$ & $\begin{array}{l}\text { Experimental Group } \\
n=20\end{array}$ & $\begin{array}{l}\text { Control Group } \\
n=22\end{array}$ \\
\hline & Mean \pm SD & Mean \pm SD & Mean \pm SD \\
\hline \multicolumn{4}{|c|}{15 m fastest walk (sec) } \\
\hline Baseline & $18.04 \pm 5.91$ & $18.65 \pm 6.27$ & $17.51 \pm 5.67$ \\
\hline Week 6 & $18.12 \pm 725$ & $16.56 \pm 6.27$ & $19.53 \pm 7.92$ \\
\hline Week 12 & $16.91 \pm 6.12$ & $15.52 \pm 6.33^{*}$ & $18.17 \pm 5.77$ \\
\hline Month 6 & $17.71 \pm 7.93$ & $15.09 \pm 6.04^{* *}$ & $20.10 \pm 8.79 * *$ \\
\hline \multicolumn{4}{|c|}{15 m normal walk (sec) } \\
\hline Baseline & $23.20 \pm 7.58$ & $24.82 \pm 8.43$ & $21.81 \pm 6.64$ \\
\hline Week 6 & $22.97 \pm 6.99$ & $21.76 \pm 6.46$ & $24.07 \pm 7.41$ \\
\hline Week 12 & $20.79 \pm 6.64$ & $19.42 \pm 5.99 *$ & $22.03 \pm 7.09$ \\
\hline Month 6 & $21.20 \pm 7.39$ & $18.60 \pm 5.45^{*}$ & $23.57 \pm 8.21$ \\
\hline $\begin{array}{l}\text { **Indicates a signific } \\
* \text { Indicates a significa } \\
\text { experimental group }\end{array}$ & $\begin{array}{l}\text { t in the time taken to } \mathrm{v} \\
\text { th in the time taken } t\end{array}$ & $\begin{array}{l}\text { alk } 15 \mathrm{~m} \text { at fastest speed bet } \\
\text { walk } 15 \mathrm{~m} \text { at fastest and } n\end{array}$ & $\begin{array}{l}\text { Neen groups } \\
\text { ormal speed in the }\end{array}$ \\
\hline
\end{tabular}


Appendix J-2 Detailed time taken to walk $15 \mathrm{~m}$ at fastest speed in OA hip, knee, both hip and knee subgroups

\begin{tabular}{|c|c|c|c|}
\hline & Participants & Experimental Group & Control Group \\
\hline & $N=42$ & $n=20$ & $n=22$ \\
\hline & Mean \pm SD & Mean \pm SD & Mean \pm SD \\
\hline \multicolumn{4}{|c|}{ Baseline $15 \mathrm{~m}$ fastest } \\
\hline Hip & $17.44 \pm 5.30$ & $20.11 \pm 6.78$ & $15.66 \pm 3.67$ \\
\hline Knee & $19.58 \pm 7.42$ & $19.93 \pm 7.73$ & $19.26 \pm 7.54$ \\
\hline Both & $16.09 \pm 2.32$ & $15.75 \pm 1.93$ & $16.44 \pm 2.81$ \\
\hline \multicolumn{4}{|l|}{ Week 6} \\
\hline Hip & $19.61 \pm 7.70$ & $22.23 \pm 9.81$ & $17.43 \pm 5.40$ \\
\hline Knee & $19.20 \pm 8.47$ & $15.80 \pm 3.65$ & $22.27 \pm 10.47$ \\
\hline Both & $15.03 \pm 3.19$ & $13.00 \pm 1.67$ & $17.07 \pm 3.11$ \\
\hline \multicolumn{4}{|l|}{ Week 12} \\
\hline Hip & $18.98 \pm 7.33$ & $21.10 \pm 9.78$ & $17.22 \pm 4.77$ \\
\hline Knee & $17.21 \pm 6.38$ & $14.75 \pm 3.99 *$ & $19.42 \pm 7.48$ \\
\hline Both & $14.53 \pm 3.66$ & $12.02 \pm 1.87^{*}$ & $17.04 \pm 3.29$ \\
\hline \multicolumn{4}{|l|}{ Month 6} \\
\hline Hip & $18.48 \pm 7.21$ & $20.46 \pm 10.03$ & $16.82 \pm 4.03$ \\
\hline Knee & $18.64 \pm 10.00$ & $13.40 \pm 2.78^{*}$ & $23.35 \pm 11.89$ \\
\hline Both & $15.54 \pm 4.05$ & $13.13 \pm 2.70$ & $17.96 \pm 3.85$ \\
\hline \multicolumn{4}{|c|}{$\begin{array}{l}\text { * Indicates a significant improvement in time taken to walk } 15 \mathrm{~m} \text { at fastest speed in experimental OA } \\
\text { knee and OA both hip and knee subgroups }\end{array}$} \\
\hline
\end{tabular}




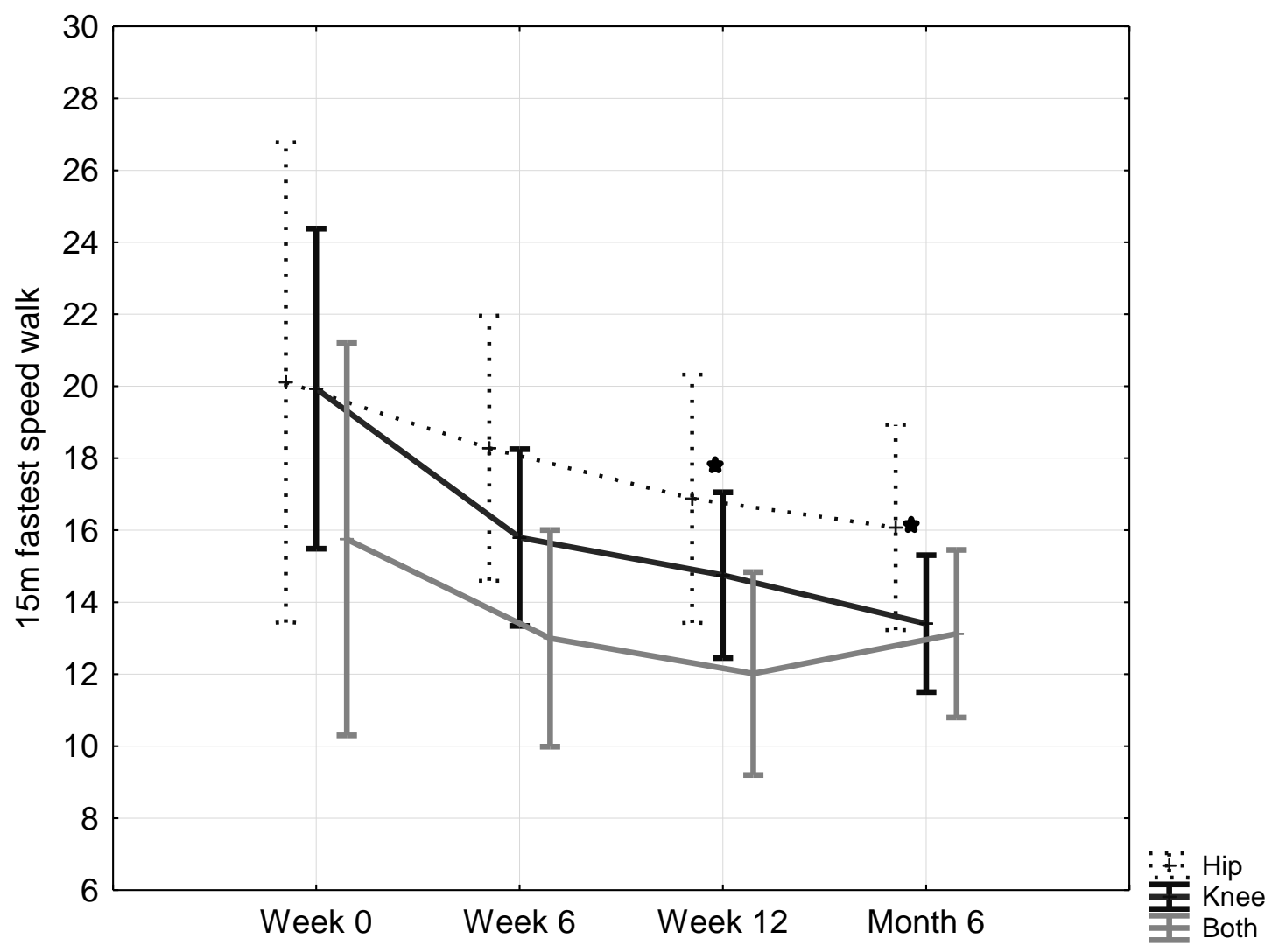

Indicates a significant improvement in the OA knee subgroup at week $12(p=0.01)$ and month $6(p<0.01)$

Appendix J-1 Change in OA hip, knee, both hip and knee subgroups in the experimental group $(n=20)$ 


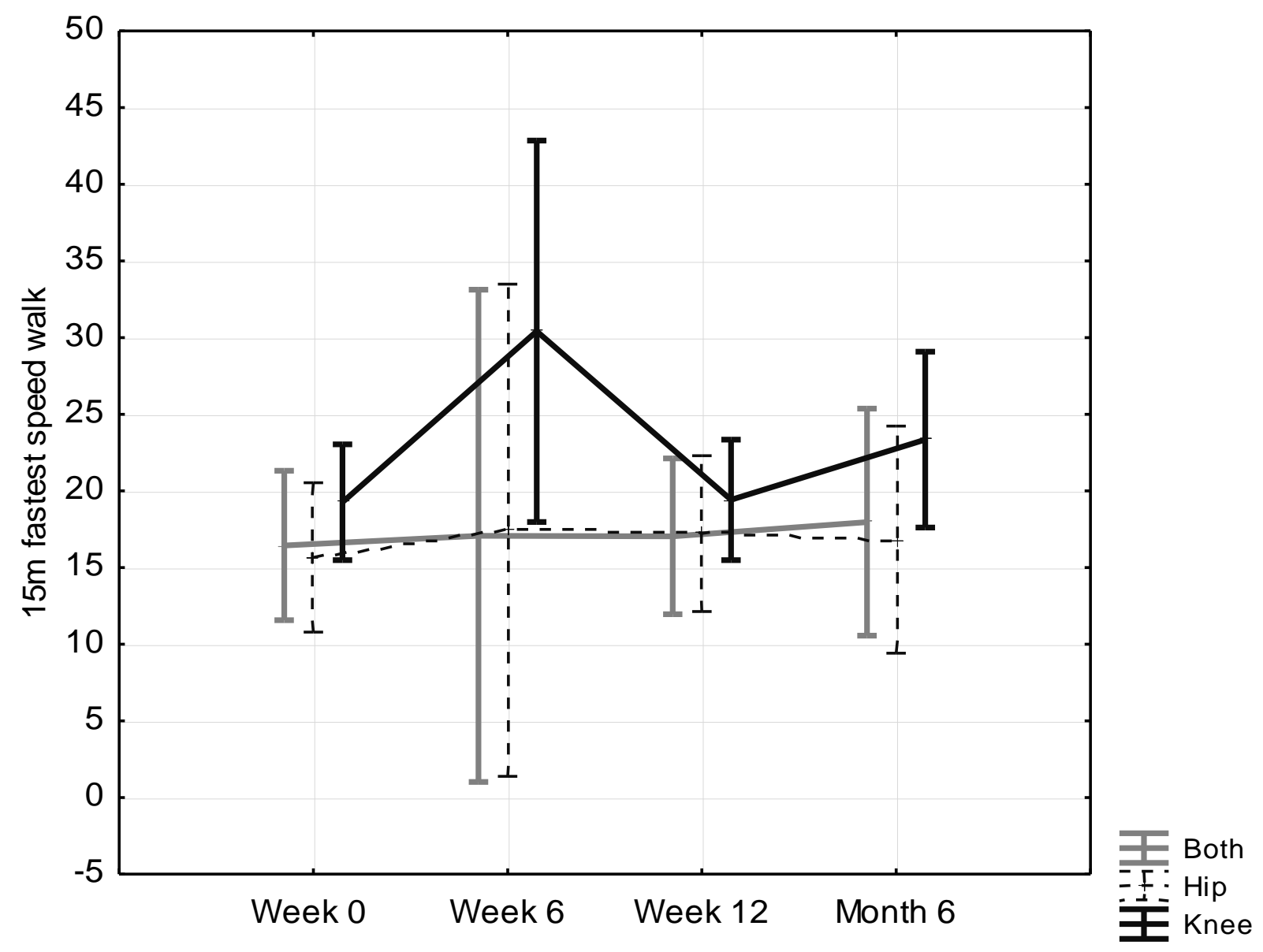

Appendix J-2 Change in OA hip, knee, both hip and knee subgroups in the control group $(n=22)$ 


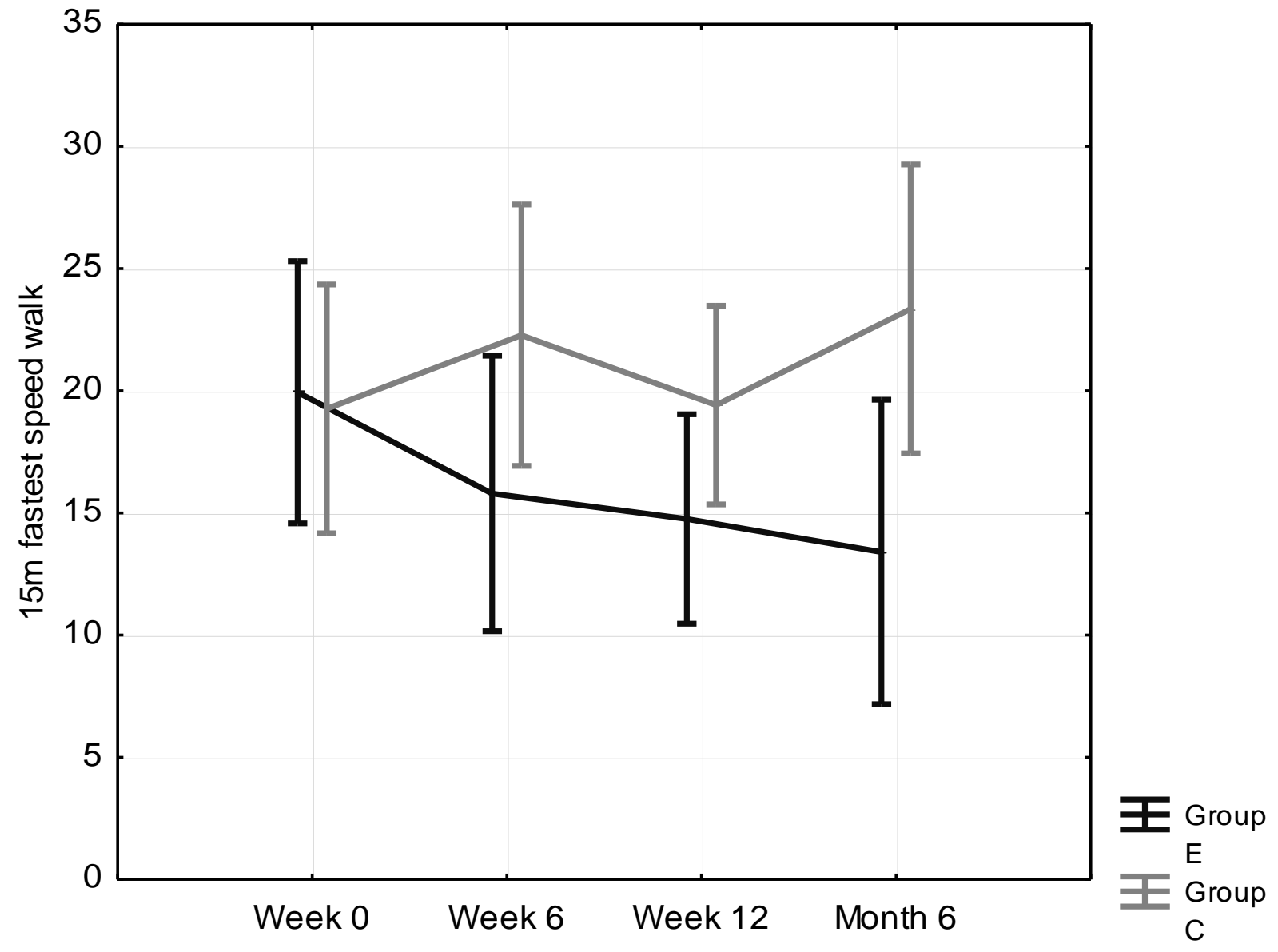

Appendix J-3 Change in time taken for $15 \mathrm{~m}$ fastest speed walk of OA knee subgroup ( $\mathrm{n}=$ 19) 


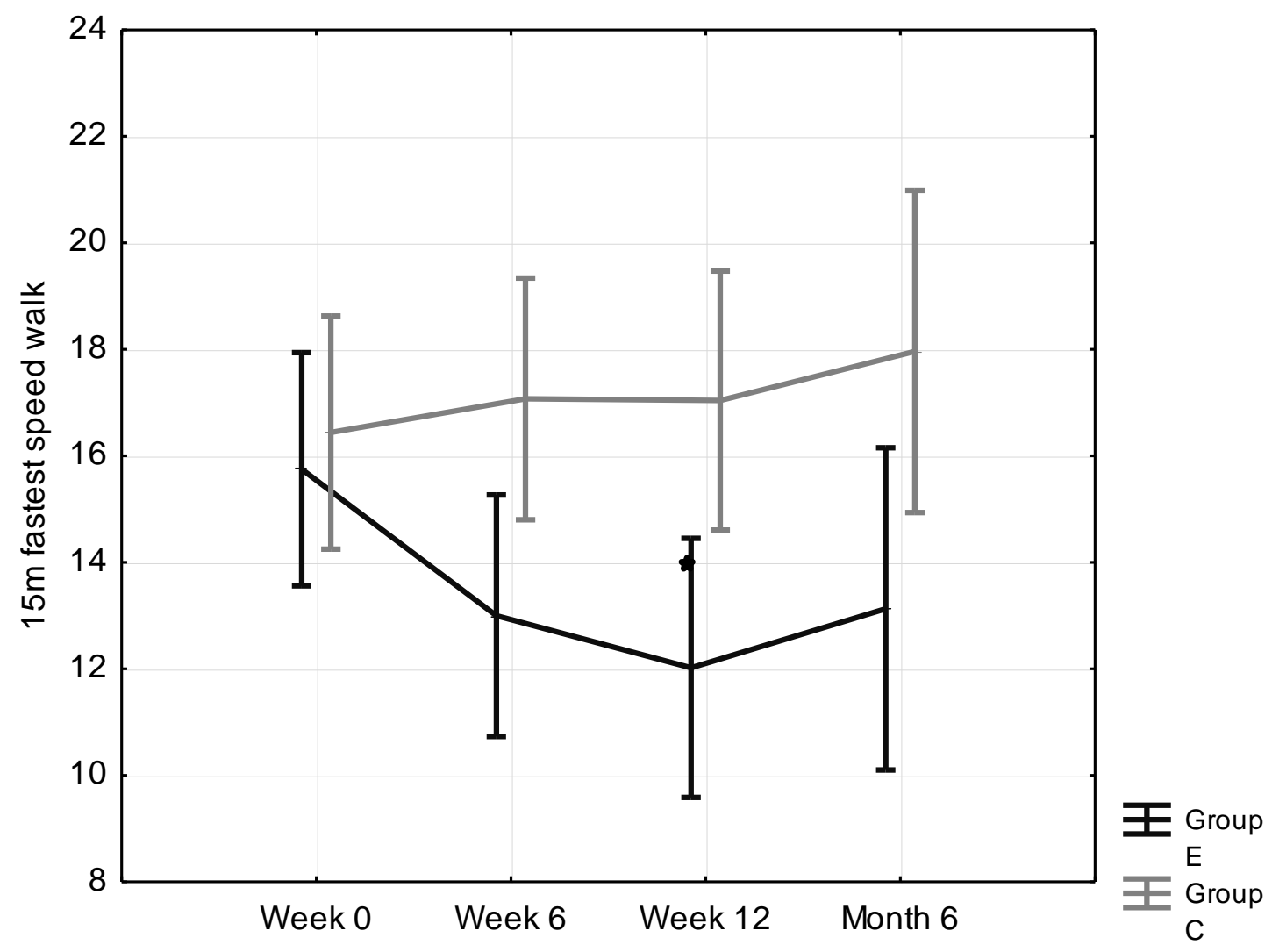

- Indicates a significant improvement in time taken in OA both hip and knee in experimental group at week 12 ( $p=0.04)$

Appendix J-4 Change in time taken for $15 \mathrm{~m}$ fastest speed walk of OA both hip and knee subgroup $(n=12)$ 


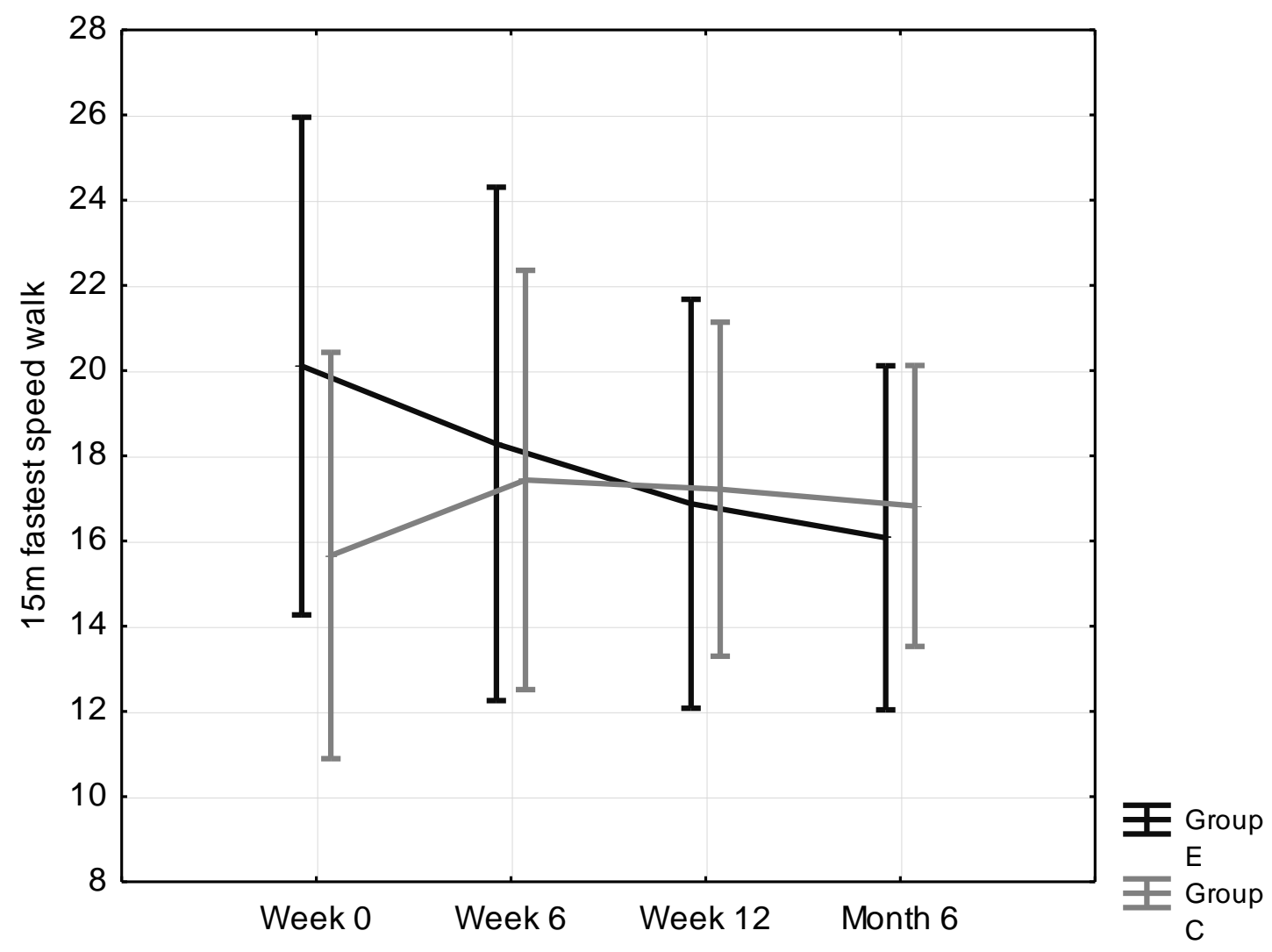

Appendix J-5 Change in time taken for $15 \mathrm{~m}$ fastest speed walk of OA hip subgroup ( $\mathrm{n}=$ 11) 
Appendix J-3 Detailed time taken to walk $15 \mathrm{~m}$ at normal speed for OA hip, knee and both hip and knee subgroups

\begin{tabular}{|c|c|c|c|}
\hline & $\begin{array}{l}\text { Participants } \\
\mathbf{N}=\mathbf{4 2}\end{array}$ & $\begin{array}{l}\text { Experimental } \\
\text { Group } \\
\mathrm{n}=\mathbf{2 0}\end{array}$ & $\begin{array}{l}\text { Control Group } \\
n=22\end{array}$ \\
\hline & Mean $\pm S D$ & Mean \pm SD & Mean \pm SD \\
\hline \multicolumn{4}{|c|}{ Baseline $15 \mathrm{~m}$ normal } \\
\hline Hip & $21.59 \pm 5.86$ & $25.95 \pm 6.78$ & $18.68 \pm 2.97$ \\
\hline Knee & $23.72 \pm 9.49$ & $26.80 \pm 11.22$ & $20.95 \pm 7.08$ \\
\hline Both & $23.72 \pm 5.52$ & $21.09 \pm 1.37$ & $26.36 \pm 6.96$ \\
\hline \multicolumn{4}{|l|}{ Week 6} \\
\hline Hip & $25.13 \pm 7.04$ & $28.24 \pm 7.74$ & $22.53 \pm 5.78$ \\
\hline Knee & $23.53 \pm 7.85$ & $21.07 \pm 4.93$ & $25.75 \pm 9.50$ \\
\hline Both & $20.08 \pm 4.65$ & $17.38 \pm 2.41$ & $22.79 \pm 4.91$ \\
\hline \multicolumn{4}{|l|}{ Week 12} \\
\hline Hip & $21.74 \pm 6.61$ & $24.09 \pm 8.43$ & $19.78 \pm 4.53$ \\
\hline Knee & $21.70 \pm 7.22$ & $19.82 \pm 4.41^{*}$ & $23.38 \pm 8.97$ \\
\hline Both & $18.48 \pm 5.60$ & $14.94 \pm 1.57$ & $22.03 \pm 6.02$ \\
\hline \multicolumn{4}{|l|}{ Month 6} \\
\hline Hip & $21.60 \pm 6.38$ & $22.78 \pm 9.09$ & $20.61 \pm 3.58$ \\
\hline Knee & $22.23 \pm 8.76$ & $18.22 \pm 2.92 *$ & $25.84 \pm 10.74$ \\
\hline Both & $19.21 \pm 5.88$ & $15.69 \pm 2.23$ & $22.73 \pm 6.42$ \\
\hline \multicolumn{4}{|c|}{$\begin{array}{l}\text { * Indicates a significant improvement in time taken to walk } 15 \mathrm{~m} \text { at fastest speed in experimental OA } \\
\text { knee subgroup }\end{array}$} \\
\hline
\end{tabular}




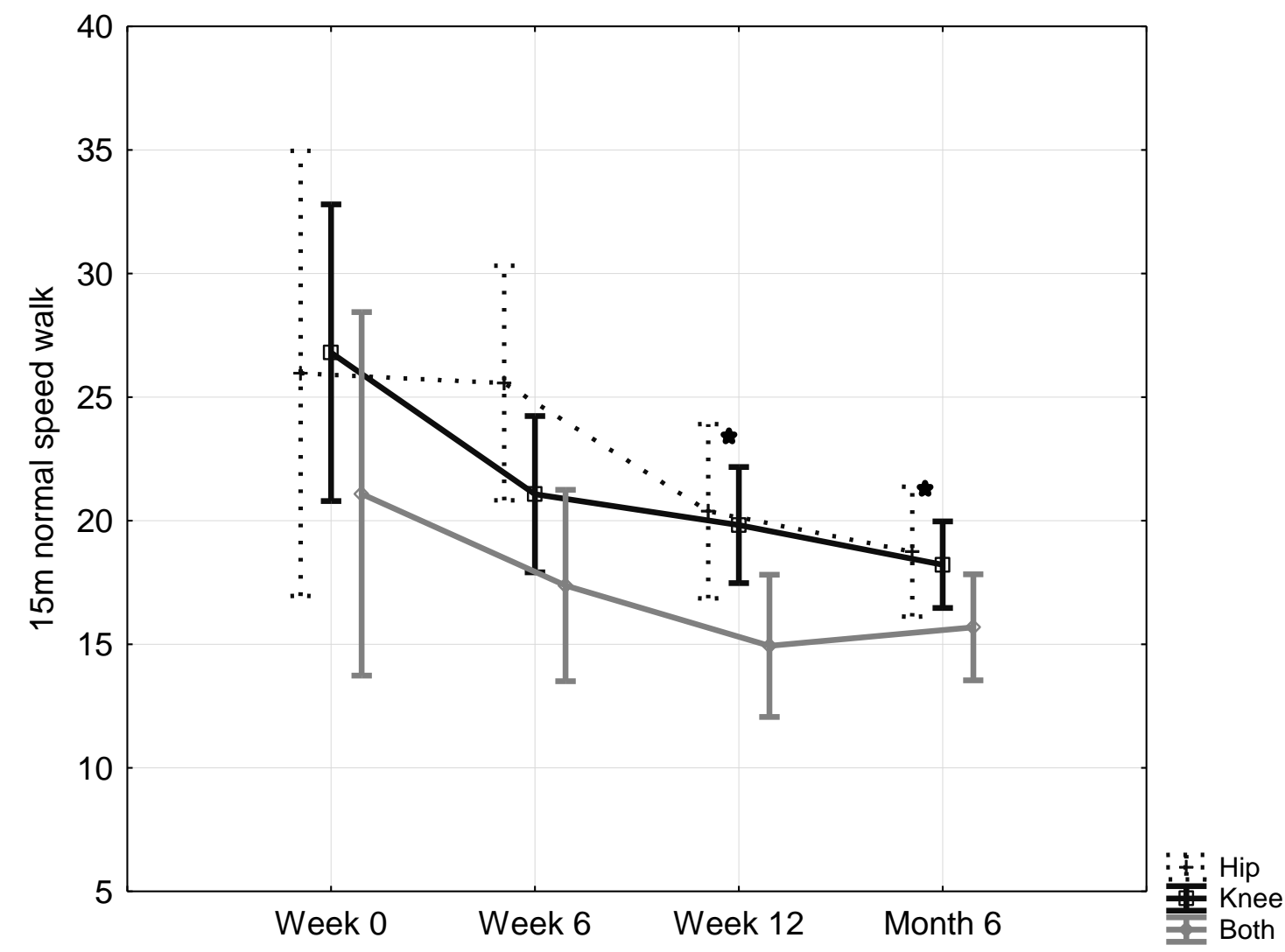

- Indicates a significant improvement in the time taken to walk $15 \mathrm{~m}$ at normal speed of OA knee subgroup at week 12 (p $=0.03)$ and month $6(p<0.01)$

Appendix J-6 Change in OA hip, knee, both hip and knee subgroups in the experimental group $(n=20)$ 


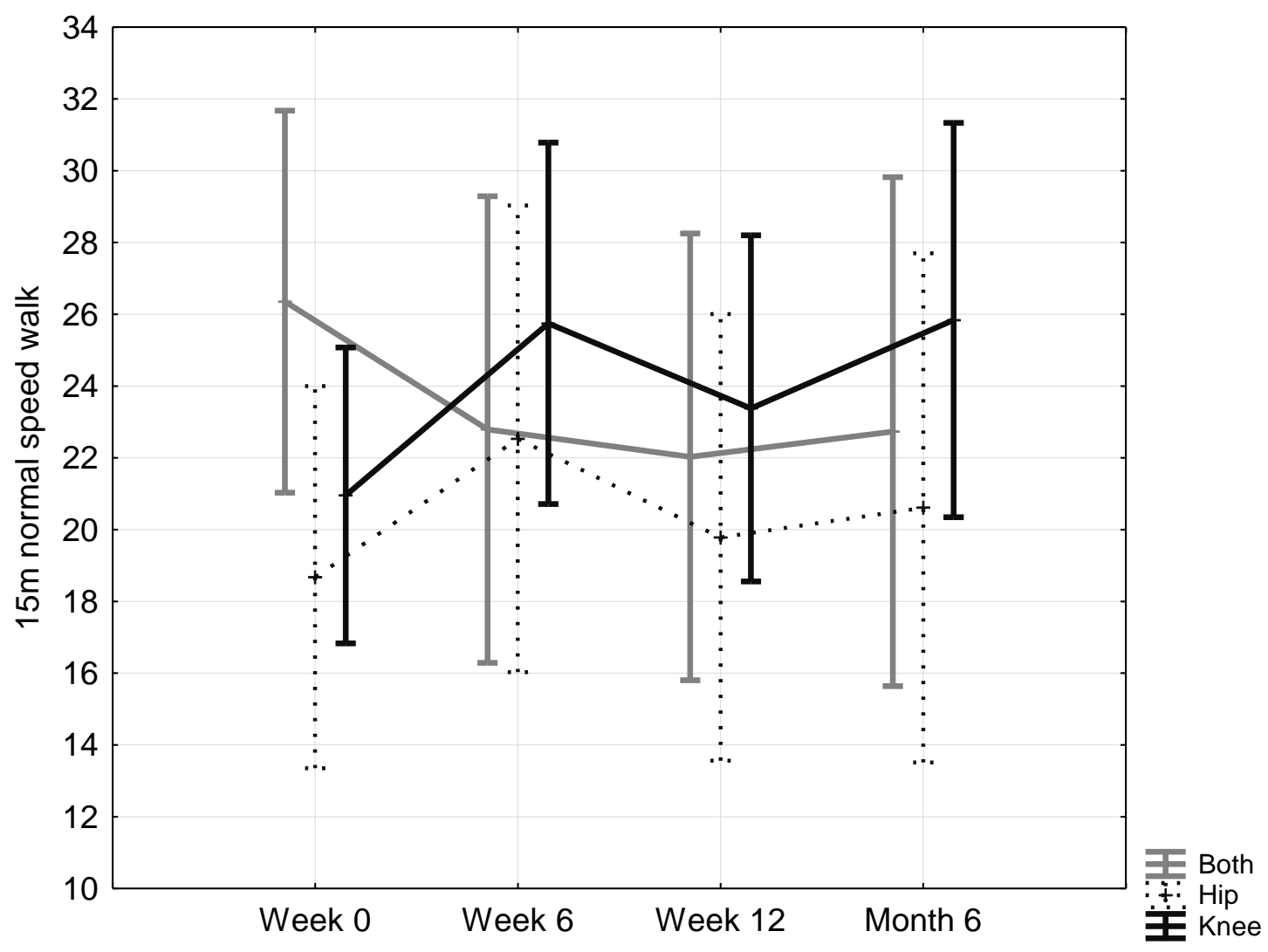

Appendix J-7 Change in OA hip, knee, both hip and knee subgroups in the control group $(n=22)$ 


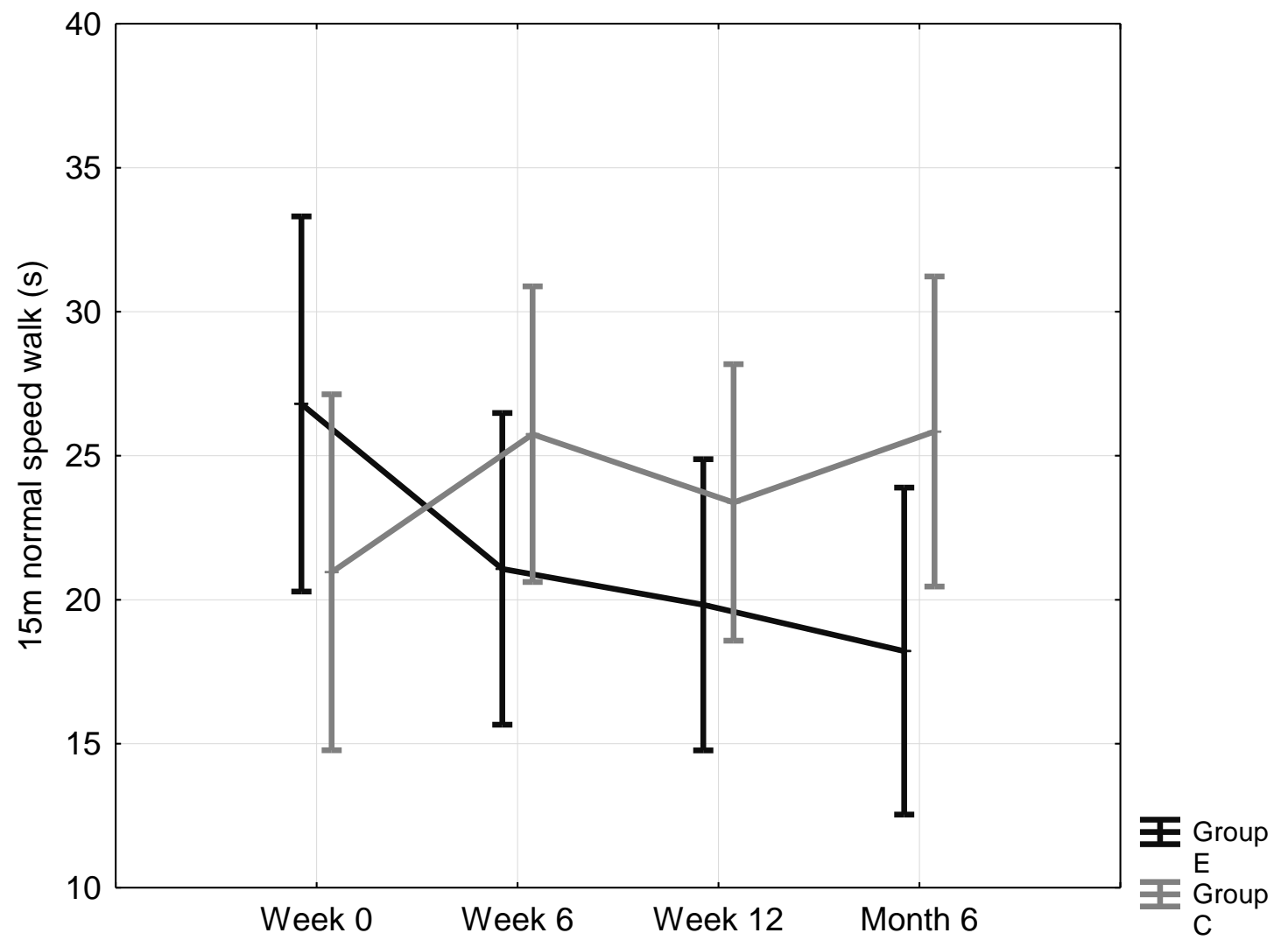

Appendix J-8 Change in OA knee subgroup $(n=19)$ 


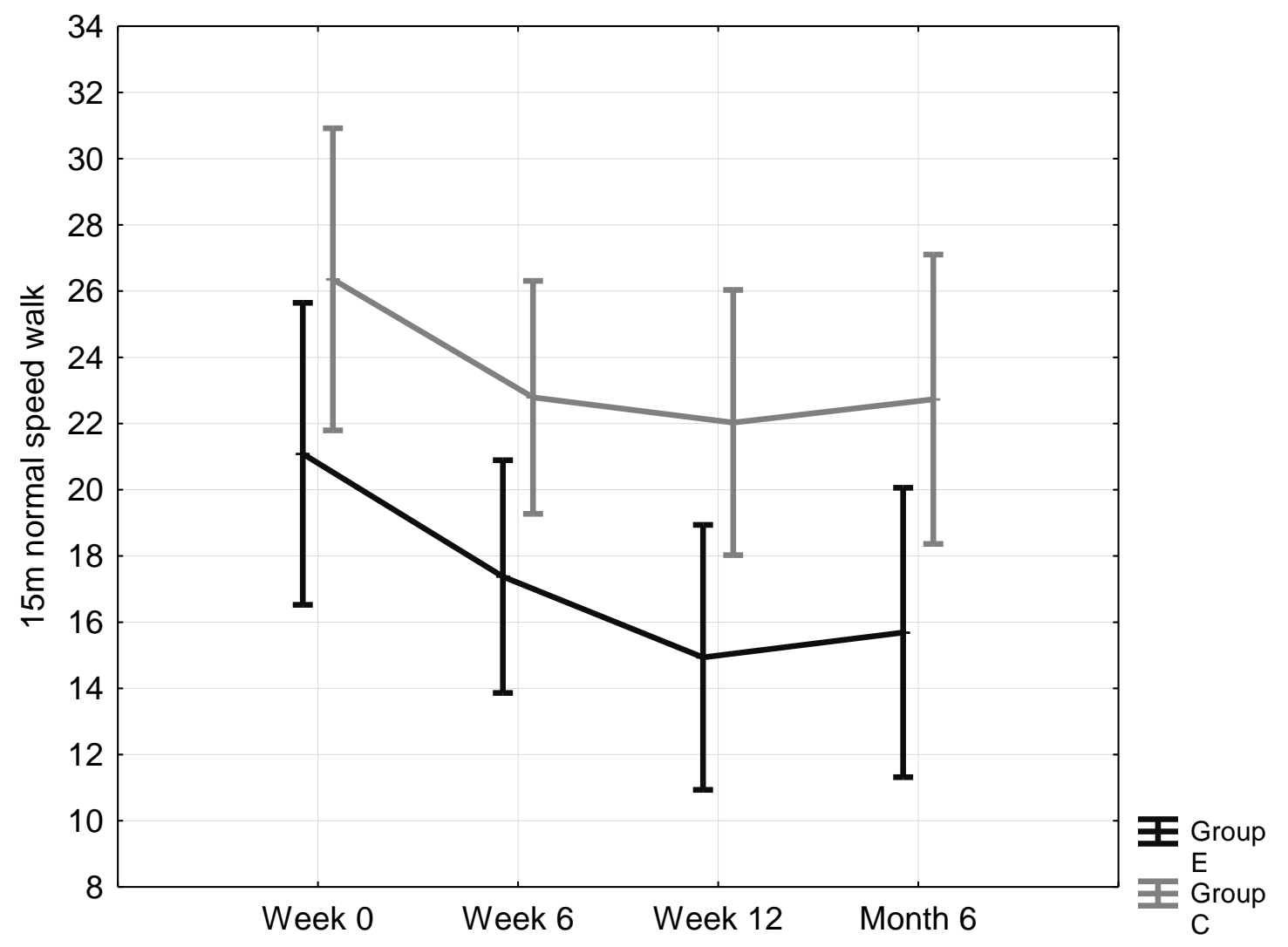

Appendix J-9 Change in OA both hip and knee subgroup $(n=12)$ 


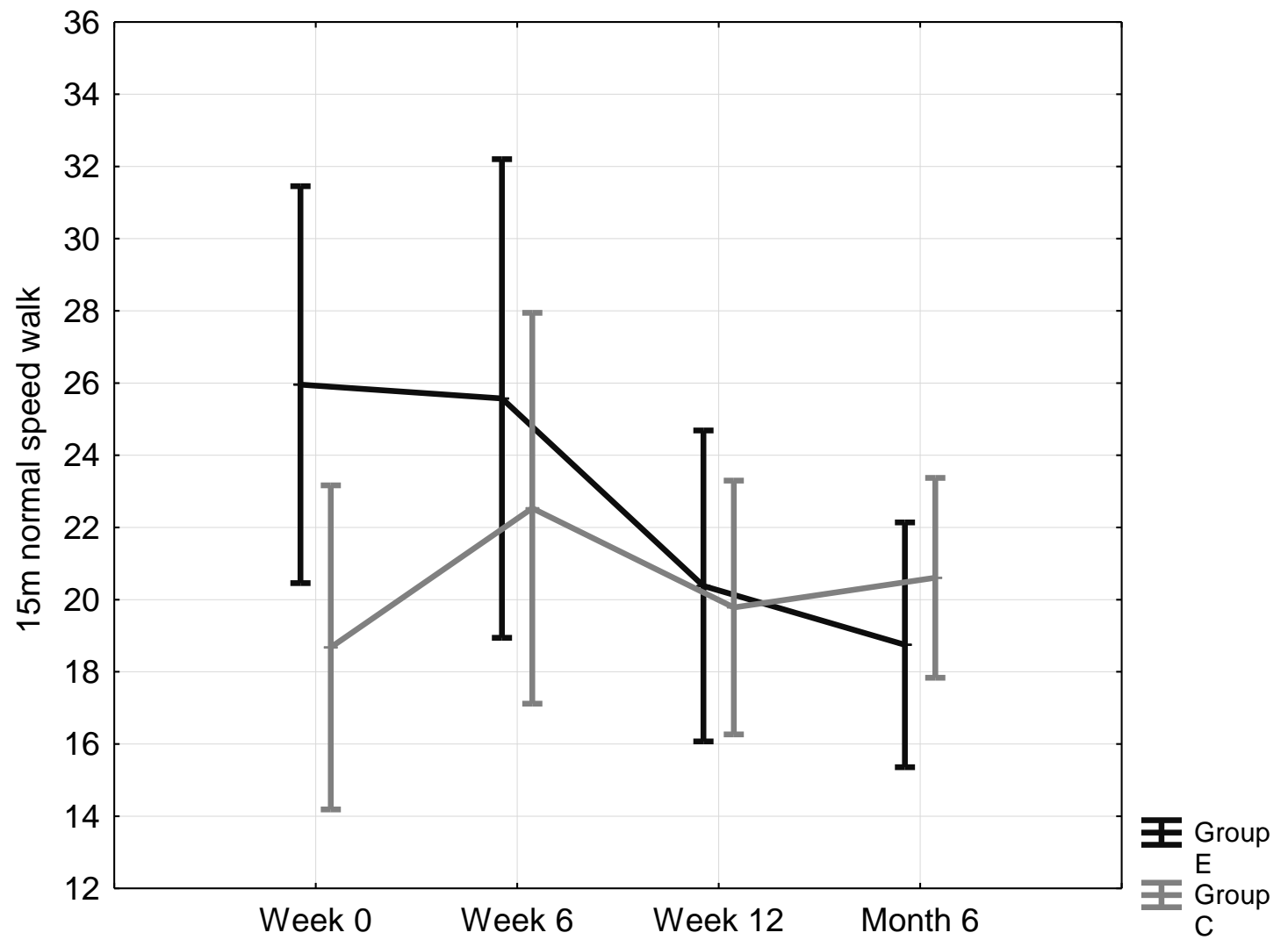

Appendix J-10 Change in OA hip subgroup $(n=11)$ 
Appendix J-4 Six minute walk test $(\mathrm{N}=42)$

\begin{tabular}{cccc} 
& Participants & Experimental Group & Control Group \\
& $\mathbf{N}=\mathbf{4 2}$ & $\mathbf{n}=\mathbf{2 0}$ & $\mathbf{n = 2 2}$ \\
\hline Mean \pm SD & Mean \pm SD & Mean \pm SD \\
Six minute walk test $(\mathbf{m})$ & & $267.80 \pm 123.90$ & $291.5 \pm 96.24$ \\
Baseline & $280.21 \pm 109.55$ & $315.65 \pm 124.81$ & $301.95 \pm 87.26$ \\
Week 6 & $308.48 \pm 105.67$ & $340.85 \pm 145.19 *$ & $286.59 \pm 101.23$ \\
Week 12 & $312.43 \pm 125.58$ & $347.85 \pm 123.55^{*}$ & $290.59 \pm 118.41$ \\
\hline Month 6 & $317.86 \pm 122.85$ & & \\
\hline *Indicates a significant improvement in six minute walk test for the experimental group
\end{tabular}


Appendix J-5 Detailed distance walked in 6 minutes for OA hip, knee, both hip and knee subgroups

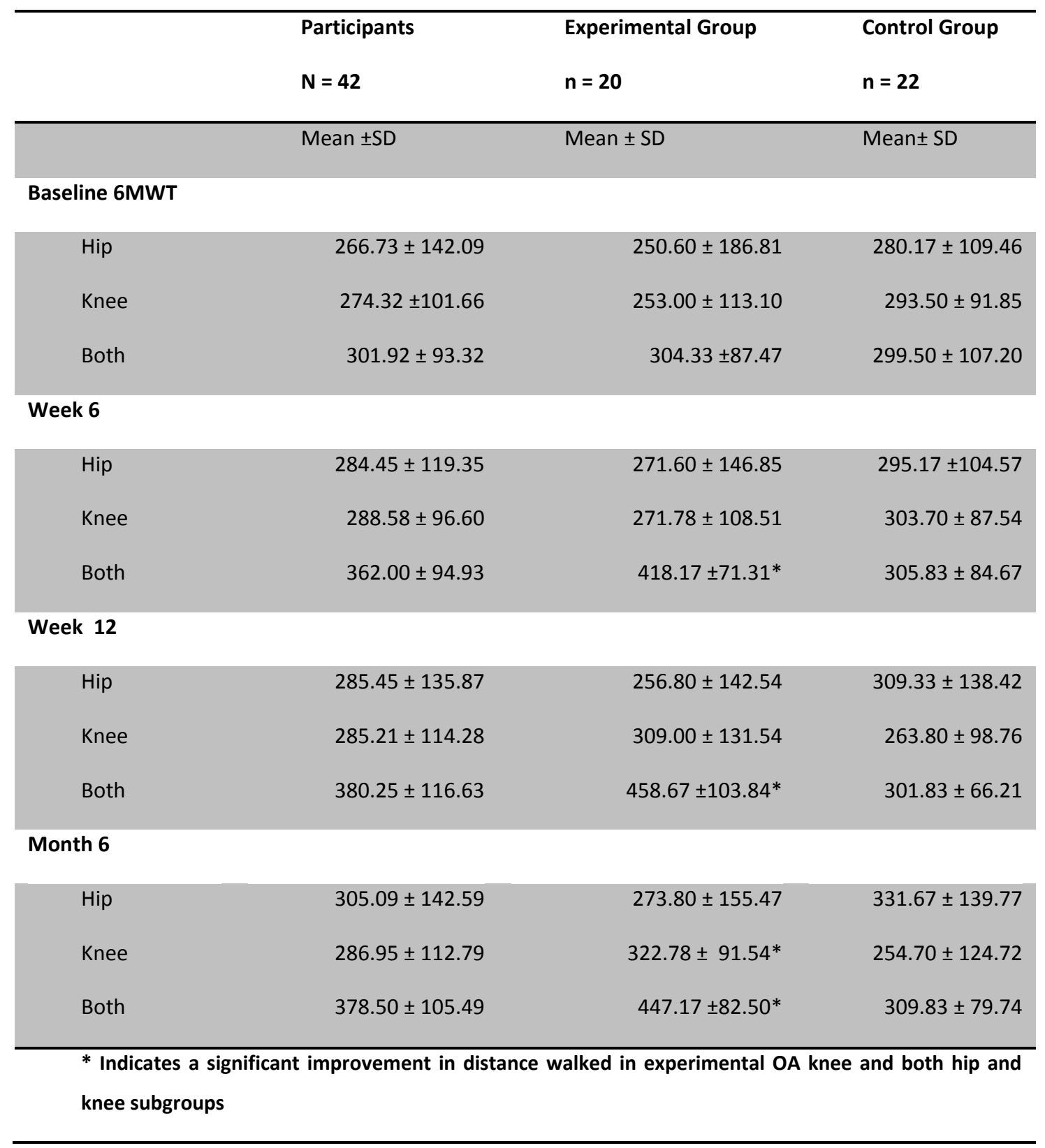




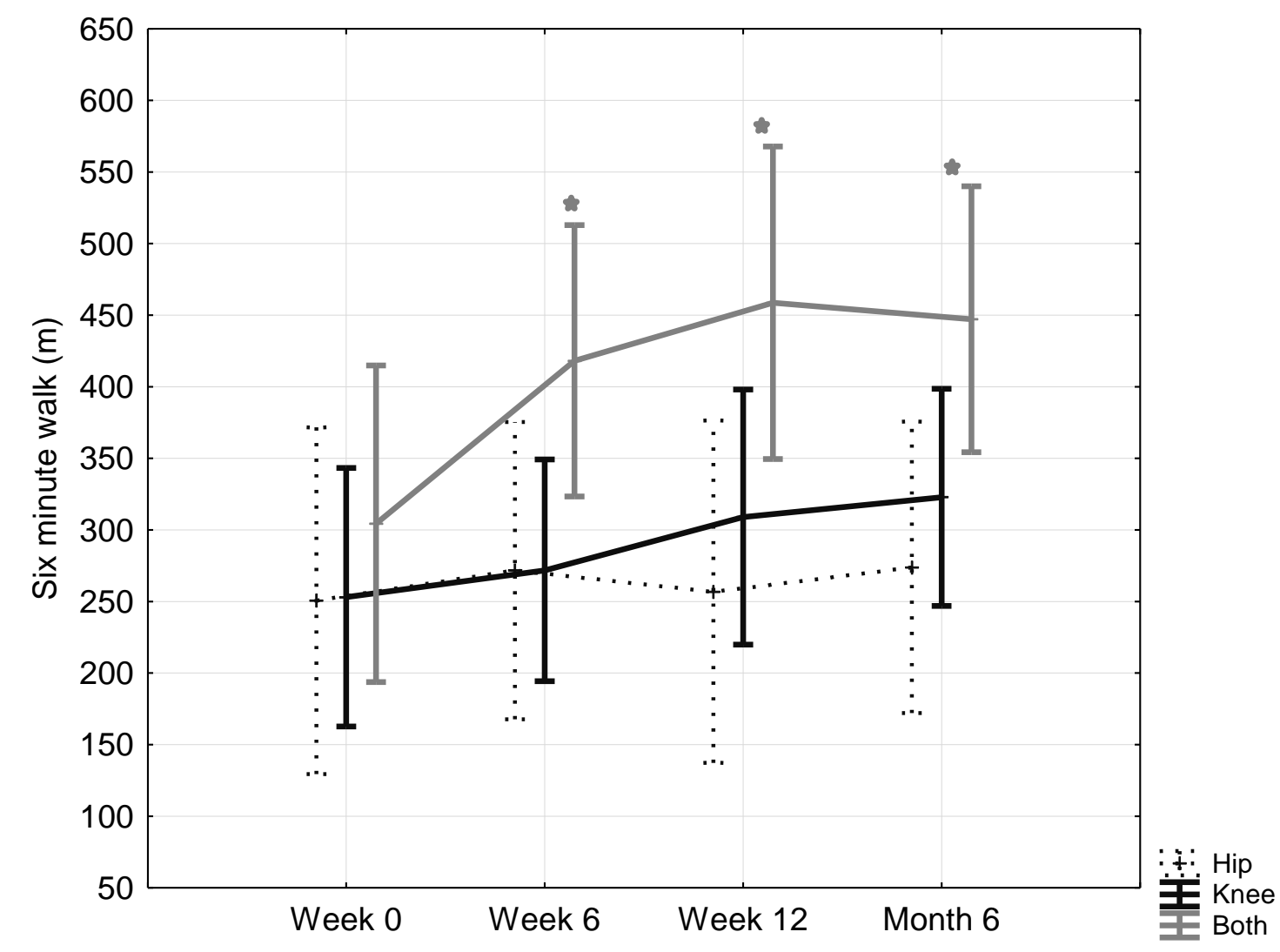

Indicates a significant improvement in the distance walked in 6 minutes for OA hip subgroup at week $6(p=0.01), 12$ and month $6(p<0.01)$

Appendix J-11 Change in OA hip, knee, both hip and knee subgroups in the experimental group $(n=20)$ 


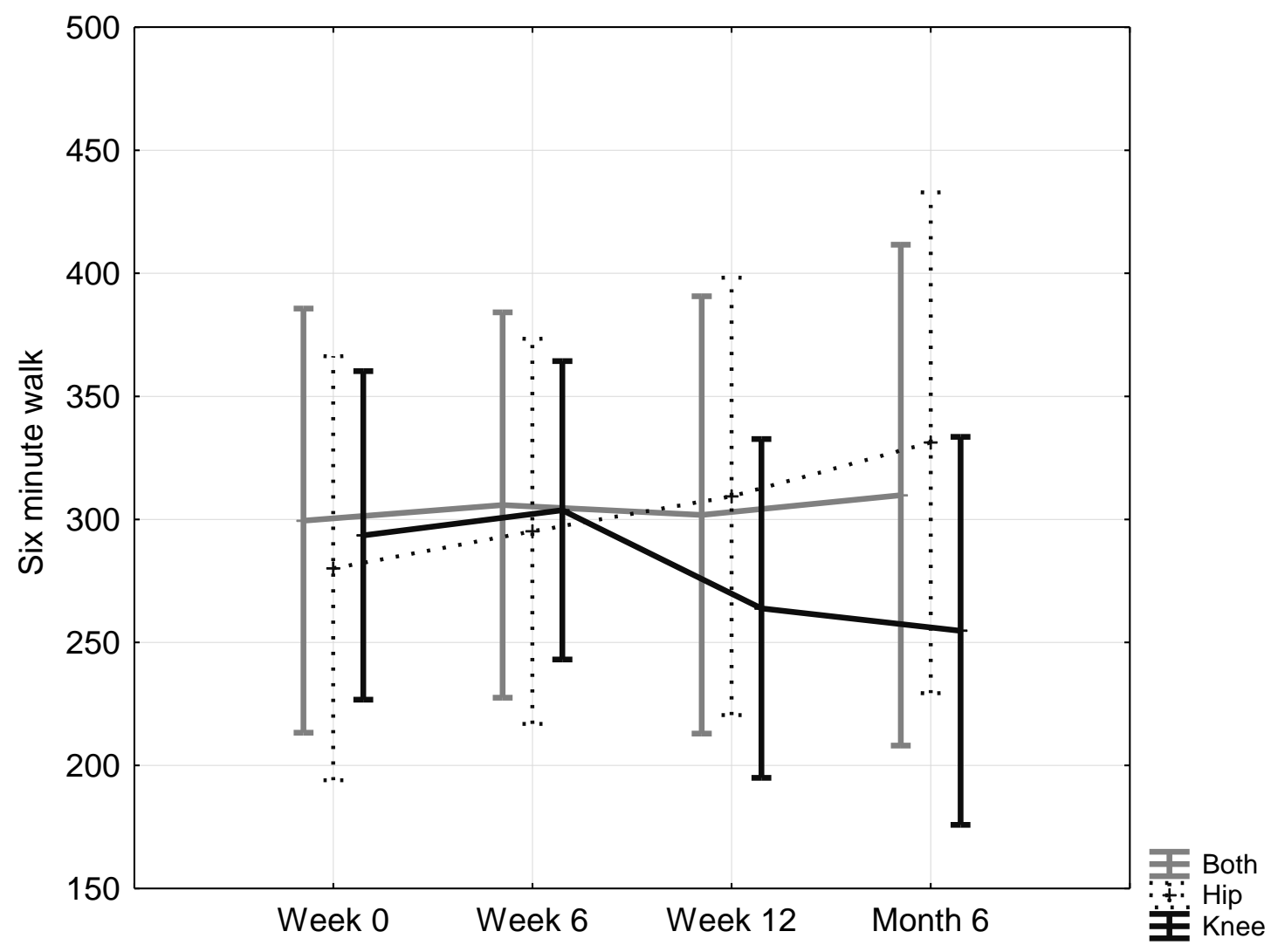

Appendix J-12 Change in OA hip, knee, both hip and knee subgroups in the control group $(n=22)$ 


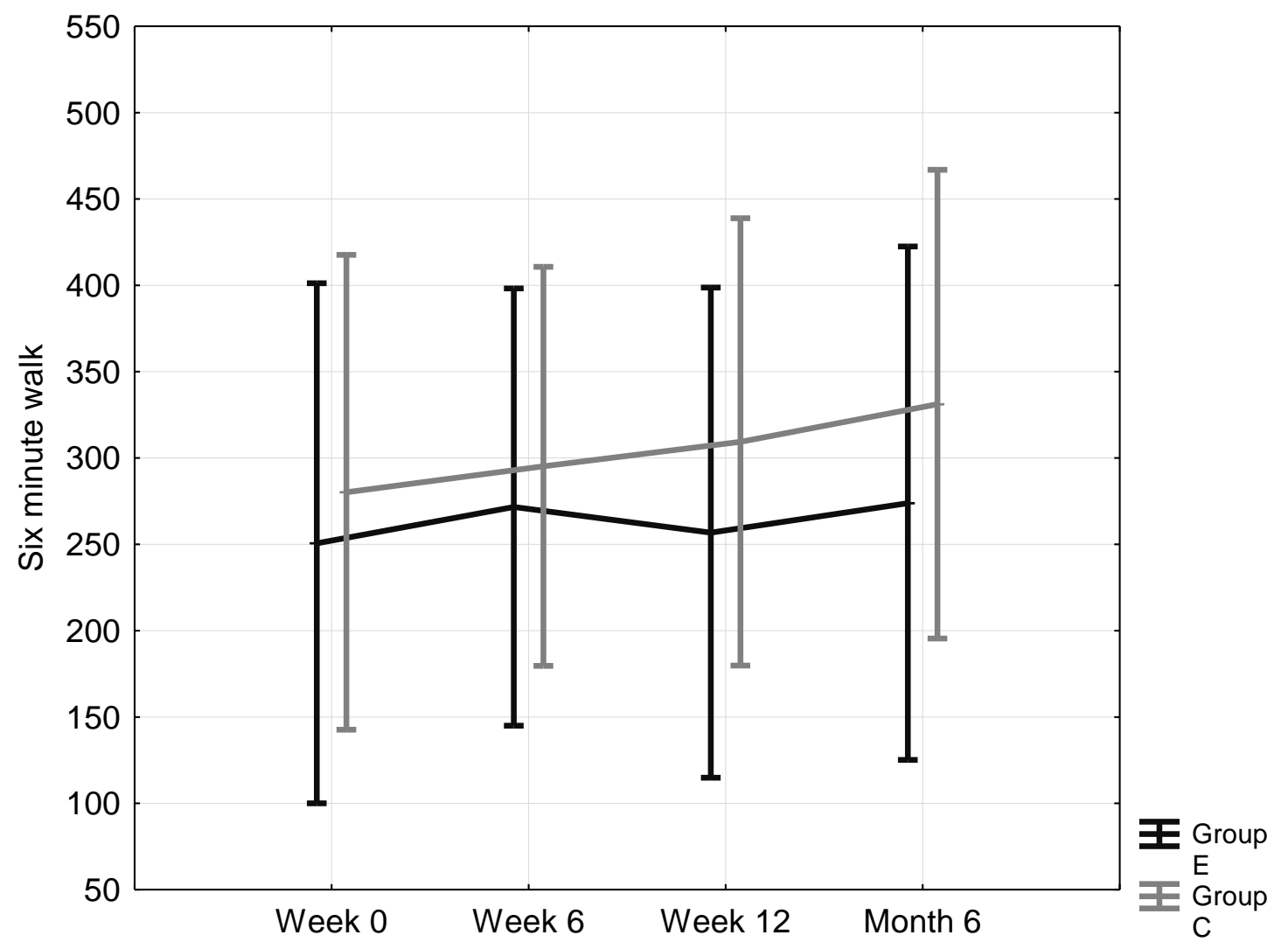

Appendix J-13 Change in OA hip subgroup $(n=11)$ 


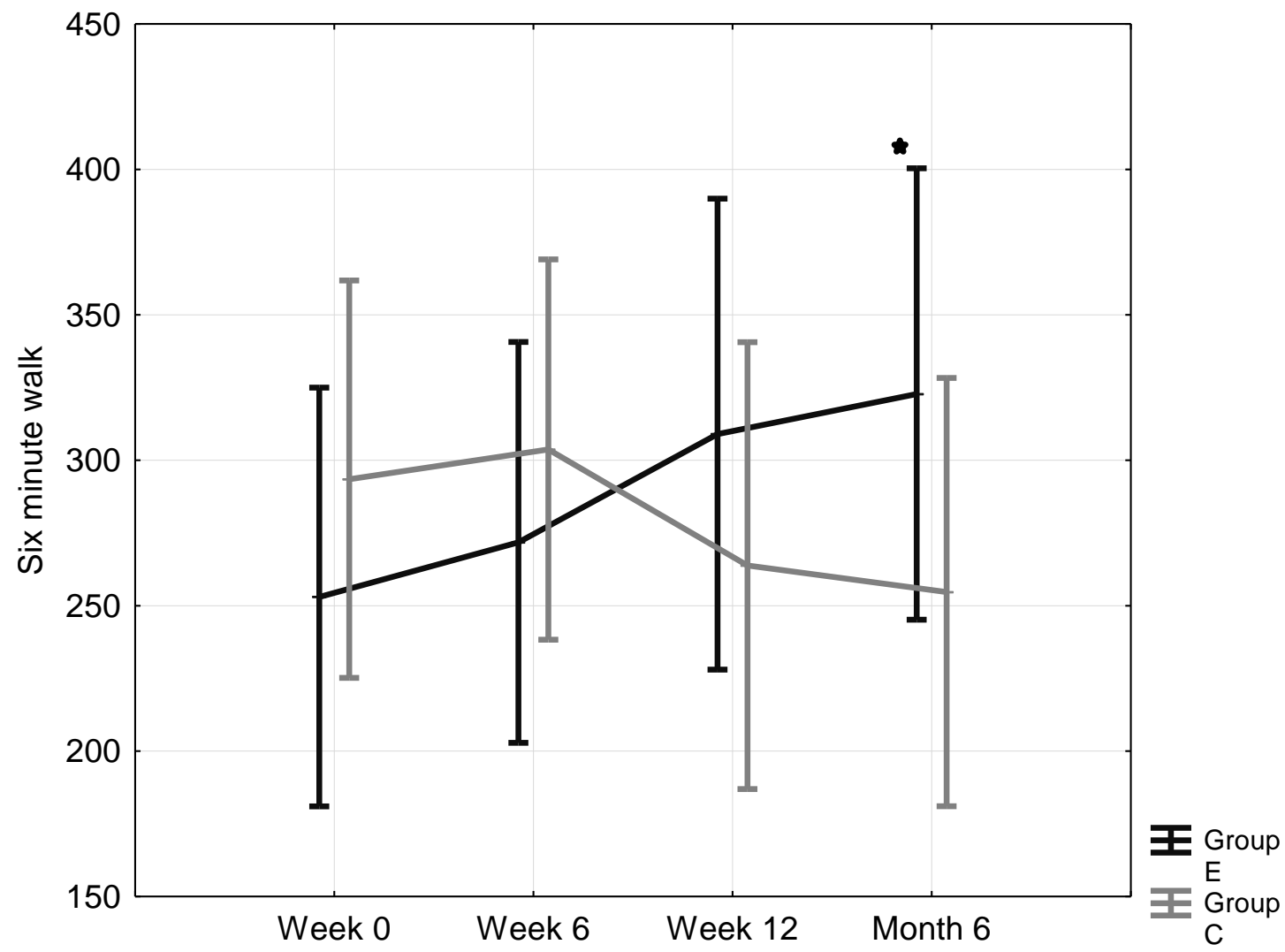

Indicates a significant improvement in the experimental OA knee subgroup at month $6(p=0.01)$

Appendix J-14 Change in OA knee subgroup $(n=19)$ 


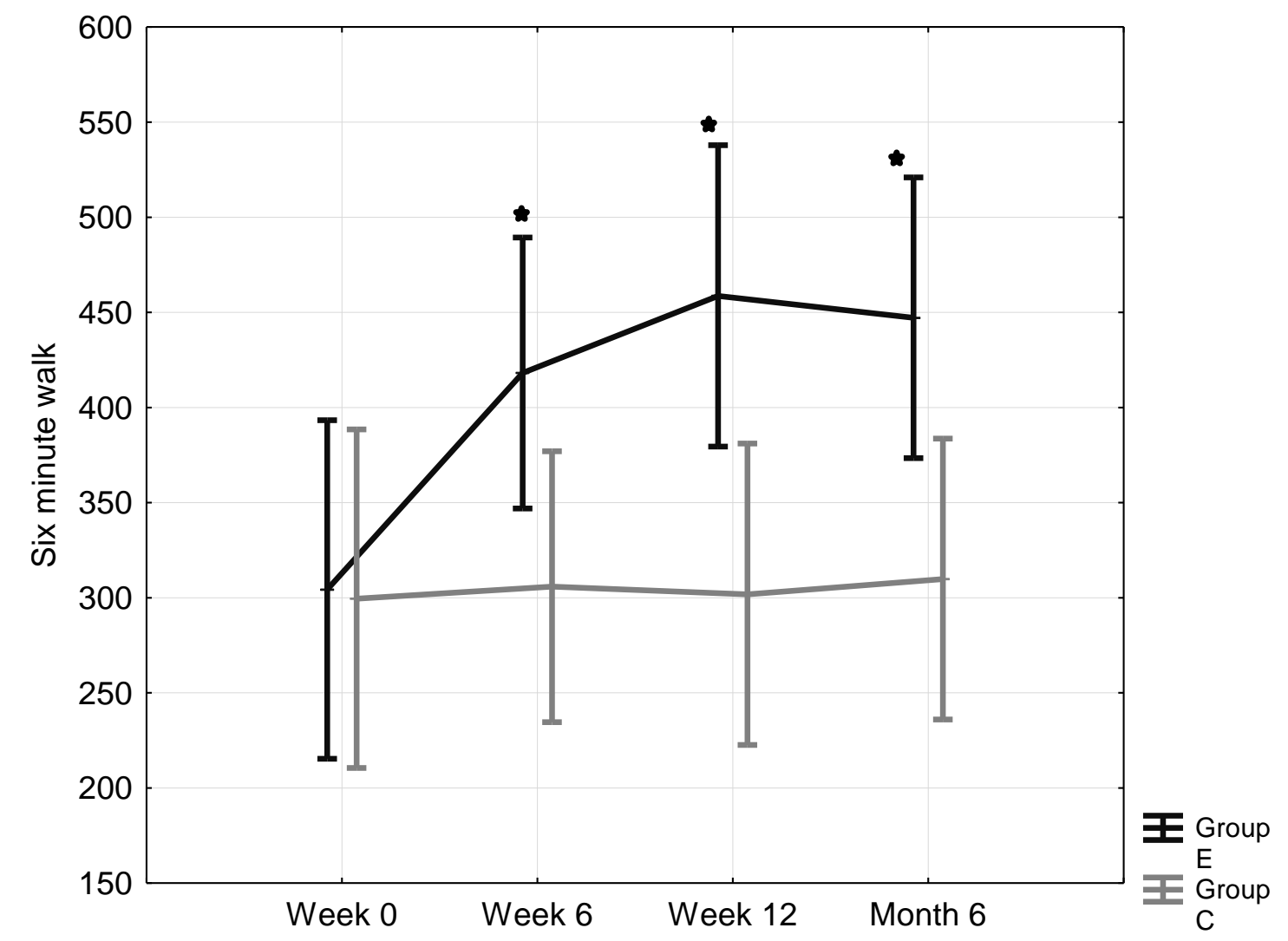

Indicates a significant improvement in experimental OA both hip and knee subgroup at week $6(p=0.02), 12$ and month 6 (p<0.01)

Appendix J-15 Change in OA both hip and knee subgroup $(n=12)$ 
Appendix J-6 Repeated sit to stand test ( $N=42)$

\begin{tabular}{|c|c|c|c|}
\hline & $\begin{array}{l}\text { Participants } \\
\mathrm{N}=42\end{array}$ & $\begin{array}{l}\text { Experimental } \\
\text { Group } \\
\mathrm{n}=\mathbf{2 0}\end{array}$ & $\begin{array}{l}\text { Control Group } \\
n=22\end{array}$ \\
\hline & Mean \pm SD & Mean \pm SD & Mean \pm SD \\
\hline \multicolumn{4}{|c|}{ Repeated sit stand (sec) } \\
\hline Baseline & $9.82 \pm 3.96$ & $10.05 \pm 4.77$ & $9.61 \pm 3.15$ \\
\hline Week 6 & $7.80 \pm 2.82$ & $6.45 \pm 2.18^{*}$ & $9.04 \pm 2.80$ \\
\hline Week 12 & $6.89 \pm 2.23$ & $6.13 \pm 1.85^{*}$ & $7.57 \pm 2.37^{*}$ \\
\hline Month 6 & $6.45 \pm 2.34$ & $5.66 \pm 1.63^{*}$ & $7.17 \pm 2.67 *$ \\
\hline
\end{tabular}


Appendix J-7 Detailed time taken for repeated sit-stand for OA hip, knee and both hip and knee subgroups

\begin{tabular}{|c|c|c|c|}
\hline & $\begin{array}{l}\text { Participants } \\
\mathbf{N}=\mathbf{4 2}\end{array}$ & $\begin{array}{l}\text { Experimental } \\
\text { Group } \\
\mathrm{n}=\mathbf{2 0}\end{array}$ & $\begin{array}{l}\text { Control } \\
\text { Group } \\
\mathrm{n}=\mathbf{2 2}\end{array}$ \\
\hline & Mean \pm SD & Mean \pm SD & Mean \pm SD \\
\hline \multicolumn{4}{|c|}{ Baseline repeated sitstand } \\
\hline Hip & $10.03 \pm 3.03$ & $11.31 \pm 2.91$ & $8.96 \pm 2.94$ \\
\hline Knee & $9.21 \pm 4.53$ & $8.85 \pm 5.38$ & $9.54 \pm 3.89$ \\
\hline Both & $6.05 \pm 3.00$ & $10.80 \pm 5.32$ & $10.36 \pm 2.19$ \\
\hline \multicolumn{4}{|l|}{ Week 6} \\
\hline Hip & $7.77 \pm 2.28$ & $7.01 \pm 1.46^{*}$ & $8.39 \pm 2.78$ \\
\hline Knee & $7.35 \pm 2.52$ & $5.95 \pm 1.24$ & $8.61 \pm 2.76$ \\
\hline Both & $8.56 \pm 3.67$ & $6.72 \pm 3.61$ & $10.40 \pm 2.91$ \\
\hline \multicolumn{4}{|l|}{ Week 12} \\
\hline Hip & $7.34 \pm 2.32$ & $6.86 \pm 1.68^{*}$ & $7.74 \pm 2.85$ \\
\hline Knee & $6.63 \pm 1.58$ & $6.21 \pm 2.00$ & $7.01 \pm 1.05$ \\
\hline Both & $6.88 \pm 3.05$ & $5.41 \pm 1.80$ & $8.34 \pm 3.47$ \\
\hline \multicolumn{4}{|l|}{ Month 6} \\
\hline Hip & $6.68 \pm 2.06$ & $7.05 \pm 1.49^{*}$ & $6.62 \pm 2.57$ \\
\hline Knee & $6.50 \pm 2.11$ & $5.80 \pm 1.45$ & $7.13 \pm 2.46$ \\
\hline Both & $6.05 \pm 3.00$ & $4.31 \pm 0.90$ & $7.80 \pm 3.41$ \\
\hline \multicolumn{4}{|c|}{$\begin{array}{l}\text { * Indicates a significant improvement in time taken for repeated sit- stand in experimental OA hip } \\
\text { subgroup }\end{array}$} \\
\hline
\end{tabular}




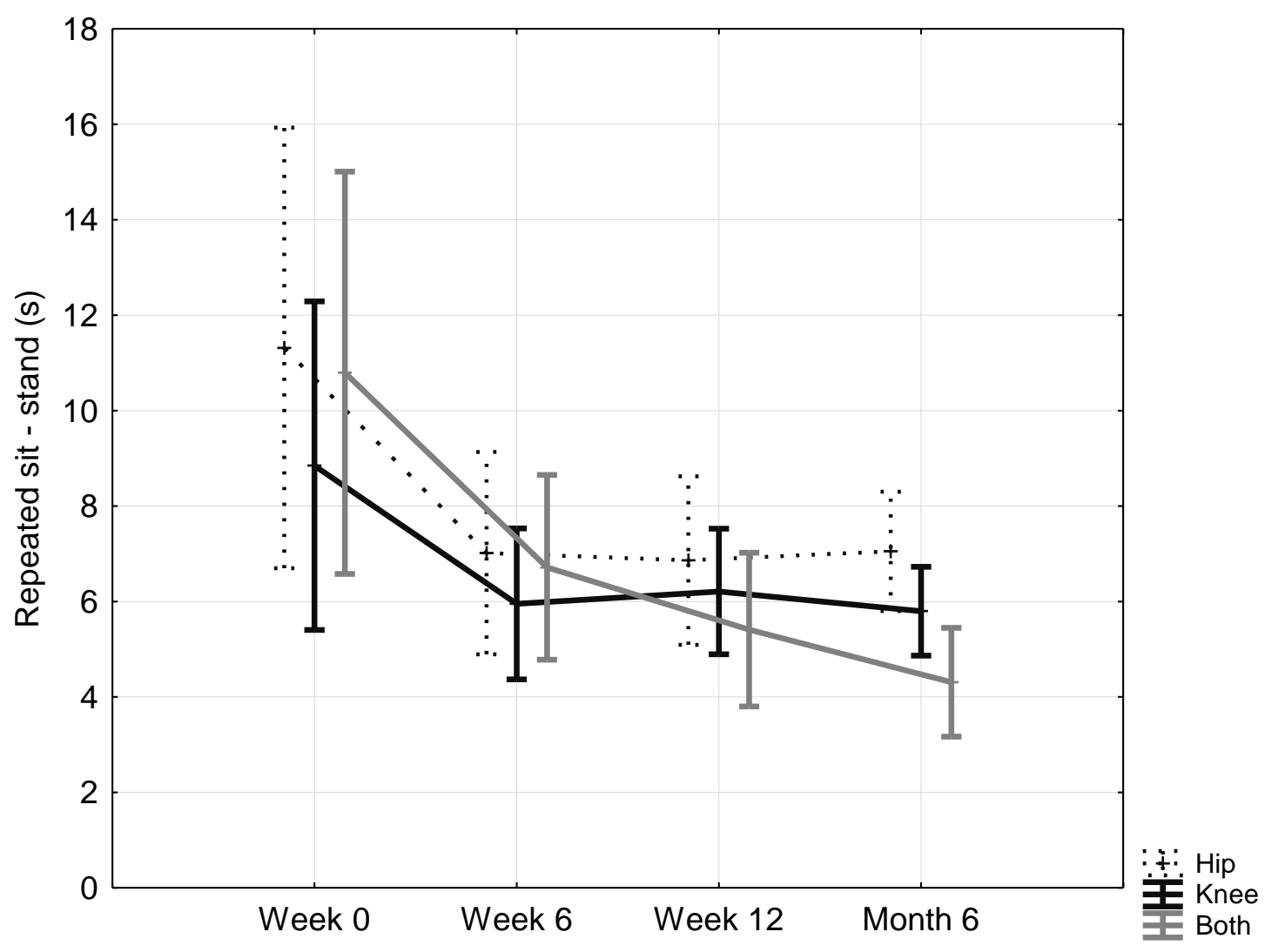

Appendix J-16 Change in OA hip, knee, both hip and knee subgroups in the experimental group $(n=20)$ 


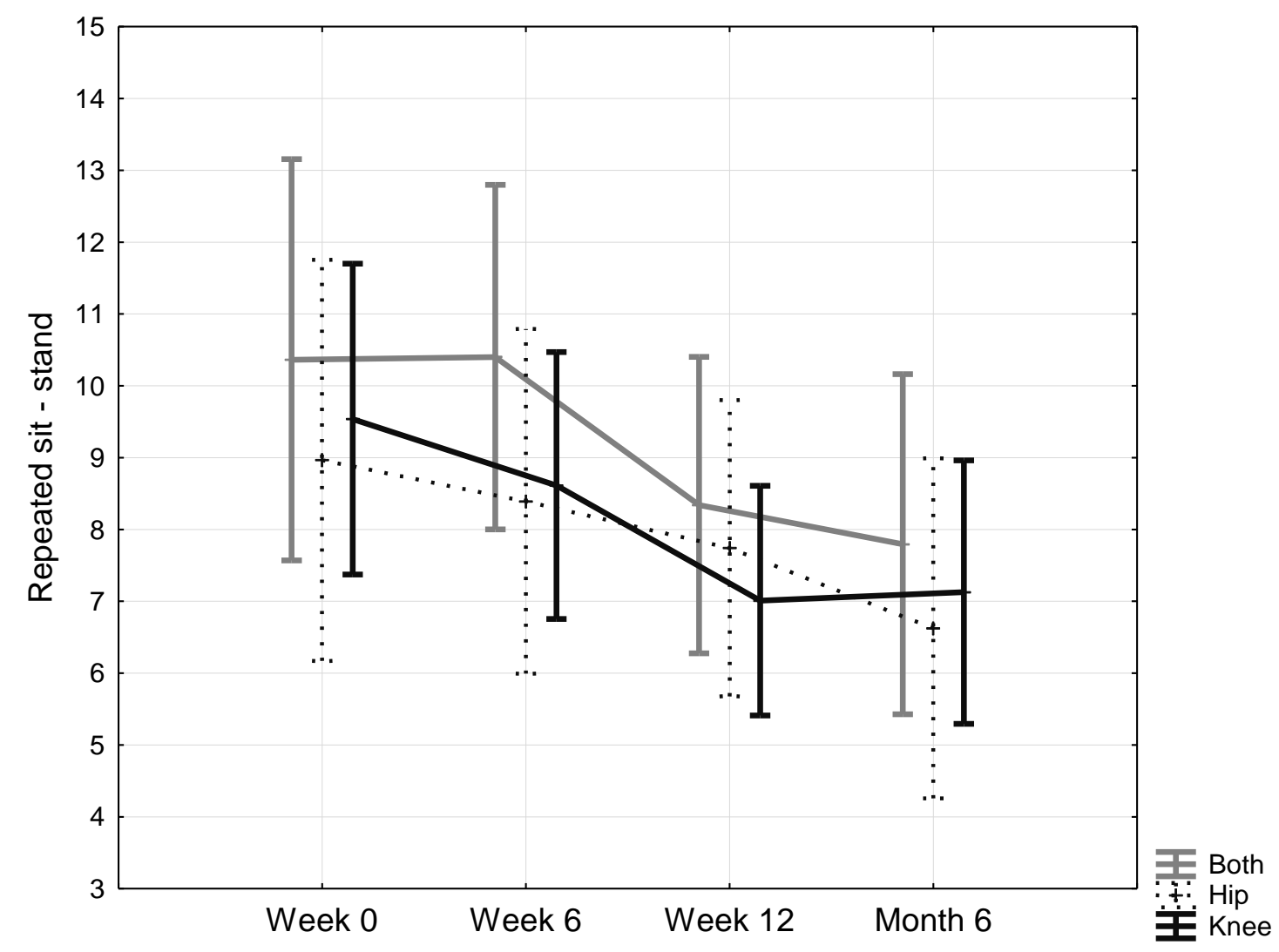

Appendix J-17 Change in OA hip, knee, both hip and knee subgroups in the control group $(n=22)$ 


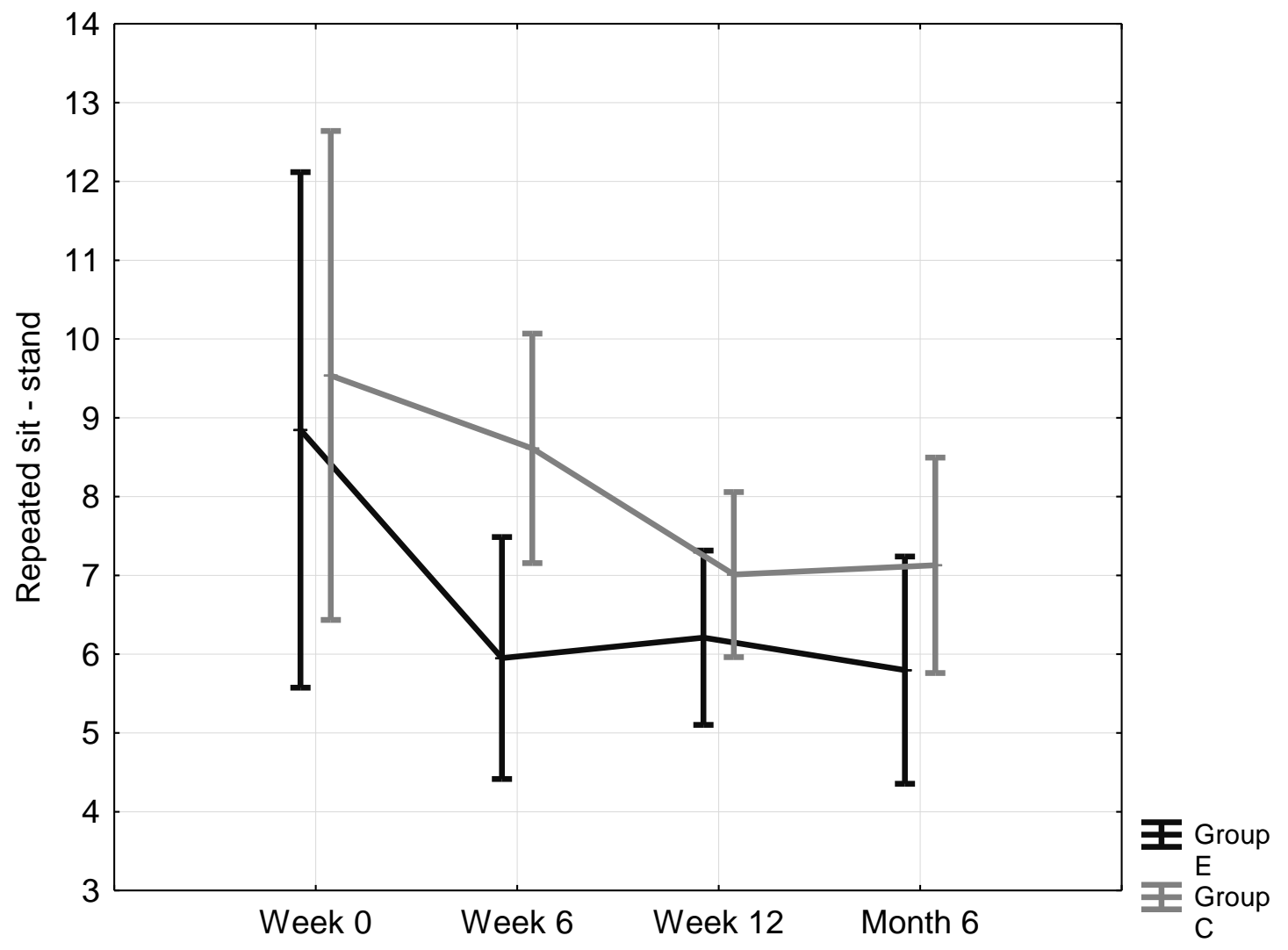

Appendix J-18 Change in OA knee subgroup $(n=19)$ 


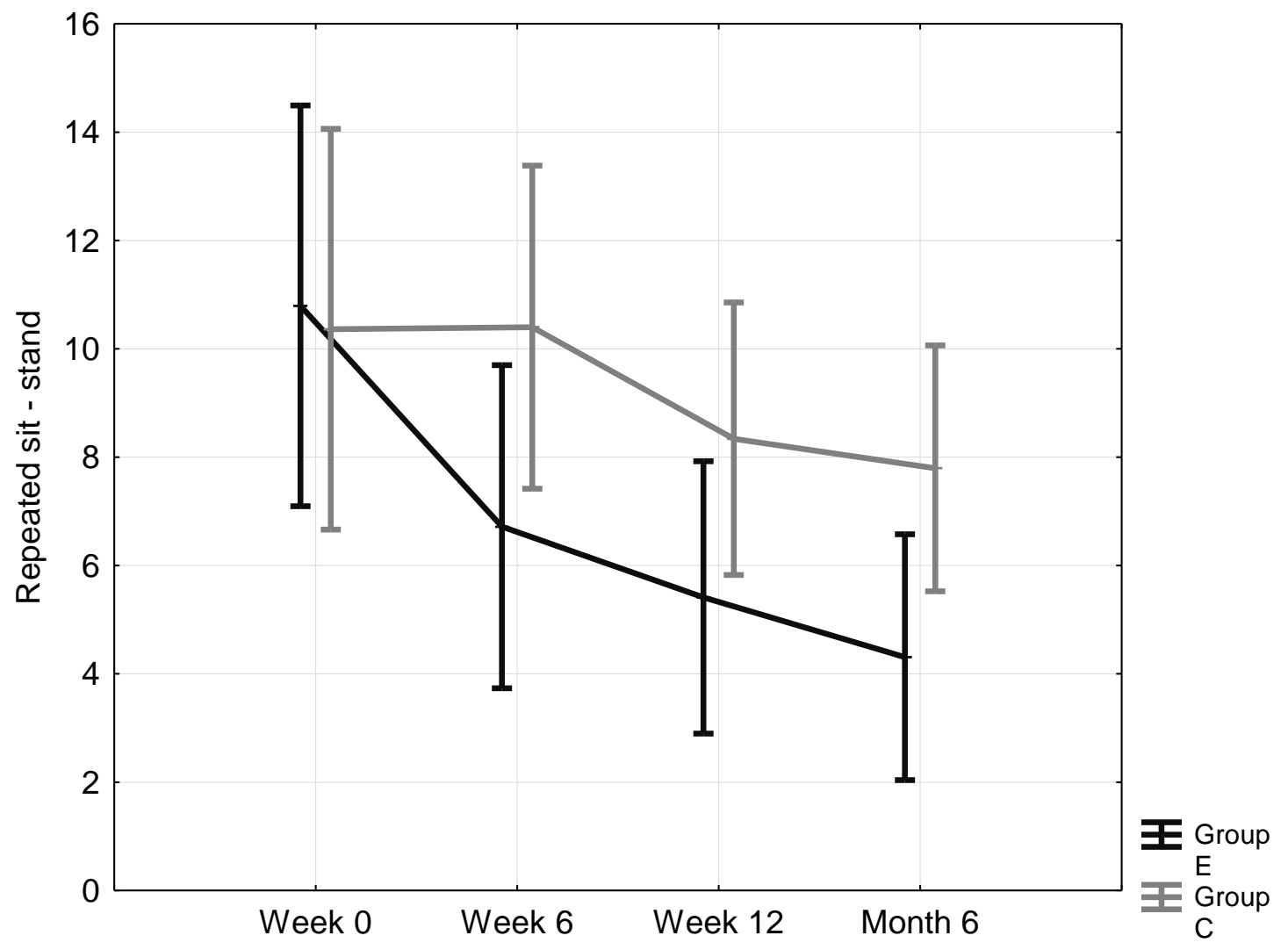

Appendix J-19 Change in OA both hip and knee subgroup $(n=12)$ 


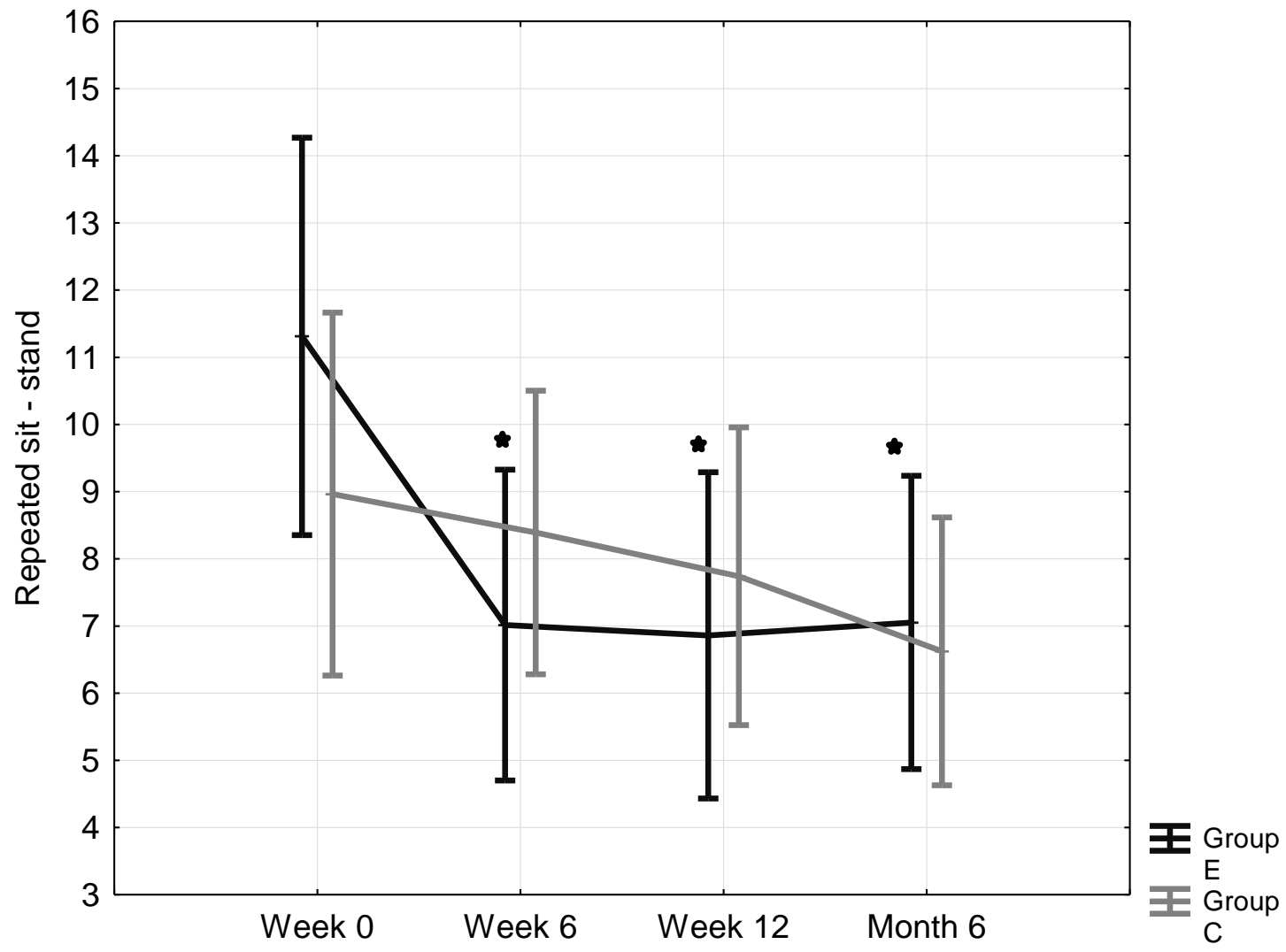

- Indicates a significant improvement in experimental OA hip subgroup at week 6, 12 and month 6 (p<0.01)

Appendix J-20 Change in OA both hip and knee subgroup $(n=12)$ 
Physical performance task battery reach tests - forwards and upwards

Appendix J-8 Forward and upward reach test $(\mathrm{N}=42)$

\begin{tabular}{|c|c|c|c|}
\hline & $\begin{array}{l}\text { Participants } \\
\mathrm{N}=42\end{array}$ & $\begin{array}{l}\text { Experimental } \\
\text { Group } \\
n=20\end{array}$ & $\begin{array}{l}\text { Control Group } \\
n=22\end{array}$ \\
\hline & Mean \pm SD & Mean \pm SD & Mean \pm SD \\
\hline \multicolumn{4}{|c|}{ Forward reach $(\mathrm{cm})$} \\
\hline Baseline & $83.69 \pm 16.51$ & $81.53 \pm 21.74$ & $85.66 \pm 9.79$ \\
\hline Week 6 & $85.27 \pm 16.46$ & $84.58 \pm 21.78$ & $85.91 \pm 9.95$ \\
\hline Week 12 & $86.01 \pm 16.61$ & $86.03 \pm 22.08$ & $86.00 \pm 9.87$ \\
\hline Month 6 & $87.27 \pm 17.45$ & $87.18 \pm 23.14$ & $87.36 \pm 10.5$ \\
\hline \multicolumn{4}{|c|}{ Upward reach (sec) } \\
\hline Baseline & $8.93 \pm 3.53$ & $8.73 \pm 3.72$ & $9.09 \pm 3.46$ \\
\hline Week 6 & $8.72 \pm 3.57$ & $7.48 \pm 3.06$ & $9.90 \pm 3.71$ \\
\hline Week 12 & $7.78 \pm 2.74$ & $7.35 \pm 3.03$ & $8.15 \pm 2.47$ \\
\hline Month 6 & $7.37 \pm 2.42$ & $6.78 \pm 2.04$ & $7.88 \pm 2.64$ \\
\hline
\end{tabular}


Appendix J-9 Ability to don a sock ( $\mathrm{N}=42$ )

\begin{tabular}{|c|c|c|c|c|}
\hline & $\begin{array}{l}\text { Participants } \\
\mathrm{N}=42\end{array}$ & $\begin{array}{l}\text { Exp Group } \\
n=20\end{array}$ & $\begin{array}{l}\text { Cont Group } \\
n=22\end{array}$ & \\
\hline & $\begin{array}{l}\text { Number } \\
(\%)\end{array}$ & $\begin{array}{l}\text { Number } \\
(\%)\end{array}$ & $\begin{array}{l}\text { Number } \\
(\%)\end{array}$ & Significance test \\
\hline \multicolumn{5}{|l|}{ Sock test } \\
\hline Baseline & & & & $\chi^{2}=0.01 ; p=0.93$ \\
\hline able & $15(35.7)$ & $7(35)$ & $8(36.4)$ & \\
\hline unable & $27(64.3)$ & $13(65)$ & $14(63.6)$ & \\
\hline Week 6 & & & & $\chi^{2}=2.96 ; p=0.09$ \\
\hline able & $33(78.6)$ & $18(90)$ & $15(68.2)$ & \\
\hline unable & $9(21.4)$ & $2(10)$ & $7(31.8)$ & \\
\hline Week 12 & & & & $\chi^{2}=0.41 ; p=0.52$ \\
\hline able & $34(80.96)$ & $17(85)$ & $17(77.3)$ & \\
\hline unable & $8(19.04)$ & $3(15)$ & $5(22.7)$ & \\
\hline Month 6 & & & & $\chi^{2}=0.31 ; p=0.58$ \\
\hline able & $32(76.2)$ & $16(80)$ & $16(72.7)$ & \\
\hline unable & $10(33.8)$ & $4(20)$ & $6(27.3)$ & \\
\hline
\end{tabular}


Appendix J-10 Time taken to don a sock on right and left foot $(\mathrm{N}=42)$

\begin{tabular}{|c|c|c|c|c|}
\hline & $\begin{array}{l}\text { Participants } \\
\mathrm{N}=42\end{array}$ & $\begin{array}{l}\text { Experimental } \\
\text { Group } \\
\mathrm{n}=\mathbf{2 0}\end{array}$ & $\begin{array}{l}\text { Control } \\
\text { Group } \\
\mathrm{n}=\mathbf{2 2}\end{array}$ & \\
\hline & Mean \pm SD & Mean \pm SD & Mean \pm SD & $\begin{array}{l}\text { Significance } \\
\text { test }\end{array}$ \\
\hline $\begin{array}{l}\text { Able: Time right } \\
\text { sock (sec) }\end{array}$ & & & & $\begin{array}{l}F(3,84)=1.13 \\
p=0.34\end{array}$ \\
\hline Baseline & $16.73 \pm 20.41$ & $12.52 \pm 17.49$ & $20.44 \pm 2.55$ & \\
\hline Week 6 & $14.48 \pm 17.22$ & $10.00 \pm 8.09$ & $18.96 \pm 22.41$ & \\
\hline Week 12 & $11.08 \pm 8.94$ & $10.20 \pm 10.97$ & $11.92 \pm 6.69$ & \\
\hline Month 6 & $8.59 \pm 5.66$ & $7.06 \pm 5.61$ & $10.22 \pm 5.40$ & \\
\hline $\begin{array}{l}\text { Able: Time left } \\
\text { sock (sec) }\end{array}$ & & & & $\begin{array}{l}F(3,78)=3.60 \\
p=0.02 *\end{array}$ \\
\hline Baseline & $14.64 \pm 11.14$ & $14.71 \pm 12.61$ & $14.56 \pm 9.90$ & \\
\hline Week 6 & $13.11 \pm 901$ & $9.43 \pm 5.74 *$ & $17.03 \pm 0.32$ & $p=0.04$ \\
\hline Week 12 & $11.76 \pm 9.18$ & $10.35 \pm 9.16$ & $13.18 \pm 9.25$ & \\
\hline Month 6 & $8.48 \pm 5.19$ & $6.27 \pm 3.54^{*}$ & $10.58 \pm 5.70$ & $p<0.01$ \\
\hline
\end{tabular}




\section{Appendix K: Full disability results}

HAQ and HAQ pain VAS scores

Appendix K-1 HAQ disability and pain VAS scores $(\mathrm{N}=42)$

\begin{tabular}{|c|c|c|c|}
\hline & Participants & Experimental Group & Control Groun \\
\hline & $N=42$ & $n=20$ & $n=22$ \\
\hline & Mean \pm SD & Mean \pm SD & Mean \pm SD \\
\hline \multicolumn{4}{|l|}{ HAQ score } \\
\hline Baseline & $1.56 \pm 0.55$ & $1.61 \pm 0.59$ & $1.51 \pm 0.52$ \\
\hline Week 6 & $1.17 \pm 0.60$ & $0.92 \pm 0.53^{*}$ & $1.39 \pm 0.59$ \\
\hline Week 12 & $1.21 \pm 0.65$ & $1.07 \pm 0.63 *$ & $1.33 \pm 0.65$ \\
\hline Month 6 & $1.23 \pm 0.73$ & $1.01 \pm 0.66^{*}$ & $1.43 \pm 0.75$ \\
\hline \multicolumn{4}{|l|}{ HAQ VAS } \\
\hline Baseline & $69.02 \pm 23.40$ & $73.35 \pm 25.45$ & $65.09 \pm 21.18$ \\
\hline Week 6 & $56.67 \pm 32.32$ & $44.30 \pm 33.70 *$ & $67.91 \pm 27.10$ \\
\hline Week 12 & $58.36 \pm 32.41$ & $53.35 \pm 34.58 *$ & $62.91 \pm 30.38$ \\
\hline Month 6 & $59.90 \pm 31.09$ & $48.30 \pm 33.95^{*}$ & $70.45 \pm 24.47$ \\
\hline
\end{tabular}

*Indicates a significant improvement in the HAQ disability and VAS score in the experimental group 

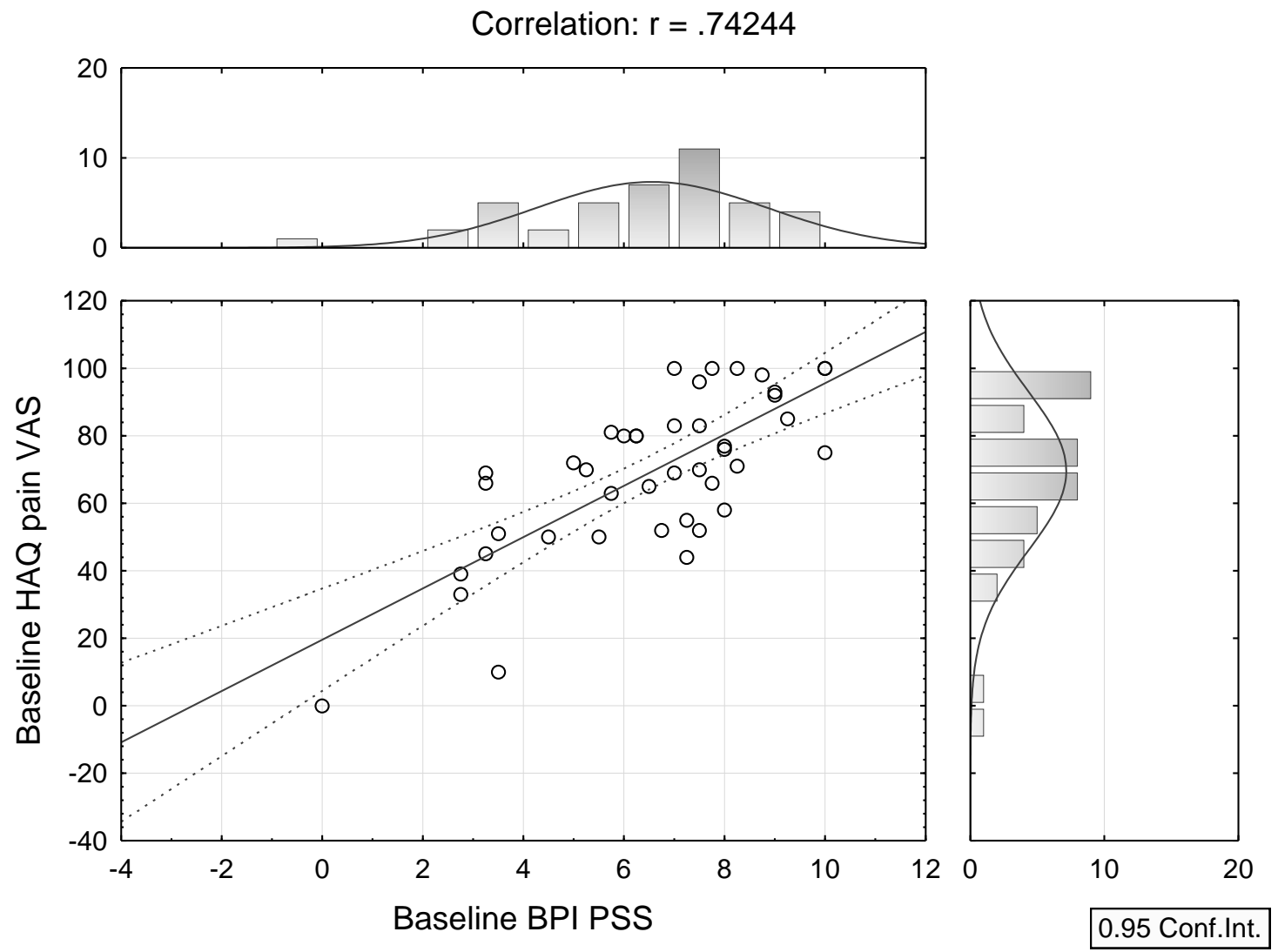

Appendix K-1 Baseline correlation between BPI and HAQ pain VAS $(N=42)$ 


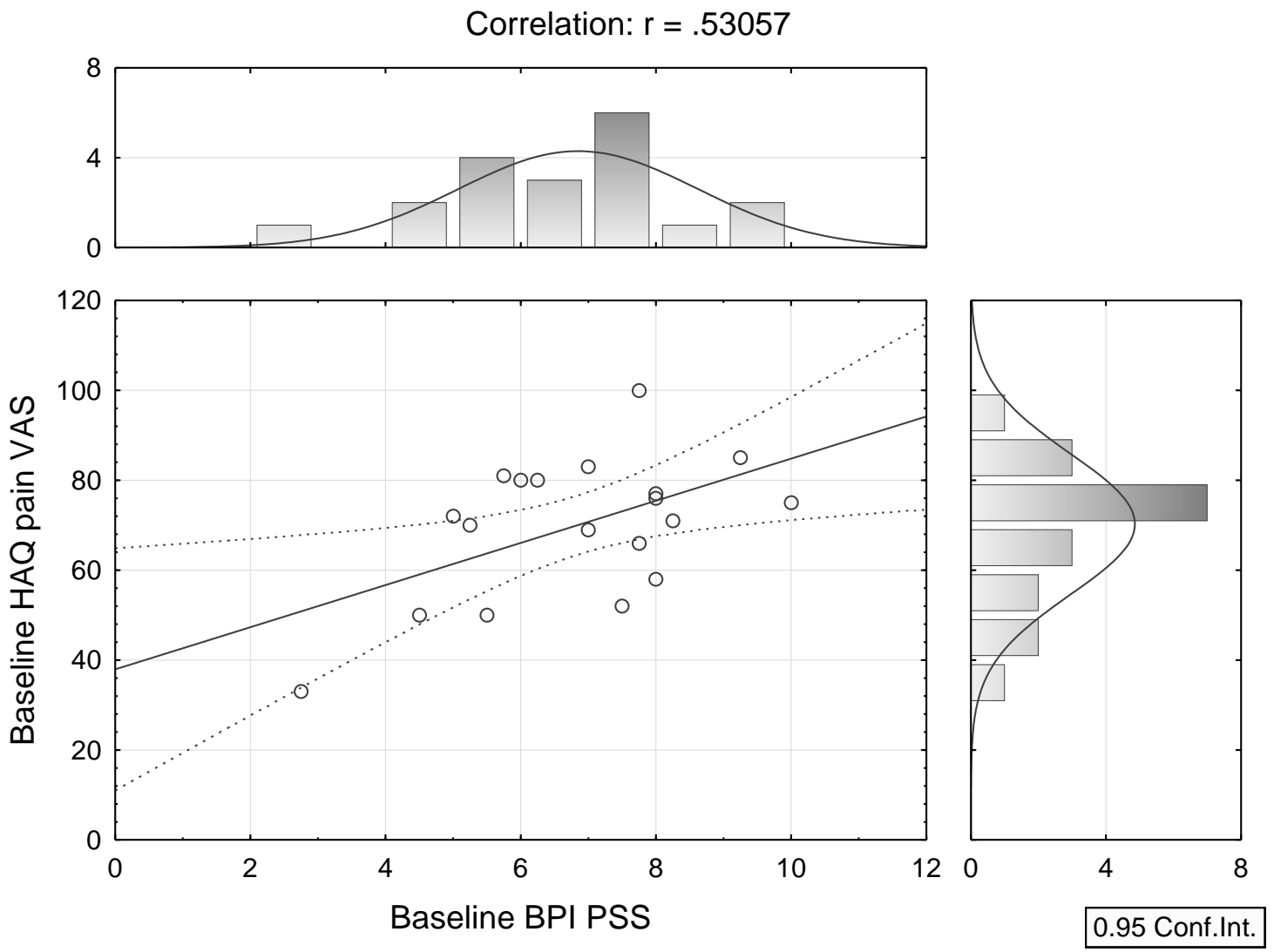

Appendix K-2 Baseline BPI and HAQ pain VAS for knee OA subgroup $(n=19)$ 
Appendix K-2 Detailed HAQ for OA hip, knee, both hip and knee subgroups ( $N=42$ )

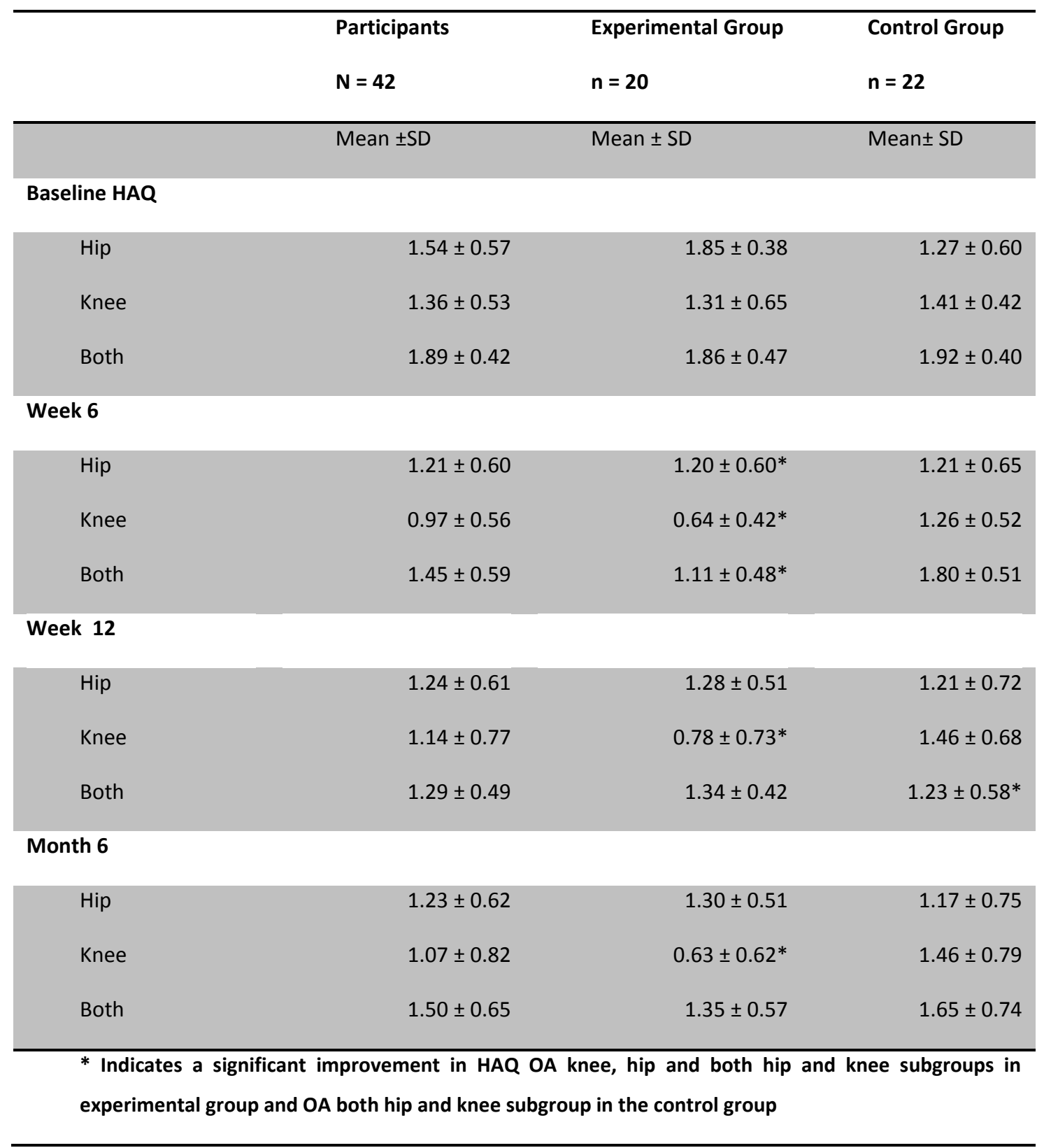




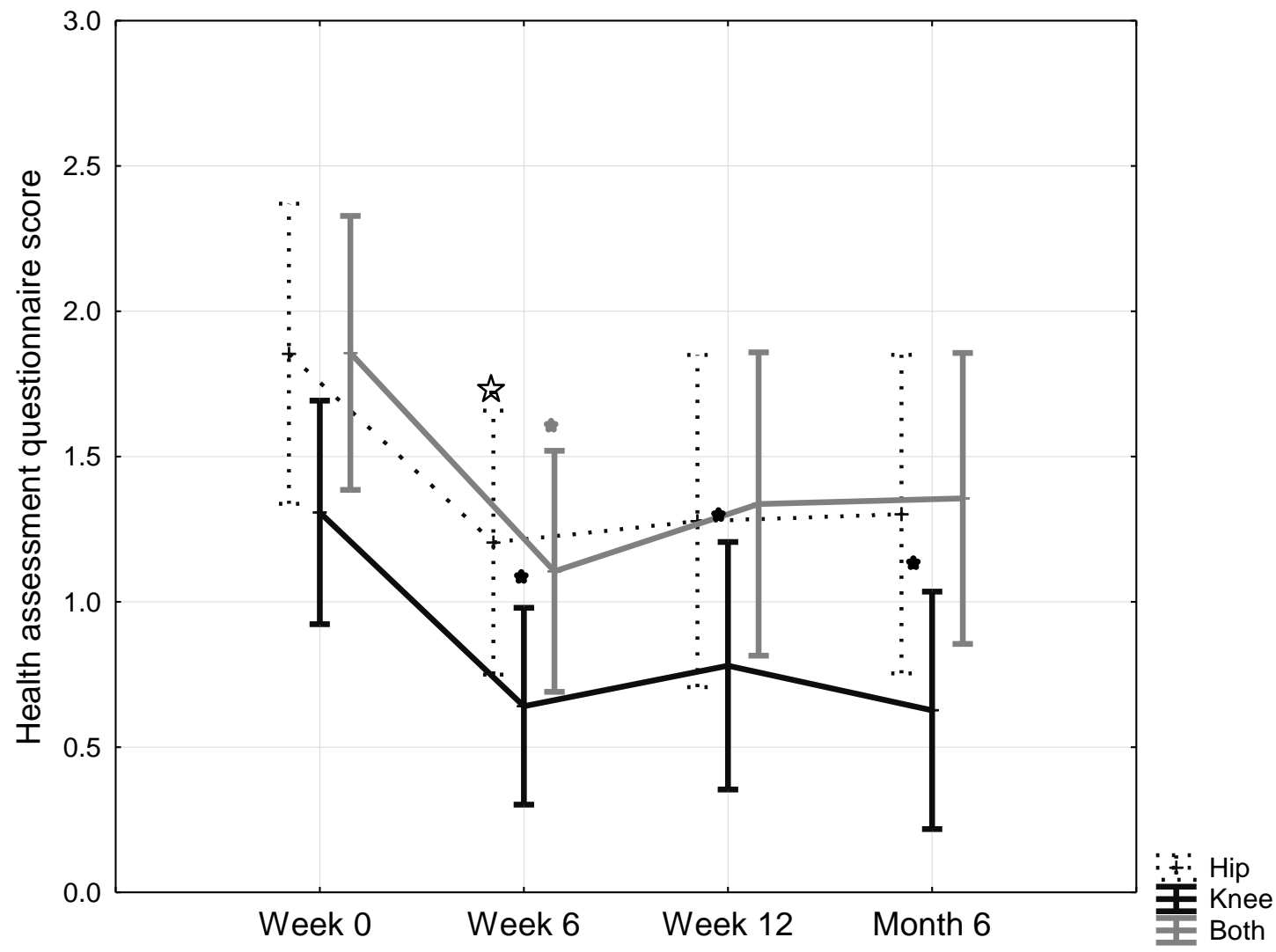

Indicates a significant improvement in HAQ of OA knee subgroup at week $6(p<0.01)$, week 12 (p=0.02) and month 6 $(p<0.01)$

Indicates a significant improvement in HAQ of OA both hip and knee subgroup at week $6(p<0.01)$

负 Indicates a significant improvement in HAQ of OA hip subgroup at week $6(p=0.048)$

Appendix K-3 Change in HAQ of OA knee, hip and both hip and knee subgroups in experimental group $(n=20)$ 


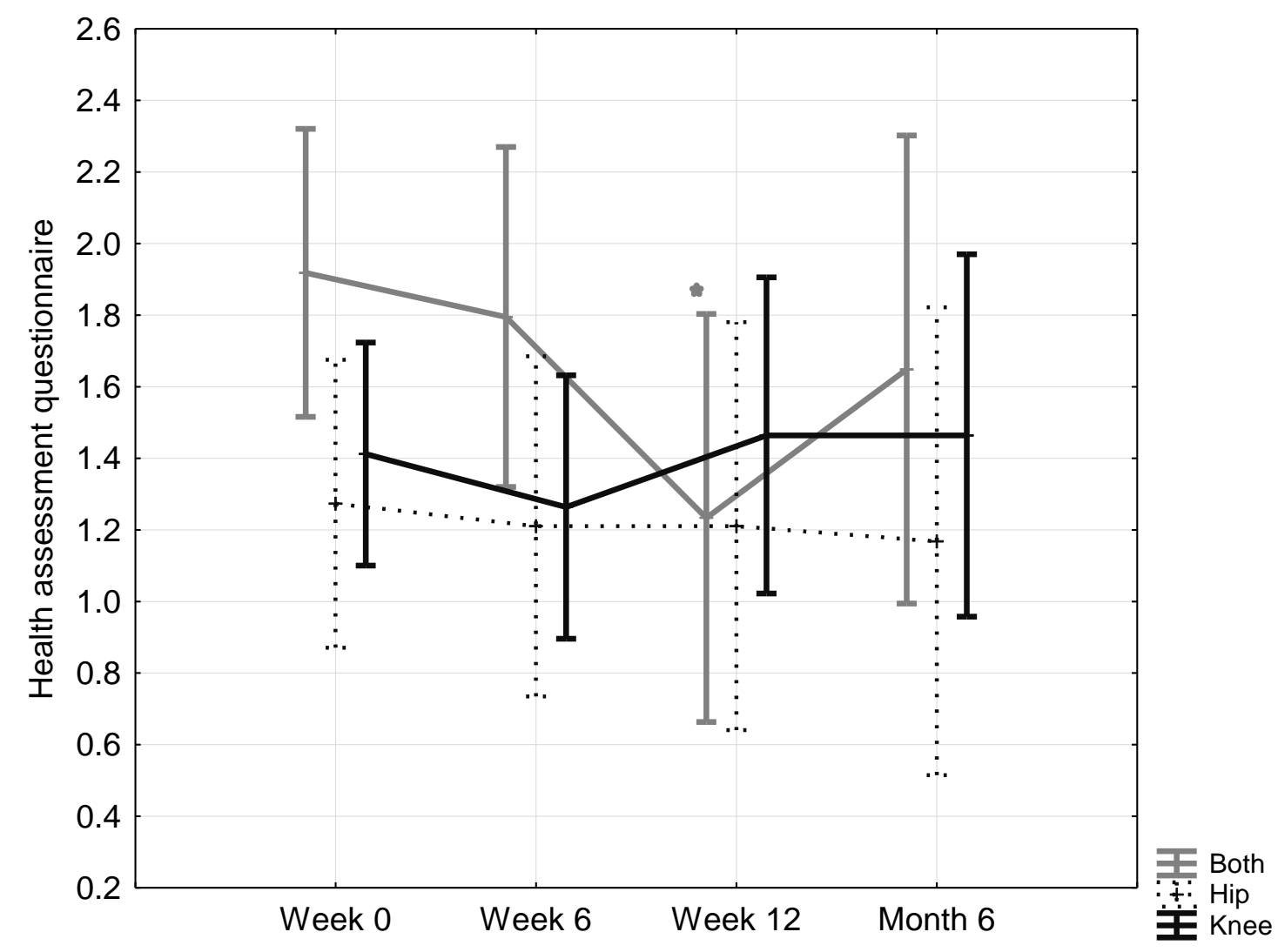

Indicates a significant improvement in HAQ of OA both hip and knee subgroup at week $12(p=0.03)$

Appendix K-4 Change in HAQ of OA knee, hip and both hip and knee subgroups in control group $(n=22)$ 


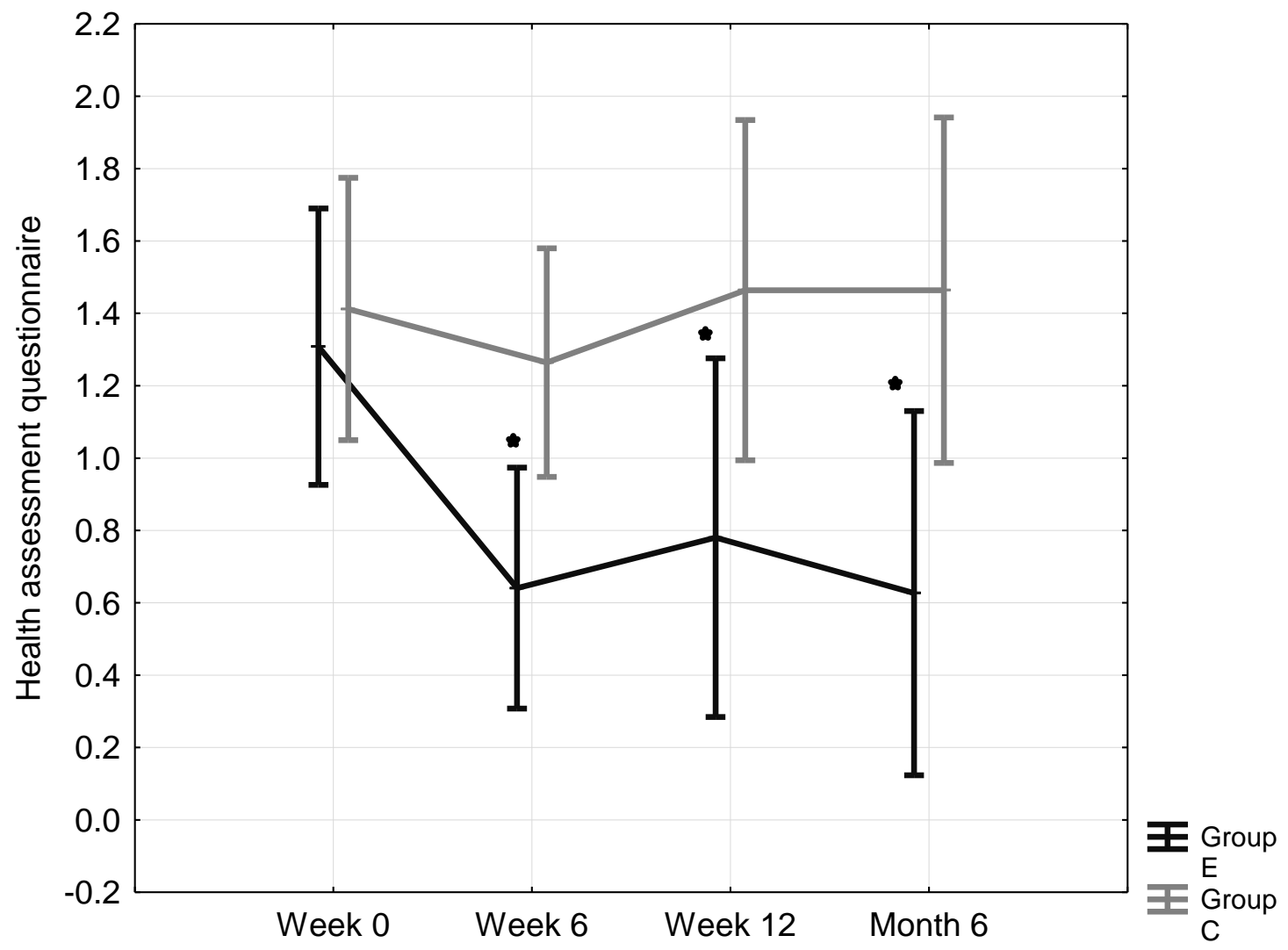

- Indicates a significant improvement in HAQ of OA knee subgroup at week $6(p<0.01)$, week $12(p=0.03)$ and month 6 $(p<0.01)$

Appendix K-5 Change in HAQ of OA knee subgroup $(n=19)$ 


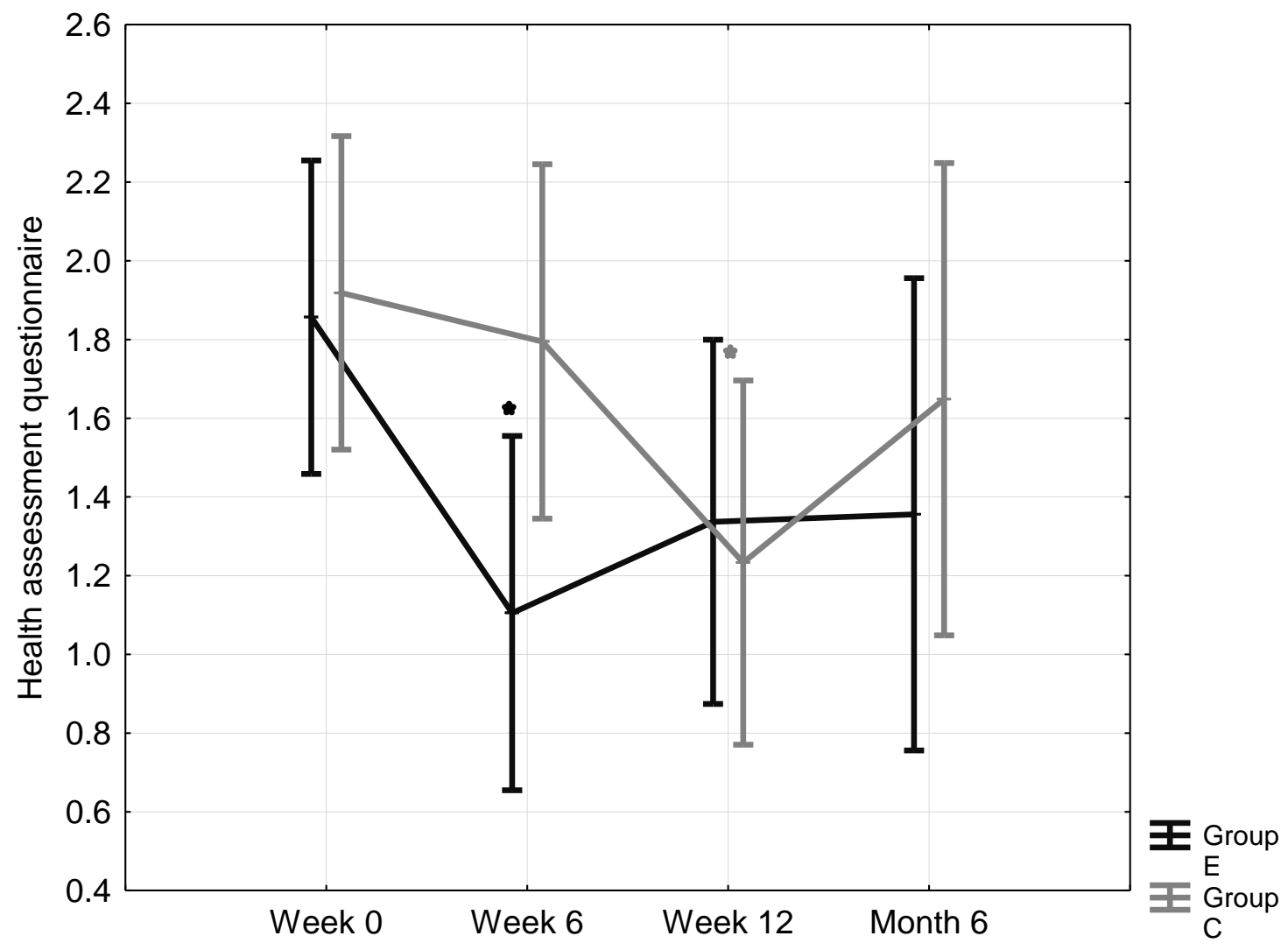

- Indicates a significant improvement in OA both hip and knee in experimental group at week $6(p<0.01)$

Indicates a significant improvement in OA both hip and knee in control group at week $12(p<0.01)$

Appendix K-6 Change in HAQ of OA both hip and knee subgroup $(n=12)$ 


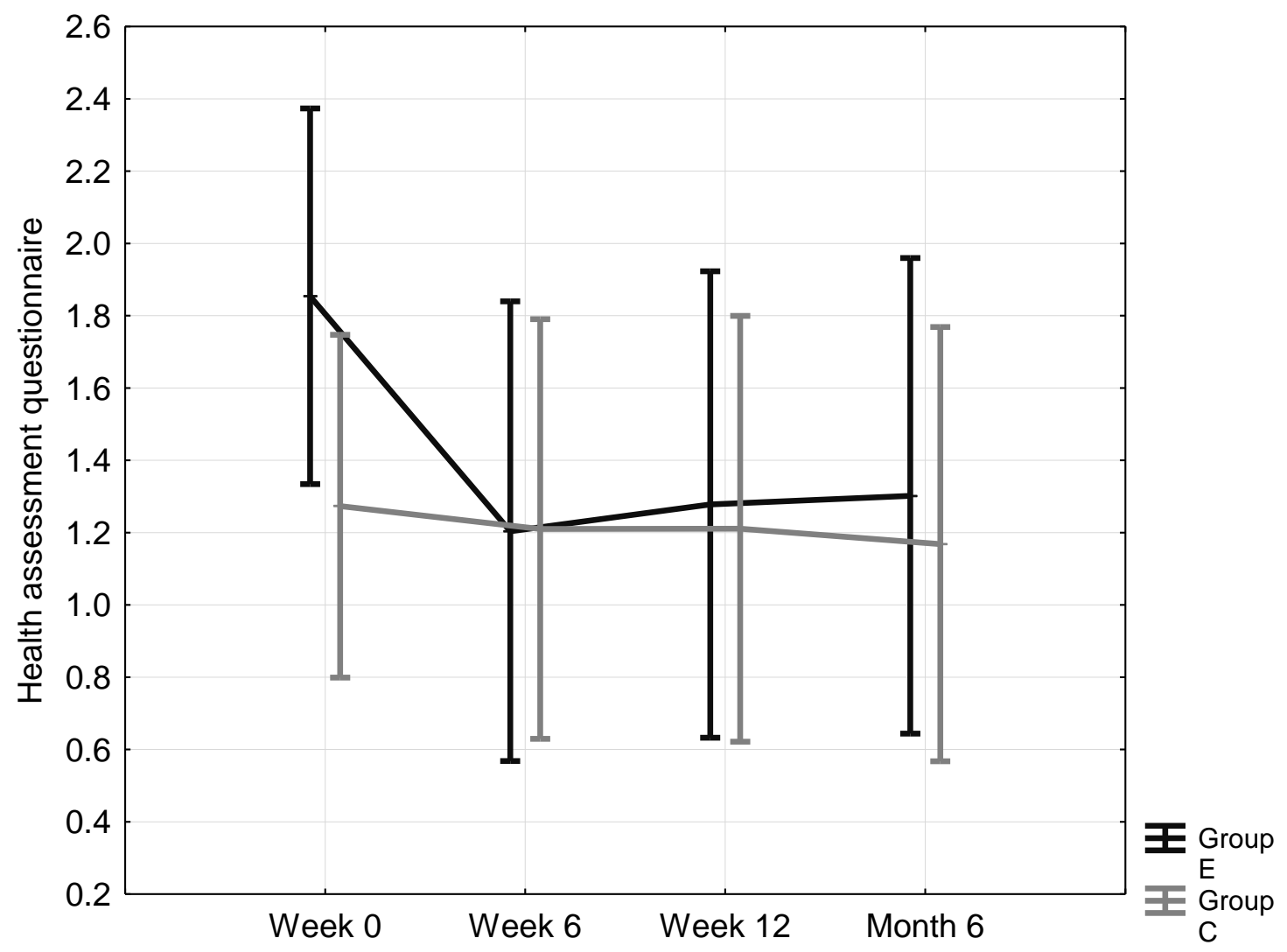

Appendix K-7 Change in HAQ of OA hip subgroup $(n=11)$ 
Appendix K-3 Detailed HAQ pain VAS of OA hip, knee, both hip and knee subgroups

\begin{tabular}{|c|c|c|c|}
\hline & $\begin{array}{l}\text { Participants } \\
\mathbf{N}=\mathbf{4 2}\end{array}$ & $\begin{array}{l}\text { Experimental } \\
\text { Group } \\
\mathrm{n}=20\end{array}$ & $\begin{array}{l}\text { Control Group } \\
n=22\end{array}$ \\
\hline & Mean \pm SD & Mean \pm SD & Mean \pm SD \\
\hline \multicolumn{4}{|c|}{ Baseline HAQ pain VAS } \\
\hline Hip & $62.36 \pm 34.89$ & $68.00 \pm 39.11$ & $57.67 \pm 33.96$ \\
\hline Knee & $69.89 \pm 15.61$ & $70.22 \pm 19.26$ & $69.60 \pm 12.54$ \\
\hline Both & $73.75 \pm 21.57$ & $82.50 \pm 22.32$ & $65.00 \pm 18.49$ \\
\hline \multicolumn{4}{|l|}{ Week 6} \\
\hline Hip & $57.45 \pm 39.37$ & $50.40 \pm 45.64$ & $63.33 \pm 36.65$ \\
\hline Knee & $57.74 \pm 28.13$ & $38.67 \pm 24.62 * *$ & $74.90 \pm 18.77^{* *}$ \\
\hline Both & $54.25 \pm 34.38$ & $47.67 \pm 39.65$ & $60.83 \pm 30.39$ \\
\hline \multicolumn{4}{|l|}{ Week 12} \\
\hline Hip & $57.64 \pm 34.46$ & $58.60 \pm 38.76$ & $56.83 \pm 34.23$ \\
\hline Knee & $61.11 \pm 30.48$ & $48.00 \pm 33.73$ & $72.90 \pm 22.93$ \\
\hline Both & $54.67 \pm 35.87$ & $57.00 \pm 37.92$ & $52.33 \pm 37.14$ \\
\hline \multicolumn{4}{|l|}{ Month 6} \\
\hline Hip & $66.64 \pm 29.73$ & $66.00 \pm 33.53$ & $67.17 \pm 29.44$ \\
\hline Knee & $54.37 \pm 34.30$ & $26.78 \pm 27.19 * *$ & $79.20 \pm 15.78^{* *}$ \\
\hline Both & $62.50 \pm 27.71$ & $65.83 \pm 27.97$ & $59.17 \pm 29.67$ \\
\hline
\end{tabular}




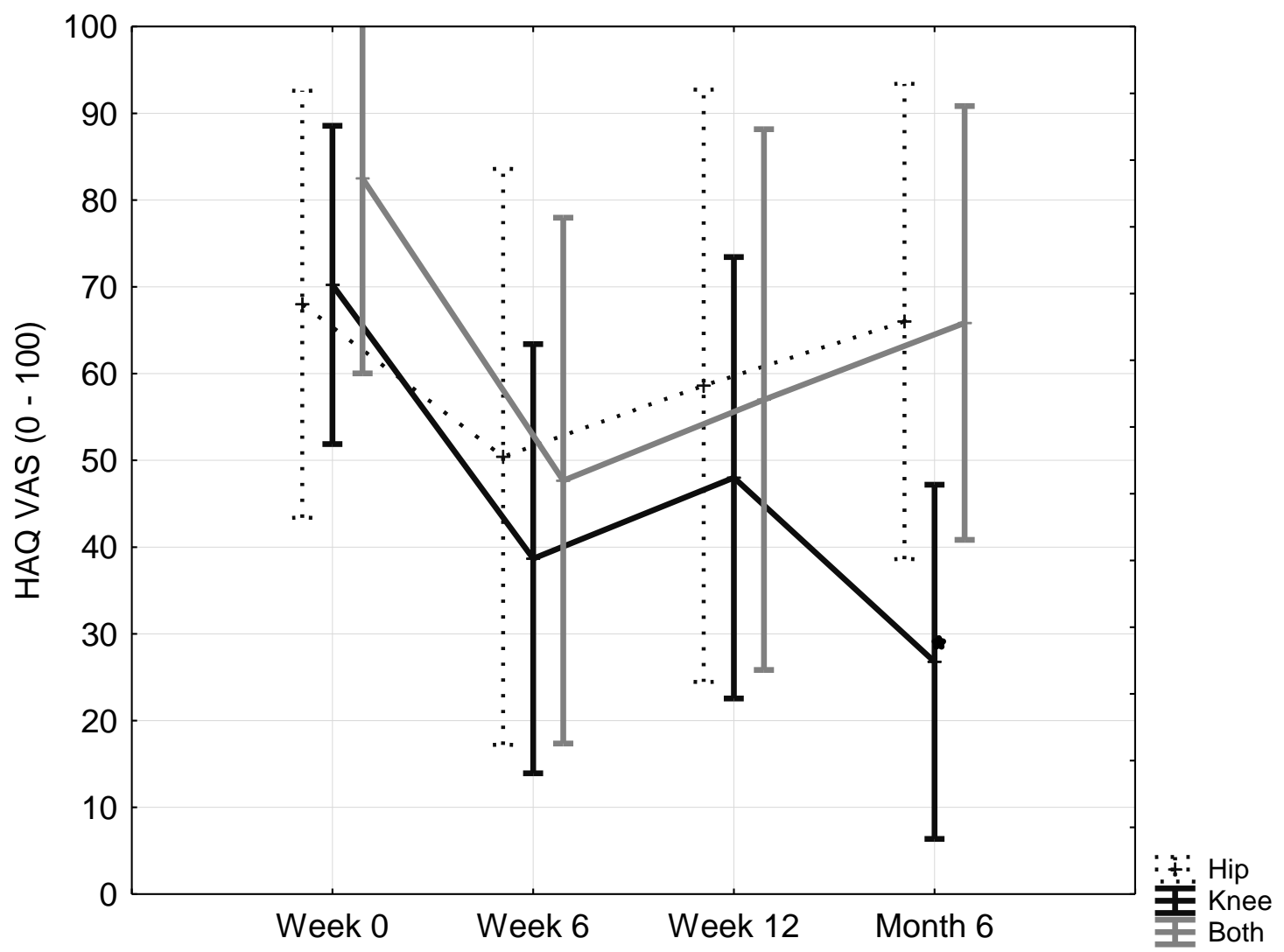

- Indicates a significant improvement in HAQ VAS of OA knee subgroup at month $6(p<0.01)$

Appendix K-8 Change in HAQ VAS of OA hip, knee, both hip and knee subgroups in the experimental group $(n=20)$ 


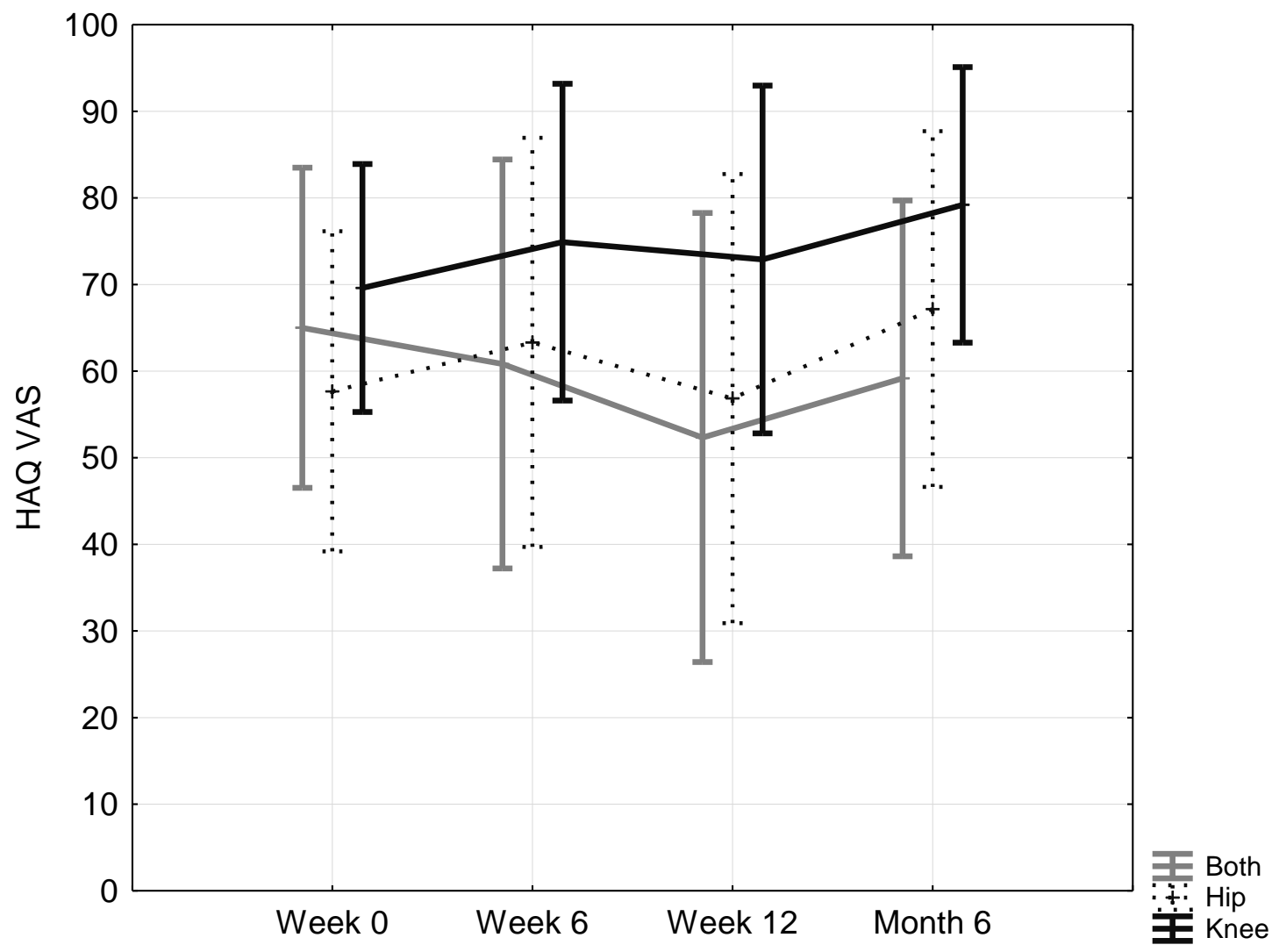

Appendix K-9 Change in HAQ VAS of OA hip, knee, both hip and knee subgroups in the control group $(n=22)$ 


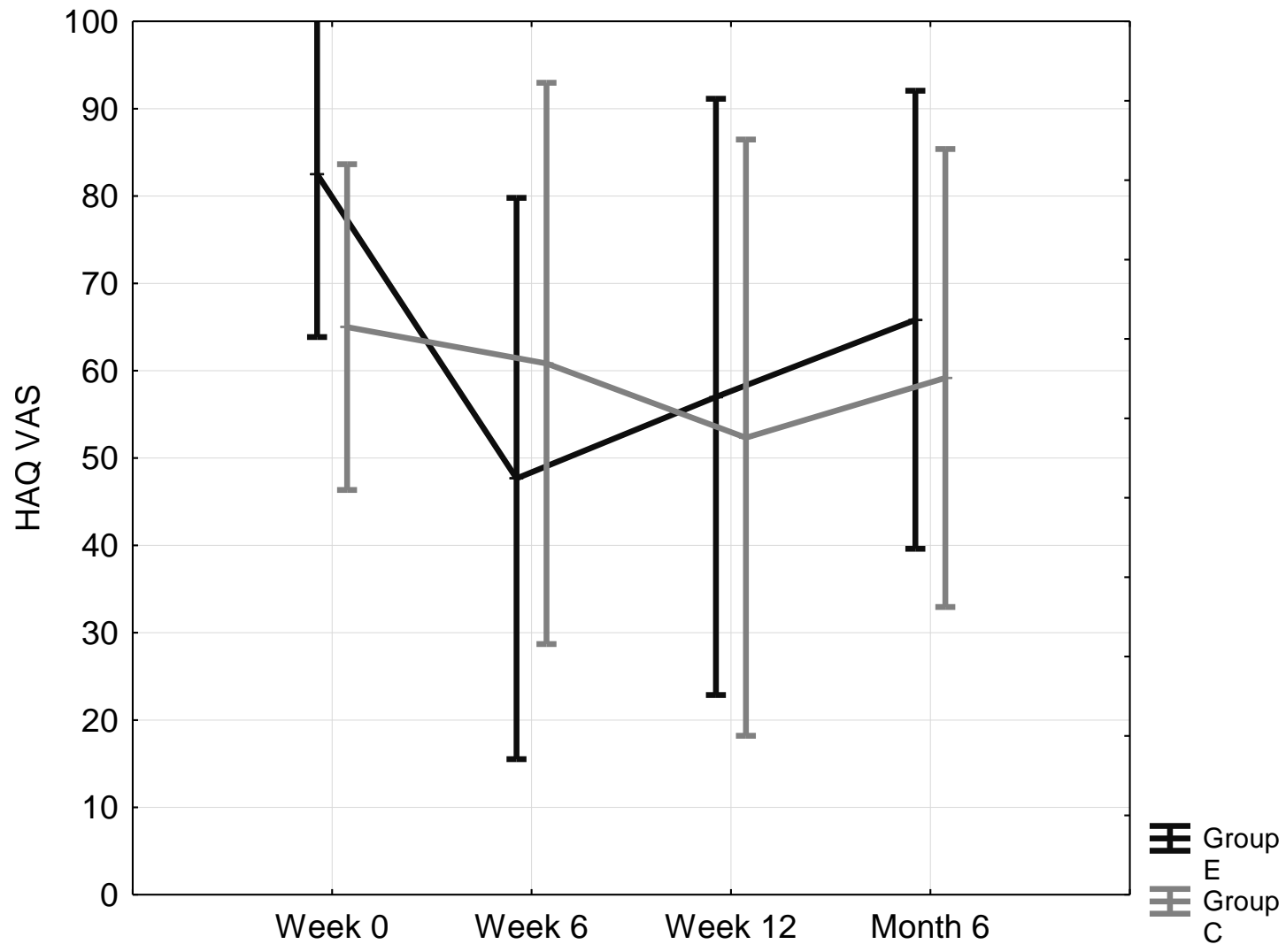

Appendix K-10 Change in HAQ VAS of OA both hip and knee subgroup $(n=12)$ 


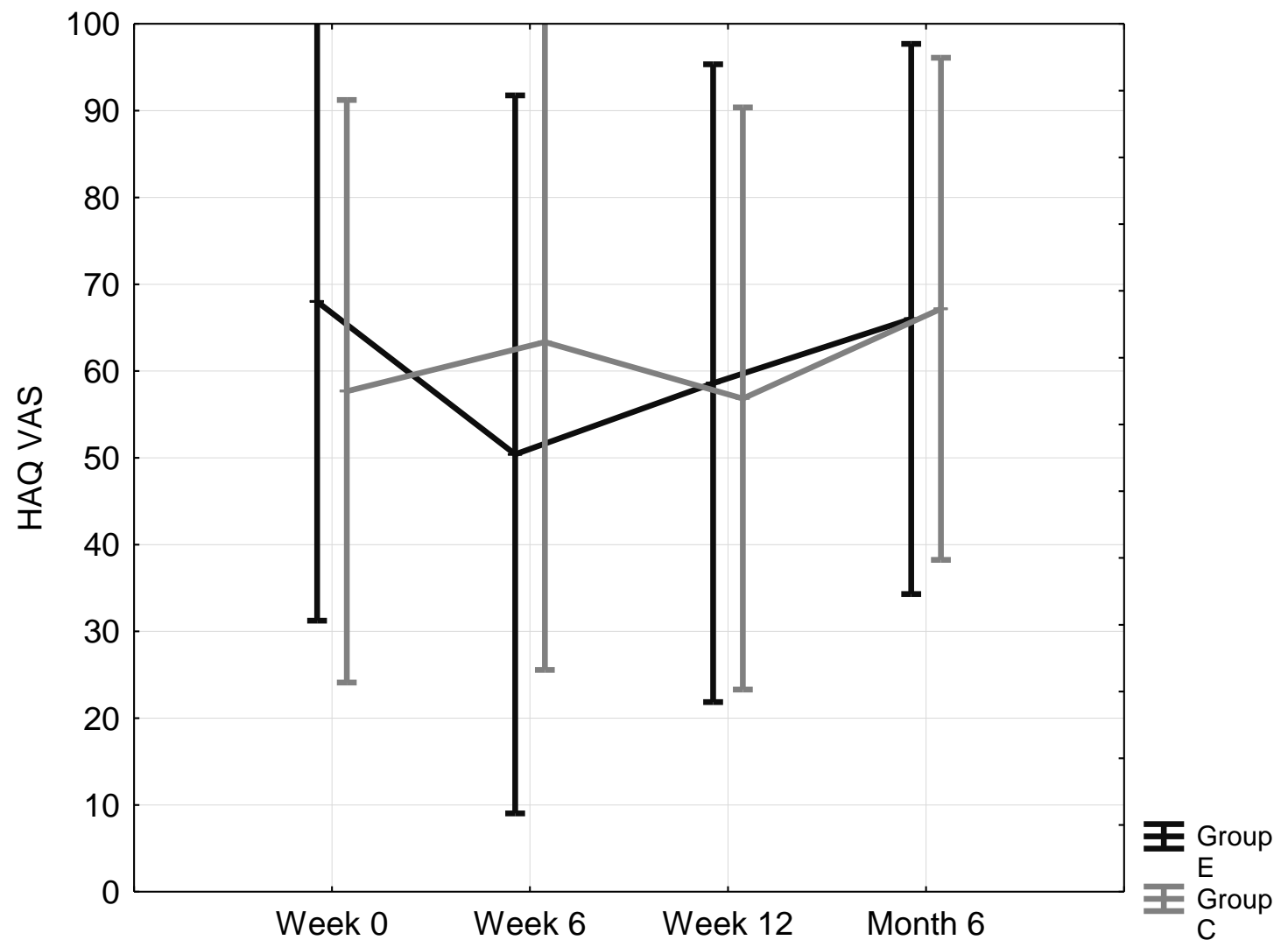

Appendix K-11 Change in HAQ VAS of OA hip subgroup $(n=11)$ 


\section{Appendix L: Full Self-efficacy results}

Appendix L-1 Self-efficacy scores ( $N=42)$

\begin{tabular}{|c|c|c|c|}
\hline & $\begin{array}{l}\text { Participants } \\
\mathrm{N}=42\end{array}$ & $\begin{array}{l}\text { Experimental } \\
\text { Group } \\
n=20\end{array}$ & $\begin{array}{l}\text { Control Group } \\
n=22\end{array}$ \\
\hline & Mean \pm SD & Mean \pm SD & Mean \pm SD \\
\hline \multicolumn{4}{|c|}{ Self-efficacy score } \\
\hline Baseline & $6.30 \pm 2.38$ & $5.75 \pm 2.71$ & $6.80 \pm 1.98$ \\
\hline Week 6 & $6.52 \pm 2.21$ & $7.15 \pm 2.32$ & $5.95 \pm 1.99$ \\
\hline Week 12 & $6.11 \pm 2.27$ & $6.68 \pm 2.33$ & $5.59 \pm 2.14$ \\
\hline Month 6 & $6.92 \pm 2.02$ & $7.33 \pm 2.13$ & $6.56 \pm 1.88$ \\
\hline
\end{tabular}




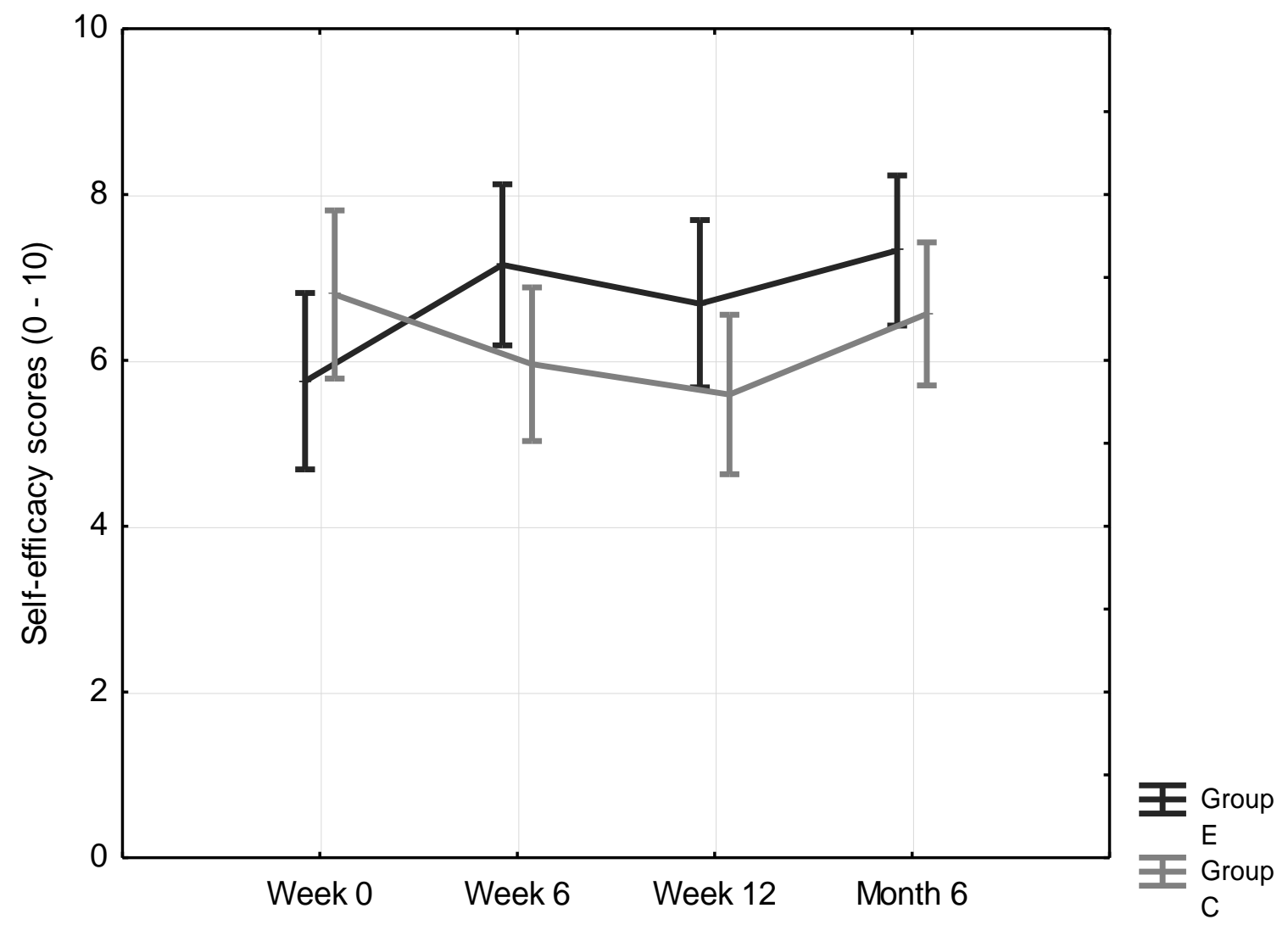

Appendix L-1 Change in self-efficacy scores $(N=42)$ 


\section{Appendix M: Full Health related quality of life results}

EQ-5D Health index and current state of health

Appendix M-1 Health index and current state of health $(N=42)$

\begin{tabular}{|c|c|c|c|}
\hline & $\begin{array}{l}\text { Participants } \\
\mathrm{N}=42\end{array}$ & $\begin{array}{l}\text { Experimental } \\
\text { Group } \\
n=20\end{array}$ & $\begin{array}{l}\text { Control Group } \\
n=22\end{array}$ \\
\hline & Mean \pm SD & Mean \pm SD & Mean \pm SD \\
\hline \multicolumn{4}{|l|}{ Health index } \\
\hline Baseline & $0.33 \pm 0.33$ & $0.31 \pm 0.34$ & $0.35 \pm 0.32$ \\
\hline Week 6 & $0.56 \pm 0.31$ & $0.58 \pm 0.36$ & $0.55 \pm 0.26$ \\
\hline Week 12 & $0.46 \pm 0.36$ & $0.53 \pm 0.36$ & $0.4 \pm 0.36$ \\
\hline Month 6 & $0.47 \pm 0.36$ & $0.51 \pm 0.38$ & $0.43 \pm 0.35$ \\
\hline \multicolumn{4}{|c|}{ Current state of health } \\
\hline Baseline & $58.26 \pm 20.46$ & $57.45 \pm 23.74$ & $59.00 \pm 17.5$ \\
\hline Week 6 & $59.67 \pm 20.07$ & $58.25 \pm 24.13$ & $60.95 \pm 16.01$ \\
\hline Week 12 & $63.12 \pm 24.07$ & $63.3 \pm 24.51$ & $62.95 \pm 24.23$ \\
\hline Month 6 & $66.19 \pm 22.49$ & $65.75 \pm 23.13$ & $66.59 \pm 22.43$ \\
\hline
\end{tabular}




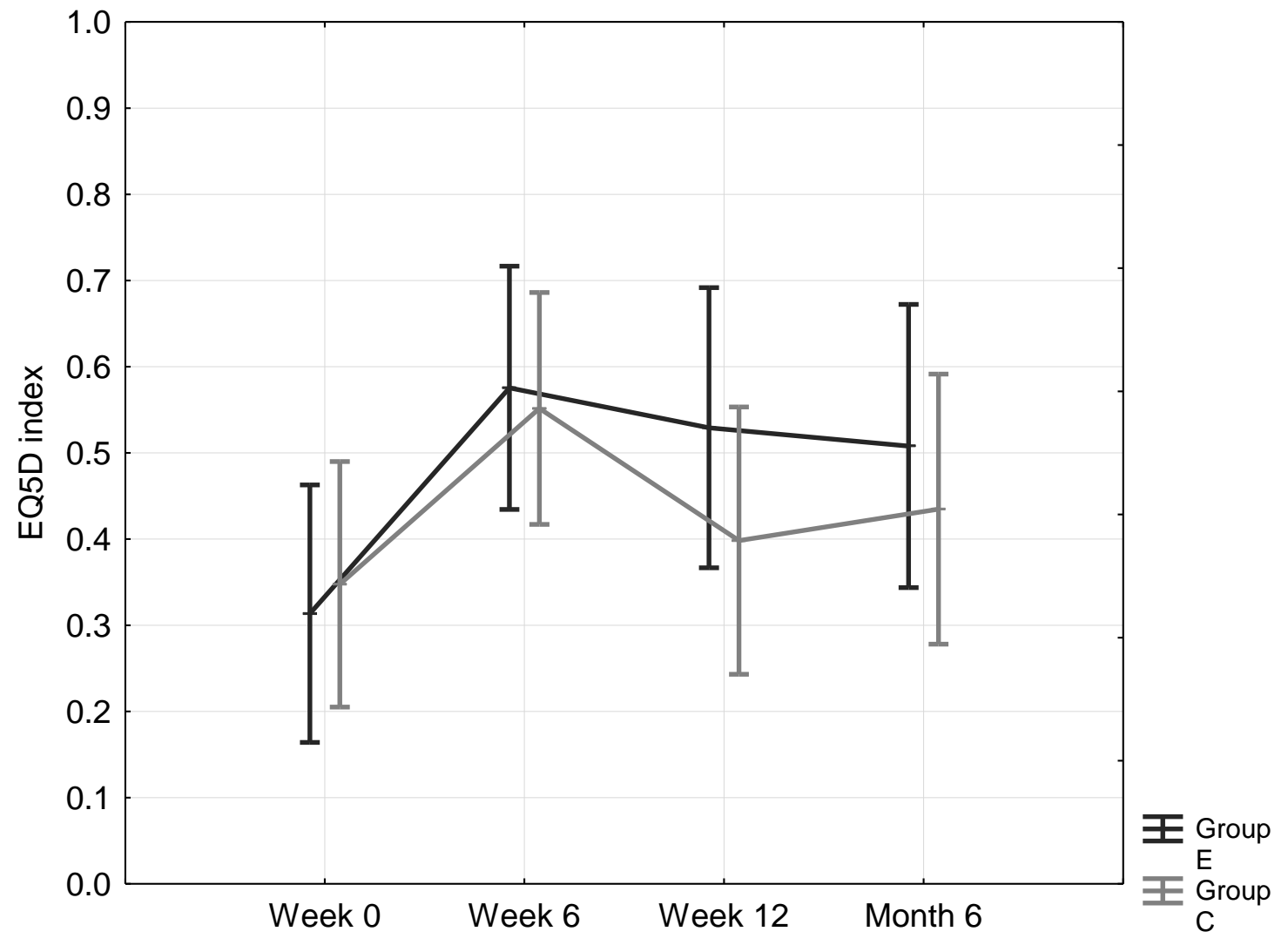

Appendix $M-1$ Change in EQ-5D index ( $N=42)$ 


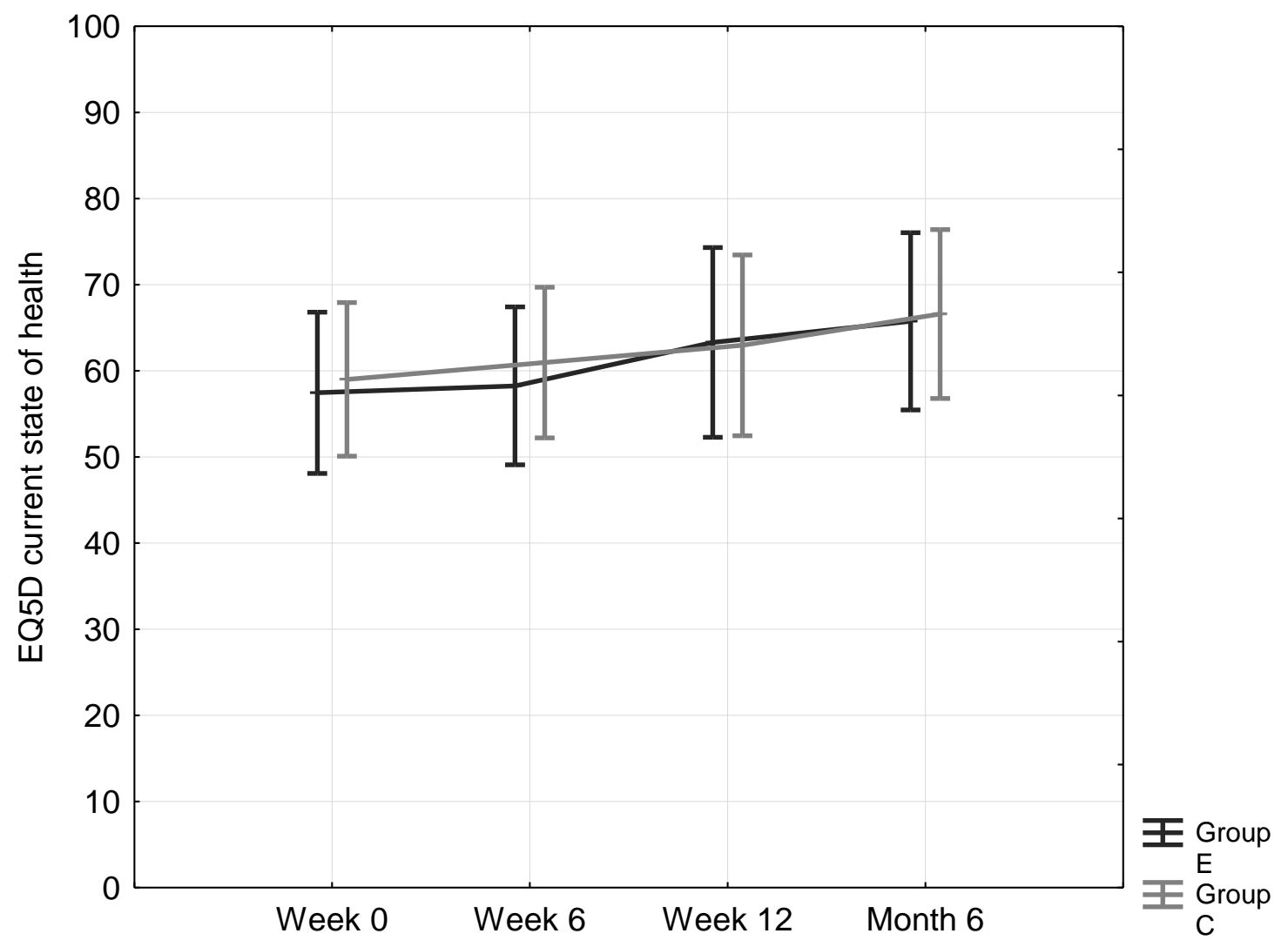

Appendix M-2 Change in EQ-5D current state of health $(N=42)$ 
Appendix M-2 Mobility, self-care, activity, pain/discomfort, anxiety/depression ( $N=42$ )

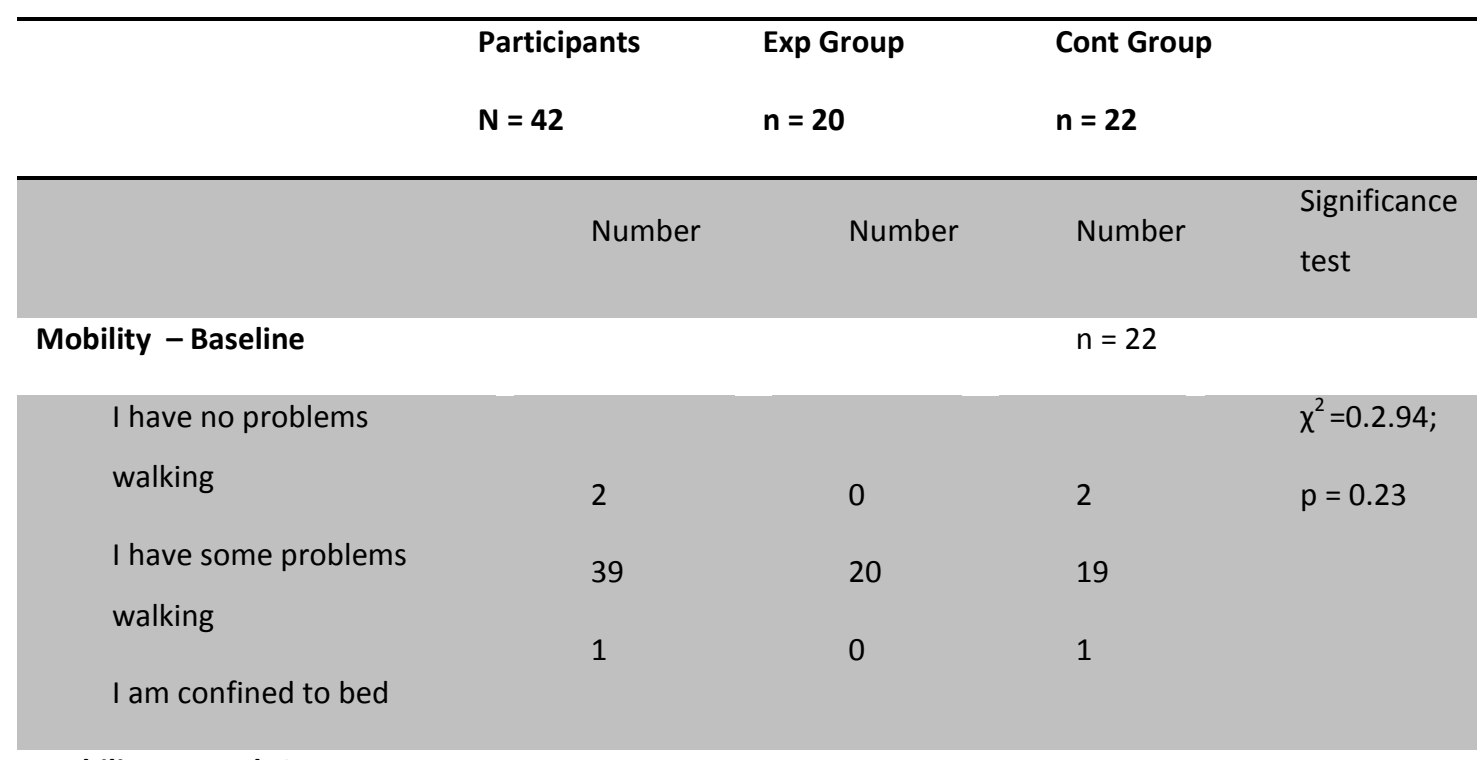

\section{Mobility - Week 6}

$\begin{array}{lcccc}\text { I have no problems } & & & & \chi^{2}=0.31 ; \\ \text { walking } & 7 & 4 & 19 & \mathrm{p}=0.58 \\ \text { I have some problems } & 35 & 16 & 0 & \\ \text { walking } & 0 & 0 & \end{array}$

Mobility - Week 12

I have no problems

walking

I have some problems

walking

I am confined to bed
Mobility - Month 6
I have no problems

walking

I have some problems

walking

I am confined to bed $\chi^{2}=1.55$

$p=0.46$

$0 \quad 1$




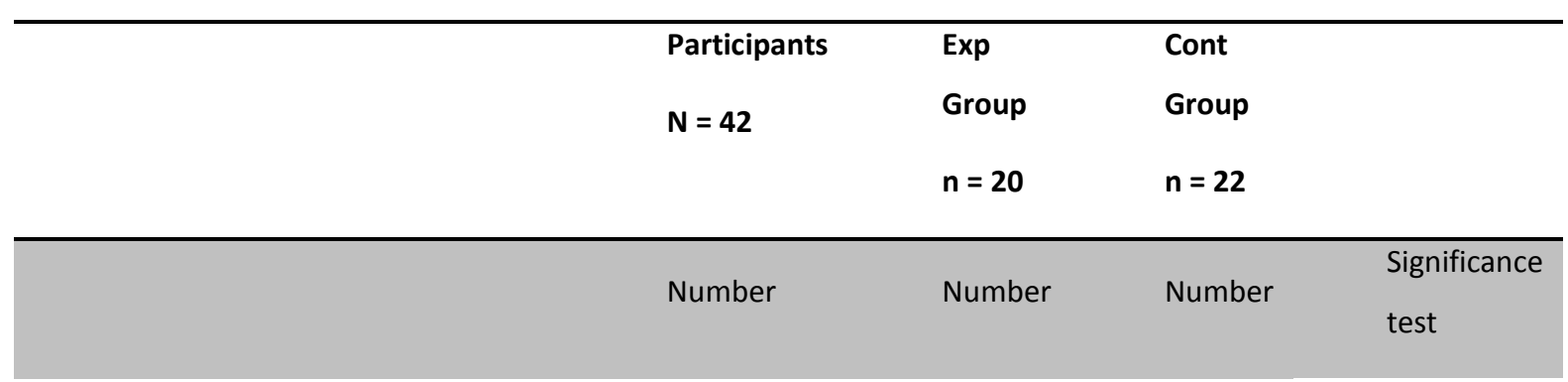

\section{Self-care - Baseline}

$\begin{aligned} & \text { I have no problems with } \\ & \text { self-care }\end{aligned}$
$\begin{aligned} & \text { I have some problems } \\ & \text { self-care }\end{aligned}$
$\begin{aligned} & \text { I am unable to wash/dress myself } \\ & \text { (1) }\end{aligned}$

Self-care Week 6

\begin{tabular}{|c|c|c|c|c|}
\hline $\begin{array}{l}\text { I have no problems with } \\
\text { self-care }\end{array}$ & 28 & 16 & 12 & $\begin{array}{l}\chi^{2}=3.41 \\
p=0.18\end{array}$ \\
\hline $\begin{array}{l}\text { I have some problems } \\
\text { self-care }\end{array}$ & 13 & 4 & 9 & \\
\hline I am unable to wash/dress myself & 1 & 0 & 1 & \\
\hline
\end{tabular}

\section{Self-care - Week 12}

I have no problems with
self-care
I have some problems
with self-care
I am unable to wash/dress myself

22

Self-care - Month 6

I have no problems with

self-care

I have some problems

with self-care

I am unable to wash/dress myself
19

1

22

20

2

19

2

0
11

9

0

$$
\begin{aligned}
& \chi^{2}=0.96 \\
& p=0.62
\end{aligned}
$$

10

11

$\chi^{2}=0.11$

$p=0.75$

11

9

0

0 


\begin{tabular}{|c|c|c|c|c|}
\hline & $\begin{array}{l}\text { Partici- } \\
\text { pants } \\
\mathrm{N}=\mathbf{4 2}\end{array}$ & $\begin{array}{l}\text { Exp } \\
\text { Group } \\
n=20\end{array}$ & $\begin{array}{l}\text { Cont } \\
\text { Group } \\
\mathrm{n}=\mathbf{2 2}\end{array}$ & \\
\hline & Number & Number & Number & Test \\
\hline \multicolumn{5}{|l|}{ Activity - Baseline } \\
\hline $\begin{array}{l}\text { I have no problems with usual } \\
\text { activities }\end{array}$ & 7 & 3 & 4 & $\begin{array}{l}\chi^{2}=1.42 \\
p=0.70\end{array}$ \\
\hline $\begin{array}{l}\text { I have some problems with usual } \\
\text { activities }\end{array}$ & 31 & 16 & 15 & \\
\hline $\begin{array}{l}\text { I am unable to perform my usual } \\
\text { activities }\end{array}$ & 3 & 1 & 2 & \\
\hline Missing answer & 1 & 0 & 1 & \\
\hline \multicolumn{5}{|l|}{ Activity - Week 6} \\
\hline $\begin{array}{l}\text { I have no problems with usual } \\
\text { activities }\end{array}$ & 11 & 8 & 3 & $\begin{array}{l}\chi^{2}=5.05 \\
p=0.08\end{array}$ \\
\hline $\begin{array}{l}\text { I have some problems with usual } \\
\text { activities }\end{array}$ & 29 & 12 & 17 & \\
\hline $\begin{array}{l}\text { I am unable to perform my usual } \\
\text { activities }\end{array}$ & 2 & 0 & 2 & \\
\hline
\end{tabular}

\section{Activity - Week 12}

$\begin{array}{lllll}\text { I have no problems with usual } & 10 & 4 & 6 & \chi^{2}=2.44 ; \\ \text { activities } & 30 & 16 & 14 & 0.29 \\ \text { I have some problems with usual } & & & 2\end{array}$

\section{Activity - Month 6}

\begin{tabular}{|c|c|c|c|c|}
\hline $\begin{array}{l}\text { I have no problems with usual } \\
\text { activities }\end{array}$ & 10 & 7 & 3 & $\begin{array}{l}\chi^{2}=4.05 \\
p=0.13\end{array}$ \\
\hline $\begin{array}{l}\text { I have some problems with usual } \\
\text { activities }\end{array}$ & 30 & 13 & 17 & \\
\hline $\begin{array}{l}\text { I am unable to perform my usual } \\
\text { activities }\end{array}$ & 2 & 0 & 2 & \\
\hline
\end{tabular}




\begin{tabular}{lcccc}
\hline Pain/discomfort - Baseline & & & & \\
I have no pain/discomfort & 2 & 1 & 1 & $\chi^{2}=0.36 ;$ \\
I have moderate pain/discomfort & 23 & 10 & 8 & $p=0.84$ \\
I have severe pain/discomfort & 17 & 9 &
\end{tabular}

\section{Pain/discomfort - Week 6}

\begin{tabular}{|c|c|c|c|c|}
\hline I have no pain/discomfort & 10 & 6 & 4 & $\chi^{2}=3.56$ \\
\hline I have moderate pain/discomfort & 25 & 9 & 16 & $p=0.17$ \\
\hline I have severe pain/discomfort & 7 & 5 & 2 & \\
\hline \multicolumn{5}{|l|}{ Pain/discomfort - Week 12} \\
\hline I have no pain/discomfort & 7 & 4 & 3 & $\chi^{2}=0.74$ \\
\hline I have moderate pain/discomfort & 22 & 11 & 11 & $p=0.69$ \\
\hline I have severe pain/discomfort & 13 & 5 & 8 & \\
\hline \multicolumn{5}{|l|}{ Pain/discomfort - Month 6} \\
\hline I have no pain/discomfort & 7 & 4 & 3 & $\chi^{2}=0.44$ \\
\hline I have moderate pain/discomfort & 23 & 10 & 13 & $p=0.80$ \\
\hline I have severe pain/discomfort & 12 & 6 & 6 & \\
\hline
\end{tabular}




\begin{tabular}{|c|c|c|c|c|}
\hline & $\begin{array}{l}\text { Participants } \\
\mathrm{N}=42\end{array}$ & $\begin{array}{l}\text { Exp Group } \\
n=20\end{array}$ & $\begin{array}{l}\text { Cont } \\
\text { Group } \\
\mathrm{n}=\mathbf{2 2}\end{array}$ & \\
\hline & Number & Number & Number & Test \\
\hline \multicolumn{5}{|c|}{ Anxiety/depression - Baseline } \\
\hline $\begin{array}{l}\text { I have no } \\
\text { anxiety/depression }\end{array}$ & 13 & 7 & 6 & $\begin{aligned} \chi^{2} & =0.35 \\
p & =0.84\end{aligned}$ \\
\hline $\begin{array}{l}\text { I have moderate } \\
\text { anxiety/depression }\end{array}$ & 24 & 11 & 13 & \\
\hline $\begin{array}{l}\text { I have severe } \\
\text { anxiety/depression }\end{array}$ & 5 & 2 & 3 & \\
\hline
\end{tabular}

\section{Anxiety/depression - Week 6}

$\begin{array}{lcccc}\text { I have no } & 23 & 14 & 9 & \chi^{2}=3.88 ; \\ \text { anxiety/depression } & 17 & 5 & 12 & \mathrm{p}=0.14 \\ \begin{array}{l}\text { I have moderate } \\ \text { anxiety/depression }\end{array} & & 1 & \\ \text { I have severe } & 2 & 1 & \end{array}$

\section{Anxiety/depression - Week 12}

$\begin{array}{lcccc}\text { I have no } & 19 & 12 & 7 & \chi^{2}=3.56 ; \\ \text { anxiety/depression } & 21 & 7 & 14 & \mathrm{p}=0.17 \\ \begin{array}{l}\text { I have moderate } \\ \text { anxiety/depression }\end{array} & & 1 & \\ \text { I have severe } & 2 & 1 & \end{array}$

\section{Anxiety/depression - Month 6}

$\begin{array}{lcccc}\text { I have no } & 18 & 11 & 7 & \chi^{2}=2.60 ; \\ \text { anxiety/depression } & 20 & 8 & 12 & p=0.27 \\ \begin{array}{l}\text { I have moderate } \\ \text { anxiety/depression }\end{array} & & 3 & \\ \text { I have severe } & 4 & 1 & \end{array}$




\section{Appendix N: Full Body mass index results}

Appendix N-1 Change in BMI $(\mathrm{kg} / \mathrm{m2})(\mathrm{N}=42)$

\begin{tabular}{|c|c|c|c|c|}
\hline & $\begin{array}{l}\text { Participants } \\
\mathrm{N}=42\end{array}$ & $\begin{array}{l}\text { Experimental } \\
\text { Group } \\
\mathrm{n}=\mathbf{2 0}\end{array}$ & $\begin{array}{l}\text { Control } \\
\text { Group } \\
\mathbf{n}=\mathbf{2 2}\end{array}$ & \\
\hline & Mean $\pm S D$ & Mean $\pm S D$ & Mean $\pm S D$ & $\begin{array}{l}\text { Significance } \\
\text { test }\end{array}$ \\
\hline BMI $\left(\mathrm{kg} / \mathrm{m}^{2}\right)$ & & & & $P=0.56$ \\
\hline Baseline & $35.78 \pm 8.81$ & $33.69 \pm 9.21$ & $37.68 \pm 8.17$ & \\
\hline Week 12 & $35.54 \pm 9.00$ & $33.48 \pm 9.22$ & $37.41 \pm 8.58$ & \\
\hline Month 6 & $35.76 \pm 8.92$ & $33.55 \pm 8.92$ & $37.77 \pm 8.63$ & \\
\hline
\end{tabular}




\section{Appendix P: Full assistive device usage}

Appendix P-1 RA recorded assistive device usage $(\mathrm{N}=42)$

\begin{tabular}{|c|c|c|c|}
\hline & $\begin{array}{l}\text { Participants } \\
\mathbf{N}=\mathbf{4 2}\end{array}$ & $\begin{array}{l}\text { Experimental Group } \\
\mathrm{n}=\mathbf{2 0}\end{array}$ & $\begin{array}{l}\text { Control Group } \\
\mathrm{n}=\mathbf{2 2}\end{array}$ \\
\hline Assistive device used & Number & Number & Number \\
\hline \multicolumn{4}{|l|}{ Baseline: } \\
\hline Yes & 23 & 14 & 9 \\
\hline No & 19 & 6 & 13 \\
\hline \multicolumn{4}{|l|}{ Type: } \\
\hline None & 19 & 6 & 13 \\
\hline 1 crutch/stick & 16 & 10 & 6 \\
\hline 2 crutches & 7 & 4 & 3 \\
\hline \multicolumn{4}{|l|}{ Week6: } \\
\hline Yes & 20 & 10 & 10 \\
\hline No & 22 & 10 & 12 \\
\hline \multicolumn{4}{|l|}{ Type: } \\
\hline None & 22 & 10 & 12 \\
\hline 1 crutch/stick & 12 & 7 & 5 \\
\hline 2 crutches & 7 & 2 & 5 \\
\hline Walking frame & 1 & 1 & 0 \\
\hline \multicolumn{4}{|l|}{ Week 12: } \\
\hline Yes & 20 & 9 & 11 \\
\hline No & 22 & 11 & 11 \\
\hline \multicolumn{4}{|l|}{ Type: } \\
\hline None & 22 & 11 & 11 \\
\hline 1 crutch/stick & 14 & 6 & 8 \\
\hline 2 crutches & 5 & 2 & 3 \\
\hline Walking frame & 1 & 1 & 0 \\
\hline
\end{tabular}




\section{Month 6:}

$\begin{array}{cccc}\text { Yes } & 22 & 11 & 11 \\ \text { No } & 20 & 9 & 11\end{array}$

Type:

\begin{tabular}{llll} 
None & 20 & 9 & 11 \\
1 crutch/stick & 14 & 7 & 7 \\
2 crutches & 5 & 1 & 4 \\
Walking frame & 3 & 3 & 0 \\
\hline
\end{tabular}

There was a significant change noted $\left(\chi^{2}=19.14, p<0.01\right)$ in usage of $A D$ between baseline and week sixand week $12(N=42)$ : fiveparticipants using an $A D$ at baseline were not using an $A D$ at week six.Two participants not using an $A D$ at baseline were using an $A D$ at week six. 35 participants usage were unchanged.

There was a significant change noted $\left(\chi^{2}=24.37, p<0.01\right)$ in usage of AD between baseline and month six $(N=42)$ : threeparticipants using an $A D$ at week 0 were still not using an $A D$ at month six.Two participants not using an $A D$ at baseline were now using an $A D$ at month six. 37 participants usage were unchanged. 


\section{Appendix Q: Themes and full responses to open ended questionnaire}

\section{Appendix Q-1 Themes emerged from participant's responses to open ended questions}

\begin{tabular}{|c|c|}
\hline & $\begin{array}{l}\text { Examples of the experimental group's answers to open ended } \\
\text { questions }\end{array}$ \\
\hline Theme: & $\begin{array}{l}\text { Question 1: Did you find the } 6 \text { week course helpful to you in } \\
\text { any way? } \\
\text { If so how did it help you? }\end{array}$ \\
\hline \multirow[t]{6}{*}{ Knowledge } & $\begin{array}{l}\text { Participant B: "Yes." } \\
\text { Participant K: "Yes the information about healthy eating and } \\
\text { that I don't have to drink pills all the time." }\end{array}$ \\
\hline & $\begin{array}{l}\text { Participant DD: "Learnt more exercises, especially with } \\
\text { equipment." }\end{array}$ \\
\hline & Participant CC: "How to live healthier... " \\
\hline & Participant NN: "To eat healthy and how to lose weight." \\
\hline & $\begin{array}{l}\text { Participant WW: "...learnt about how to control pain, eat } \\
\text { healthier..." }\end{array}$ \\
\hline & $\begin{array}{l}\text { Participant YY: "Very helpful. Helped me physically, taught me } \\
\text { exercise and better eating habits." }\end{array}$ \\
\hline \multicolumn{2}{|l|}{ Function/ } \\
\hline \multirow[t]{6}{*}{ Activity } & $\begin{array}{l}\text { Participant A: "Yes, I began with two crutches, now I walk most } \\
\text { of the time with just one crutch and short distance with none." }\end{array}$ \\
\hline & $\begin{array}{l}\text { Participant P: "This course helping me very much because I } \\
\text { moving myself every day." }\end{array}$ \\
\hline & Participant R: "It was very helpful, especially the exercising..." \\
\hline & $\begin{array}{l}\text { Participant S: "It was helpful; I can go to the shops now when I } \\
\text { wake up. When I feel pain I don't need to take pain pills } \\
\text { anymore, instead to rather exercise or walk." }\end{array}$ \\
\hline & $\begin{array}{l}\text { Participant NN: "Yes, much more mobile which decreases pain. } \\
\text { Exercises." }\end{array}$ \\
\hline & Participant XX: "It helped a lot to exercise regularly... \\
\hline
\end{tabular}




\section{Pain relief}

Participant A: "Much better with pain and sometimes without pain."

Participant N: "Yes, pain relief."

Participant R: "...and now I don't have to rely on pain pills anymore."

Participant V: "All the pain was gone."

Participant AA: "It gave my motivation again because my life was all about pain before. Now I use my workbook and it helps

Personal a lot."

benefits

Participant CC: "...and it decreased my pain."

Participant XX: "...and to decrease pain."

Participant L: "It gave me the opportunity to get out and meet people with the same sort of problem"

Participant UU: "Made me very positive."

Participant WW: "It made me very positive... very motivating to exercise in a group instead of alone."

\section{Question 2: What did you like about the course?}

\section{Knowledge}

FunctionAct ivity

Pain relief

\section{Participant A: "I learnt a lot."}

Participant B: "The people and Melissa who helped me a lot."

Participant P: "I like this course because I learn a lot and I'm so happy"

Participant R: "Learnt a lot about stress management and diet."

Participant NN: "Everything that Melissa taught us..."

Participant UU: "Everything that we learnt."

Participant C: "everything, I can walk better and I feel better."

Participant K: "I enjoyed it a lot. The exercise was very nice, at the hospital and at home."

Participant V: “...exercise.”

Participant S: "I learnt how to put ice on my knee to relieve the pain."

Participant WW: "how to be more positive and to control pain." 


\begin{tabular}{ll}
\hline $\begin{array}{l}\text { Personal } \\
\text { benefits }\end{array}$ & Participant A: "Fun. Built my self-confidence" \\
& Participant L: "Exercise with people, it was fun." \\
& Participant V: "The people who came together to help." \\
& Participant N: "Fun of it all." \\
& Participant AA: "A lot. I am now part of an exercise group. I have \\
& more friends and am not alone with pain anymore." \\
& Participant NN: “...every week's meeting and to exercise in a \\
& group as we motivate each other." \\
& Participant WW: "Learnt how to be more sociable." \\
& Participant XX: "The group exercises were better than exercising \\
& alone."
\end{tabular}

\section{Question 5: What did you like about the workbook?}

$\begin{array}{ll}\text { Knowledge } & \text { Participant K: "Diet, healthy eating for myself." } \\ & \text { Participant L: "Helpful for myself and others at home and } \\ \text { around me." } & \text { Participant N: "Everything." } \\ & \text { Participant P: "I'm just teaching others from the book." } \\ & \text { Participant S: "Learnt about a lot of things I didn't know } \\ & \text { before." } \\ & \text { Participant V: "The nutrition section." } \\ & \text { Participant AA: "Yes." } \\ & \text { Participant CC: "That we had to write our illnesses down." } \\ & \text { Participant NN: "All the knowledge that I gained." } \\ & \text { Participant UU: "Everything." } \\ & \text { Participant WW: "it was very interesting. Learnt a lot of } \\ \text { knowledge from the experience." } & \text { Participant XX: "All the knowledge that I gained. It was } \\ & \text { fantastic." } \\ & \text { Participant YY: "it was clear and understandable." } \\ \text { Punction/ } & \text { Participant A: "Exercises." } \\ \text { Activity } & \text { Participant R: "...also the section about pain management and } \\ \text { nefits } & \text { Participant B: "Everything I learnt, especially about self- } \\ & \text { confidence." } \\ & \end{array}$




\section{Appendix R: CONSORT table}

Table R-1 CONSORT guidelines and indication of chapter where items were addressed

\begin{tabular}{|c|c|c|c|}
\hline Section/Topic & $\begin{array}{l}\text { Item } \\
\text { No }\end{array}$ & Checklist item & Reported in \\
\hline Title and abstract & $1 b$ & $\begin{array}{l}\text { Identification as a randomised trial in the title } \\
\text { Structured summary of trial design, methods, results, and conclusions }\end{array}$ & Abstract \\
\hline $\begin{array}{l}\text { Introduction } \\
\qquad \text { Background/objective }\end{array}$ & $\begin{array}{l}2 a \\
2 b\end{array}$ & $\begin{array}{l}\text { Scientific background and explanation of rationale } \\
\text { Specific objectives or hypotheses }\end{array}$ & $\begin{array}{l}\text { Chapter } 1.1 \text { and } \\
2.1 \\
\text { Chapter } 1.2\end{array}$ \\
\hline \multicolumn{4}{|l|}{ Methods } \\
\hline Trial design & $\begin{array}{l}3 a \\
3 b\end{array}$ & $\begin{array}{l}\text { Description of trial design (such as parallel, factorial) including allocation ratio } \\
\text { Important changes to methods after trial commencement (such as eligibility criteria), with reasons }\end{array}$ & Chapter 3.1 \\
\hline Participants & $\begin{array}{l}4 a \\
4 b\end{array}$ & $\begin{array}{l}\text { Eligibility criteria for participants } \\
\text { Settings and locations where the data were collected }\end{array}$ & $\begin{array}{l}\text { Chapter } 3.2 \\
\text { Chapter } 3.2 \text { and } \\
3.5\end{array}$ \\
\hline Interventions & 5 & $\begin{array}{l}\text { The interventions for each group with sufficient details to allow replication, including how and when } \\
\text { they were actually administered }\end{array}$ & $\begin{array}{l}\text { Chapter } 3.5 \text { and } \\
\text { Appendix } F \text { and } \\
\text { G }\end{array}$ \\
\hline Outcomes & $6 a$ & $\begin{array}{l}\text { Completely defined pre-specified primary and secondary outcome measures, including how and when } \\
\text { they were assessed } \\
\text { Any changes to trial outcomes after the trial commenced, with reasons }\end{array}$ & $\begin{array}{l}\text { Chapter } 3.4 \text { and } \\
\text { Appendix E } \\
\text { Chapter } 3.4,3.6 \\
\text { and Appendix E } \\
\text { (pg. 219) }\end{array}$ \\
\hline Sample size & $\begin{array}{l}7 \mathrm{a} \\
7 \mathrm{~b}\end{array}$ & $\begin{array}{l}\text { How sample size was determined } \\
\text { When applicable, explanation of any interim analyses and stopping guidelines }\end{array}$ & Chapter 3.3 \\
\hline $\begin{array}{l}\text { Randomisation: } \\
\text { Sequence generation }\end{array}$ & $\begin{array}{l}8 a \\
8 b\end{array}$ & $\begin{array}{l}\text { Method used to generate the random allocation sequence } \\
\text { Type of randomisation; details of any restriction (such as blocking and block size) }\end{array}$ & Chapter 3.2 \\
\hline $\begin{array}{l}\text { Allocation } \\
\text { concealment } \\
\text { mechanism }\end{array}$ & 9 & $\begin{array}{l}\text { Mechanism used to implement the random allocation sequence (such as sequentially numbered } \\
\text { containers), describing any steps taken to conceal the sequence until interventions were assigned }\end{array}$ & $\begin{array}{l}\text { Chapter } 3.6 \text { and } \\
3.7\end{array}$ \\
\hline
\end{tabular}




\begin{tabular}{|c|c|c|c|}
\hline Section/Topic & $\begin{array}{l}\text { Item } \\
\text { No }\end{array}$ & Checklist item & Reported in \\
\hline Implementation & 10 & $\begin{array}{l}\text { Who generated the random allocation sequence, who enrolled participants, and who assigned } \\
\text { participants to interventions }\end{array}$ & Chapter 3.6 \\
\hline Blinding & $11 a$ & $\begin{array}{l}\text { If done, who was blinded after assignment to interventions (for example, participants, care providers, } \\
\text { those assessing outcomes) and how } \\
\text { If relevant, description of the similarity of interventions }\end{array}$ & Chapter 3.6 \\
\hline Statistical methods & $\begin{array}{l}12 a \\
12 b\end{array}$ & $\begin{array}{l}\text { Statistical methods used to compare groups for primary and secondary outcomes } \\
\text { Methods for additional analyses, such as subgroup analyses and adjusted analyses }\end{array}$ & Chapter 3.8 \\
\hline \multicolumn{4}{|c|}{ с } \\
\hline $\begin{array}{l}\text { Participant flow (a } \\
\text { diagram is strongly } \\
\text { recommended) }\end{array}$ & $13 a$ & $\begin{array}{l}\text { For each group, the numbers of participants who were randomly assigned, received intended } \\
\text { treatment, and were analysed for the primary outcome } \\
\text { For each group, losses and exclusions after randomisation,together with reasons }\end{array}$ & $\begin{array}{l}\text { Error! } \\
\text { source } \quad \text { not } \\
\text { found.1 }\end{array}$ \\
\hline Recruitment & $\begin{array}{l}14 a \\
14 b\end{array}$ & $\begin{array}{l}\text { Dates defining the periods of recruitment and follow-up } \\
\text { Why the trial ended or was stopped }\end{array}$ & $\begin{array}{l}\text { Chapter } 4.3 \text { and } \\
\text { Appendix C }\end{array}$ \\
\hline Baseline data & 15 & A table showing baseline demographic and clinical characteristics for each group & $\begin{array}{l}\text { Error! Reference } \\
\text { source not } \\
\text { found. }-4.3\end{array}$ \\
\hline Numbers analysed & 16 & $\begin{array}{l}\text { For each group, number of participants (denominator) included in each analysis and whether the } \\
\text { analysis was by original assigned groups }\end{array}$ & $\begin{array}{l}\text { Figure } 4.1 \text { and } \\
\text { Chapter } 4.2\end{array}$ \\
\hline $\begin{array}{l}\text { Outcomes } \\
\text { estimation }\end{array}$ & $17 \mathrm{a}$ & $\begin{array}{l}\text { For each primary and secondary outcome, results for each group, and the estimated effect size and } \\
\text { its precision (such as } 95 \% \text { confidence interval) } \\
\text { For binary outcomes, presentation of both absolute and relative effect sizes is recommended }\end{array}$ & $\begin{array}{l}\text { Chapter } 4.3-4.8 \\
\text { and } 4.10 \text { and } \\
\text { Appendix I-Q }\end{array}$ \\
\hline Ancillary analyses & 18 & $\begin{array}{l}\text { Results of any other analyses performed, including subgroup analyses and adjusted analyses, } \\
\text { distinguishing pre-specified from exploratory }\end{array}$ & $\begin{array}{l}\text { Chapter } 4.3-4.8 \\
\text { and } 4.10 \text { and } \\
\text { Appendix I-Q }\end{array}$ \\
\hline Harms & 19 & All important harms or unintended effects in each group & \\
\hline \multicolumn{4}{|l|}{ Discussion } \\
\hline Limitations & 20 & $\begin{array}{l}\text { Trial limitations, addressing sources of potential bias, imprecision, and, if relevant, multiplicity of } \\
\text { analyses }\end{array}$ & Chapter 5.8.2 \\
\hline Generalizability & 21 & Generalizability (external validity, applicability) of the trial findings & $\begin{array}{l}\text { Chapter } \quad 5.8 .1 \\
\text { and } 5.9\end{array}$ \\
\hline Interpretation & 22 & $\begin{array}{l}\text { Interpretation consistent with results, balancing benefits and harms, and considering other relevant } \\
\text { evidence }\end{array}$ & $\begin{array}{l}\text { Chapter } 5.9 \text { and } \\
6\end{array}$ \\
\hline
\end{tabular}




\begin{tabular}{|c|c|c|c|}
\hline Section/Topic & $\begin{array}{l}\text { Item } \\
\text { No }\end{array}$ & Checklist item & Reported in \\
\hline Registration & 23 & Registration number and name of trial registry & $\begin{array}{lr}\text { Pan } & \text { African } \\
\text { Clinical } & \text { Trial } \\
\text { Registry } & \\
\text { (PACTR20140900 } \\
\text { 0885765) }\end{array}$ \\
\hline Protocol & 24 & Where the full trial protocol can be accessed, if available & N/A \\
\hline Funding & 25 & Sources of funding and other support (such as supply of drugs), role of funders & $\begin{array}{l}\text { Acknowledgeme } \\
\text { nts }\end{array}$ \\
\hline
\end{tabular}


